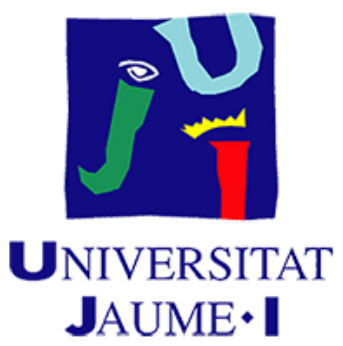

UNIVERSITAT JAUME I DE CASTELLÓ

ESCUELA SUPERIOR DE TECNOLOGÍA Y CIENCIAS EXPERIMENTALES

Departamento de Ingeniería Química

\title{
SINTERIZACIÓN DE COMPACTOS DE VIDRIO Y DE COMPOSITES VIDRIO-CIRCÓN. MECANISMO Y CINÉTICA DEL PROCESO.
}

\author{
MEMORIA \\ que para optar al grado de \\ Doctora en Ingeniería Química presenta \\ Encarnación Blasco Roca
}

Castellón 2017 



\section{JOSÉ LUIS AMORÓS ALBARO, CATEDRÁTICO DE INGENIERÍA QUÍMICA DE LA UNIVERSITAT JAUME I DE CASTELLÓN, Y ARNALDO VICENTE MORENO BERTO, PROFESOR TITULARDE INGENIERÍA QUÍMICA DE LA UNIVERSITAT JAUME I DE CASTELLÓN.}

CERTIFICAN: Que $D^{a}$ Encarnación Blasco Roca ha realizado bajo su dirección, en los laboratorios del Instituto de Tecnología Cerámica, dentro del programa de doctorado del Departamento de Ingeniería Química, el trabajo que bajo el título "SINTERIZACIÓN DE COMPACTOS DE VIDRIO Y DE COMPOSITES VIDRIO-CIRCÓN. MECANISMO Y CINÉTICA DEL PROCESO" presenta en esta Memoria y que constituye su Tesis para optar al grado de Doctora en Ingeniería Química.

Y para que conste a los efectos oportunos, firman el presente certificado en Catellón, a 25 de abril de 2017.

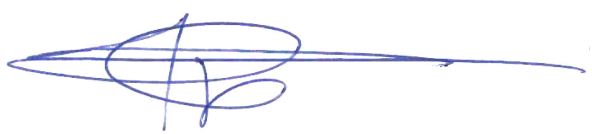

Fdo. José Luis Amorós Albaro Catedrático de Ingeniería Química

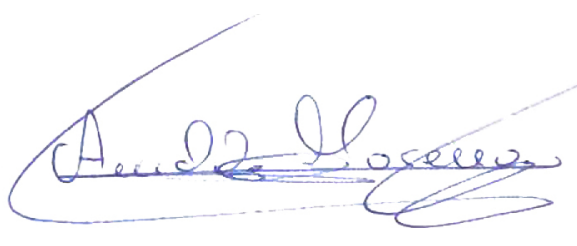

Fdo. Arnaldo V. Moreno Berto

Profesor Titular de Ingeniería Química 

A mis padres Rafael y Encarna,

luchadores y mis referentes. 



\section{Agradecimientos}

El trabajo que se presenta en esta memoria ha sido realizado, en su totalidad, en los laboratorios del Instituto de Tecnología Cerámica de la Universitat Jaume I de Castelló. En consecuencia, me gustaría manifestar mi agradecimiento al centro, por poner a mi disposión los medios humanos y materiales necesarios para su elaboración.

Mi más sincero agradecimiento a los doctores José Luis Amorós Albaro y Arnaldo Moreno Berto, por su esfuerzo y completa implicación en la dirección y desarrollo de este estudio.

Quiero hacer extensiva mi gratitud a todos mis compañeros del Instituto de Tecnología Cerámica que han contribuido en la realización de este estudio, en especial a Rosa Pérez Forner, Silvia Arrufat Arandes y Eulalia Zumaquero Silvero, por su apoyo personal y técnico, sin cuya colaboración no habría sido capaz de abordar todo el trabajo experimental de esta memoria.

Por último, quisiera dedicar especialmente este trabajo a mis hijos, Raúl y Carla, marido y familia, por su comprensión, apoyo, cariño y confianza. 



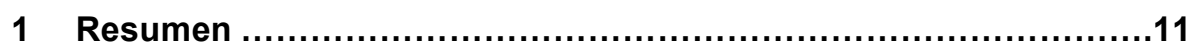

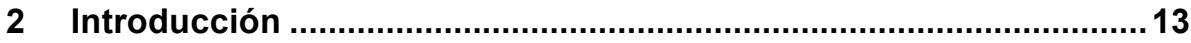

2.1 Sinterización de mezclas de partículas de vidrio y de fases

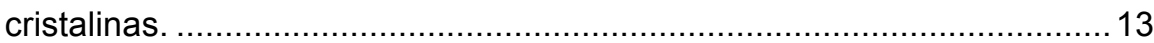

2.1.1 Sinterización viscosa. Efecto de las características físicas del polvo de vidrio (tamaño, forma, distribución y empaquetamiento de las

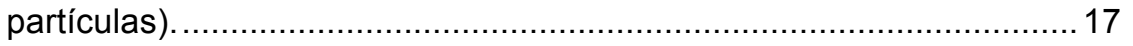

2.1.1.1 Tamaño de partícula......................................................... 17

2.1.1.2 Forma de las partículas. ............................................ 18

2.1.1.3 Distribución del tamaño de las partículas (PSD) ............... 18

2.1.1.4 Aproximación estereológica. ...........................................19

2.1.1.5 Empaquetamiento heterogéneo de partículas. Sinterización heterogénea y anisotrópica............................................................ 19

2.1.1.5.1 Distribuciones de tamaños de partícula (PSD)

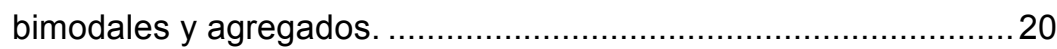

2.1.1.5.2 Empaquetamientos anisotrópicos de partículas. .........21

2.1.2 Fenómenos y procesos que retrasan la sinterización viscosa.. 22

2.1.3 Sinterización de mezclas de partículas de vidrio y pequeñas

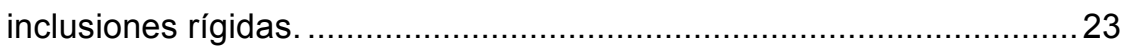

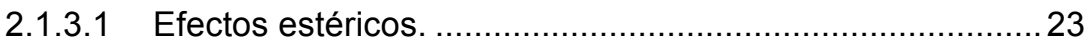

2.1.3.2 Disolución parcial de inclusiones rígidas ...........................25

2.1.3.3 Densificación y crecimiento de grano por solución-

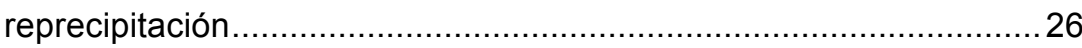

2.1.4 Modelos cinéticos de sinterización por flujo viscoso.................30

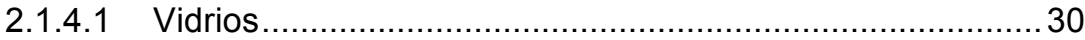

2.1.4.1.1 Modelos que conducen a cinéticas de orden 1............30 
2.1.4.1.2 Modelos teóricos que conducen a cinéticas más complejas.

2.1.4.1.3 Modelos reológicos basados en la teoría continua de la sinterización 35

2.1.4.1.3.1 Presión de sinterización, $\mathrm{P}_{\mathrm{L}}$, en función del tamaño de partícula inicial, $r_{0}$, y la densidad relativa, 36

2.1.4.1.3.2 Módulo viscoso global $\left(\mathrm{K}_{\mathrm{p}}\right)$ en función de la viscosidad del vidrio poroso, $\eta_{p}$, y de la densidad relativa, $\rho . .41$

2.1.4.1.3.3 Viscosidad de vidrios porosos, $\eta_{p}$. Efecto de la compacidad del vidrio, $\rho$ 42

2.1.4.1.3.4 Modulo viscoso adimensional, $\Psi$ 43

2.1.4.1.3.5 Modelos de sinterización basados en la teoría continua de la sinterización viscosa. Su relación con el modelo de Avrami-Erofeev 45

2.1.4.2 Composites de matriz vítrea con inclusiones rígidas de pequeño tamaño de partícula (GMC's) 50

2.1.4.2.1 Modelos de Scherer 50

2.1.4.3 Modelos de Bordia y Raj [46] .52

2.1.4.3.1 Modelo de Müller [30] .54

2.1.4.3.2 Modelo empírico. Ecuación de Avrami de orden n. ....57

2.1.4.3.3 Modelo reológico basado en la teoría continua de la sinterización. Efecto del crecimiento de grano sobre la densificación [75].

2.2 Análisis de los métodos de determinación de los parámetros cinéticos de una transformación mediante análisis térmico. Su adaptación al estudio de la sinterización 60 
2.2.2 Ventajas e inconvenientes de la experimentación a velocidad de calentamiento constante sobre la isoterma ...................................6 64

2.2.3 Métodos isoconversionales ......................................66

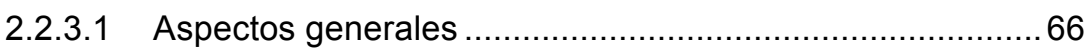

2.2.3.2 Método isoconversional diferencial ...........................6 67

2.2.3.3 Método isoconversional integral ............................69

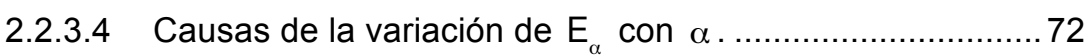

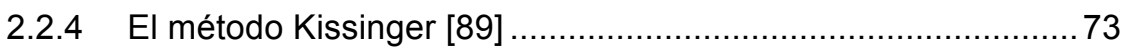

2.2.5 Métodos de ajuste al modelo ..................................... 75

2.2.6 Resumen de las recomendaciones del "Kinetics Committee of the International Confederation of Thermal Analysis and Calorimetry (ICTAC)" para el cálculo de parámetros cinéticos de datos de análisis

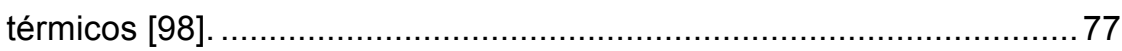

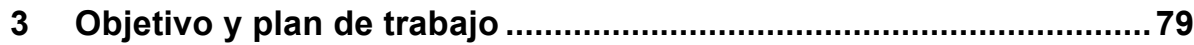

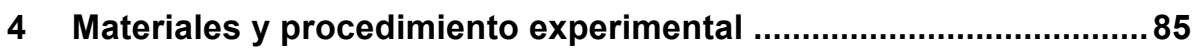

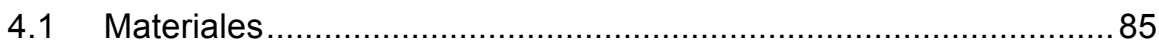

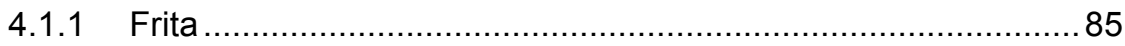

4.1.2 Micronizado y harina de circón ..................................... 87

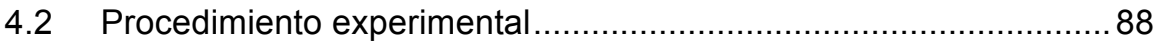

4.2.1 Molienda de la frita y determinación granulométrica del material resultante 88

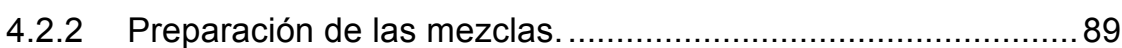

4.2.3 Conformado de probetas por colado. ............................... 89

4.2.4 Conformado de probetas por prensado. ........................... 90 
4.2.5.1 Microscopio de calefacción. ……………........................ 90

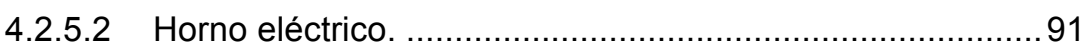

4.2.6 Caracterización textural y microestructural de las probetas. ....92

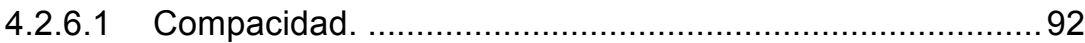

4.2.6.2 Porosidad abierta. Distribución de tamaños de poro de

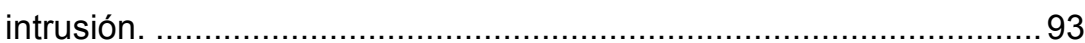

4.2.6.3 Observación por MEB. ....................................................... 94

4.2.6.3.1 Porosidad cerrada. Distribución del tamaño de los

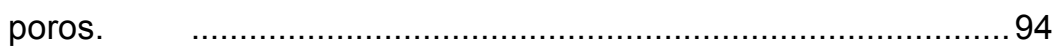

4.2.6.3.2 Distribución del tamaño de grano del circón.................94

4.2.6.3.3 Zona de difusión vidrio-circón. ......................................94

4.2.6.3.4 Cuantificación de estructuras cristalinas y determinación

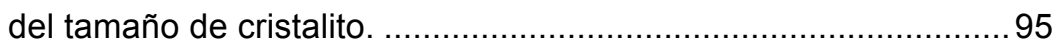

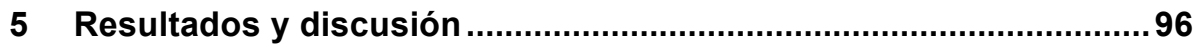

5.1 Sinterización de compactos de partículas de frita..........................96

5.1.1 Procesos físico-químicos que se desarrollan en el material durante su tratamiento térmico.......................................................96

5.1.1.1 Análisis térmico diferencial (ATD). ..................................96

5.1.1.2 Sinterización, reblandecimiento y "spreading" (HSM)........96

5.1.1.3 Curva de viscosidad-temperatura de la matriz vítrea. ........98

5.1.1.4 Evolución de las características texturales y microestructurales de la pieza con el avance del proceso de

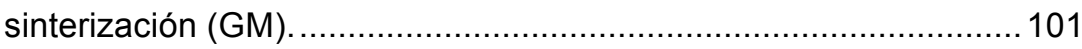

5.1.1.4.1 Análisis cualitativo (micrografías SEM).................... 101 
5.1.1.4.2 Compacidad, $\rho$, y grado de avance de la sinterización, a (calculado a partir de la compacidad). ..................................... 105

5.1.1.4.3 Razón porosidad abierta/porosidad total, $\varepsilon_{\text {abierta }} / \varepsilon_{\text {total.. }} 110$ 5.1.1.4.4 Distribución de tamaño de los poros abiertos. Diámetro medio de intrusión. Presión de sinterización. 113

5.1.1.4.5 Porosidad cerrada. Distribución de tamaño de poros.......

5.1.2 Análisis cinético de la sinterización. Determinación de los parámetros cinéticos. 121

5.1.2.1 Experimentos a velocidad de calentamiento constante (granulometría GM) 121

5.1.2.1.1 Métodos isoconversionales 122

5.1.2.1.2 Método de Kissinger 124

5.1.2.1.3 Método de ajuste al modelo 125

5.1.2.1.3.1 Selección del modelo. Determinación del triplete cinético. 127

5.1.2.2 Experimentos isotermos. 129

5.1.3 Influencia de la compacidad en crudo sobre la sinterización.. 132

5.1.3.1 Contracción superficial de la silueta, $\left(\ln A / A_{0}\right)_{s}$ 133

5.1.3.2 Anisotropía de la sinterización 136

5.1.3.3 Cinética del proceso. 139

5.2 Sinterización de composites. Efecto del contenido en circón. .......142

5.2.1 Procesos físico-químicos que se desarrollan en los materiales durante su tratamiento térmico 142

5.2.1.1 Curvas de sinterización no isotermas. 142

5.2.1.1.1 Contracción superficial de la silueta máxima, $\varepsilon_{\mathrm{A}, \max } . .145$ 
5.2.1.1.2 Influencia de la presión de compactación sobre la sinterización

5.2.1.2 Evolución de las características texturales y microestructurales de composites con el avance del proceso de sinterización. 154

5.2.1.2.1 Análisis cualitativo (micrografías SEM) 154

5.2.1.2.2 Compacidad máxima, $\rho_{\max }$, e inicial, $\rho_{0}$ 161

5.2.1.2.3 Distribución del tamaño de los poros abiertos. Diámetro medio de intrusión. Presión de sinterización. 164

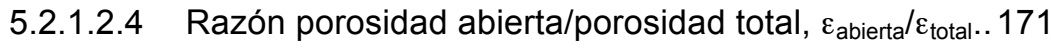

5.2.1.3 Solución-reprecipitación de granos de circón. 178

5.2.1.3.1 Efecto de la temperatura sobre la cantidad, distribución de tamaños y forma del circón. 178

5.2.1.3.2 Efecto de la temperatura sobre la zona de difusión circón-vidrio 188

5.2.1.3.3 Curvas de sinterización no isoterma. Efecto del proceso solución-reprecipitación. 189

5.2.2 Análisis cinético de la sinterización. Determinación de parámetros cinéticos. 190

5.2.2.1 Experimentos a velocidad de calentamiento constante. .. 190 5.2.2.1.1 Determinación de la energía de activación por el método de Friedman (apartado 2.2.3.2)............................... 194

5.2.2.1.2 Selección del modelo. Determinación del triplete cinético. 198

5.2.2.1.2.1 Composites con contenidos bajos y moderados de circón $(\phi \leq 0,43)$ 198 
5.2.2.1.2.2 Composite con alto contenido en circón $(\phi=0,525) \ldots$

200

5.2.2.2 Experimentos isotermos. 203

5.2.2.2.1 Selección del modelo. Determinación del triplete cinético.

203

5.2.2.2.1.1 Composites con bajos y moderados contenidos en circón $(\phi \leq 0,43)$.

203

5.2.2.2.1.2 Composites con alto contenido en circón, F2 $(\phi=0,525)$. 209

5.2.2.3 Influencia del contenido en circón sobre los parámetros cinéticos 212

5.2.2.3.1 Composites con bajos o moderados contenidos en circón $(\phi \leq 0,43)$.

5.2.2.3.2 Composite con alto contenido en circón, F2 $(\phi=0,525) \ldots$

5.3 Sinterización de composites. Efecto de la distribución de tamaños de partícula del circón y del vidrio. 223

5.3.1 Procesos físico-químicos que se desarrollan en los materiales durante su tratamiento térmico 227

5.3.1.1 Curvas de sinterización no isotermas. 227

5.3.1.1.1 Contracción superficial máxima de la silueta, $\varepsilon_{\mathrm{A}, \max . ~} 234$

5.3.1.2 Evolución de las características texturales y microestructurales de los composites con el avance del proceso de sinterización. 237

5.3.1.2.1 Análisis cualitativo (micrografías SEM) 237

5.3.1.2.2 Compacidad máxima, $\rho_{\max }$, e incial, $\rho_{0}$ 244 
5.3.2 Análisis cinético de la sinterización. Determinación de los parámetros cinéticos

5.3.2.1 Compactos de partículas de vidrio de la granulometría GF...

5.3.2.2 Composites con moderados contenidos en circón: serie 1 $(\phi=0,322)$. 254

5.3.2.2.1 Experimentos a velocidad de calentamiento constante... 254

5.3.2.2.1.1 Selección del modelo. Determinación del triplete cinético.

5.3.2.2.2 Experimentos isotermos. 258

5.3.2.2.2.1 Selección del modelo. Determinación del triplete cinético. 261

5.3.2.3 Composites con elevados contenidos en circón $(\phi=0,525) \ldots$

264

5.3.2.3.1 Experimentos a velocidad de calentamiento constante...

264

5.3.2.3.1.1 Selección del modelo. Determinación del triplete cinético.

268

5.3.2.3.2 Experimentos isotermos. 271

5.3.2.3.2.1 Selección del modelo. Determinación del triplete cinético.

5.3.2.4 Influencia de la granulometría del circón y del vidrio sobre los parámetros cinéticos. La importancia de la razón $\frac{d_{z}^{v}}{d_{g}^{v}}$ 273

5.3.2.4.1 Composites con moderados contenidos en circón $(\phi=0,322)$. 277 
5.3.2.4.2 Composites con elevados contenidos en circón $(\phi=0,525)$.

284

5.4 Sinterización no isoterma de composites asociada a reordenación de partículas por flujo viscoso. Efecto combinado del contenido en circón y de las granulometrías del vidrio y del circón 286

5.4.1 Modelo cinético desarrollado. ................................................. 286

5.4.2 Validación del modelo. 292

5.5 Interpretación de los parámetros cinéticos basándose en la teoría continua de la sinterización.

294

5.5.1 Viscosidad efectiva del composite $\left(\eta_{\mathrm{c}}\right)$ y viscosidad relativa $\left(\eta_{\mathrm{r}}\right)$. 295

5.5.2 Modelo de sinterizacion, $f(\alpha)$ 298

6 Conclusiones 302

6.1 Sinterización de compactos de partículas de frita 302

6.2 Sinterización de composites. Efecto del contenido en circón. .......306

6.3 Sinterización de composites. Efecto de la distribución de tamaños de partícula. 312

6.4 Sinterización no isoterma de composites asociada a la reordenación de partículas por flujo viscoso. Efecto combinado del contenido en circón, $\phi$, y de la razón, $d_{z}{ }^{v} / d_{g}{ }^{v}$ 316

6.5 Interpretación de los parámetros cinéticos basándose en la teoría continua de la sinterización.

7 Apéndice 319

7.1 Relación entre el grado de avance de la sinterización, $\alpha$, la densidad relativa, $\rho, y$ las dimensiones (silueta) de la probeta 
7.2 Determinación del error experimental en la determinación de las curvas de sinterización a velocidad de calentamiento constante. Selección del procedimiento.

7.3 Estimación de los valores de la diferencia entre la temperatura del horno y la temperatura media de la pieza y distribución interna de temperaturas. 327

7.3.1 Desarrollo del modelo. 327

7.3.2 Resultados. 329

7.3.2.1 Calentamiento a velocidad de calentamiento constante de probetas cilíndricas pequeñas, $D_{0}=5 \mathrm{~mm}$ y $h_{0}=3 \mathrm{~mm}$. 329

7.3.2.2 Calentamiento a $5 \mathrm{~K} / \mathrm{min}$ de probetas cilíndricas grandes, $D_{0}=20 \mathrm{~mm}$ y $h_{0}=20 \mathrm{~mm}$ en horno eléctrico. 332

7.3.2.3 Tratamientos isotermos de probetas cilíndricas grandes $\left(D_{0}=20 \mathrm{~mm}\right.$ y $\left.h_{0}=20 \mathrm{~mm}\right)$ en horno eléctrico. 334

8 Nomenclatura 337

9 Bibliografía 347 


\section{Resumen}

En esta memoria se exponen los resultados obtenidos al estudiar el proceso de sinterización de compactos de vidrio y de composites de vidrio-circón. Partiendo de un vidrio de borosilicato estándar, que no desvitrifica, y de un circón industrial se han preparado polvos de cada componente de distintas granulometrías y mezclas con diferentes fracciones volumétricas de circón y con distintas granulometrías. Con estos materiales se han conformado, por colado y prensado, probetas de vidrio y de los diferentes composites que se han tratado térmicamente, en un horno de laboratorio y en un microscopio de calefacción, mediante experimentos a velocidad de calentamiento constante (con velocidades comprendidas entre 0,5 y $60 \mathrm{~K} / \mathrm{min}$ ) e isotermos.

Se han caracterizado probetas cocidas mediante porosimetría de mercurio, microscopia electrónica de barrido (MEB) (con EDS y análisis de imagen) y difracción de rayos $\mathrm{X}$, con vistas a determinar la evolución de las características texturales y microestructurales de los diferentes materiales con el avance de la sinterización y para estudiar el proceso de soluciónreprecipitación del circón.

Se comprueba que, en etapas iniciales e intermedias de la sinterización, conforme la porosidad abierta disminuye, el tamaño medio de los poros, generalmente irregulares, crece. Este fenómeno, junto con la formación de porosidad cerrada y la porosidad máxima alcanzable en el composite, dependen del contenido en circón y de la distribución granulométrica de los componentes. El proceso de solución-reprecipitación del circón comienza a ser operativo a temperaturas elevadas y/o tiempos de tratamiento largos, por lo que solo contribuye a la densificación de composites de alto contenido en circón, que son los que requieren estas condiciones de operación para alcanzar la máxima compacidad.

Se ha comprobado que la sinterización de composites con moderados contenidos en circón se desarrolla únicamente mediante el mecanismo de reordenación de partículas por flujo viscoso. En cambio, para altos contenidos en circón se requiere, además, la intervención del proceso de 
solución-reprecipitación del circón. La cinética del primer mecanismo queda perfectamente descrita mediante el modelo de Avrami- Erofeev. Los valores del índice de potencia de Avrami, "n", y el factor preexponencial, A, que se ha comprobado que son independientes del tipo de tratamiento térmico, se han relacionado con el contenido en circón y con los tamaños medios volumétricos del circón y del vidrio. Por el contrario, el efecto de la temperatura sobre la velocidad del proceso se ha comprobado que es el mismo que el debido a la viscosidad del vidrio, y, por consiguiente, independiente de todas las variables de operación ensayadas. Por último, se ha desarrollado y validado un modelo cinético que describe muy bien el efecto que ejercen todas las variables de operación estudiadas (velocidad de calentamiento, distribución de tamaño de partículas de los componentes, contenido en circón) sobre el grado de avance de la sinterización no isoterma de composites asociada a la reordenación de partículas por flujo viscoso. 


\section{Introducción}

\subsection{Sinterización de mezclas de partículas de vidrio y de fases cristalinas.}

La presencia de fases líquidas viscosas de alto contenido en sílice es crucial para algunas rutas de sinterización que parten de mezclas de partículas de vidrio y cristales. Estas tecnologías, basadas en el procesado de sistemas particulados, permiten bajas temperaturas de fabricación, la obtención de formas complejas y de recubrimientos [1] (Figura 2.1).

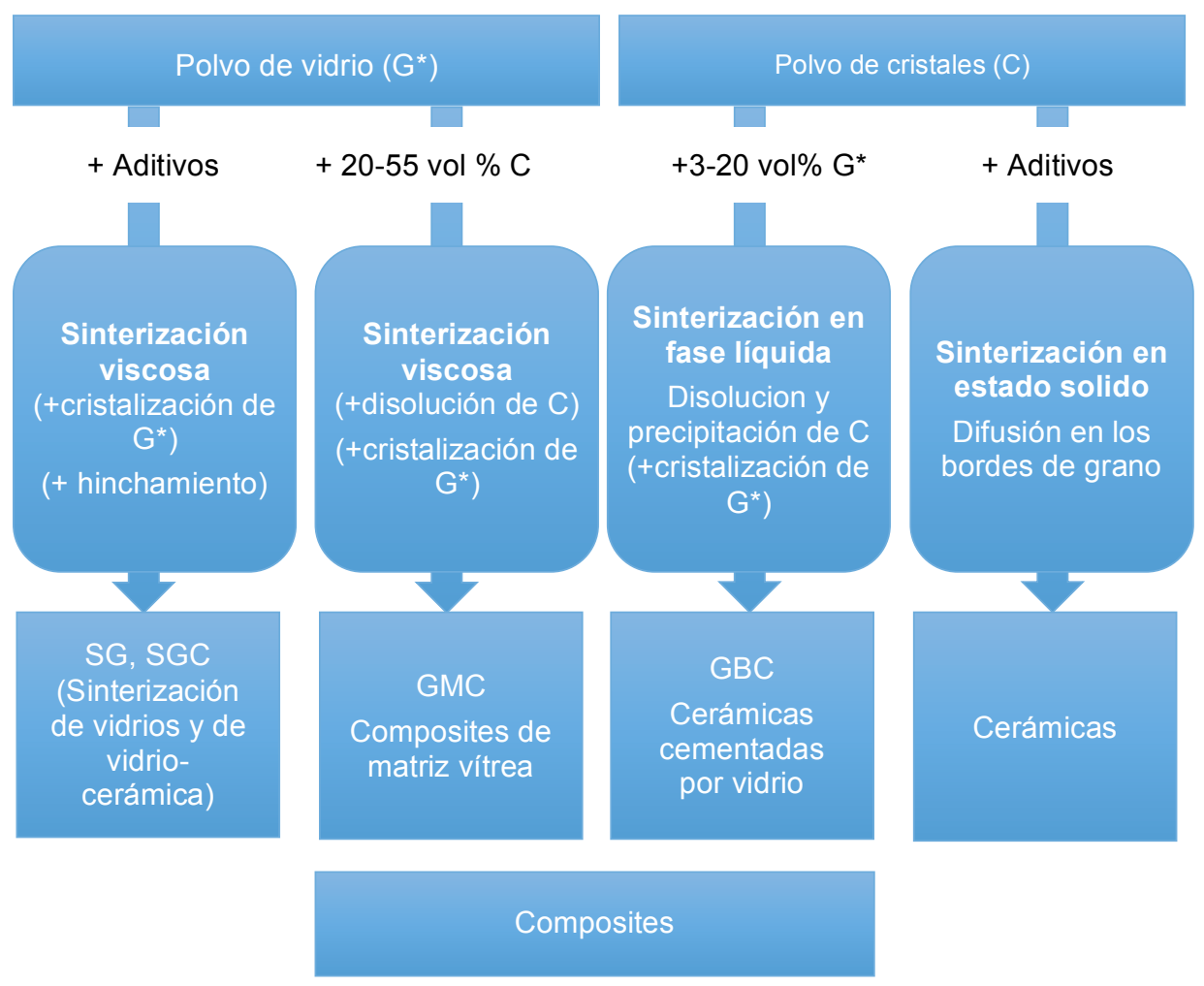

Figura 2.1. Rutas para el procesado de polvos clasificadas de acuerdo con la mezcla inicial de fase cristalina $(C)$ y vidrio $\left(G^{*}\right)$. La fase cristalina inicial y la fracción volumétrica de vidrio de GMC y GBC son típicos para materiales cerámicos cocidos a baja temperatura.

Las piezas de vidrios sinterizados (SG) y vitrocerámicos sinterizados (SGC) se obtienen a partir de partículas de vidrio (frita), obtenidas por molienda, que se conforman utilizando las tecnologías habituales (prensado, extrusión, 
colado, colado en banda etc.). Asimismo, los recubrimientos de estas mezclas también se obtienen por las técnicas habituales de preparación. La densificación de estos materiales se desarrolla por sinterización viscosa (flujo viscoso) impulsada por la energía superficial del fundido y limitada por su viscosidad. Esta última propiedad controla todos los fenómenos importantes de la densificación, tales como: formación de cuellos, redistribución de partículas de vidrio y contracción de poro. En el caso de productos SGC densos, la sinterización debe completarse antes de que comience la cristalización. En efecto, si los tamaños de partícula del vidrio son pequeños y su distribución estrecha, puede obtenerse una distribución quasi-homogénea de cristales, incluso sin el uso de agentes nucleantes, debido a la fuerte tendencia a la nucleación de la superficie de las partículas de vidrio. Las rutas de procesado de polvo SG y SGC también se utilizan para la obtención de recubrimientos vitrocerámicos. Por otra parte, mediante la ruta SG, pueden obtenerse vidrios de sílice y derivados de sol-gel a temperaturas mucho más bajas que las que se necesitarían a partir de fundidos.

En el caso de GMCs ("glass matrix composites"), su elevada fracción volumétrica de vidrio, $\mathrm{G}^{*}$, (entre $20 \% \leq \mathrm{G}^{*} \leq 55 \%$ en volumen) facilita que la matriz vítrea rodee completamente las inclusiones cristalinas rígidas como fibras o láminas u otras formas de partículas dispersas. Debido a que la sinterización es impulsada por las energías interfaciales de los poros y de los cristales dispersos en el fundido viscoso y que este proceso se desarrolla mediante un transporte de materia por flujo viscoso, a este mecanismo de sinterización también se le denomina "sinterización viscosa", incluso aún cuando no se produzca un buen mojado de las partículas rígidas por el vidrio [2]. La Figura 2.2 ilustra esquemáticamente los fenómenos clave de la sinterización viscosa de GMCs. Además de la sinterización, los fenómenos de disolución parcial de fases cristalinas, cristalización y crecimiento en el borde de grano pueden desarrollarse simultáneamente, influenciando decisivamente la cinética de la sinterización, la evolución microestructural y las propiedades del material final. 

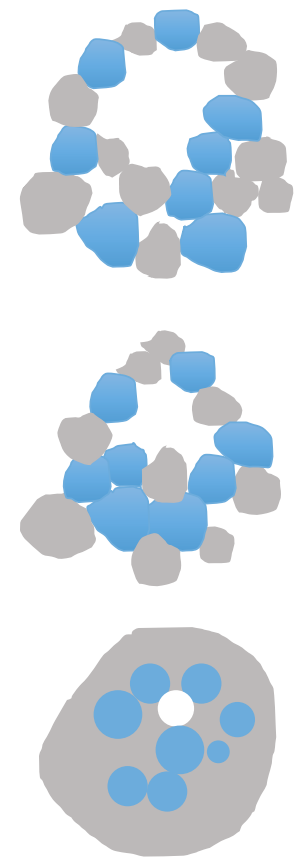

Contracción de los poros y reordenación de partículas hasta alcanzar la máxima densificación,

Figura 2.2. Ilustración esquemática de la sinterización viscosa de GMC. Gris claro: vidrio fundido; azul: fase cristalina; blanco: poros. Esta representación esquemática no tiene en cuenta la disolución parcial y la cristalización, la cual puedo ocurrir o no.

Los GMCs se caracterizan por su fácil densificación, bajo coste y altas prestaciones [3][4]. Otras ventajas derivan del hecho de la gran variedad de posibles combinaciones de partículas de vidrio y de "filler", en lo referente a la naturaleza físico-química, fracción volumétrica, tamaños de partícula, lo que posibilita una variación casi continua de las propiedades del producto.

En el caso de partículas cristalinas cementadas por vidrios, GBCs (glass bonded ceramics), se añaden fracciones volumétricas moderadas de vidrio, $\mathrm{G}^{*}$, (generalmente $6 \% \leq \mathrm{G}^{*} \leq 20 \mathrm{vol}$. \%), de baja temperatura de fusión, a partículas cristalinas, para conseguir propiedades superiores en el producto a temperaturas de densificación más bajas. El mecanismo por el que densifican se denomina sinterización con presencia de fase líquida [5][6]. Este mecanismo combina redistribución del líquido y de las fases cristalinas y los procesos de disolución-precipitación en el líquido de baja viscosidad. El 
líquido, una vez formado durante el calentamiento, moja las partículas cristalinas y penetra en la estructura de poros, rodeando los granos, por una combinación de reacción y capilaridad [6]. Durante la primera etapa de la sinterizacion, la densificación procede por capilaridad, fragmentación de agregados de partículas y reordenación por flujo viscoso (Figura 2.3). Durante la segunda etapa, de disolución y reprecipitación, los contactos se aplanan, la forma de los granos se acomoda en el espacio disponible y se agrandan (coarsening) y se desarrolla una segunda reordenación de partículas. Durante el estado final, la red del esqueleto sólido densifica mediante crecimiento del cuello intergranular y mediante el llenado de poros por redistribución del líquido.

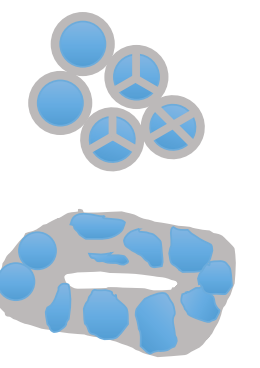

Mojado y redistribución

del líquido.

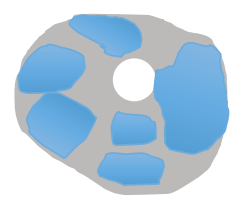

Fragmentación de

partículas y reordenación.

Disolución y reprecipitación, contacto y reordenación de partículas secundario.

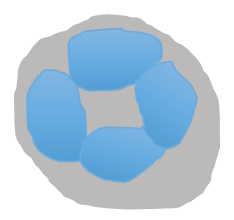

Contacto y crecimiento de grano.

Figura 2.3. Ilustración esquemática de la sinterización en fase líquida de GBC. Gris claro: vidrio fundido; azul: fase cristalina; blanco: poros. Esta representación esquemática no tiene en cuenta la disolución parcial y la cristalización, la cual puedo ocurrir o no. 
2.1.1 Sinterización viscosa. Efecto de las características físicas del polvo de vidrio (tamaño, forma, distribución y empaquetamiento de las partículas).

En esta sección se trata de ilustrar en que medida el procesado del polvo de vidrio anterior a la cocción influye sobre la sinterización viscosa.

\subsubsection{Tamaño de partícula.}

De los parámetros del procesado en polvo, el tamaño de partícula es quizás el más importante, puesto que el exceso de energía libre del vidrio en forma pulverulenta es la fuerza impulsora de la sinterización, y ésta es tanto mayor cuanto menor es el tamaño de partícula del vidrio.

De acuerdo con el modelo de Frenkel [7] la velocidad inicial de sinterización, expresada como velocidad de contracción lineal, $\frac{\mathrm{ds}}{\mathrm{dt}}$, de un compacto de vidrio, formado por partículas esféricas de radio, $r_{0}$, es directamente proporcional a su energía superficial inicial por unidad de volumen, $\frac{3 \gamma}{r_{0}}$, e inversamente proporcional a la viscosidad del vidrio, $\eta$, de acuerdo con la ecuación:

$\frac{\mathrm{ds}}{\mathrm{dt}}=\frac{3 \gamma}{8 \eta r_{0}}$ ec. 2.1

En la etapa final de la sinterización, la velocidad de sinterización también es inversamente proporcional al radio de la partícula (directamente proporcional a su energía superficial inicial por unidad de volumen), tal como predice el modelo de Mackenzie and Shuttleworth [8]. Según estos autores, la velocidad de sinterización de un vidrio, cuando todos los poros son esféricos y del mismo tamaño, viene dada por: 
$\frac{d \rho}{d t}=\left(\frac{3 \gamma}{r_{0}}\right) \cdot \frac{O^{\frac{1}{3}}}{2 \eta} \cdot(1-\rho)^{\frac{2}{3}} \cdot \rho^{\frac{1}{3}}$

siendo $\rho$ la compacidad de la probeta o densidad relativa de la misma y $O$ el número de poros por partícula.

Otros modelos, como se verá posteriormente (apartado 2.1.4.1), también predicen un efecto similar.

\subsubsection{Forma de las partículas.}

La forma de las partículas de vidrio obtenidas por molienda es irregular, lo que, según la bibliografía [9][10][11][12], aumenta la velocidad de sinterización respecto a las partículas esféricas. Distintos investigadores han cuantificado el efecto de dicha característica sobre la etapa inicial de sinterización, introduciendo en la ec. 2.1 un parámetro ajustable. Dicho parámetro, igual a 1 en el caso de partículas esféricas, aumenta conforme lo hace la anisotropía de las partículas. Según la bibliografía varía entre 1,3 [9] y $3,5[13]$.

\subsubsection{Distribución del tamaño de las partículas (PSD).}

Para PSD relativamente estrechas y monomodales, el empleo de un tamaño de partícula promedio (superficial o volumétrico) suele ser suficiente para describir el efecto de la PSD sobre la sinterización. Otros modelos [14], más sofisticados y laboriosos, consideran que la sinterización de una pieza es la suma de las contribuciones debidas a cada fracción de tamaños en la que puede dividirse la distribución, de acuerdo con la expresión:

$\mathbf{s}=\sum_{\mathrm{j}} \psi_{\mathrm{j}}\left(\mathrm{r}_{0 \mathrm{j}}\right) \cdot \mathrm{s}_{\mathrm{j}}$

siendo $\psi_{\mathrm{j}}\left(\mathrm{r}_{0 \mathrm{j}}\right)$ la frecuencia relativa, en peso, de partículas de tamaño $r_{0 \mathrm{j}} \mathrm{y}$ $\mathrm{s}_{\mathrm{j}}$ la contracción debida a la fracción de partículas j. El valor de $\mathrm{s}_{\mathrm{j}}$ se calcula a partir de la integración de la ec. 2.1 y la ec. 2.2. Este modelo, que 
desprecia cualquier interacción entre partículas de tamaño diferente, no es apropiado para PSD anchas o polimodales. En efecto, en estas últimas, las partículas pequeñas forman cuellos sobre las partículas más grandes a las que rodean, pudiendo contribuir o no a la contracción de la probeta.

\subsubsection{Aproximación estereológica.}

Según Exner y Giess [15], la velocidad de sinterización es inversamente proporcional a un parámetro $\lambda_{0}$, que representaría un radio efectivo de partícula y que combina el efecto del tamaño, forma y distribución de tamaños de las partículas. $\lambda_{0}$ se define como:

$\lambda_{0}=\frac{4 V_{s}}{S_{V}}$

siendo $V_{s}$ y $S_{v}$ el volumen de sólido y la superficie interfacial del compacto.

En el caso de partículas esféricas $\lambda_{0}=(4 / 3) \cdot r_{0}$. Para partículas irregulares $\lambda_{0}<(4 / 3) \cdot r_{0}$

2.1.1.5 Empaquetamiento heterogéneo de partículas. Sinterización heterogénea y anisotrópica.

El procesado anterior a la cocción puede generar microestructuras y propiedades heterogéneas $\mathrm{y} / \mathrm{o}$ anisotrópicas en crudo, que suelen exagerarse, todavía más, durante la sinterización. Así, las técnicas de conformado de piezas como prensado uniaxial, extrusión, colado en banda, etc., conducen a una orientación preferente de la interfase sólido-poro y/o a una distribución heterogénea de la densidad en crudo. La sinterización también puede estar condicionada o restringida, en alguna dirección, por la presencia de inclusiones rígidas, cargas externas o adhesión a un sustrato, como es el caso de los recubrimientos.

Los fenómenos antes descritos pueden provocar que la sinterización no sea isotrópica, la deformación de la pieza, la aparición de laminaciones o la 
formación de grietas. Aun cuando estas heterogeneidades microestructurales sean pequeñas respecto al tamaño de la muestra, éstas pueden generar tensiones locales, las cuales acabarán convirtiéndose en defectos o grietas durante la sinterización o simplemente retrasarán la densificación [16].

2.1.1.5.1 Distribuciones de tamaños de partícula (PSD) bimodales y agregados.

A menudo se obtienen PSD bimodales de partículas al mezclar polvos con tamaños medios distintos, con vistas a conseguir compacidades en crudo más altas que las que se obtendrían con una PSD monomodal estrecha. Los efectos de PSD bimodales sobre la sinterización son complejos. Por una parte, la sinterización más pronta y rápida de las partículas más pequeñas causa su agregación o la formación de grietas tal y como puede apreciarse en la Figura 2.4. Por otra parte, las partículas más grandes, al formar un esqueleto rígido debido a su sinterización más lenta, pueden constreñir la densificación de las partículas pequeñas intersticiales incrementando el tamaño de poro, retrasando el final de la densificación o generando porosidad residual.
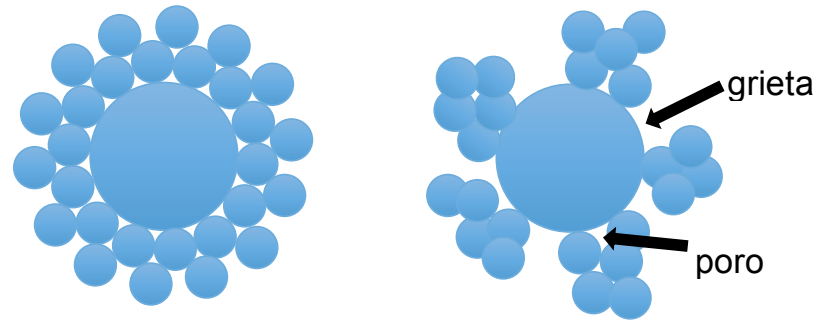

Figura 2.4. Fuerza local y formación de agregados durante la sinterización de polvos bimodales.

Análogamente, los agregados de partículas, generalmente formados por aglomeración de partículas submicroscópicas en crudo o al inicio de la cocción, durante las etapas iniciales e intermedias de la sinterización, conducen a la formación de grandes poros que, como en el caso anterior, retardan considerablemente el final de la densificación o provocan porosidad residual. 
Se ha constatado que, excepto para distribuciones de tamaño de poro muy anchas, el efecto de la PSD en la sinterización es pequeño. Por el contrario, en el caso de distribuciones de tamaño de poro bimodales, originadas por la presencia de agregados y/o grandes partículas, su efecto sobre las etapas intermedias o finales de sinterización es sustancial [17].

2.1.1.5.2 Empaquetamientos anisotrópicos de partículas.

En la densificación de vidrios frecuentemente se ha observado la sinterización anisotrópica, aun cuando sobre la probeta no actúe ninguna carga [18][19][20][21][22][23][24]. Este fenómeno, aunque suele presentarse en muchos casos de relevancia práctica, a menudo se ha ignorado, debido a que el estudio de la sinterización se ha efectuado con la dilatometría convencional [25], y ésta no es una técnica adecuada para la cuantificación de este fenómeno.

La microscopía de calentamiento (HSM) [26] y otros métodos de dilatometría óptica [27][28] son los que generalmente se emplean para el estudio de este fenómeno. Para cuantificar la anisotropía que se desarrolla durante la sinterización de compactos cilíndricos se utiliza la razón: contracción axial/contracción radial, $\mathrm{k}_{\mathrm{A}}$.

De forma general, durante la cocción de compactos de partículas irregulares de vidrios, obtenidos por prensado uniaxial, se produce una anisotropía pronunciada, especialmente en las primeras etapas de la densificación. Exner y Giess [18] observan un aumento de $k_{A}$, de 0,4 a 0,7 , conforme avanza la densificación de la pieza (la anisotropía se reduce). Dicho comportamiento se atribuye a una orientación preferencial de poros y partículas perpendicular a la dirección del prensado. Esta interfase sólidoporo, altamente orientada, ha sido también observada en el prensado de partículas esféricas, lo que indica que la forma y el tamaño de las partículas parecen no ser determinantes en la aparición de este fenómeno [18]. E efecto dominante del conformado sobre la anisotropía en la orientación también ha sido demostrado por Boccaccini y Conradt [23], quienes 
observaron un comportamiento isotrópico en piezas obtenidas mediante prensado isostático, aun cuando el polvo contenía partículas laminares.

En lo que respecta a los restantes métodos de moldeo (en plástico o en suspensión), conviene señalar, en primer lugar, que las partículas anisotrópicas son más susceptibles a orientarse cuando están dispersas en fluidos y se ven forzadas a fluir. En efecto, se ha comprobado que la contracción en la dirección del colado es siempre más pequeña que en la perpendicular y que un reducido tamaño de las partículas agudiza el fenómeno [29].

\subsubsection{Fenómenos y procesos que retrasan la sinterización viscosa.}

La sinterización viscosa (o por flujo viscoso) de compactos de partículas de vidrio puede ralentizarse o incluso anularse por la cristalización concurrente de la fase vítrea o por la presencia de inclusiones rígidas (partículas cristalinas). Algunos de los fenómenos que mayoritariamente influyen en la velocidad de sinterización, retrasando la densificación del compacto o incluso llegando a paralizar o impedir el proceso son los siguientes (Figura 2.5):

a) La disminución de la superficie vítrea de las partículas debido a su cristalización superficial (Figura 2.5a).

b) La cristalización parcial, másica o volumétrica, de las partículas de vidrio (Figura 2.5b).

c) La presencia de pequeñas inclusiones rígidas, de tamaño similar al de la matriz vítrea (Figura 2.5c).

d) La presencia de grandes inclusiones rígidas de tamaño superior al de la matriz vítrea (Figura 2.5d). 
a)

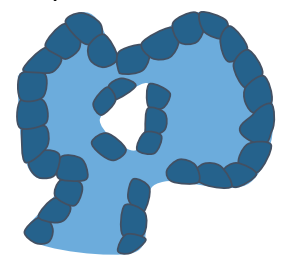

b)

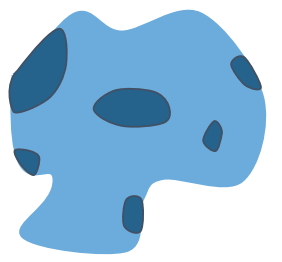

c)

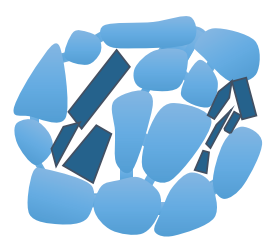

d)

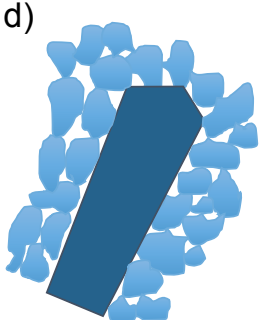

Figura 2.5. Fenómenos de retardo de la sinterización viscosa. a) Disminución de la superficie vitrea de las partículas debido a su cristalización superficial. b) Incremento de la viscosidad efectiva por la cristalización parcial, másica o volumétrica, de las partículas de vidrio. c) Incremento de la viscosidad efectiva por la presencia de pequeñas inclusiones rígidas. d) Inclusiones rígidas grandes.

\subsubsection{Sinterización de mezclas de partículas de vidrio y pequeñas inclusiones rígidas.}

Muchas productos, tales como esmaltes, vidriados, materiales de sellado y soldadura, LTCC (Low Temperature Cofired Ceramics) emplean inclusiones rígidas dispersas en una matriz vítrea para obtener las propiedades deseadas (expansión térmica, color, viscosidad a alta temperatura, dureza, propiedades dieléctricas, etc.). Para conseguir una buena homogeneidad y una densificación prácticamente completa, el tamaño medio de las partículas de ambos materiales, vidrio e inclusión, debe ser pequeño.

\subsubsection{Efectos estéricos.}

Los efectos estéricos de pequeñas inclusiones rígidas han sido recientemente estudiados en composites de vidrio de borosilicato de bario/alúmina, empleando tamaños de partícula medio, tanto para el vidrio como para la alúmina de $3 \mu \mathrm{m}$, y comprobando que la disolución de alúmina y la cristalización de fases podía considerarse despreciable [30][31][32]. En estos trabajos, efectuados en condiciones no isotermas, se comprobó que para fracciones volumétricas de cristales, $\phi$, pequeñas, $\phi<0,15$, el efecto de la presencia de éstos sobre la velocidad de sinterización era muy pequeño, de acuerdo con los resultados obtenidos por otros autores [33][34]. En 
cambio, un incremento de $\phi$ por encima del valor antes señalado conduce a lo siguiente:

a) Una marcada reducción de la velocidad de sinterización, lo que se traduce en un aumento de la temperatura final de sinterización.

b) Una disminución de la contracción máxima de cocción. Esta última se comprobó que era proporcional a $\phi^{3}$ y se atribuyó a los poros residuales que ya se forman en crudo en el interior de aglomerados constituidos por, al menos, tres partículas cristalinas, en los que no ha penetrado el fundido. Dicha hipótesis se apoya en que, para un mezclado ideal de partículas, la probabilidad de formación de tales tripletes (clusters o agregados de tres partículas cristalinas) es $\phi^{3}$.

En lo que respecta a la influencia de las inclusiones rígidas sobre la velocidad de sinterización, dicho efecto se explica y cuantifica utilizando el concepto de viscosidad efectiva del composite que, según algunos autores, aumenta conforme lo hace $\phi$ y el de avance de la sinterización [30][31][32][35]. El efecto del progreso de la sinterización sobre esta viscosidad se debe al aumento de la fracción de los cristales que son mojados por el fundido con el avance del proceso. El mojado de las inclusiones rígidas por el vidrio fundido y la forma de estas determinan el tamaño y la forma de las cavidades entre cristales y, en consecuencia, aquellas en las que puede penetrar el fundido, afectando, por tanto, a la porosidad residual del composite.

En este sentido, Pascual et al. [35], al estudiar la sinterización de composites de fibras de circona/borosilicato de sodio, comprobó que las fibras se mojaban mal y que la porosidad residual estaba prácticamente asociada a las fibras. Análogamente, Boccaccini et al. [23], empleando inclusiones en forma de láminas, observaron una reducción notable de la contracción (hasta un $10 \%$ para $\phi=0,10$ ). Estos autores atribuyen este efecto al límite de percolación de las inclusiones rígidas, puesto que este límite depende no solo de $\phi$ sino también de la forma de las partículas e, incluso, de la de sus agregados. 
Por otra parte, la presencia de inclusiones rígidas en un compacto de polvo de vidrio provoca que la velocidad de sinterización no sea homogénea, lo que genera tensiones [36]. Estas tensiones, que son tanto mayores cuanto mayor es el tamaño de la inclusión, no sólo ralentizan la velocidad de sinterización de la pieza sino que pueden llegar a generar grietas [37][38].

\subsubsection{Disolución parcial de inclusiones rígidas}

Con la disminución del tamaño de partícula y el aumento de la temperatura de cocción, que generalmente conlleva el incremento de la fracción volumétrica de inclusiones rígidas, $\phi$, la disolución parcial de cristales puede llegar a ser significativa, alterando la fase vítrea, tanto en su contenido como en su composición, lo que puede modificar de forma considerable la cinética de la sinterización e incluso favorecer la cristalización de nuevas fases. En efecto, la disolución parcial de cristales tales como corindón, circón, circona o cuarzo enriquece la composición del vidrio en estos componentes, lo que incrementa notablemente la viscosidad del fundido. Esto se traduce a su vez en una reducción de la velocidad de sinterización, aún cuando el contenido en fase líquida aumente. Sin embargo, este fenómeno puede tener efectos positivos, al evitar, en algunos casos, la aparición de fenómenos tales como: separación de fases, cristalizaciones indeseadas, o hinchamiento de la matriz vítrea. 


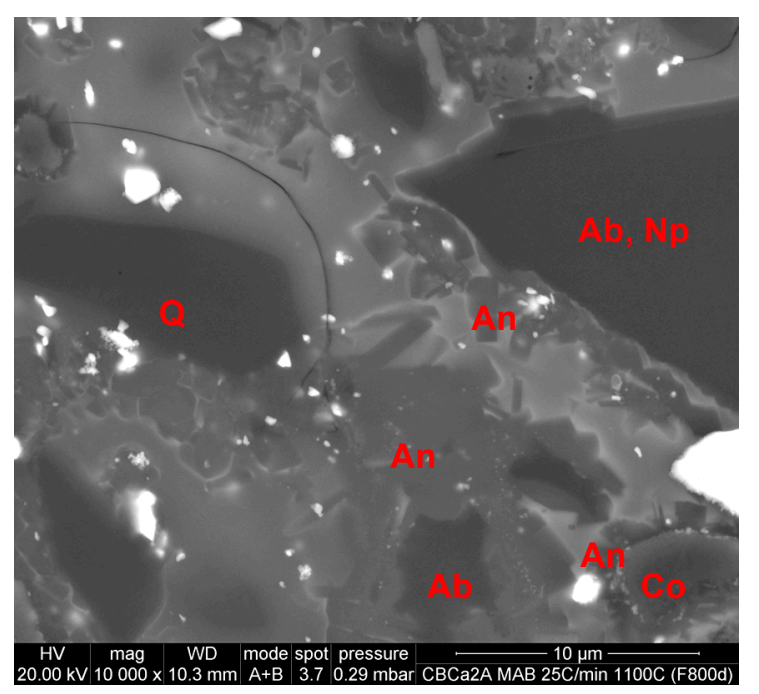

Figura 2.6. Micrografía SEM de un vidriado cocido a $1100^{\circ} \mathrm{C}$. Quarzo (Q), Anortita (An), Corindón (Co), Albita (Ab), Nefelina (Np).

Especialmente a temperaturas elevadas, el mojado de la inclusión por el vidrio es elevado, incluso completo, y la disolución de los cristales y difusión de los iones resultantes en la matriz vítrea son también altos, lo que provoca un cambio sustancial de la composición de ésta última, facilitando con ello la precipitación de cristales secundarios. Dicho fenómeno se ha comprobado en composites de borosilicato de calcio/alúmina, en los que se forma anortita, hasta un 60\%, coincidiendo con la disolución de corindón, a temperaturas comprendidas entre 900 y $1050^{\circ} \mathrm{C}$ [39]. También son frecuentes los procesos de disolución de fases cristalinas en el fundido y la posterior cristalización de otras durante la cocción de vidriados cerámicos. En efecto, en la Figura 2.6 se aprecia la formación de cristales de anortita, por cristalización del fundido alrededor de las partículas de corindón, albita y nefelina, parcialmente disueltas durante la cocción del vidriado a $1100^{\circ} \mathrm{C}$ [66].

\subsubsection{Densificación y crecimiento de grano por solución-reprecipitación}

i) Cuando la fracción volumétrica de vidrio es insuficiente para llenar todos los poros, que se formaron en un hipotético empaquetamiento compacto de 
partículas rígidas, la densificación completa del composite solo puede alcanzarse por solución-reprecipitación [40] de las partículas rígidas.

Este proceso de transporte de materia comprende tres etapas:

i) El sólido se disuelve en el líquido, preferiblemente desde las superficies de más alta energía tales como: asperidades, puntos convexos, granos pequeños o en áreas bajo compresión.

ii) El sólido disuelto se difunde en el seno del líquido.

iii) El sólido disuelto precipita en las superficies de menor energía tales como: regiones cóncavas, grandes granos o áreas bajo tensión, resultando un crecimiento de grano y un cambio de forma

Los tres mecanismos que intervienen en esta última etapa son los siguientes: aplanamiento del contacto, disolución de pequeños granos y enlace en estado sólido [41].

En el primer mecanismo, aplanamiento del contacto, la tensión capilar comprime los puntos de contacto entre granos lo que provoca la disolución preferencial del sólido con reprecipitación en áreas alejadas del mismo. Sin embargo, este mecanismo no provoca un crecimiento de grano ni disminución del número de granos.

El segundo mecanismo implica la disolución de pequeños granos con reprecipitación sobre los grandes. Los granos pequeños desaparecen mientras los grandes crecen y modifican la forma para conseguir una mejora de la compacidad. El crecimiento de grano, únicamente, no conduce a la densificación. La difusión suele ser la etapa controlante del proceso.

El tercer mecanismo implica el crecimiento del contacto entre granos por difusión del soluto a través de la frontera de grano. El agrandamiento de la zona de contacto cambia la forma del grano, incrementando la contracción. Este mecanismo no involucra crecimiento de grano.

Estos tres mecanismo difieren en la fuente del soluto y en el camino que sigue su transporte, pero conjuntamente explican los fenómenos de 
acomodación de la forma del grano y crecimiento de grano, que de forma concurrente se desarrollan en la densificación por este proceso (sinterización-reprecipitación). En efecto, se ha comprobado que la densificación correlaciona con el crecimiento de grano, observándose en algunos sistemas que la disolución de pequeños granos es paralela a la reducción de la porosidad [42]. Análogamente, también se ha comprobado que la esferoidización del grano ocurre por disolución de los pequeños granos y reprecipitación sobre los grandes [43].

El hecho de que los tres mecanismos antes citados, junto con el crecimiento de grano por coalescencia, se den a menudo de forma simultánea, dificulta, e incluso impide la formulación de modelos teóricos que describan la sinterización por solución-reprecipitación en estados avanzados del proceso, recurriendo, generalmente, a modelos reológicos y a otros métodos semiempíricos.

En cualquier caso, se ha comprobado que los siguientes parámetros clave controlan la velocidad de sinterización:

- Solubilidad, S, y difusividad, $D_{\mathrm{S}}$, cantidad de soluto en el líquido y mojado del sólido por el líquido. Características altamente dependientes de la temperatura.

- Tamaños de partículas del vidrio y de grano de la inclusión rígida que controlan la curvatura y el área de la superficie y la distania de difusión.

ii) La marcada influencia que ejerce el tamaño de grano sobre la sinterización implica la necesidad de considerar el proceso de crecimiento de grano a la hora de comprender y modelizar la densificación del composite, incluso empleando modelos semiempíricos (MSC).

El crecimiento de grano provoca que el volumen de grano medio cambie linealmente con el tiempo de sinterización. Para un grano de tamaño, G, la velocidad de cambio $\mathrm{dG} / \mathrm{dt}$, depende de la superficie del grano $\left(\alpha \mathrm{G}^{2}\right)$ y de 
la diferencia entre su tamaño y el tamaño medio $\left(\Delta G / G_{M}\right)$, siendo $\left(\Delta \mathrm{G}=\mathrm{G}-\mathrm{G}_{\mathrm{M}}\right)$, mediante la ecuación:

$\frac{d G}{d t}=\frac{p \cdot K_{S L}}{G^{2}} \cdot \frac{\Delta G}{G_{M}}$

Donde $\mathrm{p}$ es una constante geométrica y $\mathrm{K}_{\mathrm{SL}}$ la constante de velocidad que depende, a su vez, de las propiedades termodinámicas y de transporte del sistema [40]. Para el caso idealizado de un composite homogéneo de partículas esféricas y exento de porosidad, se tiene:

$K_{S L}=\frac{g \cdot D_{S} \cdot S \cdot \Omega \cdot \gamma_{S L}}{R T(1-\rho)^{\frac{2}{3}}}$

Donde la constante geométrica $g \approx 7, D_{S}$ es la difusividad del soluto en el líquido, $\mathrm{S}$ es la solubilidad del sólido en el líquido, $\Omega$ es el volumen molar del sólido y $\gamma_{S L}$ es la energía superficial sólido-líquido. A efectos prácticos se asume que la dependencia de $\mathrm{K}_{\mathrm{SL}}$ con la temperatura es del tipo Arrhenius.

De acuerdo con la ec. 2.5, el cambio de tamaño de cada grano individual depende de su relación con el tamaño medio de los granos, $G_{M}$. No obstante, se ha comprobado que en sistemas reales en los que la microestructura no es completamente homogénea, debe aplicarse dicha ecuación al entorno local del grano considerado. Así pues, un grano más pequeño que el tamaño medio del entorno, $G_{M}$, contrae $\left(\frac{\Delta G}{G_{M}}<0\right)$, mientras que uno más grande que la media del entorno $\left(G>G_{M}\right)$, crece $\left(\frac{\Delta G}{G_{M}}>0\right)$.

De este modo, es el tamaño de los granos vecinos el que determina que un determinado grano crezca o disminuya, y no el tamaño medio global del sistema. 
En el caso de una microestructura idealizada, la integración de la ec. 2.5 conduce a la variación del tamaño de grano con el tiempo [40]:

$$
\mathrm{G}^{3}-\mathrm{G}_{0}^{3}=\mathrm{K}_{\mathrm{SL}} \cdot \mathrm{t}
$$

Siendo $G_{0}$ el tamaño inicial.

\subsubsection{Modelos cinéticos de sinterización por flujo viscoso.}

\subsubsection{Vidrios}

2.1.4.1.1 Modelos que conducen a cinéticas de orden 1.

Se ha comprobado, mediante experimentos isotermos, que la velocidad de sinterización de compactos de partículas de vidrio [44][45][46][47][48][49][50] puede expresarse como:

$$
\frac{d \alpha}{d t}=k^{*}(1-\alpha)
$$

que convenientemente integrada se transforma en:

$-\ln (1-\alpha)=k^{*} \cdot t$

siendo $\alpha$ el grado de avance de la sinterización $y k^{*}$ la constante de velocidad que depende de las características físicas (tamaño de partícula, compacidad de la probeta...) y químicas (composición química...) y de la temperatura del tratamiento. En la Tabla 2.1 se detallan las expresiones de $\alpha \mathrm{y} \mathrm{k}^{*}$ correspondientes a algunos modelos. 
Tabla 2.1. Modelos teóricos sencillos de sinterización de partículas de vidrio que conducen a cinéticas de orden 1.

\begin{tabular}{|l|c|c|}
\hline Modelo & $\begin{array}{l}\text { Expresión del grado } \\
\text { de avance de la } \\
\text { sinterización, } \boldsymbol{a}\end{array}$ & $\begin{array}{l}\text { Expresión de la constante } \\
\text { de velocidad, } \mathrm{k}^{*}\end{array}$ \\
\hline Bordia \& Raj [46] & $\frac{\Delta \rho}{\Delta \rho_{\max }}=\frac{\rho-\rho_{0}}{1-\rho_{0}}$ & $\mathrm{k}^{*}=\left(\frac{4 \cdot \pi}{3}\right)^{\frac{-1}{3}} \cdot \frac{\gamma \cdot \ln \left(1 / \rho_{0}\right)}{r_{0} \cdot \eta}$ \\
\hline Giess [44] & $\frac{\mathrm{s}}{\mathrm{s}_{\max }}$ & $\mathrm{k}^{*}=\frac{3 \cdot \gamma}{2 \cdot r_{0} \cdot \eta}$ \\
\hline Exner \& Giess & $\frac{\mathrm{s}}{\mathrm{s}_{\max }}$ & $\mathrm{k}^{*}=\frac{\pi \cdot \gamma}{2 \cdot r_{0} \cdot \eta \cdot\left(1-\rho_{0}^{1 / 3}\right)}$ \\
\hline [47] &
\end{tabular}

donde $\eta$ y $\gamma$ : viscosidad y tensión superficial del vidrio, en $(\mathrm{Pa} \cdot \mathrm{s})$ y $(\mathrm{N} / \mathrm{m}), \mathrm{r}_{0}$ : radio de la partícula incial y $\rho_{0}$ : densidad relativa inicial del compacto.

Como puede comprobarse, todos estos modelos predicen una proporcionalidad directa entre $\mathrm{k}^{*} \mathrm{y}$ la tensión superficial del vidrio, $\gamma$, y una relación, también proporcional, pero inversa, entre $k^{*}$ y la viscosidad del vidrio, $\eta$, y entre $k^{*}$ y el radio de partícula $r_{0}$.

Dado que los valores de radio de partícula, $r_{0}$, radio de poro, $r_{p 0}$ y compacidad inicial, $\rho_{0}$, están relacionados entre sí, de forma unívoca, una vez establecida la microestructura teórica inicial del modelo (empaquetamiento regular de la partículas esféricas, poros esféricos, etc) todas las relaciones de $k^{*}$ de la Tabla 2.1 pueden expresarse en la forma:

$k^{*} \propto \frac{\gamma}{r_{0} \cdot \eta}$ ec. 2.10 
2.1.4.1.2 Modelos teóricos que conducen a cinéticas más complejas.

Otros modelos, como el de Scherer [51][52][53], el de Mackenzie y Shuttleworth (ec. 2.2), e incluso algunos modelos que resultan de modificaciones de este último [54][55][56], Tabla 2.2, no se ajustan a cinéticas de orden 1. En la Tabla 2.2 se detallan las expresiones que resultan al expresar la velocidad de sinterización, $d \alpha / d t$, a partir de los modelos originales.

El modelo de Scherer, desarrollado para describir la sinterización de compactos poco densos de partículas de vidrio, considera que su microestructura está formada por celdas cúbicas, cuyas aristas son cilindros, de radio "as" y longitud " $\mathrm{L}_{s}$ ", cuyas dimensiones van modificándose conforme avanza la sinterización. 
Tabla 2.2. Modelos teóricos que conducen a modelos cinéticos más complejos.

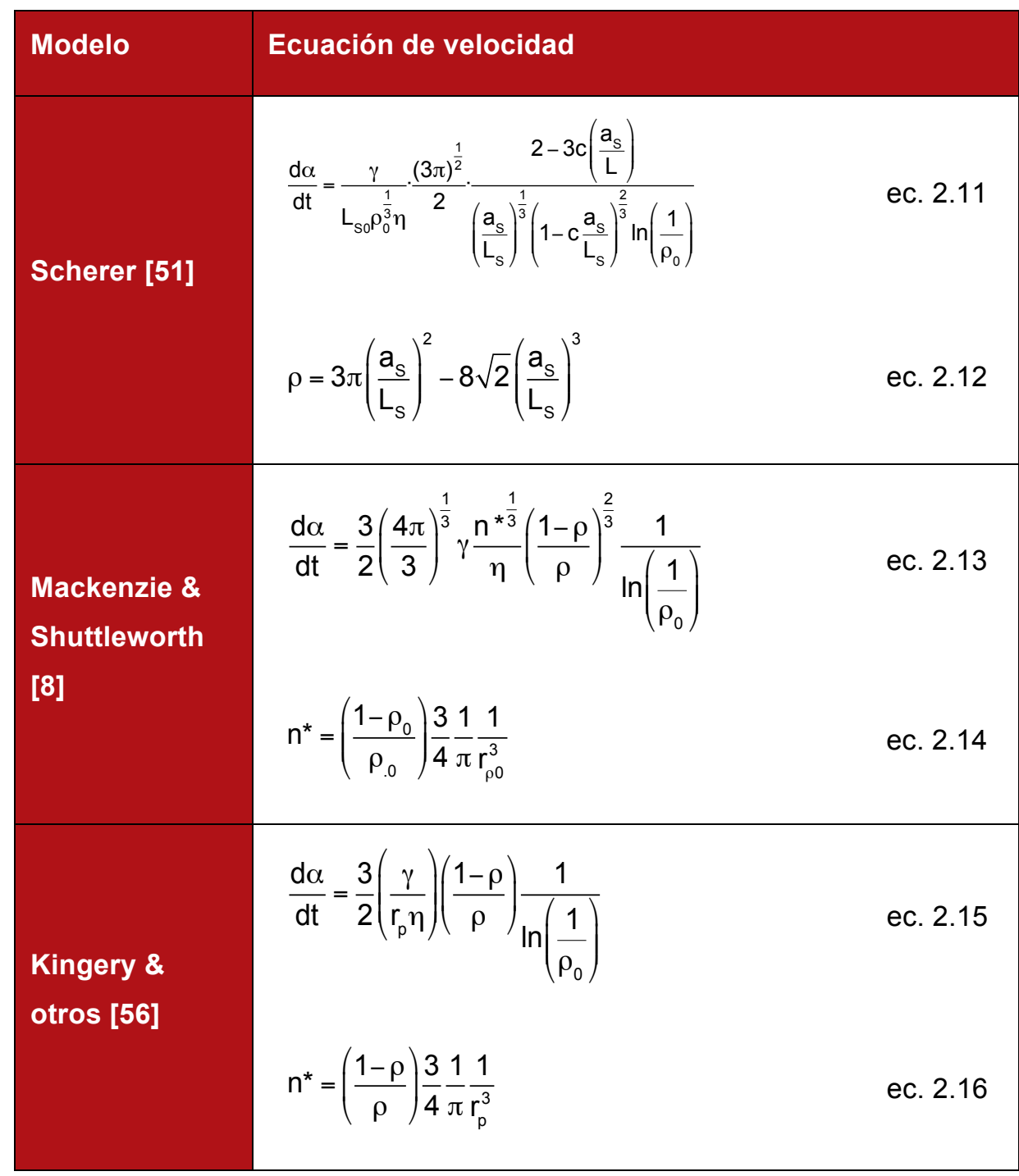

$\gamma=$ tensión superficial del vidrio; $\eta=$ viscosidad del vidrio; $c=8(2)^{0,5} / 3 \pi ; a_{s} y L_{s}=$ radio y longitud del cilindro del modelo Scherer; $n^{*}=$ concentración numérica de poros; $r_{p}=$ radio del poro y el subíndice " 0 " indica el inicio de sinterización.

La relación entre la densidad relativa $\rho$, y las dimensiones del cilindro, viene dado por la ec. 2.12 de la Tabla 2.2 y la ecuación de velocidad del proceso en la ec. 2.11. 
El modelo de Mackenzie y Shuttleworth, cuya ecuación de velocidad viene dada por la ec. 2.13 (Tabla 2.2) considera una distribución homogénea de poros esféricos en el seno de una matriz vítrea. Según este modelo, a medida que la sinterización progresa el tamaño de los poros se reduce, sin que se altere su concentración numérica, $n^{*}$. Dicho parámetro, $n^{*}$, por una parte, está relacionado con el radio de poro inicial, $r_{p 0}$, y la densidad relativa inicial, $\rho_{0}$, mediante la ec. 2.14 (Tabla 2.2). Por otra, también se relaciona con el parámetro $O$ de la ec. 2.2 mediante la expresión $n^{*}=30 / 4 \pi r_{0}^{3}$.

Kingenry [56], al igual que otros autores [54][55], sustituye en la expresión de Mackenzie y Shuttleworth (ec. 2.13 de la Tabla 2.2), la concentración numérica de poros $\mathrm{n}^{*}$ por la ec. 2.16, que relaciona $\mathrm{n}^{*}$ por la ec. 2.16, que relaciona $n^{*}$ con $\rho$ y con el radio del poro, $r_{p}$, con lo que obtiene la ecuación de velocidad buscada ec. 2.15 (Tabla 2.2). Ahora bien, a diferencia del modelo anterior, estos autores especifican, que el tamaño de poro permanece constante, en vez de contraer. Como resultado de esta hipótesis, la disminución de la velocidad de sinterización con el avance del proceso que predice la ec. 2.15 es mayor que la de los modelos anteriores, como puede apreciarse en la Figura 2.7. En ésta se representa la variación de la velocidad de sinterización que predicen los modelos de la Tabla 2.2, con el avance de la sinterización, $\alpha$, en forma normalizada. La velocidad normalizada se ha definido como la razón entre la velocidad de sinterización, $\frac{\mathrm{d} \alpha}{\mathrm{dt}}$ ó $\dot{\alpha}$, y la velocidad de sinterización para $\alpha=0,5, \frac{\mathrm{d} \alpha(0,5)}{\mathrm{dt}}$ ó $\dot{\alpha}(0,5)$. Dichas magnitudes se han calculado de la ec. 2.13 a ec. 2.16 , tomando como densidad relativa en crudo $\rho_{0}=0,4$.

En efecto, se comprueba que este modelo predice un efecto del grado de conversión, $\alpha$, sobre la velocidad de sinterización algo mayor que la que resulta de una cinética de orden 1 , especialmente a valores bajos de $\alpha$, mientras que para otros modelos, el efecto es menor. 


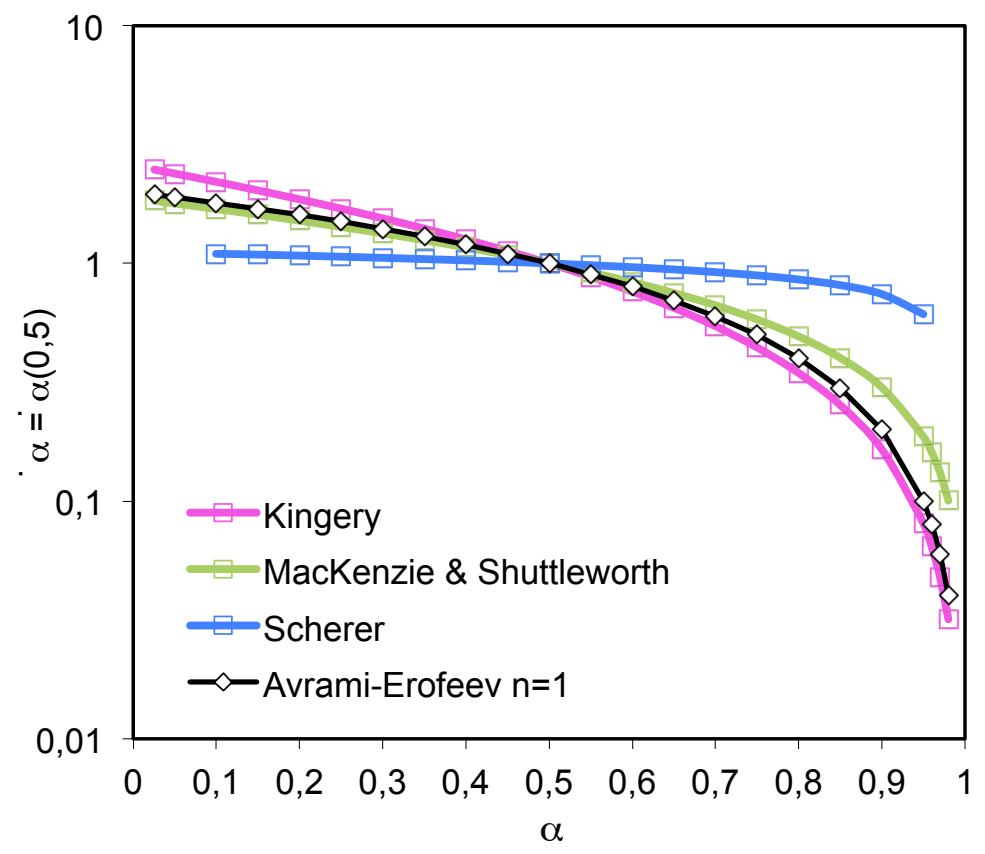

Figura 2.7.Variación de la velocidad de sinterización normalizada, $\dot{\alpha} / \dot{\alpha}(0,5)$, frente al grado de avance, $\alpha$, según los modelos de Scherer (línea azul), Mackenzie \& Shuttleworth (línea verde), Kingery (línea rosa) y orden 1.

2.1.4.1.3 Modelos reológicos basados en la teoría continua de la sinterización

Para un material poroso cuyas propiedades reológicas pueden considerarse visco-lineales, la velocidad de sinterización, $\frac{d \varepsilon_{v}}{d t}$, cuando sobre el sistema no actúa ninguna tensión externa, de acuerdo con la teoría continua de la sinterización [57][58] viene dada por:

$\frac{d \varepsilon_{V}}{d t}=-\frac{P_{L}}{K_{p}}$

Donde $\varepsilon_{\mathrm{V}}=\ln \left(\mathrm{V} / \mathrm{V}_{0}\right)$ siendo $\mathrm{V}$ y $\mathrm{V}_{0}$ el volumen de la probeta, instantáneo e 
inicial respectivamente, $P_{L}$ es la presión efectiva de Laplace, también denominada tensión de sinterización, que representa la acción colectiva de todas las tensiones capilares en un material poroso, $\mathrm{K}_{\mathrm{p}}$ es la viscosidad global de un material poroso ("bulk viscosity") que representa la resistencia viscosa a la densificación.

Ambas propiedades dependen, por una parte, de las características intrínsecas del material exento de porosidad, es decir, de su esqueleto sólido, y por otra, de su estructura porosa. En consecuencia, tanto $P_{L}$ como $K_{p}$, suelen expresarse en función de la porosidad $(1-\rho)$ del material, siendo la relación de dependencia entre cada propiedad de comportamiento $P L$ y $K_{p}$ y dicha característica diferente, según el modelo estructural elegido para representar el material.

2.1.4.1.3.1 Presión de sinterización, $P_{L}$, en función del tamaño de partícula inicial, $r_{0}$, y la densidad relativa, $\rho$.

En la Tabla 2.3, se resumen los distintos modelos que se analizarán en el presente apartado. 
Tabla 2.3. Modelos reológicos que relacionan la presión de sinterización, $P_{L}$, con el radio de partícula inicial, $r_{0}$, y la densidad relativa, $\rho$. Se incluye en esta tabla la expresión de la presión de Laplace adimensional, \& $\wp_{L}$.

\begin{tabular}{|c|c|c|c|c|}
\hline Modelo & \multicolumn{2}{|l|}{ Expresión de $\mathrm{P}_{\mathrm{L}}$} & \multicolumn{2}{|c|}{$\wp_{\mathrm{L}}=\mathrm{P}_{\mathrm{L}} /\left(3 \gamma / \mathrm{r}_{0}\right)$} \\
\hline Ducamp y Raj [59] & $\frac{2 \gamma}{r_{0}} \cdot\left(\frac{\rho}{1-\rho}\right)^{\frac{1}{3}}$ & ec. 2.18 & $\frac{2}{3} \cdot\left(\frac{\rho}{1-\rho}\right)^{\frac{1}{3}}$ & ec. 2.19 \\
\hline Skovohod [57] [58] & $\frac{3 \gamma}{r_{0}} \cdot \rho^{2}$ & ec. 2.20 & $\rho^{2}$ & ec. 2.21 \\
\hline $\begin{array}{l}\text { Bouvard \& } \\
\text { McMeeking [57] }\end{array}$ & $\frac{3 \gamma}{r_{0}} \cdot \rho^{\frac{5}{3}}$ & ec. 2.22 & $\rho^{\frac{5}{3}}$ & ec. 2.23 \\
\hline Ashby [57] & $\frac{3 \gamma}{r_{0}} \cdot\left(\frac{6 \rho}{1-\rho}\right)^{\frac{1}{3}}$ & ec. 2.24 & $\left(\frac{6 \rho}{1-\rho}\right)^{\frac{1}{3}}$ & ec. 2.25 \\
\hline Scherer [60] & $\frac{\gamma}{L_{0} o_{0}^{\frac{1}{3}}} \cdot \frac{3 \pi^{4}\left(a_{s} / L_{s}\right)^{5}}{\left(\frac{1-8 \sqrt{2}}{3 \pi\left(a_{s} / L_{s}\right.}\right)^{2}} \mid$ & ec. 2.26 & --- & \\
\hline
\end{tabular}

$\gamma=$ tensión superficial del vidrio; $a_{S}$ y $L_{S}=$ radio y longitud del cilindro del modelo de Scherer; $r_{0}=$ radio inicial de la partícula (su relación con $\rho$ viene dada por la ec. 2.12 de la Tabla 2.2).

La expresión general de la presión de sinterización, $P_{L}$, para la sinterización de compactos de partículas de vidrio, con un tamaño de poro, $r_{p}$, viene dada por: 
$P_{L}=\frac{2 \gamma}{r_{p}}$

ec. 2.27

Ahora bien, puesto que no se sigue la sinterización determinando $r_{p}$ sino $\rho$ ó $\alpha, P_{L}$ debe expresarse en términos de $\rho$ ó $\alpha$. Para ello, debe de recurrirse a considerar una estructura porosa ideal.

i. Modelo de Ducamp y Raj [59]

Este modelo asume que todos los poros son idénticos y que hay un poro, de radio $r_{p}$, en cada una de las celdas cúbicas, que forman la estructura del compacto de partículas de vidrio. El volumen de cada una de estas celdas, es la suma del volumen de sólido y del poro. Para relacionar $\rho$ con $r_{p}$ se considera que el vidrio forma una capa esférica que rodea al poro. Esta aproximación conduce a la relación:

$$
\frac{r_{p}}{r_{0}}=\left(\frac{1}{\rho}-1\right)^{\frac{1}{3}}
$$

De la ec. 2.27 y ec. 2.28 se obtiene la expresión buscada:

$$
P_{L}=\frac{2 \gamma}{r_{0}}\left(\frac{\rho}{1-\rho}\right)^{\frac{1}{3}}
$$

De acuerdo con este modelo, la presión de sinterización incrementa monotónicamente con la densidad relativa, $\rho, y$ tiende a infinito cuando $\rho$ tiende a 1 . Estos autores comprobaron que el buen acuerdo entre los valores de $P_{L}$, medidos y teóricos, para valores de $\rho<0,95$. Por encima de esta densidad, los poros más pequeños desaparecen y los más grandes crecen. Ambos procesos conducen a una marcada dispersión en los valores de $P_{L}$. 
ii. Modelo de Skovohod [57][58]

Este modelo asume que:

$P_{L}=\frac{d F_{S}}{d V_{p}}$

ec. 2.30

Donde $F_{S}$ es la energía libre superficial del sistema y $V_{p}$ el volumen de los poros.

Introduciendo el área superficial específica, $S_{V}=$ superficie de los poros/volumen de sólido, y la tensión superficial del vidrio, $\gamma$, y operando, la ec. 2.30 se transforma en:

$P_{L}=\gamma \rho^{2}\left(\frac{-d S_{V}}{d \rho}\right)$

Considerando las partículas esféricas, los poros cuasi esféricos e isométricos y basándose en un análisis probabilístico, Skovohod, obtuvo:

$\mathrm{S}_{\mathrm{v}}=\frac{3 \gamma}{\mathrm{r}_{0}}(1-\rho)$

De la ec. 2.31 y ec. 2.32 se obtiene:

$\mathrm{P}_{\mathrm{L}}=\frac{3 \gamma}{\mathrm{r}_{0}} \rho^{2}$

De acuerdo con este modelo, $\mathrm{P}_{\mathrm{L}}$, aumenta con el cuadrado de la densidad relativa, $\rho$, hasta un valor máximo de $\left(3 \gamma / r_{0}\right)$ cuando se completa la sinterización $\rho=1$.

Olevsky, en su artículo de revisión sobre sinterización, enumera algunas expresiones de $P_{L}$ [57], que junto a la desarrollada por Scherer [60] se detallan en la Tabla 2.3. En esta misma tabla se incluye, también, la 
expresión correspondiente a la presión de Laplace adimensional, sugerida por este autor:

$$
\wp_{\mathrm{L}}=\frac{\mathrm{P}_{\mathrm{L}}}{3 \gamma / \mathrm{r}_{0}}
$$

Para determinar la variación que sigue la presión de sinterización adimensional, $\wp_{L}$, con el grado de sinterización, $\alpha$, que predicen los distintos modelos, se han calculado los valores de $\wp_{L}$ para distintos valores de $\alpha$, mediante las expresiones de la Tabla 2.3 y la ec. A1. 9, tomando como $\rho_{0}=0,4$ y $\rho_{\max }=1$. Los resultados se detallan en la Figura 2.8 en la forma $P_{L}(\alpha) / P_{L}(0,5)$

Se comprueba que, prácticamente durante todo el proceso de sinterización, $0,05 \leq \alpha \leq 0,95$, la presión de sinterización adimensional aumenta con $\alpha$, aún cuando existen diferencias cualitativas entre los diferentes modelos. 


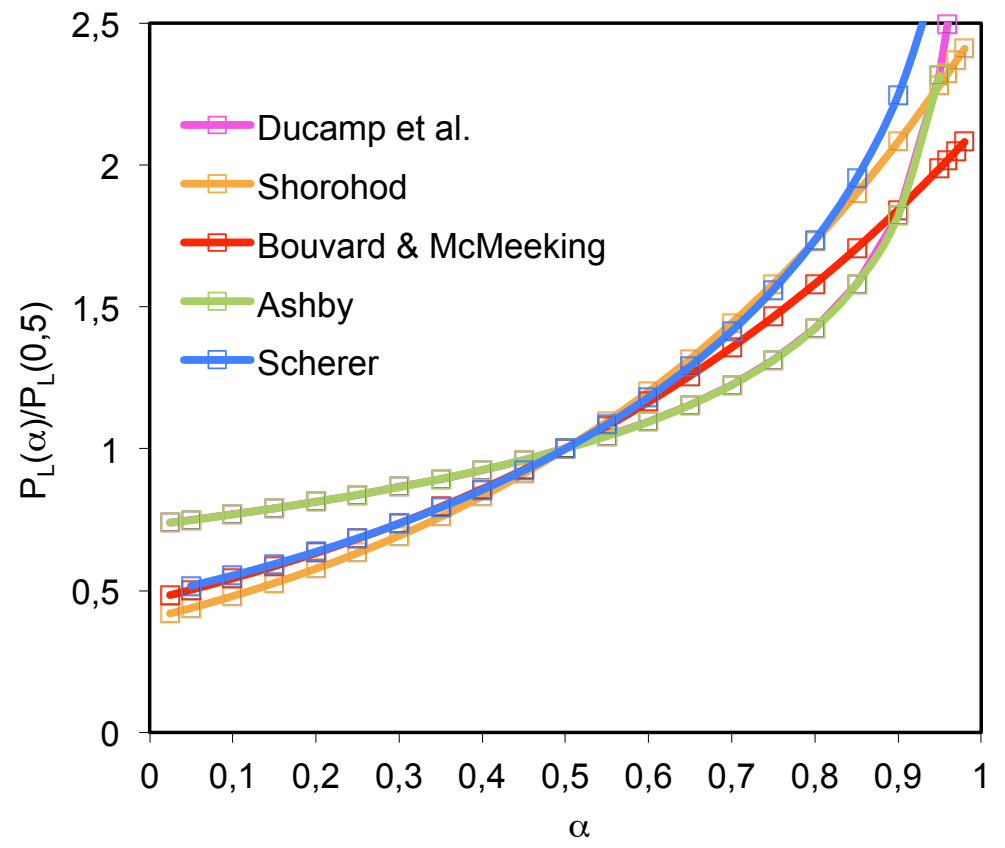

Figura 2.8. Variación de la presión de sinterización normalizada frente al grado de avance de la sinterización, $\alpha$, según distintos modelos.

2.1.4.1.3.2 Módulo viscoso global $\left(K_{p}\right)$ en función de la viscosidad del vidrio poroso, $\eta_{p}$, y de la densidad relativa, $\rho$.

Ducamp [59], Shorohod [58] y Olesky [57] han llegado a la misma expresión:

$\mathrm{K}_{\mathrm{P}}=\frac{4}{3} \eta_{\mathrm{p}}\left(\frac{\rho}{1-\rho}\right)$

ec. 2.35

En su desarrollo se basaron en el modelo de Mackenzie \& Shuttleworth [8] pero con una diferencia sustancial. En el modelo de estos últimos, el elemento representativo del sistema para el cálculo de $\mathrm{K}_{\mathrm{p}}$ se basa en un poro esférico rodeado por una capa de sólido incompresible al que le corresponde una viscosidad del medio exento de porosidad, $\eta$. En cambio, para los primeros, el material que rodea al poro es compresible, por lo que corresponde a una viscosidad de un vidrio poroso, $\eta_{\mathrm{p}}$. 
2.1.4.1.3.3 Viscosidad de vidrios porosos, $\eta_{p}$. Efecto de la compacidad del vidrio, $\rho$.

Estudios teóricos y experimentales predicen un aumento de la viscosidad, $\eta_{p}$, con la compacidad, $\rho$, del vidrio. De entre las muchas expresiones existentes, en la Tabla 2.4 se detallan las más utilizadas.

Tabla 2.4. Expresiones que describen el efecto de la compacidad del vidrio, $\rho$, sobre su viscosidad, $\eta_{p}$.

\begin{tabular}{|c|c|c|}
\hline Modelo & Expresión de $\eta_{p}$ & \\
\hline Mackenzie [61] & $\frac{\eta_{P}}{\eta}=\left(1-\frac{5}{3}(1-\rho)\right)$ & ec. 2.36 \\
\hline Shorohod [58] & $\frac{\eta_{p}}{\eta}=\rho^{2}$ & ec. 2.37 \\
\hline Scherer [60] & $\frac{\eta_{P}}{\eta}=\frac{3 \rho}{2+(1+2(1-\rho))+\sqrt{1+(1-\rho)-2(1-\rho)^{2}}}$ & ec. 2.38 \\
\hline Ducamp et al. [59] & $\frac{\eta_{p}}{\eta}=\exp \left(-a \cdot \frac{1-\rho}{\rho}\right)$ & ec. 2.39 \\
\hline $\begin{array}{l}\text { Rahaman et al. } \\
\text { [62] }\end{array}$ & $\frac{\eta_{P}}{\eta}=\exp (-11,2 \cdot(1-\rho))$ & ec. 2.40 \\
\hline $\begin{array}{l}\text { Sura \& Panda }{ }^{(*)} \\
{[63]}\end{array}$ & $\frac{\eta_{P}}{\eta}=\frac{3 \rho^{2}}{3 \rho+2(1-\rho)}$ & ec. 2.41 \\
\hline
\end{tabular}

$\eta_{\mathrm{P}}=$ viscosidad del vidrio poroso $;=$ viscosidad del vidrio exento de poros; $\rho=$ compacidad del vidrio; $a=2,4$ a altas porosidades y $a=4$ para valores de $\rho>0,75$. ${ }^{(*)}$ Para poros esféricos. 
2.1.4.1.3.4 Modulo viscoso adimensional, $\Psi$.

El modelo de sinterización viscoso de Shorohod-Olevsky (SOVS) ("Shorohod-Olevsky Viscous Sintering Model") define un módulo viscoso adimensional, $\Psi$, mediante la ecuación:

$\Psi=\frac{K_{\mathrm{P}}}{2 \eta}$

que al introducir en la ec. 2.35 se convierte en:

$\Psi=\frac{2}{3}\left(\frac{\rho}{1-\rho}\right)\left(\frac{\eta_{\mathrm{P}}}{\eta}\right)$

que, de acuerdo con la ec. 2.36 a ec. $2.43, \Psi$ sólo depende de la densidad relativa del material. De la ec. 2.36 a ec. 2.41 y la ec. A1. 9 del apéndice, tomando $\rho_{0}=0,4$ y $\rho_{\max }=1$, se han calculado los valores de $\Psi$ que predicen los diferentes modelos para distintos valores de $\alpha$ (Figura 2.9). 


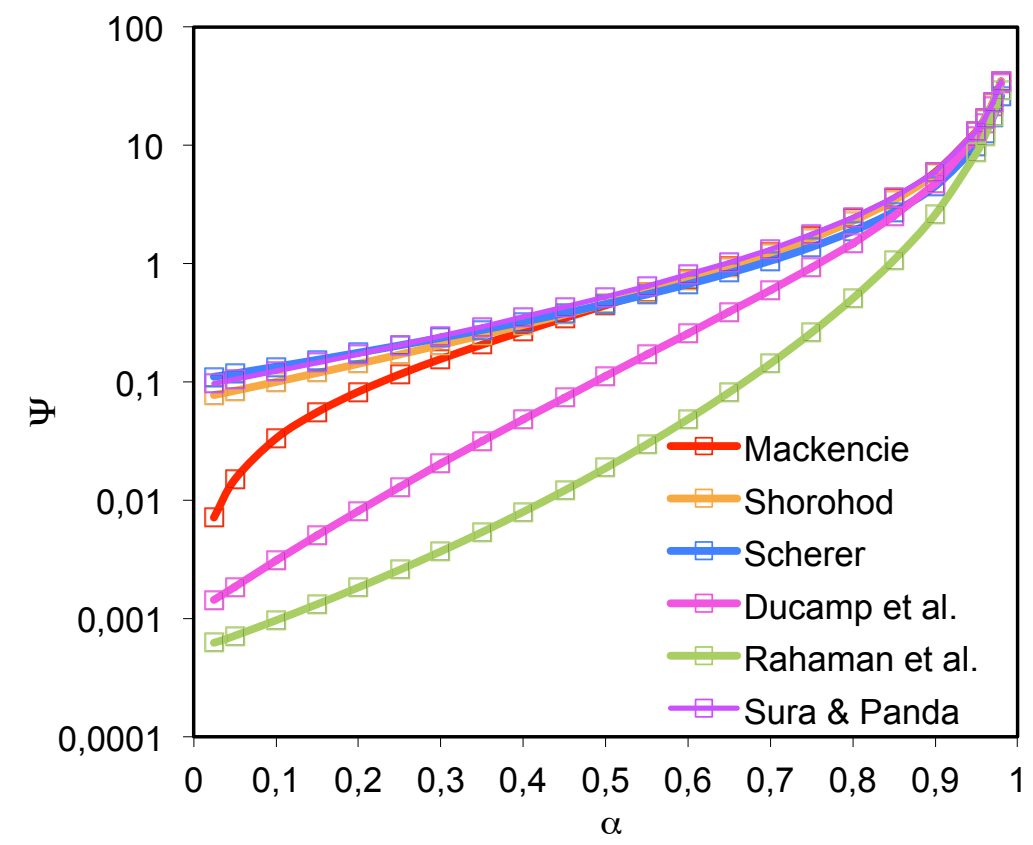

Figura 2.9. Variación del módulo viscoso adimensional, $\Psi$, frente al grado de avance de la sinterización, $\alpha$, según distintos modelos.

Se comprueba que para todos los modelos de viscosidad ensayados, el módulo viscoso adimensional $\Psi$, que expresa la resistencia adimensional que opone el material a la sinterización, aumenta conforme avanza la sinterización, $\alpha$, hasta que el material se vuelve incompresible, $\Psi=\infty$, cuando su sinterización ya es completa, $\alpha=1$. Ahora bien, el efecto cuantitativo que ejerce $\alpha$ sobre $\Psi$, es muy diferente si se utiliza un modelo para la estimación de la viscosidad u otro.

Asimismo, se puede comprobar fácilmente, que las dos expresiones empíricas, ec. 2.40 y ec. 2.41 , implican un efecto mayor del grado de sinterización, $\alpha$, sobre le módulo viscoso adimensional $\Psi$, que los restantes modelos de viscosidad de vidrios porosos tanto teóricos (ec. 2.36 a ec. 2.38) como semi-empíricos (ec. 2.39). 
2.1.4.1.3.5 Modelos de sinterización basados en la teoría continua de la sinterización viscosa. Su relación con el modelo de Avrami-Erofeev.

i) De forma generalizada, la velocidad de un proceso se puede expresar por:

$\frac{\mathrm{d} \alpha}{\mathrm{dt}}=\mathrm{K}(\mathrm{T}) \cdot \mathrm{f}(\alpha)$

siendo $f(\alpha)$ un modelo genérico de sinterización, que expresa la dependencia de la velocidad del proceso con el grado de avance del mismo.

De las definiciones de presión de sinterización adimensional $\wp_{\mathrm{L}}$ (ec. 2.34), y de módulo viscoso adimensional $\Psi$ (ec. 2.43) se obtiene:

$\frac{\mathrm{d} \alpha}{\mathrm{dt}}=\frac{1}{\ln \left(\frac{\rho_{\max }}{\rho_{0}}\right)} \cdot \frac{3 \gamma}{2 r_{0} \eta} \cdot \frac{\wp_{\mathrm{L}}}{\Psi}$

que al compararla con la ec. 2.44 se desprende la siguiente correspondencia:

$f(\alpha)=\frac{\wp_{L}}{\Psi}$

y:

$K(T)=\frac{1}{\ln \left(\frac{\rho_{\max }}{\rho_{0}}\right)} \cdot \frac{3 \gamma}{2 r_{0} \eta}$

En consecuencia, se pueden obtener tantas expresiones concretas para el modelo de sinterización genérico, $f(\alpha)$, como combinaciones pueden establecerse entre las cuatro expresiones de $\wp_{L}$ (Tabla 2.3) y las seis de $\Psi$, 
que resultan de introducir la ec. 2.36 a ec. 2.41 de la Tabla 2.4, en la ec. 2.43.

ii) Por otra parte, uno de los modelos de mayor versatilidad y sencillez a la hora de describir procesos cinéticos es el modelo de Avrami-Erofeev [64], cuya ecuación es:

$f(\alpha)=n(1-\alpha)[-\ln (1-\alpha)]^{\frac{n-1}{n}}$

Este modelo ha sido usualmente aplicado para describir cineticas de reacciones químicas y transiciones de fases, debido, principalmente, a la simplicidad de la forma integrada de la ec. 2.48.

$g(\alpha)=[-\ln (1-\alpha)]^{\frac{1}{n}}$

Además, este modelo es capaz de describir apropiadamente muchos tipos de curvas de sinterización: $\alpha$ vs t, simplemente modificando el índice de potencia de Avrami "n". Sin embargo, su implementación para la descripción de cinéticas de sinterización ha sido escaso, aunque hace veinte años ya fue utilizado con éxito para describir la evolución de la porosidad con el tiempo de sinterización, en experimentos isotermos, en el estado intermedio del proceso de densificación de pavimentos de gres rojo [65]. Además, recientemente dicho modelo ha sido utilizado con éxito por nosotros, en la descripción de las cinéticas de sinterización de esmaltes [66][67][68][69][70].

iii) A continuación, analizamos algunos modelos de sinterización que resultan de introducir distintas expresiones de $\wp_{\mathrm{L}}$ y $\Psi$ en la ec. 2.46, comparando sus resultados con los que se obtienen de la ec. 2.48. En efecto, combinando las expresiones de Ducamp \& Raj [59] para $\wp\llcorner$ y $\Psi$, ambas comprobadas experimentalmente, se llega a un modelo de sinterización que también queda perfectamente descrito mediante el modelo de Avrami con n=0,55 (Figura 2.10). 
Manteniendo la expresión de Ducamp y Raj [59] para $\wp_{\mathrm{L}}$, pero tomando para $\Psi$ la expresión que resulta del modelo empírico de viscosidad de Rahaman [62] (ec. 2.40), se obtiene un modelo cinético que, excepto para pequeños valores de $\alpha, \alpha<0,2$, se adecua perfectamente al modelo de Avrami con $\mathrm{n}=0,35$ (Figura 2.11).

Siguiendo este mismo procedimiento, pero utilizando en el cálculo de $\Psi$ los modelos de viscosidad de vidrios porosos de Mackenzie (ec. 2.36), Scherer (ec. 2.37) y Sura \& Panda (ec. 2.41) se obtienen los respectivos modelos cinéticos (Figura 2.12 a Figura 2.14). En todos los casos, excepto para los valores de $\alpha$ pequeños, $\alpha<0,15$, los modelos cinéticos se ajustan muy bien al modelo de Avrami con valores de $\mathrm{n}$ de 0,6, 0,85 y 0,9 , respectivamente.

En resumen, puede concluirse que la práctica totalidad de modelos cinéticos de sinterización analizados, tanto teóricos como empíricos, conducen a cinéticas que pueden también describirse con el modelo de Avrami-Erofeev con valores de $\mathrm{n}$ menores que 1 , generalmente $0,35 \leq n \leq 1$. 


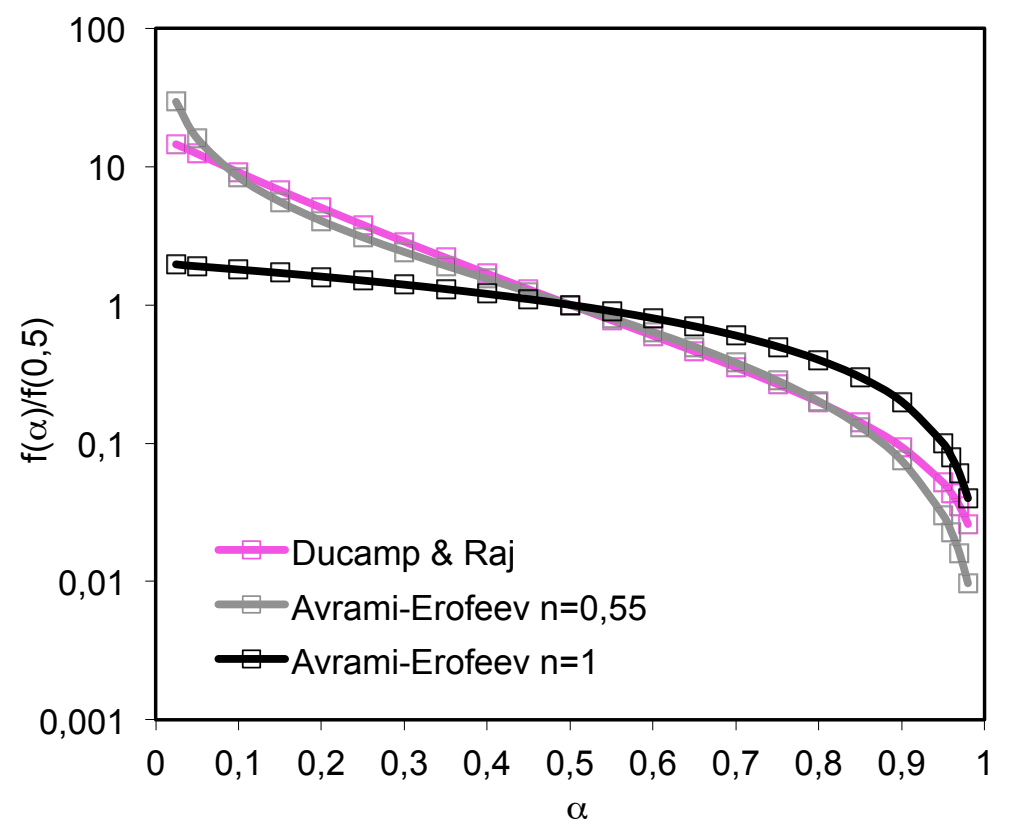

Figura 2.10. Comparación entre el modelo teórico de Ducamp \& Raj [59] y AvramiErofeev para $n=0,55$ y $n=1$.

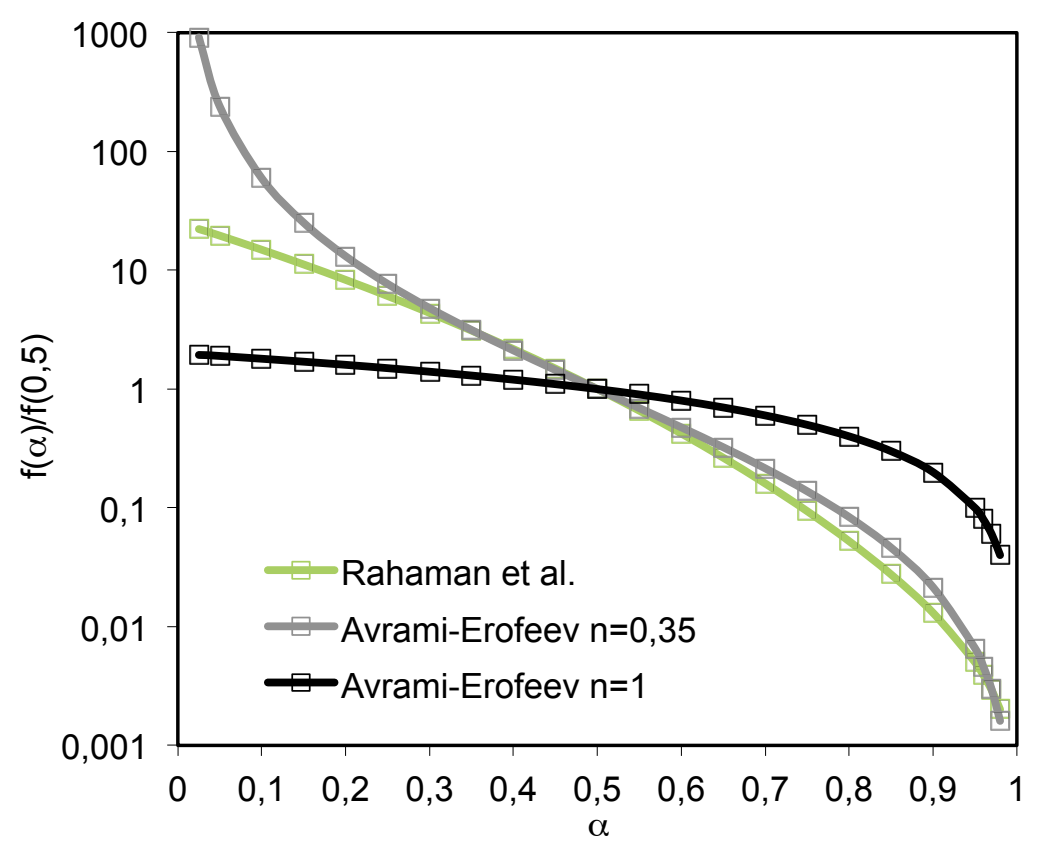

Figura 2.11. Comparación entre el modelo teórico de Rahaman et al. [62] y AvramiErofeev para $n=0,35$ y $n=1$. 


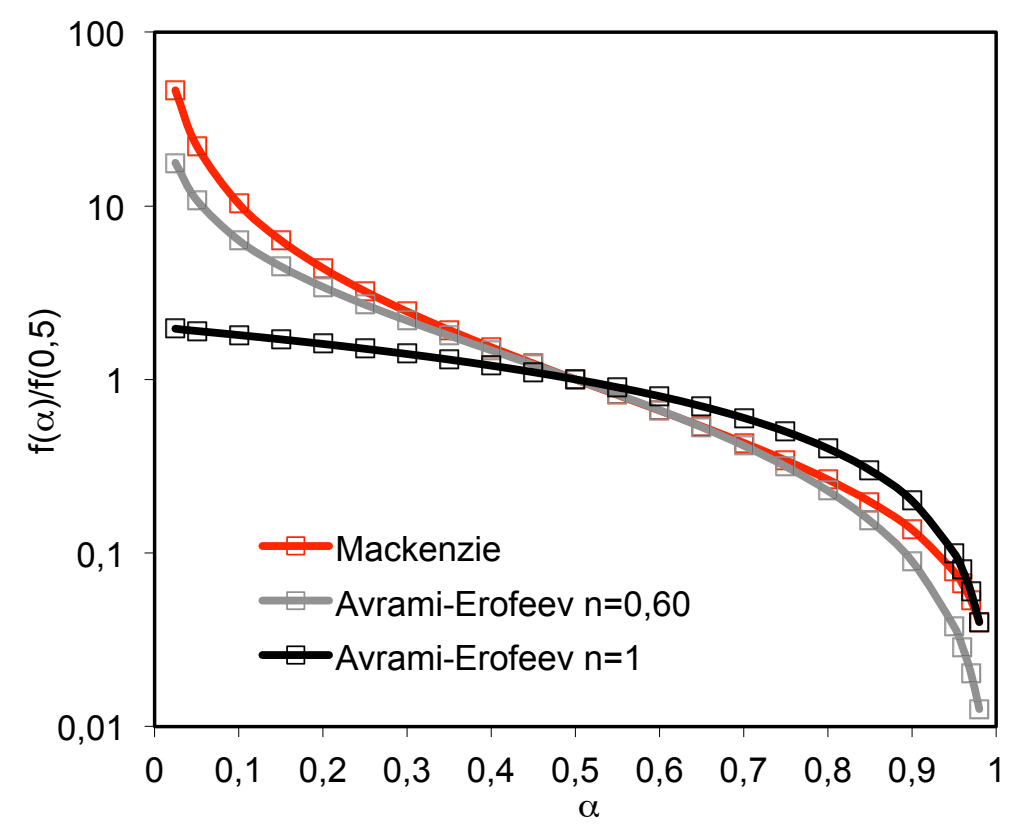

Figura 2.12. Comparación entre el modelo teórico de Mackenzie [61] y AvramiErofeev para $n=0,60$ y $n=1$.

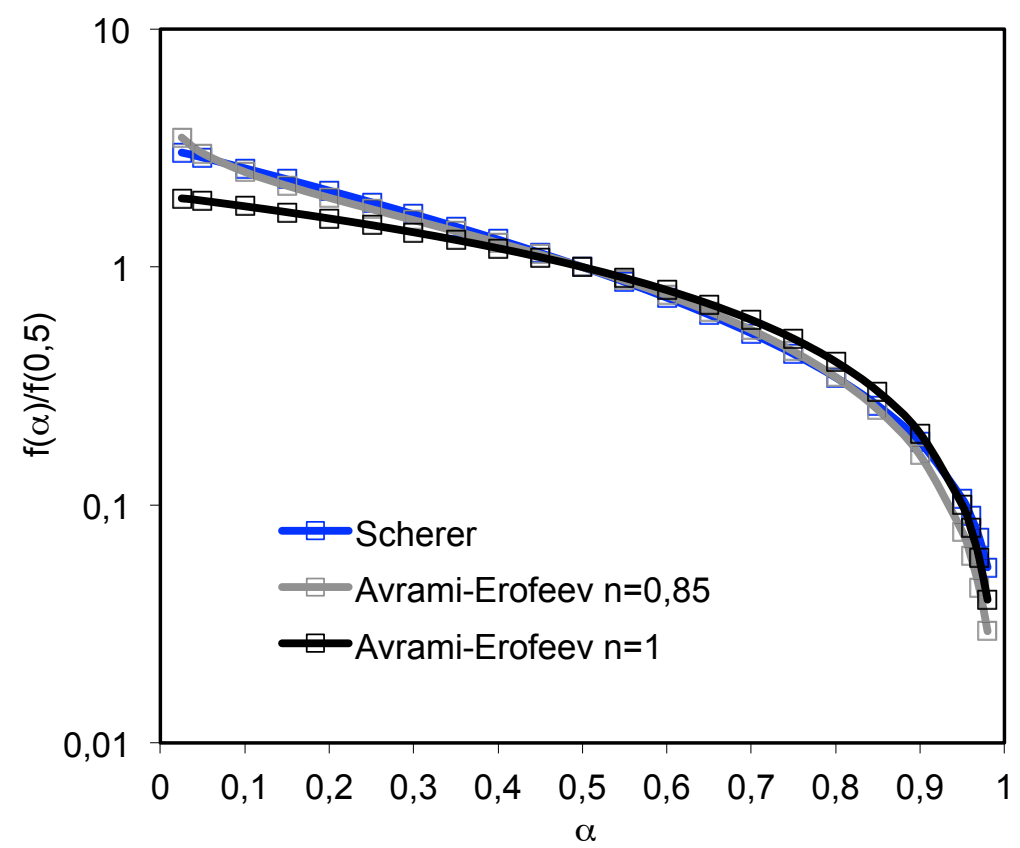

Figura 2.13. Comparación entre el modelo teórico de Scherer [60] y Avrami-Erofeev para $n=0,85$ y $n=1$. 


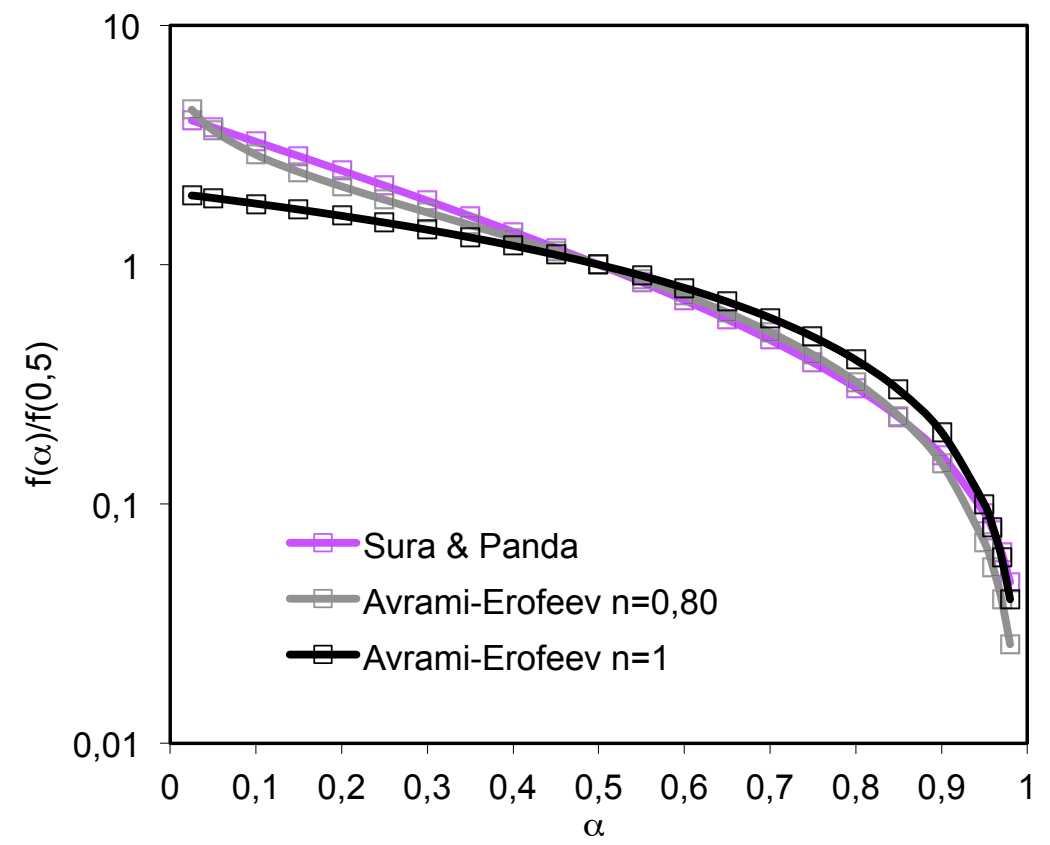

Figura 2.14. Comparación entre el modelo teórico de Sura \& Panda [63] y AvramiErofeev para $n=0,80$ y $n=1$.

2.1.4.2 Composites de matriz vitrea con inclusiones rígidas de pequeño tamaño de partícula (GMC's)

\subsection{Modelos de Scherer}

Scherer estudió teóricamente el efecto de las inclusiones rígidas sobre la sinterización viscosa, desarrollando dos modelos reológicos basados en la teoría continua de la sinterización. En el primero [71], el composite se representaba como una esfera compuesta por una inclusión rígida rodeada de una matriz de partículas vítreas sinterizables. La aplicación de dicho modelo se circunscribe a composites con bajos contenidos en inclusiones, $\phi<0,20$, ya que se supone que las tensiones provocadas por las inclusiones no se solapan. El segundo modelo [72], llamado "self-consistent", puede ser aplicado hasta el valor límite de $\phi$ para el que las inclusiones forman una 
red continua que impide la contracción de la probeta. Para valores de $\phi<0,20$ ambos modelos conducen al mismo resultado.

En su primer modelo [71], en el que se considera una inclusión esférica embebida en una matriz de pequeñas partículas vítreas, Scherer obtuvo para la velocidad de contracción lineal del composite, $\frac{\mathrm{d} \varepsilon_{\mathrm{C}}}{\mathrm{dt}}{ }^{(*)}$, la expresión:

$\frac{d \varepsilon_{C}}{d t}=\left[1+v_{i}\left(\frac{4 \eta_{p m}}{3 K_{p m}}\right)\right]^{-1} \cdot\left(1-v_{i}\right) \cdot \frac{d \varepsilon_{f m}}{d t}$

donde:

$\frac{\mathrm{d} \varepsilon_{\mathrm{fm}}}{\mathrm{dt}}$ es la velocidad de contracción lineal de la matriz exenta de inclusiones, $\eta_{p m}$ y $K_{p m}$ son la viscosidad de cizalla y el modulo viscoso o global de la matriz porosa, respectivamente, y $v_{i}$ es la fracción volumétrica instantánea de las inclusiones en la pieza (se incluyen poros). Esta última característica, $\mathrm{v}_{\mathrm{i}}$, varía con la compacidad, $\rho$, y con la fracción volumétrica de inclusiones en la mezcla de materiales, $\phi$, según la ecuación:

$V_{i}=\rho\left[\rho+\frac{1-\phi}{\phi}\right]^{-1}$

Los dos factores del segundo miembro de la ec. 2.50 contribuyen a reducir la velocidad de sinterización de la matriz, $\frac{d \varepsilon_{\mathrm{fm}}}{\mathrm{dt}}$. El término $\left(1-\mathrm{v}_{\mathrm{i}}\right)$ representa la contribución de la matriz a la sinterización de la pieza, de ${ }^{*} \varepsilon_{\mathrm{C}}=\frac{1}{3} \ln \frac{\mathrm{V}}{\mathrm{V}_{0}}$ siendo $\mathrm{V}$ y $\mathrm{V}_{0}$ el volumen instantáneo e inicial del compacto de composite. 
acuerdo con la regla de las mezclas. El término entre corchetes, $\left[1+v_{i}\left(\frac{4 \eta_{p m}}{3 K_{p m}}\right)\right]^{-1}$, representa la presión hidrostática que ejercen las inclusiones sobre la matriz, oponiéndose a la sinterización, la cual depende de las propiedades reológicas de la matriz, $\eta_{p m}$ y $K_{p m}$, y de la fracción volumétrica de las inclusiones en la pieza.

En el segundo modelo [72] ("self-consistent"), Scherer sustituye en la ec. 2.50 la viscosidad de cizalla de la matriz, $\eta_{\mathrm{pm}}$, por la viscosidad de cizalla del composite, lo que incrementa el efecto retardante de las inclusiones en la sinterización. Aun así, el efecto que predice el modelo, incluso para valores de $\phi=0,40$, es muy pequeño, ya que la razón entre la velocidad de sinterización del composite y de la matriz, $\frac{\mathrm{d} \varepsilon_{\mathrm{c}}}{\mathrm{dt}} / \frac{\mathrm{d} \varepsilon_{\mathrm{fm}}}{\mathrm{dt}}$, es mucho mayor que la que se observa experimentalmente. En consecuencia, se puede concluir que la presión hidrostática generada por las inclusiones no es la única causa de la reducción de la viscosidad de sinterización de un composite por efecto de las inclusiones.

Rahaman et al. [128][129] observaron un buen acuerdo entre los resultados experimentales y los que predice el modelo "self-consistent" para valores de $\phi \leq 0,12$ en composites de SiC [128] y para valores de $\phi \leq 0,15$ cuando las inclusiones eran esféricas y de $\mathrm{Ni}$ [129]. Pascual et al. [35], empleando como inclusiones fibras de circona, comprobaron que los resultados no se adaptaban al modelo, incluso para valores de $\phi$ tan bajos como 0,09 .

El mismo Scherer, en un trabajo posterior al desarrollo de sus modelos [74], al estudiar la sinterización de un composite comercial de matriz vítrea de borosilicato con $\phi=0,33$, comprobó que su modelo no funcionaba bien en este caso.

\subsubsection{Modelos de Bordia y Raj [46]}

Estos autores, en el desarrollo de su modelo, consideraron la simultaneidad de la densificación y la deformación viscosa durante la sinterización, dando 
gran importancia a la deformación viscosa para la relajación de las tensiones que provocan la presencia de inclusiones en la matriz vítrea. Según estos autores, la sinterización de un composite depende, en gran medida, de la relación: velocidad de deformación/velocidad de densificación, B. En su desarrollo consideraron que la ecuación cinética de la sinterización de la matriz exenta de inclusiones sigue una cinética de orden 1 , como ya se vió en el apartado 2.1.4.1.1 (Tabla 2.1). La expresión original de estos autores de la ecuación cinética es:

$\left(\frac{\Delta \rho}{\Delta \rho_{\max }}\right)_{\phi=0}=1-\exp \left(\frac{-\mathrm{t}}{\tau}\right)$

Donde $\Delta \rho$ es el aumento de densidad relativa de la pieza a tiempo t, $\Delta \rho_{\max }$ el incremento máximo de compacidad y $\tau$ una constante de tiempo, numéricamente igual a $\left(\mathrm{k}^{*}\right)^{-1}$ de la Tabla 2.1.

Para el caso de una matriz vítrea que contenga partículas inertes obtuvieron la siguiente ecuación cinética:

$\left(\frac{\Delta \rho}{\Delta \rho_{\max }}\right)_{\phi \neq 0}=1-\exp \left(\frac{-9 B}{4 \phi+9 B} \cdot \frac{-t}{\tau}\right)$

El parámetro de relajación, $B$, es el más relevante, puesto que si es elevado $(B>1)$ se produce la relajación de tensiones, por lo que las inclusiones rígidas ejercen muy poco efecto sobre la densificación de la matriz. En estos casos, el comportamiento en la sinterización seguiría la regla de las mezclas. Por el contrario, si $\mathrm{B}<1$, la velocidad de sinterización se ralentiza considerablemente por la presencia de inclusiones y el comportamiento en la sinterización no seguiría la regla de las mezclas. Estos autores, para el caso de matrices vítreas, basándose en los modelos de Mackenzie and Shuttleworth [8] y Scherer [42], obtuvieron que $B>1$, para compacidades iniciales usuales de las probetas $\left(\rho_{0}>0,3\right)$, y que B aumentaba conforme lo hacía $\rho_{0}$. 
Así pues, según este modelo, la adición de inclusiones a una matriz vítrea sólo incrementaría la constante de tiempo, de $\tau$ a $\frac{\tau(4 \phi+9 B)}{9 B}$ en la ec. 2.52 y ec. 2.53, que seguiría siendo de orden 1. En consecuencia, la representación del $\ln \left[\frac{\Delta \rho}{\Delta \rho_{\max }}-1\right]_{\phi \neq 0}$ vs el tiempo de sinterización, $t$, debería ser una línea recta, de cuya pendiente se obtendrían los valores de $\tau$ y $\mathrm{B}$ al realizar diferentes experimentos de sinterización en mezclas con diferentes contenidos en inclusiones.

Pascual et al. [35] al aplicar este modelo, a los resultados obtenidos en el estudio de la sinterización isoterma de mezclas de vidrio de este borosilicato y fibras de circona, no obtuvieron líneas rectas, sino curvas de pendiente decreciente. $\mathrm{Y}$, además, al ajustar dichas curvas a rectas, obtuvieron valores de $\mathrm{B}$ que dependían considerablemente del contenido en fibra, $\phi$, y del tamaño de partícula del vidrio, $r_{0}$, contrariamente a lo que predice el modelo. En un trabajo posterior, Raj et al. [59] comprobaron que para una matriz vítrea se cumple que $B>1$ y que este parámetro aumentaba conforme avanza el proceso de sinterización. De acuerdo con esta variación de B con el aumento de la densidad relativa del vidrio, $\rho$, del tipo exponencial, la representación antes citada daría curvas de pendiente creciente, contradiciendo a los resultados observados por Pascual et al. [35].

\subsection{Modelo de Müller [30]}

Este modelo fue expresamente desarrollado para describir la sinterización mezclas de partículas de vidrio y de inclusiones rígidas de pequeño tamaño, ambas con distribuciones de tamaño de partícula estrechas y similares.

Como ya se indicó en el apartado 2.1.3.1, la presencia de partículas rígidas en una matriz vítrea conduce frecuentemente a una porosidad residual en el compacto sinterizado. Para tener en cuenta este efecto, se considera que el compacto es un empaquetamiento de cuatro tipo diferentes de "clusters" constituidos cada uno por tres partículas (Figura 2.15). Asumiendo un 
mezclado ideal de las partículas, la probabilidad de ocurrencia de cada uno de los cuatro tipos de "clusters" depende únicamente de la fracción volumétrica del inerte o inclusión, $\phi$, (Figura 2.15). La contracción global de la probeta, s, es el resultado de la suma de las de los diferentes clusters, donde cada tipo de cluster sinteriza a una velocidad que es proporcional al $\mathrm{n}^{\circ}$ de contactos vidrio-vidrio. Es decir, en los "clusters" de tres partículas de vidrio el factor es 1 mientras que en los clusters de 2 partículas de vidrio es de 1/3. Los demás tipos de clusters no contribuyen. Con estas consideraciones y suponiendo que el polvo presenta una determinada distribución de tamaños de partículas, la ec. 2.3 se convierte:

$\mathrm{s}=\sum_{\mathrm{ij}} \Psi_{\mathrm{j}}\left(\mathrm{r}_{0 \mathrm{j}}\right) \cdot P_{\mathrm{i}} \cdot \mathrm{s}_{\mathrm{ij}}$ ec. 2.54

En esta ecuación: $\Psi_{j}\left(r_{0 j}\right)$, al igual que en la ec. 2.3, es la frecuencia relativa de cada fracción de tamaño de partícula, $r_{0 j} ; P_{i}$ es la probabilidad de ocurrencia de cada tipo de cluster (Figura 2.15); $\mathrm{s}_{\mathrm{ij}}$ es la sinterización de cada tipo de cluster "i" formado por partículas de tamaño $r_{0 j}$. Los valores de $\mathrm{s}_{\mathrm{ij}}$ se calculan como en el apartado 2.1.1.3. pero teniendo en cuenta el factor de corrección correspondiente a cada tipo de cluster y la sustitución de la viscosidad del vidrio, $\eta$, por una viscosidad efectiva del composite, dependiente del grado de avance de la sinterización, $\frac{\Delta \rho}{\Delta \rho_{\max }}$, y de la fracción volumétrica de inclusiones, $\phi$, de acuerdo con las ecuaciones:

$$
\begin{aligned}
& \frac{\eta_{c}^{\infty}}{\eta}=\left(1-\frac{\phi}{\phi_{\max }}\right)^{-n} \\
& \frac{\eta_{c}}{\eta}=1+\left(\frac{\Delta \rho}{\Delta \rho_{\max }}\right)^{\lambda^{*}} \cdot\left[\frac{\eta_{\mathrm{c}}^{\infty}}{\eta}-1\right]
\end{aligned}
$$


a)

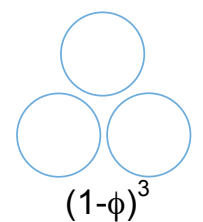

c)

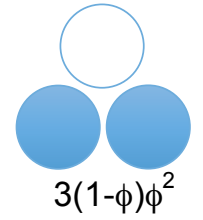

b)

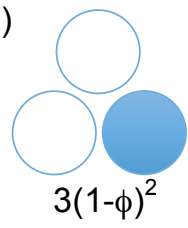

d)

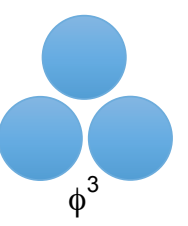

Figura 2.15. Tipos de agrupaciones de tres partículas vidrio-inclusión. Probabilidad de formación. $\phi$, fracción volumétrica de inclusiones rígidas.

La ec. 2.55 expresa el conocido efecto del contenido en sólidos, $\phi$, sobre la viscosidad de una suspensión de partículas dispersas, $\eta_{c}^{\infty}$, en un líquido o fundido de viscosidad $\eta$, es decir, $\eta_{c}^{\infty}$ es la viscosidad del composite exento de porosidad, cuando todas las inclusiones están completamente mojadas y dispersas en el fundido. En la ec. 2.56, el factor $\left(\frac{\Delta \rho}{\Delta \rho_{\max }}\right)^{\lambda^{*}}$ representa la fracción de la superficie de las inclusiones mojada por el fundido, y $\lambda^{*}$ un parámetro ajustable. De acuerdo con estas expresiones, la viscosidad efectiva relativa del composite, $\eta_{c} / \eta$, aumenta con el contenido en sólidos, $\phi$, de acuerdo con la expresión clásica de Kriegger-Dogerty (ec. 2.55) y con el avance de la sinterización, $\left(\frac{\Delta \rho}{\Delta \rho_{\max }}\right)$.

Según los autores, para que el modelo sea aplicable es imprescindible que se cumplan las siguientes restricciones:

i. La distribución de los tamaños de las partículas de las inclusiones debe ser similar a la del polvo de vidrio. 
ii. La homogeneidad de la muestra debe ser elevada.

Aun cumpliéndose estas condiciones, los autores comprobaron [30], mediante experimentos de sinterización a velocidad de calentamiento constante de composites vidrio-corindón, que el modelo era sólo aplicable para valores de $\phi \leq 0,35$. Recientemente [73], se ha aplicado este modelo al estudio de la sinterización de mezclas de partículas de vidrio y de hidroxiapatito, también mediante experimentos a velocidad de calentamiento constante. Al igual que en el caso anterior, los resultados sólo se adecuan aceptablemente bien al modelo para $\phi<0,35$. Así pues, en ambos casos, el modelo falla para valores de $\phi>0,35$, incluso al aplicarlo en experimentos no isotermos que, como se verá en el apartado 2.2.2, son mucho menos exigentes que los isotermos. Además, el modelo requiere cuatro parámetros de ajuste y una considerable complejidad de cálculo (debe recurrirse a aproximaciones numéricas). Todas estas razones cuestionan la utilidad práctica del modelo.

\subsection{Modelo empírico. Ecuación de Avrami de orden n.}

Mikeska, Scherer y Bordia [74] en una comunicación, en 1990, algunos años después de haber desarrollado sus respectivos modelos (apartados 2.1.4.2.1 y 2.1.4.3) describieron los resultados obtenidos al estudiar la sinterización de un composite comercial con un $33 \%$ de inclusiones cerámicas, mediante experimentos isotermos y a velocidad de calentamiento constante, registrando en continuo las dimensiones del compacto. Tras intentar analizar los resultados con sus respectivos modelos comprobaron la incapacidad de éstos para describir el comportamiento de composites constituidos por una matriz vítrea e inclusiones rígidas, cuando el volumen de estas últimas supera el límite de percolación, que situaron en el 16\%. En cambio, los resultados se ajustaron muy bien a la ecuación de Avrami de orden $\mathrm{n}$ (ec. 2.36), utilizando la nomenclatura original de los autores se tiene, para experimentos isotermos: 
$\frac{\Delta \rho}{\Delta \rho_{\max }}=1-\exp \left[-\left(\frac{\mathrm{t}}{\tau}\right)^{\mathrm{n}}\right]$

ec. 2.57

Y para no isotermos:

$\frac{\Delta \rho}{\Delta \rho_{\max }}=1-\exp \left[-\left(\int_{0}^{t^{\prime}} \frac{d t^{\prime}}{\tau}\right)^{n}\right]$

ec. 2.58

En ambos casos se consideraron una dependencia de la constante de tiempo, $\tau$, con la temperatura, $\mathrm{T}$, tipo Arrhenius:

$\tau=\tau_{0} \cdot \exp \left(\frac{Q_{v}}{R T}\right)$

Según estos autores, los resultados obtenidos en experimentos isotermos y no isotermos se ajustaron muy bien a la ec. 2.57 , ec. 2.58 y ec. 2.59, empleando los mismos valores para los parámetros $n, \tau_{0}$ y $Q_{v}$ en ambos casos. Además, el valor de $Q_{v}(\approx 470 \mathrm{~kJ} / \mathrm{mol})$ utilizado era el de la energía de activación correspondiente al flujo viscoso obtenido para la matriz vítrea, determinando experimentalmente su viscosidad a diferentes temperaturas.

2.1.4.3.3 Modelo reológico basado en la teoría continua de la sinterización. Efecto del crecimiento de grano sobre la densificación [75].

i) La presión de sinterización o de Laplace, $P_{L}$, cuando la densificación se desarrolla simultáneamente al crecimiento de grano por el mecanismo de solución-reprecipitación, empleada en el caso de metales y aleaciones con bajo contenido en líquido, es:

$P_{L}=\frac{2 \gamma}{G} F\left(\rho ; \rho_{0}\right)$

Siendo: $\gamma$ la energía superficial, $G$ el tamaño de grano y $F\left(\rho, \rho_{0}\right)$ el potencial de sinterización. Este último parámetro es una función, más o menos 
compleja, que expresa la influencia del avance de la sinterización sobre la presión de Laplace.

El módulo viscoso global, $\mathrm{K}_{\mathrm{p}}$, se expresa como:

$$
K_{p}=H\left(\rho ; \alpha^{\prime}, \rho_{0}\right) \frac{T G^{3}}{\exp \left(\frac{-Q_{d}}{R T}\right)}
$$

Siendo $H\left(\rho ; \alpha^{\prime}, \rho_{0}\right)$ una función que describe el efecto del aumento de la compacidad con el avance de la sinterización sobre $\mathrm{K}_{\mathrm{p}}$. Dicho parámetro depende también de la compacidad en crudo, $\rho_{0}$, y de un parámetro efectivo del material, $\alpha$, que incluye propiedades físicas y geométricas del mismo. $Q_{d}$ es la energía de activación del proceso de densificación del material.

ii) Puesto que tanto $P_{L}$ como $K_{p}$ dependen del tamaño de grano, $G$, para determinar la cinética de densificación mediante este modelo es imprescindible disponer de la ecuación que describe el crecimiento del tamaño de grano. Una de las expresiones más comunes es:

$$
\frac{d G}{d t}=\frac{k^{\prime}}{G^{2}}
$$

Ecuación análoga a la ec. 2.5 donde k' es un parámetro dependiente del material y de la temperatura:

$k^{\prime}=k_{0}^{\prime} \exp \left(\frac{-Q_{G}}{R T}\right)$

Siendo $k_{0}^{\prime}$ y $Q_{G}$ el factor preexponencial y la energía de activación para el crecimiento cristalino.

La integración de la ec. 2.62 a lo largo del ciclo térmico, T(t), conduce a la expresión: 
$\mathrm{G}^{3}=\mathrm{G}_{0}^{3}+3 \int_{0}^{\mathrm{t}} \mathrm{k}_{0}^{\prime} \exp \left(\frac{-\mathrm{Q}_{\mathrm{G}}}{\mathrm{RT}}\right) \mathrm{dt}$

ec. 2.64

Siendo $\mathrm{G}_{0}$ el tamaño de grano inicial.

iii) De la ec. 2.17 , ec. 2.60 y ec. 2.61 se obtiene la expresión de la velocidad de sinterización:

$\frac{-d \varepsilon_{V}}{d t}=\frac{P_{L}}{K_{p}}=\frac{2 \gamma}{T G^{4}} \frac{F\left(\rho ; \rho_{0}\right)}{H\left(\rho ; \alpha, \rho_{0}\right)} \exp \left(\frac{-Q_{d}}{R T}\right)$

en la que para su integración se necesita disponer de la ec. 2.64 de forma integrada.

\subsection{Análisis de los métodos de determinación de los parámetros cinéticos de una transformación mediante análisis térmico. Su adaptación al estudio de la sinterización.}

\subsubsection{Aspectos generales.}

i) La mayoría de los métodos cinéticos usados en el área de análisis térmico (ATD, DSC, TG, HSM etc.) consideran que la velocidad del proceso, $\frac{\mathrm{d} \alpha}{\mathrm{dt}}$, es sólo función de los valores de la temperatura, T, y del grado de conversión $\alpha$, de acuerdo con la ec. 2.44. En ella, la dependencia de la velocidad del proceso con la temperatura, está representada por la constante de velocidad $\mathrm{K}(\mathrm{T})$, y la dependencia del grado de sinterización por el modelo de sinterización, $f(\alpha)$.

La ec. 2.44, describe la velocidad del proceso que ocurre en una sola etapa. El grado de sinterización, $\alpha$, varía entre 0 y 1 , conforme el proceso progresa desde el inicio hasta que se completa. Debe resaltarse que la propiedad física que medimos, cambio de tamaño de la pieza, usualmente no puede relacionarse directamente con transformaciones o reacciones específicas. 
Por esta razón, el valor de $\alpha$ típicamente refleja el proceso global de la densificación del material, el cual, generalmente, involucrará más de una transformación sencilla, o en otras palabras, múltiples etapas. Una de la principales recomendaciones del Comité de Cinética del ICTAC (International Confederation for Thermal Analysis and Calorimetry) para que los modelos cinéticos sean reproducibles es que sean capaces de detectar y tratar cinéticas multietapa.

Conviene recalcar que, aunque un proceso quede bien descrito con la ec. 2.44, ello no supone necesariamente que el mecanismo del proceso consiste en una única etapa. Muy probablemente, lo que ocurre es que el mecanismo del proceso involucra algunas etapas, pero sólo una de ellas, la controlante, determina la velocidad del mismo.

La dependencia con la temperatura de la velocidad del proceso típicamente se parametriza con la ecuación de Arrhenius:

$K(T)=A \cdot \exp \left(\frac{-E}{R T}\right)$

Donde $A$ y $E$ son los parámetros cinéticos, factor pre-exponencial y energía de activación, respectivamente, y $\mathrm{R}$, la constante universal de los gases. No obstante, se ha constatado [76] que algunos procesos no obedecen la ley de Arrhenius. Los parámetros cinéticos determinados experimentalmente se denominan apropiadamente "efectivos", "aparentes" o "globales" para recalcar el hecho que estos, frecuentemente, no corresponden a los parámetros intrínsecos de ciertas etapas individuales.

En efecto, la complejidad del proceso de sinterización de composites, estudiado por el HSM (microscopio de calefacción), imposibilita que se puedan obtener los parámetros cinéticos intrínsecos de una etapa, como por ejemplo, de la sinterización propiamente dicha por flujo viscoso, sin que éstos estén afectados por las contribuciones cinéticas de otras etapas, como la disolución parcial de partículas más refractarias o la cristalización de fases en el fundido, etc. Así pues, en general, los parámetros cinéticos efectivos 
que se obtienen, son una combinación de los parámetros cinéticos intrínsecos de las etapas individuales que intervienen en el proceso.

La relación entre la velocidad del proceso, da/dt, y el grado de sinterización, $\alpha$, se puede expresar utilizando un modelo cinético de sinterización genérico $f(\alpha)$.

De todos aquellos modelos que son del tipo decelerante, y que, por tanto, en condiciones isotermas, $\mathrm{T}=$ constante, describen procesos cuya velocidad disminuye, conforme el grado de avance progresa, los más empleados se detallan en la Tabla 2.5. Nótese, que para $n=1$ los modelos de Avrami y de orden de reacción coinciden.

ii) El tratamiento térmico puede ser isotermo, $\mathrm{T}=$ cte., o no isotermo. Para cualquier tratamiento térmico, combinando la ec. 2.44 y ec. 2.66 , se obtiene:

$\frac{d \alpha}{d t}=A \cdot \exp \left(\frac{-E}{R T}\right) \cdot f(\alpha)$

En esta forma, la ecuación es aplicable a cualquier programa de temperatura, sea isotermo o no isotermo, e incluso permite introducir la variación de la temperatura verdadera de la muestra $T(t)$, cuando ésta se desvía significativamente de la temperatura del horno. En condiciones de velocidad de calentamiento constante, $\beta=\frac{\mathrm{dT}}{\mathrm{dt}}$, la ec. 2.67 se transforma en:

$\beta \cdot \frac{d \alpha}{d T}=A \cdot \exp \left(\frac{-E}{R T}\right) \cdot f(\alpha)$

Se deduce fácilmente que, en un experimento a velocidad de calentamiento constante, puesto que $T$ y $\alpha$, y por supuesto $f(\alpha)$, varían simultáneamente, y su efecto sobre la velocidad del proceso es antagónica, la representación de (d $\alpha / d T)$ vs T o $\alpha$ presenta siempre un máximo. Y, en consecuencia, la curva de sinterización $\alpha$ vs T es una curva sigmoidal. 
Obviamente, la introducción del valor explícito de la velocidad de calentamiento, $\beta$, reduce la aplicabilidad de la ec. 2.68 a procesos en los que la temperatura de la muestra no se desvía significativamente de la temperatura de referencia, lo cual es cuestionable para tamaños de muestra o velocidades de calentamiento grandes.

La integración de la ec. 2.67 conduce a:

$g(\alpha)=\int_{0}^{\alpha} \frac{d \alpha}{f(\alpha)}=A \int_{0}^{t} \exp \left(\frac{-E}{R T}\right) d t$

Donde $g(\alpha)$ es la forma integral del modelo de reacción $f(\alpha)$. La ec. 2.69 es aplicable a cualquier programa de temperatura, sustituyendo $\mathrm{T}$ por $\mathrm{T}(\mathrm{t})$. Para condiciones de velocidad de calentamiento constante, la integral con respecto al tiempo normalmente se sustituye por la integral respecto a la temperatura.

$g(\alpha)=\int_{0}^{\alpha} \frac{d \alpha}{f(\alpha)}=\frac{A}{\beta} \int_{0}^{T} \exp \left(\frac{-E}{R T}\right) d T$

Uno de los propósitos de análisis cinético del proceso de sinterización, es establecer la relación matemática entre la velocidad de sinterización, (da/dt), el avance del proceso, $\alpha, y$ la temperatura, T. Para procesos sencillos, que se desarrollan en una sola etapa, o que sólo una etapa del proceso es la controlante, la determinación de $\mathrm{A}$, E y $\mathrm{f}(\alpha) \circ \mathrm{g}(\alpha)$, también denominado "triplete cinético" debería ser suficiente para predecir la cinética del proceso para cualquier programa de temperatura $\mathrm{T}(\mathrm{t})$. En el caso de procesos de sinterización más complejos, integrados por una combinación de etapas individuales (transformaciones), consecutivas y/o paralelas, se deberían de determinar múltiples tripletes cinéticos. 
Tabla 2.5. Tipos de modelos cinéticos decelerantes de sinterización.

\begin{tabular}{|l|l|l|}
\hline Modelo & $f(\alpha)$ & $g(\alpha)$ \\
\hline \multirow{2}{*}{ Ley de la potencia } & $\alpha^{-n}$ & $\frac{1}{n+1} \alpha^{n+1}$ \\
\hline \multirow{2}{*}{ Orden de reacción } & $(1-\alpha)^{n}$ & $\frac{1}{n-1} \cdot\left(\frac{1}{\left.(1-\alpha)^{n-1}-1\right)}\right.$ \\
\cline { 2 - 3 } & $(1-\alpha)$ & y $n \neq 1)$ \\
\hline \multirow{2}{*}{ Avrami-Erofeev } & $(n=1)$ & $-\ln (1-\alpha)$ \\
\cline { 2 - 4 } & $(0<n<1)$ & {$[-\ln (1-\alpha)]^{\frac{1}{n}}$} \\
\cline { 2 - 4 } & $(1-\alpha)$ & $-\ln (1-\alpha)$ \\
\hline
\end{tabular}

\subsubsection{Ventajas e inconvenientes de la experimentación a velocidad de} calentamiento constante sobre la isoterma

En todo experimento isotermo, siempre existe un periodo asociado al calentamiento de la muestra en el que no se cumple la condición de temperatura constante. De ahí, que sólo puedan obtenerse buenos datos en un limitado intervalo de temperatura. En efecto, a bajas temperaturas, a las que corresponde una baja velocidad de sinterización, puede que no se alcance la conversión completa en un tiempo razonable. En otros casos, el desarrollo de otras transformaciones concurrentes, como la desvitrifivación de fases cristalinas, que pueden darse a velocidades apreciables, puede llegar a paralizar la sinterización. A temperaturas elevadas, a las que la 
velocidad de sinterización es alta, el periodo de calentamiento de la muestra se vuelve comparable al tiempo característico del proceso, por lo que la mayor parte de la sinterización de la muestra ocurre en estado no estacionario. Además, en este último caso, se potencia la formación de porosidad cerrada, impidiendo que se complete la densificación. Estos problemas son fácilmente evitables en experimentos a velocidad de calentamiento constante, si el calentamiento comienza a temperaturas muy por debajo de la que empieza a producirse la sinterización.

Una de las desventajas de este último método, reside en que la velocidad de calentamiento es una variable crítica, que figura en todas las expresiones para el cálculo de los parámetros cinéticos, por lo que cualquier desviación entre la velocidad de calentamiento programada y la que experimenta la muestra puede ocasionar grandes errores en la determinación de E y A. Estas desviaciones son tanto mayores cuanto mayores son las dimensiones de la muestra y la velocidad de calentamiento. Para tratar de minimizar estos problemas conviene, además de reducir al máximo el tamaño de la muestra, realizar más de tres experimentos a velocidades de calentamiento diferentes, analizando en su caso cualquier desviación sistemática.

La mayor desventaja de los experimentos a velocidad de calentamiento constante es la dificultad de seleccionar o identificar el modelo cinético, $f(\alpha)$ o $g(\alpha)$, que sigue el proceso ya que en este tipo de ensayos, el avance del proceso con la temperatura o la variación de la velocidad de sinterización con esta variable depende simultáneamente de la constante de velocidad, que varía con la temperatura según la ecuación de Arrhenius, y del modelo cinético, que también varía con la temperatura, puesto que $\alpha$ también varía con $\mathrm{T}$. Ahora bien, el efecto de la temperatura sobre $\mathrm{K}(\mathrm{T})$ es mucho mayor que sobre $f(\alpha) \circ g(\alpha)$, en este tipo de experimentos, por lo que, a menudo, varios modelos cinéticos ajustan bien los resultados experimentales, si solo se emplean una velocidad de calentamiento y se elije individualmente la energía de activación.

En consecuencia, se recomienda realizar al menos una serie de 
experimentos isotermos, a una temperatura determinada, además de varias series de experimentos a velocidad de calentamiento constante (al menos a tres velocidades). De este modo, la serie isoterma facilitaría la selección del modelo de reacción y también podría ayudar a verificar la validez de los tripletes cinéticos obtenidos de las series a velocidad de calentamiento constante comprobando si los valores de estos parámetros, $f(\alpha)$, E y $A$ pueden ser utilizados satisfactoriamente para predecir series isotermas. De hecho, el verdadero buen modelo debería ajustar simultáneamente los dos tipos de experimentos con los mismos parámetros cinéticos. Solo obteniendo una buena correspondencia, utilizando los mismos parámetros cinéticos, en un amplio rango de temperaturas $y / o$ velocidades de calentamiento podemos estar seguros de su certeza.

\subsubsection{Métodos isoconversionales}

\subsubsection{Aspectos generales}

Todos estos métodos se basan en el siguiente principio:

"La velocidad de reacción (sinterización) para un grado de conversión (sinterización) constante sólo depende de la temperatura".

Es decir, que la velocidad de sinterización, en lo que al programa térmico se refiere, sólo depende de la temperatura $(T)$ y no de la historia térmica. Dicha hipótesis suele no cumplirse en el caso de que concurran simultáneamente otras transformaciones (separación de fases, cristalizaciones, etc.) frecuentes en la cocción de vidrios y composites.

En el supuesto que se considere válido este principio, tomando logaritmos naturales en la ec. 2.44 y derivando respecto de $\mathrm{T}^{-1}$, para $\alpha=$ constante, se obtiene:

$$
\left[\frac{\partial \ln \left(\frac{d \alpha}{d t}\right)}{\partial T^{-1}}\right]_{\alpha}=\left[\frac{\partial \ln K(T)}{\partial T^{-1}}\right]_{\alpha}+\left[\frac{\partial \ln f(\alpha)}{\partial T^{-1}}\right]_{\alpha}
$$


Donde $\alpha$ indica el valor del grado sinterización fijado. Ahora bien, puesto que $\alpha=$ constante, $f(\alpha)$, también lo será, por lo que su derivada se hace cero. Así pues:

$\left[\frac{\partial \ln \left(\frac{d \alpha}{d t}\right)}{\partial T^{-1}}\right]_{\alpha}=-\frac{E}{R}$ ec. 2.72

De la ec. 2.72 se desprende que determinando la velocidad del proceso puede obtenerse un valor de la energía de activación para un determinado valor de $\alpha$, sin necesidad de asumir a priori ningún modelo de sinterización. Por este motivo, a estos métodos se les denomina también "model-free", o de modelo libre. Ahora bien, aunque estos métodos no necesitan identificar el modelo de sinterización, $f(\alpha)$ o $g(\alpha)$, es imprescindible, para su aplicación correcta, que en todo el proceso se sigua el mismo modelo.

Para determinar $E_{\alpha}$, o en su caso la variación de $E_{\alpha}$ con $\alpha, E_{\alpha}(\alpha)$, se programan series de experimentos a varias velocidades de calentamiento, y/o series isotermas a varias temperaturas $y$, de acuerdo con la ec. 2.72, se determinan los valores de $\mathrm{E}_{\alpha}$ dentro de un amplio intervalo de $\alpha$ $(0,05 \leq \alpha \leq 0,95)$. La dependencia $E_{\alpha}(\alpha)$ es importante para detectar, $y$ en su caso tratar, los procesos complejos con múltiples etapas. Una variación significativa de $\mathrm{E}_{\alpha}$ con $\alpha$ implica que el proceso es complejo, y que, por tanto, no puede describirse adecuadamente mediante una ecuación sencilla del tipo de la ec. 2.44 , ec. 2.67 o ec. 2.68 .

\subsubsection{Método isoconversional diferencial}

Este método, también denominado de Friedman, está basado en la ecuación: 
$\ln \left[\left(\frac{d \alpha}{d t}\right)_{\alpha, i}\right]=\ln \left[f(\alpha) \cdot A_{\alpha}\right]-\frac{E_{\alpha}}{R T_{\alpha, i}}$

Ecuación que resulta de aplicar el principio isoconversional a la ec. 2.67. Como la ec. 2.67, la ec. 2.73 es aplicable a cualquier programa de temperatura.

A cada valor de $\alpha$ el valor de $\mathrm{E}_{\alpha}$ se determina de la pendiente de la representación de $\ln (d \alpha / d t)_{\alpha, i}$ frente $1 / T_{\alpha, i}$. El subíndice $i$ se introduce para indicar varios programas de temperatura. $T_{\alpha, i}$ es la temperatura a la que el grado de sinterización $\alpha$ ha sido alcanzado, empleando el programa i. Para programas isotermos, i, identifica la temperatura de cada serie. Para series de velocidad de calentamiento constante, i, identifica la velocidad individual de calentamiento de cada serie. En este caso, la ec. 2.73 se transforma en:

$\ln \left[\beta_{\mathrm{i}}\left(\frac{\mathrm{d} \alpha}{\mathrm{dt}}\right)_{\alpha, \mathrm{i}}\right]=\ln \left[\mathrm{f}(\alpha) \cdot \mathrm{A}_{\alpha}\right]-\frac{\mathrm{E}_{\alpha}}{\mathrm{RT} \mathrm{T}_{\alpha, \mathrm{i}}}$

Esta ecuación asume que $T_{\alpha, i}$ cambia linealmente con el tiempo de acuerdo con la velocidad de calentamiento, $\beta_{i}$. Esto implica que no puede sustituirse la temperatura real de la probeta por $T_{\alpha, i}$ en la ec. 2.74 para tener en cuenta el efecto del autoenfriamiento que se produce en reacciones endotérmicas, como la sinterización.

En principio este método no hace uso de ninguna aproximación, a excepción del principio de isoconversión (ec. 2.71), por lo que potencialmente es más fiel o exacto que el método integral que se considerará a continuación. Ahora bien, puesto que los datos que obtenemos del microscopio de calefacción (HSM), son en forma integral (contracción en función de la temperatura), es decir $\alpha=\alpha(T)$, la aplicación de métodos de diferenciación 
numérica a los datos originales en forma integrada, introducen imprecisión (o ruido) en los valores de ( $d \alpha / d t$ ) vs $t$, por lo que al tratar de suavizar estas curvas, (reducir el ruido), podemos perder exactitud. Así pues, en nuestro caso, el método diferencial no supone, en principio, mayor precisión o exactitud que el integral.

\subsubsection{Método isoconversional integral}

Los métodos isoconversionales integrales derivan de la aplicación del principio de isoconversional a la integral de la ec. 2.69. Ahora bien, dicha integral no tiene solución analítica para cualquier programa de temperatura. No obstante, para tratamientos isotermos conduce a:

$g(\alpha)=A \cdot \exp \left(\frac{-E}{R T}\right) \cdot t$

que, por aplicación del principio isoconversional y reordenando términos, se convierte:

$\operatorname{lnt} t_{\alpha, i}=\ln \left[\frac{g(\alpha)}{A_{\alpha}}\right]+\frac{E_{\alpha}}{R T_{i}}$ ec. 2.76

donde $t_{\alpha, i}$ es el tiempo al que se alcanza un determinado grado de sinterización a diferentes temperaturas, $T_{i}$. Para cada valor de $\alpha$, el valor de $E_{\alpha}$ se determina de la pendiente que resulta de representar $\operatorname{lnt}_{\alpha, \mathrm{i}}$ frente a $1 / T_{i}$.

Como ya se ha indicado anteriormente, en experimentos a velocidad de calentamiento constante la ec. 2.70 debe integrarse. La integral del segundo miembro de dicha igualdad no tiene solución analítica. Por esta razón se pueden obtener expresiones ligeramente distintas, que sólo difieren en las aproximaciones utilizadas en el cálculo de la integral de la temperatura. En 
efecto, la integración de la ec. 2.70 conduce a la expresión:

$g(\alpha)=\frac{A}{\beta} \int_{0}^{T} \exp \left(\frac{-E}{R T}\right) d T=\frac{A}{\beta} T E_{2}^{*}\left(\frac{E}{R T}\right)$

Donde $\mathrm{E}_{2}^{*}$ es la función exponencial de orden 2, definida como:

$E_{2}^{*}\left(\frac{E}{R T}\right)=\left[\frac{\exp \left(\frac{-E}{R T}\right)}{\left(\frac{E}{R T}+2\right)}\right) \cdot(1+\Re)$

siendo $\Re$ un desarrollo en serie, cuyo primer término es: $\Re \cong 2 \cdot \frac{1}{\left(\frac{\mathrm{E}}{\mathrm{RT}}+2\right)^{2}}$

para valores de $\frac{E}{R T}>>2$, que es lo usual en estos procesos. $\Re<<$ por lo que la ec. 2.77 se convierte en:

$g(\alpha)=\frac{A R T^{2}}{\beta E} \exp \left(\frac{-E}{R T}\right)$

reordenando términos y tomando logaritmos se tiene:

$\ln \left(\frac{\beta}{T^{2}}\right)=\ln \frac{A R}{E \cdot g(\alpha)}-\frac{E}{R T}$

que al aplicar el principio isoconversional se convierte en:

$\ln \left(\frac{\beta_{i}}{T_{\alpha, i}^{2}}\right)=\operatorname{cte}-\frac{E_{\alpha}}{R T_{\alpha, i}}$

Así pues, como en el método anterior, para cada valor de $\alpha$, el valor de $\mathrm{E}_{\alpha}$ 
se determina de la pendiente que resulta al representar los valores de $\ln \left(\frac{\beta_{i}}{T_{\alpha, i}^{2}}\right)$ frente a los de $1 / T_{\alpha, i}$. Como en el apartado anterior, el subíndice " $\mathrm{i}$ " hace referencia a la velocidad de calentamiento. A esta expresión se le denomina frecuentemente ecuación de Kissinger-Akahira-Sunose (KAS) [77].

La aproximación utilizada por Doyle [78] para resolver la integral de la temperatura conduce a la misma expresión que la conocida ecuación de Ozawa [79] o de Flynn and Wall [80]:

$\ln \left(\beta_{\mathrm{i}}\right)=\operatorname{cte}-\frac{\mathrm{E}_{\alpha}}{\mathrm{RT} \mathrm{T}_{\alpha, \mathrm{i}}}$

Los valores $E_{\alpha}$ obtenidos por esta ecuación son bastante inexactos, debido a que la resolución de la integral exponencial que conduce a la ec. 2.82 es poco rigurosa.

Más recientemente Starink [81] ha comprobado que la mayor exactitud en la estimación de $E_{\alpha}$ se obtiene mediante la ecuación:

$\ln \left(\frac{\beta_{\mathrm{i}}}{\mathrm{T}_{\alpha, \mathrm{i}}^{1,92}}\right)=\operatorname{cte}-1,0008 \cdot \frac{\mathrm{E}_{\alpha}}{\mathrm{RT}_{\alpha, \mathrm{i}}}$ ec. 2.83

que como puede comprobarse, prácticamente coincide con la ec. 2.81.

Los métodos isoconversionales integrales que acabamos de describir (ec. 2.81, ec. 2.82 y ec. 2.83 ) están basados en la resolución de la integral de temperatura, suponiendo que el valor de $\mathrm{E}_{\alpha}$ permanece constante sobre el intervalo de integración, es decir $\mathrm{E}_{\alpha}$ es independiente de $\alpha$. En la práctica, comúnmente, $\mathrm{E}_{\alpha}$ varía con $\alpha$ [76][82], lo que introduce un error sistemático en la determinación de $\mathrm{E}_{\alpha}$, que puede ser superior al 20-30\% si la variación 
de $E_{\alpha}$ con $\alpha$ es grande [83]. Esta fuente de error no se da en el método diferencial de Friedman (apartado 2.2.3.2).

Así pues, a modo de resumen, se puede concluir que de los métodos isoconvercionales, el método diferencial de Friedman es uno de los más universales en su aplicación. Sin embargo, en muchos casos, los métodos integrales (ec. 2.40 , ec. 2.44 , ec. 2.45 y ec. 2.46), más sencillos, son suficientes. Ahora bien, en aquellos casos en los $E_{\alpha}$ varía significativamente con $\alpha$, es decir, cuando la diferencia entre los valores máximo y mínimo de $E_{\alpha}$, sea mayor que el $20-30 \%$ del valor de $E_{\alpha, \text { medio }}$, debe recurrirse al método de Friedman u otros más avanzados [84]

\subsubsection{Causas de la variación de $\mathrm{E}_{\alpha}$ con $\alpha$.}

Si $E_{\alpha}$ es rigurosamente constante en todo el intervalo de sinterización y no se observan ni picos ni hombros en la curva de sinterización, a velocidad de calentamiento constante, $\alpha=\alpha(T)_{\beta}$, o en la curva de velocidad de sinterización, $\frac{d \alpha}{d t}$ vs t ó T en experimentos isotermos o no isotermos, es muy probable que el proceso esté controlado por una sola etapa de transformación (física o química) y que, por tanto, pueda describirse por un modelo de etapa única ("single-step"). Sin embargo, es de preveer, al tratar la sinterización de algunos composites, que $\mathrm{E}_{\alpha}$ varíe con $\alpha$ y que la curva de sinterización, $\alpha=\alpha(T)_{\beta}$, se aparte de la forma sigmoidal. En este caso, se trata de un proceso controlado por múltiples etapas paralelas y/o consecutivas.

En general, una variación sistemática, más o menos pronunciada, de $\mathrm{E}_{\alpha}$ con $\alpha$ en la sinterización de vidrios, composites de matriz vítrea, y en general, de materiales que sinterizan por un mecanismo de flujo viscoso (porcelanas, gres, porcelánico, etc) indica un cambio de mecanismo controlante del 
proceso. Así por ejemplo, un aumento de $\mathrm{E}_{\alpha}$ con $\alpha$ en la sinterización de vidrios con desvitrificación concurrente se explica basándose en el aumento de la viscosidad aparente o efectiva del sistema [85][86]. Análogamente, en la sinterización de esmaltes, en experimentos isotermos y no isotermos, se aprecia un cambio importante en la energía de activación al pasar de los estados iniciales del proceso, en el que el mecanismo controlante de la densificación es la reordenación de las partículas en presencia de fase líquida, a la de los estados intermedios, controlado por el mecanismo de solución-precipitación [70]. No obstante, en otros casos, como en la sinterización de vidrios, que no desvitrifican en todo el proceso, se ha comprobado que $\mathrm{E}_{\alpha}$ disminuye con $\alpha$ de forma suave pero progresiva. Dicha variación se asocia con que la fluidez del vidrio (inversa de su viscosidad), directamente proporcional a la constante de velocidad de sinterización por flujo viscoso de vidrios, no se ajusta exactamente a Arrhenius.

Así pues, exceptuando este último caso, y algunos casos que se detallan en la bibliografía [87][88], en el que la variación de $\mathrm{E}_{\alpha} \operatorname{con} \alpha$ no supone un proceso complejo en varias etapas, la variación progresiva o brusca de $\mathrm{E}_{\alpha}$ con $\alpha$ fácilmente perceptible en la curva de sinterización debe tratarse como procesos multi-etapa.

\subsubsection{El método Kissinger [89]}

De los métodos de velocidad de calentamiento múltiple, éste ha sido el más utilizado, a pesar de que presenta algunas limitaciones importantes, que derivan de que en su desarrollo se asumen algunas hipótesis simplificativas de dudosa validez. El método se basa en aplicar la condición matemática de máximo, indicado por el subíndice m, a la ecuación de velocidad (ec. 2.68). En este punto: 
$\left(\frac{d^{2} \alpha}{d T^{2}}\right)=\beta^{2} \cdot\left(\frac{d^{2} \alpha}{d T^{2}}\right)_{m}=0$

ec. 2.84

Con lo que la ec. 2.68 se transforma en la ec. 2.85 :

$\beta^{2}\left(\frac{d^{2} \alpha}{d T^{2}}\right)_{m}=\beta \cdot\left(\frac{d \alpha}{d T}\right)\left[\frac{E \beta}{R T_{m}^{2}}+A\left(\frac{d f(\alpha)}{d \alpha}\right) \cdot \exp \left(\frac{-E}{R T_{m, i}}\right)\right]=0$

Reordenando términos y tomando logaritmos se transforma:

$\ln \left(\frac{\beta_{i}}{T_{m, i}^{2}}\right)=\ln \left(\frac{-A R}{E}\left(\frac{d f(\alpha)}{d \alpha}\right)_{m}\right)-\frac{E}{R T_{m, i}}$

Al representar el primer miembro de la ecuación frente a $\frac{1}{T_{m, i}}$ se obtiene una recta de pendiente, $\frac{E}{R}$, de donde se calcula la energía de activación.

Una de las limitaciones de este método, deriva del hecho de que $\left(\frac{\mathrm{df}(\alpha)}{\mathrm{d} \alpha}\right)_{\mathrm{m}}$ debe ser independiente de la velocidad de calentamiento, $\beta_{\mathrm{i}}$.

En efecto, si el primer término del segundo miembro de la ec. 2.86 no fuera constante, la representación de $\ln \frac{\beta_{\mathrm{i}}}{\mathrm{T}_{\mathrm{m}, \mathrm{i}}^{2}}$ vs $\frac{1}{\mathrm{~T}_{\mathrm{m}, \mathrm{i}}}$ debería desviarse consecuentemente de una línea recta, provocando un error sistemático en la pendiente $\frac{E}{R}$. El término $\left(\frac{d f(\alpha)}{d \alpha}\right)_{m}$ sólo es estrictamente independiente de $\beta$ para transformación de orden 1 , es decir, $f(\alpha)=(1-\alpha)$.

Para otros modelos cinéticos, la variación de $\alpha$ en el máximo, $\alpha=\alpha_{m}$, con 
la velocidad de calentamiento, $\beta$, puede modificar el valor de $\left(\frac{\mathrm{df}(\alpha)}{\mathrm{d} \alpha}\right)_{\mathrm{m}}$ con $\beta$. La variación de $\alpha_{m}$ con $\beta$ es también despreciable para los modelos de Avrami-Erofeev [90][91]. Sin embargo, $\alpha_{m}$ puede variar significativamente para otros modelos [92][93].

De forma general, un cambio significativo de la forma del pico en la curva, $\frac{\mathrm{d} \alpha}{\mathrm{dT}}$ vs $\mathrm{T}$, con la velocidad de calentamiento, $\beta$, implica una variación también significativa de $\alpha_{\mathrm{m}}$ con $\beta$ [94]. También hay que resaltar que el error sistemático que se comete por este concepto disminuye conforme aumenta $\frac{E}{R T}$, de tal modo que para valores de $\frac{E}{R T}>10$, el error de $E$ no excede del 5\% en la mayoría de los modelos cinéticos [93][95][96].

\subsubsection{Métodos de ajuste al modelo}

La obtención de los parámetros cinéticos mediante estos métodos está asociada al modelo de sinterización, $f(\alpha) \circ g(\alpha)$, seleccionado. Estos métodos difieren significativamente en su fiabilidad. En particular, los resultados basados en el ajuste a un modelo de los datos obtenidos a una única velocidad de calentamiento, $\beta$, son muy poco fiables [97]. Por el contrario, cuando series de datos, obtenidos a diferentes velocidades de calentamiento, se ajustan simultáneamente al modelo apropiado, los resultados obtenidos son bastante fiables, tanto o más que los modelos isoconversionales [98]. Además, éste es el procedimiento idóneo para la descripción de cinéticas complejas que requieren modelos multietapa, y el único aplicable cuando no se cumple el principio de isoconversión.

La primera etapa, y quizás la más importante, de estos métodos es la selección del modelo apropiado.

En primer lugar, es esencial, a la vista de las curvas de sinterización, $g(\alpha)$ vs t o $f(\alpha)$ vs $t$, constatar la existencia o no de etapas múltiples, basada en la 
presencia de puntos de inflexión y hombros en la curvas de sinterización. Los datos en forma diferencial, $\frac{\mathrm{d} \alpha}{\mathrm{dt}}$ vs $\alpha, \frac{\mathrm{d} \alpha}{\mathrm{dt}}$ vs $\mathrm{T}$, son más sensibles al efecto.

Si se detecta la coexistencia de transformaciones múltiples paralelas, parcialmente solapadas o no, que es lo que ocurre frecuentemente, entonces resulta imprescindible utilizar métodos de regresión no lineal. En este caso se suele recurrir, si es posible, a un mecanismo multietapa, formado por una combinación en paralelo del mínimo número posible de etapas individuales de cinéticas sencillas.

$\mathrm{Si}$, por el contrario, no hay evidencia de un proceso multietapa, al examinar las curvas de sinterización, preferiblemente en forma diferencial, y además los valores de energía de activación, $\mathrm{E}_{\alpha}$, obtenidos por métodos isoconversionales, no varían significativamente con el avance de la sinterización, $\alpha$, los datos experimentales deben de tratar de ajustarse a los modelos cinéticos sencillos. Ahora bien, en aquellos casos en los que no son aplicables los métodos isoconversionales, porque la velocidad de sinterización, $\frac{\mathrm{d} \alpha}{\mathrm{dt}}$, depende no sólo de la temperatura y del grado de sinterización, $\alpha$, sino también de la historia térmica, también es necesario probar distintos modelos, $\mathrm{j}$, con los datos experimentales obtenidos a velocidades de calentamiento diferentes.

Por otra parte, reordenando factores y tomando logaritmos naturales de la ec. 2.79 se tiene:

$\ln \left(\frac{g_{j}(\alpha)}{T^{2}}\right)=\ln \left(\frac{A_{j} R}{\beta E_{j}}\right)-\frac{E_{j}}{R T}$

el subíndice "j" se refiere al modelo $g_{j}(\alpha)$ ensayado.

En el tratamiento de los datos resultantes de los ensayos a una velocidad de 
calentamiento constante, es frecuente, que varios modelos describan adecuadamente los resultados [97], y, lo que es peor, que los valores de $A_{j}$ y $E_{j}$ sean muy dependientes del modelo $g_{j}(\alpha)$ elegido. Para reducir esta ambigüedad, como ya se ha indicado anteriormente, es imprescindible que los resultados de varías series de experimentos a velocidad de calentamiento constante conduzcan al mismo valor de los parámetros cinéticos. Además, en muchos casos, es recomendable recurrir a ensayos isotermos. La bondad del ajuste obtenido al modelo seleccionado no garantiza que la descripción cinética que se hace del proceso sea adecuada. Una variación sustancial con la velocidad de calentamiento, $\beta$, de los parámetros cinéticos, $A_{j}$ y $E_{j}$, incluso del intervalo de grado de sinterización en que los resultados se adaptan a un determinado modelo $g_{j}(\alpha)$, ponen claramente de manifiesto la existencia de transformaciones concurrentes dependientes de la historia térmica.

\subsubsection{Resumen de las recomendaciones del "Kinetics Committee of} the International Confederation of Thermal Analysis and Calorimetry (ICTAC)" para el cálculo de parámetros cinéticos de datos de análisis térmicos [98].

Para el cálculo de parámetros cinéticos de datos de análisis térmicos se recomiendan las siguientes etapas:

i. La obtención de datos de calidad, utilizando diferentes programas térmicos, al menos tres experimentos a velocidad de calentamiento constante y/o algunos isotermo.

ii. En segundo lugar, se debe de aplicar al menos, un método isoconversional, para obtener la relación entre la energía de activación, $E_{\alpha}$, y el grado de avance del proceso, $\alpha$.

iii. Si $\mathrm{E}_{\alpha}$ no varía significativamente con $\alpha$, el proceso de sinterización puede ser, generalmente, adecuadamente descrito por un modelo 
cinético sencillo, consistente en una única etapa. En este caso, utilizando un modelo de ajuste lineal o no, se determinará el triplete cinético, es decir, el modelo cinético, $f(\alpha) \circ g(\alpha)$, el factor preexponencial, $A$, y la energía de activación, $E$.

iv. Si $E_{\alpha}$ varía significativamente con $\alpha$, el proceso debe ser descrito mediante un modelo multietapa, requiriendo múltiples tripletes cinéticos. En este caso, es necesario recurrir a modelos de ajuste no lineal, y es recomendable seguir los siguientes pasos:

a) Seleccionar el apropiado esquema cinético. Para ello se requiere decidir el mecanismo formal del modelo (etapas paralelas, consecutivas o combinación de unas y otras o ambas). Siempre que se pueda, se recomienda recurrir a una combinación de etapas en paralelo, ya que su solución numérica es más sencilla.

b) Decidir el número de etapas individuales a incluir en el esquema cinético. En general, cada punto de inflexión y/u hombro en la curva ( $\alpha$ vs $T, \frac{d \alpha}{d t}$ vs $T \circ \frac{d \alpha}{d t}$ vs $t$ ), representa al menos una etapa de transformación.

c) Seleccionar el modelo cinético apropiado a cada etapa individual del proceso de sinterización. En este sentido siempre que se pueda, deben de elegirse entre los modelos cinéticos apropiados, aquellos más sencillos, y si es posible, el mismo para todas las etapas. 


\section{Objetivo y plan de trabajo}

Las principales razones que han motivado la selección del tema, la elaboración del plan de trabajo y su ejecución, objeto de esta Tesis, son, entre otros, los siguientes:

i) La información de que se dispone sobre el tema es incompleta. En efecto, muchos materiales cerámicos se adecuan mejor a sus funciones si son compuestos; es decir, son el resultado de la adición controlada de una o varias fases cristalinas a una matriz de naturaleza vítrea (GMC). Sin embargo, la presencia de estas inclusiones rígidas (y frecuentemente inertes a la temperaturas de trabajo) reduce drásticamente la velocidad de densificación de los "composites", llegando incluso a impedir su densificación completa, lo que conduce, en la mayoría de los casos, a un deterioro de sus propiedades. A pesar de la marcada importancia que tiene la sinterización de estos materiales, tanto desde el prisma tecnológico como científico, no se ha localizado en la bibliografía que describa con éxito la cinética de este proceso para GMCs con altos contenidos en inclusiones rígidas (fracciones volumétricas mayores del $0,3)$ que son las más interesantes. Además, tampoco se han encontrado trabajos que estudien de forma sistemática el efecto que las granulometrías de los dos componentes (inclusión y matriz vítrea) y el contenido en inclusiones ejercen sobre la cinética de sinterización de estos materiales y sobre sus características microestructurales.

ii) Los vidriados cerámicos, cuyo sector industrial es de gran importancia socio-económica en la provincia de Castellón, son un grupo más o menos complejo de GMCs, y su sinterización ,aun siendo un proceso 
crítico, ha sido poco estudiada. $Y$ en los pocos casos que se ha estudiado este fenómeno en vidriados industriales [66][67][68][69], la excesiva complejidad de sus formulaciones dificulta la interpretación racional y científica de los resutados. En consecuencia, el estudio de este proceso en sistemas más simples no solo facilitará la interpretación de los resultados, sino también establecer de forma más clara el efecto de algunas variables (proceso y composición) sobre la cinética de la sinterización. Este nuevo conocimiento generado debe ser fácilmente transferible a la fabricación de vidriados cerámicos.

iii) El circón es quizá el "filler" más utilizado en vidriados cerámicos, debido a que su introducción en cantidades apropiadas en la formulación de estos materiales conduce a una mejora sustancial de las propiedades químicas y mecánicas.

iv) A escala de laboratorio, se ha comprobado que los composites del tipo circón-matriz vítrea presentan muy buenas propiedades mecánicas (Microdureza Vickers: 8GPa; Resistencia a la flexión: 220MPa; Resistencia a la abrasión profunda: $51 \mathrm{~mm}^{3}$ ), según la bibliografía [99].

v) Los resultados que se espera conseguir de la realización de esta Tesis son imprescindibles para lograr recubrimientos de alta dureza, basados en el sistema circón-vidrio, en la fabricación de materiales multicapa. Así pues, este trabajo es una parte significativa de un proyecto financiado [100].

Así pues, el objetivo general de este trabajo es, por una parte, el estudio del mecanismo y la cinética de la densificación de compactos de partículas del vidrio matriz y de composites circón-vidrio, con vistas a establecer en estos materiales la ecuación cinética que adecuadamente describa el proceso; por otra, se pretende determinar el efecto que sobre los diferentes parámetros del modelo cinético y sobre las características microstructurales ejercen el contenido en circón y la distribución del tamaño de las partículas del circón y de vidrio seleccionado.

En consecuencia, se ha planificado el siguiente trabajo 
a) Puesta a punto de las técnicas experimentales necesarias para la caracterización físico-química de los materiales de partida, productos intermedios y acabados, así como de los procedimientos requeridos para realizar las siguientes acciones y operaciones:

i) Selección de la composición de la matriz vítrea a utilizar, fusión de la correspondiente frita y obtención, por molienda en vía húmeda, de las distintas distribuciones granulométricas.

ii) Preparación por vía húmeda de las mezclas homogéneas circónvidrio; es decir, exentas, en lo posible, de aglomerados de alguno de los dos componentes y reduciendo al máximo la aparición de segregaciones.

iii) Conformado de probetas por colado y por prensado a diferentes presiones de compactación.

iv) Tratamientos térmicos, isotermos y a velocidad de calentamiento constante, de las probetas conformadas, en el microscopio de calefacción y en horno eléctrico de laboratorio.

b) Estudio de la sinterización de compactos de partículas de frita, partiendo de una distribución granulométrica similar a la industrial (GM). Su realización comprende a su vez:

i) La determinación de la evolución de las características texturales y microestructurales de la pieza con el avance de la densificación. Para ello, en probetas sometidas a diferentes tratamientos térmicos se determinarán: la compacidad, la porosidad abierta y su distribución por tamaños, la razón: porosidad abierta/porosidad cerrada y la porosidad cerrada y su distribución por tamaños. Se ha realizado también una caracterización microestructural de las probetas, cualitativa, mediante su observación por microscopia electrónica de barrido (MEB).

ii) Análisis cinético de proceso, lo que supone, por una parte, la selección del modelo cinético apropiado y la determinación de los parámetros cinéticos, utilizando métodos de calculo diferentes; y por otra, la posterior comparación de los resultados obtenidos por los 
diferentes métodos. Para ello, utilizando un microscopio de calefacción, se realizarán una serie de experimentos a velocidad de calentamiento constante, con valores de ésta comprendidos entre 0,5 y $60 \mathrm{~K} / \mathrm{min}$ y un conjunto de experimentos isotermos a diferentes temperaturas.

iii) Determinar el efecto de la compacidad en crudo de la probeta sobre: su contracción superficial, la razón: contracción axial/contracción diametral y sobre la cinética de la sinterización. Para ello, se realizarán una serie de experimentos a la velocidad de calentamiento de $15 \mathrm{~K} / \mathrm{min}$ en probetas conformadas a diferentes presiones de compactación, comprendidas entre 5 y $50 \mathrm{MPa}$.

c) Efecto del contenido en circón sobre la sinterización de composites. Para la realización de esta tarea se partirá de siete mezclas obtenidas a partir de polvo de vidrio de granlometría similar a la industrial (GM) y un circón micronizado comercial. El estudio comprende a su vez:

i) Determinar el efecto del contenido en circón sobre las curvas de sinterización no isotermas y el efecto combinado de esta variable y la presión de presado de las probetas sobre: las curvas de sinterización, la anisotropía de la contracción y sobre la compacidad máxima alcanzable.

ii) Estudio del efecto del contenido en circón sobre las características texturales y micro-estructurales de los composites a diferentes grados de avance de la sinterización. Además de la observación por MEB, las características a determinar serán: la compacidad, la porosidad abierta y su distribución por tamaños y la razón: porosidad abierta/porosidad cerrada. Todo ello con el objetivo de establecer o identificar los mecanismos que intervienen en el proceso de sinterización.

iii) Estudio del proceso de solución-reprecipitación de granos de circón y su influencia en la sinterización. Para un composite de alto contenido en circón, se determinará, en probetas tratadas térmicamente a $5 \mathrm{~K} / \mathrm{min}$ hasta distintas temperaturas máximas, el 
efecto de dicha variable sobre: la cantidad, tamaño medio de cristalito, distribución de tamaños de grano y forma del circón. Todo ello con vistas a establecer el efecto de este mecanismo de sinterización en las curvas de densificación no isotermas.

iv) Selección de modelo o modelos para composites con bajos, moderados y altos contenidos en circón y determinación del efecto de la fracción volumétrica de circón sobre los parámetros cinéticos. Para ello, utilizando un microscopio de calefacción, se realizarán, para cada composite (o contenido en circón), series de experimentos, isotermos y no isotermos, utilizando diferentes métodos de calculo de los parámetros cinéticos.

d) Efecto de la distribución del tamaño de las partículas del circón y del vidrio sobre la sinterización de composites. Para la realización de esta tarea se partirá de dos distribuciones de tamaño de partícula para el vidrio y tres para el circón. Con estos materiales se prepararán dos series de composites: una con un moderado contenido en circón $(\phi=0,322)$ y otra con un alto contenido en circón $(\phi=0,525)$, modificando dentro de cada serie la granulometría de cada componente; de este modo, se dispondrá, para cada serie de seis composites, con una razón: tamaño medio del circón/tamaño medio del vidrio, comprendida entre 0,3 y 9,6. El estudio comprende a su vez:

i. Determinar el efecto de la granulometría de los componentes sobre las curvas de sinterización no isotermas y sobre la compacidad máxima alcanzable, para composites con moderados y altos contenidos en circón.

ii. Estudio de la evolución microstructural de la probeta con el grado de avance de la sinterizacion, para algunos composites seleccionados, con el objeto de identificar o confirmar los mecanismos que intervienen en la densificación y su relación con la granulometría de los componentes y contenido en circón. 
iii. Selección del modelo para composites con moderado y alto contenido en circón y determinación del efecto de la granulometría de los componentes sobre los parámetros cinéticos. Para la realización de esta tarea, para cada composite, se realizaran una serie de experimentos a velocidad de calentamiento constante. También se realizaran, series de experimentos isotermos, pero solo para aquellos composites de interés tecnológico, es decir, que conduzcan a compacidades finales altas (porosidades bajas). Todo ello con vistas a disponer de un modelo o modelos cinéticos capaces de describir el efecto combinado del contenido en circón y de la granulometría del circón y del vidrio sobre el proceso de sinterización.

e) Validación del modelo o modelos seleccionados. Para ello, se prepararán probetas de un composite, con un contenido en circón y granulometría del vidrio distintos a los utilizados en las tareas anteriores, que serán tratadas también a una velocidad de calentamiento diferente a las empledas. Los resultados experiementales obtenidos serán comparados con los que predice el modelo o modelos desarrollados en el apartado d. 


\section{Materiales y procedimiento experimental}

\subsection{Materiales}

\subsubsection{Frita}

Se han preparado partículas de frita de borosilicato de composición próxima los vidrios SRM 717 [101] y SRM 731 [102], utilizados como materiales de referencia para la determinación de la curva viscosidad-temperatura y para la determinación de la expansión térmica, respectivamente.

La selección de esta composición se ha basado, principalmente, en las siguientes razones: i) Durante el calentamiento de las partículas de vidrio de esta composición no se produce ni separación de fases ni desvitrificación de cristales, lo que facilita la interpretación de los resultados. ii) El intervalo de fusión del vidrio es amplio y la curva de viscosidad-temperatura es conocida.

Aunque la composición química exacta de este vidrio no es conocida su composición nominal en óxidos aproximada, dada por los respectivos informes [101][102], se muestra en la Tabla 4.1.

Para conseguir unos $250 \mathrm{gr}$ de frita de composición similar a la indicada, se partieron de los materiales y en la proporción que se indica en la Tabla 4.2. Éstos, en la proporción indicada, se mezclaron en seco y se introdujeron en un crisol de alúmina para su calcinación y fusión en un horno eléctrico de laboratorio. El ciclo térmico utilizado fue el siguiente: calentamiento desde $500^{\circ} \mathrm{C}$ hasta $1550^{\circ} \mathrm{C}$ a una velocidad de $10 \mathrm{~K} / \mathrm{min}$ y $30 \mathrm{~min}$ de permanencia a la temperatura máxima. Transcurrido este periodo de tiempo, el crisol con la masa fundida se extrae del horno y se vierte sobre agua a temperatura ambiente. La frita resultante era transparente y homogénea. 
Tabla 4.1. Composición en óxidos del vidrio SRM nominal y obtenido, G (\% en peso).

\begin{tabular}{|c|c|c|}
\hline Óxidos & SRM & G \\
\hline $\mathrm{SiO}_{2}$ & 68 & 68,3 \\
\hline $\mathrm{Al}_{2} \mathrm{O}_{3}$ & 3,5 & 4,4 \\
\hline $\mathrm{B}_{2} \mathrm{O}_{3}$ & 18,5 & 16,6 \\
\hline $\mathrm{Fe}_{2} \mathrm{O}_{3}$ & & 0,05 \\
\hline $\mathrm{CaO}$ & & 0,13 \\
\hline $\mathrm{MgO}$ & 1 & 0,07 \\
\hline $\mathrm{Na}_{2} \mathrm{O}$ & 0,91 \\
\hline $\mathrm{K}_{2} \mathrm{O}$ & 8 & 7,80 \\
\hline $\mathrm{TiO}_{2}$ & & 0,05 \\
\hline $\mathrm{ZrO}_{2}$ & & 0,01 \\
\hline $\mathrm{Li}_{2} \mathrm{O}$ & & 0,66 \\
\hline $\mathrm{PbO}$ & & $<0,01$ \\
\hline $\mathrm{ZnO}$ & & $<0,01$ \\
\hline $\mathrm{HfO}_{2}$ & & $<0,01$ \\
\hline $\mathrm{P}_{2} \mathrm{O}_{5}$ & & 0,04 \\
\hline $\mathrm{SrO}$ & & $<0,01$ \\
\hline
\end{tabular}

Tabla 4.2. Fórmula de carga de la frita (\% en peso).

\begin{tabular}{|c|c|}
\hline Materia prima & \% peso \\
\hline Ácido Bórico & 25,2 \\
\hline Carbonato Potásico & 9,8 \\
\hline Carbonato de Litio & 0,9 \\
\hline Cuarzo & 58,8 \\
\hline Alúmina & 3,4 \\
\hline Carbonato Sódico & 1,6 \\
\hline
\end{tabular}

La composición química de la frita obtenida, G, como puede comprobarse en la Tabla 4.1, aunque es muy parecida a la SRM, presenta un mayor 
contenido en $\mathrm{Al}_{2} \mathrm{O}_{3}$, un menor contenido en los óxidos más volátiles $\left(\mathrm{B}_{2} \mathrm{O}_{3}\right.$, $\mathrm{Na}_{2} \mathrm{O}, \mathrm{K}_{2} \mathrm{O}$ y $\mathrm{LiO}_{2}$ ) y la presencia de pequeñas cantidades de los elementos introducidos como impurezas en las materias primas.

\subsubsection{Micronizado y harina de circón}

Se han empleado dos polvos de circón con granulometría distinta suministrados por la firma Mario Pilato Blat, con los nombres comerciales de harina de circón y circón micronizado. La harina de circón utilizada, fue tamizada por vía húmeda con un tamiz de $40 \mu \mathrm{m}$ de luz de malla. Su composición química se detalla en la Tabla 4.3 y sus distribuciones granulométricas, determinadas por difracción láser, en la Figura 4.1.

Tabla 4.3. Composición en óxidos de la harina de circón y del circón micronizado (\% en peso).

\begin{tabular}{|c|c|c|}
\hline Óxido & Harina de circón & Circón micronizado \\
\hline $\mathrm{SiO}_{2}$ & 32,8 & 33,0 \\
\hline $\mathrm{ZrO}_{2}$ & 64,8 & 64,5 \\
\hline $\mathrm{HfO}_{2}$ & 1,30 & 1,30 \\
\hline $\mathrm{Al}_{2} \mathrm{O}_{3}$ & 0,20 & 0,20 \\
\hline $\mathrm{Fe}_{2} \mathrm{O}_{3}$ & 0,14 & 0,11 \\
\hline $\mathrm{CaO}$ & 0,16 & 0,16 \\
\hline $\mathrm{MgO}$ & $<0,01$ & $<0,01$ \\
\hline $\mathrm{Na}_{2} \mathrm{O}$ & $<0,01$ & 0,01 \\
\hline $\mathrm{K}_{2} \mathrm{O}$ & 0,02 & 0,02 \\
\hline $\mathrm{TiO}_{2}$ & 0,26 & 0,26 \\
\hline p.p.c. & 0,32 & 0,34 \\
\hline
\end{tabular}

Por difracción de rayos $\mathrm{X}$ se determinó que en ambos casos el circón era de alta pureza, $99 \pm 1$. A la vista de los análisis químicos se comprueba que se trata de minerales del mismo origen. 


\subsection{Procedimiento experimental}

4.2.1 Molienda de la frita y determinación granulométrica del material resultante.

Las partículas de frita se han molturado, durante $30 \mathrm{~min}$, en molinos de bolas de alúmina de laboratorio hasta conseguir la distribución granulométrica GM. Para conseguir una distribución más fina, GF, una suspensión de partículas de frita, GM, se molturó de nuevo en un molino de microbolas de circona de la firma FRYMA.

La distribución del tamaño de partículas del material resultante de la molienda y del circón se han determinado mediante un equipo de difracción láser (MASTERSIZER de Malvern) provisto de 32 detectores pararecoger la luz dispersada por las partículas. La interpretación de la señal de dispersión se ha realizado utilizando el modelo de difracción de Fraunhofer.

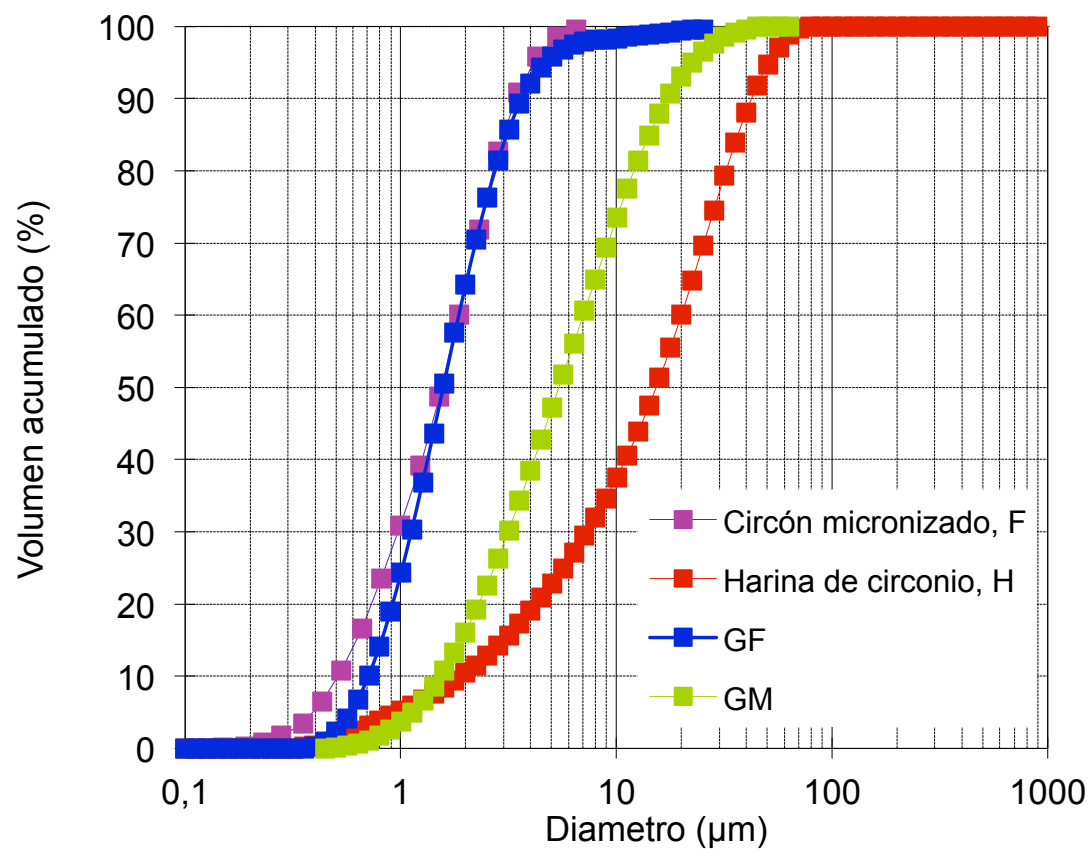

Figura 4.1. Distribuciones granulométricas de la frita y del circón. 
La distribución granulométrica GM ha sido utilizada para la determinación de la curva de viscosidad-temperatura ya que se adecua a la recomendada por distintos autores [103][104][105][106]. Las curvas GM y GF se ajustan aceptablemente bien a distribuciones normal logarítmicas cuyos parámetros son: diámetro medio $\mathrm{d}=5,9 \mu \mathrm{m}$ y desviación estándar $=2,65$ para la distribución $\mathrm{GM}$ y d=1,6 $\mathrm{m}$ y desviación estándar $=1,9$ para la GF. Sus diámetros medios volumétricos, $d_{g}^{v}$, fueron: $7,8 \mu \mathrm{m}$ y $2,1 \mu \mathrm{m}$, respectivamente. GM se asemeja a la distribución granulométrica de las fritas que se emplean en la industria, es más gruesa que la granulometría del circón micronizado y, a su vez, mucho más fina que la harina de circón. La distribución GF, mucho más fina, es similar a la del circón micronizado. Así pues, con estas dos granulometrías de frita y las dos granulometrías de circón se puede estudiar el efectos de la razón tamaño medio de circón/tamaño medio de frita sobre la densificación y propiedades de los composites.

\subsubsection{Preparación de las mezclas.}

La preparación de las mezclas se realizó por vía húmeda, en un molino rápido de bolas de alúmina de laboratorio, con pocas bolas (el nivel de éstas era aproximadamente $1 / 4$ de la altura de la suspensión). El contenido en sólidos de todas las mezclas fue del $70 \%$ en peso aproximadamente. En todos los casos se añadía como desfloculante a la suspensión $0,3 \%$ en peso respecto al sólido de tripolifosfato sódico. La duración del mezclado era de unos $15 \mathrm{~min}$.

\subsubsection{Conformado de probetas por colado.}

Con las suspensiones de los composites resultantes del mezclado se prepararon probetas cilíndricas, por colado, vertiendo el fluido en el interior de un anillo de bronce, de $2 \mathrm{~cm}$ de diámetro interno y $2 \mathrm{~cm}$ aproximadamente de altura, apoyado sobre un soporte cerámico poroso. La rápida succión del agua de la suspensión por los capilares del material cerámico provoca la formación y crecimiento de una capa consolidada de partículas, y un aumento de la viscosidad de la suspensión remanente, también rápido. 
Ambos fenómenos impiden la segregación de las partículas por tamaño y/o densidad. Una vez conformadas las probetas, se extrajeron del anillo y se secaron en estufa a $110^{\circ} \mathrm{C}$, durante al menos dos horas. Con este procedimiento de mezclado y conformado, se obtuvieron probetas homogéneas, a escala del tamaño de las partículas.

\subsubsection{Conformado de probetas por prensado.}

En los experimentos en el microscopio de calefacción (HSM), destinados a determinar los puntos fijos de viscosidad, se han utilizado probetas cilíndricas de $3 \times 5 \mathrm{~mm}^{2}$. Para determinar la cinética de sinterización de los composites se emplearon discos de $5 \mathrm{~mm}$ de diámetro y $3 \mathrm{~mm}$ de espesor obtenidos, también, por prensado en una matriz de acero inoxidable, compactando el polvo ligeramente húmedo (1-2\%) en una máquina de ensayos universales a desplazamiento constante de $2 \mathrm{~mm} / \mathrm{min}$ hasta la presión requerida. Excepto en aquellos casos en los que se pretendía estudiar el efecto de la presión de prensado sobre el proceso de sinterización, la presión de prensado utilizada fue de 50MPa.

\subsubsection{Tratamientos térmicos.}

\subsubsection{Microscopio de calefacción.}

Se ha utilizado un microscopio de calefacción MISURA 3 (Expert system solutions) con análisis de imagen y software de tratamiento de datos. El microscopio proyecta la imagen de la muestra a través de una ventana de cuarzo sobre el dispositivo de grabación. Un sistema de análisis de imagen computarizado registra y analiza la geometría de la muestra durante el calentamiento. El sistema calcula el porcentaje de disminución de altura, anchura y área de las imágenes de la silueta, relativa a su tamaño inicial.

Las probetas, de $3 \mathrm{~mm}$ de diámetro y $5 \mathrm{~mm}$ de altura, utilizadas en la determinación de los puntos fijos de viscosidad y los discos de $D=5 \mathrm{~mm}$ de diámetro y $\mathrm{h}=3 \mathrm{~mm}$ de altura, empleados para estudiar la sinterización, se situaban sobre un soporte de $10 \times 15 \times 1 \mathrm{~mm}^{3}$ de alúmina $(>99,5 \%)$. La 
temperatura era medida con termopar $\mathrm{Pt} / \mathrm{Rh}(6 / 30)$ situado bajo el soporte de alúmina, muy próximo a éste, pero sin tocarlo.

Para estudiar la cinética de la sinterización se han realizado dos tipos de tratamientos térmicos: a velocidad de calentamiento constante, $\beta$, e isotermo. En el primero, la velocidad de calentamiento utilizada variaba de $\beta=0,5$ a $60 \mathrm{~K} / \mathrm{min}$. En todos los casos, la temperatura máxima del ciclo era superior a la de máxima compacidad de la pieza.

En el tratamiento isotermo, la probeta introducida en el horno era calentada a una velocidad de $\beta=25 \mathrm{~K} / \mathrm{min}$ hasta la temperatura del tratamiento fijada para cada ensayo. La duración del tratamiento isotermo era superior a los $400 \mathrm{~min}$.

Si durante el tratamiento isotermo la probeta no alcanzaba la máxima compacidad, el tratamiento se prolongaba, utilizando un ciclo de calentamiento de $25 \mathrm{~K} / \mathrm{min}$ hasta una temperatura $50^{\circ} \mathrm{C}$ más alta, seguido de un tratamiento isotermo, de $60 \mathrm{~min}$ adicionales. De este modo, para cada experimento se dispone de la altura y área al inicio de la sinterización de la probeta, $h_{0}$ y $A_{0}$, y los valores correspondientes a la máxima compacidad, $h_{\min }$ y $A_{\min }$.

A partir de estos valores, de acuerdo con el apendice 7.1, se pueden calcular la contracción superficial de la silueta de la probeta, $\varepsilon_{A}$ (ec. A1. 2), y el grado de avance de la sinterización, $\alpha$ (ec. A1. 6) en otras características. En el apendice 7.2 se comprueba que al utilizar como valores iniciales $h_{0} y$ $\mathrm{A}_{0}$ los del inicio de la sinterización en vez de los que proporciona el equipo a $\mathrm{T}=25^{\circ} \mathrm{C}$ se mejora sustancialmente la dispersión de los resultados. Lo propio sucede cuando en vez de emplear contracciones superficiales, $\varepsilon_{A}=\ln \left(A / A_{0}\right)_{S}$, para describir la sinterización, se trabaja con el grado de avance de la sinterización, $\alpha=\varepsilon_{A} / \varepsilon_{A, \max }$.

\subsubsection{Horno eléctrico.}

Con vistas a determinar la evolución de la textura porosa (distribución del tamaño de intrusión por porosimetría, porosidad abierta y cerrada), tamaño 
de cristalito y de grano de circón y porcentaje de fase cristalina en los materiales, las probetas obtenidas por colado (apartado 4.2.2) han sido tratadas térmicamente en horno eléctrico de laboratorio en atmósfera de aire. Se han utilizado dos tipos de tratamientos: a velocidad de calentamiento constante de $\beta=5 \mathrm{~K} / \mathrm{min}$ e isotermo. En ambos casos la probeta cilíndrica estaba apoyada sobre un soporte refractario de cordierita. La temperatura del horno era registrada por un termopar $\mathrm{Pt} / \mathrm{Rh}(6 / 30)$ protegido de la radiación directa por una funda de alúmina.

Con los tratamientos isotermos, un conjunto de probetas apoyadas sobre el soporte se introducen rápidamente en el horno, que ya se encontraba a la temperatura deseada. Dicha operación ocasionaba una disminución de la temperatura (alrededor de $20-30^{\circ} \mathrm{C}$ ) que se recuperaba al cabo de muy poco tiempo (2-3min). Para cada tiempo de permanencia establecido se extraía la probeta del horno, lo que ocasionaba una alteración de la temperatura de esta similar a la anterior

En los experimentos a velocidad de calentamiento constante, $\beta=5 \mathrm{~K} / \mathrm{min}$, al menos dos probetas se situaban en el horno, antes de iniciarse el ciclo de calentamiento. Una vez finalizado el calentamiento, se enfriaba la pieza en el interior del horno, por convección forzada, gracias a unos ventiladores (la duración del enfriamiento variaba entre 10-15 min).

\subsubsection{Caracterización textural y microestructural de las probetas.}

\subsubsection{Compacidad.}

A partir de los valores de la densidad aparente, $\rho_{a p}$, y de los de la densidad real del sólido, $\rho_{\text {real, }}$ se han calculado los de la compacidad, mediante la ecuación:

$\rho=\frac{\rho_{\mathrm{ap}}}{\rho_{\text {real }}}$ ec. 4.1

La densidad real, $\rho_{\text {real, }}$, se ha calculado mediante la expresión: 
$\frac{1}{\rho_{\text {real }}}=\frac{w_{z}}{\rho_{\text {circón }}}+\frac{1-w_{z}}{\rho_{\text {vidrio }}}$

ec. 4.2

Siendo $\rho_{\text {circón }}$ y $\rho_{\text {vidrio }}$ los valores de la densidad del circón y del vidrio respectivamente. Éstas se han determinado experiementalmente mediante un pignémetro de helio (Quantachromme Ultrapygnometer 1000), resultando los valores de $\rho_{\text {circón }}=4610 \pm 50$ y $\rho_{\text {vidrio }}=2210 \pm 40 \mathrm{~kg} / \mathrm{m}^{3}$.

En el caso de probetas obtenidas por colado, la densidad aparente, $\rho_{\mathrm{ap}}$, tanto de la probeta seca como cocida se ha determinado mediante inmersión de mercurio.

En el caso de las probetas obtenidas por prensado, su densidad aparente después de cocida se ha determinado de sus dimensiones y de su masa.

4.2.6.2 Porosidad abierta. Distribución de tamaños de poro de intrusión.

La distribución del tamaño de los poros de la probeta se ha determinado con un porosímetro de mercurio de la marca Micromeritics Autopore IV 9500. El método se basa en la medida del volumen de mercurio que e introduce en el sólido poroso a medida que se va incrementando la presión aplicada $(P)$. Par el cálculo del radio capilar de intrusión, $r_{c}$, se asume que los poros son cilíndricos y que se cumple la ecuación de Washbourn:

$r_{c}=\frac{2 \gamma \cos \theta}{P}$ ec. 4.3

donde: $\gamma=$ tensión superficial del mercurio $=474 \cdot 10^{3} \mathrm{~N} / \mathrm{m}$ y $\theta=$ ángulo de contacto mercurio-sólido $=130^{\circ}$.

La porosidad abierta total se obtiene a partir del volumen de mercurio que a penetrado a la máxima presión, $\mathrm{P}=227,5 \mathrm{MPa}$.

La cantidad de material necesario para realizar el ensayo era de $1 \mathrm{~g}$ aproximadamente por lo que se ha utilizado probetas obtenidas por colado. 


\subsubsection{Observación por MEB.}

Las muestras se observaron con un microscopio electrónico de barrido de emisión de campo, modelo Quanta 200F de la marca FEI, conectado a un equipo de microanálisis por dispersión de energías de rayos $\mathrm{X}$ (EDX).

4.2.6.3.1 Porosidad cerrada. Distribución del tamaño de los poros.

Las muestras se han preparado seccionando transversalmente las porobetas cocidas, embutidas en una resina tipo Epoxi y puliéndose hasta un tamaño de grano inferior a $3 \mu \mathrm{m}$.

La medida de la porosidad cerrada implica la observación y toma de imágenes por MEB y posterior tratamiento por análisis de imagen.

Se han utilizado los siguientes parámetros para caracterizar la porosidad cerrada:

- Porcentaje del área observada ocupada por poros.

- Porcentaje del área observada ocupada por poros de radio menor de $1 \mu \mathrm{m}$.

- Porcentaje del área observada ocupada por poros de radio mayor de $10 \mu \mathrm{m}$.

- $\quad \mathrm{n}^{\circ}$ de poros $/ \mathrm{mm}^{2}$ de área observada.

- $\mathrm{n}^{\circ}$ de poros de radio menor $1 \mu \mathrm{m} / \mathrm{mm}^{2}$ de área observada.

- $\mathrm{n}^{\circ}$ de poros de radio mayor $10 \mu \mathrm{m} / \mathrm{mm}^{2}$ de área observada.

Para cada una de las muestras se han medido más de 30 pantallas.

4.2.6.3.2 Distribución del tamaño de grano del circón.

La distribución del tamaño de grano del circón y su forma se han determinado también por MEB y análisis de imagen. Para cada muestra el $\mathrm{n}^{\circ}$ de partículas analizadas fue siempre superior a 2500.

4.2.6.3.3 Zona de difusión vidrio-circón.

Se ha determinado el gradiente de composición con la distancia mediante EDS. Se ha calculado la evolución con la distancia a la superficie de la 
partícula de circón, $x$, del gradiente del $\mathrm{n}^{\circ}$ de cuentas de circonio, $d($ cuentasZr)/dx. Para ello se ha utilizado el método de diferenciación de Savitzky-Golay, empleando un ancho de ventana de 59 puntos (alrededor de $3 \mu \mathrm{m})$, para suavizar la curva.

4.2.6.3.4 Cuantificación de estructuras cristalinas y determinación del tamaño de cristalito.

Ambas características se han determinado mediante difracción de rayos $\mathrm{X}$, empleando un equipo Theta-Theta modelo D8 ADVANCE de la firma Bruker. La determinación de circón, la única forma cristalina detectada, se ha realizado por el método Rietveld [107][108], empleando para los cálculos el programa comercial TOPAS-4 (Bruker) y utilizando fluorita como patrón interno.

El tamaño promedio del cristalito de circón se ha determinado aplicando la ecuación de Scherrer [109], según la cual, para una muestra monodispersa, el tamaño medio del cristalito, $\mathrm{C}$, es inversamente proporcional al ensanchamiento del pico, $\beta_{\mathrm{p}}$ :

$C=\frac{\kappa^{\prime} \cdot \lambda_{\text {radiación }}}{\beta_{\mathrm{p}} \cdot \cos \vartheta}$ ec. 4.4

siendo: $\vartheta=$ ángulo de difracción, $\lambda_{\text {radiación }}=$ longitud de onda de la radiación $\mathrm{k} \alpha$ y $\mathrm{K}^{\prime}=$ constante que depende de las condiciones de medida.

Para los cálculos también se utilizó el programa comercial TOPAS-4 (Bruker). 


\section{Resultados y discusión}

5.1 Sinterización de compactos de partículas de frita.

\subsubsection{Procesos físico-químicos que se desarrollan en el material durante su tratamiento térmico.}

5.1.1.1 Análisis térmico diferencial (ATD).

En la curva de ATD (Figura 5.1) solo se distingue claramente sobre los $510^{\circ} \mathrm{C}-520^{\circ} \mathrm{C}$ un cambio en la pendiente asociado a la transición vítrea del vidrio. No se aprecian picos exotérmicos ociados a procesos tales como separación de fases o cristalizaciones, tal como se señala en la bibliografía.

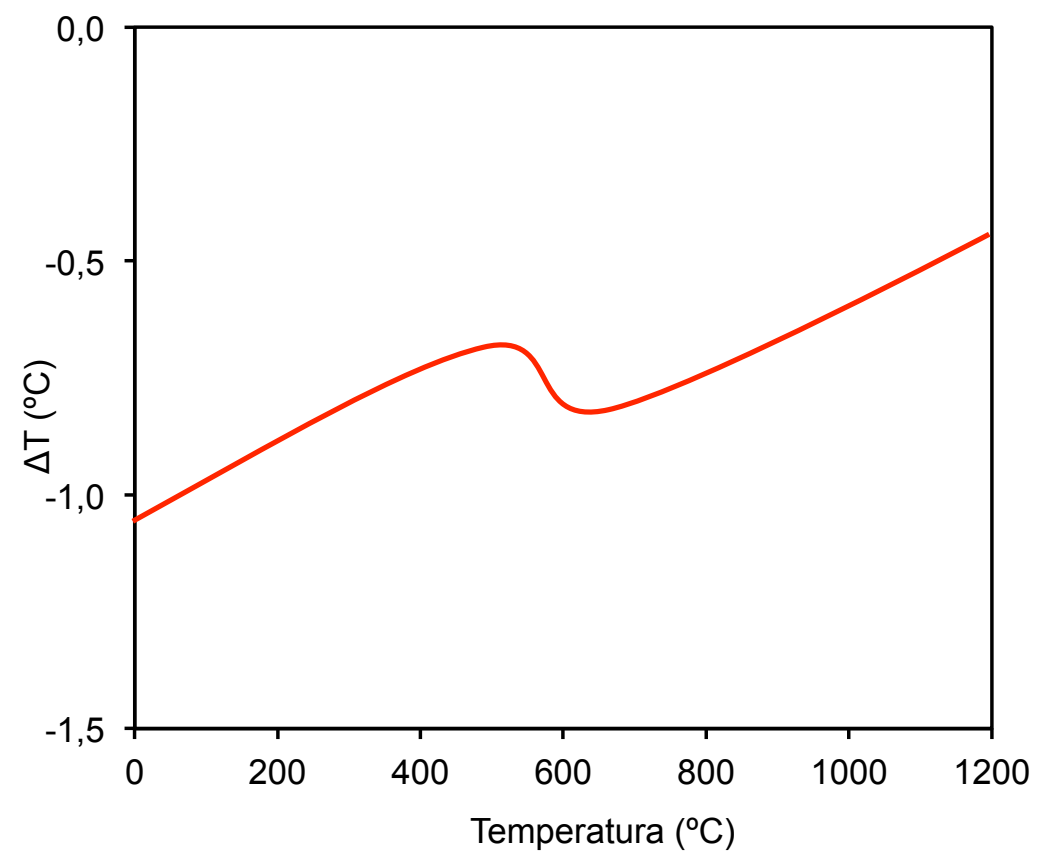

Figura 5.1. Análisis térmico diferencial del vidrio. Velocidad de calentamiento $10 \mathrm{~K} / \mathrm{min}$

5.1.1.2 Sinterización, reblandecimiento y "spreading" (HSM).

En la Figura 5.2 se ha representado, para una probeta cilíndrica de $3 \times 5 \mathrm{~mm}^{2}$ prensada a mano a partir de la granulometría GM, la contracción de la 
superficie de la silueta de la probeta cilíndrica, $\varepsilon_{A}=\ln \left(A / A_{0}\right)_{S}$, en función de la temperatura. Se incluyen, en esta misma figura, las formas geométricas correspondientes a los puntos fijos de viscosidad.

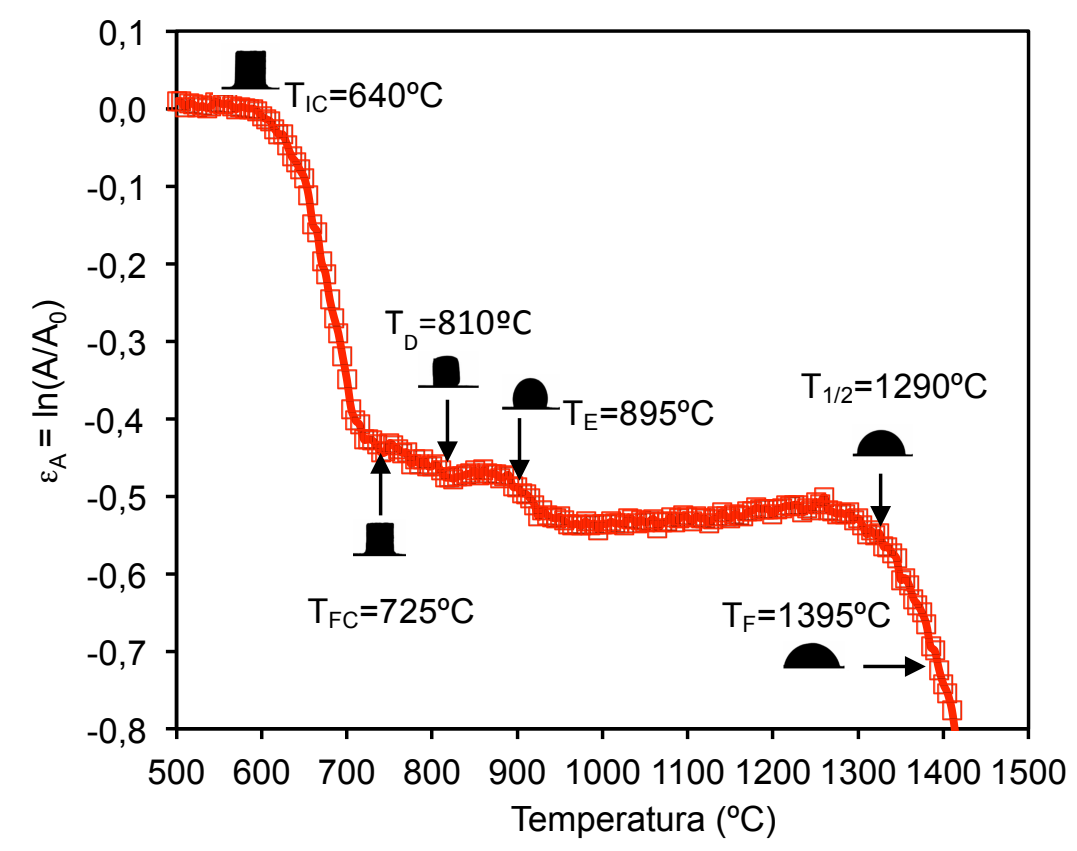

Figura 5.2. Temperaturas características y evolución de la contracción superficial de la silueta con la temperatura para la muestra GM. Velocidad de calentamiento $10^{\circ} \mathrm{C} / \mathrm{min}$.

Se comprueba que la sinterización comienza a temperaturas $\left(T_{1 C} \approx 600^{\circ} \mathrm{C}\right)$, ligeramente superiores a la temperatura de transformación, $T_{g}=510^{\circ} \mathrm{C}, y$ alcanza la máxima densidad sobre $\mathrm{T}_{\mathrm{FC}}=725^{\circ} \mathrm{C}$. Un posterior calentamiento provoca un cambio de la forma de la silueta de la probeta. La forma sigmoidal de las curvas de contracción frente a la temperatura, entre $T_{I C} y$ $\mathrm{T}_{\mathrm{FC}}$, es típica de la sinterización de compactos de partículas de naturaleza exclusiva o mayoritariamente vítrea, durante la cual no se produce la cristalización de fases del fundido o lo hace en muy poca extensión. 


\subsubsection{Curva de viscosidad-temperatura de la matriz vitrea.}

En la Tabla 5.1 se detallan los valores de las temperaturas correspondientes a los puntos fijos de viscosidad para esta frita, obtenidos por dilatometría y HSM. Se incluyen, además, los valores de la viscosidad, expresados como $\log \eta$ en $\mathrm{Pa} \cdot \mathrm{s}$, que asignan a los puntos fijos obtenidos por distintos autores. Como puede comprobarse existe diferencias significativas entre los valores de viscosidad asignados a cada punto por los diferentes autores (Tabla 5.1). En la Figura 5.3 se han representado estos resultados junto con las curvas: viscosidad-temperatura, para esta composición según la SRM [101][102]. Dichas curvas son la representación de log $\eta$ vs $T$ obtenidas al ajustar a la ecuación de Vogel-Fulcher-Tamman (ec. 5.1) los resultados experimentales obtenidos por diferentes laboratorios.

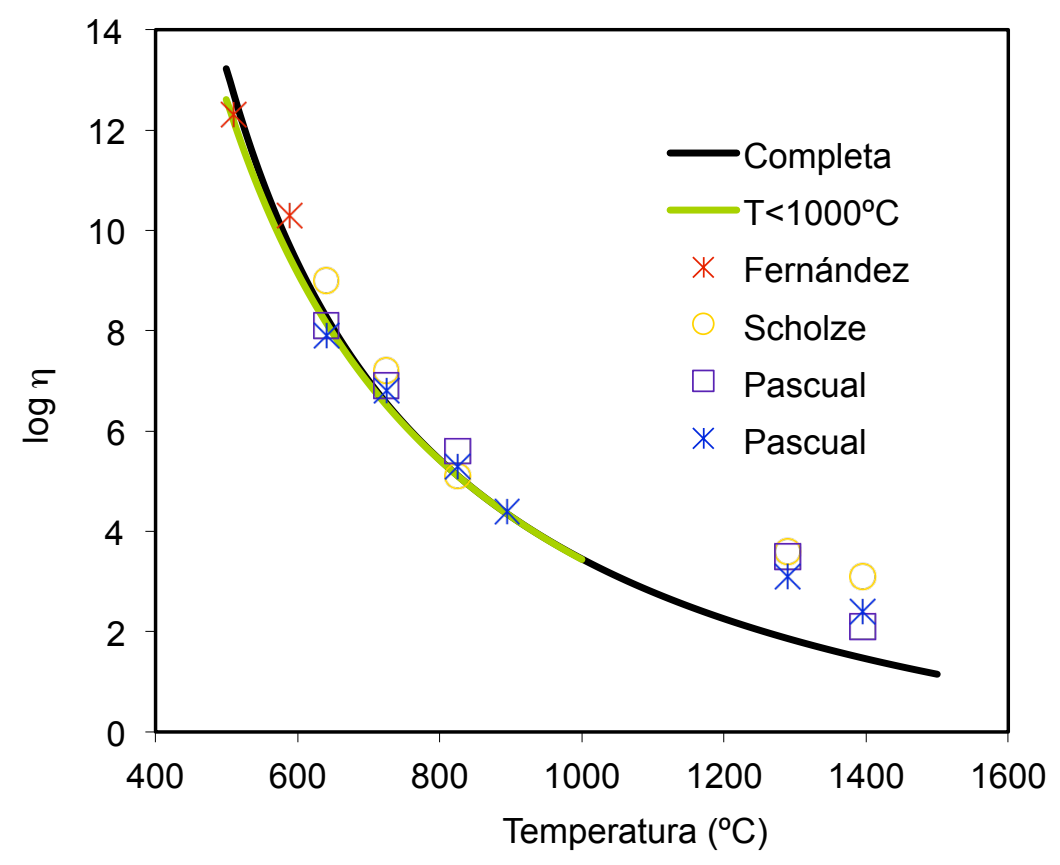

Figura 5.3. Curvas VTF según NBS y puntos fijos de viscosidad. 
$\log \eta=A^{*}+\frac{B}{T-T_{0}}$

ec. 5.1

donde: $\mathrm{T}=$ temperatura en ${ }^{\circ} \mathrm{C}, \eta=$ viscosidad en Pa.s y $\mathrm{A}^{*}$, B y $\mathrm{T}_{0}$, parámetros de ajuste cuyos valores se detallan en la Tabla 5.2. El error estándar estimado por la SRM en $\log \eta$ es $\sigma(\log \eta)= \pm 0.029$.

La única diferencia apreciable entre estas dos curvas se observa en el intervalo de bajas temperaturas. En efecto, para temperaturas inferiores a $1000^{\circ} \mathrm{C}$ los valores de la viscosidad correspondientes a la curva recomendada para este intervalo de temperaturas son ligeramente menores.

Tabla 5.1. Temperaturas $\left({ }^{\circ} \mathrm{C}\right)$ correspondientes a los puntos fijos y valores de la viscosidad asignadas por diferentes autores.

\begin{tabular}{|c|c|c|c|c|c|}
\hline Puntos fijos & $\mathbf{T}\left({ }^{\circ} \mathbf{C}\right)$ & $\begin{array}{c}\text { Scholze } \\
\log \eta \pm \sigma \\
(\mathbf{P a} \cdot \mathbf{s}) \\
{[110]}\end{array}$ & $\begin{array}{c}\text { Pascual } \\
\log \eta \pm \sigma \\
\mathbf{( P a} \cdot \mathbf{s}) \\
{[103]}\end{array}$ & $\begin{array}{c}\text { Pascual } \\
\log \eta \pm \sigma \\
(\mathbf{P a} \cdot \mathbf{s}) \\
{[105]}\end{array}$ & $\begin{array}{c}\text { Fernández } \\
\log \eta \pm \sigma \\
\mathbf{( P a} \cdot \mathbf{s}) \\
{[111]}\end{array}$ \\
\hline $\begin{array}{c}\text { Transformación } \\
\text { Reblandecimiento } \\
\text { dilatométrico }\end{array}$ & $510 \pm 3$ & - & - & - & 12,3 \\
\hline $\begin{array}{c}\text { Inicio de } \\
\text { contracción }\end{array}$ & $688 \pm 3$ & - & - & - & 10,3 \\
\hline $\begin{array}{c}\text { Máxima contracción } \\
\text { Deformación }\end{array}$ & $725 \pm 5$ & $7,2 \pm 0,5$ & $6,9 \pm 0,2$ & $6,8 \pm 0,1$ & - \\
\hline Esfera & $895 \pm 5$ & - & - & $4,4 \pm 0,1$ & - \\
\hline Semiesfera & $1290 \pm 5$ & $3,6 \pm 0,1$ & $3,5 \pm 0,1$ & $3,1 \pm 0,1$ & - \\
\hline Flujo & $1395 \pm 10$ & $3,1 \pm 0,1$ & $2,1 \pm 0,1$ & $2,4 \pm 0,1$ & - \\
\hline
\end{tabular}


Tabla 5.2. Parámetros de la ecuación de Vogel-Fulcher-Tamman (VFT) (ec. 5.1), correspondientes a la curva SRM.

\begin{tabular}{|c|c|c|c|}
\hline $\mathbf{A}^{*}(\log \eta(\mathrm{Pa} \cdot \mathbf{s}))$ & $\mathbf{B}\left({ }^{\circ} \mathrm{C}\right)$ & $\mathrm{T}_{0}\left({ }^{\circ} \mathrm{C}\right)$ & $\begin{array}{c}\text { Intervalo de } \\
\text { temperatura } \\
\left({ }^{\circ} \mathrm{C}\right)\end{array}$ \\
\hline$-2,5602$ & 4852,2 & 192,462 & $510<\mathrm{T}<1550$ \\
\hline$-3,012$ & 5495,3 & 148,1 & $\mathrm{~T}<1000^{\circ} \mathrm{C}$ \\
\hline
\end{tabular}

Se comprueba que los resultados obtenidos por HSM se adaptan aceptablemente bien a la curva SRM, excepto para altas temperaturas o bajas viscosidades. En general, la asignación de la viscosidad a cada punto fijo del HSM recomendada por Pascual [105] parece la más adecuada de acuerdo con lo observado en otros trabajos [103][110]. En lo referente al intervalo de bajas viscosidades, correspondientes al punto de semiesfera y flujo, los valores de la viscosidad que asignan a estos puntos los diferentes investigadores, obtenidos por el HSM, son significativamente más elevados que los correspondientes a la curva SRM experimental.

Esta diferencia puede deberse a que la forma de la probeta a estas temperaturas no solo depende de su viscosidad, sino también de los fenómenos de mojado y "spreading", los cuales dependen de la naturaleza del sustrato. En este sentido, se ha comprobado que los puntos fijos de semiesfera y flujo en vidrios se pueden desplazar hasta $50^{\circ} \mathrm{C}$ dependiendo del sustrato sobre el que se apoye la muestra.

A partir de los valores de los parámetros de la ecuación de VFT (ec. 5.1, Tabla 5.2), correspondiente al ajuste de la SRM en el intervalo de bajas temperaturas, se han calculado los valores de la energía de activación de flujo viscoso en función de la temperatura para este vidrio, definido como:

$$
\eta=\eta_{0} \cdot \exp \left(\frac{E_{\eta}}{R T}\right)
$$




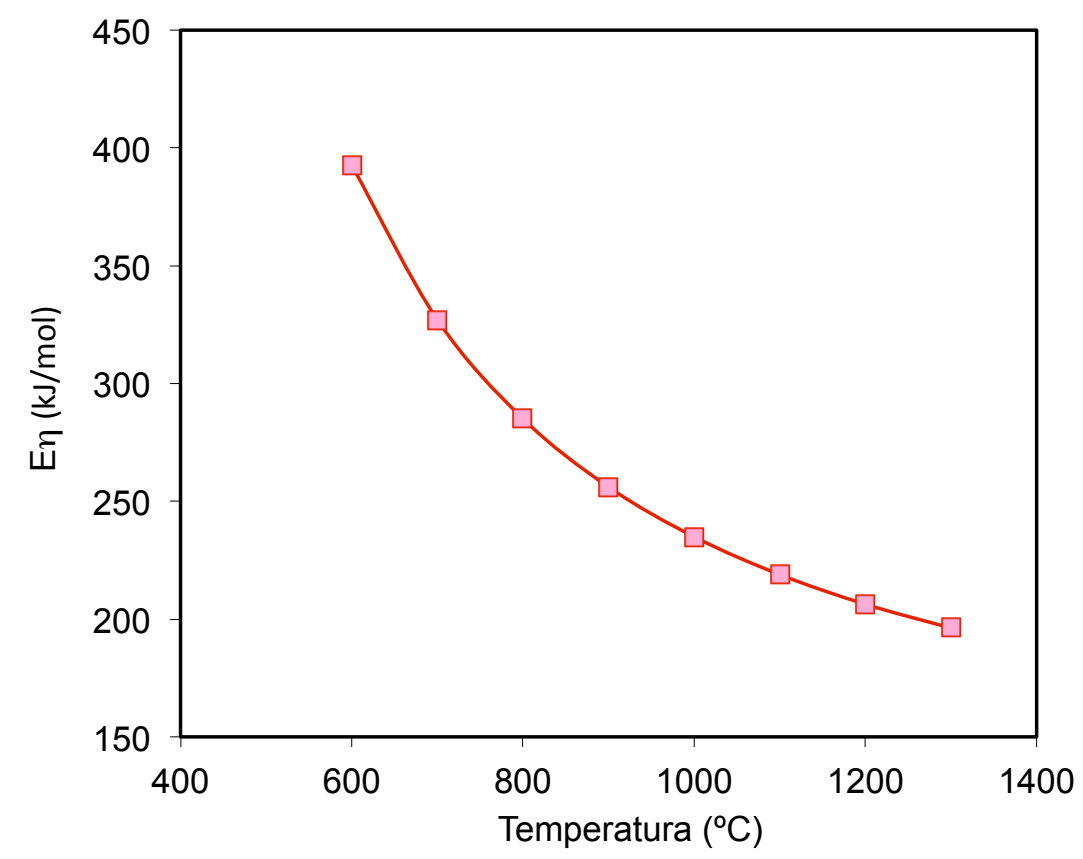

Figura 5.4. Variación de la energía de activación de flujo viscoso, Eq, con la temperatura para el vidrio.

Se confirma (Figura 5.4) una disminución continua de $E_{\eta}$ con el aumento de la temperatura. No obstante, para intervalos cortos de temperatura puede utilizarse una energía de activación media $\bar{E}_{\eta}$ con vistas a comporarla con la que se obtienen para el proceso de sinterización, $\overline{\mathrm{E}}$.

5.1.1.4 Evolución de las características texturales y microestructurales de la pieza con el avance del proceso de sinterización (GM).

5.1.1.4.1 Análisis cualitativo (micrografías SEM).

Las micrografías, a dos aumentos diferentes (Figura 5.5 y Figura 5.6), de las secciones pulidas de las probetas correspondientes a un experimento isotermo a $637^{\circ} \mathrm{C}$ (Figura 5.23) ilustran la evolución de la microestructura de los compactos de partículas de vidrio con el avance del grado de sinterización, $\alpha$, con el tiempo. 
Del examen se deduce lo siguiente:

- Las imágenes de las microestructuras de los estados iniciales de la sinterización ( $\alpha=0,2$ a $\mathrm{t}=5 \mathrm{~min}$ y $\alpha=0,35$ a $\mathrm{t}=15 \mathrm{~min}$ ) confirman que la distribución de tamaños de partícula es ancha y que la forma de las partículas es irregular, especialmente las de mayor tamaño. En este estado inicial solo las partículas pequeñas presentan los cantos redondeados. En este estado de la sinterización, la coalescencia de partículas solo se presenta en las más pequeñas.

- En el estado intermedio de la sinterización $(\alpha=0,5$ a $t=30$ min y $\alpha=0,65$ a $t=1 \mathrm{~h}$ ), la coalescencia entre partículas de vidrio conduce a que la microestructura pueda considerarse como una matriz vítrea continua en la que se distribuyen de forma bastante homogénea los poros irregulares. Especialmente la microestructura correspondiente a la probeta con $\alpha=0,5$ a $\mathrm{t}=30 \mathrm{~min}$ muestra poros muy irregulares, debido a la coalescencia de tres o cuatro partículas o de agregados de partículas muy pequeños que ya han coalescido. A mayores grados de sinterización $\alpha=0,65$ a $t=1 \mathrm{~h}$, las áreas densas de vidrio son más grandes y los poros menos numerosos, aun siendo irregulares, son más esféricos que en la muestra anterior.

- A un grado de avance de la sinterización de $\alpha=0,8$ a $t=2 h$ empieza la etapa final de la sinterización, aunque todavía muchos de los poros son irregulares, ya comienzan a esferoidizarse algunos de ellos y a convertirse en cerrados como se verá posteriormente (Figura 5.11). A las $8 \mathrm{~h}$ de sinterización la compacidad de la pieza es máxima $(\alpha=1)$, la mayoría de los poros presentan una sección circular o en forma de elipse y son cerrados (Figura 5.11) 

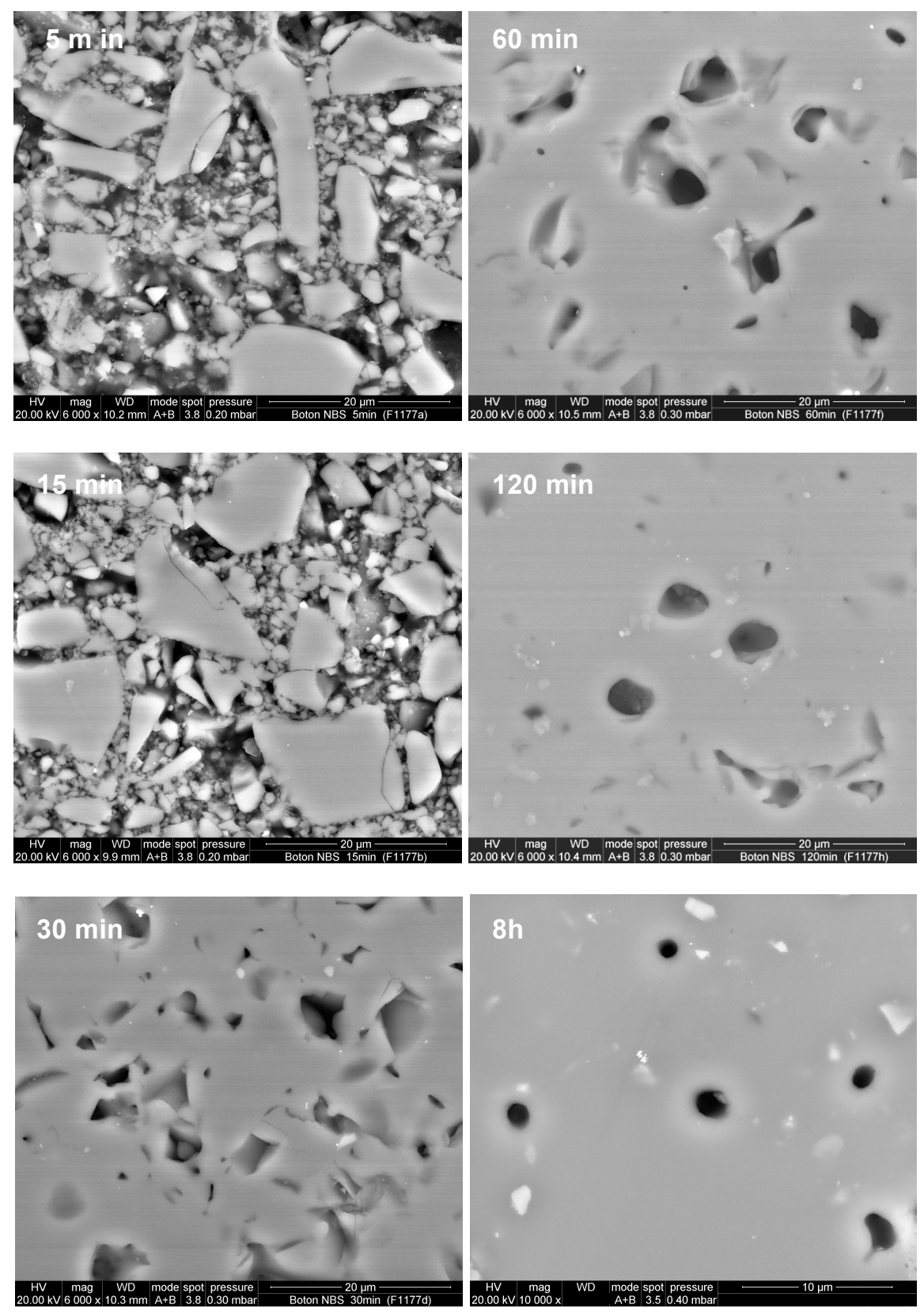

Figura 5.5. Evolución de la microestructura (x6000 aumentos) de los compactos de partículas de vidrio, a $637^{\circ} \mathrm{C}$ y $50 \mathrm{MPa}$, con el avance del grado de sinterización, $\alpha$, con el tiempo: $\alpha=0,20$ a $t=5 \mathrm{~min}, \alpha=0,35$ a $t=15 \mathrm{~min}$, $\alpha=0,5$ a $t=30 \mathrm{~min}, \alpha=0,65$ a $t=1 h, \alpha=0,80$ a $t=2 h$ y $\alpha=1$ a $t=8 h$. 

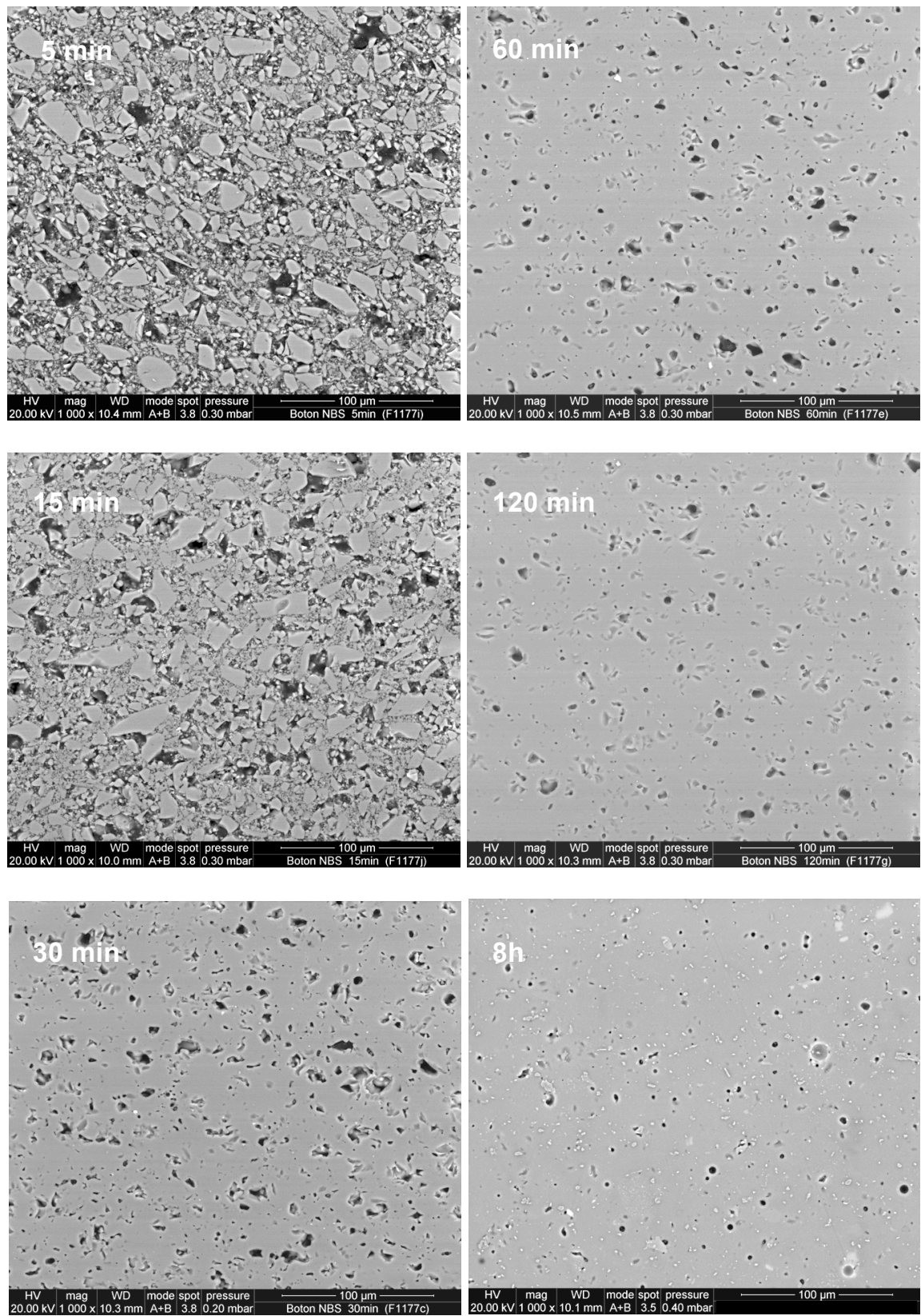

Figura 5.6. Evolución de la microestructura (x1000 aumentos) de los compactos de partículas de vidrio, a $637^{\circ} \mathrm{C}$ y $50 \mathrm{MPa}$, con el avance del grado de sinterización, $\alpha$, con el tiempo: $\alpha=0,20$ a $t=5 \mathrm{~min}, \alpha=0,35$ a $t=15 \mathrm{~min}$, $\alpha=0,5$ a $t=30 \mathrm{~min}, \alpha=0,65$ a $t=1 \mathrm{~h}, \alpha=0,80$ a $t=2 \mathrm{~h}$ y $\alpha=1$ a $t=8 \mathrm{~h}$. 
5.1.1.4.2 Compacidad, $\rho, y$ grado de avance de la sinterización, $\alpha$ (calculado a partir de la compacidad).

En el desarrollo de este apartado se han utilizado probetas de forma cilíndrica, de aproximadamente $2 \mathrm{~cm}$ de diámetro y $2 \mathrm{~cm}$ de altura, obtenidas por colado, con vistas a determinar en las piezas crudas y cocidas su compacidad, $\rho$, y su distribución de tamaños de poro, DTP, por porosimetría de mercurio. Se han realizado dos tipos de tratamientos térmicos: isotermo, a tres temperaturas diferentes, y a velocidad de calentamiento contante $(5 \mathrm{~K} / \mathrm{min})$. La temperatura máxima alcanzada en este segundo tratamiento has sido $100^{\circ} \mathrm{C}$ más alta que la de máxima compacidad de la pieza, con vistas a determinar la evolución de la porosidad cerrada.

En la Figura 5.7 se han representado los valores de la compacidad, $\rho$, de las piezas cocidas frente al tiempo de permanencia en el interior del horno, $t^{\star}$, a tres temperaturas diferentes y en la Figura 5.8 la variación de $\rho$ con la temperatura, en el tratamiento a velocidad de calentamiento constante. 


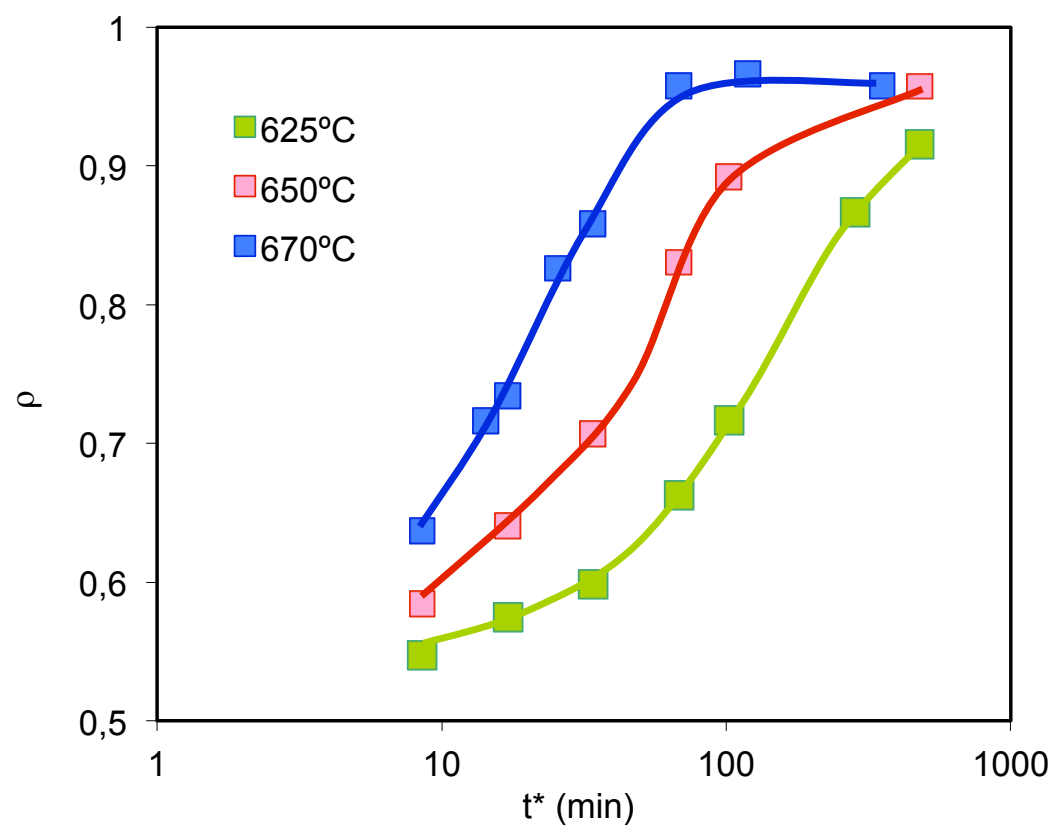

Figura 5.7. Variación de la compacidad, $\rho$, de las piezas cocidas frente al tiempo de permanencia en el interior del horno, $t^{*}$ ( $\left.\mathrm{min}\right)$, a tres temperaturas diferentes.

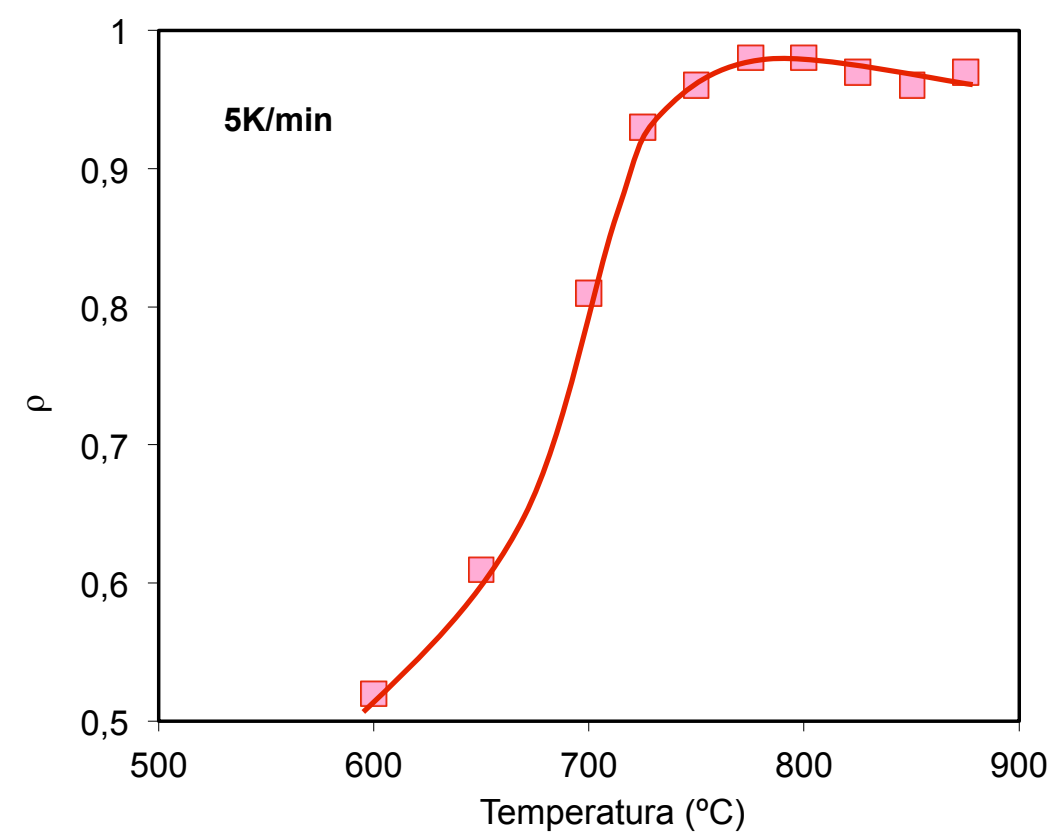

Figura 5.8. Variación de la compacidad de las probetas cocidas, $\rho$, con la temperatura. Experimentos a una velocidad de calentamiento de $\beta=5 \mathrm{~K} / \mathrm{min}$. 
Para comprobar si se cumple el principio de isoconversionalidad (apartado 2.2.3) en la sinterización isoterma de compactos de partículas de vidrio, se han tratado de agrupar todos los resultados en una única curva. Para ello se ha definido un tiempo adimensional mediante la expresión:

$\frac{t}{t_{c}}=\frac{t \cdot \gamma}{d \cdot \eta}$

donde:

t es el tiempo de permanencia de la pieza en el horno a la temperatura de sinterización.

$t_{c}=\frac{d \cdot \eta}{\gamma}$ es un tiempo característico, función del tamaño medio de la partícula de vidrio, $d$, de la viscosidad, $\eta$, y de la tensión superficial del vidrio, $\gamma$. De todas estas propiedades únicamente la viscosidad depende significativamente de la temperatura.

Este tiempo adimensional combina el efecto de la temperatura y el tiempo sobre la densificación.

Ahora bien, puesto que cuando se introduce la probeta de vidrio en el horno la temperatura de ésta (alrededor de $300^{\circ} \mathrm{C}$ ) es mucho menor que la de sinterización, debe de transcurrir un periodo de tiempo hasta que la temperatura de la pieza sea prácticamente uniforme y similar a la de sinterización. Basándose en las dimensiones de la probeta y en los valores de su porosidad y propiedades térmicas, dicho periodo no debe ser inferior a los 5 minutos, de acuerdo con los resultados obtenidos por simulación en el apéndice (apartado 7.3.2.3). Así pues, en primera aproximación, se ha estimado el tiempo de residencia a la temperatura de sinterización, $t$, restando 5 minutos al tiempo de permanencia real en el horno, $t^{*}$. Es evidente que dicho método, para minimizar el efecto del problema del calentamiento de la muestra en tratamientos isotermos, es muy aproximado. Ahora bien, como ya se ha indicado antes, el objetivo de este apartado no 
es estudiar la cinética del proceso sino relacionar la evolución de la microestructura de la pieza con el avance del grado de sinterización, $\alpha$.

Tomando los valores de $\eta$ correspondientes a cada temperatura, de acuerdo con los valores de VFT de la Tabla 5.2 para $\mathrm{T}<1000^{\circ} \mathrm{C}$, el valor de $\gamma=0,277$ $\mathrm{N} / \mathrm{m}$ (estimado a partir de la composición del vidrio mediante la ecuación de Dietzel) y para d, el valor del diámetro medio geométrico de 5,9 $\mu \mathrm{m}$ (obtenido de la distribución de tamaños de partícula experimental) (Figura 4.1), los resultados de la Figura 5.7 se han agrupado bastante bien a una única curva (Figura 5.9), lo que confirma la isoconversionalidad del proceso.

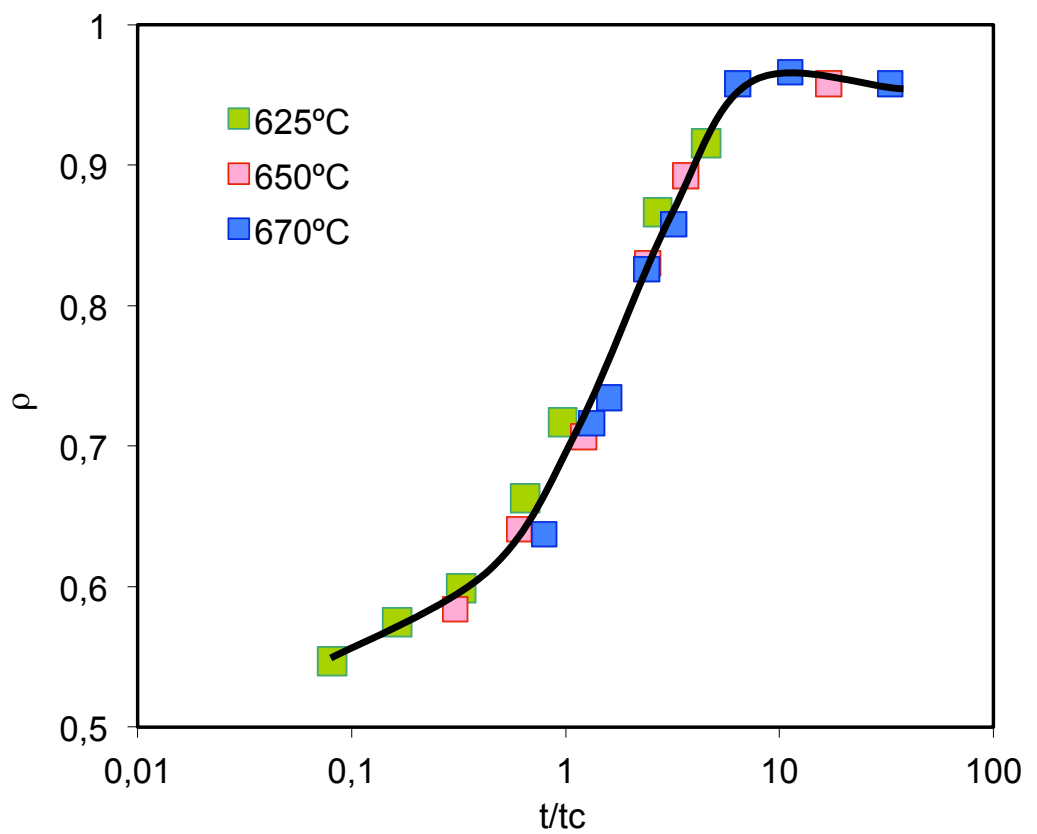

Figura 5.9. Compacidad, $\rho$, de las piezas cocidas frente al tiempo adimensional, $t / t_{c}$.

Experimentos isotermos. Compactos de vidrio GM.

Se comprueba, además, que, independientemente del tratamiento térmico, todas las probetas presentan una porosidad residual cerrada, de alrededor del $4 \%(\rho \approx 0,96)$. Este es un problema recurrente de la sinterización de materiales por flujo viscoso, que incluso en ausencia de cristalización y de inclusiones rígidas, impide que se pueda alcanzar la densificación completa 
utilizando el procedimiento de sinterización convencional en atmósfera de aire. En efecto, la baja solubilidad del vapor de agua, y del aire (particularmente del nitrógeno), en vidrios ricos en sílice conduce a que, a las temperaturas de sinterización, los gases previamente disueltos en el vidrio durante su fabricación (alrededor de $1500^{\circ} \mathrm{C}$ ) pasen a la fase gas. Además, los poros al cerrarse ocluyen aire. A elevadas temperaturas, cuando la viscosidad del vidrio es baja y la porosidad prácticamente cerrada, conforme aumenta la temperatura se reduce la viscosidad del vidrio a la vez que los poros ocluidos expanden provocando un crecimiento de los poros $y$, con ello, la dilatación de la probeta.

A partir de los valores de la compacidad en crudo, $\rho_{0}$, y en cocido, $\rho$, de cada probeta se han calculado los valores de un grado de avance de la sinterización, $\alpha^{\dagger}, \quad$ definido como la razón: contracción volumétrica/contracción volumétrica máxima, que al introducir los valores de compacidad inicial, $\rho_{0}$, a un tiempo t, $\rho$, y máxima, $\rho_{\max }$, se transforma en:

$$
\alpha=\frac{\rho-\rho_{0}}{\rho_{\max }-\rho_{0}} \cdot \frac{\rho_{\max }}{\rho}
$$

Los resultados se han representado en función del tiempo adimiensional, $t / t_{c}$ (Figura 5.10). Se confirma, como era de esperar, el principio de isoconversionalidad.

† Los valores de $\alpha$ calculados mediante la ec. 5.4, para las probetas conformadas por colado, que no son exactamente cilíndricas, son muy parecidos a los que se obtiene a partir de la ec. A1. 6, para compactos cilíndricos de pequeñas dimensiones, tratados térmicamente en el HSM. 


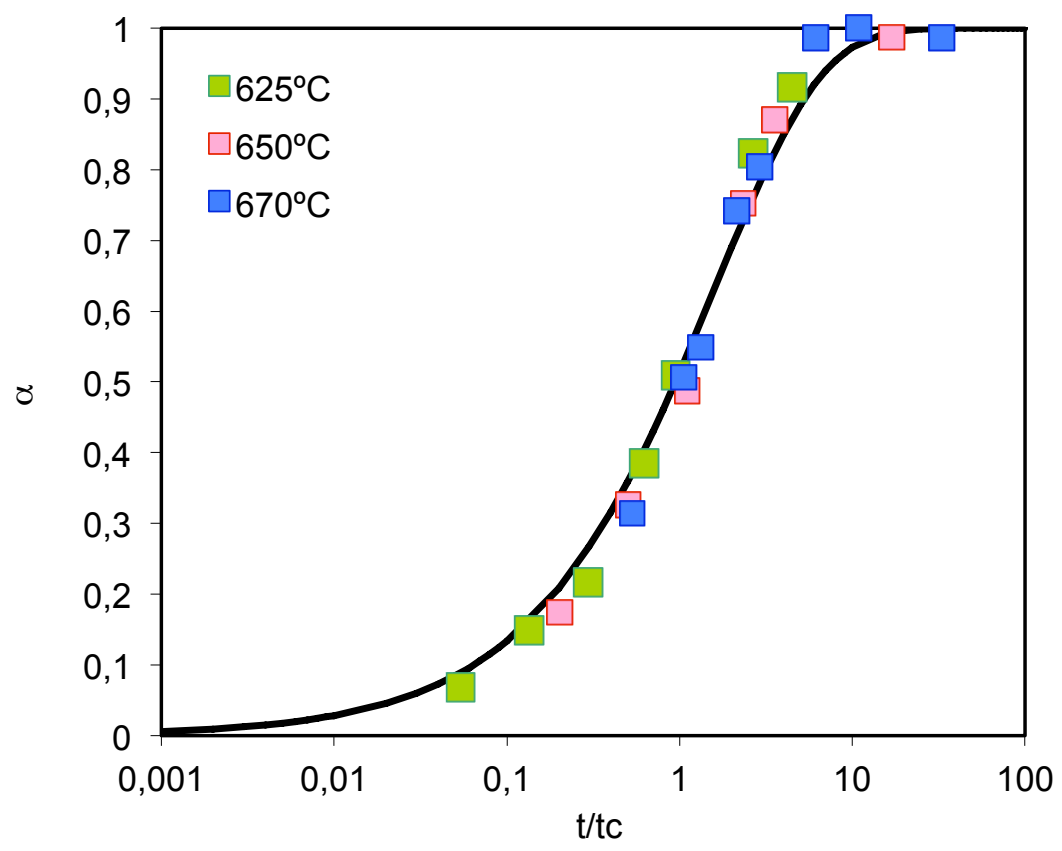

Figura 5.10. Variación del grado de avance de la sinterización, $\alpha$, frente al tiempo adimensional, $\mathrm{t} / \mathrm{t}_{\mathrm{c}}$. Experimentos isotermos. Compactos del vidrio $\mathrm{GM}$.

Además, los resultados, a pesar de las aproximaciones antes citadas, se han adaptado aceptablemente bien a una única curva representativa del modelo cinético de sinterización de este material (que se estudiará con mayor detalle en el apartado siguiente). Concretamente, los resultados se ajustaron a la ecuación:

$\alpha=1-\exp \left(-\frac{\lambda_{g} t}{t_{c}}\right)^{0.7}$

con un valor de $\lambda_{g}=0,73$.

5.1.1.4.3 Razón porosidad abierta/porosidad total, $\varepsilon_{\text {abierta }} / \varepsilon_{\text {total }}$.

A partir de los valores del volumen máximo de intrusión de mercurio y la compacidad de cada probeta cocida, se han calculado (teniendo en cuenta 
la densidad real de sólido) los valores de la razón porosidad abierta/porosidad total, $\varepsilon_{\text {abierta }} / \varepsilon_{\text {total }}$. En la Figura 5.11 y la Figura 5.12 se han representado estos valores frente a $\alpha$ y $\rho$ respectivamente.

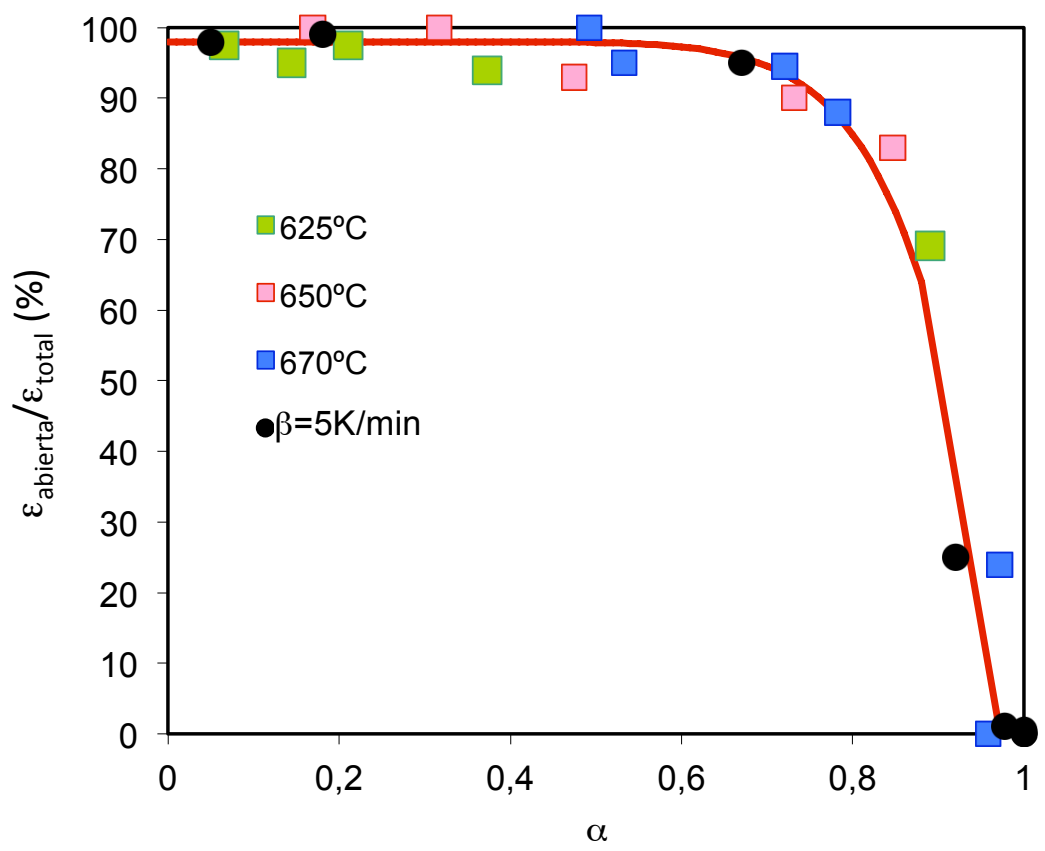

Figura 5.11. Variación de la razón $\varepsilon_{a b i e r t a} \varepsilon_{\text {total }}(\%)$ frente al grado de avance de la sinterización, $\alpha$. Compactos de vidrio GM. 


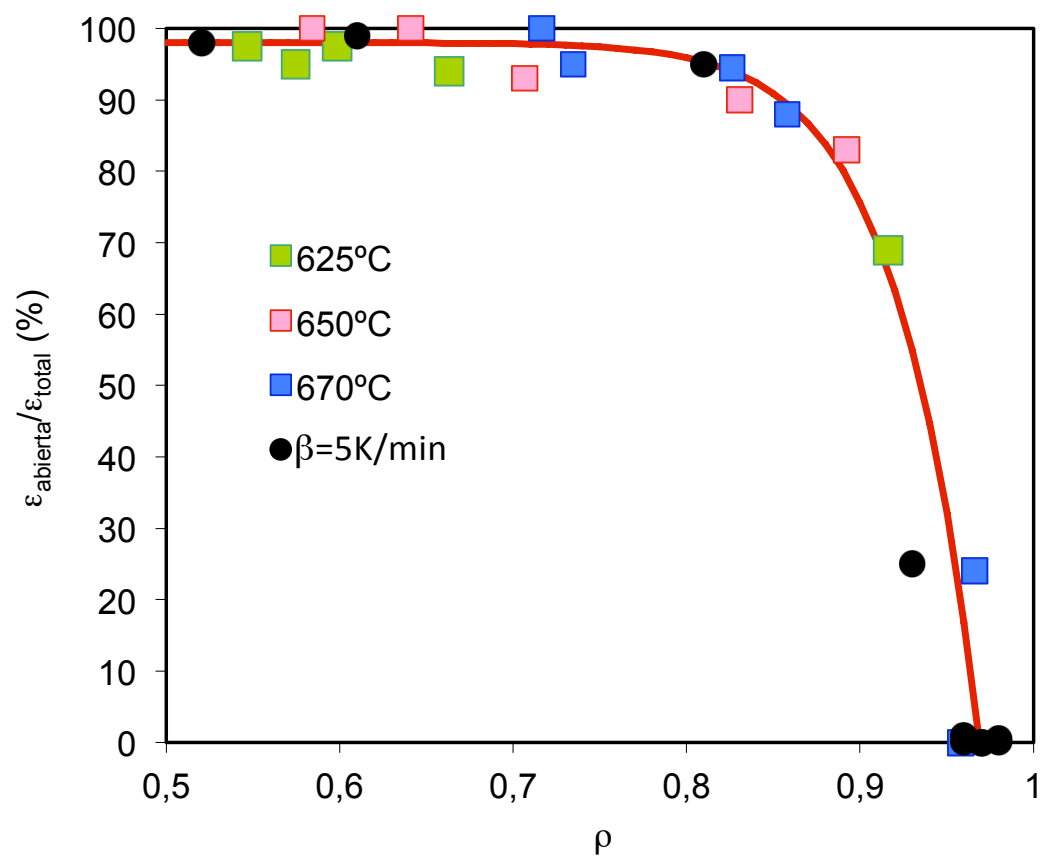

Figura 5.12. Variación de la razón $\varepsilon_{a b i e r t a} / \varepsilon_{\text {total }}(\%)$ frente a $\rho$. Compactos de vidrio GM.

Se comprueba que, independientemente del tratamiento térmico seguido, para cada representación todos los valores se ajustan a una única curva, de ecuaciones:

$\varepsilon_{\text {abierta }} / \varepsilon_{\text {total }}=0,98-\left(\frac{\alpha}{0,98}\right)^{10} y \varepsilon_{\text {abierta }} / \varepsilon_{\text {total }}=0,98-\left(\frac{\rho}{0,97}\right)^{20}$ ec. 5.6

Se aprecia claramente que para estados de sinterización iniciales e intermedios, $\alpha \leq 0,70$ y $\rho \leq 0,80$, la práctica totalidad de la porosidad es abierta. En cambio, para valores de $\alpha$ y $\rho$ más altos, la porosidad cerrada comienza a ser significativa, aumentando considerablemente conforme avanza el proceso de sinterización, hasta convertirse en la única presente para valores de $\alpha \approx 0,98$ y $\rho \approx 0,97$. 
5.1.1.4.4 Distribución de tamaño de los poros abiertos. Diámetro medio de intrusión. Presión de sinterización.

En la Figura 5.13 se ha representado, para todas las probetas estudiadas, la variación que sigue con el grado de avance de sinterización, $\alpha$, el diámetro medio de intrusión (poro), obtenido por porosimetría de mercurio. Éste se ha definido como la razón 4V/A, siendo $\mathrm{V}$ el volumen de intrusión correspondiente a un determinado tamaño de poro y $A$ su superficie, suponiendo que éste es cilíndrico. Se comprueba que todos los valores se ajustan a una única curva.

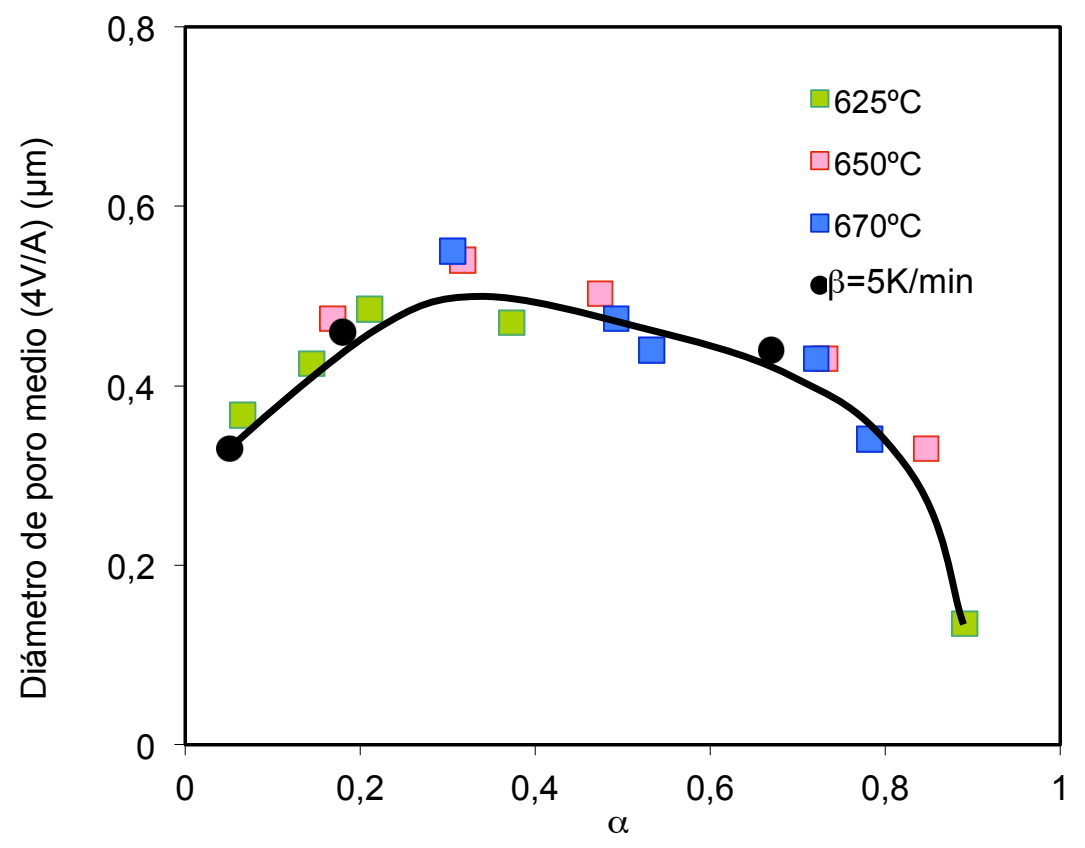

Figura 5.13. Variación del diámetro medio de intrusión (4V/A) frente al grado de avance de la sinterización, $\alpha$. Compactos de vidrio GM.

Al analizar la variación que sigue el diámetro medio de poro, 4V/A, con el grado de avance de la sinterización, $\alpha$, se aprecia que en los estados iniciales del proceso el tamaño medio de poro aumenta hasta alcanzar un máximo para $\alpha \sim 0,30$. Para valores de $0,3<\alpha<0,7$, el diámetro del poro va 
disminuyendo ligeramente conforme avanza el proceso de forma prácticamente lineal. En cambio, para valores de $\alpha \geq 0,8$ (cuando la porosidad cerrada empieza a ser significativa), la disminución del tamaño de poro es más brusca. En efecto, los poros más pequeños contraen a mayor velocidad, y por tanto, se van eliminando antes que los más grandes, debido a la mayor presión capilar de los primeros (inversamente proporcional al tamaño de poro). En consecuencia, conforme avanza el proceso de sinterización no solo se reduce el tamaño de los poros sino también su número ya que los poros inicialmente más pequeños desaparecen antes. El efecto combinado de ambas características conduce a que el tamaño medio de los poros alcance un máximo. Dicho comportamiento también ha sido comprobado por simulación numérica [112]. Por otra parte, conviene señalar que, en este caso, el crecimiento del tamaño medio de los poros, 4V/A, no requiere que los poros más grandes crezcan al inicio del proceso de sinterización (Figura 5.14), a diferencia de lo que ocurre en la densificación de las cerámicas tradicionales [113], y en composites, como se verá posteriormente. 


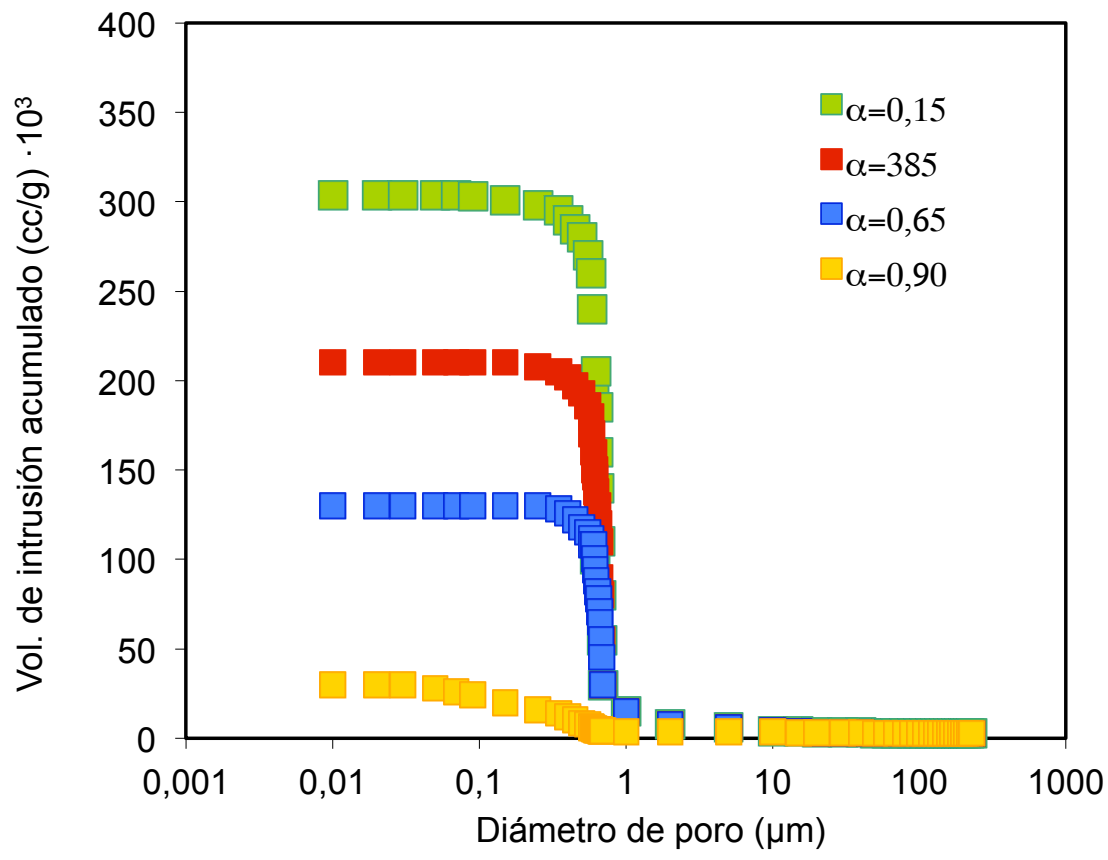

Figura 5.14. Volumen de intrusión acumulado frente al diámetro de poro para distintos grados de avance de la sinterización, $\alpha$. Compactos de vidrio GM.

Por otra parte, se ha calculado la presión de sinterización, $\Sigma^{\mathcal{S}}$, de acuerdo con la ec. 5.7, obtenida por simulación para distribuciones de tamaño de poro y formas complejas, por Wakai [114], a partir de los valores del diámetro medio de poros obtenido experimentalmente, 4V/A, por porosimetría de mercurio, Figura 5.13. En efecto, sustituyendo en la ecuación desarrollada por este autor el diámetro medio de poro por 4V/A, resulta la expresión:

$\Sigma^{S}=\frac{8}{3} \gamma\left(\frac{4 \mathrm{~V}}{\mathrm{~A}}\right)^{-1}$

En la Figura 5.15 se representan estos valores frente al grado de avance de la sinterización, $\alpha$. Como era de esperar, al igual que ocurre con el diámetro medio de poro, la variación de $\Sigma^{S}$ con $\alpha$ es independiente del ciclo térmico. 
Además, se aprecia que, debido a un aumento inicial del diámetro medio de poro, la presión de sinterización disminuye ligeramente hasta alcanzar un mínimo, para valores de $\alpha \approx 0,3$. A partir de dicho valor, estados intermedios y finales de la sinterización, $\sum^{S}$ aumenta con el grado de avance de la sinterización.

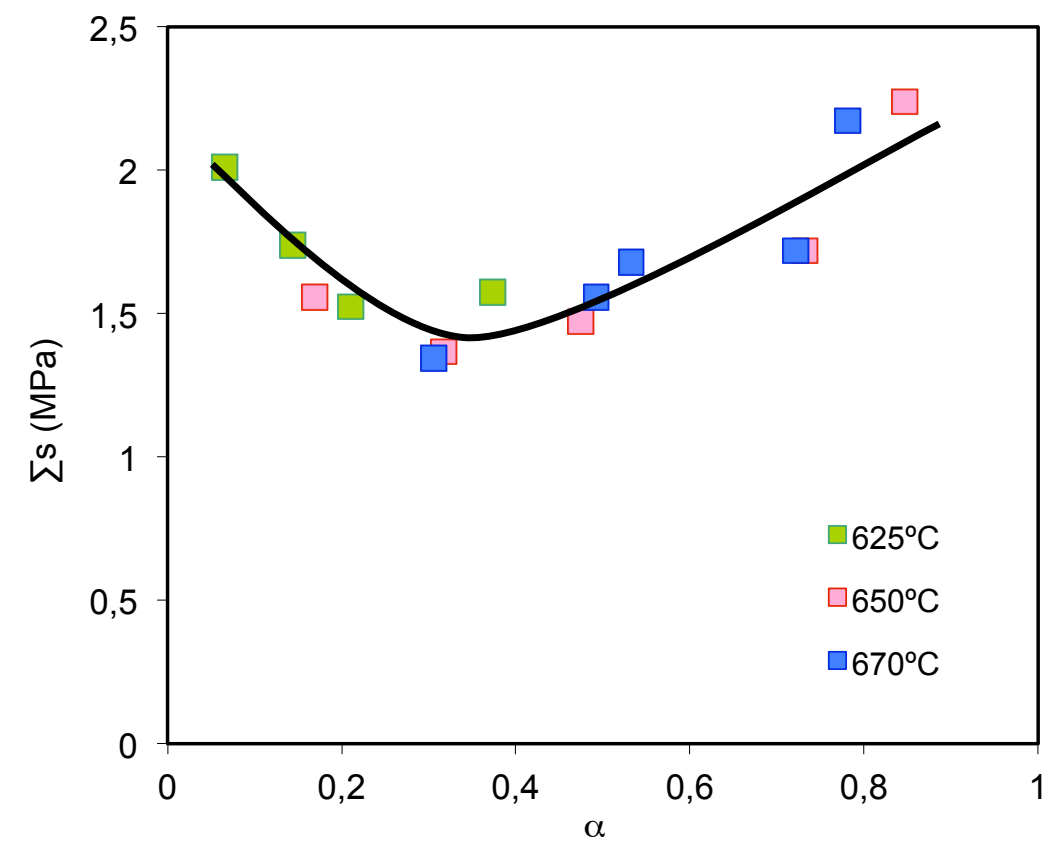

Figura 5.15. Variación de la presión de sinterización, $\sum^{S}$ (ec. 5.7) frente al grado de avance de la sinteirzación, $\alpha$. Compactos de vidrio GM.

Estos resultados se han comparado con la relación $P_{L}$ vs $\rho$ que resulta de aplicar los diferentes modelos teóricos que se detallan en la Tabla 2.3 para estos compactos de vidrio (Figura 5.16). 


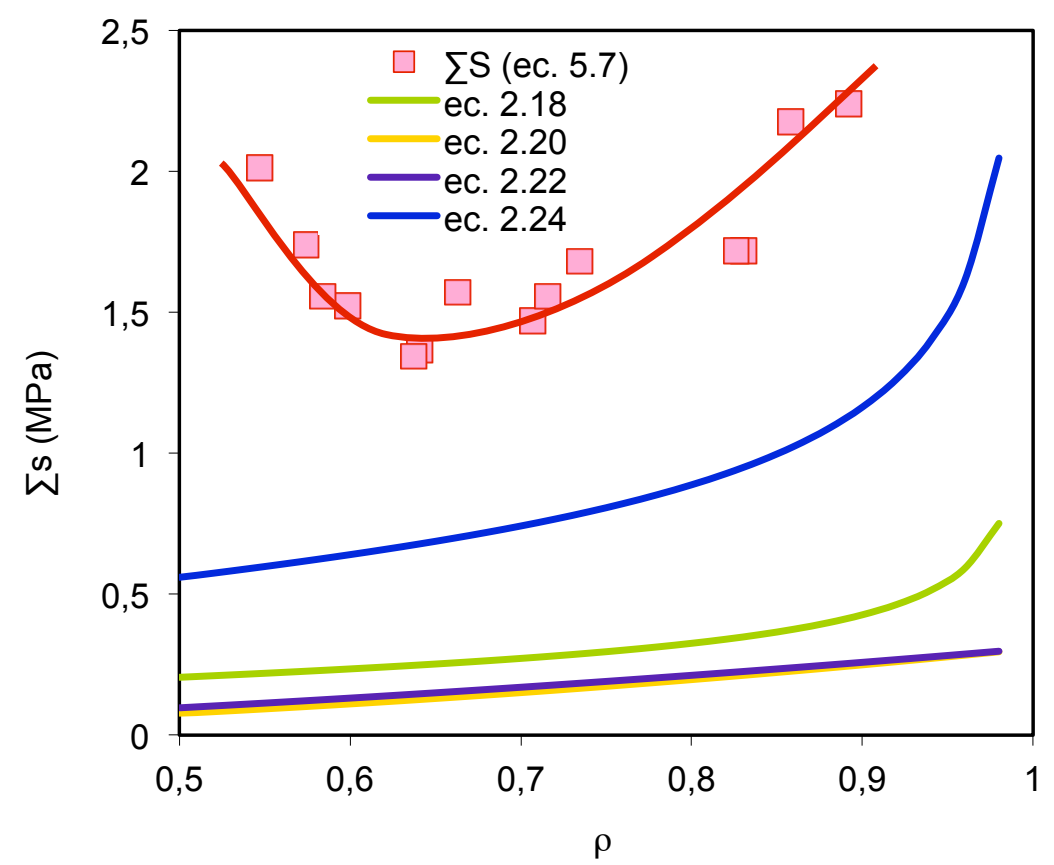

Figura 5.16. Variación de la presión de sinterización calculada según (ec. 5.7), $\sum^{S}, y$ la resultante de aplicar distintos modelos $\left(P_{L}\right)$ con el grado de avance de la sinterización, $\alpha$. Compactos de vidrio $G M$.

Se confirma, en primer lugar, que existe una gran disparidad entre los valores obtenidos a partir de uno y otro modelo, debido a las simplificaciones e hipótesis que utiliza cada uno. No obstante, para estados intermedios y finales de la sinterización, el efecto cualitativo de $\rho$ sobre la presión de sinterización $\left(\Sigma^{S} \circ \mathrm{P}_{\mathrm{L}}\right)$ es similar.

Por otra parte, conviene señalar que aunque el modelo de Wakai, en principio, es de mayor validez y aplicabilidad que los otros, debido a que tiene en cuenta en su deducción características microestructurales del sistema poroso más acordes con la realidad (formas y distribución de tamaños) su utilización requiere disponer simultáneamente de los valores del volumen y del área superficial de los poros para distintos grados de sinterización. En nuestro caso hemos considerado que la razón: superficie de poros/volumen de poros, es la que se obtiene a partir de la porosimetría 
de mercurio, que asume que todos los poros son cilíndricos, y su diámetro es el de intrusión. Ambas hipótesis son burdas simplificaciones de la distribución porosa real (Figura 5.5 y Figura 5.6).

Por otra parte, en ninguno de los modelos de estimación de la presión de sinterización se tiene en cuenta el efecto de la presión de los gases ocluidos en los poros cerrados, que debe reducir considerablemente dicha característica en la etapa final de la sinterización. En consecuencia, debe considerarse que todos los modelos de estimación de la presión de sinterización son inapropiados.

5.1.1.4.5 Porosidad cerrada. Distribución de tamaño de poros.

En este apartado, la porosidad cerrada se ha estudiado mediante observación al MEB y análisis de imagen, de las probetas cocidas a la temperatura de máxima densificación y superiores (a velocidad de calentamiento constante).

Los resultados se detallan en la Figura 5.17, en la forma porcentaje del área ocupada por poros, y en la Figura 5.18, en la forma de $\mathrm{n}^{\circ}$ deporos $/ \mathrm{mm}^{2}$. En ambas figuras se consideran los poros totales, los poros de radio superior a $10 \mu \mathrm{m}$ y los de radio inferior al micrómetro. Seleccionando estos tamaños es posible comparar estos resultados con los que se obtienen con otras matrices vítreas (fritas) empleadas en la industria y estudiadas por nosotros en el ITC. 


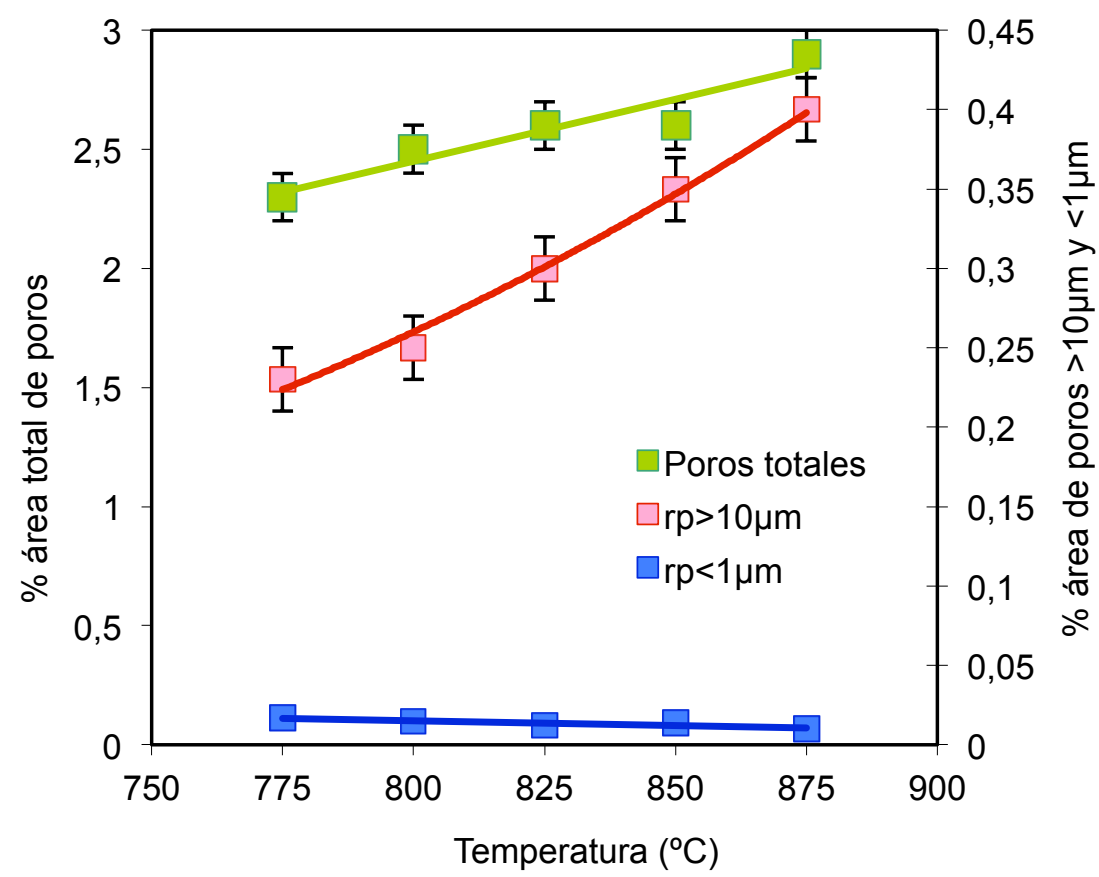

Figura 5.17. Variación porcentual del área ocupada por poros en la sección de una probeta frente a la temperatura de cocción. Experimentos a velocidad de calentamiento constante, $\beta=5 \mathrm{~K} / \mathrm{min}$. Compactos de vidrio $\mathrm{GM}$.

Se comprueba un aumento prácticamente lineal de la porosidad total de la muestra con el incremento de la temperatura de cocción (Figura 5.17) debido, en parte, al incremento del número (Figura 5.18) y volumen (Figura 5.17) de los poros más grandes $\left(r_{p}>10 \mu m\right)$.

Por el contrario, el área ocupada por los poros más pequeños $\left(r_{p}<1 \mu \mathrm{m}\right)$ (Figura 5.17) y su número (Figura 5.18) se reducen con el aumento de la temperatura. 


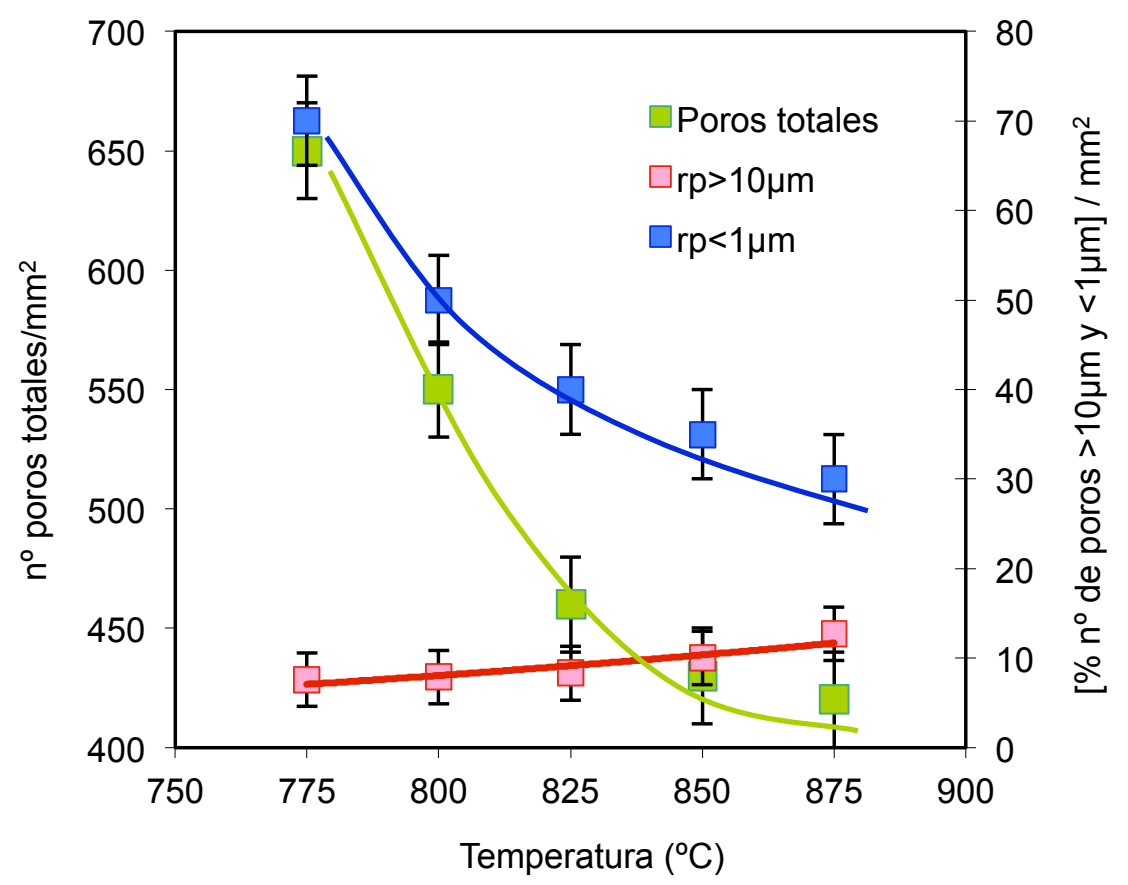

Figura 5.18. Variación del número de poros $/ m^{2}$ de sección la probeta frente a la temperatura de cocción. Experimentos a velocidad de calentamiento constante, $\beta=5 \mathrm{~K} / \mathrm{min}$. Compactos de vidrio $\mathrm{GM}$.

El continuo aumento del número y volumen de poros grandes $\left(r_{p}>10 \mu m\right)$, y la progresiva disminución del número y volumen de los más pequeños $\left(r_{p}<1 \mu \mathrm{m}\right)$ con el aumento de la temperatura, es consistente con la variación que sigue con dicha variable la tensión de sinterización para un poro esférico y cerrado, cuya expresión viene dada por:

$\left(P_{L}\right)_{\text {cerrado }}=\left(2 \gamma-P_{g} \cdot r_{p}\right)$

siendo: $\gamma$ la tensión superficial del vidrio, $\mathrm{P}_{\mathrm{g}}$ la presión del gas en el interior del poro y $r_{p}$ el radio del poro.

Así pues, si el producto $P_{g} \cdot r_{p}$, dependiendo de la temperatura y el tamaño del poro, es mayor que $2 \gamma$ (prácticamente constante) $P_{L}$ es negativo por lo 
que el poro tiene a aumentar de tamaño. En el caso contrario, $P_{g} \cdot r_{p}<2 \gamma$ el poro reduce su tamaño. En nuestro caso, a una temperatura dada, la distribución de tamaños de poros cerrador es muy heterogénea, al igual que la de $\mathrm{P}_{\mathrm{g}}$, debido a que el fenómeno de cerrado de los poros es gradual (los que se cierran a temperaturas más bajas tendrán un valor más alto según la ley de los gases perfectos).

En consecuencia, para poros pequeños de $r_{p}<1 \mu \mathrm{m}$, incluso para $P_{g}$ altos, $\left(\mathrm{P}_{\mathrm{L}}\right)_{\text {cerrado }}$ es positivo y los poros se van reduciendo de tamaño, tal como reflejan los resultados de la Figura 5.17 y Figura 5.18. Por el contrario, para $r_{p}>10 \mu m,\left(P_{L}\right)_{\text {cerrado }}<0$, incluso para valores de $P_{g}$ pequeños.

Por otra parte, el aumento de la temperatura no solo incrementa el valor del producto $P_{g} \cdot r_{p}$ sino que también reduce la viscosidad del fundido lo que acentúa el proceso de crecimiento o reducción del tamaño de poros.

\subsubsection{Análisis cinético de la sinterización. Determinación de los parámetros cinéticos.}

5.1.2.1 Experimentos a velocidad de calentamiento constante (granulometría GM).

Se han preparado compactos de partículas de vidrio de la distribución granulométrica GM. Sus curvas de sinterización ( $\alpha$ vs T), obtenidas a diferentes velocidades de calentamiento (Figura 5.19), son claramente sigmoidales, lo que indica que, en principio, pueden ser descritas mediante modelos de una sola etapa. 


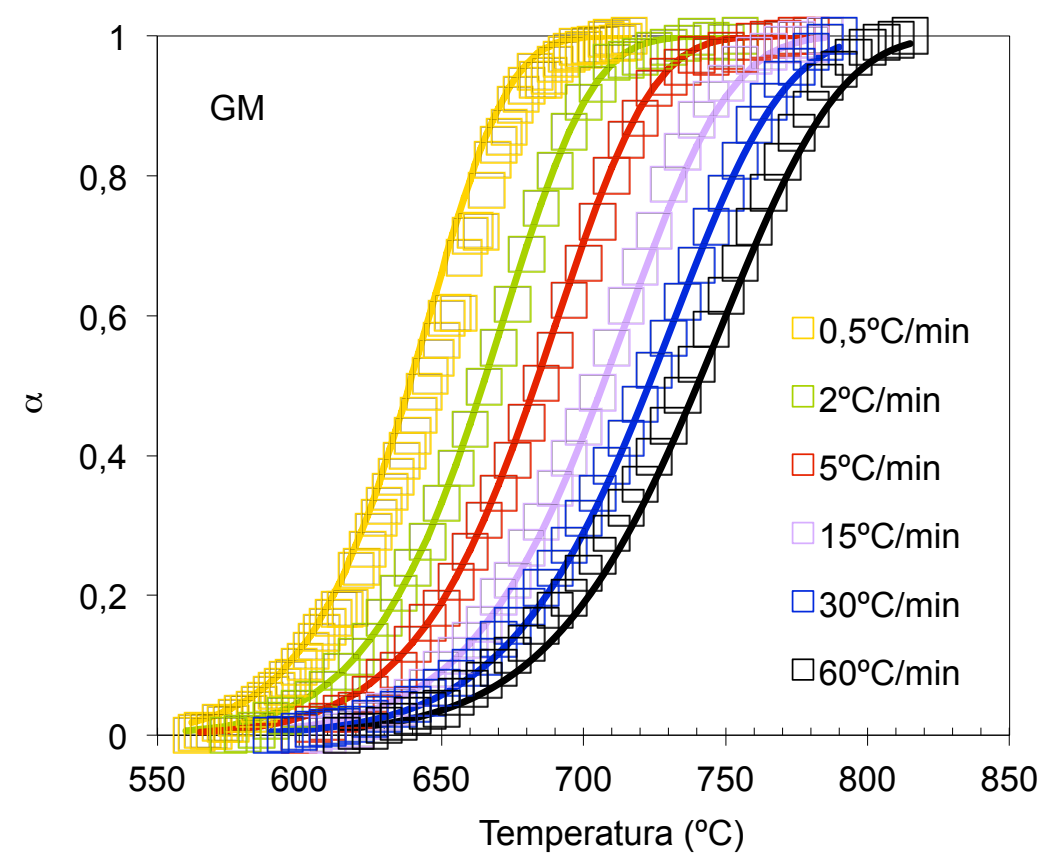

Figura 5.19. Curvas de sinterización no isotermas de compactos de vidrio de la granulometría GM, a distintas velocidades de calentamiento. Comparación entre los valores experimentales y los calculados según el ajuste 3 de la Tabla 5.4 .

\subsection{Métodos isoconversionales}

En la Figura 5.20 se han representado, frente al grado de sinterización, $\alpha$, los valores de la energía de activación aparente, $\mathrm{E}_{\alpha}$, que resultan de aplicar el método de K.A.S. (ec. 2.80) y el método de Friedman (ec. 2.73). Los valores de $\left(\frac{d \alpha}{d T}\right)_{\alpha, i}$ requeridos para aplicar este último método, se han calculado aplicando el método de Savitzchi-Golay. 


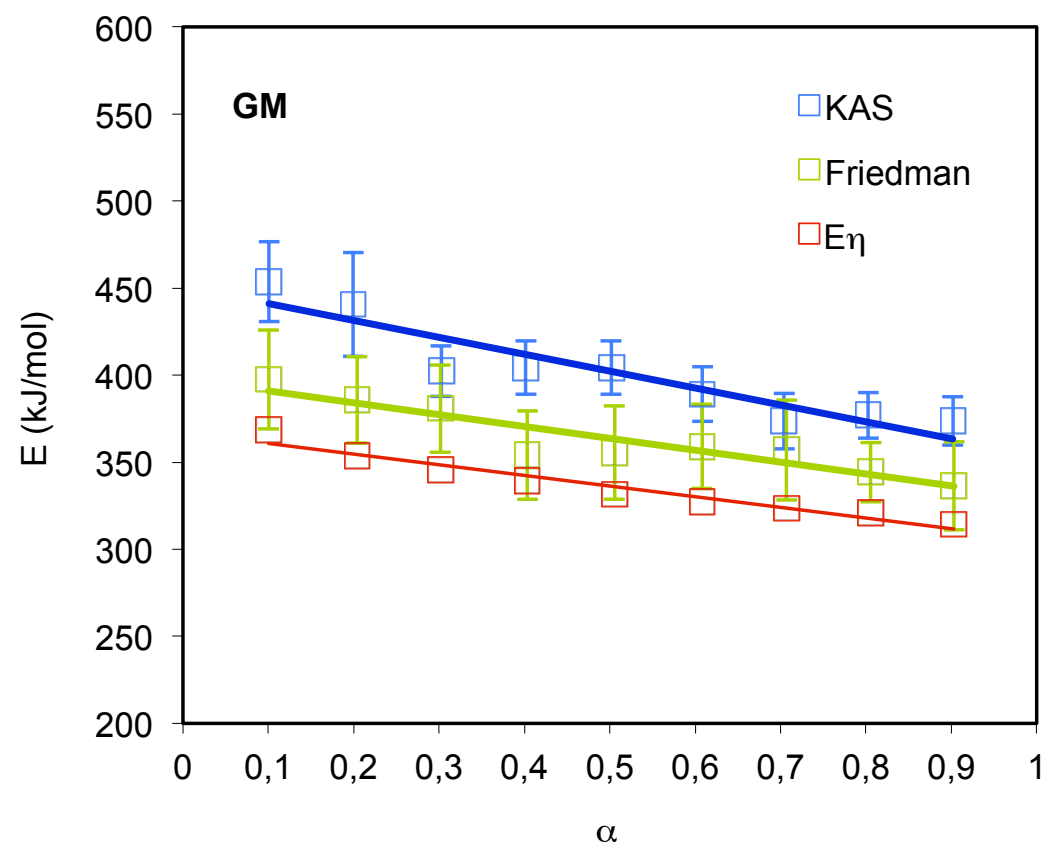

Figura 5.20. Variación de la energía de activación de la sinterización, E $\alpha$, y de flujo

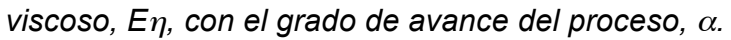

Se ha incluido en esta figura los valores de la energía de activación de flujo viscoso, $E_{\eta}$, obtenidos a partir de la curva de viscosidad que resulta del ajuste a la ecuación de Vogel-Fulcher-Tamman (VFT), tomando como parámetros los de la Tabla 5.2 para $\mathrm{T}<1000^{\circ} \mathrm{C}$. Para ello, los valores de la viscosidad correspondientes al intervalo de temperaturas que comprende cada valor de $\alpha$ se han ajustado a la ecuación de Arrhenius (ec. 5.2).

Se comprueba que tanto los valores de $\mathrm{E}_{\alpha}$, calculados por uno $\mathrm{u}$ otro método, como los de $E_{\eta}$ van disminuyendo ligeramente con el aumento de $\alpha$, con una pendiente muy parecida. Dicho comportamiento indica que la variación de $\mathrm{E}_{\alpha}$ con $\alpha$ no se debe a ningún cambio progresivo del mecanismo de sinterización, sino a que la ecuación de Arrhenius solo 
describe aceptablemente bien el efecto de la temperatura sobre la viscosidad para pequeños intervalos de temperatura.

Asimismo, se aprecia que los valores de $E_{\alpha}$ calculados por el método de Friedman son, para cualquier valor de $\alpha$, más parecidos a los del flujo viscoso, $E_{\eta}$, que los calculados por el método de K.A.S. En efecto, el valor medio de $E_{\alpha}$ calculado por el método de K.A.S. es $\bar{E}_{\alpha}=402 \pm 29 \mathrm{~kJ} / \mathrm{mol}$, sensiblemente mayor que el calculado por el método de Friedman, $\overline{E_{\alpha}}=363 \pm 20 \mathrm{~kJ} / \mathrm{mol}, y$ notablemente mayor que el de flujo viscoso, $\overline{\mathrm{E}_{\eta}}=336 \pm 17 \mathrm{~kJ} / \mathrm{mol}$.

Estos resultados ponen claramente de manifiesto, por una parte, que la sinterización de partículas de vidrio es un proceso controlado exclusivamente por un mecanismo de flujo viscoso. $\mathrm{Y}$, por otro, que el método de Friedman, que no asume ninguna simplificación en el cálculo de $E_{\alpha}$, es el más apropiado. El hecho que los valores de $E_{\alpha}$, para cualquier valor de $\alpha$, tanto si son calculados por el método de Friedman como por el de K.A.S., sean siempre más dispersos y altos que los de $E_{\eta}$ (Figura 5.20) también puede deberse a que la temperatura de la muestra y la del horno son ligeramente diferentes, especialmente a altas velocidades de calentamiento (apartado 7.3.2.1).

\subsection{Método de Kissinger.}

Al aplicar este método (apartado 2.2.4) se ha obtenido como resultado una recta de ajuste (Figura 5.21), de acuerdo con la ec. 2.85, con un coeficiente de correlación aceptable $\left(r^{2}=0.9892\right)$. El valor de la energía de activación ha sido de $384 \pm 20 \mathrm{~kJ} / \mathrm{mol}$. Se comprueba que el valor obtenido por este método es intermedio al obtenido por los dos métodos isoconversionales. Asimismo, la incertidumbre asociada al resultado es igual al obtenido por el método de Friedman. 


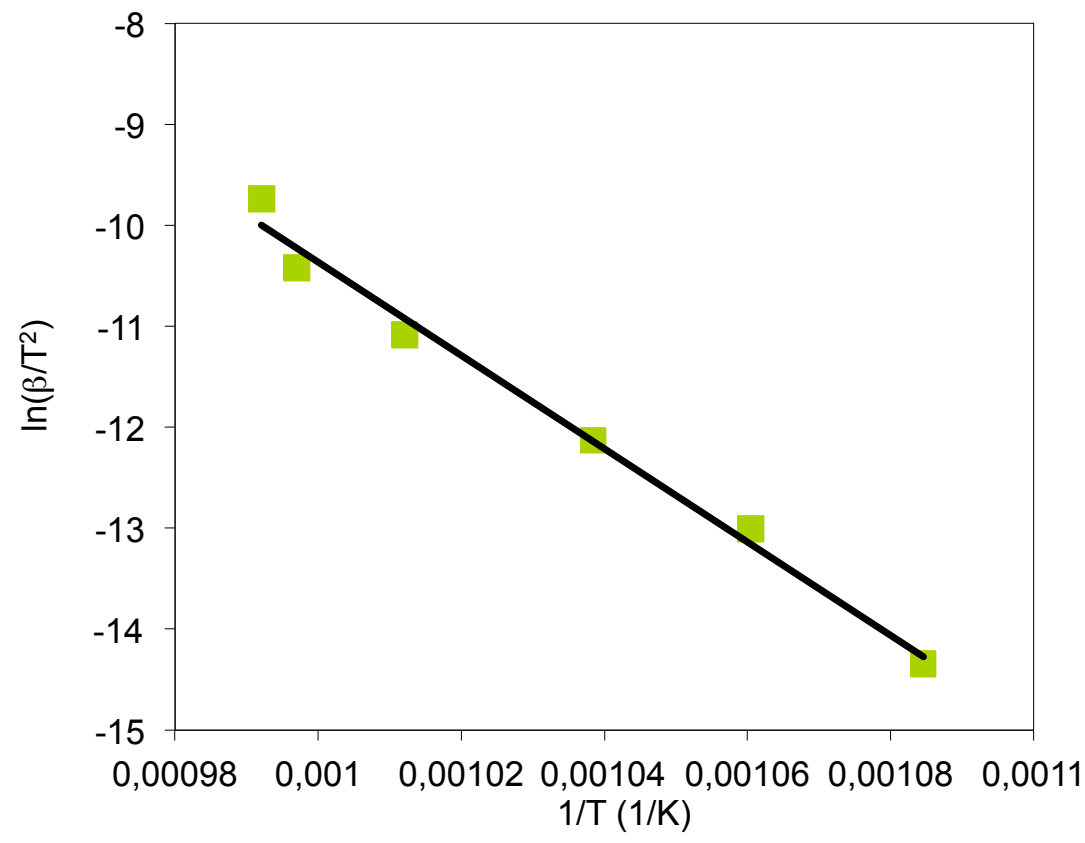

Figura 5.21. Determinación de la energía de activación por el método de Kissinger.

5.1.2.1.3 Método de ajuste al modelo.

Insertando cada una de las expresiones integradas, $g(\alpha)$, de los distintos modelos (Tabla 2.5) en la ec. 2.87 y ajustando a la ecuación resultante por regresión lineal, los pares de valores $\alpha$ y $\mathrm{T}$, correspondientes a la velocidad de calentamiento, $\beta$, se obtienen los parámetros de Arrhenius y la bondad del ajuste $\left(r^{2}\right)$. El ajuste de los resultados experimentales al modelo de Avrami-Erofeev, para diferentes valores de $n$, con $0<n<1$, fueron buenos siendo el valor de $\mathrm{E}$ obtenido dependiente del valor del índice de potencia de Avrami "n". En efecto, al insertar la ec. 2.49 en la ec. 2.87, resulta la expresión:

$\ln [-\ln (1-\alpha)]=n \cdot \ln \frac{A R T^{2}}{\beta E}-\frac{n E}{R T}$

por lo que $\mathrm{nE}=$ cte, si los resultados se ajustan a la ecuación de Avrami. 
En nuestro caso, éste varía muy poco con la velocidad de calentamiento; de $\mathrm{nE}=255 \mathrm{~kJ} / \mathrm{mol}$ para $\beta=0,5 \mathrm{~K} / \mathrm{min}$ a $\mathrm{nE}=248 \mathrm{~kJ} / \mathrm{mol}$ para $\beta=60 \mathrm{~K} / \mathrm{min}$.

El ajuste correspondiente a los restantes modelos de la Tabla 5.2, en todos los casos, fue muy pobre $r^{2}<0.96$.

Estos resultados confirman que, con este método, se pueden obtener, ajustando a diferentes modelos de sinterización (o con diferentes valores de n), resultados buenos y equivalentes, desde el punto de vista estadístico, por lo que no es posible, solamente mediante el empleo de este método, seleccionar el triplete cinético correcto, en nuestro caso, una vez fijado el mejor modelo cinético, el valor de $n, E$ y $\ln A$.

Para resolver esta ambigüedad, dado que la energía de activación obtenida por los métodos isoconversionales, $E_{\alpha}$, varía muy poco con $\alpha$, para $0,05 \leq \alpha \leq 0,95$ resulta más efectivo tomar como correcto este valor de la energía de activación, $\overline{\mathrm{E}}_{\alpha}$, y determinar por ajuste al modelo los valores de $\mathrm{n}$ $y \ln A$.

Al comparar los valores de $\mathrm{nE}$ obtenidos por el método de ajuste al modelo, con los valores medios obtenidos por los métodos isoconversionales, $\overline{\mathrm{E}}_{\alpha}$, se aprecia que el valor de $\mathrm{n}$ del modelo de Avrami que conduce a valores de $\mathrm{n} \overline{\mathrm{E}}_{\alpha}$ más próximos a $\mathrm{nE}$ está comprendido entre 0,60 y 0,75 , dependiendo del valor de $\overline{\mathrm{E}}_{\alpha}$ utilizado y la velocidad de calentamiento, $\beta$. Este resultado es consistente con lo indicado en el apartado 2.1.4.1.3.5 en lo que respecta a la validez de este modelo para describir todo el proceso de sinterización de vidrios, con valores de $\mathrm{n}$ próximos a los que resultan de aplicar la teoría continua de la sinterización a los modelos teóricos de Mackenzie (Figura 2.12) y Sura \& Panda (Figura 2.14), en la que se confirma la validez del modelo Avrami-Erofeev con valores de $\mathrm{n} \leq 1$ para describir la cinética de sinterización por flujo viscoso de materiales con alto contenido en fase vítrea. 


\subsection{Selección del modelo. Determinación del triplete cinético.}

El ajuste del modelo de Avrami-Erofeev se realiza mediante regresión no lineal, que minimiza la diferencia entre los valores de $\alpha$ obtenidos experimentalmente, $\alpha_{\mathrm{exp}}$, y calculados, $\alpha_{\mathrm{cal}}$, mediante la expresión:

$\mathrm{RCS}=\sum\left(\alpha_{\text {exp }}-\alpha_{\text {cal }}\right)^{2}=\min$ ec. 5.10

Los valores de $\alpha_{\text {cal }}$ son los que resultan de ajustar los resultados experimentales a la expresión:

$\alpha_{\text {cal }}=1-\exp \left\{-\left[\left(\frac{A}{\beta}\right)\left(\frac{R T^{2}}{E}\right) \exp \left(-\frac{E}{R T}\right)\right]^{n}\right\}$

En la Tabla 5.3 se detallan los valores del triplete cinético, $\mathrm{E}, \mathrm{n}$ y $\ln \mathrm{A}$ (en este caso $\ln A_{g}$ ), y de la varianza, $S^{2}$, que resultan al ajustar los valores experimentales a la ec. 5.11, con las siguientes condiciones: sin fijar ningún parámetro (ajuste 1), fijando el valor de $n$ (ajuste 2), fijando $n$ y $E$ (ajuste 3 ) y con los valores promedio de $\mathrm{n}, \mathrm{E}$ y $\ln \mathrm{A}_{\mathrm{g}}$ (ajuste 4). Obviamente, conforme aumente el número de parámetros que se fijan se incrementa el valor de la varianza. No obstante, en todos los casos, dicho valor es bajo.

Tabla 5.3. Valores del triplete cinético: $E, n$ y $\ln A_{g}$, y de la varianza $S^{2}$ que resulta del ajuste de los valores de $\alpha$ a la ec. 5.11.

\begin{tabular}{|c|c|c|c|c|}
\hline Ajuste & $\mathbf{E}(\mathbf{k J} / \mathbf{m o l})$ & $\mathbf{n}$ & $\operatorname{In} \mathbf{A}_{\mathbf{g}}\left(\mathbf{s}^{-1}\right)$ & $\mathbf{S}^{2}$ \\
\hline 1 & $340 \pm 16$ & $0.71 \pm 0.23$ & $36.33 \pm 2.36$ & 0.000088337 \\
\hline 2 & $349 \pm 21$ & 0.71 & $37.03 \pm 2.47$ & 0.0001222 \\
\hline 3 & 349 & 0.71 & $37.84 \pm 0.226$ & 0.0002103 \\
\hline 4 & 349 & 0.71 & 37.84 & 0.0013713 \\
\hline
\end{tabular}

En la Figura 5.22 se ha representado frente a la velocidad de calentamiento, los valores de la energía de activación, E, que se obtienen de los ajustes, 
fijando el valor de $n=0,71$, y los de flujo viscoso correspondientes a los respectivos intervalos de temperatura.

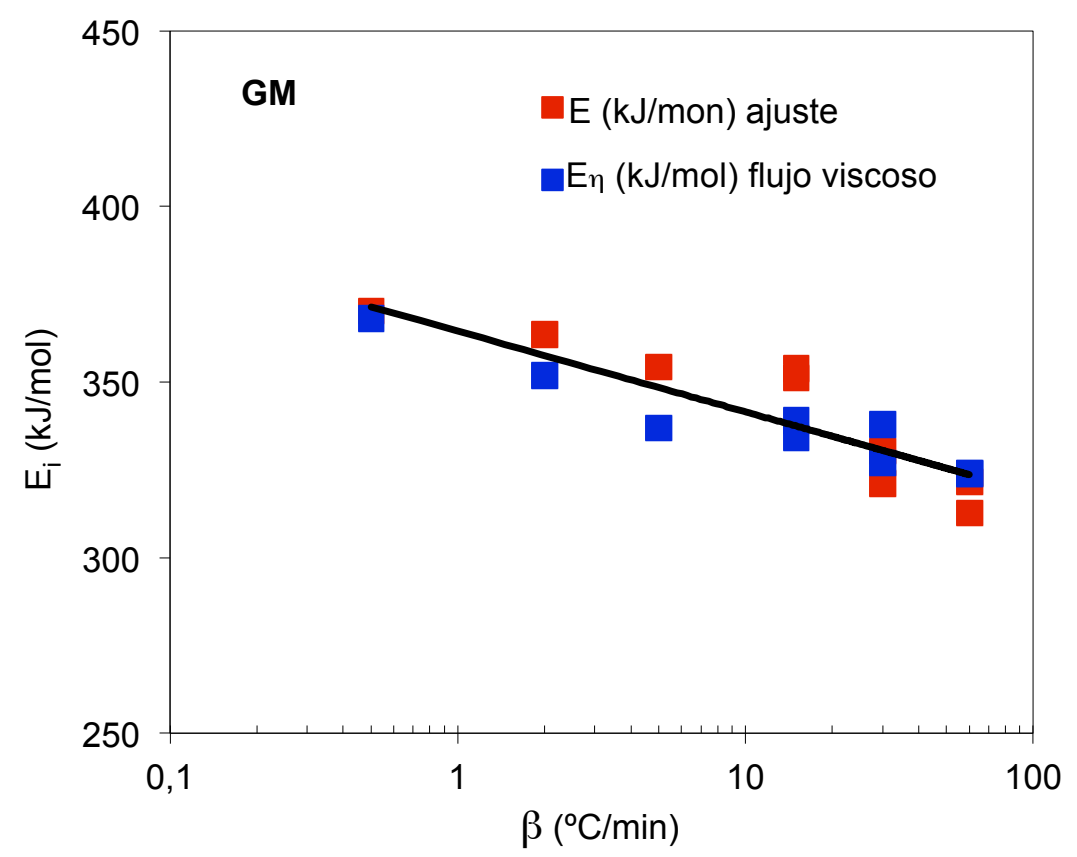

Figura 5.22. Variación de la energía de activación de la sinterización, E, y de flujo viscoso, $\mathrm{E}_{\eta}$, con la velocidad de calentamiento, $\beta$.

Se confirma que los valores de $E$ y $E_{\eta}$ son en general muy parecidos a cualquier velocidad de calentamiento, y, además, los dos valores van disminuyendo conforme aumenta la velocidad de calentamiento, siguiendo la misma tendencia. En vista de esta buena concordancia entre $E$ y $E_{\eta}$ se ha considerado sustituir la dependencia de la velocidad del proceso con la temperatura dada por la ecuación de Arrhenius, por la relación que sigue la inversa de la viscosidad con la temperatura dada por la ecuación de V.F.T. (ec. 5.1), tomando como valores de $\mathrm{B}$ y $\mathrm{T}_{0}$ los que resultan de ajustar los valores de la viscosidad recomendados por la NBS para el intervalo de baja temperatura (Tabla 5.2).

Es decir: 
$\alpha_{\text {cal }}=1-\exp \left\{-\left[\left(\frac{A}{\beta}\right)\left(\frac{\left(T-T_{0}\right)^{2}}{B}\right) \exp \left(-\frac{B}{\left(T-T_{0}\right)}\right)\right]^{n}\right\}$

ec. 5.12

Como en el caso anterior se han realizado tres ajustes modificando el número de parámetros fijos. Se comprueba que el ajuste 3 de la Tabla 5.4 que mantiene fijos todos los parámetros y que considera que la constante de velocidad varía de acuerdo con la inversa de la viscosidad experimental da muy buenos resultados (Figura 5.19). El hecho de que las curvas correspondientes a los valores más extremos de velocidad de calentamiento ( $\beta=0,5$ y $60 \mathrm{~K} / \mathrm{min}$ ) sean los que menos se ajustan al modelo, puede ser debido al efecto que dicha variable, $\beta$, ejerce sobre la diferencia de temperatura entre el horno y la muestra (apartado 7.3.2.1).

Tabla 5.4. Valores de los parámetros $\ln A_{g}, n$ y de la varianza, $S^{2}$, que resultan de ajustar los valores de $\alpha$ a la ec. 5.12. Valores de la ecuación de V.F.T. (ec. 5.1): $B=5495 \mathrm{~K}$ y $T_{0}=421 \mathrm{~K}$.

\begin{tabular}{|c|c|c|c|}
\hline Ajuste & $\mathbf{n}$ & $\operatorname{InA}_{\mathbf{g}}\left(\mathbf{s}^{-1}\right)$ & $\mathbf{S}^{2}$ \\
\hline 1 & $0,71 \pm 0,019$ & $17,51 \pm 0,16$ & 0,0001058 \\
\hline 2 & 0,71 & $17,52 \pm 0,16$ & 0,0001228 \\
\hline 3 & 0,71 & 17,52 & 0,0009562 \\
\hline
\end{tabular}

\subsubsection{Experimentos isotermos.}

Debido a que cuando la pieza alcanza la temperatura de sinterización, la probeta ya ha experimentado una cierta contracción, para ajustar los resultados experimentales al modelo es necesario integrar la ecuación cinética en forma diferencial (ec. 5.13), teniendo en cuenta esta condición inicial: 
$\frac{\mathrm{d} \alpha}{\mathrm{dt}}=\mathrm{k} \cdot \mathrm{n} \cdot(1-\alpha)[-\ln (1-\alpha)]^{\mathrm{n}-1}$

Para ello, se ha tomado como límite de integración las condiciones iniciales, para $\mathrm{t}=\mathrm{t}_{0} \alpha=\alpha_{0}$, y un valor genérico, $\mathrm{t}=\mathrm{t} \alpha=\alpha$, resultando:

$$
\alpha=1-\frac{1}{\exp \left\{k \cdot t+\left[-\ln \left(1-\alpha_{0}\right)\right]^{\frac{1}{n}}\right\}^{n}}
$$

En la Figura 5.23 se representan los resultados experimentales y calculados correspondientes a seis temperaturas diferentes.

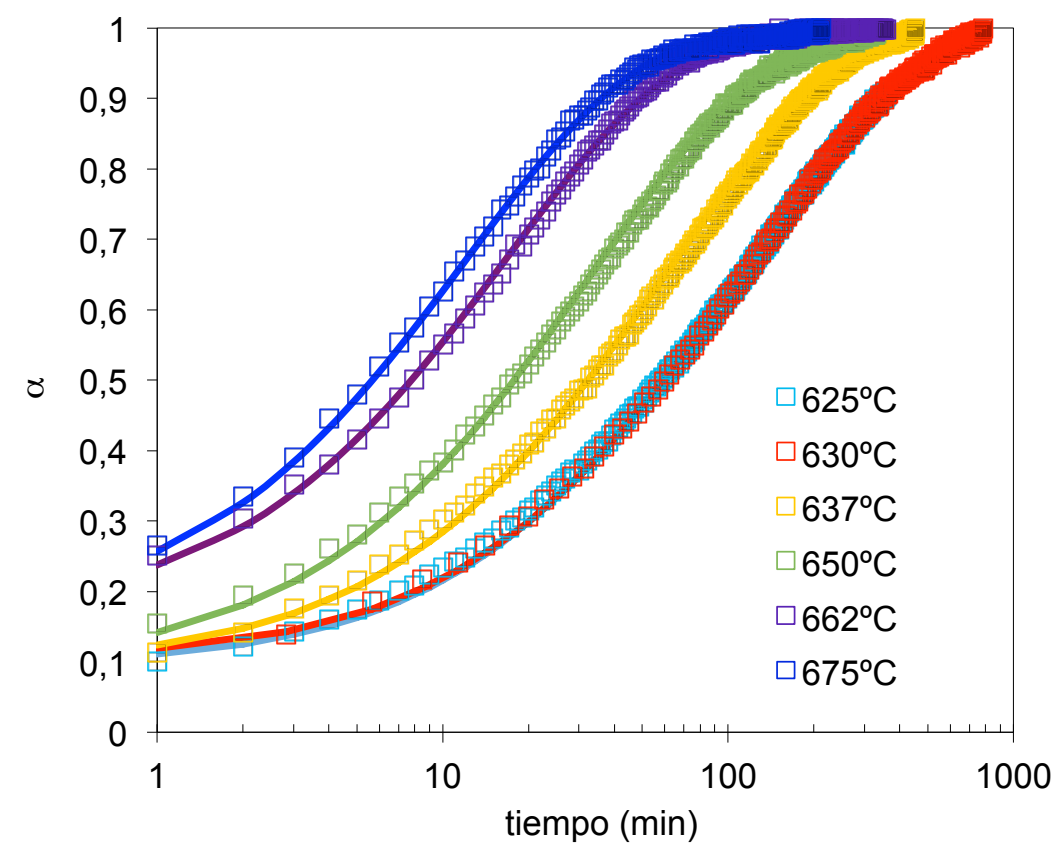

Figura 5.23. Curvas de sinterización isotermas de compactos de vidrio de la granulometría GM. Comparación entre los valores experimentales y los calculados mediante la ec. 5.14 y los parámetros de la Tabla 5.5 (ajuste2). 
En primer lugar, los resultados correspondientes a cada temperatura se han ajustado a la ec. 5.14, sin fijar ningún parámetro. En este caso los valores de $\mathrm{n}$ varían un poco y de forma aleatoria de una a otra temperatura (ajuste 1) (Tabla 5.5). Fijando los valores de $n=0.7$, para todas las temperaturas, los valores de la varianza, $\mathrm{S}^{2}$, aun siguen siendo muy bajos y la concordancia entre los valores experimentales y los calculados muy buena (Figura 5.23). Además, los valores de la energía de activación del proceso, E, son prácticamente coincidente con la energía de activación de flujo viscoso, $\mathrm{E}_{\eta}$.

Además, al representar en escala doble logarítmica la constante de velocidad del proceso, $\mathrm{k}$, (en este caso $\mathrm{k}_{\mathrm{g}}$ ) para cada temperatura, frente a la inversa del tiempo característico, $\gamma / \mathrm{d} \cdot \eta$, se aprecia una buena proporcionalidad entre ambos parámetros de acuerdo con los modelos teóricos de sinterización de vidrios (apartado 2.1.4.1) (Figura 5.24). Se aprecia que el valor de $\lambda_{g}=0,95$ en este apartado es muy parecido al que se ha obtenido en al apartado anterior, $\lambda_{g}=0,63$, utilizando un procedimiento experimental distinto.

Estos resultados, además, son concordantes con los obtenidos en los experimentos a velocidad de calentamiento constante.

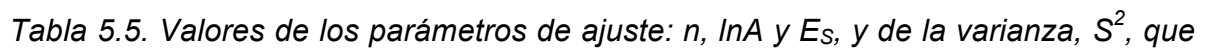
resultan del ajuste simultáneo a la ec. 5.14 y ec. 2.66 .

\begin{tabular}{|c|c|c|c|c|c|c|}
\hline Ajuste & $\mathbf{n}$ & $\mathbf{n}^{\mathbf{0}}$ datos & $\mathbf{S}^{\mathbf{2}}$ & $\left.\mathbf{I n A}_{\mathbf{g}} \mathbf{s}^{-1}\right)$ & $\mathbf{E}(\mathrm{KJ} / \mathbf{m o l})$ & $E \eta(\mathbf{K J} / \mathbf{m o l})$ \\
\hline 1 & $0.70 \pm 0.035$ & 1786 & $3.24 \cdot 10^{-5}$ & $42.7 \pm 3.4$ & $354 \pm 18$ & $360 \pm 2$ \\
\hline 2 & 0.70 & 1786 & $4.28 \cdot 10^{-5}$ & $41.9 \pm 3.1$ & $346 \pm 25$ & $360 \pm 2$ \\
\hline
\end{tabular}




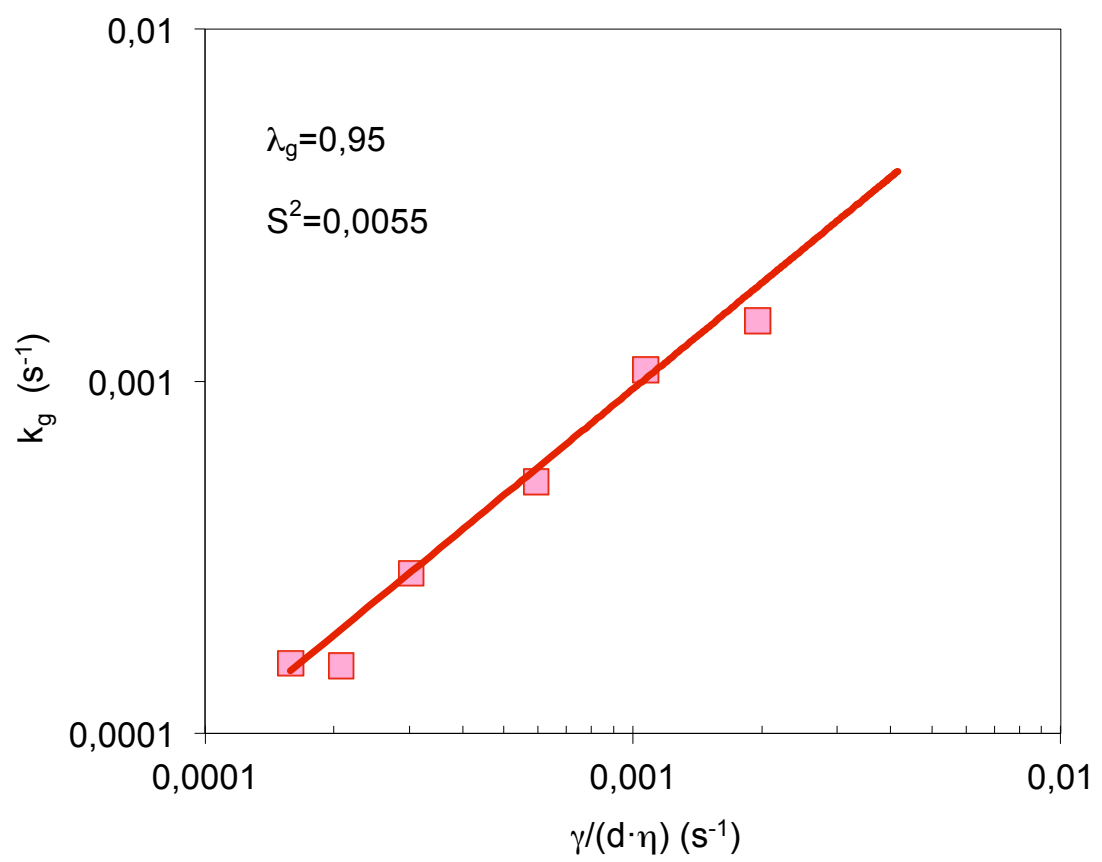

Figura 5.24. Variación de la constante de velocidad isoterma, $k_{g}$, frente a la inversa del tiempo característico, $\gamma / d \eta$. Compactos de vidrio de la granulometría GM.

\subsubsection{Influencia de la compacidad en crudo sobre la sinterización.}

Como se ha indicado en el apartado 2.1.4.1.3, tanto la presión de sinterización adimensional, $\wp_{\mathrm{L}}$, como el modulo viscoso adimensional, $\psi, \mathrm{y}$ la viscosidad de un vidrio poroso, $\eta_{p}$, son función de la compacidad, $\rho$. En consecuencia, al modificar el valor de la compactación inicial, $\rho_{0}$, debe alterarse el valor de $\rho$ para un determinado valor del grado de avance de la sinterización, $\alpha$, lo que puede modificar a su vez la variación de la razón $\frac{\wp_{L}}{\psi}$ con $\alpha$ (ya que el efecto de $\rho$ sobre $P_{L}$ y $\psi$ son contrarios) y por consiguiente, el modelo cinético.

Al efecto, se han preparado probetas por prensado (dos a cada presión) a 6 presiones de compactación diferentes (entre 2,2 y $50 \mathrm{MPa}$ ). Las probetas se 
han sometido a sinterización no isoterma, a una velocidad de calentamiento de $15 \mathrm{~K} / \mathrm{min}$ en el HSM, hasta la temperatura a la que la pieza alcanza la máxima densificación $\left(\sim 800^{\circ} \mathrm{C}\right)$,

De las dimensiones de las probetas y de su masa se ha calculado su densidad aparente y su densidad relativa, $\rho_{\max }$. En todos los casos los valores de $\rho_{\max }=0,99 \pm 0,02$.

\subsubsection{Contracción superficial de la silueta, $\left(\operatorname{In} A / A_{0}\right)_{s}$.}

En la Figura 5.25 se han representado los valores de la contracción superficial de la silueta de la probeta, $\varepsilon_{A}$, frente a la temperatura, únicamente para tres presiones de prensado (los dos extremos y la intermedia), con el fin de no complicar la figura.

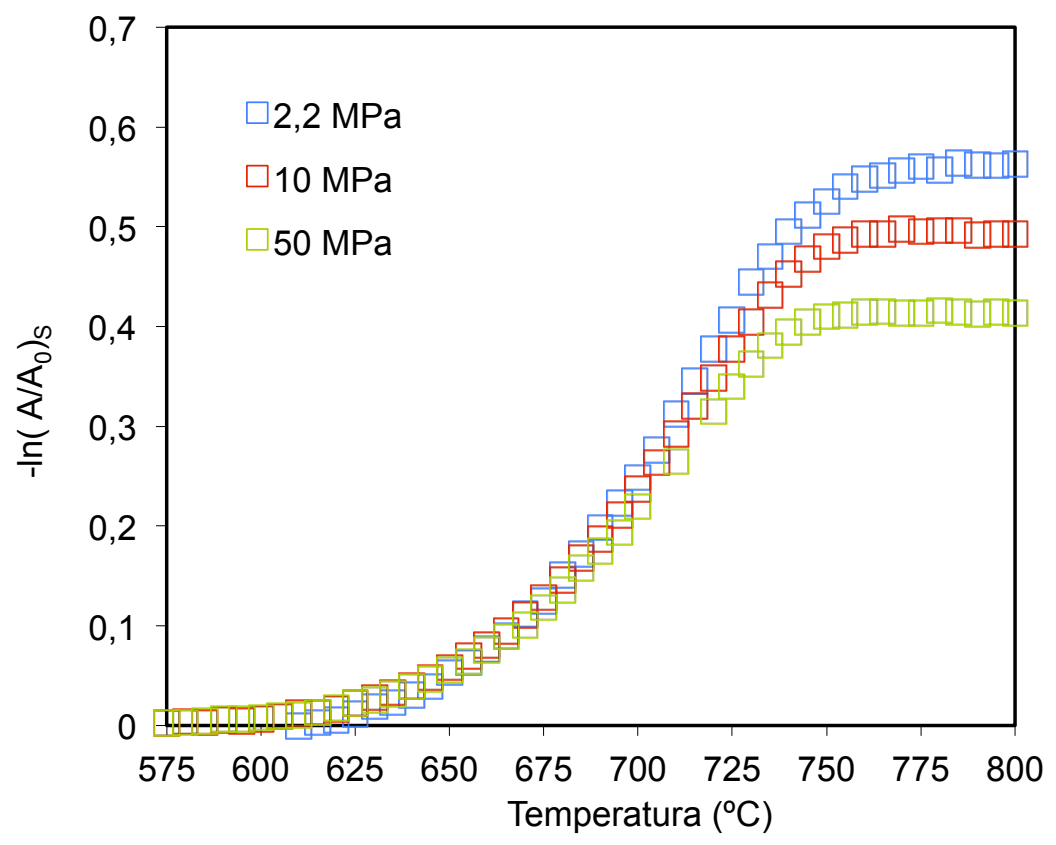

Figura 5.25. Influencia de la presión de compactación de las probetas sobre la curva: $-\operatorname{In}\left(A / A_{0}\right)_{s}$ vs $T$. Compactos de vidrio GM. Velocidad de calentamiento $\beta=15 \mathrm{~K} / \mathrm{min}$. 
Se aprecia un aumento de $\varepsilon_{\mathrm{A}}$ con la disminución de la presión de compactación, $\mathrm{P}_{\text {comp }}$, siendo este efecto tanto mayor cuanto mayor es la temperatura o el grado de sinterización. No obstante, la temperatura (o intervalos de temperaturas $750-800^{\circ} \mathrm{C}$ ) en la probeta alcanza la máxima densificación $\left(\rho_{\max }\right)$, a la contracción máxima $\varepsilon_{\mathrm{A} \text {, max }}$, es independiente de la

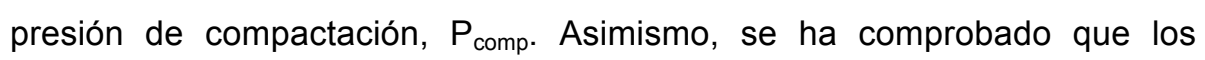
valores de $-\varepsilon_{\mathrm{A}, \max }$, aunque dispersos, varían linealmente con el logaritmo de la $\mathrm{P}_{\text {comp }}$ (Figura 5.26), tal como ya ha sido observado en la sinterización de distintos tipos de materiales [115][116]. Dicha relación es consecuencia de que la porosidad en crudo y al inicio de la sinterización, $\rho_{0}$, directamente relacionada con la $-\varepsilon_{A, \max }$, disminuye con el logaritmo de la presión de compactación, $P_{\text {comp }}$, como ya se verá a continuación (Tabla 5.6).

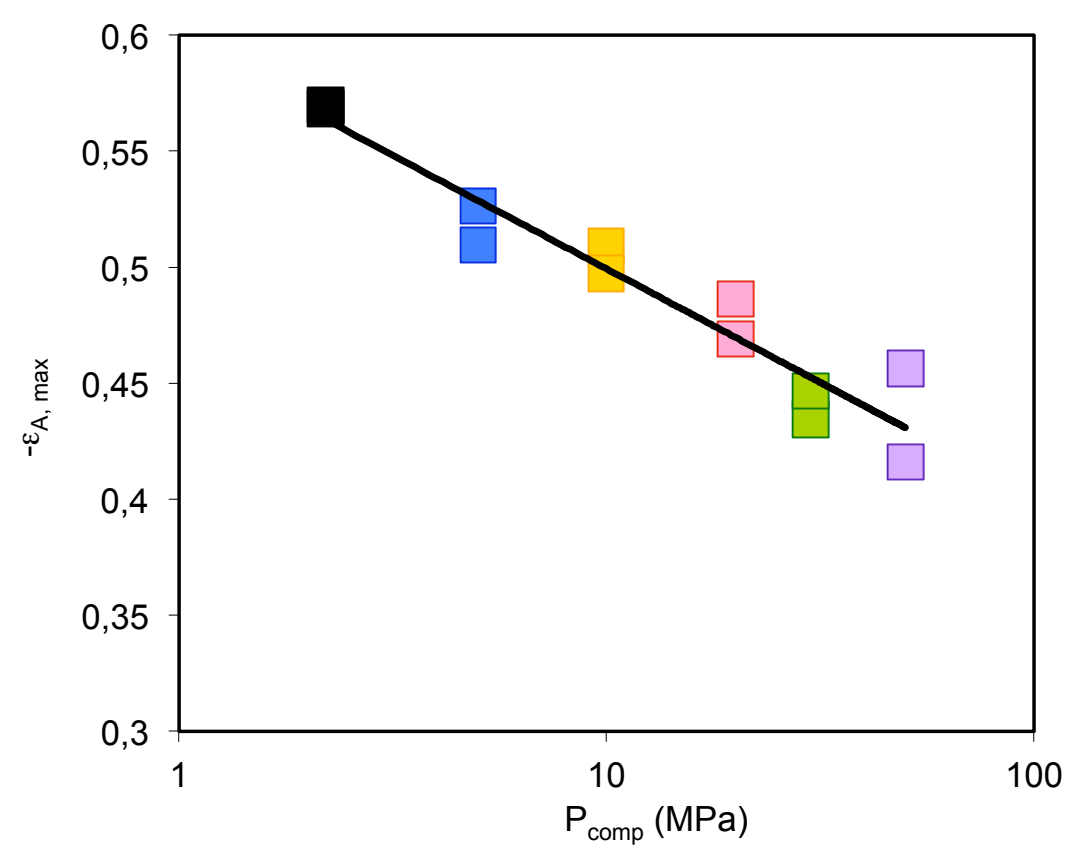

Figura 5.26. Variación de la contracción máxima de la silueta, - $\varepsilon_{A}$, max, con la presión

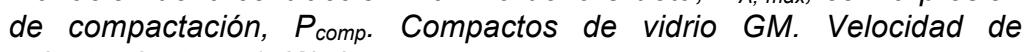
calentamiento $\beta=15 \mathrm{~K} / \mathrm{min}$. 
Tabla 5.6. Relación entre $\varepsilon_{A, \max } y \rho_{0}$ con la presión de compactación, $P_{\text {comp. }}$

\begin{tabular}{|l|l|l|}
\hline Relación & $\mathbf{r}^{2}$ & $\mathbf{S}^{2}$ \\
\hline$-\varepsilon_{\mathrm{A}, \mathrm{max}}=0,5972-0,0979 \cdot \log \mathrm{P}_{\text {comp }}$ & 0,924 & 0,000204 \\
\hline$\rho_{0}=0,4027+0,0709 \cdot \log \mathrm{P}_{\text {comp }}$ & 0,918 & 0,000116 \\
\hline
\end{tabular}

En efecto, de la ec. A1. 4 y la ec. A1. 8 se obtiene la relación:

$$
\rho_{0}=\rho_{\max } \cdot \exp \left(-\frac{3}{2}\left|\varepsilon_{A, \max }\right|\right)
$$

Sustituyendo $\rho_{\max }=0,99$ e introduciendo los valores experimentales de $\varepsilon_{\mathrm{A}, \max }$ se obtienen los valores de $\rho_{0}$ para cada probeta.

Al representar para todas las probetas los valores de $\rho_{0}$ frente al logaritmo de $P_{\text {comp }}$ (Figura 5.27) (Tabla 5.6) se comprueba que los valores, aunque dispersos, se ajustan aceptablemente bien a una recta. 


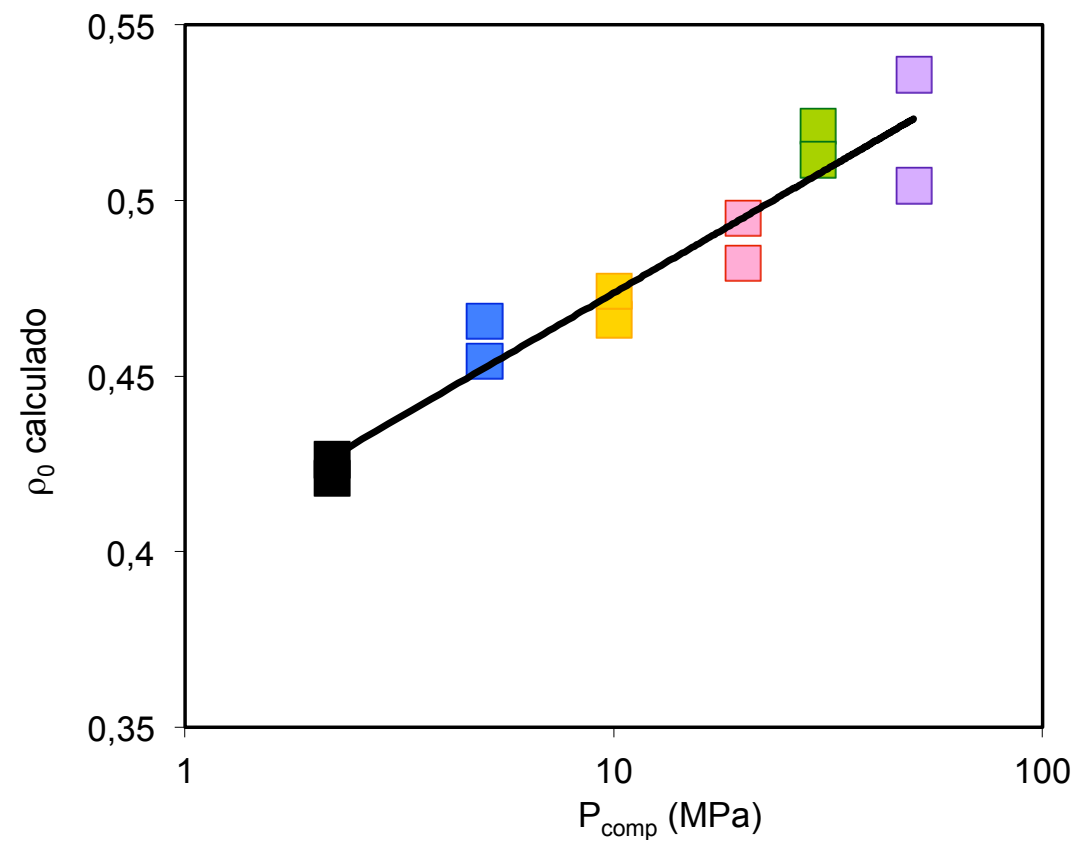

Figura 5.27. Variación de la compacidad al inicio de la sinterización, $\rho_{0}$ calculada (ec.

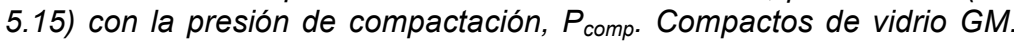
Velocidad de calentamiento $\beta=15 \mathrm{~K} / \mathrm{min}$.

\subsubsection{Anisotropía de la sinterización.}

Para cuantificar la anisotropía que se desarrolla durante la sinterización y el efecto que ejerce la presión de prensado, $\mathrm{P}_{\text {comp }}$, o la compactación en crudo, $\rho_{0}$, sobre dicha característica se ha utilizado el factor de anisotropía definido como la razón de la contracción axial/contracción diametral:

$\mathrm{k}_{\mathrm{A}}=\frac{\ln \left(\mathrm{h} / \mathrm{h}_{0}\right)}{\ln \left(\mathrm{D} / \mathrm{D}_{0}\right)}$

siendo: h y D la altura y el diámetro del cilindro respectivamente.

Los valores instantáneos de $\mathrm{h}$ son los registrados directamente por el equipo, mientras que los valores instantáneos de $D$ se obtienen a partir a partir de los valores del área de la silueta del cilindro. En la Figura 5.28 se 
representan los valores de $\ln \left(A / A_{0}\right)_{S}, \ln \left(h / h_{0}\right), \ln \left(D / D_{0}\right)$ y $k_{A}$ en función de la temperatura, para la probeta compactada a $5 \mathrm{MPa}$. Para otras compactaciones la forma de las curvas son similares.

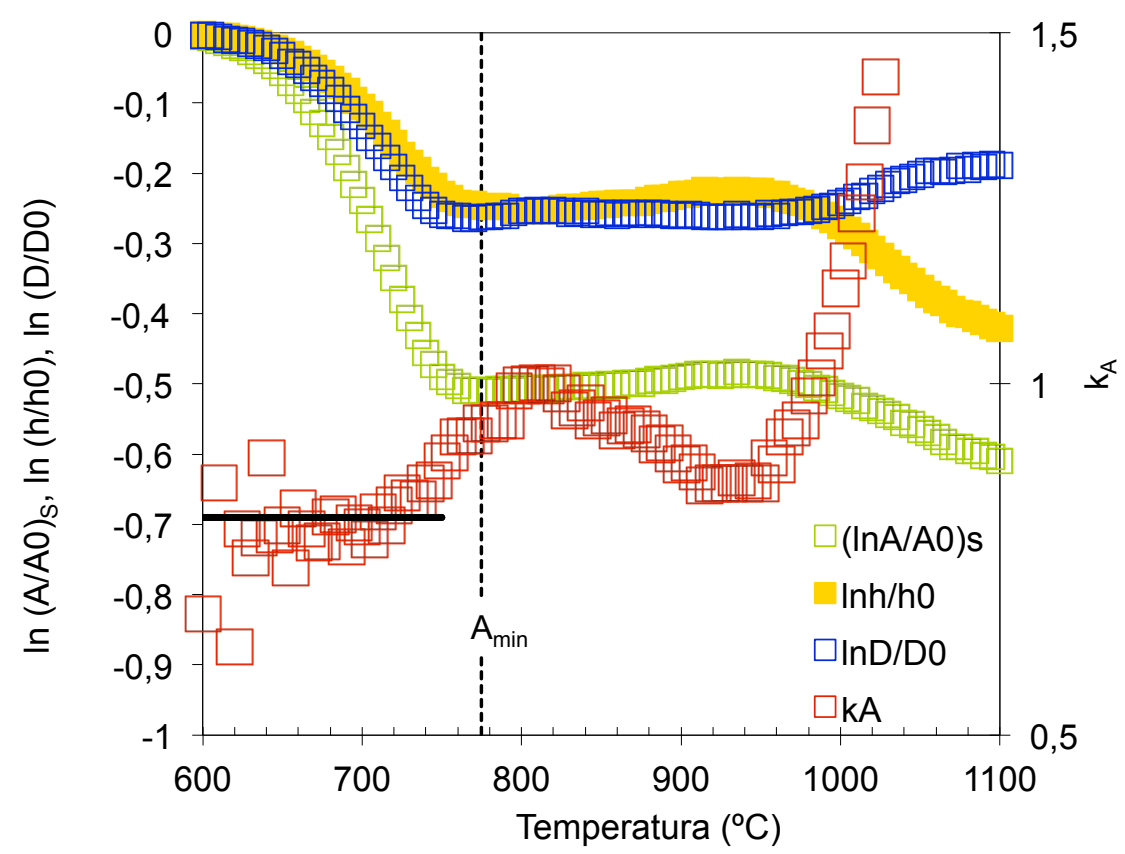

Figura 5.28. Variación de la contracción superficial, $\ln \left(A / A_{0}\right)_{s}$, axial, $\ln \left(h / h_{0}\right), y$ diametral, $\ln \left(D / D_{0}\right)$, frente a la temperatura. Compacto de vidrio $G M$ obtenido a $P_{\text {comp }}=5 \mathrm{MPa}$. Velocidad de calentamiento $\beta=15 \mathrm{~K} / \mathrm{min}$.

En la Figura 5.29 se han representado para las probetas prensadas a $5 \mathrm{MPa}$ y $50 \mathrm{MPa}$ los valores de $\ln \left(\mathrm{h} / \mathrm{h}_{0}\right)$ frente $\ln \left(\mathrm{D} / \mathrm{D}_{0}\right)$. Del examen de ambas representaciones se desprende lo siguiente:

En estados iniciales e intermedios de la sinterización $(\alpha \leq 0,6)$ a los que corresponden temperaturas inferiores a $750^{\circ} \mathrm{C}$ aproximadamente, el factor de anisotropía es prácticamente constante (Figura 5.28) y su valor depende ligeramente de la presión de compactación (Figura 5.29). En efecto, un aumento en un orden de magnitud de esta variable ( $5 \mathrm{MPa}$ a $50 \mathrm{MPa})$ solo reduce el valor de $k_{A}$ de 0,83 a 0,72 (Figura 5.29). Estos valores de $k_{A}$ son similares a los obtenidos por Boccaccini $\left(k_{A} \approx 0,70\right)$ en probetas obtenidas por 
prensado de partículas irregulares de vidrio de borosilicato [24] y silicoaluminato [20].

A temperaturas más elevadas correspondientes al estado final de sinterización, conforme aumenta la temperatura y, por consiguiente, con el avance de la sinterización, el factor de anisotropía va aumentando hasta alcanzar prácticamente la unidad (sinterización isotrópica).

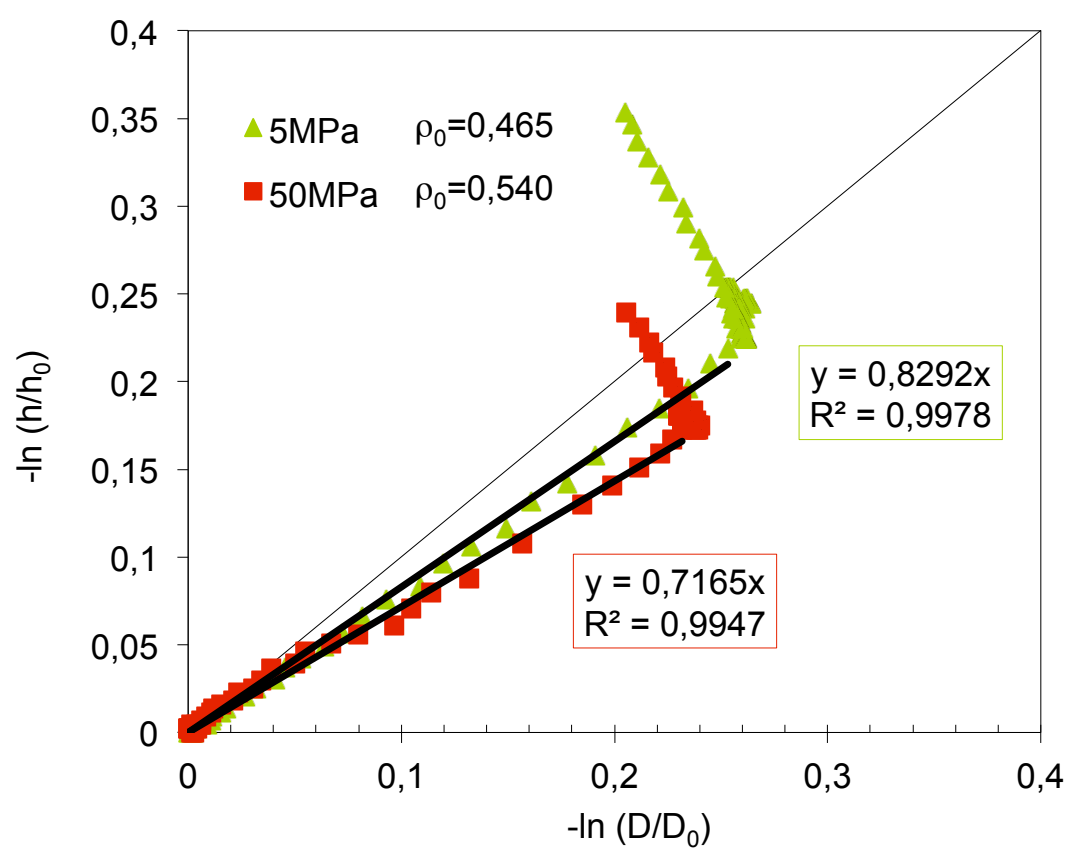

Figura 5.29. Variación de la contracción axial, $-\ln \left(h / h_{0}\right)$, frente a la contracción diametral, $-\ln \left(D / D_{0}\right)$, para un compacto de vidrio $G M$, denso $\left(P_{\text {comp }}=50 M P a\right.$ y $\left.\rho_{0}=0,540\right)$ y muy poco denso $\left(P_{\text {comp }}=5 M P a\right.$ y $\left.\rho_{0}=0,465\right)$. Velocidad de calentamiento $\beta=15 \mathrm{~K} / \mathrm{min}$.

A temperaturas ligeramente superiores a la de máxima densificación, el valor de $\mathrm{k}_{\mathrm{A}}$ vuelve a disminuir de nuevo, debido a que la expansión axial de la muestra, debido a la dilatación de los gases ocluidos en los poros cerrados, es mayor que la diametral. A temperaturas elevadas, a las que la viscosidad del vidrio es suficientemente baja para que la deformación piroplástica debido a la acción de la gravedad sea importante, $\mathrm{k}_{\mathrm{A}}$ vuelve a aumentar considerablemente con la temperatura. 


\subsubsection{Cinética del proceso.}

Cada una de las 12 curvas de sinterización ( $\alpha$ vs T) obtenidas a 6 presiones de prensado diferentes se han ajustado a la ec. 5.12 dejando como parámetros variables del ajuste el parámetro $n$ y $\mathrm{A}_{\mathrm{g}}$. En todos los casos se cumplió que $S^{2}<9 \cdot 10^{-5}$, el valor $n$ obtenido fue de $n=0,713 \pm 0,0107$ y el factor preexponencial aumentaba ligeramente con el incremento de la presión de prensado. Al ajustar todas las curvas fijando $n=0,71$ se obtuvieron muy buenos resultados $\left(S^{2}<9 \cdot 10^{-5}\right)$. Además, los valores de $A_{g}$ y $P_{\text {comp }}$, aunque dispersos, se ajustaron aceptablemente bien a una relación potencial (Figura 5.30, Tabla 5.7).

Tabla 5.7. Efecto de $\rho_{0}$ y $P_{\text {comp }}$ sobre $\ln A_{g}$ del modelo (ec. 5.12). Compacto de vidrio GM. Velocidad de calentamiento $\beta=15 \mathrm{~K} / \mathrm{min}$.

\begin{tabular}{|l|l|l|}
\hline Relación & $\mathbf{r}^{2}$ & $\mathbf{s}^{2}$ \\
\hline $\ln \mathrm{A}_{\mathrm{g}}=17,4+0,135 \cdot \ln \mathrm{P}_{\text {comp }}$ & 0,9012 & 0,0027 \\
\hline $\ln \mathrm{A}_{\mathrm{g}}=15,6+4,32 \cdot \rho_{0}$ & 0,9617 & 0,00105 \\
\hline
\end{tabular}




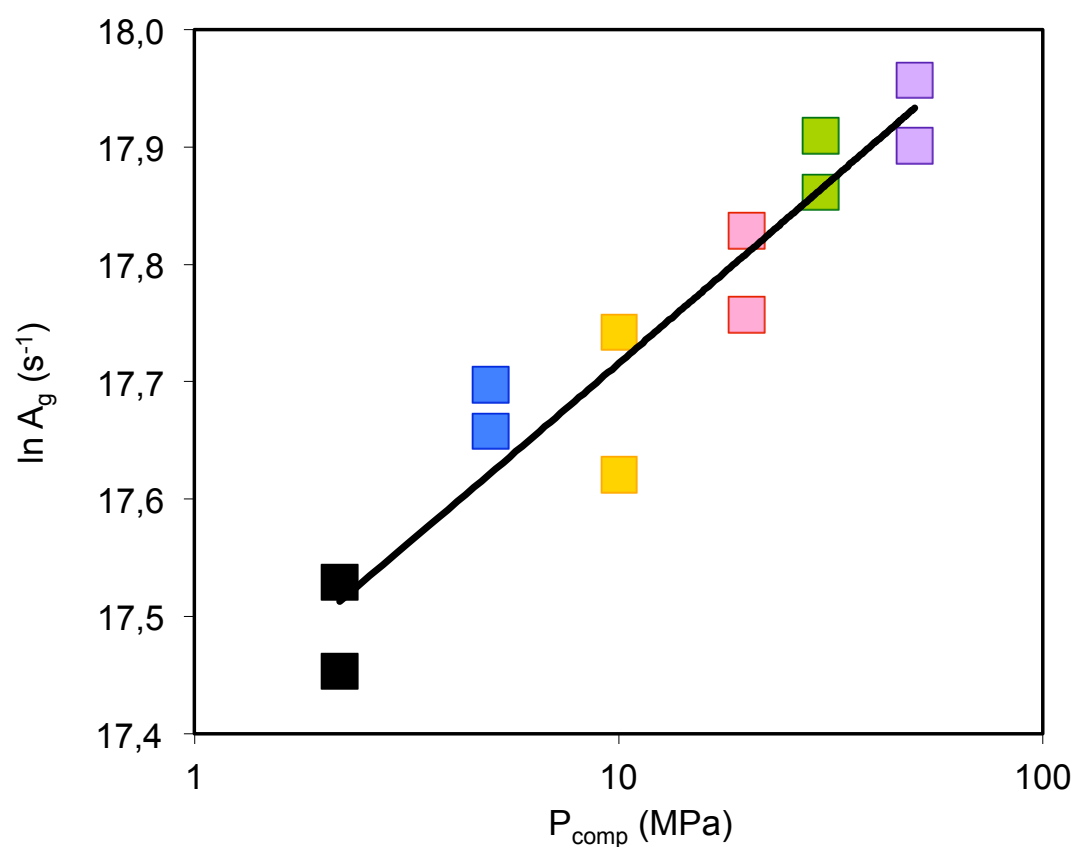

Figura 5.30. Efecto de la presión de compactación, $P_{\text {comp, sobre el factor }}$ preexponencial, In $A_{g}$, del modelo cinético (ec. 5.12). Compacto de vidrio GM. Velocidad de calentamiento $\beta=15 \mathrm{~K} / \mathrm{min}$.

La relación fue todavía mejor cuando se ajustan los valores de $A_{g}$ con los de la compacidad al inicio de la sinterización, $\rho_{0}$, (Tabla 5.7.) (Figura 5.31). 


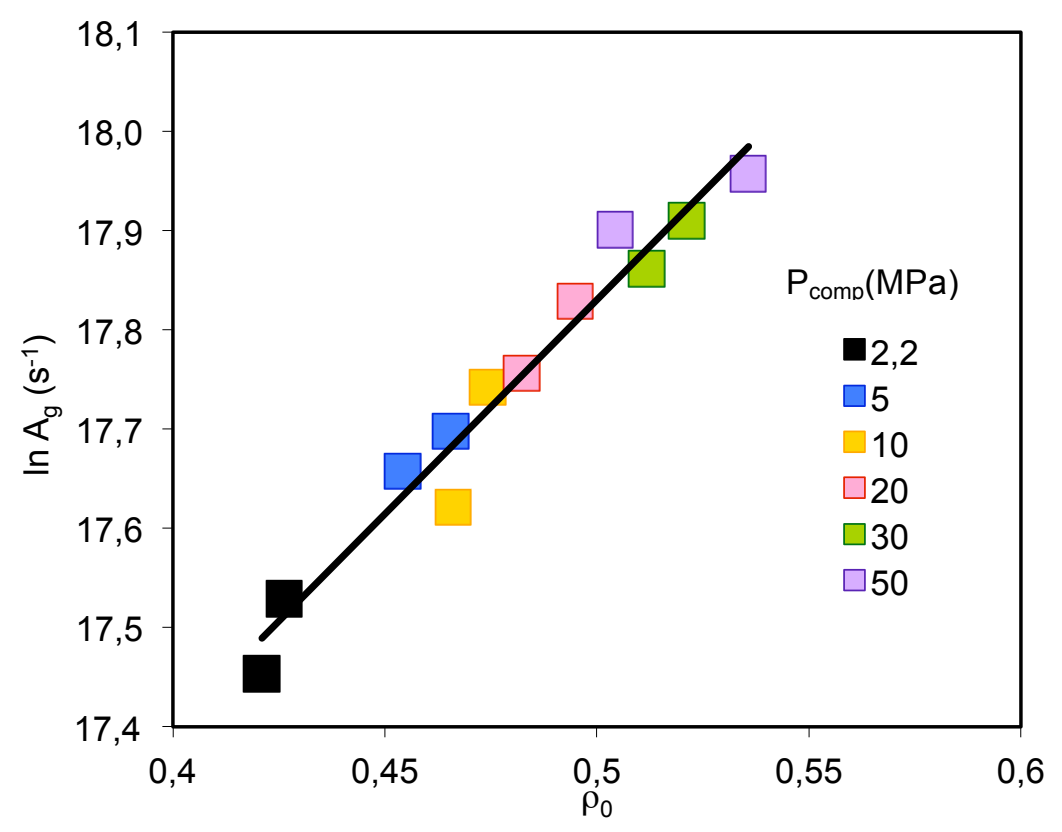

Figura 5.31. Efecto de la compacidad al inicio de la sinterización, $\rho_{0}$, sobre el factor preexponencial, $\ln A_{g}$, del modelo cinético (ec. 5.12). Compacto de vidrio GM. Velocidad de calentamiento $\beta=15 \mathrm{~K} / \mathrm{min}$.

Se comprueba que la influencia de la compacidad en crudo, $\rho_{0}$, sobre la cinética de la sinterización queda perfectamente descrita mediante el efecto que dicha característica, $\rho_{0}$, ejerce sobre el factor preexponencial, $A_{g}$, manteniendo constante los restantes parámetros cinéticos $B, T_{0}$ y $n$ (Figura 5.32).

No obstante, el efecto que ejerce la presión de prensado (o la compacidad en crudo, $\rho_{0}$ ) sobre la cinética de la sinterización es pequeño, ya que un aumento considerable de esta variable (de 2,2 a 50MPa) apenas desplaza la curva de sinterización unos $5^{\circ} \mathrm{C}$ a temperatura más bajas (Figura 5.32). 


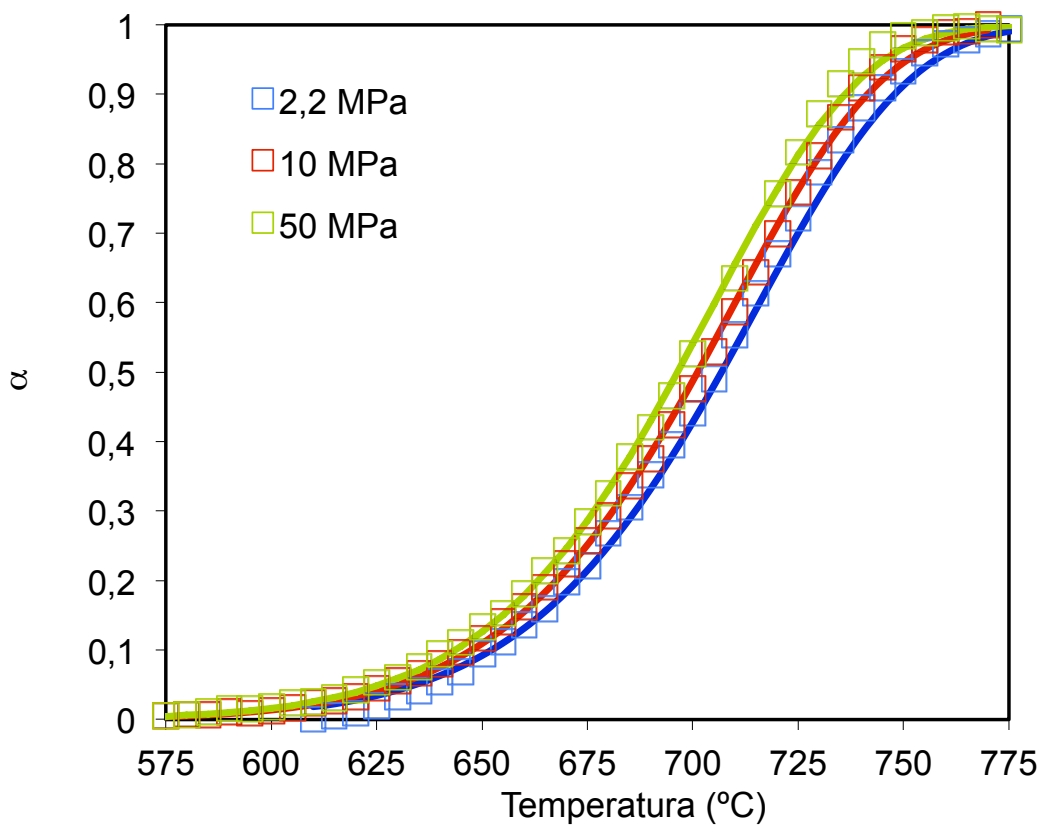

Figura 5.32. Curvas de sinterización experimentales y calculadas según la ec. 5.12, considerando la relación $\operatorname{In} A_{g}$ y $P_{\text {comp }}$ de la Tabla 5.7. Compacto de vidrio GM. Velocidad de calentamiento $\beta=15 \mathrm{~K} / \mathrm{min}$.

\subsection{Sinterización de composites. Efecto del contenido en circón.}

Para determinar el efecto de la fracción volumétrica de circón, $\phi$, sobre la sinterización de composites de vidrio-circón, se han preparado mezclas de ambos materiales con las granulometrías GM para el vidrio y micronizado para el circón (Figura 4.1). A esta serie de composites se les denominará F.

\subsubsection{Procesos físico-químicos que se desarrollan en los materiales durante su tratamiento térmico.}

\subsubsection{Curvas de sinterización no isotermas.}

En la Figura 5.33 se aprecia que conforme aumenta la fracción volumétrica de circón, $\phi$, la contracción superficial de la silueta, $\ln \left(A / A_{0}\right)_{s}$, y la variación de ésta con la temperatura disminuyen (pendiente de la curva de sinterización). Por el contrario, la adición de circón al vidrio supone un aumento de la temperatura a la que el composite empieza a sinterizar y 
también a la que alcanza la máxima densificación. Para compactos con bajos contenidos en circón, $\phi \leq 0,17$, el efecto de $\phi$ sobre la curva de sinterización es pequeño. Para valores de $\phi>0,17$, el efecto sobre la curva de sinterización ya es significante, mayor en los último estados de la sinterización que en los iniciales. En efecto, para un contenido en $\phi=0,53$, el composite empieza a sinterizar a una temperatura próxima a los $725^{\circ} \mathrm{C}$, mientras que el vidrio lo hace a temperatura inferiores a los $600^{\circ} \mathrm{C}$. Este retraso en el inicio de la sinterización se debe, probablemente, a que la presencia de partículas de circón rodeando las partículas de vidrio reducen el número de contactos vidrio-vidrio más fundentes. En cambio, la temperatura a la que este composite $(\phi=0,53)$ alcanza la máxima densificación, $T_{c}{ }^{\max }$, es de alrededor de $1600^{\circ} \mathrm{C}$ mucho mayor que el vidrio que lo hace sobre los $700^{\circ} \mathrm{C}$.

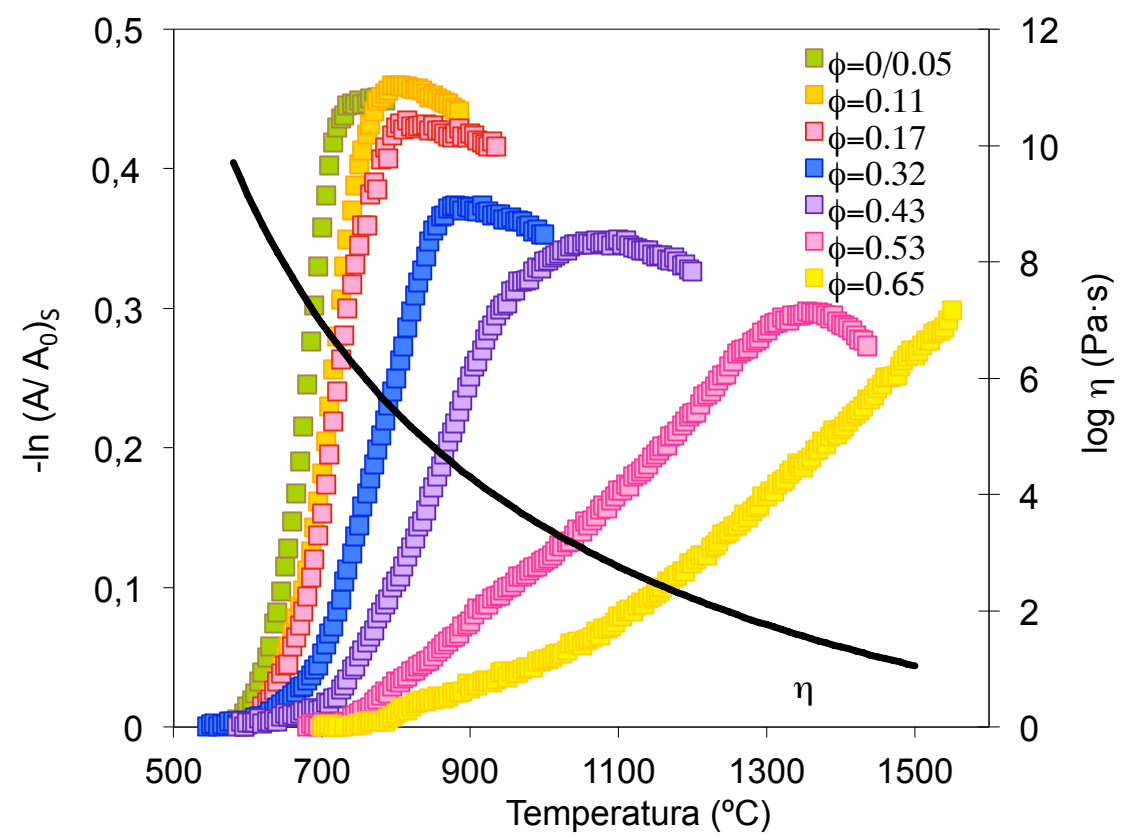

Figura 5.33. Efecto de la fracción volumétrica de circón, $\phi$, sobre la curva de sinterización del composite $F$. Velocidad de calentamiento $5 \mathrm{~K} / \mathrm{min}$. Curva de viscosidad de la matriz vítrea, $\eta$, con la temperatura. 
De forma general, la diferencia, $\Delta T$, entre la temperatura de máxima densificación del composite, $\mathrm{T}_{\mathrm{c}}{ }^{\max }$, y la del vidrio, $\mathrm{T}_{\mathrm{g}}{ }^{\max }$, prácticamente despreciable para pequeñas adiciones de circón, $\phi<0,2$, se hace tanto más acusada cuanto mayor es el contenido en circón (Figura 5.34). Así pues, para valores de $\phi<0,42$ la disminución del contenido en fundido, que se produce con el aumento de $\phi$, aumenta la viscosidad efectiva del sistema,

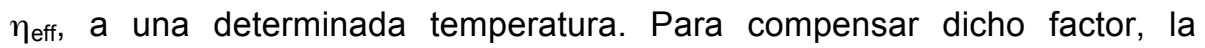
viscosidad del vidrio debe reducirse aumentando la temperatura; de este modo la máxima densificación del composite se alcanza, probablemente por el mecanismo de reordenación de partículas, a temperaturas cada vez más altas. En efecto, a la vista de la Figura 5.34, se comprueba que la viscosidad del fundido a la que el composite alcanza la máxima densificación, $\eta^{*}$, para contenidos en $\phi<0,42$, es especular a la que sigue $\Delta$ T con esta variable $(\phi)$.

Al comparar las curvas de sinterización correspondientes a $\phi=0,43$ y $\phi=0,53$ se aprecia un marcado desplazamiento hacia temperaturas más altas de esta última (alrededor de $300^{\circ} \mathrm{C}$ ). Además, el efecto de la temperatura sobre la sinterización (pendiente de las curvas) se reduce considerablemente, y la forma de la curva deja de ser sigmoidal. Todo ello parece indicar que para este composite el mecanismo que mayoritariamente contribuye en la etapa final de la densificación es el de solución-reprecipitación como se verá posteriormente. 


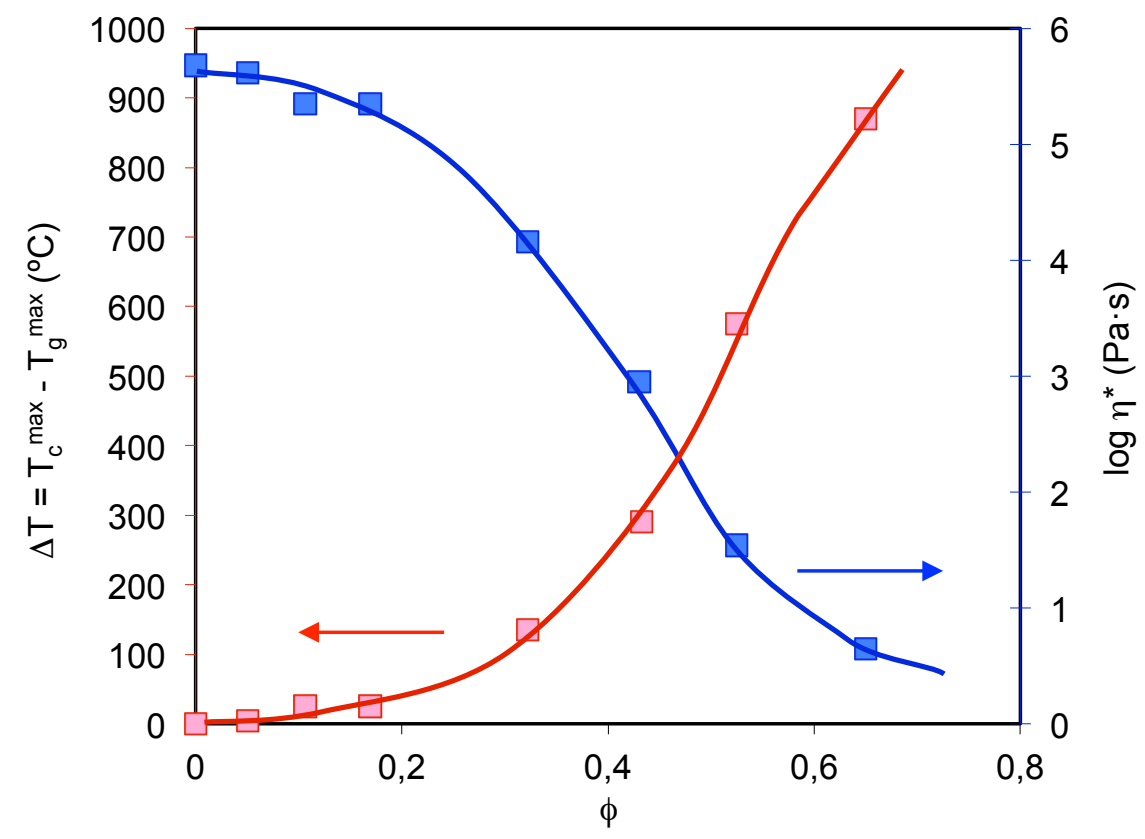

Figura 5.34. Efecto del contenido en circón, $\phi$, sobre el incremento de la temperatura de máxima densificación del composite, $\Delta T$, y sobre la viscosidad del fundido, $\eta^{*}$, a $T_{c}^{\max }$. Compacto $F$.

\subsection{Contracción superficial de la silueta máxima, $\varepsilon_{\mathrm{A}, \max }$.}

A partir de las curvas de sinterización se ha determinado, para cada valor de $\phi$, y para las cinco velocidades de calentamiento ensayadas, el valor de la contracción máxima promedio, $\varepsilon_{\mathrm{A}, \max }$, y su desviación estándar (Figura 5.35). Se comprobó que, únicamente, para el composite con el contenido en circón más alto, $\phi=0,65$, que no alcanzaba la densificación completa, a la temperatura máxima del ensayo, una disminución de la velocidad de calentamiento conducía a una mayor densificación, en consecuencia, a un aumento de $\varepsilon_{\mathrm{A}, \max }$. Para los restantes composites, el valor individual $\varepsilon_{\mathrm{A}, \max }$ variaba aleatoriamente con la velocidad de calentamiento, indicando con ello que la desviación estándar de las medidas, se debía al error experimental 
del método, en el que se incluyen variaciones de la densidad en crudo de las probetas.

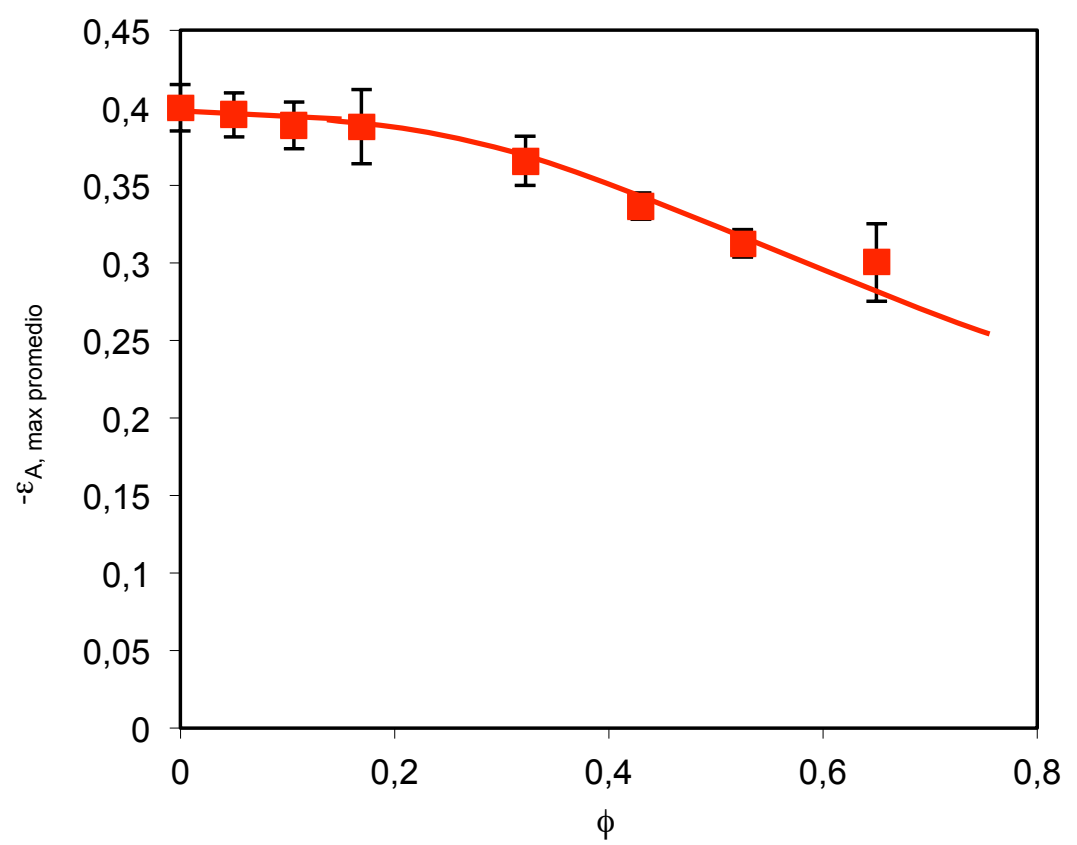

Figura 5.35. Efecto de la fracción volumétrica de circón, $\phi$, sobre la contracción máxima promedio de las cinco velocidades, $\varepsilon_{A}$, max. Compacto $F$.

De forma general, se ha comprobado una disminución de $\bar{\varepsilon}_{A \text {, max }}$ con el incremento del contenido en circón en el composite. Los resultados son similares a los obtenidos por Eberstein et al. en composites de $\mathrm{Al}_{2} \mathrm{O}_{3}$-vidrio [31]. En efecto, tanto en este caso como en aquel, para valores bajos $(\phi \leq 0,17)$ el efecto de las inclusiones rígidas sobre el desplazamiento de la curva de sinterización (Figura 5.33 y Figura 5.34) y sobre la contracción máxima (Figura 5.35) es pequeño. En cambio, para valores de $\phi \geq 0,17$ el efecto sobre la curva de sinterización y sobre $\varepsilon_{\mathrm{A}, \max }$ es significativo. Para cuantificar este último efecto, se ha recurrido a utilizar un grado de reducción 
de la contracción superficial máxima, $\Delta \varepsilon_{\mathrm{A}, \max }$, análoga al grado de reducción de la contracción lineal máxima, utilizado por Eberstein [31], definido como:

$\Delta \varepsilon_{\mathrm{A}, \max }=1-\frac{\varepsilon_{\mathrm{A}, \max }}{\varepsilon_{\mathrm{A}, \max }(0)}$

Siendo $\varepsilon_{A, \max }(0)$ la contracción máxima de la silueta correspondiente al vidrio. Los valores de $\Delta \varepsilon_{A, \max }$ obtenidos a partir de los valores $\varepsilon_{\mathrm{A}, \max }$ de la Figura 5.35 se han representado en la Figura 5.36. Se incluyen en esta figura los resultados obtenidos por Eberstein para $\mathrm{GMC}$ de $\mathrm{Al}_{2} \mathrm{O}_{3}$ [31] y por Jean et al. para GMC de cordierita [117].

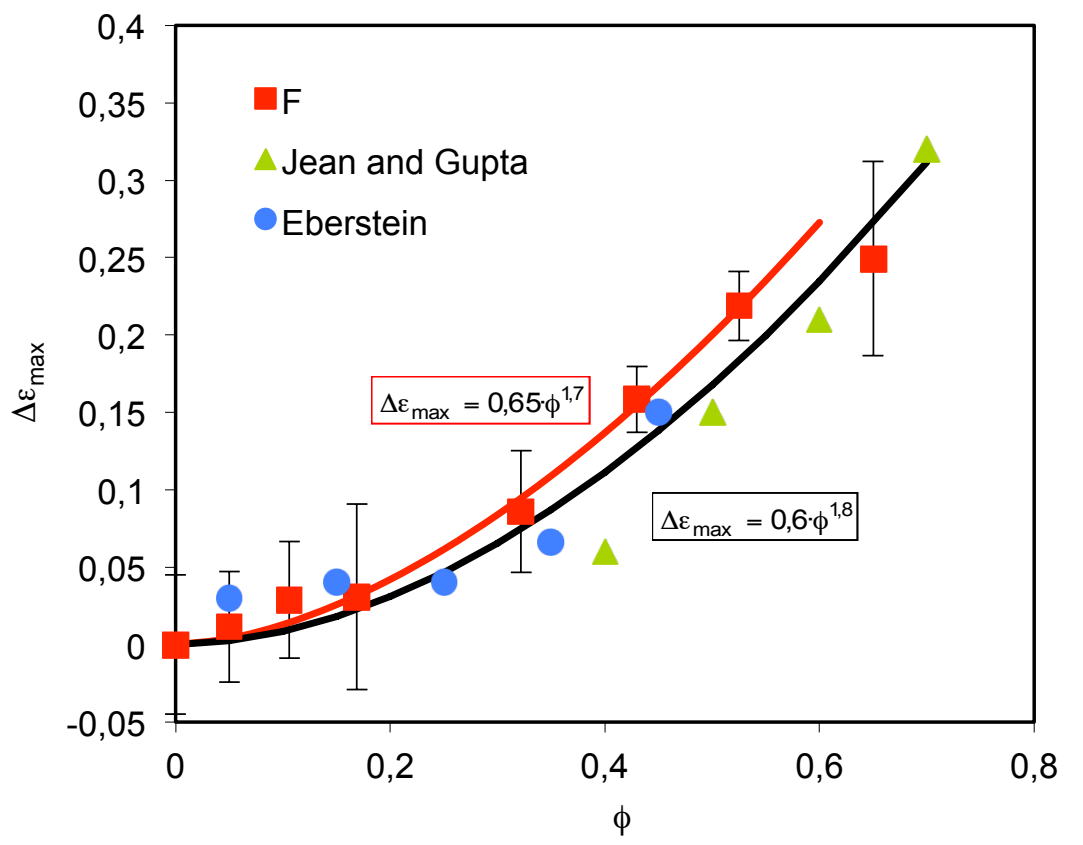

Figura 5.36. Variación del grado de reducción de la contracción máxima, $\Delta \varepsilon_{A}$, max, con a $\phi$. Cuadrados: este trabajo. Esferas: datos de Eberstein [31]. Triángulos: datos de Jean et al. [117]. Compacto F. 
Para todos los GMC, independientemente de la naturaleza del sistema y de la distribución de tamaño de las partículas, se comprueba que el grado de reducción de la contracción lineal máxima debida a la adición de partículas rígidas, $\Delta \varepsilon_{\mathrm{A}, \max }$, aumenta de forma potencial con $\phi$.

Tabla 5.8. Efecto de $\phi$ sobre el grado de reducción de la contracción máxima, $\Delta \varepsilon_{\mathrm{A}, \max }$. Compacto $F$.

\begin{tabular}{|l|l|l|}
\hline Datos & Relación & $\mathbf{S}^{2}$ \\
\hline Este trabajo & $0,65 \cdot \phi^{1,7}$ & $7,27 \cdot 10^{-5}$ \\
\hline Todos los valores & $0,60 \cdot \phi^{1,8}$ & $5,97 \cdot 10^{-4}$ \\
\hline
\end{tabular}

El efecto de $\phi$ sobre $\Delta \varepsilon_{\mathrm{A}, \max }$ se debe, probablemente, a la influencia de dicha variable sobre la formación de poros estables, asociados a la presencia, ya en crudo o en estados iniciales de la sinterización, de aglomerados de partículas cristalinas, que reduce la densificación de la pieza. No obstante, dado que la contracción lineal máxima está directamente relacionada con la diferencia entre la porosidad inicial y final del composite, para confirmar esta hipótesis, se recurrirá a determinar la compacidad inicial y final y al análisis de la microestructura de la pieza en los apartados siguientes.

5.2.1.1.2 Influencia de la presión de compactación sobre la sinterización.

i) Curvas de sinterización.

En la Figura 5.37 se representan, para el composite con $\phi=0,322$, F1, las curvas de sinterización en la forma $\varepsilon_{A}$ vs $T$ correspondientes a las probetas conformadas a $20 \mathrm{MPa}$ y $80 \mathrm{MPa}$, tratadas a $5 \mathrm{~K} / \mathrm{min}$. Las curvas de 
sinterización, expresadas en la forma $\alpha$ vs $T$, se han representado en la Figura 5.38. Se comprueba que el efecto de la presión de compactación de las probetas sobre la sinterización de un compacto con un contenido moderado de circón, $\phi=0,322$, es análogo al que ejerce esta misma variable en el caso de un compacto de vidrio. En efecto, un aumento de la presión de compactación de 20 a $80 \mathrm{MPa}$, supone un aumento de la compacidad en crudo del composite, lo que se traduce, a su vez, en una disminución de la contracción (Figura 5.37). Ahora bien, dicho efecto es prácticamente despreciable sobre el grado de avance de la sinterización, $\alpha$ y sobre su variación con la temperatura (Figura 5.38).

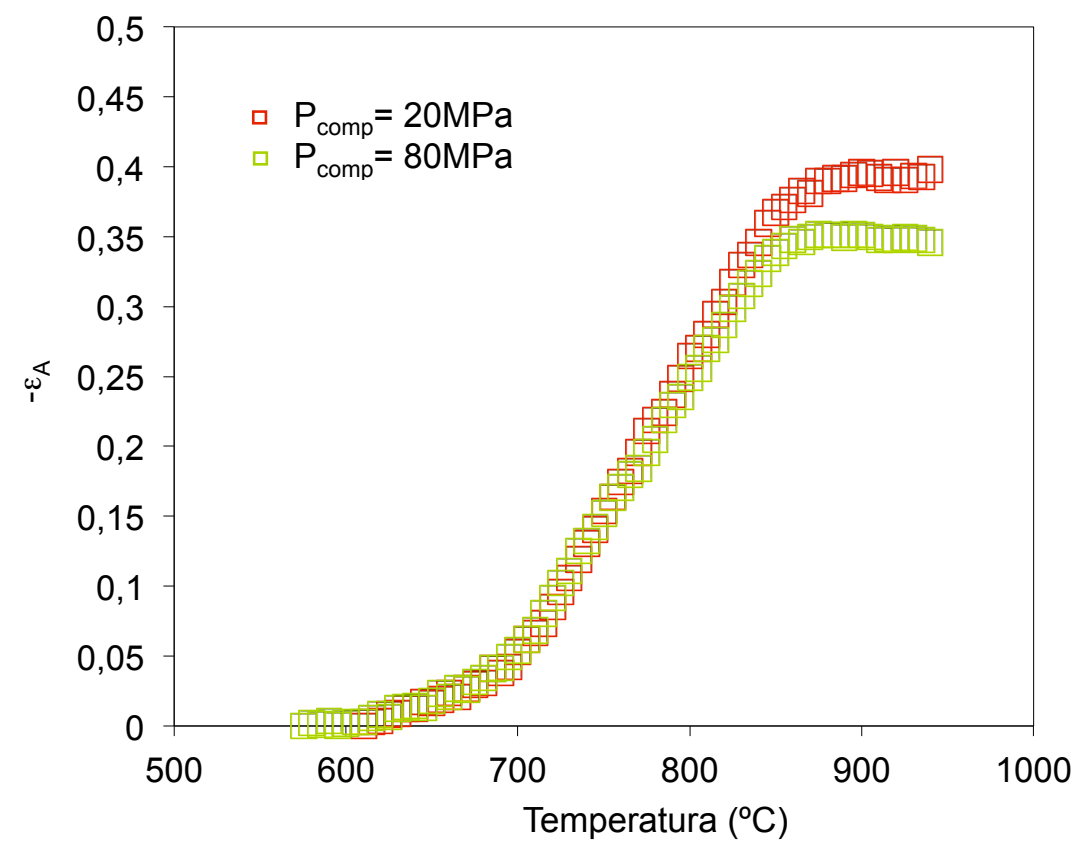

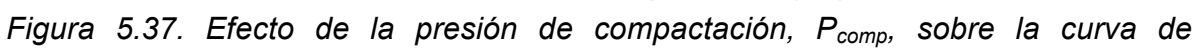
sinterización: $-\varepsilon_{A}$ vs T. Composite $F$ con $\phi=0,322$, F1. Velocidad de calentamiento $\beta=15 \mathrm{~K} / \mathrm{min}$. 


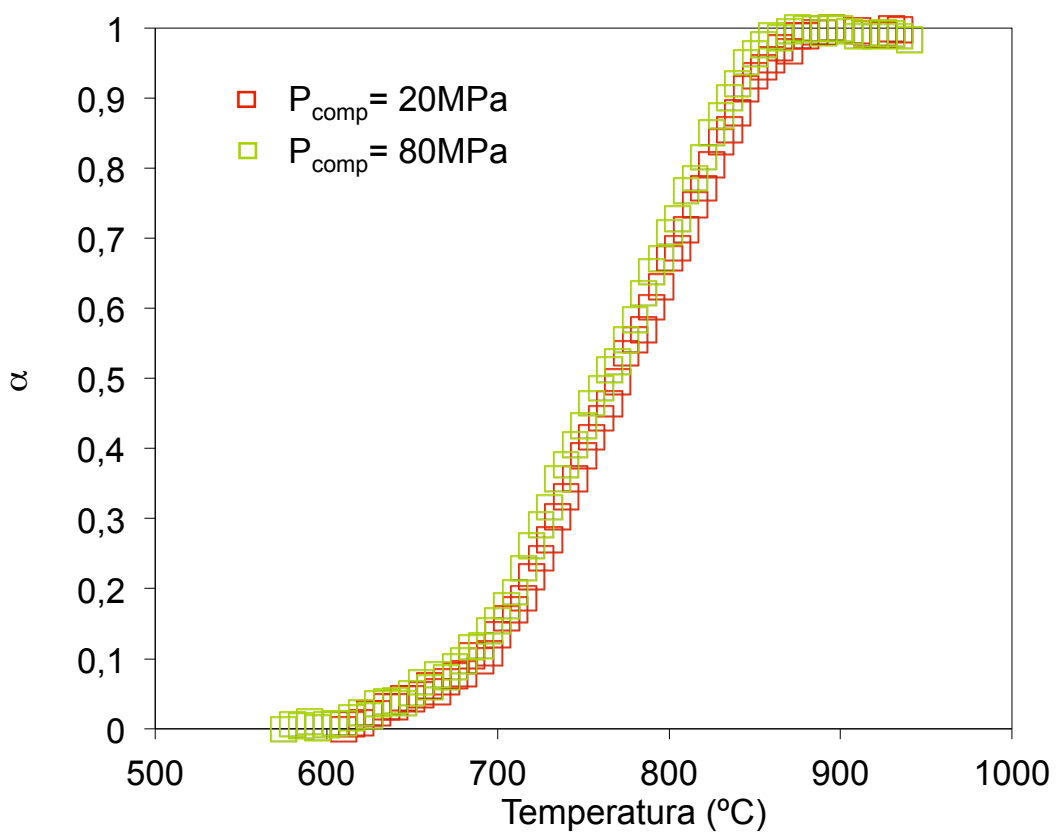

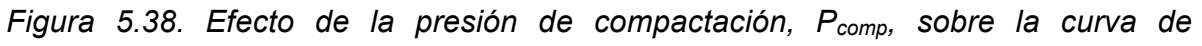
sinterización: $\alpha$ vs T. Composite $F$ con $\phi=0,322$, F1. Velocidad de calentamiento $\beta=15 \mathrm{~K} / \mathrm{min}$.

Lo propio sucede al determinar el efecto de la presión de compactación sobre las curvas de sinterización, $\varepsilon_{A}$ vs $T$ y $\alpha$ vs $T$, correspondientes a un composite $\phi=0,525$, F2, (Figura 5.39 y Figura 5.40). 


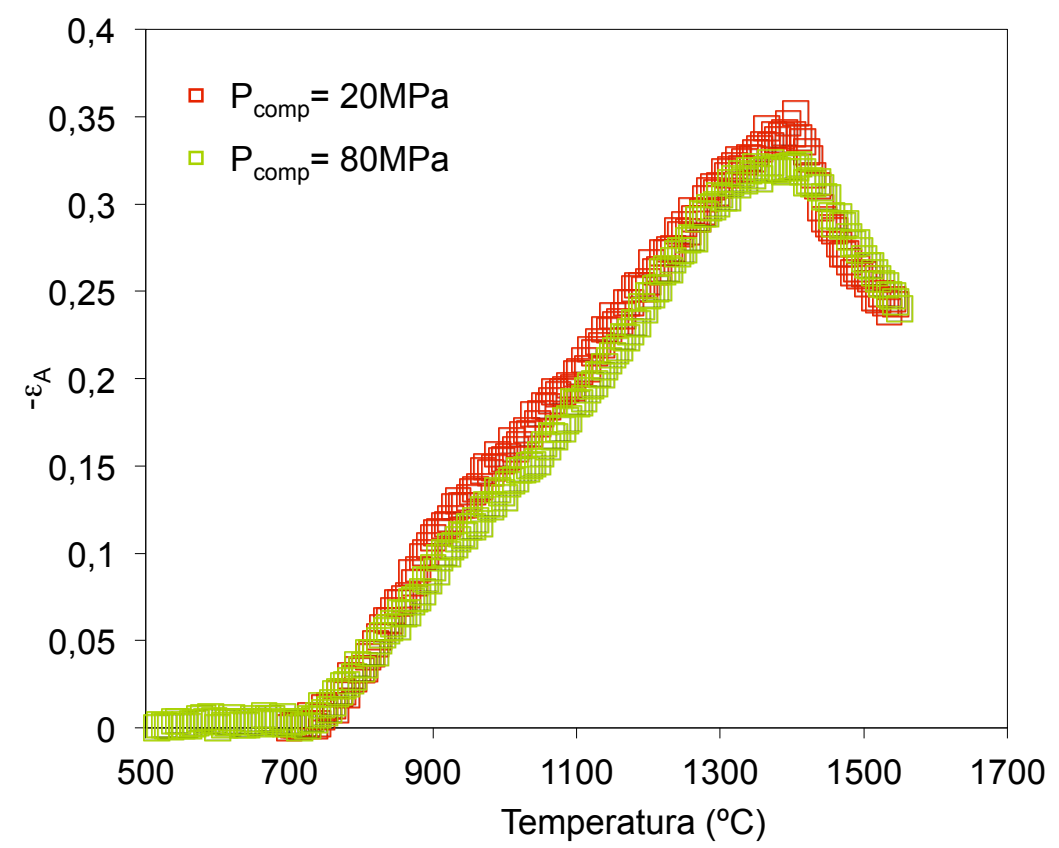

Figura 5.39. Efecto de la presión de compactación, $P_{\text {comp, sobre la curva de }}$ sinterización: $-\varepsilon_{A}$ vs $T$. Composite $F$ con $\phi=0,525$, F2. Velocidad de calentamiento $\beta=15 \mathrm{~K} / \mathrm{min}$.

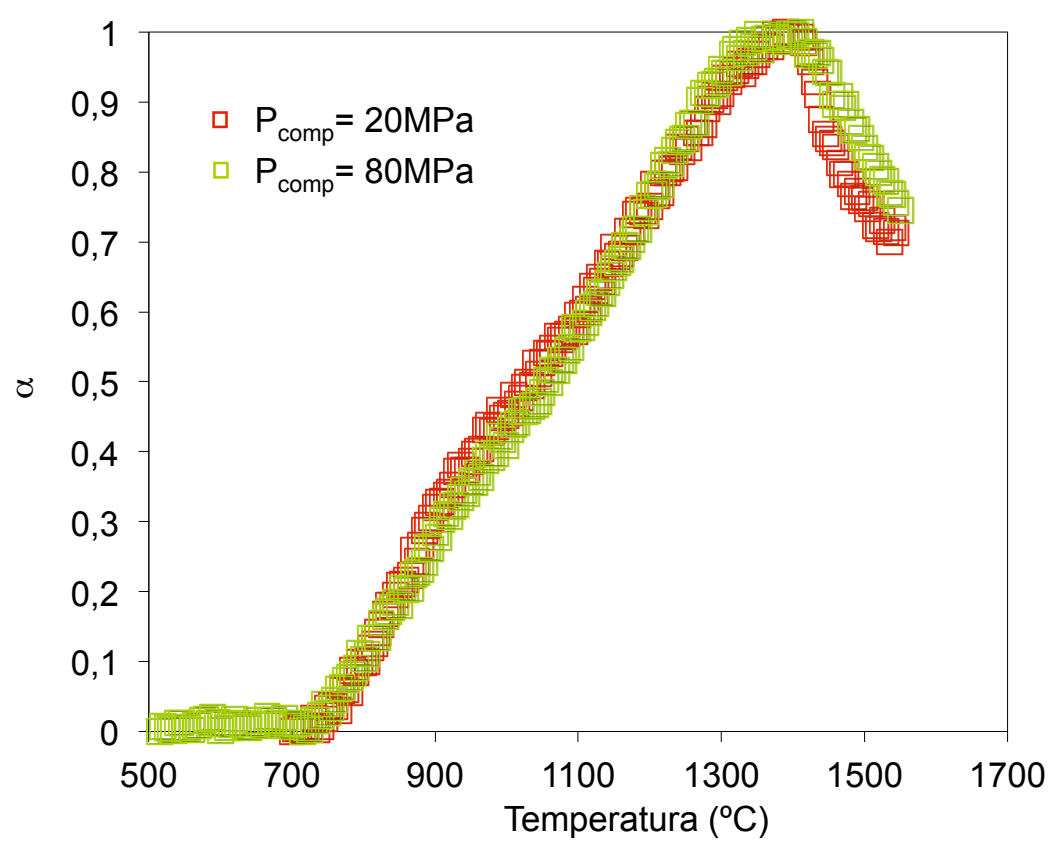

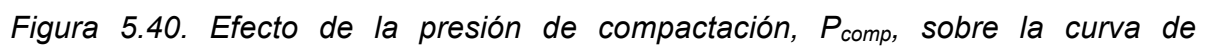
sinterización: $\alpha$ vs T. Composite $F$ con $\phi=0,525$, F2. Velocidad de calentamiento $\beta=15 \mathrm{~K} / \mathrm{min}$. 
ii) Anisotropia de la sinterización.

Siguiendo el mismo procedimiento que el empleado en el apartado 5.1.3.2 se han determinado, para las probetas de composite $\phi=0,322$, F1, compactadas a $20 \mathrm{MPa}$ y $80 \mathrm{MPa}$, los valores de $\ln \left(\frac{\mathrm{A}}{\mathrm{A}_{0}}\right)_{\mathrm{S}}, \ln \frac{\mathrm{h}}{\mathrm{h}_{0}}$ y $\ln \frac{\mathrm{D}}{\mathrm{D}_{0}}$ en función de la temperatura (Figura 5.41 y Figura 5.42). En la Figura 5.43 se han representado los valores de $-\ln \frac{h}{h_{0}}$ vs $-\ln \frac{D}{D_{0}}$ correspondientes a cada presión. El valor de la pendiente de cada recta es el factor de anisotropía, $\mathrm{k}_{\mathrm{A}}$, definido por la ec. 5.16. Se comprueba que, contrariamente a lo que ocurría en el caso de compactos de vidrio, $\mathrm{k}_{\mathrm{A}}$ es mayor o igual que $1 \mathrm{y}$ dicho parámetro aumenta con la presión de compactación. Esta misma tendencia se ha obtenido en el composite con $\phi=0,525, F 2$. Estos resultados son consistentes con lo obtenidos por Eberstein et al. [31] y Boccaccini et al. [25] en la medida en que dichos investigadores observar en $\mathrm{GMC}$ de $\mathrm{Al}_{2} \mathrm{O}_{3}$ factores de anisotropía mayores que 1 (Figura 5.43). 


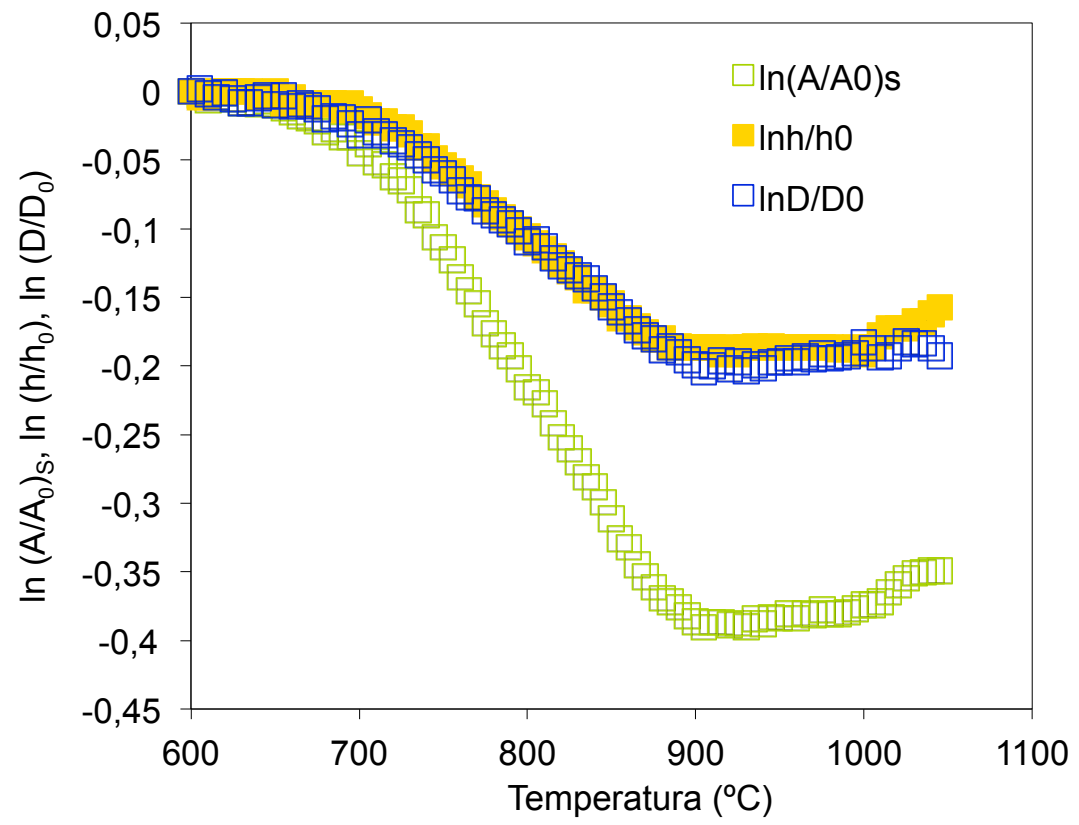

Figura 5.41. Variación de la contracción superficial, $\ln \left(A / A_{0}\right)$ s, axial, $\ln \left(h / h_{0}\right)$ y diametral, $\operatorname{In}\left(D / D_{0}\right)$, frente a la temperatura. Composite $F$ con $\phi=0,322$, F1. $P_{\text {comp }}=20 \mathrm{MPa}$. Velocidad de calentamiento $\beta=5 \mathrm{~K} / \mathrm{min}$.

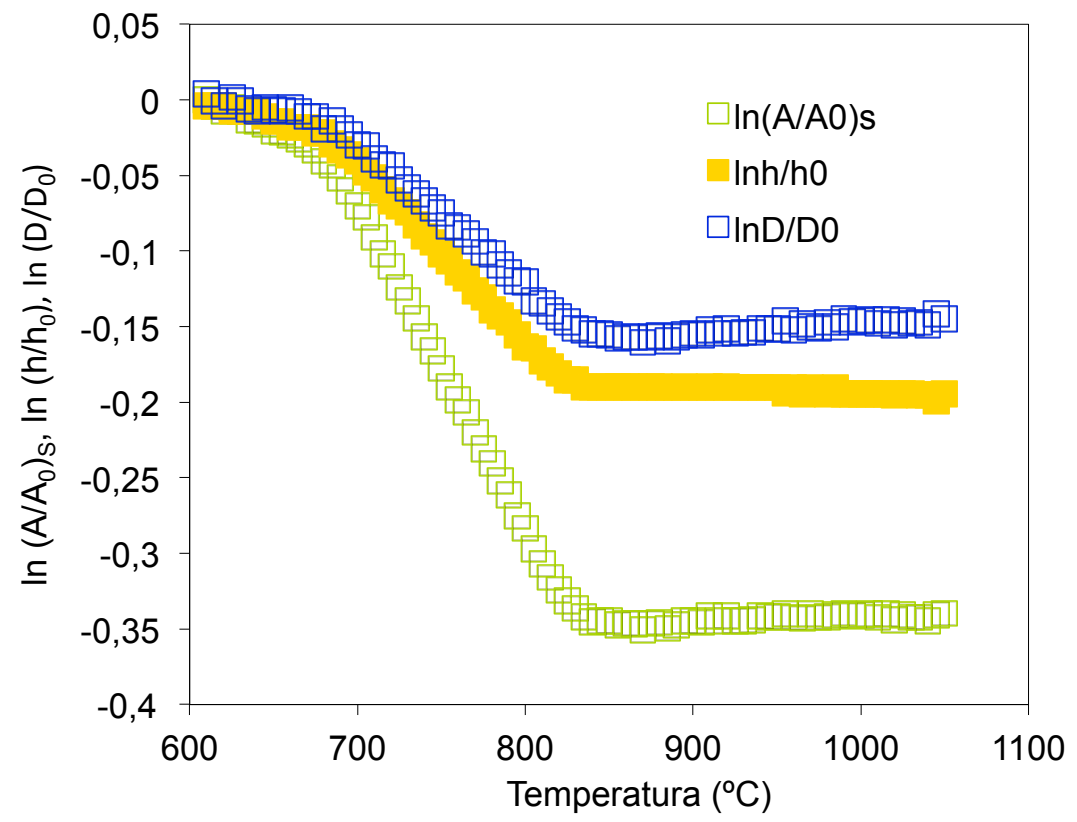

Figura 5.42. Variación de la contracción superficial, $\ln \left(A / A_{0}\right)$ s, axial, $\ln \left(h / h_{0}\right)$ y diametral, $\ln \left(D / D_{0}\right)$, frente a la temperatura. Composite $F$ con $\phi=0,322$, F1. $P_{\text {comp }}=80 \mathrm{MPa}$. Velocidad de calentamiento $\beta=5 \mathrm{~K} / \mathrm{min}$. 


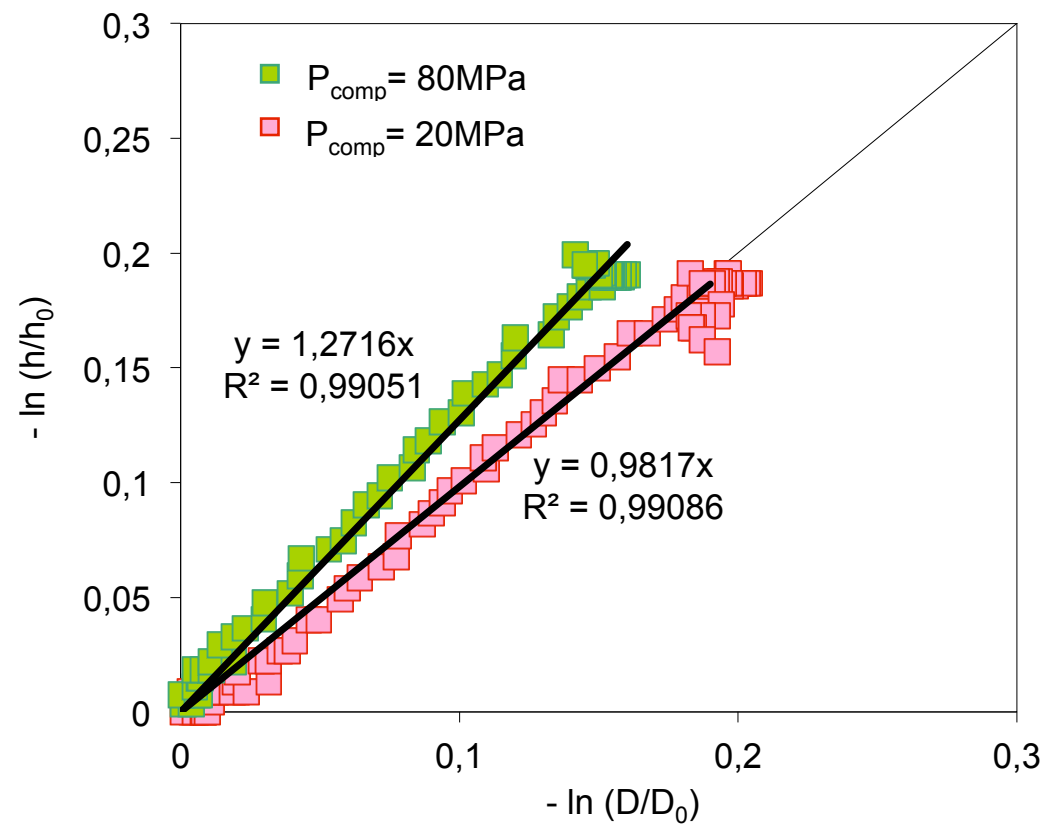

Figura 5.43. Variación de la contracción axial, $-\ln \left(h / h_{0}\right)$ frente a la diametral, $-\ln \left(D / D_{0}\right)$. Composite $F$ con $\phi=0,322, F 1 . P_{\text {comp }}=20 M P a$ y $80 \mathrm{MPa}$. Velocidad de calentamiento $\beta=5 \mathrm{~K} / \mathrm{min}$.

5.2.1.2 Evolución de las características texturales y microestructurales de composites con el avance del proceso de sinterización.

5.2.1.2.1 Análisis cualitativo (micrografías SEM).

Las micrografías de las secciones pulidas de diferentes GMC que se han seleccionado en la Figura 5.44 a la Figura 5.48 , ilustran la evolución de la microestructura de los composites con el avance de la sinterización, para diferentes valores de $\phi$. De forma general, se ha observado, cualitativamente, que la relación entre microestructura y grado de avance de la sinterización, no parece depender del tipo de tratamiento térmico, a excepción de las probetas sobrecocidas. En consecuencia, se han elegido piezas tratadas de forma isoterma y a velocidad de calentamiento constante. Las probetas crudas o bien son tratadas isotérmicamente a diferentes temperaturas, manteniendo el tiempo de permanencia a $300 \mathrm{~min}$ (Figura 5.44, Figura 5.46 y Figura 5.48), o son cocidas a una velocidad de 
calentamiento de $5 \mathrm{~K} / \mathrm{min}$ hasta diferentes temperaturas máximas (Figura 5.45 y Figura 5.47).

Los resultados más significativos se resumen a continuación:

i) De forma general, considerando los aglomerados de cristales nanométricos de circón como partículas individuales, la mayoría de las partículas de circón en estados iniciales y/o intermedios de la sinterización, están en contacto y/o rodeando áreas de vidrio (partículas de vidrio con los cantos redondeados o agregados resultantes de la coalescencia de partículas), como se aprecia más claramente, para GMC con valores de $\phi \leq 0,322$ (Figura 5.44).Esta distribución de las partículas es indicativa de un buen mezclado, al menos a escala del tamaño medio de partículas de vidrio.

ii) En el estado intermedio de sinterización y para valores de $\phi \leq 0,322$ (Figura 5.44) se aprecia que el área de contacto entre partículas de vidrio es mayor y su forma es más redondeada conforme disminuye el contenido en circón. En este estado del proceso, la forma de los poros es irregular y la porosidad principalmente abierta. Las pequeñas partículas de circón están adheridas a las partículas de vidrio o embebidas en áreas de vidrio más o menos irregulares, dependiendo del contenido en circón y de la temperatura. En cambio, para las más grandes, el área de contacto entre estas y el vidrio es generalmente pequeña.

iii) Para todos los GMC, conforme avanza el proceso de sinterización (Figura 5.45 a Figura 5.47), la matriz vítrea viscosa va mojando progresivamente las partículas de circón y/o penetrando por capilaridad en los poros que forman esas partículas. La cantidad de estos poros y clústers de circón, en estados intermedios y avanzados de la sinterización, depende del contenido en circón del composite. Ahora bien, este efecto es menos marcado en la etapa final de cocción ya que con el aumento de la temperatura/tiempo de cocción disminuyen la viscosidad y tensión superficial del vidrio lo 
que favorece la densificación y crecimiento de los poros. En efecto, a temperaturas/tiempos ligeramente más altos que los necesarios para alcanzar la máxima densificación, $\alpha$, los compactos con $\phi=0,106$ (Figura 5.46) y $\phi=0,322$ (Figura 5.45), contienen menos poros, aunque esféricos y cerrados, y, en general, de mayor tamaño, que los irregulares formados por clústers de circón. Este comportamiento de cerrado o llenado progresivo de poros, es decir, según su tamaño, se debe a que la presión capilar, fuerza impulsora del proceso, depende del ángulo de mojado y del radio capilar del poro (relacionado con el tamaño de las partículas de circón y forma en la que se agrupan. La mayor parte de los poros presentes en el composite en la etapa final de sinterización son esféricos y/o seudoesféricos, con sus paredes constituidas casi exclusivamente de partículas de circón. No obstante, también se observan, especialmente en aquellos composites con menor contenido en circón, poros esféricos en el seno de las áreas irregulares de matriz vítrea.

iv) Únicamente en los composites con $\phi \geq 0,525$ y a temperaturas elevadas (Figura 5.47, $\mathrm{T}=1200$ y $1300^{\circ} \mathrm{C}$ ) y/o tiempos de permanencia elevados (Figura $5.48, \mathrm{~T}=1100^{\circ} \mathrm{C}$ ) se aprecia un cambio en el tamaño y forma de las partículas de circón debido al mecanismo de solución-reprecipitación, como se verá en el apartado 5.2.1.3. 

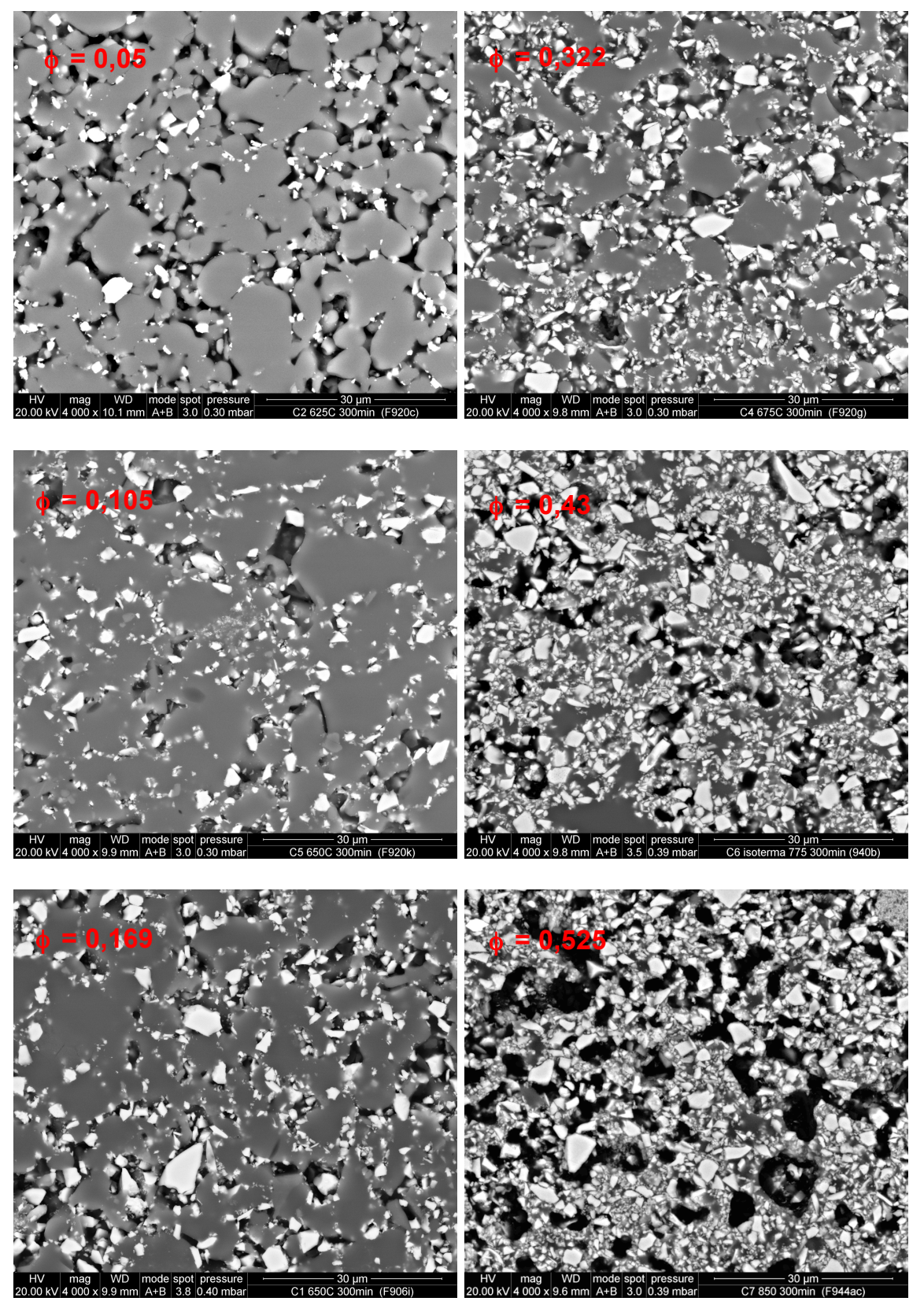

Figura 5.44. Etapa intermedia de la sinterización. Influencia de la fracción volumétrica de circón: $\phi=0,05\left(\alpha=0,65\right.$ a $\left.T=625^{\circ} \mathrm{C}\right) ; \phi=0,105\left(\alpha=0,80\right.$ a $\left.T=650^{\circ} \mathrm{C}\right)$; $\phi=0,169\left(\alpha=0,73\right.$ a $\left.T=650^{\circ} \mathrm{C}\right) ; \phi=0,322\left(\alpha=0,55\right.$ a $\left.T=675^{\circ} \mathrm{C}\right) ; \phi=0,43$ $\left(\alpha=0,65\right.$ a $\left.T=775^{\circ} \mathrm{C}\right) ; \phi=0,525\left(\alpha=0,35\right.$ a $\left.T=850^{\circ} \mathrm{C}\right)$. Tratamiento isotermo, tiempo de permanencia 300min. (x4000). 

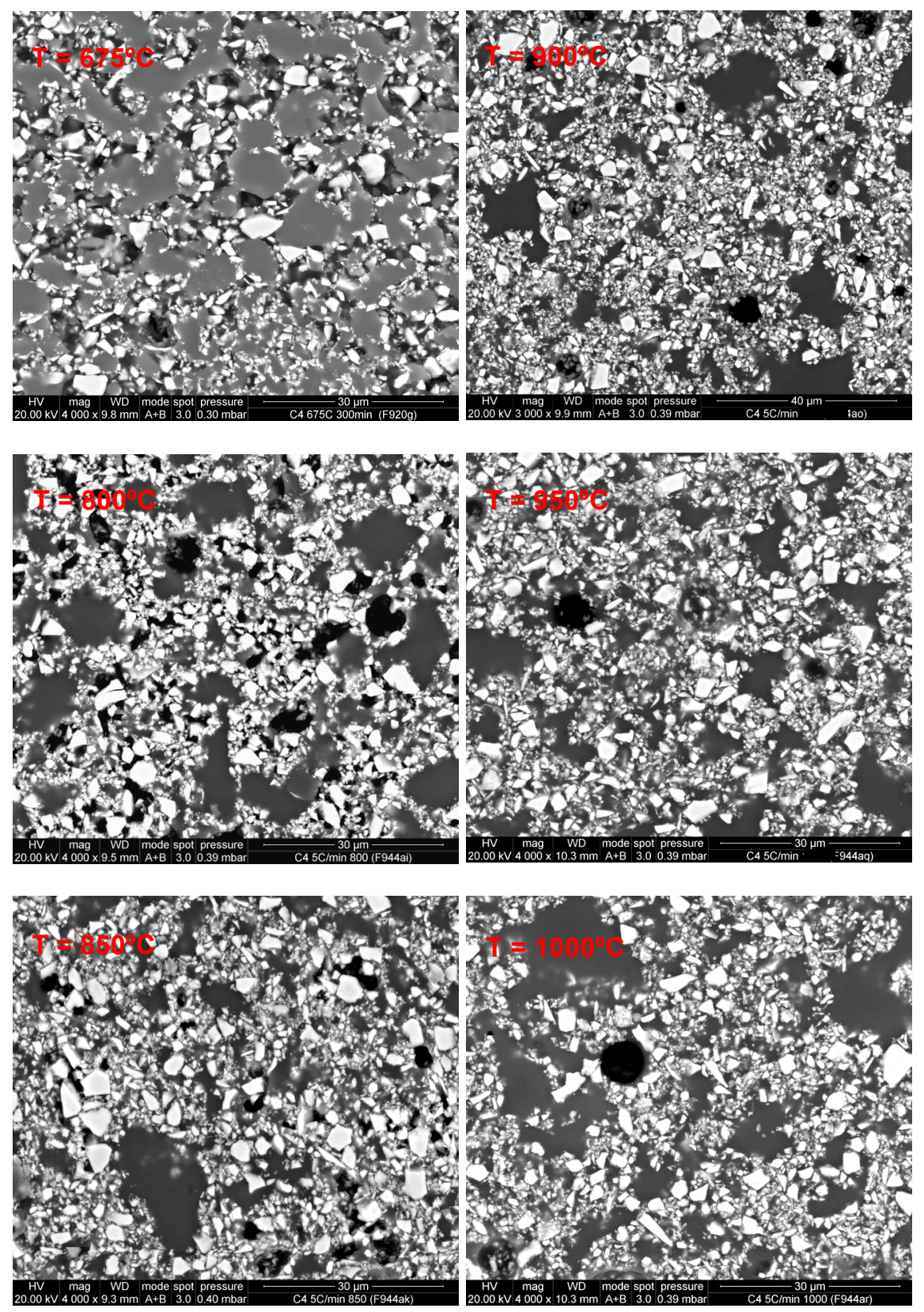

Figura 5.45. Evolución de la microestructura ( $x 4000$ aumentos) del composite $F$, $\phi=0,322, F 1$, con el avance del grado de sinterización, $\alpha: \alpha=0,40$ a $T=800^{\circ} \mathrm{C}, \alpha=0,94$ a $T=850^{\circ} \mathrm{C}, \alpha=1$ a $T=900^{\circ} \mathrm{C}, \alpha=0,97$ a $T=950^{\circ} \mathrm{C}$, $\alpha=0,91$ a $T=1000^{\circ} \mathrm{C}$. Velocidad de calentamiento $5 \mathrm{~K} / \mathrm{min}$. 

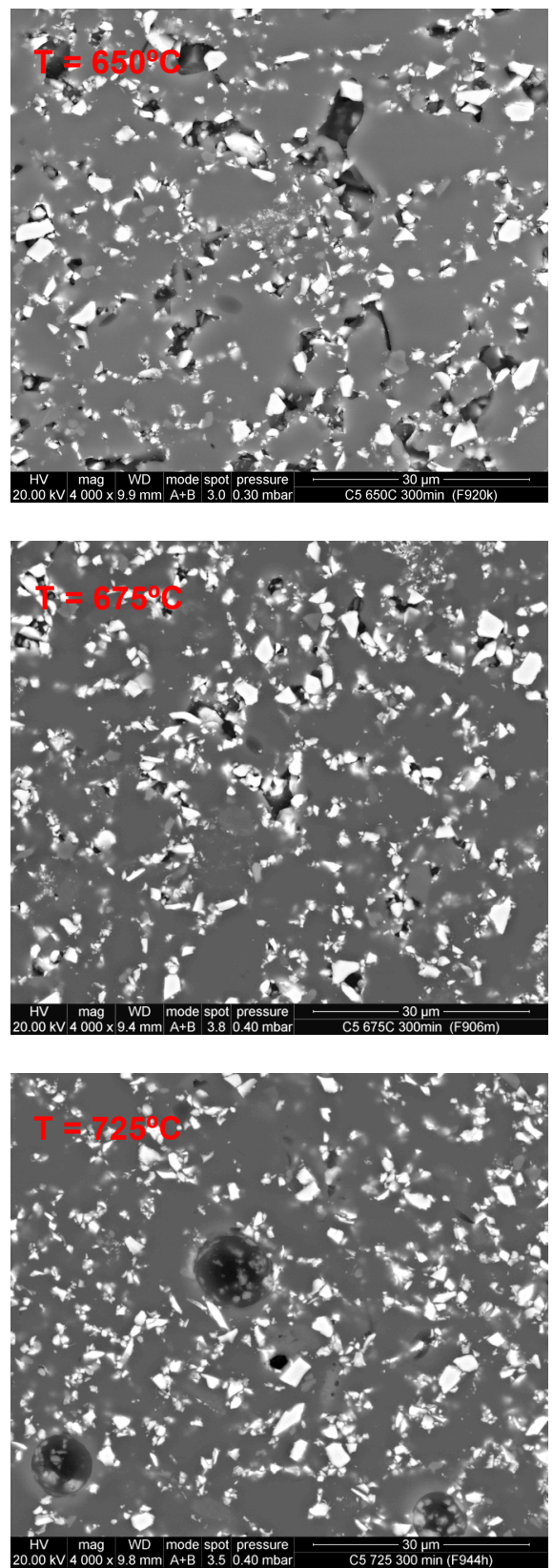

Figura 5.46. Evolución de la microestructura (x4000 aumentos) del composite $F$, $\phi=0,106$, con el avance del grado de sinterización, $\alpha: \alpha=0,72$ a $T=650^{\circ} \mathrm{C}$, $\alpha=0,93$ a $T=675^{\circ} \mathrm{C}, \alpha=0,97$ a $T=725^{\circ} \mathrm{C}$. Tratamiento isotermo, tiempo de permanencia $300 \mathrm{~min}$ 

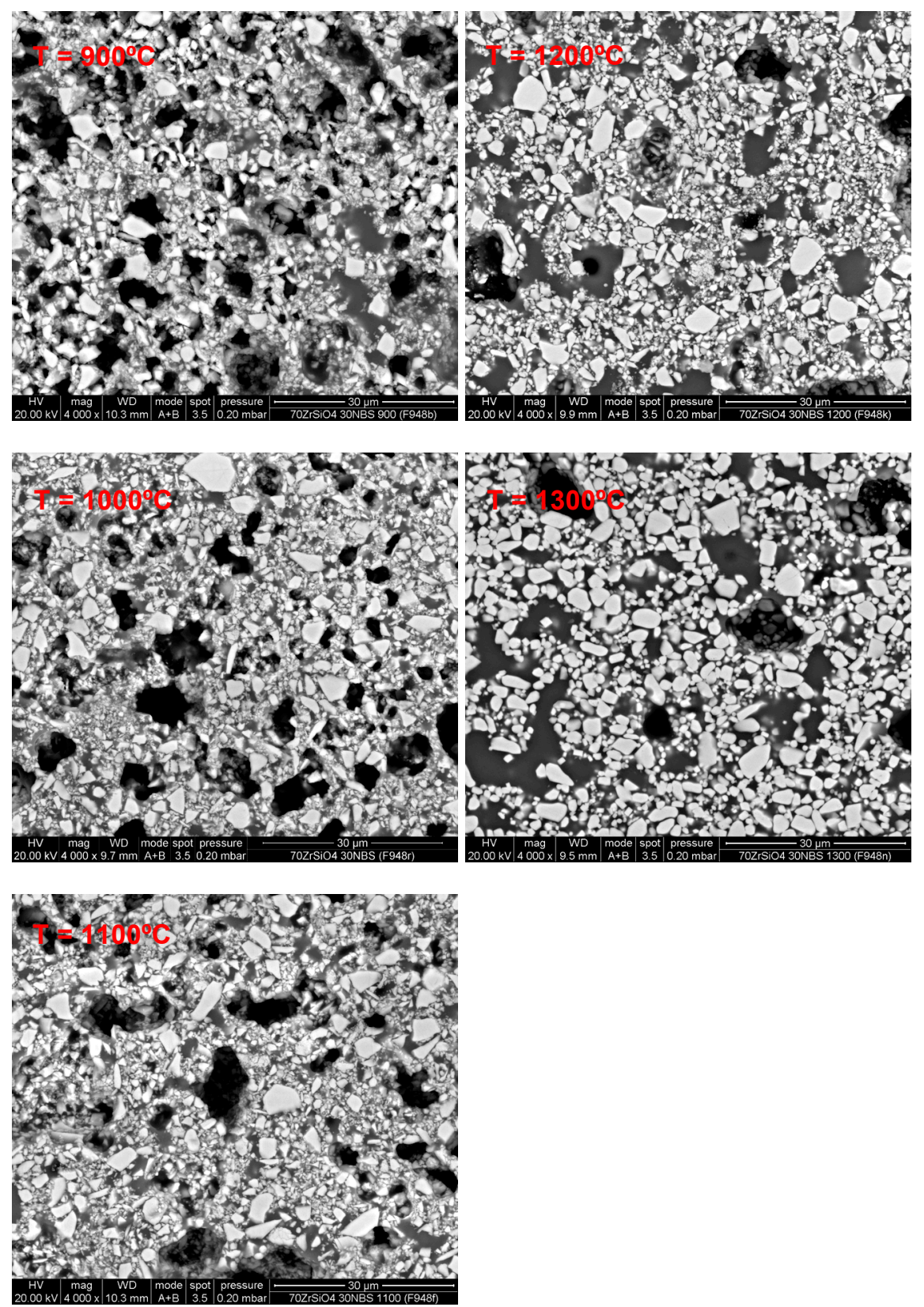

Figura 5.47. Evolución de la microestructura (x4000 aumentos) del composite $F$, $\phi=0,525, F 2$, con el avance del grado de sinterización, $\alpha: \alpha=0,22$ a $T=900^{\circ} \mathrm{C}, \quad \alpha=0,40$ a $T=1000^{\circ} \mathrm{C}, \quad \alpha=0,54$ a $T=1100 \mathrm{~min}, \alpha=0,73$ a $T=1200^{\circ} \mathrm{C}$ y $\alpha=0,92$ a $T=1300^{\circ} \mathrm{C}$. Velocidad de calentamiento $5 \mathrm{~K} / \mathrm{min}$. 


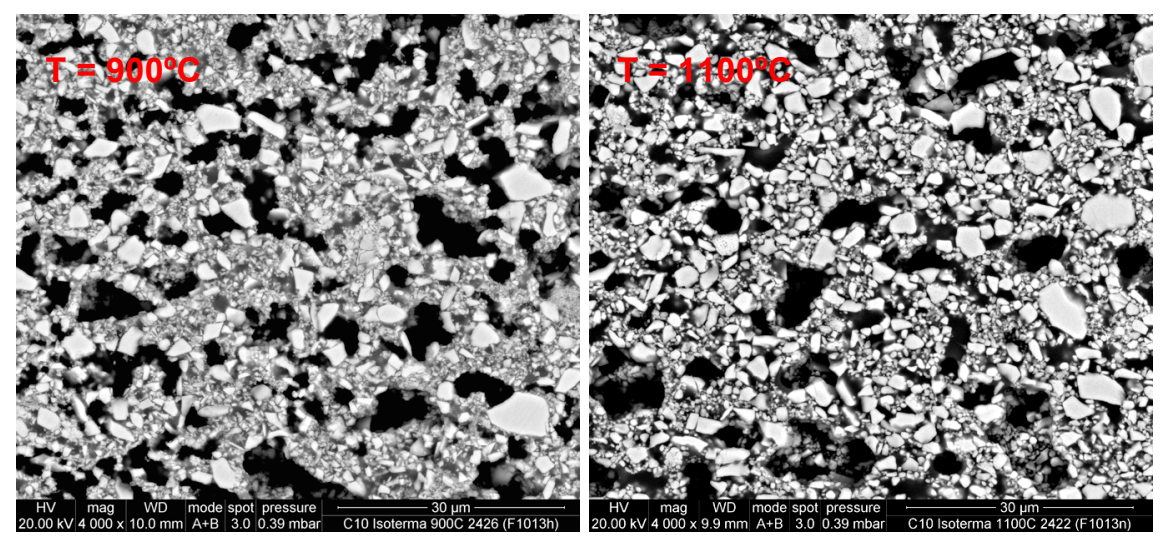

Figura 5.48. Evolución de la microestructura (x4000 aumentos) del composite $F$, $\phi=0,525, \mathrm{~F} 2$, con el avance del grado de sinterización, $\alpha: \alpha=0,47$ a $T=900^{\circ} \mathrm{C}$ y $\alpha=0,87$ a $T=1100^{\circ} \mathrm{C}$. Isotermas $300 \mathrm{~min}$.

\subsection{Compacidad máxima, $\rho_{\max }$, e inicial, $\rho_{0}$.}

De las dimensiones finales de la probeta y de la variación que sigue la superficie de su silueta y su altura en el ensayos de HSM, se ha calculado el volumen de la probeta a la máxima compacidad. De éste valor, de la masa de la probeta obtenida por pesada y de la densidad real de sólido calculada con la ec. 4.2 se ha calculado la compacidad máxima, $\rho_{\max }$, mediante la ec. 4.1. En la Figura 5.49 se representa, en función de $\phi$, para cada composite de la serie $F$, el valor medio que resulta de promediar los valores de $\rho_{\max }$ individuales obtenidos a cada velocidad de calentamiento. Al igual que ocurre con $\varepsilon_{\mathrm{A}, \max }$, no se aprecia, en ningún caso, una variación sistemática de $\rho_{\max }$ con la velocidad de calentamiento. En todos los casos la desviación estándar era menor que $\pm 0,02$, error que se estima en las medidas, y que además es coincidente con el hallado en otros trabajos [31][118]. Estos resultados son consistentes con los obtenidos en un estudio sobre la porosidad de vidriados cerámicos [119], en el que se confirma que la porosidad final mínima era independiente del ciclo térmico empleado, y que esta aumentaba con el contenido en fases cristalinas añadidas en su preparación. 


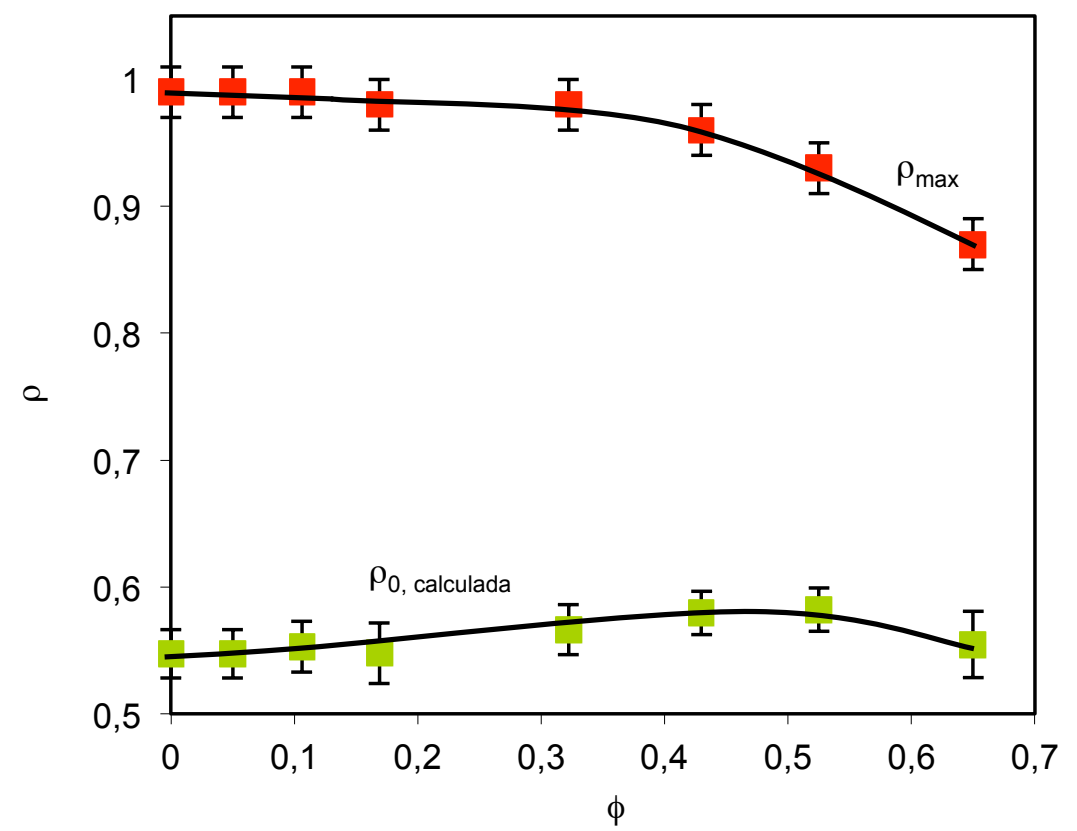

Figura 5.49. Influencia de $\phi$ sobre la compacidad máxima promedio, $\rho_{\max }$ y sobre la inicial calculada, $\rho_{0}$, calculada. Experimentos a velocidad de calentamiento $\beta=2,5,15,25$ y 60K/min. Compactos serie $F$.

En esta misma figura se han representado los valores, también promedio de la compacidad de las probetas al inicio de la sinterización, $\rho_{0, \text { calculada, }}$ calculados a partir de los valores de $\rho_{\max }$ y de los valores de $\varepsilon_{\mathrm{A}, \max }$, mediante la ec. 5.15. Se comprueba una disminución progresiva de $\rho_{\max }$ con el aumento del contenido en circón en el composite, $\phi$, siendo dicho efecto mucho más marcado para valores de $\phi \geq 0,322$. Para valores inferiores de $\phi$ la variación es pequeña, del orden de la desviación estándar de las medidas.

La variación de $\rho_{\max }$ vs $\phi$, aun siendo similar a la de $\varepsilon_{\mathrm{A}, \max }$ vs $\phi$ (Figura 5.35), es ligeramente diferente debido a que la compacidad inicial, $\rho_{0}$, al inicio de la sinterización, varía con $\phi$, debido a dos factores: la compacidad en crudo de la probeta prensada, dependiente de la distribución de tamaños 
de partícula y a su expansión térmica. Ambos son dependiente del contenido en circón.

Para cuantificar el efecto de $\phi$ sobre la disminución de la compacidad máxima, $\rho_{\max }$, se ha utilizado un grado de reducción de la compacidad máxima, análogo al empleado en el aparado 5.2.1.1.1, definido como:

$\Delta \rho_{\max }^{*}=1-\frac{\rho_{\max }}{\rho_{\max }(0)}$

Siendo $\rho_{\max }(0)$ la compacidad media máxima correspondiente a los compactos de vidrio.

Se comprueba que el efecto de la adición de circón sobre la reducción de la compacidad máxima que puede alcanzar el composite (o el aumento de la porosidad), $\Delta \rho_{\max }^{*}$, aumenta de acuerdo con una ecuación del tipo (Figura 5.50):

$\Delta \rho_{\max }^{*}=0,5 \cdot \phi^{3,3} \quad S^{2}=5,71 \cdot 10^{-6}$ ec. 5.19 


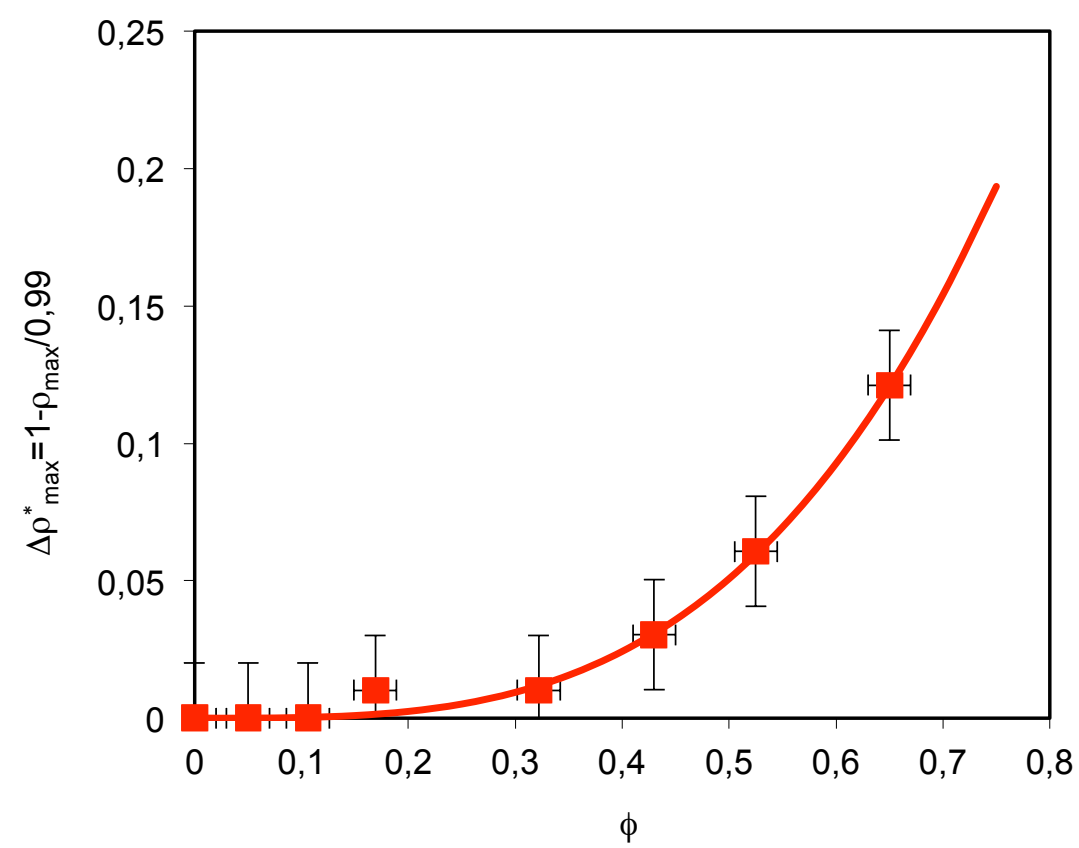

Figura 5.50. Influencia de $\phi$ sobre el grado de reducción de la compacidad máxima, $\Delta \rho_{\text {max. }}^{*}$ Experimentos a velocidad de calentamiento $\beta=2,5,15,25$ y 60 $K / \min$. Compactos serie $F$. (Solo se incluyen algunas barras de error ya que éste es prácticamente constante).

El hecho de que $\Delta \rho_{\max }^{*} \alpha \phi^{m}$ con $m>3$ debe relacionarse, según Eberstein [31], al menos con tres factores característicos: i) la presencia, en el composite sinterizado, de poros o cavidades formadas por la agrupación de más de tres partículas rígidas (circón); ii) un buen mezclado de los componentes y; iii) un buen mojado de las partículas de circón por el vidrio, es decir, un ángulo de mojado pequeño.

En efecto, un valor de $\mathrm{m}$ más alto implica un menor efecto de la adición de partículas rígidas sobre la porosidad final. $\mathrm{Y}$, dicha situación se produce, como en nuestro caso, cuando el mezclado a escala de tamaño medio de partícula es bueno, el número de partículas de circón que forman cavidades porosas es generalmente elevado y el ángulo de mojado pequeño. Como puede comprobarse en las micrografías de la Figura 5.44 a Figura 5.48.

5.2.1.2.3 Distribución del tamaño de los poros abiertos. Diámetro medio de 
intrusión. Presión de sinterización.

En el desarrollo de este apartado también se han utilizado probetas cilíndricas, de aproximadamente $2 \mathrm{~cm}$ de diámetro y $2 \mathrm{~cm}$ de altura, obtenidas por colado. Se han realizado dos tipos de tratamiento: no isotermo, a $5 \mathrm{~K} / \mathrm{min}$, e isotermo, a distintas temperaturas según el composite, $700^{\circ} \mathrm{C}$ para el F1 y $1100^{\circ} \mathrm{C}$ para el F2.

En la Figura 5.51 y Figura 5.52 se representan, para los composites de la serie $F$ con $\phi=0,322, F 1$ y $\phi=0,525, F 2$, las curvas de distribución de tamaños de poro, correspondientes a probetas obtenidas a diferentes temperaturas de cocción, en experimentos a velocidad de calentamiento de $5 \mathrm{~K} / \mathrm{min}$.

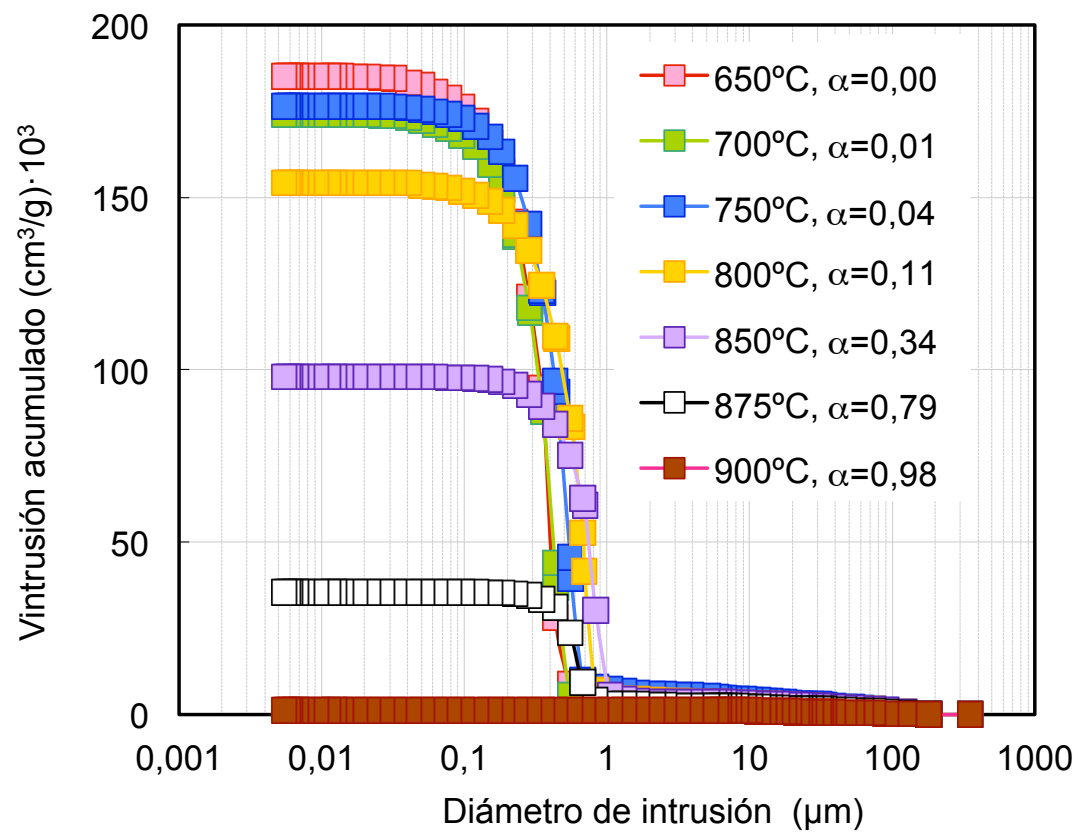

Figura 5.51. Evolución de la distribución de poros abiertos de las probetas cocidas con el avance de la sinterización, $\alpha$, y la temperatura, T. Composite $F$ con $\phi=0,322$, F1. Velocidad de calentamiento $\beta=5 \mathrm{~K} / \mathrm{min}$. 


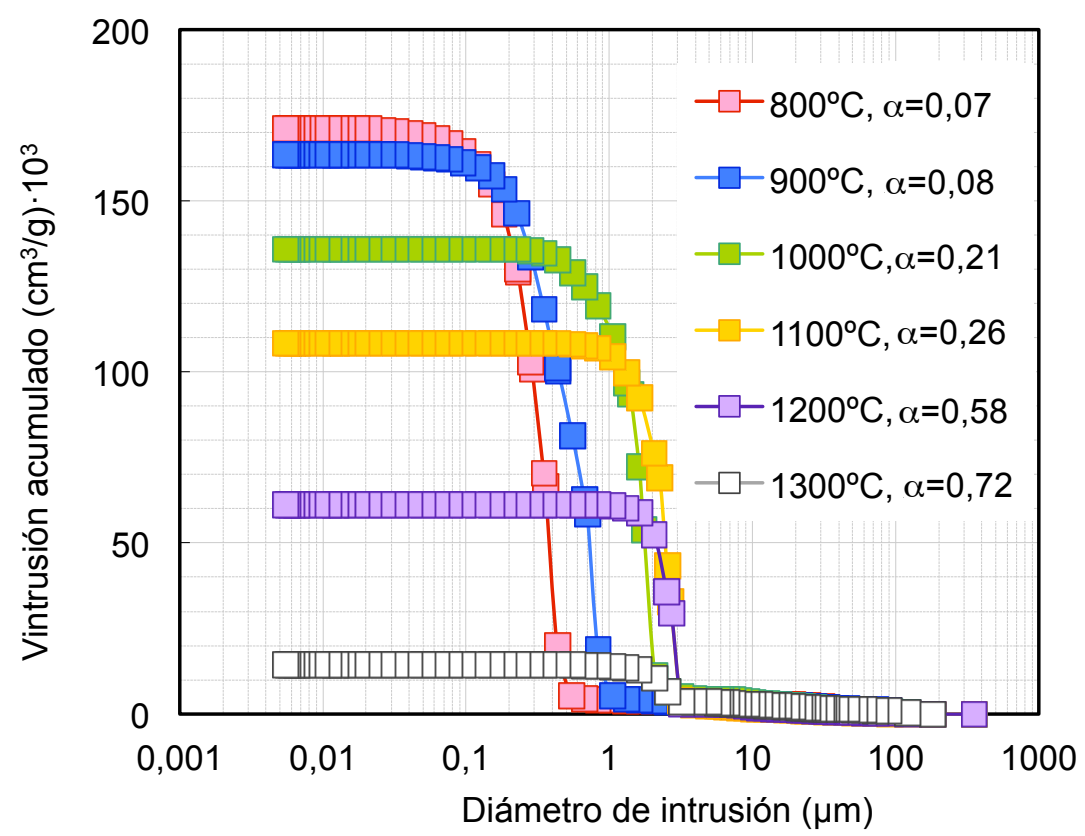

Figura 5.52. Evolución de la distribución de poros abiertos de las probetas cocidas con el avance de la sinterización, $\alpha$, y la temperatura, T. Composite $F$ con $\phi=0,525$, F2. Velocidad de calentamiento $\beta=5 \mathrm{~K} / \mathrm{min}$.

En la Figura 5.53 se han representado, para todas las probetas obtenidas en experimentos a una velocidad de calentamiento de $5 \mathrm{~K} / \mathrm{min}$, e isotermos a $700^{\circ} \mathrm{C}$ y $1100^{\circ} \mathrm{C}$, para $\phi=0,322$ y $\phi=0,525$, la variación del diámetro de intrusión, $d_{16}(\mu \mathrm{m})$, con el grado de avance de la sinterización. Se incluyen en esta representación, los resultados correspondientes a las probetas de vidrio del apartado 5.1.1.4.4. El $d_{16}$ representa el diámetro de intrusión por encima del cual se encuentra el $16 \%$ del volumen de poros abiertos. Se obtiene ajustando la curva porosimétrica a una distribución logarítmonormal. Generalmente, se usa como una medida estadística de los poros más grandes [113][120]. 


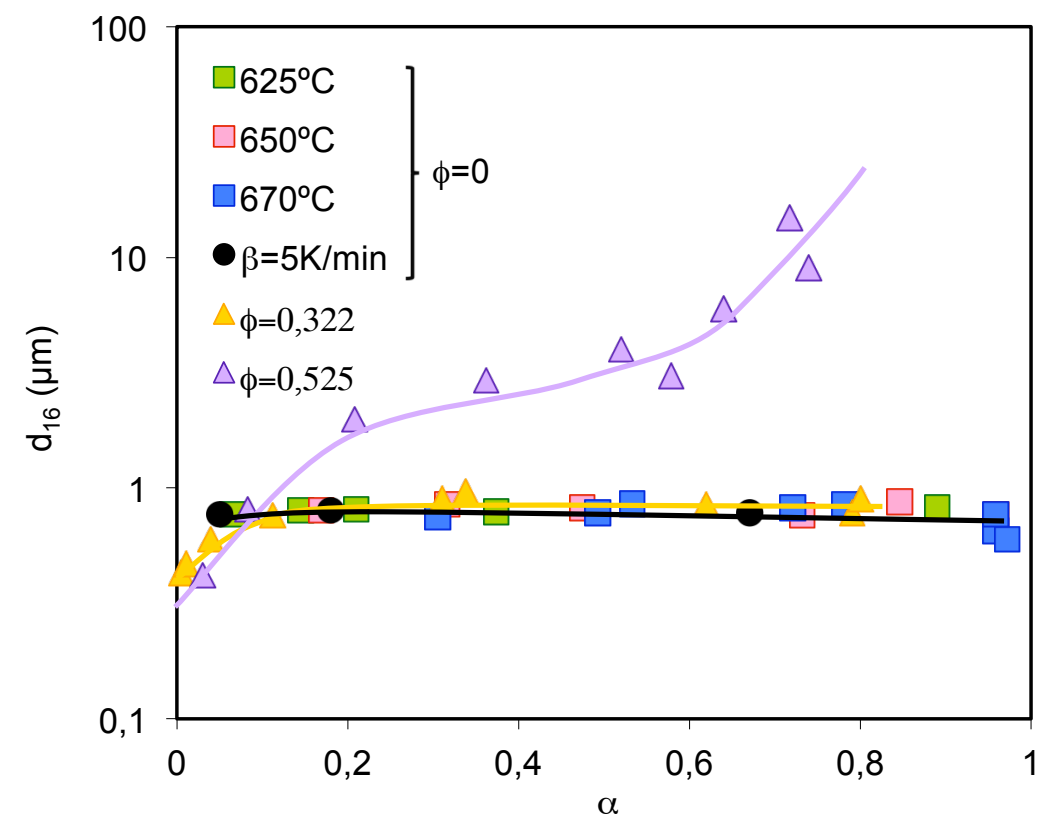

Figura 5.53. Variación del diámetro de intrusión, $d_{16}$, de probetas cocidas con el grado de avance de la sinterización, $\alpha$, para vidrio GM y para composites $F$ con $\phi=0,322, F 1, y \phi=0,525, F 2$. Tratamientos isotermos y no isotermos.

En la Figura 5.54 se han representado para las mismas probetas que las empleadas en la Figura 5.53, la variación del diámetro medio de intrusión, $\frac{4 \mathrm{~V}}{\mathrm{~A}}$, frente al grado de avance de la sinterización, para valores de $\phi=0$, $\mathrm{GM}, \phi=0,322, \mathrm{~F} 1, \mathrm{y} \phi=0,525, \mathrm{~F} 2$.

Del examen del conjunto de estas representaciones se desprende:

i) La variación que sigue el diámetro medio de poro, $\frac{4 \mathrm{~V}}{\mathrm{~A}}$, frente al grado de avance de la sinterizaión depende del contenido en circón (Figura 5.54). En efecto, para las probetas de vidrio, $\phi=0$, como ya se vio en el apartado 5.1.1.4.4, el $\frac{4 \mathrm{~V}}{\mathrm{~A}}$ crece inicialmente muy poco. Para valores de 
$\alpha \geq 0,3$, el tamaño medio de poro va disminuyendo con el avance de la sinterización. Para composites con contenidos intermedios de circón, $\phi=0,322$, el crecimiento inicial del tamaño medio de poro se prolonga hasta valores de $\alpha \approx 0,4$ y se estabiliza hasta valores próximos a $\alpha=0,8$. En cambio, para contenidos en circón altos, el tamaño de poro medio va creciendo continuamente con el avance del proceso. Este crecimiento de tamaño medio de poro con $\alpha$ es considerable (tipo exponencial) en etapas iniciales de la sinterización, $\alpha \leq 0,4$, y más moderada en etapas intermedias del proceso, $0,4 \leq \alpha \leq 0,8$.

ii) A diferencia de lo que ocurría con las probetas de vidrio (Figura 5.14), en las que no se apreciaba un crecimiento de poros más grandes con el avance de la sinterización, $\alpha$, en los composites F1 y F2, paralelamente a la eliminación de los poros más pequeños, los más grandes crecen, siendo dicho fenómeno dependiente del contenido en circón (Figura 5.51 y Figura 5.52). En efecto, en el composite $\mathrm{F} 1(\phi=0,322)$ el crecimiento de los poros más grandes solo es significativo en las etapas iniciales de la sinterización (Figura 5.51 y Figura 5.53). En cambio, para el composite F2 $(\phi=0,525)$, el tamaño de poros más grande crece desde valores iniciales de $d_{16}=0,6 \mu \mathrm{m}$ hasta valores de $d_{16}=20 \mu \mathrm{m}$ para grados de avance de la sinterización de $\alpha \approx 0,8$ (Figura 5.52 y Figura 5.53). Esta evolución de la porosidad abierta con el avance del proceso es consistente con el observado para otros materiales que densifican por sinterización en presencia de fase líquida, con contenidos apreciables en fases cristalinas en su composición de partida, como vidriados [119], baldosas de baja porosidad [113] y de gres porcelánico [120]. Dicho comportamiento debe asociarse, como ya se ha indicado en la introducción (apartado 2.1.1.5.1), a la diferencia de velocidad de sinterización entre los distintos contactos y/o empaquetamientos que se establecen entre partículas de distinto tamaño y/o naturaleza (vidrio o inclusión). 


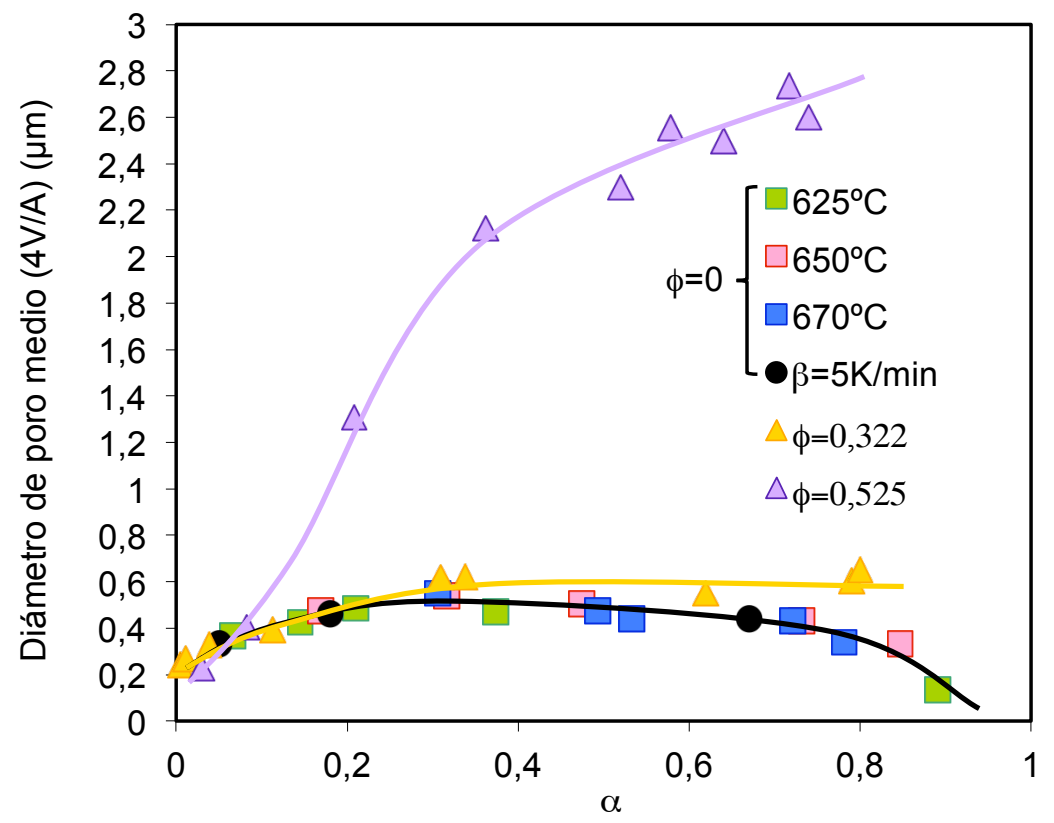

Figura 5.54. Variación del diámetro medio de intrusión, 4V/A, de probetas cocidas con el grado de avance de la sinterización, $\alpha$, para vidrio GM y para composites $F$ con $\phi=0,322, F 1, y \quad \phi=0,525, F 2$. Tratamientos isotermos $y$ no isotermos.

A partir de los valores del diámetro medio de poro (Figura 5.54) se han calculado, mediante la ec. 5.7, los valores de la presión de sinterización, $\Sigma^{S}$, según Wakai [114] (Figura 5.55). Se observa que, a diferencia de lo que ocurría en el vidrio, en el que la curva $\sum^{S}$ vs $\alpha$ presentaba un mínimo, para los composites, $\Sigma^{S}$ siempre disminuye con el avance de la sinterización, $\alpha$, de forma asintótica. En efecto, para valores de $\alpha \leq 0,3$, la disminución de $\Sigma^{S}$ con $\alpha$ es muy marcada debido a la eliminación de los poros más pequeños (Figura 5.51 y Figura 5.52), al igual que ocurría con el compacto de vidrio, y dependiente del contenido en circón. En cambio, para valores de $\alpha \geq 0,3$, solo en los composites $F 1$ y $F 2$, en los que sigue habiendo un crecimiento de los poros más grandes (Figura 5.53) y de tamaño medio (Figura 5.54) con el avance de la sinterización, $\Sigma^{S}$ sigue disminuyendo pero 
a menor velocidad. Las curvas $\Sigma^{S}$ vs $\alpha$ correspondientes a $\alpha=0,322, F 1$, y $\alpha=0,525, F 2$, se han ajustado a una ecuación del tipo:

$\Sigma^{S}=\mathrm{a} \cdot \alpha^{-\mathrm{b}}$

ec. 5.20

resultando los valores que se detallan en la Tabla 5.9. Estos resultados son consistentes con los obtenidos por German [121][122][123], quien observa que la presión capilar en la sinterización en fase líquida disminuye con el avance del proceso.

Tabla 5.9. Parámetros de ajuste a la ec. 5.20, para los composites $F 1(\phi=0,322)$ y $F 2$ $(\phi=0,525)$.

\begin{tabular}{|c|c|c|c|}
\hline$\phi$ & $\mathrm{a}$ & $\mathrm{b}$ & $\mathrm{r}^{2}$ \\
\hline 0,322 & 1,01 & 0,26 & 0,963 \\
\hline 0,525 & 0,18 & 0,85 & 0,963 \\
\hline
\end{tabular}

Se aprecia que conforme aumenta el contenido en circón se reduce el parámetro "a", valor al que tiende $\Sigma^{S}$ cuando $\alpha \rightarrow 1$. Por contra, el efecto del avance de la sinterización sobre la reducción de $\Sigma^{S}$, parámetro "b", aumenta con $\phi$. 


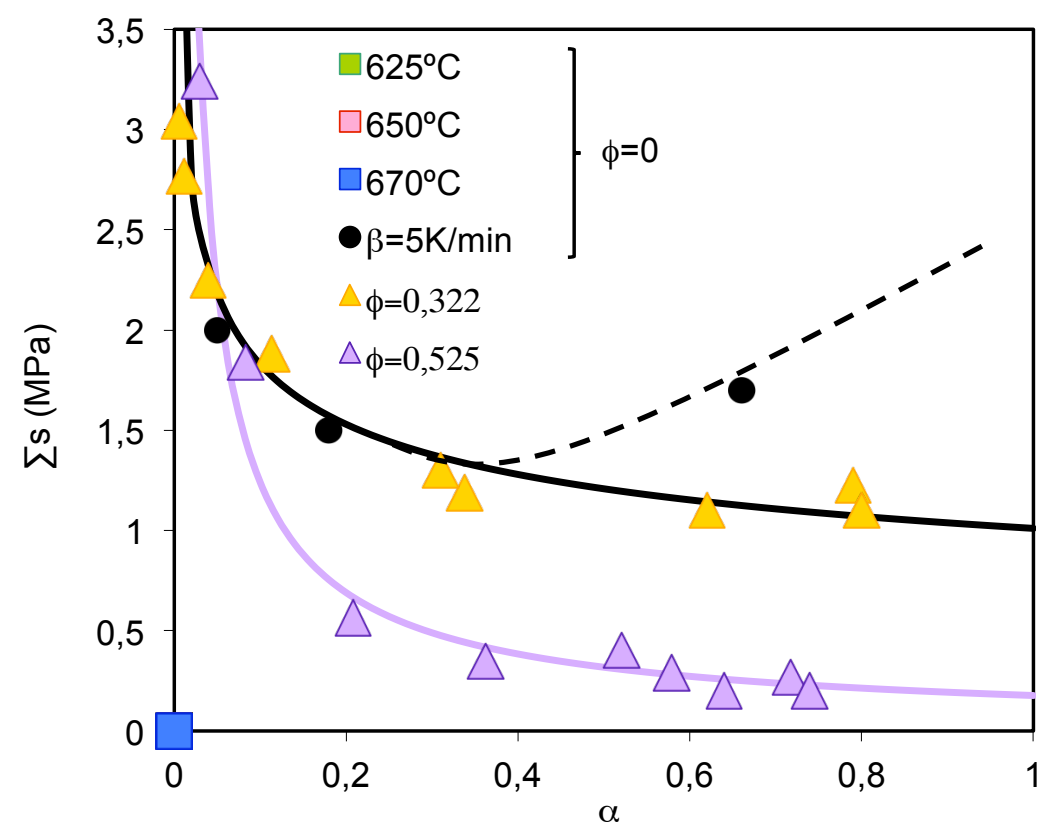

Figura 5.55. Variación de la presión de sinterización calculada, $\Sigma^{S}$, con el grado de avance de la sinterización, $\alpha$, para vidrio GM y para composites $F$ con $\phi=0,322, F 1, y \phi=0,525, F 2$. Tratamientos isotermos y no isotermos.

La marcada reducción que experimenta $\Sigma^{S}$, para estados intermedios y finales de la sinterización, con el aumento del contenido en circón, $\phi$, es el otro factor importante que, junto con el aumento de la viscosidad efectiva, contribuye a desplazar las curvas de sinterización a temperaturas cada vez más altas.

5.2.1.2.4 Razón porosidad abierta/porosidad total, $\varepsilon_{\text {abierta }} / \varepsilon_{\text {totall }}$.

A partir de los valores del volumen máximo de intrusión de mercurio y de la compacidad de cada probeta, se han calculado los valores de $\varepsilon_{\text {abierta }} / \varepsilon_{\text {total }}$ para los composites $\phi=0,322$, F1, y $\phi=0,525$, F2. En la Figura 5.56 y Figura 5.57 se han representado esto valores frente a $\alpha$ y $\rho$, respectivamente. Se incluyen en estas figuras las curvas correspondientes al compacto de vidrio, GM. Se comprobó que independientemente del tipo de tratamiento, los 
resultados, para cada composite y característica, se ajustan a la ecuación del tipo:

$$
\begin{aligned}
& \frac{\varepsilon_{\text {abierta }}}{\varepsilon_{\text {total }}}=\left(\frac{\varepsilon_{\text {abierta }}}{\varepsilon_{\text {total }}}\right)_{\alpha=0}-\left(\frac{\alpha}{\alpha_{\varepsilon_{\text {abierta }}=0}}\right)^{n} \\
& \frac{\varepsilon_{\text {abierta }}}{\varepsilon_{\text {total }}}=\left(\frac{\varepsilon_{\text {abierta }}}{\varepsilon_{\text {total }}}\right)_{\alpha=0}-\left(\frac{\rho}{\rho_{\varepsilon_{\text {abierta }}=0}}\right)^{m}
\end{aligned}
$$

En la Tabla 5.10 se detallan los valores de los parámetros de ajuste, para el compacto de vidrio, y para los dos composites.

Tabla 5.10. Parámetros de ajuste de la ec. 5.21 y la ec. 5.22 para el vidrio $G M(\phi=0)$ y para los composites $F 1(\phi=0,322)$ y $F 2(\phi=0,525)$.

\begin{tabular}{|c|c|c|c|c|c|c|c|}
\hline & $\left(\varepsilon_{\text {abierta }} / \varepsilon_{\text {total }}\right) \alpha=0$ & $\alpha_{\text {abierta }=0}$ & $n$ & $S^{2}$ & $\rho_{\text {abierta }=0}$ & $m$ & $S^{2}$ \\
\hline$\phi=0$ & 0,98 & 0,98 & 10 & 0,0028 & 0,97 & 20 & 0,0048 \\
\hline$\phi=0,322$ & 1 & 0,97 & 7 & 0,0054 & 0,96 & 15 & 0,0041 \\
\hline$\phi=0,525$ & 1 & 0,85 & 3 & 0,0014 & 0,87 & 12 & 0,0042 \\
\hline
\end{tabular}

Se aprecia que, para todos los materiales, en estados iniciales de la sinterización, $\alpha \leq 0,4$, toda la porosidad es prácticamente abierta. Ahora bien, los valores tanto del grado de avance de la sinterización, $\alpha_{\varepsilon}$ como de la compacidad $\rho_{\varepsilon_{\text {abierta }}=0}$, a la que la porosidad es prácticamente cerrada, disminuyen muy poco con la adición moderada de circón $\phi=0,322$, $\mathrm{F} 1$, pero es considerable para valores de $\phi=0,525, \mathrm{~F} 2$.

Además, para altos contenidos en circón, $\phi=0,525$, F2, la formación de porosidad cerrada comienza a ser apreciable para valores de $\alpha$ y $\rho$ mucho 
más bajos que los correspondientes al composite con $\phi=0,322, \mathrm{~F} 1$, y al vidrio. Del mismo modo, para estos últimos (F1), la formación de la porosidad cerrada es más brusca: $\mathrm{n}$ y $\mathrm{m}$ son más altos que los composites $\mathrm{F} 2(\phi=0,525)$.

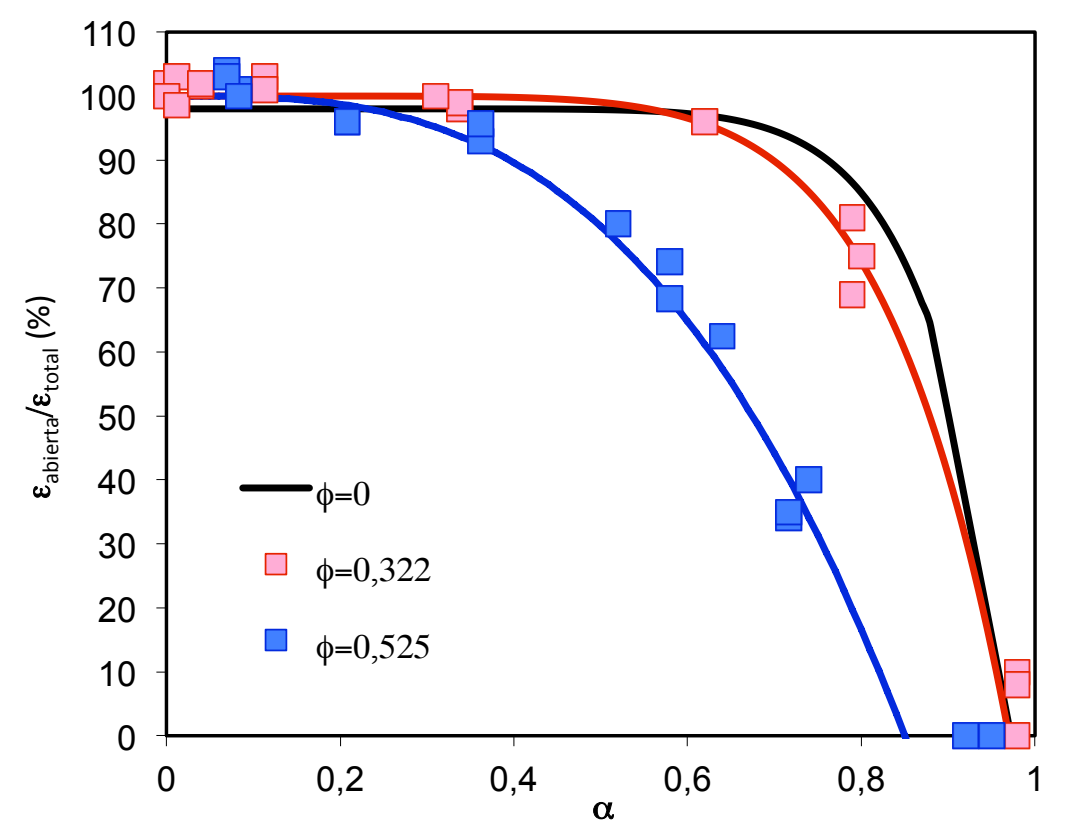

Figura 5.56. Variación $\varepsilon_{a b i e r t a} / \varepsilon_{\text {total }}$ con el grado de avance de la sinterización, $\alpha$, para probetas de vidrio $G M(\phi=0)$ y de los composites F1 $(\phi=0,322)$ y $F 2$ $(\phi=0,525)$. Tratamientos isotermos y no isotermos.

Estos resultados parecen indicar que el proceso de formación de poros cerrados, no solo depende del grado de avance del proceso de sinterización, sino también de la viscosidad de la matriz vítrea, dependiente de la temperatura del tratamiento térmico, de acuerdo con Eberstein [31]. En efecto, a temperaturas próximas a $800^{\circ} \mathrm{C}$, a una velocidad de calentamiento de $5 \mathrm{~K} / \mathrm{min}$, toda la porosidad del vidrio es prácticamente cerrada y a $875^{\circ} \mathrm{C}$, todos los poros ya son de un radio superior a las $10 \mu \mathrm{m}$ (Figura 5.17). En cambio, a $900^{\circ} \mathrm{C}$, el composite $\mathrm{F} 2, \phi=0,525$, sólo ha alcanzado un valor de $\alpha=0,22$ y en las zonas más vítreas ya se pueden apreciar algún poro cerrado 
de sección casi circular (Figura 5.58). Dicho efecto, la esfericidad de los poros, se hace más evidente a temperaturas más altas, aun cuando el grado de avance de la sinterización en algunas probetas no sea muy elevado (Figura 5.58 a Figura 5.60). Así pues, se confirma que mientras las condiciones del flujo macroscópico del material, dependientes del contenido en partículas rígidas, son las responsables de la densificación del composite, el flujo de material local, en las áreas vítreas, únicamente dependiente de la viscosidad del fundido, es solo función de la temperatura.

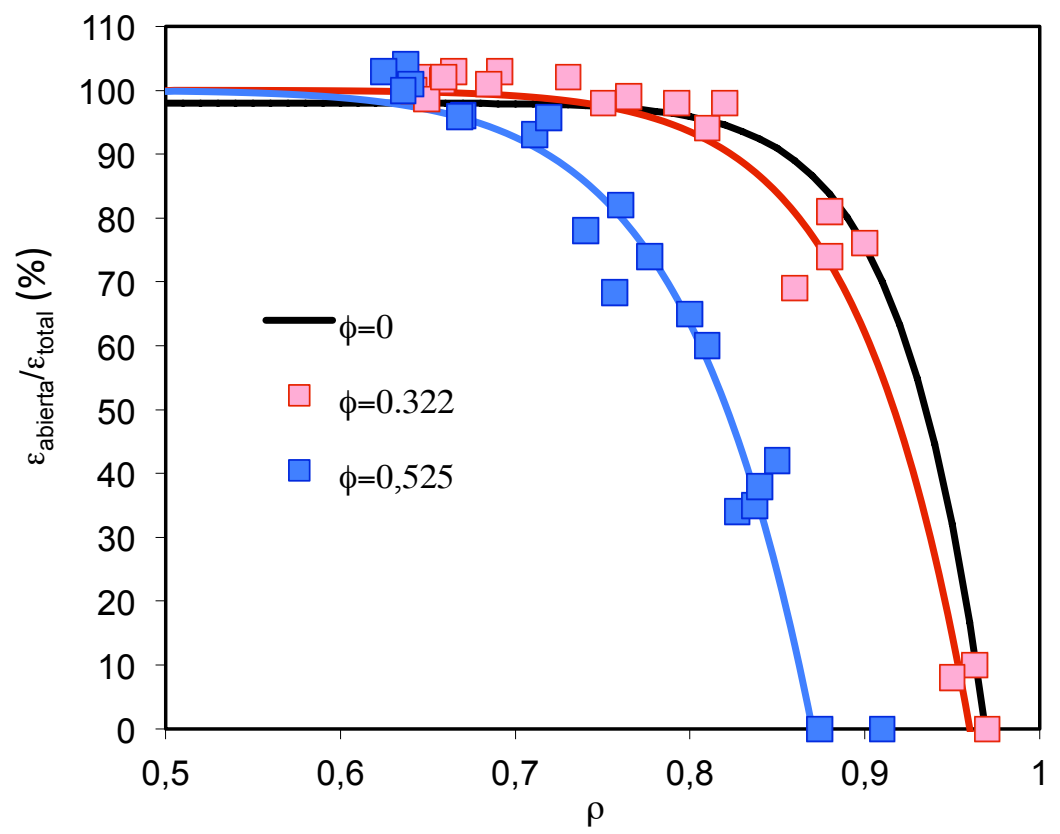

Figura 5.57. Variación $\varepsilon_{a b i e r t a} / \varepsilon_{\text {total }}$ con la compacidad, $\rho$, de las probetas cocidas para probetas de vidrio $G M(\phi=0)$ y de los composites $F 1 \quad(\phi=0,322)$ y $F 2$ $(\phi=0,525)$. Tratamientos isotermos y no isotermos. 

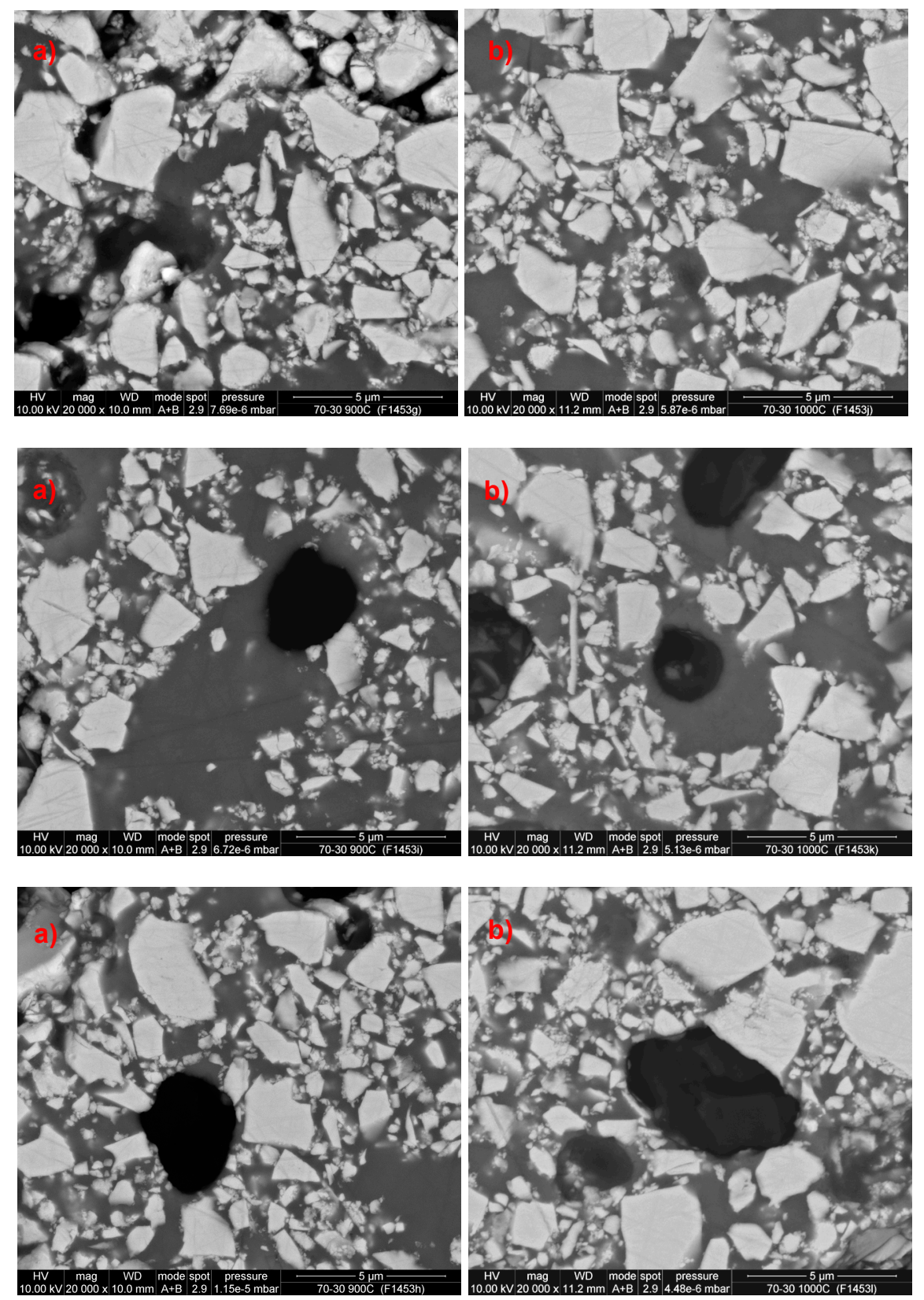

Figura 5.58. Micrografías $(x 20000)$ corresponidentes al composite $F 2(\phi=0,525)$ : a) cocido a $900^{\circ} \mathrm{C}(\alpha=0,22)$ (izquierda) y b) a $1000^{\circ} \mathrm{C}(\alpha=0,40)$ (derecha). Velocidad de calentamiento $\beta=5 \mathrm{~K} / \mathrm{min}$. 

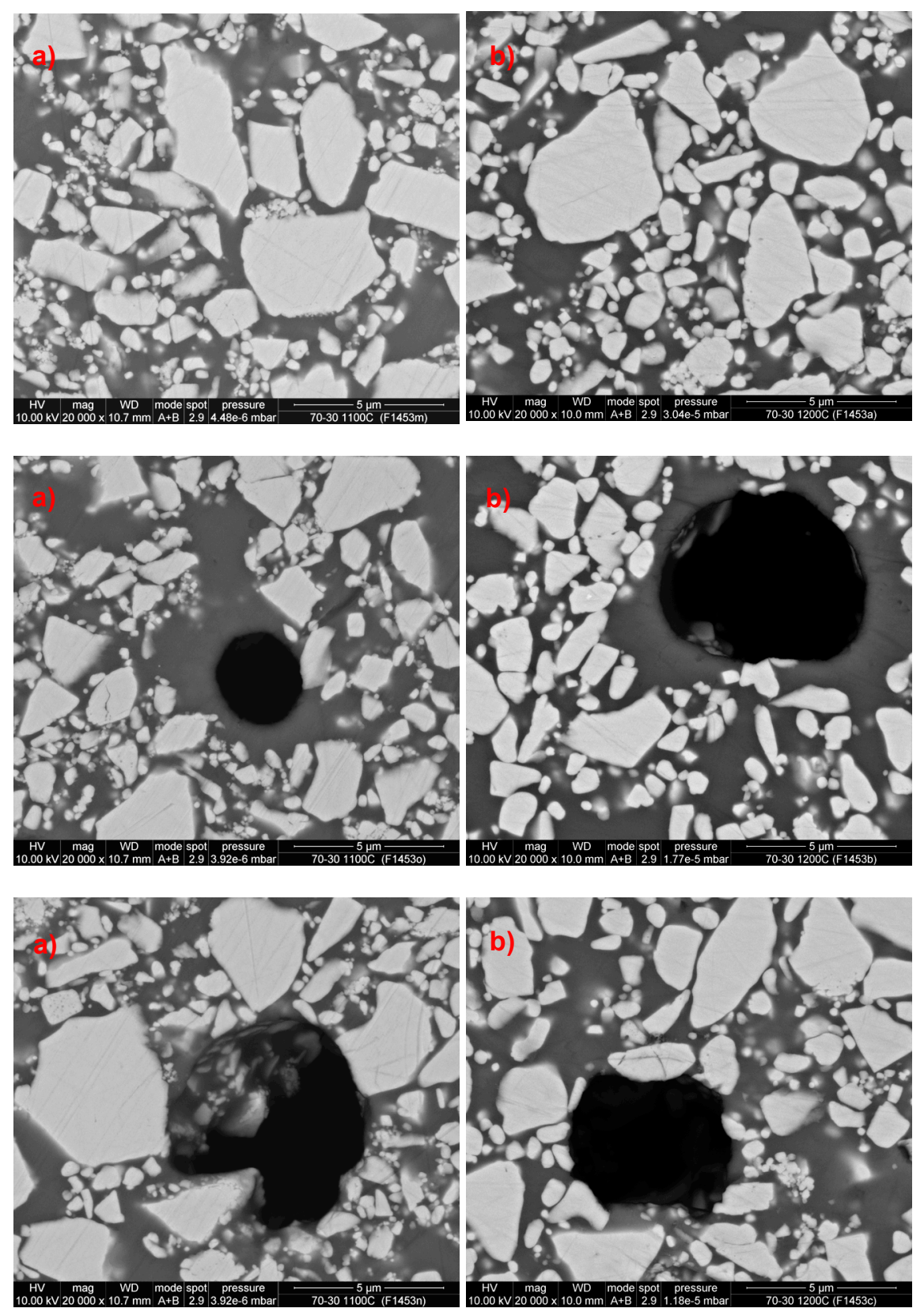

Figura 5.59. Micrografías (x20000) corresponidentes al composite $F 2(\phi=0,525)$ : a) cocido a $1100^{\circ} \mathrm{C}(\alpha=0,54)$ (izquierda) y b) a $1200^{\circ} \mathrm{C}(\alpha=0,73)$ (derecha). Velocidad de calentamiento $\beta=5 \mathrm{~K} / \mathrm{min}$. 

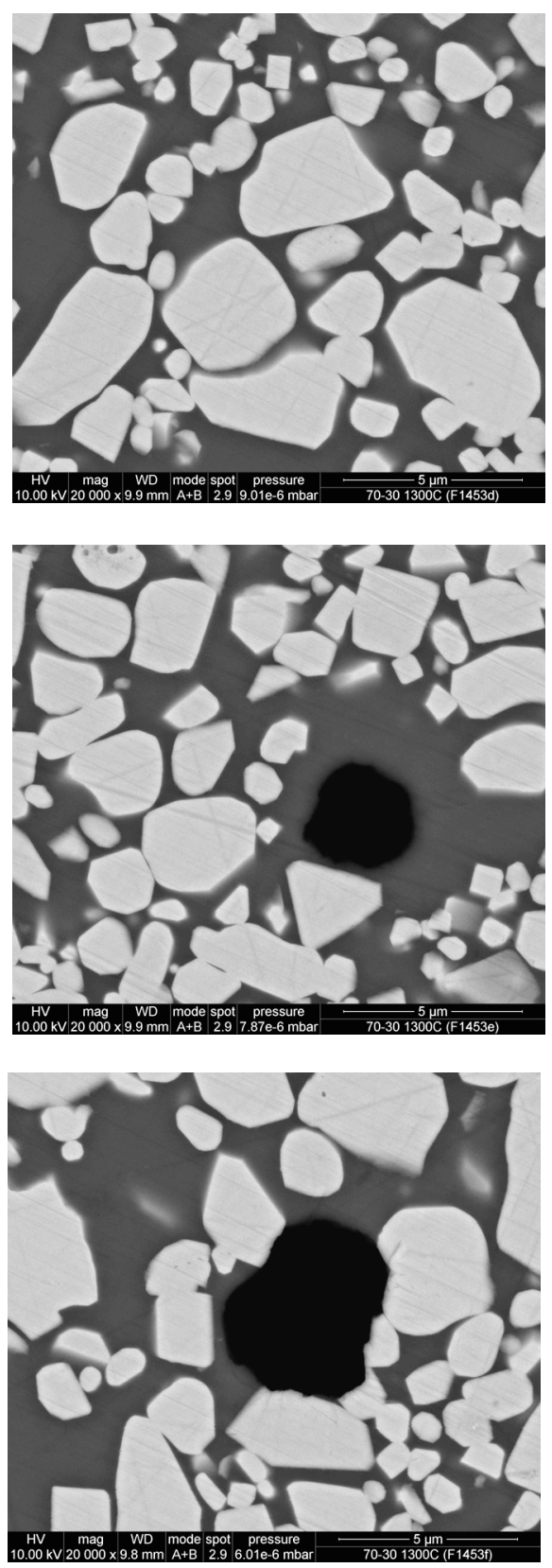

Figura 5.60. Micrografias (x20000) corresponidentes al composite $F 2(\phi=0,525)$ cocido a $1300^{\circ} \mathrm{C}(\alpha=0,92)$. Velocidad de calentamiento $\beta=5 \mathrm{~K} / \mathrm{min}$. 


\subsubsection{Solución-reprecipitación de granos de circón.}

5.2.1.3.1 Efecto de la temperatura sobre la cantidad, distribución de tamaños y forma del circón.

La distribución de tamaño de los granos de circón, DTG, se ha determinado siguiendo el procedimiento descrito en el apartado 4.2.6.3.2. Los resultados obtenidos para los composites F2 $(\phi=0,525)$ y F1 $(\phi=0,322)$, cocidos a una velocidad de calentamiento de $\beta=5 \mathrm{~K} / \mathrm{min}$ hasta distintas temperaturas (Figura 5.61) muestran que las DTG correspondientes a los composites obtenidos a temperaturas bajas $\left(900\right.$ y $\left.950^{\circ} \mathrm{C}\right)$, independientemente de la fracción volumétrica inicial de circón, son anchas y muy parecidas entre si. Dicho comportamiento se debe a la baja solubilidad del circón en un fundido de borosilicato alcalino, como se verá posteriormente. A temperaturas más altas (superiores a $1100^{\circ} \mathrm{C}$ ), a las que la solubilidad de las partículas más finas comienza a ser apreciable, el mecanismo de solución-reprecipitación empieza a ser importante, lo que se manifiesta en la eliminación progresiva de las partículas más pequeñas y el crecimiento de las intermedias y grandes (Figura 5.59 y Figura 5.60).

Estas DTG's obtenidas en 2D (Figura 5.61), como diámetro de círculo equivalente, Gc, se han transformado en DTG's 3D (Figura 5.62), utilizando la relación de German [124]:

$\mathrm{G}=1,27 \cdot \mathrm{Gc}$

ec. 5.23

De acuerdo con este mismo autor las últimas DTG's (Figura 5.62) se han ajustado a la función de distribución de Weibull:

$F(G)=1-\exp \left[-\ln 2\left(\frac{G}{G_{M}}\right)\right]^{M}$ ec. 5.24 
Siendo $F(G)$ la fracción acumulada de granos de tamaño menor que $G$ y los parámetros $G_{M}$ y $M$ el tamaño medio y el índice de uniformidad, respectivamente. Los resultados de los ajustes se detallan en la Tabla 5.11. Además, de la bondad de los ajustes se confirma un aumento del tamaño medio, $G_{M}$, y del índice de uniformidad, $M$, con el incremento de la temperatura, de tipo exponencial (Figura 5.63).

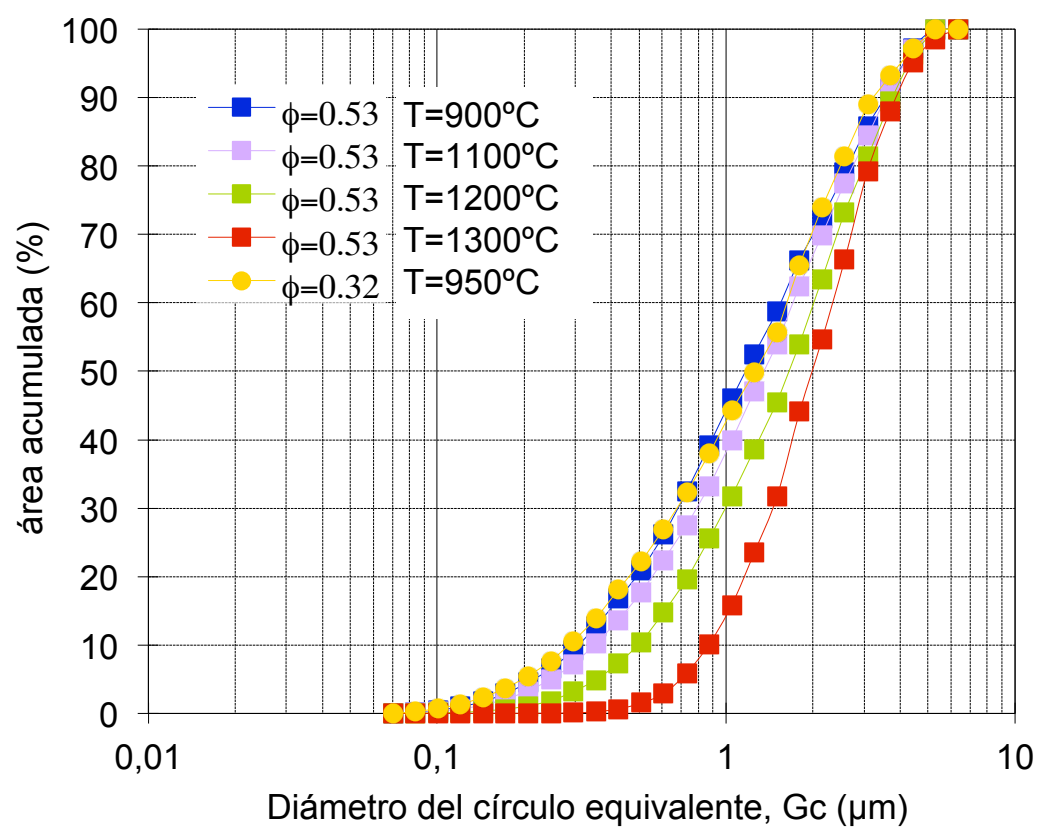

Figura 5.61. Evolución de la distribución del tamaño de grano de circón (diámetro del círculo equivalente, Gc) con la temperatura. Probetas $F 1(\phi=0,322)$ y F2 $(\phi=0,525)$. Velocidad de calentamiento $\beta=5 \mathrm{~K} / \mathrm{min}$. 
Tabla 5.11. Distribución del tamaño de grano del circón con la temperatura. Parámetros de la ecuación de Weibull (ec. 5.24).

\begin{tabular}{|c|c|c|c|c|c|}
\hline \multirow{2}{*}{ Parámetro } & \multicolumn{5}{|c|}{ Temperatura $\left({ }^{\circ} \mathrm{C}\right)$} \\
\cline { 2 - 6 } & 900 & 950 & 1100 & 1200 & 1300 \\
\hline $\mathrm{G}_{\mathrm{M}}(\mu \mathrm{m})$ & 1,40 & 1,45 & 1,60 & 1,90 & 2,40 \\
\hline $\mathrm{M}$ & 1,20 & 1,25 & 1,30 & 1,50 & 2,05 \\
\hline $\mathrm{S}^{2}$ & 0,000515 & 0,000265 & 0,000250 & 0,000213 & 0,000209 \\
\hline
\end{tabular}

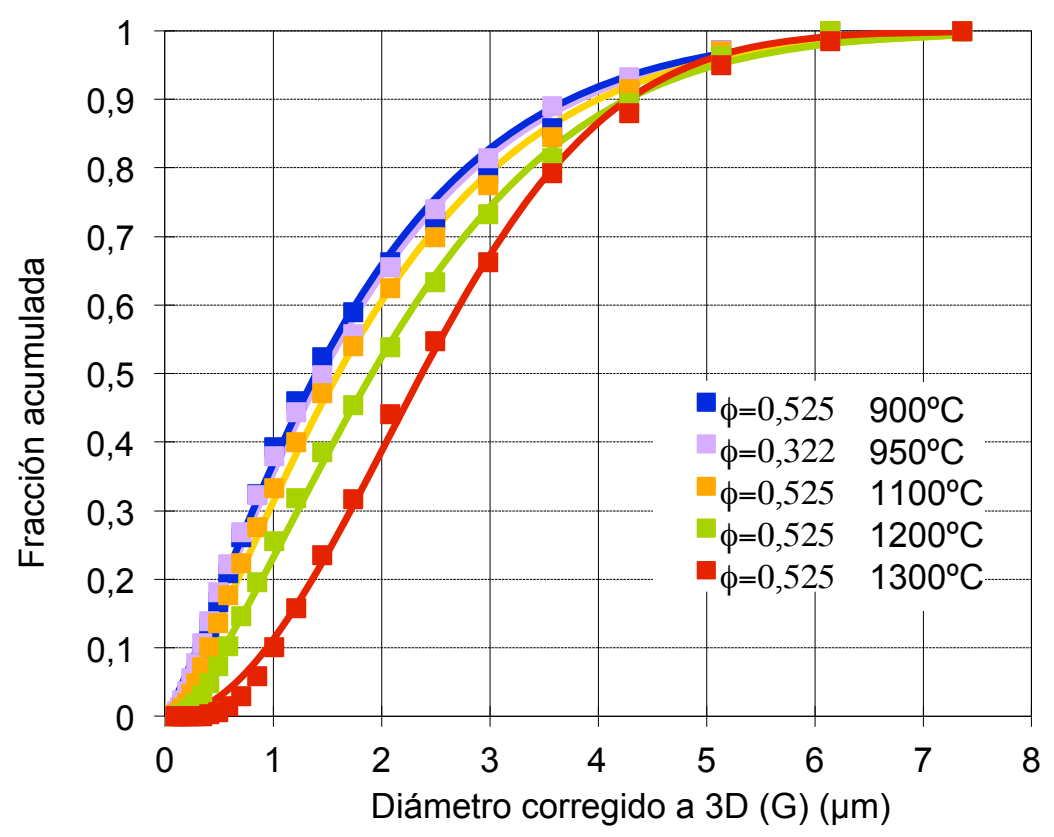

Figura 5.62. Evolución de la distribución de tamaño de grano del circón (diámetro corregido a $3 D$, ec. $5.23, G)$ con la temperatura. Probetas $F 1(\phi=0,322)$ y F2 $(\phi=0,525)$. Velocidad de calentamiento $\beta=5 \mathrm{~K} / \mathrm{min}$. Ajuste de los resultados a la ec. 5.24 .

Para tratar de calcular la energía de activación del proceso de crecimiento de grano a partir de los valores medios del tamaño de grano, $G_{M}$, se ha 
tenido en cuenta que en un experimento a velocidad de calentamiento constante, $\beta$, la ec. 2.7 del apartado 2.1 .3 .3 se transforma en:

$G_{M}^{3}-G_{M_{0}}^{3}=\frac{3 K_{S L}}{\beta} \int_{0}^{T} \exp \left(\frac{-Q_{G}}{R T}\right) d T$

Expresión que al integrarla, de acuerdo con el apartado 2.2.3.3, se convierte en:

$G_{M}^{3}-G_{M_{0}}^{3}=\frac{3 K_{S L} R T^{2}}{\beta Q_{G}} \exp \left(\frac{-Q_{G}}{R T}\right)$

siendo el inicial de $G_{M}, G_{M_{0}}$, el correspondiente a la temperatura de $900^{\circ} \mathrm{C}$.

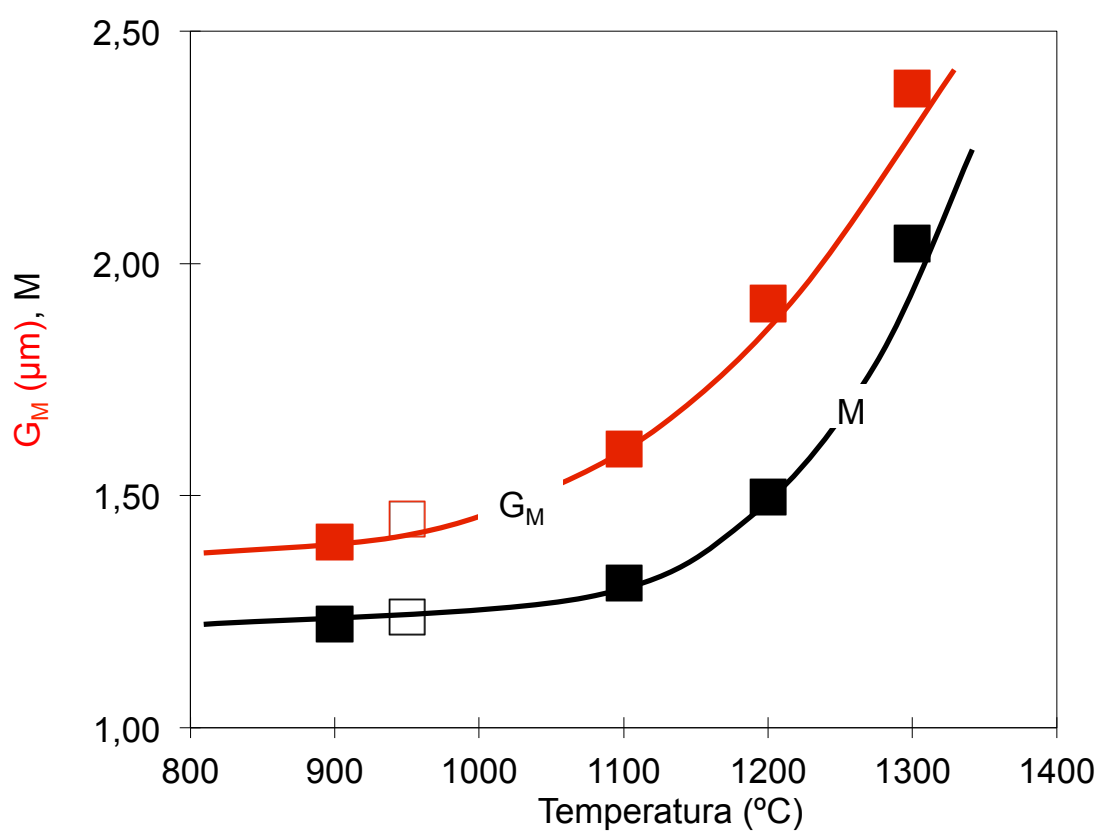

Figura 5.63. Evolución de los parámetros de Weibull, $G_{M}$ y $M$, de las distribuciones de tamaño de grano de circón con la temperatura (Tabla 5.11). Símbolos huecos, F1 $(\phi=0,322)$ y símbolos llenos $F 2(\phi=0,525)$. Velocidad de calentamiento $\beta=5 \mathrm{~K} / \mathrm{min}$. 
Al representar el logaritmo neperiano del primer miembro de la ec. 5.26 frente a la inversa de la temperatura absoluta se ha obtenido una muy buena recta, de la que resulta un valor de $Q_{G}=168 \pm 2,9 \mathrm{KJ} / \mathrm{mol}$ (Figura 5.64).

El efecto de la temperatura sobre la velocidad de crecimiento del grano, determinado como $Q_{G}$, está relacionada, según la ec. 2.6, con la influencia positiva que dicha variable ejerce sobre la solubilidad del circón en el líquido y la difusividad del soluto en el líquido, $D_{s}$. Por el contrario, la temperatura, también ejerce un efecto negativo sobre el crecimiento de grano al incrementar el contenido en fase líquida.

Así pues, el valor de $Q_{G}$ obtenido es consecuencia del efecto combinado que ejerce la temperatura sobre los tres factores antes citados. En la bibliografía no se han encontrado trabajos que traten sobre el efecto combinado de la temperatura sobre la solubilidad, $S$, y difusividad, $D_{S}$, del circón en fundido de composición parecida a las que se han estudiado. No obstante, la solubilidad del circón en fundidos magmáticos ha sido un tema recurrente, por lo que, aun cuando los valores que se van a manejar no son del todo apropiados, debido, principalmente, al alto contenido en boro del vidrio empleado, exento en líquidos magmáticos, en primera aproximación pueden considerarse aplicables. 


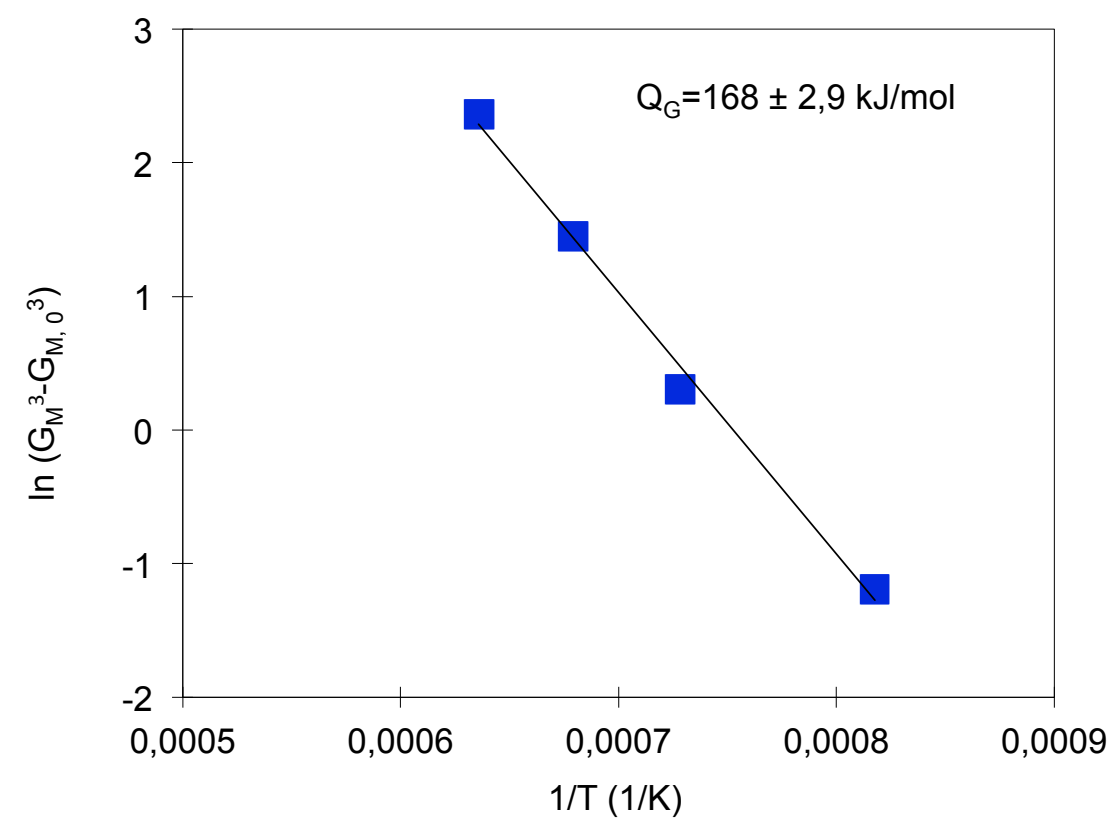

Figura 5.64. Determinación de la energía de activación del proceso de crecimiento de grano, $Q_{G}$. Probeta F2 $(\phi=0,525)$. Velocidad de calentamiento $\beta=5 \mathrm{~K} / \mathrm{min}$.

En lo referente a la S, para fundidos magmáticos, a partir del coeficiente de distribución de $\mathrm{Zr}$, entre el circón y el fundido, definido como $\mathrm{D}_{\mathrm{Zr}}=\frac{[\mathrm{Zr}]_{\text {circón }}}{[\mathrm{Zr}]_{\text {fundido }}}$, expresando cada concentración en p.p.m., se puede calcular la solubilidad como:

$\mathrm{S}(\mathrm{ppm})=\frac{[\mathrm{Zr}]_{\text {circón }}}{\mathrm{D}_{\mathrm{Zr}}}=\frac{5 \cdot 10^{5}}{\mathrm{D}_{\mathrm{Zr}}}$

Se ha tomado para $D_{z r}$ la relación propuesta más reciente [125]:

$\ln D_{\mathrm{Zr}}=\frac{10108 \pm 32}{\mathrm{~T}(\mathrm{~K})}-(1,16 \pm 0,16)\left(\mathrm{M}^{*}-1\right)-(1,48 \pm 0,09)$ 
Siendo $M^{*}=\left(\frac{N a+K+L i}{S i \cdot A l}\right)$, en donde cada elemento debe expresarse en atomos-kg en el vidrio.

Teniendo en cuenta que para nuestro vidrio $\mathrm{M}^{*}=2,45$ y la ec. 2.28 , la solubilidad, en tanto por ciento (\%), resulta:

$\ln S(\%)=-\frac{84}{R T}+2,44$

Así pues, con un valor de la energía de activación de $84 \mathrm{~kJ} / \mathrm{mol}$, resulta a $1200^{\circ} \mathrm{C}$ un valor de la solubilidad $\mathrm{S}=1,2 \%$.

En lo que respecta al coeficiente de difusión del circonio en fundidos, $D_{\mathrm{S}}$, solo se han localizado unos pocos trabajos sobre fundidos de composición próximas a las magmáticas graníticas [126][127]. Para estas composiciones más ricas en $\mathrm{Al}_{2} \mathrm{O}_{3}$ que el vidrio utilizado y exentas de $\mathrm{B}_{2} \mathrm{O}_{3}$, en el intervalo de temperatura comprendido entre $1000^{\circ} \mathrm{C}$ y $1400^{\circ} \mathrm{C}$, se obtienen coeficientes de difusión, $D_{S}$, que varían hasta dos órdenes de magnitud, $10^{-13,5}<D_{\mathrm{s}}<10^{-11,5} \mathrm{~m}^{2} / \mathrm{s}$, con valores de la energía de activación también variables, comprendidos entre 140 y $250 \mathrm{~kJ} / \mathrm{mol}$.

Si se considera que la energía superficial sólido-líquido, $\gamma_{\mathrm{SL}}$, disminuye con la temperatura al igual que el contenido en sólidos, lo que dificulta el crecimiento cristalino, y que, por contra, $D_{S}$ y $S$ aumentan con la temperatura, el valor de la energía aparente de activación obtenida por el crecimiento cristalino es consistente con los escasos resultados que figuran en la bibliografía.

La variación con la temperatura de la fracción másica de circón (Figura 5.65), determinada por $\mathrm{DRX}$, para los composites $\mathrm{F} 2(\phi=0,525)$ y $\mathrm{F} 1$ $(\phi=0,322)$, muestra que solo a temperaturas superiores a $1100^{\circ} \mathrm{C}$ comienza a apreciarse una disminución de cristales de circón por disolución en el 
fundido, debido al aumento de su solubilidad con la temperatura, como se acaba de analizar. A $1300^{\circ} \mathrm{C}$, esta última ya es significativa.

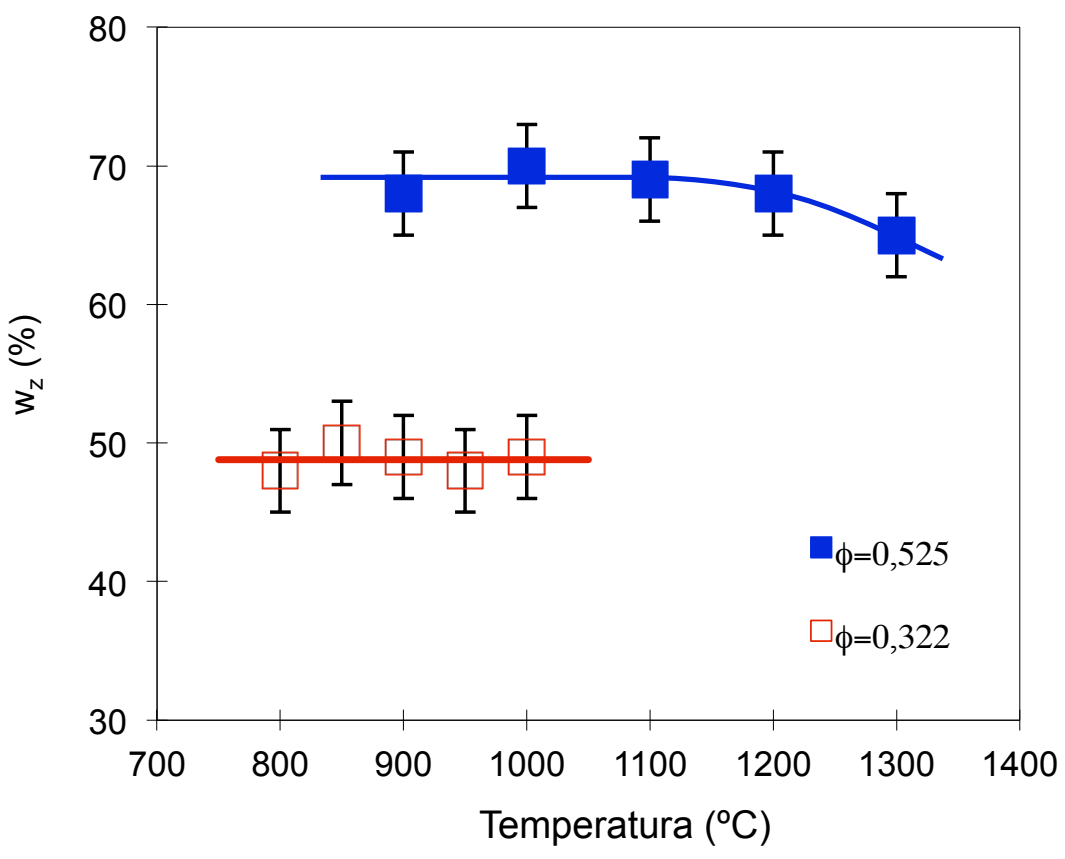

Figura 5.65. Variación de la fracción másica de circón, $W$, con la temperatura de cocción, para los composites $F 1(\phi=0,322)$ y $F 2(\phi=0,525)$. Velocidad de calentamiento $\beta=5 \mathrm{~K} / \mathrm{min}$.

A partir de los resultados de la difracción de rayos $\mathrm{X}$, mediante el método descrito en el apartado 4.2.6.3.4, se ha calculado el tamaño de cristalito, C, para el silicato de circonio micronizado $(F)$ y para distintos composites de la serie $\mathrm{F}$ obtenidos con diferentes valores de $\phi$ y a diferentes temperaturas máximas de cocción. En la Figura 5.66 se detallan los resultados. De su examen se comprueba que el aumento del tamaño de cristalito, C, con la temperatura, es prácticamente independiente del contenido en circón y sigue una variación paralela a la del tamaño de grano obtenida por MEB-Análisis de Imagen. Además, se aprecia que únicamente a temperaturas superiores a los $900^{\circ} \mathrm{C}$, los fenómenos de transporte de materia comienzan a ser efectivos. En efecto, a temperaturas inferiores a los $900^{\circ} \mathrm{C}$, el tamaño de 
cristalito es prácticamente independiente de la temperatura y coincidente con el del silicato de circonio micronizado $(F)$ sin tratar térmicamente.

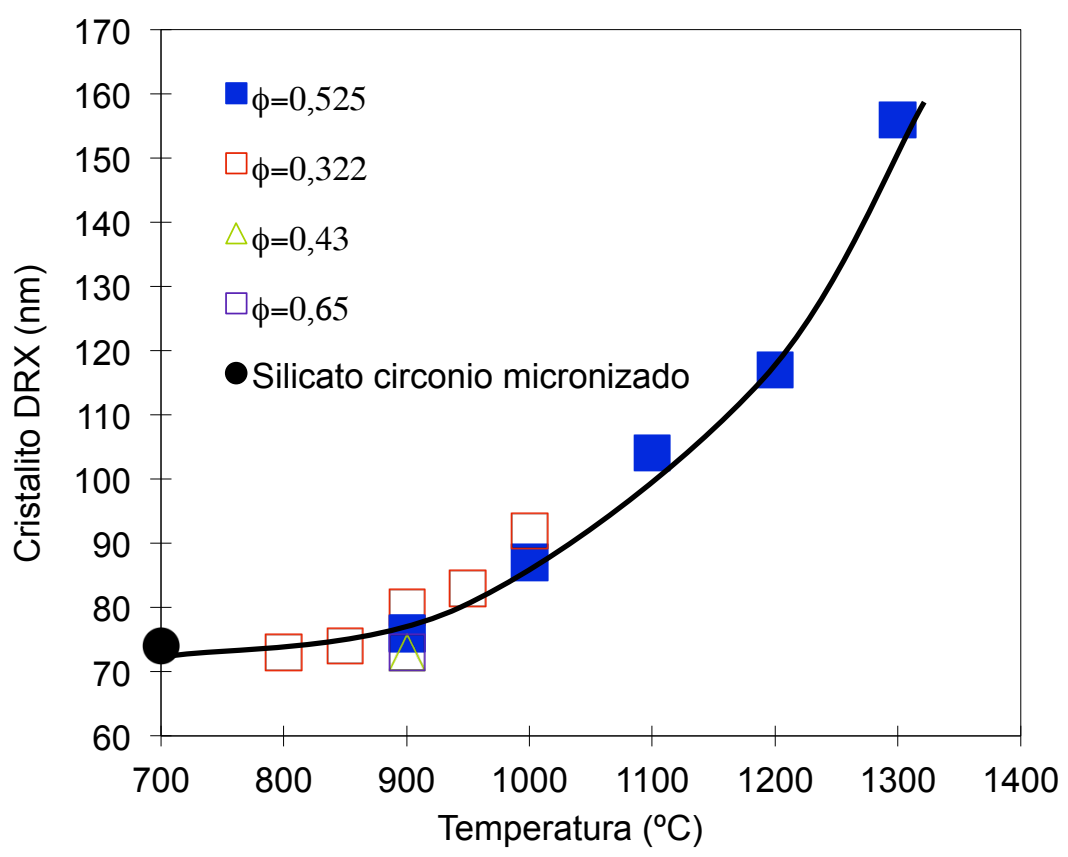

Figura 5.66. Variación del tamaño de cristalito, C, con la temperatura de cocción para distintos composites de la serie $F$ y para el silicato de circonio micronizado (F) sin tratar térmicamente. Velocidad de calentamiento $\beta=5 \mathrm{~K} / \mathrm{min}$.

A la vista de estos resultados (Figura 5.66) se ha considerado que el crecimiento del tamaño de cristalito sigue la misma cinética que el crecimiento de grano y se ha calculado, siguiendo el mismo procedimiento, la energía aparente de activación del proceso, Q (Figura 5.67). Los resultados, aunque dispersos, se ajustan a una recta de Arrhenius, obteniendo un valor para $Q_{C}=157 \pm 15 \mathrm{KJ} / \mathrm{mol}$. La gran similiutd existente entre $Q_{G}=168 \pm 2,9 \mathrm{KJ} / \mathrm{mol}$ y de $Q_{C}$, confirma que ambos fenómenos están controlados por las mismas propiedades: una termodinámica, S, y la otra de transporte, $D_{S}$. 


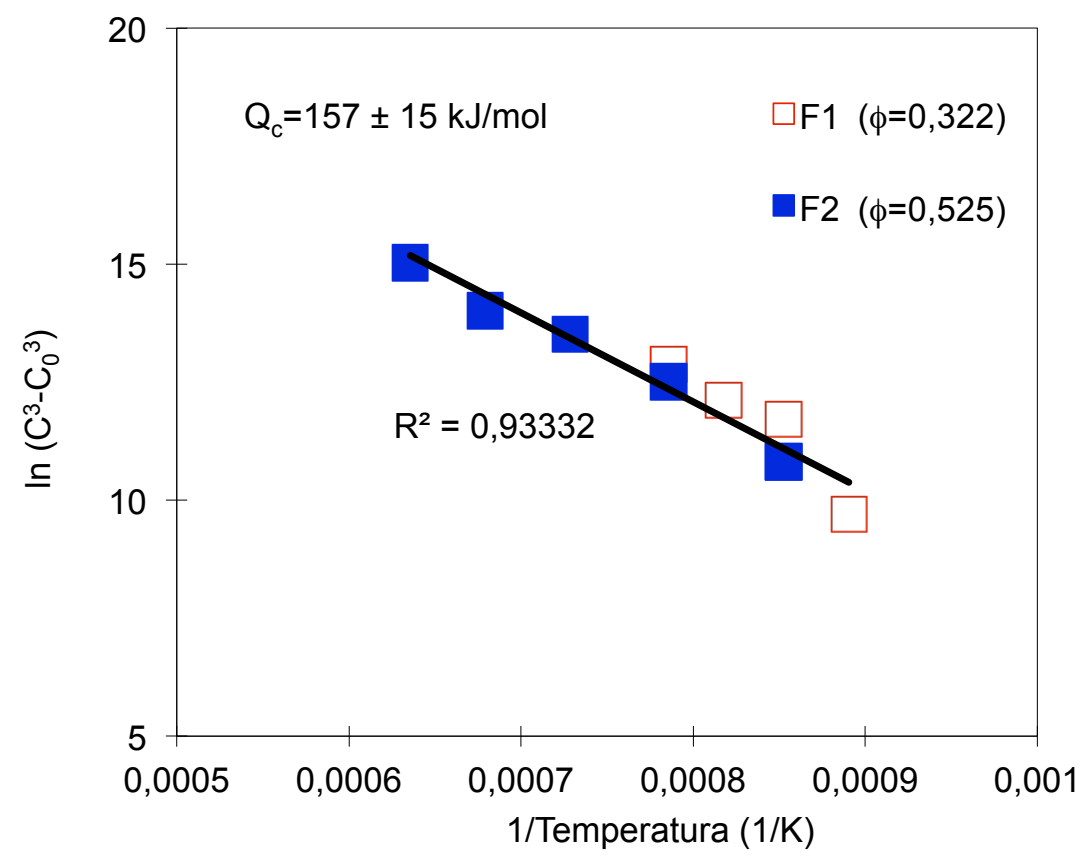

Figura 5.67. Determinación de la energía de activación del proceso de crecimiento del cristalito, $Q_{c}$. Probetas F1 $(\phi=0,322)$ y F2 $(\phi=0,525)$. Velocidad de calentamiento $\beta=5 \mathrm{~K} / \mathrm{min}$.

Paralelamente al crecimiento del tamaño medio de grano, asociado principalmente a la eliminación de los más pequeños, los granos que crecen van cambiando su morfología, especialmente a temperaturas superiores a $1100^{\circ} \mathrm{C}$ (Figura 5.59 y Figura 5.60 ).

En general, las partículas más pequeñas se van redondeando (Figura 5.59), excepto a la temperatura máxima ensayada (Figura 5.60), en la que algunos su sección es rectangular o cuadrada, lo que podría indicar que la reacción interfacial es la etapa controlante del proceso. Para las granos intermedios y grandes se aprecia que, excepto en aquellos casos en los que los granos adaptan su forma para mejorar el empaquetamiento o están formando poros, el perfil del perímetro suele ser liso y la curvatura de su superficie convexa. 
5.2.1.3.2 Efecto de la temperatura sobre la zona de difusión circón-vidrio.

Para estudiar el tamaño de la zona de difusión circón-vidrio, se ha determinado la variación del número de cuentas de circonio con la distancia, en las proximidades de un grano de circón, mediante EDS, siguiendo el procedimiento descrito en el apartado 4.2.6.3.3.

Se ha comprobado que a $1000^{\circ} \mathrm{C}$ la capa de difusión es prácticamente inexistente al igual que el porcentaje de circonio en la fase vítrea. Los resultados a $1300^{\circ} \mathrm{C}$ indican que la disolución en el vidrio es considerable confirmando los resultados obtenidos por los otros procedimientos.

En la Figura 5.58 se ha representado el gradiente del número de cuentas de circonio, $\frac{d Z r}{d x}$, con la distancia, $x$, para el composite $F 2(\phi=0,525)$ calentado hasta $1000^{\circ} \mathrm{C}$ y $1300^{\circ} \mathrm{C}$, a una velocidad de $\beta=5 \mathrm{~K} / \mathrm{min}$.

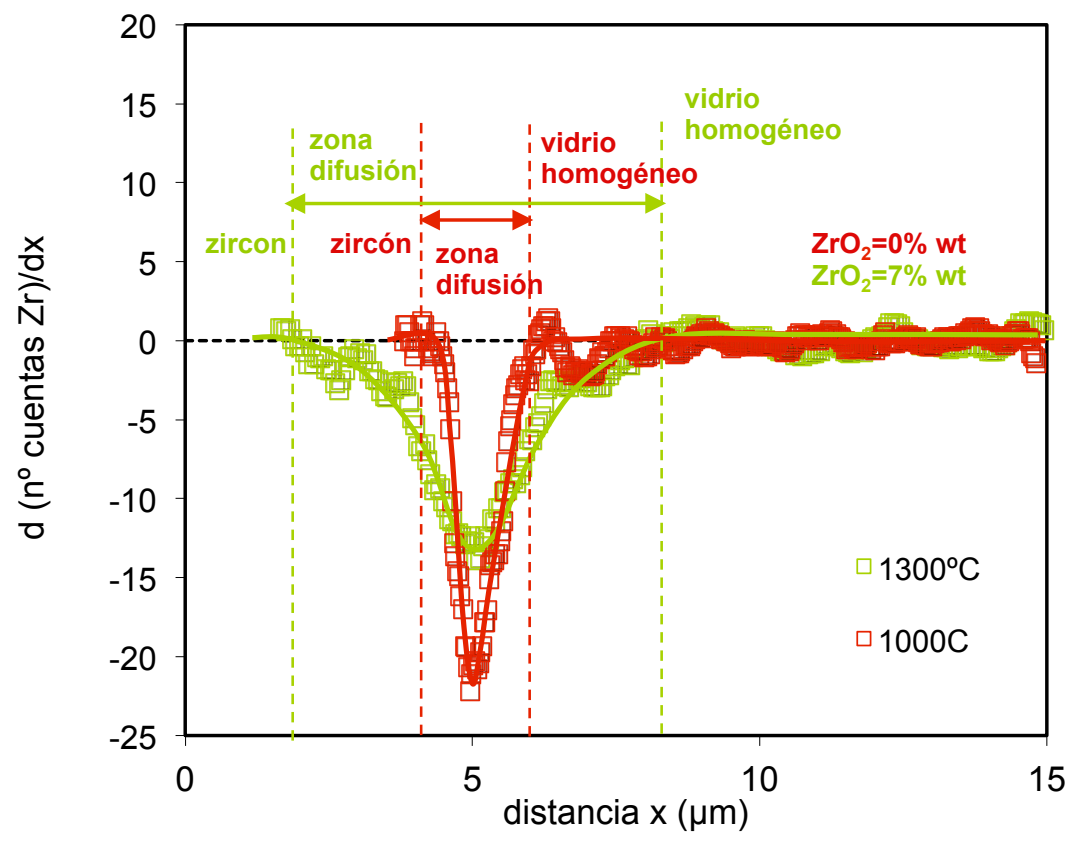

Figura 5.68. Efecto de la temperatura sobre la zona de difusión: circón-matriz vítrea. Composite $F 2(\phi=0,525)$. Velocidad de calentamiento $\beta=5 \mathrm{~K} / \mathrm{min}$. 
5.2.1.3.3 Curvas de sinterización no isoterma. Efecto del proceso soluciónreprecipitación.

Para el composite F2 $(\phi=0,525)$ se observó, en todas las curvas de sinterización, independientemente de la velocidad de calentamiento (Figura 5.75), una ligera inflexión que parece relacionarse con un cambio del mecanismo de sinterización.

Al diferenciar la curva de $\alpha$ vs T correspondiente a $\beta=5 \mathrm{~K} / \mathrm{min}$ se obtiene la curva velocidad de sinterización, $\frac{d \alpha}{d t}$, frente a la temperatura (Figura 5.69) en la que se aprecian claramente dos tramos convexos con sus respectivos máximos, parcialmente solapados, asociados a dos mecanismos de sinterización. La curva correspondiente al intervalo de temperaturas más bajo se asocia al proceso de reordenación de partículas por flujo viscoso. La que se presenta a temperaturas más altas se asocia a la densificación por el proceso de solución-reprecipitación.

Esta hipótesis se confirma, por una parte, al superponer la curva que sigue el tamaño medio de grano de circón con la temperatura (Figura 5.69). En efecto, a temperaturas inferiores a $900^{\circ} \mathrm{C}$ la solubilidad del circón y, en consecuencia, el crecimiento del grano son despreciables por lo que el único proceso de densificación es la reordenación de partículas por flujo viscoso. Por el contrario, a temperaturas superiores a los $900^{\circ} \mathrm{C}$, a los que la solubilidad del circón comienza a ser apreciable, el proceso de soluciónreprecipitación ya es operativo, lo que se manifiesta con un crecimiento del tamaño de grano.

Por otra parte, como ya se indicado en el apartado 5.2.1.3.1, paralelamente al crecimiento del tamaño de grano, a temperaturas superiores a los $900^{\circ} \mathrm{C}$, los cambios microestructurales observados confirman la densificación por el mecanismo de solución-reprecipitación. 


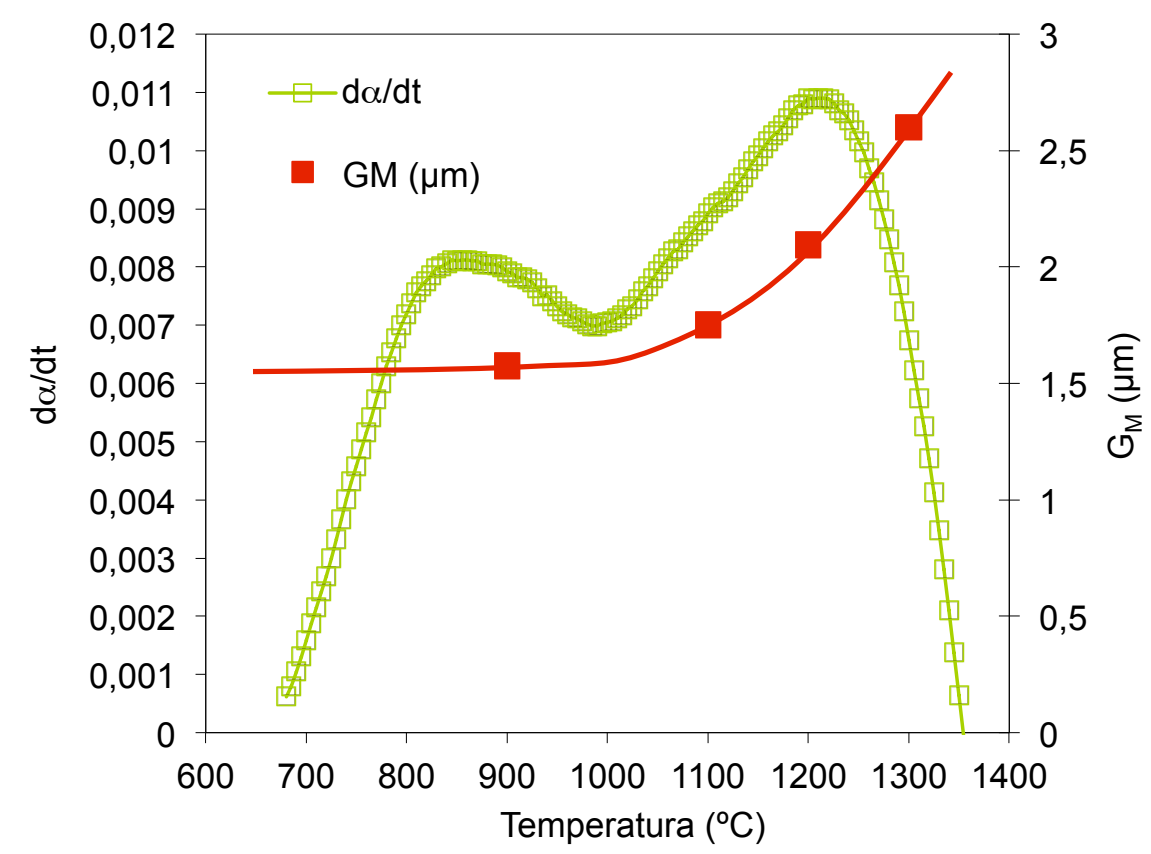

Figura 5.69. Variación de la velocidad de sinterización, $d \alpha / d t$, y del tamaño medio del grano de circón, $G_{M}$, con la temperatura, para el composite $F 2(\phi=0,525)$. Velocidad de calentamiento $\beta=5 \mathrm{~K} / \mathrm{min}$.

\subsubsection{Análisis cinético de la sinterización. Determinación de parámetros cinéticos.}

\subsubsection{Experimentos a velocidad de calentamiento constante.}

Para cada uno de los composites de la serie $F$ se han determinado sus curvas de sinterización a 5 velocidades de calentamiento: $\beta=2,5,15,25$ y 60 $\mathrm{K} / \mathrm{min}$. Para los composites con $\phi \leq 0,43$ (Figura 5.70 a Figura 5.74) las curvas son claramente sigmoidales por lo que, en principio, pueden ser descritas mediante modelos de una sola etapa. Por el contrario, para el composite F2 $(\phi=0,525)$ (Figura 5.75) se requiere al menos dos etapas para describir la cinética del proceso. 


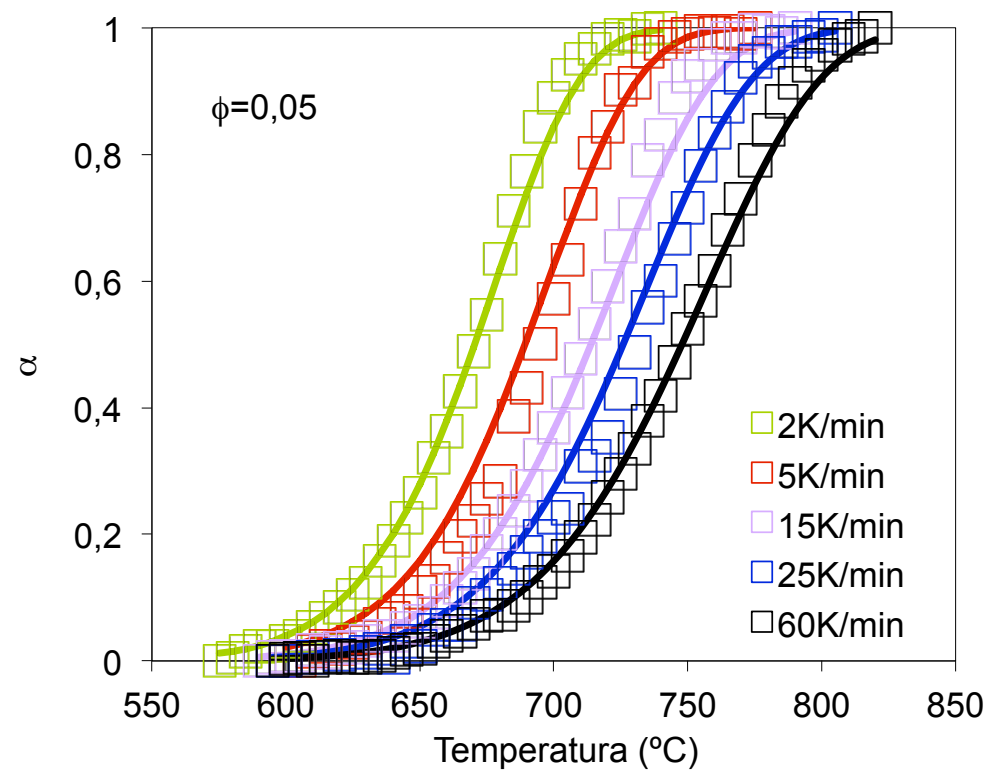

Figura 5.70. Curvas de sinterización a velocidades de calentamiento: $\beta=2,5,15,25$ y $60 \mathrm{~K} / \mathrm{min}$, correspondientes al composite de la serie $F$ con $\phi=0,05$. Comparación entre los resultados experimentales y los calculados mediante la ec. 5.12 y la Tabla 5.12.

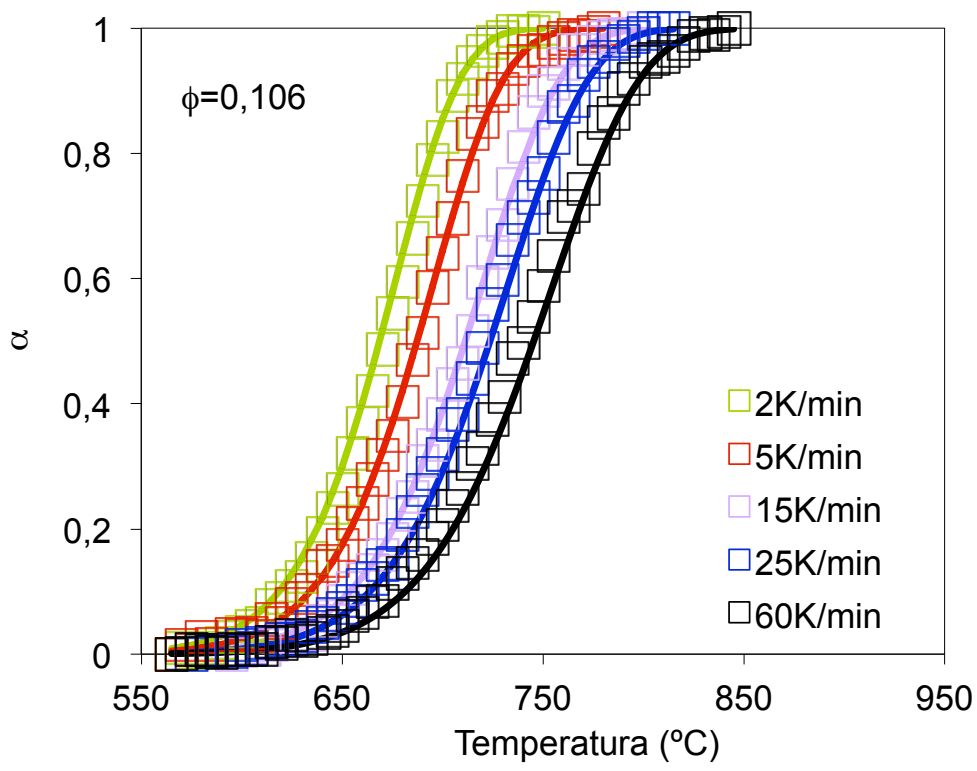

Figura 5.71. Curvas de sinterización a velocidades de calentamiento: $\beta=2,5,15,25$ y $60 \mathrm{~K} / \mathrm{min}$, correspondientes al composite de la serie $F$ con $\phi=0,106$. Comparación entre los resultados experimentales y los calculados mediante la ec. 5.12 y la Tabla 5.12. 


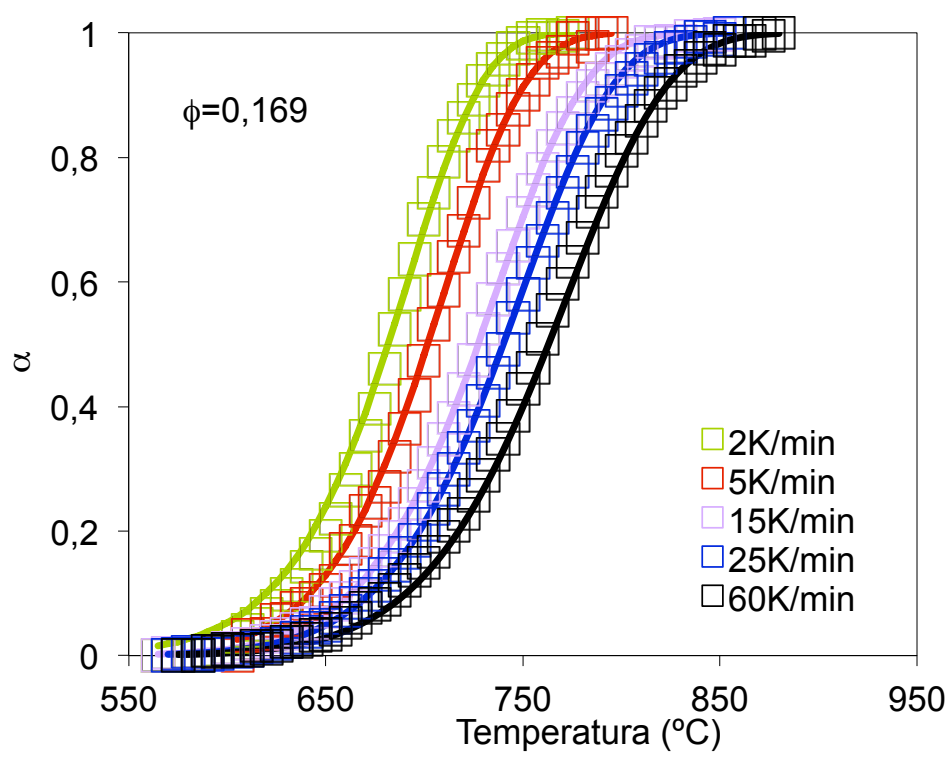

Figura 5.72. Curvas de sinterización a velocidades de calentamiento: $\beta=2,5,15,25$ y $60 \mathrm{~K} / \mathrm{min}$, correspondientes al composite de la serie $F$ con $\phi=0,169$. Comparación entre los resultados experimentales y los calculados mediante la ec. 5.12 y la Tabla 5.12.

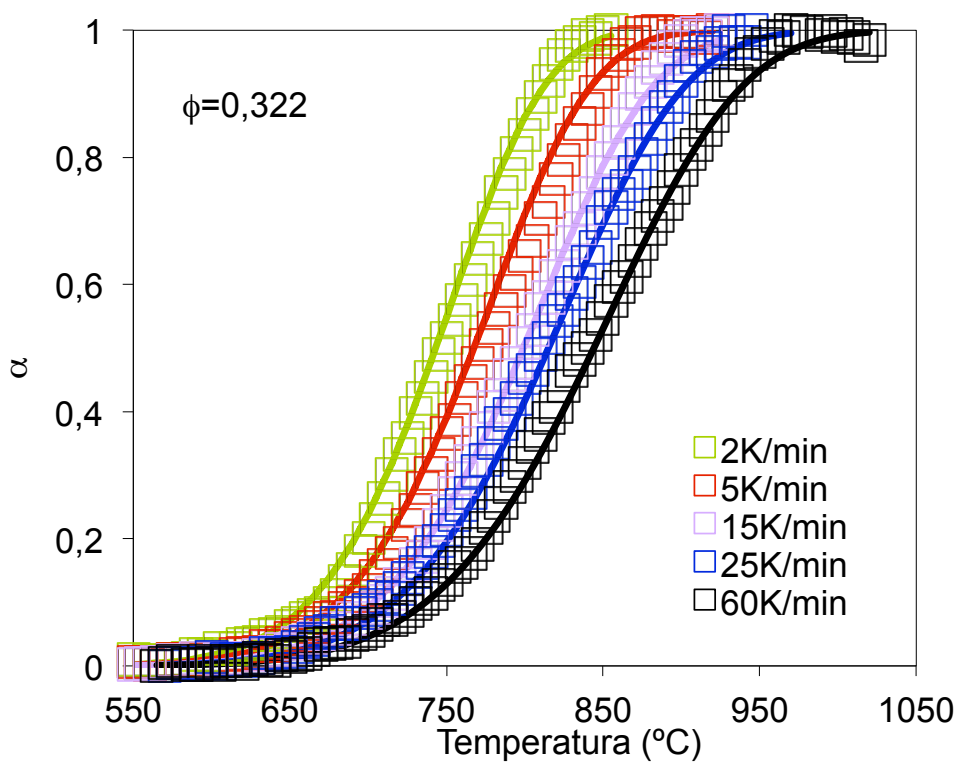

Figura 5.73. Curvas de sinterización a velocidades de calentamiento: $\beta=2,5,15,25$ y $60 \mathrm{~K} / \mathrm{min}$, correspondientes al composite de la serie $F$ con $\phi=0,322$. Comparación entre los resultados experimentales y los calculados mediante la ec. 5.12 y la Tabla 5.12. 


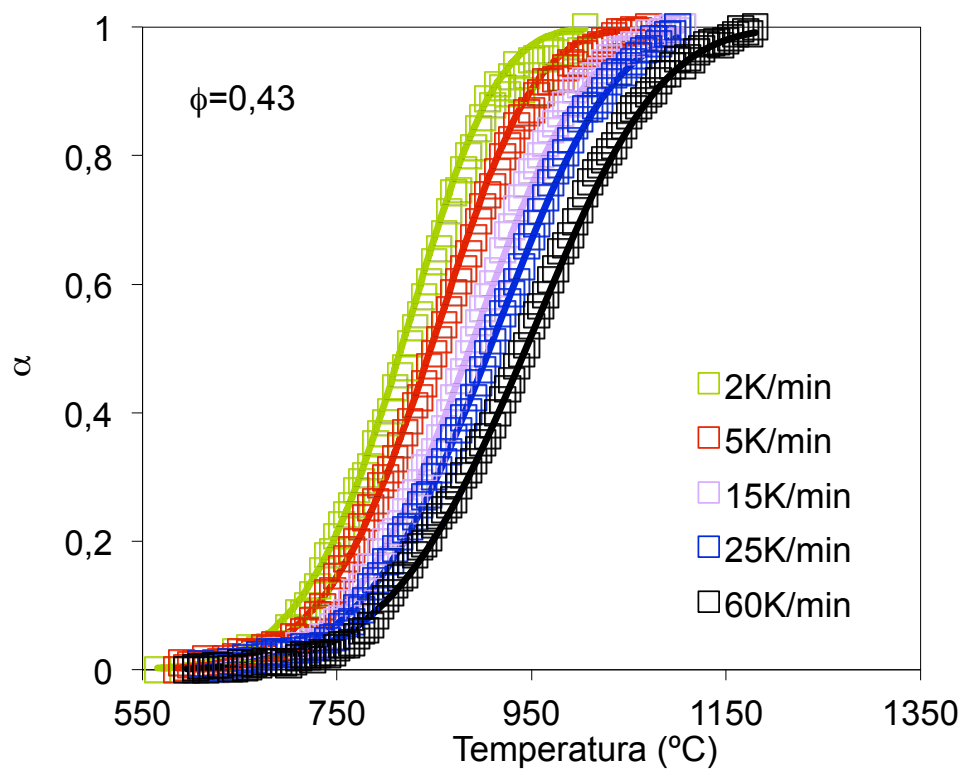

Figura 5.74. Curvas de sinterización a velocidades de calentamiento: $\beta=2,5,15,25$ y $60 \mathrm{~K} / \mathrm{min}$, correspondientes al composite de la serie $F$ con $\phi=0,43$. Comparación entre los resultados experimentales y los calculados mediante la ec. 5.12 y la Tabla 5.12 .

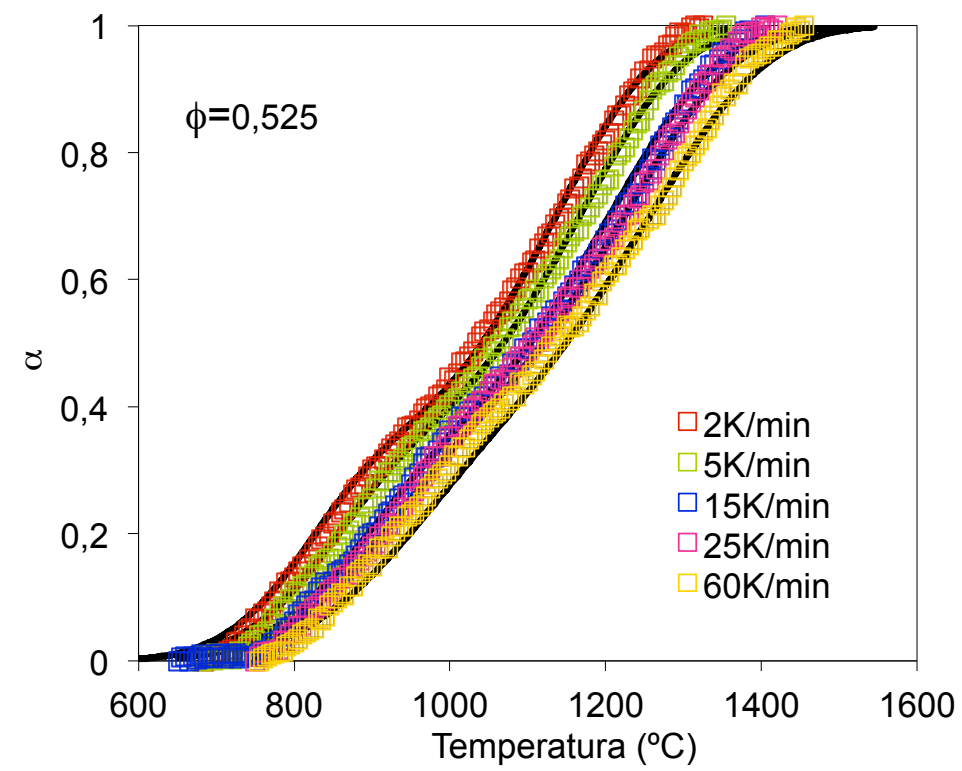

Figura 5.75. Curvas de sinterización a velocidades de calentamiento: $\beta=2,5,15,25$ y $60 \mathrm{~K} / \mathrm{min}$, correspondientes al composite de la serie $F$ con $\phi=0,525$. Comparación entre los resultados experimentales y los calculados mediante el modelo desarrollado en el apartado 5.2.2.1.2.2. 
5.2.2.1.1 Determinación de la energía de activación por el método de Friedman (apartado 2.2.3.2).

En el apartado 5.1.2.1.1, para compactos de vidrio se comprobó que los valores de la energía de activación de la sinterización, $\mathrm{E}_{\alpha}$, calculados con el método de Friedman, para cualquier valor de $\alpha$, eran más parecidos a los de flujo viscoso, $E_{\eta}$, calculados a partir de la curva de viscosidad, que los que se obtienen por el método de K.A.S. Además, también se apreció una disminución continua de los valores de $E_{\alpha}$ y $E_{\eta}$ con $\alpha$. Lo propio se ha comprobado para algunos composites con $\alpha \leq 0,43$.

En consecuencia, siguiendo el mismo procedimiento que el empleado en el apartado 5.1.2.1.1 se han calculado, para todos los composites, los valores de $E_{\alpha}$ y $E_{\eta}$ para diferentes valores de $\alpha$. Los resultados para valores de $\alpha \leq 0,43$ presentaban la misma tendencia que la que se observó para el vidrio GM (Figura 5.20).

A partir de estos valores individuales de $E_{\alpha}$ y $E_{\eta}$, para cada uno de los composites, se ha calculado su valor medio $\overline{E_{\alpha}}$ y $\overline{E_{\eta}}$. Los resultados para valores de $\alpha \leq 0,43$ se representan frente a $\phi$ en la Figura 5.76. Se comprueba una disminución progresiva y continua de $\bar{E}_{\alpha}$ y $\bar{E}_{\eta}$ con el aumento del contenido en circón, $\phi$. Esto se debe a que con el incremento de dicha variable, $\phi$, las curvas de sinterización se desplazan hacia intervalos de temperatura cada vez mayores, lo que debido a la relación $\eta$ vs $\mathrm{T}$ implica una disminución de $\overline{\mathrm{E}}_{\alpha}$ y $\overline{\mathrm{E}}_{\eta}$ con el aumento de $\mathrm{T} \mathrm{y}$, por consiguiente, con $\alpha$. Para los composites con $\alpha \leq 0,43$, el valor medio de $\mathrm{E}_{\alpha}, \overline{\mathrm{E}}_{\alpha}$, y su dispersion son un poco mayores que los correspondientes a 
$E_{\eta}$. De forma general, los valores de $\overline{E_{\alpha}}$ suelen ser de alrededor del $10 \%$ más altos que los de $\bar{E}_{\eta}$ (Figura 5.77).

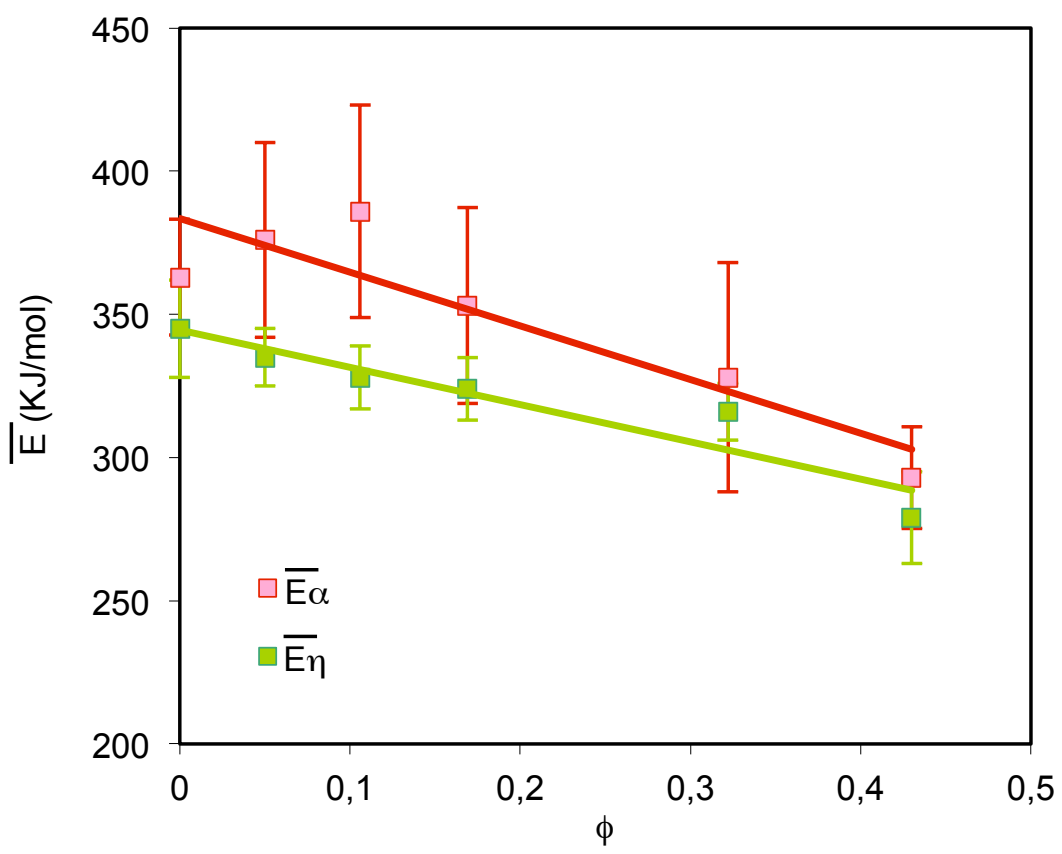

Figura 5.76. Variación de los valores promedio de las energías de activación de sinterización, $\overline{\mathrm{E}}_{\alpha}$, y de flujo viscoso, $\overline{\mathrm{E}}_{\eta}$, con la fracción volumétrica de circón, $\phi$. Composites de la serie $F, \phi \leq 0,43$. Experimentos a velocidad de calentamiento $\beta=2,5,15,25$ y $60 \mathrm{~K} / \mathrm{min}$. 


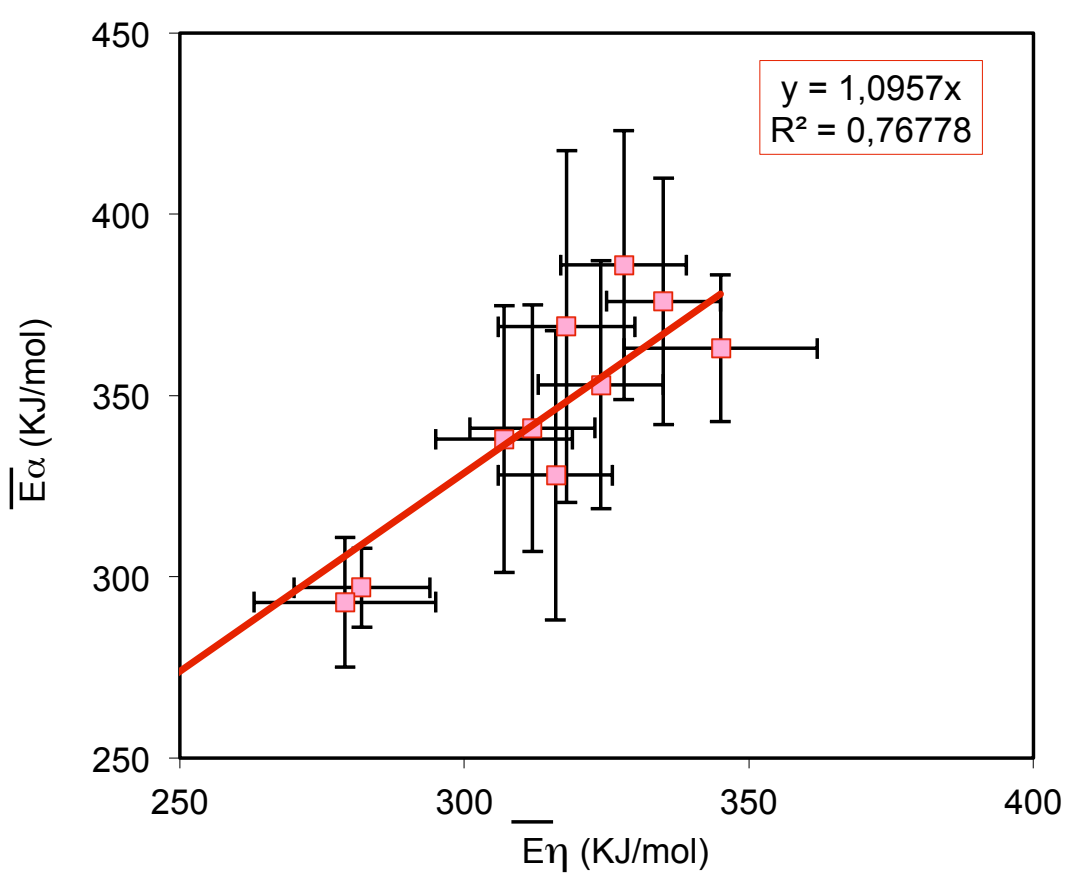

Figura 5.77. Relación entre los valores promedio de la energía de activación de sinterización, $\overline{\mathrm{E}}_{\alpha}$, y los de flujo viscoso, $\overline{\mathrm{E}}_{\eta}$. Composites de la serie $F$, $\phi \leq 0,43$. Experimentos a velocidad de calentamiento $\beta=2,5,15,25$ y 60 $\mathrm{K} / \mathrm{min}$.

Estos resultados vienen a confirmar que para composites vidrio-circón, con valores moderados de este último, $\phi \leq 0,43, y$ con las distribuciones granulométricas empleadas, la densificación es un proceso controlado exclusivamente por un mecanismo de flujo viscoso, en el que el efecto de la temperatura sobre la velocidad del proceso, $\mathrm{E}_{\alpha}$, es el debido al efecto de esta misma variable sobre la viscosidad del vidrio, $\mathrm{E}_{\eta}$.

Por otra parte, para el composite con F2 $(\phi=0,525)$, al representar los valores de $\mathrm{E}_{\alpha}$ frente a $\alpha$ se obtienen claramente dos tramos (Figura 5.78): uno a bajos valores de $\alpha, \alpha \leq 0,30$, con prácticamente un valor de $\overline{E_{\alpha}}$ próximo a los $370 \mathrm{~kJ} / \mathrm{mol}$, ligeramente más alto que $\overline{\mathrm{E}}_{\eta}$. El otro tramo se 
presenta a valores de $\alpha \geq 0,30$, con un valor de $\overline{E_{\alpha_{2}}} \approx 500 \mathrm{~kJ} / \mathrm{mol}$, muy superiores a $\overline{E_{\eta}}\left(\overline{E_{\alpha}} \approx 2 \bar{E}_{\eta}\right)$.

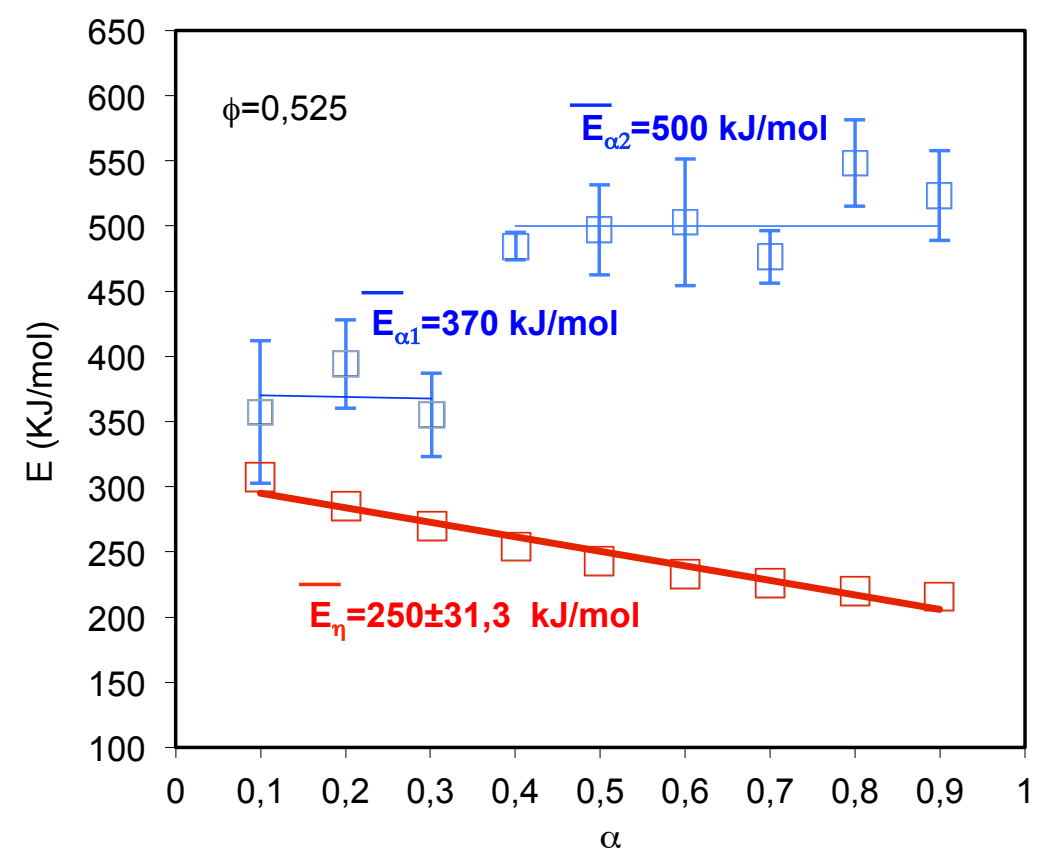

Figura 5.78. Variación de las energías de activación correspondientes a las dos etapas, $E \alpha_{1}$ y $E \alpha_{2}$, de la sinterización y la de flujo viscoso, Eq, con el avance del proceso, $\alpha$. Composite F2 $(\phi=0,525)$. Velocidad de calentamiento $\beta=2,5,15,25$ y $60 \mathrm{~K} / \mathrm{min}$.

Estos resultados son consistentes con los obtenidos en el apartado 5.2.1.3.3. En efecto, la densificación, mediante la primera etapa, para valores de $\alpha \approx 0,30$, se produce mediante un mecanismo de flujo viscoso, la reordenación de partículas, por lo que $\overline{\mathrm{E}_{\alpha_{1}}} \approx \overline{\mathrm{E}_{\eta}}$. En cambio, cuando finaliza esta etapa, para seguir densificando el composite es imprescindible la disolución y posterior reprecipitación del circón, principalmente mediante los mecanismos simultáneos de crecimiento de grano y acomodación de su forma. 
En este proceso, a diferencia del de la reordenación de partículas, el efecto de la temperatura sobre la velocidad de densificación, $E_{\alpha_{2}}$, no depende únicamente del efecto de dicha variable sobre la viscosidad de la matriz vítrea, $E_{\eta}$, sino del efecto que la temperatura ejerce sobre un conjunto de propiedades termodinámicas (solubilidad del circón en el vidrio, energía superficial del sistema) y de transporte (difusividad del Zr en el vidrio) y sobre el mecanismo controlante del crecimiento de grano (difusión del soluto en el fundido, reacción interfacial) y de la densificación (aplanamiento del contacto, disolución de pequeños granos, crecimiento del contacto entre granos) y sobre algunos otros fenómenos que alteran la microestructura del material, como la coalescencia entre granos.

5.2.2.1.2 Selección del modelo. Determinación del triplete cinético.

5.2.2.1.2.1 Composites con contenidos bajos y moderados de circón $(\phi \leq 0,43)$.

A la vista de la forma sigmoidal de las curvas de sinterización, $\alpha$ vs T, y la concordancia entre los valores de $\bar{E}_{\alpha}$ y $\bar{E}_{\eta}$, correspondientes a cada composite, se ha considerado utilizar el modelo de Avrami-Erofeev para describir la cinética del proceso, sustituyendo, como se hizo para el caso del vidrio (apartado 5.1.2.1.3.1), la ecuación de Arrhenius por la ecuación de V. F. T. (ec. 5.1), tomando los valores recomendados por la NBS: $B=5495$ y $\mathrm{T}_{0}=421 \mathrm{~K}$.

Así pues, para cada valor de $\phi$, los pares de valores $(\alpha, T)$ correspondientes a las cinco velocidades ensayadas se han ajustado a la ec. $5.12 \mathrm{y}$, siguiendo el mismo procedimiento que se ha descrito en el apartado 5.1.2.1.3.1, se han calculado los valores de los parámetros cinéticos, $n$ y $\operatorname{lnA}$, y de la varianza, $S^{2}$. En la Tabla 5.12 se detallan los resultados: 
Tabla 5.12. Valores de los parámetros cinéticos, $n$ y $\ln A$, y de la varianza, $S^{2}$, obtenidos al ajustar las curvas de sinterización, $\alpha$ vs $T$, a la ec. 5.12 con $B=5495$ y $T_{0}=421 \mathrm{~K}$. Composites $F$.

\begin{tabular}{|c|c|c|c|}
\hline$\phi$ & $\mathbf{n}$ & $\ln \mathbf{A}\left(\mathbf{s}^{-1}\right)$ & $\mathbf{s}^{2}$ \\
\hline 0,05 & $0,700 \pm 0,022$ & $17,2 \pm 0,15$ & 0,0007474 \\
\hline 0,106 & $0,680 \pm 0,033$ & $17,3 \pm 0,12$ & 0,0004036 \\
\hline 0,169 & $0,630 \pm 0,020$ & $16,6 \pm 0,14$ & 0,0000344 \\
\hline 0,322 & $0,515 \pm 0,021$ & $13,8 \pm 0,16$ & 0,0000747 \\
\hline 0,43 & $0,465 \pm 0,014$ & $11,2 \pm 0,14$ & 0,0000434 \\
\hline $0,525^{\ddagger}$ & $0,405 \pm 0,013$ & $7,8 \pm 0,11$ & 0,0006868 \\
\hline
\end{tabular}

Se comprueba (Tabla 5.12 y Figura 5.70 a Figura 5.75 ) que, para valores de $\phi \leq 0,43$, el modelo cinético ensayado (ec. 5.12), considerando que la constante de velocidad del proceso, varía con la temperatura del mismo modo que la viscosidad experimental, describe muy bien el proceso, aun cuando los parámetros cinéticos se han mantenido fijos, independientemente de la velocidad de calentamiento, $\beta$, a pesar de que dicha variable se ha modificado considerablemente ( $\beta=2$ a $60 \mathrm{~K} / \mathrm{min})$.

Para el composite con $\phi=0,525$, el modelo desarrollado solo ajusta bien el primer tramo de la curva, el correspondiente a la reordenación de partículas por flujo viscoso.

‡ Solo se han ajustado, para cada velocidad, el primer tramo de la curva $(\alpha \leq 0,3)$. 
5.2.2.1.2.2 Composite con alto contenido en circón $(\phi=0,525)$.

Lo anteriormente expuesto en los apartados 5.2.1.3 y 5.2.2.1.1 y la forma de las curvas de sinterización (Figura 5.75) revelan la necesidad de utilizar un modelo cinético compuesto de dos etapas o contribuciones, que se desarrollan en paralelo, parcialmente solapadas.

La primera etapa, que representa la contribución a la densificación asociada a la reordenación de partículas por flujo viscoso, puede describirse mediante el mismo modelo que el empleado para los composites con $\phi \leq 0,43$. Ahora bien, mediante este proceso solo se consigue una parte de la densificación, es decir, un grado de avance de sinterización máximo, $w_{1}<1$.

En consecuencia, para la primera etapa se tendrá:

$$
\frac{\alpha_{1}}{w_{1}}=\left[1-\exp \left\{\left[\frac{A_{1}}{\beta}\left(\frac{\left(T-T_{0}\right)^{2}}{B}\right) \exp \left(\frac{-B}{T-T_{0}}\right)\right]^{n_{1}}\right]\right\}
$$

O bien:

$$
\frac{\alpha_{1}}{w_{1}}=\left[1-\exp \left\{\left[\frac{A_{1}}{\beta}\left(\frac{R T^{2}}{E_{1}}\right) \exp \left(-\frac{E_{1}}{R T}\right)\right]^{n_{1}}\right]\right\}
$$

La segunda etapa, que representa la contribución a la densificación asociada al proceso de solución-reprecipitación, se ha considerado que también puede describirse mediante el modelo de Avrami-Erofeev debido a que dicha ecuación ha funcionado bien para describir la densificación de vidriados complejos y soportes, como ya se ha indicado en el apartado 2.1.4.1.3.5.

Así pues, para la segunda etapa, la ecuación a utilizar será: 
$\frac{\alpha_{2}}{1-\mathrm{w}_{1}}=\left[1-\exp \left\{\left[\frac{\mathrm{A}_{2}}{\beta}\left(\frac{R T^{2}}{\mathrm{E}_{2}}\right) \exp \left(-\frac{\mathrm{E}_{2}}{\mathrm{RT}}\right)\right]^{\mathrm{n}_{2}}\right\}\right]$

Puesto que solo se desarrollan dos etapas en paralelo:

$\alpha_{\text {cal }}=\alpha_{1}+\alpha_{2}$

El ajuste del modelo se ha realizado mediante regresión no lineal, que minimiza el cuadrado de la diferencia entre los valores de $\alpha$ obtenidos experimentalmente y los calculados, $\alpha_{\text {cal }}$, mediante la ec. 5.30 , ec. 5.31 , ec. 5.32 y ec. 5.33 .

En el ajuste se han tenido en cuenta los siguientes requisitos:

i) Los valores de los parámetros cinéticos de ajuste: $w_{1}, A_{1}, E_{1}$ y $n_{1}$, correspondientes a la etapa 1, y $A_{2}, E_{2}$ y $n_{2}$, de la etapa 2, deben ser independientes de la velocidad de calentamiento, $\beta$.

ii) Los valores de estos parámetros obtenidos en experimentos a velocidad de calentamiento constante tienen que ser iguales o muy parecidos a los obtenidos mediante métodos isotermos. $\mathrm{Y}$, por supuesto, los valores de $E_{1}$ y $E_{2}$ que se obtienen por uno y otro método de ajuste al modelo deben ser muy parecidos a los que se han obtenido por el método de Friedman (apartado 5.2.2.1.1).

Los resultados obtenidos correspondientes al experimento a $\beta=5 \mathrm{~K} / \mathrm{min}$ se detallan en la Figura 5.79. En ella, además de comprobarse una buena concordancia entre los valores experimentales y los calculados, se han representado, para las dos etapas de sinterización, la variación de sus respectivos grados de avance de la sinterización, $\alpha_{1}$ y $\alpha_{2}$, calculados mediante el modelo con la temperatura. En la Tabla 5.13 se detallan los valores de los parámetros cinéticos correspondientes a todas las velocidades de calentamiento ensayadas. 
Tabla 5.13. Parámetros cinéticos y varianza obtenidos al ajustar las curvas de sinterización del composite F2 $(\phi=0,525)$ a la ec. 5.30, ec. 5.31, ec. 5.32 y ec. 5.33.

\begin{tabular}{|c|c|c|c|c|c|c|}
\hline Etapa & $\mathbf{w}_{\mathbf{i}}$ & $\mathbf{n}_{\mathbf{i}}$ & $\ln \mathbf{A}_{\mathbf{i}}\left(\mathbf{s}^{-1}\right)$ & $\mathbf{E}_{\mathbf{i}}(\mathbf{k J} / \mathbf{m o l})$ & Ecuación & $\mathbf{s}^{2}$ \\
\hline 1 & 0,3 & 0,45 & 11,5 & - & ec. 5.30 & 0,000646 \\
\hline 1 & 0,3 & 0,45 & 24,4 & 290 & ec. 5.31 & 0,000727 \\
\hline 2 & 0,7 & 0,3 & 33,5 & 480 & ec. 5.32 & 0,000275 \\
\hline
\end{tabular}

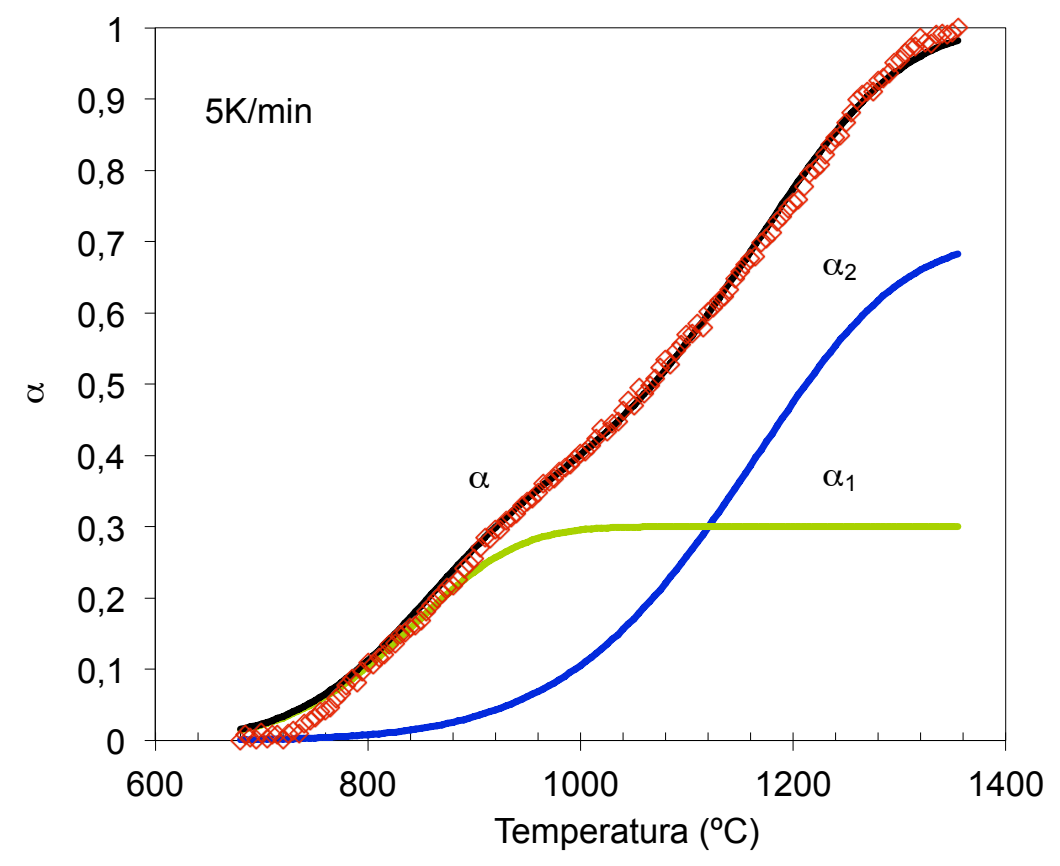

Figura 5.79. Curva de sinterización correspondiente al composite $F 2(\phi=0,525)$ a $\beta=5 \mathrm{~K} / \mathrm{min}$. Comparación de los resultados experimentales con los calculados mediante el modelo desarrollado en dos etapas (apartado 5.2.2.1.2.2).

Al comparar los valores de $E_{i}$ de la Tabla 5.13 con los obtenidos por Friedman (apartado 5.2.2.1.1) se confirma que ambos son muy similares, de acuerdo con el requisito autoimpuesto. Además, se comprueba (Tabla 5.13 y 
Figura 5.75 y Figura 5.79) que el modelo cinético desarrollado describe muy bien la sinterización no isoterma (a velocidad de calentamiento constante) del composite con un alto contenido en circón, F2 $(\phi=0,525)$.

\subsubsection{Experimentos isotermos.}

Para cada uno de los composites de la serie $\mathrm{F}$ se han realizado una serie de al menos cuatro experimentos isotermos, a diferentes temperaturas. Las temperaturas utilizadas para un determinado composite son, en general, diferentes a las empleadas para otro, debido a que el intervalo de temperaturas de sinterización de un composite en el que la velocidad del proceso se desarrolla de forma apropiada para su estudio, depende mucho del contenido en circón. Por otra parte, para el composite F2 $(\phi=0,525)$, cuya densificación se desarrolla mediante dos procesos distintos, y en el que cada uno de ellos predomina en un intervalo de temperaturas distinto se han realizado 12 experimentos isotermos. Los resultados se detallan en la Figura 5.80 a Figura 5.85).

5.2.2.2.1 Selección del modelo. Determinación del triplete cinético.

5.2.2.2.1.1 Composites con bajos y moderados contenidos en circón $(\phi \leq 0,43)$.

Para los composites con $\phi \leq 0,43$, los pares de valores $(\alpha, t)$ correspondientes a cada experimento isotermo se han ajustado a la ec. 5.14 que es la forma integrada del modelo Avrami-Erofeev para este tipo de tratamiento térmico. Al igual que ocurría para los compactos de vidrio GM (apartado 5.1.2.2), si en el ajuste no se fijaba ningún parámetro ( $\left.n, k, x_{0}\right)$, para cada composite, el valor de $\mathrm{n}$ variaba muy poco y de forma aleatoria de una temperatura a otra. Al fijar el valor de $\mathrm{n}$ al valor medio de todos los experimentos isotermos correspondientes a cada serie de composites, se obtuvieron muy buenos resultados, con valores de $S^{2}$ muy bajos $\left(S^{2}<0,0002\right)$ y una buena concordancia entre los valores calculados y los experimentales (Figura 5.80 a Figura 5.85). Para cada composite la energía de activación, E, se ha 
obtenido ajustando los pares de valores k, T obtenidos a la ecuación de Arrhenius (ec. 2.66).

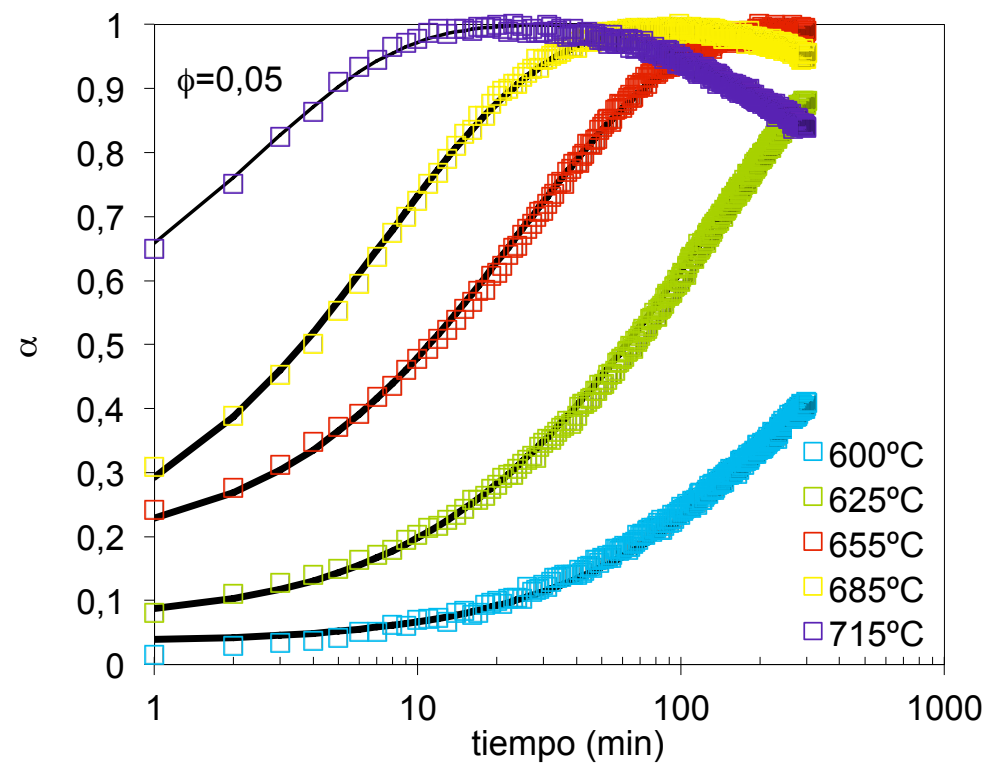

Figura 5.80. Curvas de sinterización isotermas a distintas temperaturas. Composite de la serie $F, \phi=0,05$. Comparación entre los resultados experimentales y los calculados mediante la ec. 5.14, con los valores de la Tabla 5.14. 


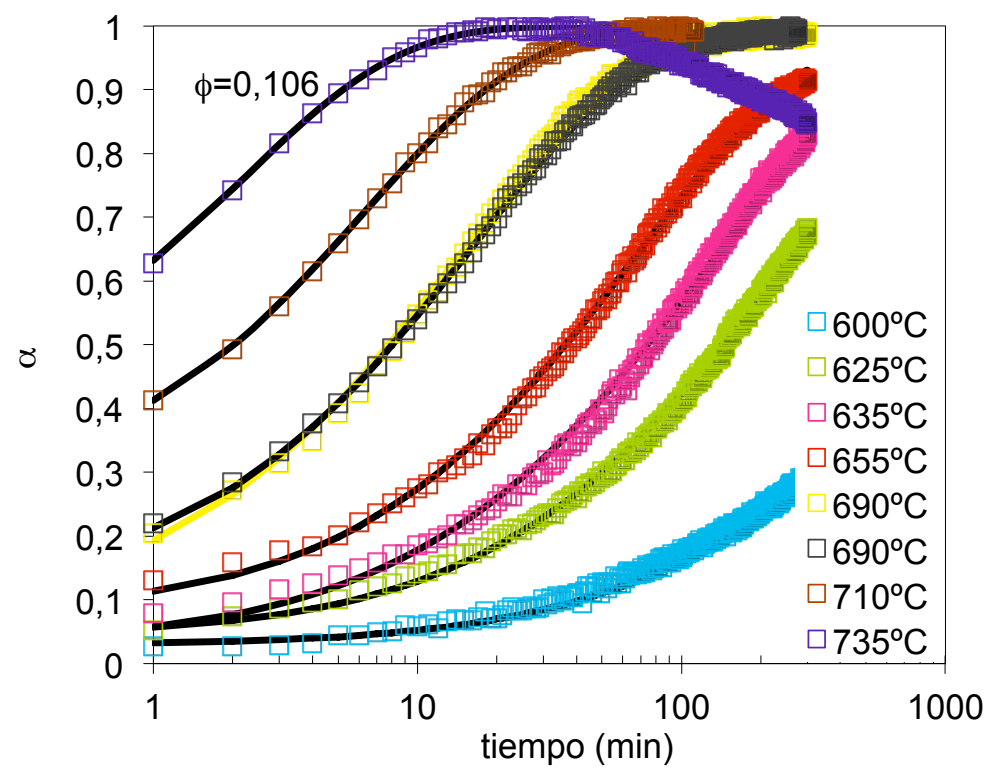

Figura 5.81. Curvas de sinterización isotermas a distintas temperaturas. Composite de la serie $F, \phi=0,106$. Comparación entre los resultados experimentales y los calculados mediante la ec. 5.14, con los valores de la Tabla 5.14.

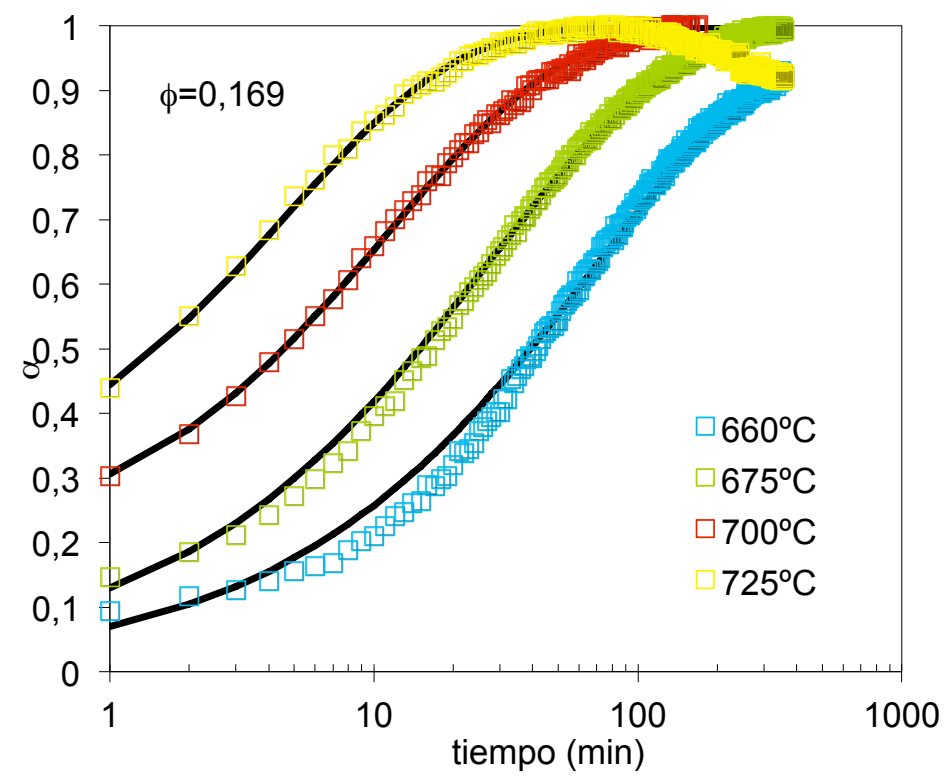

Figura 5.82. Curvas de sinterización isotermas a distintas temperaturas. Composite de la serie $F, \phi=0,169$. Comparación entre los resultados experimentales y los calculados mediante la ec. 5.14, con los valores de la Tabla 5.14. 


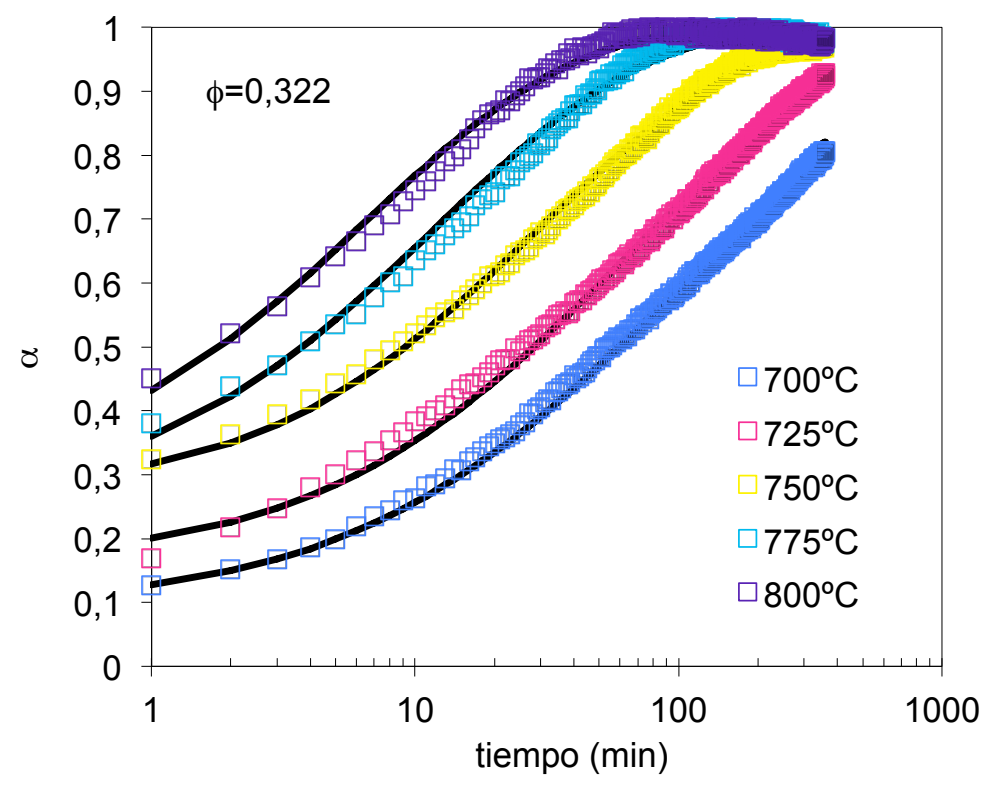

Figura 5.83. Curvas de sinterización isotermas a distintas temperaturas. Composite $F 1, \phi=0,322$. Comparación entre los resultados experimentales y los calculados mediante la ec. 5.14, con los valores de la Tabla 5.14.

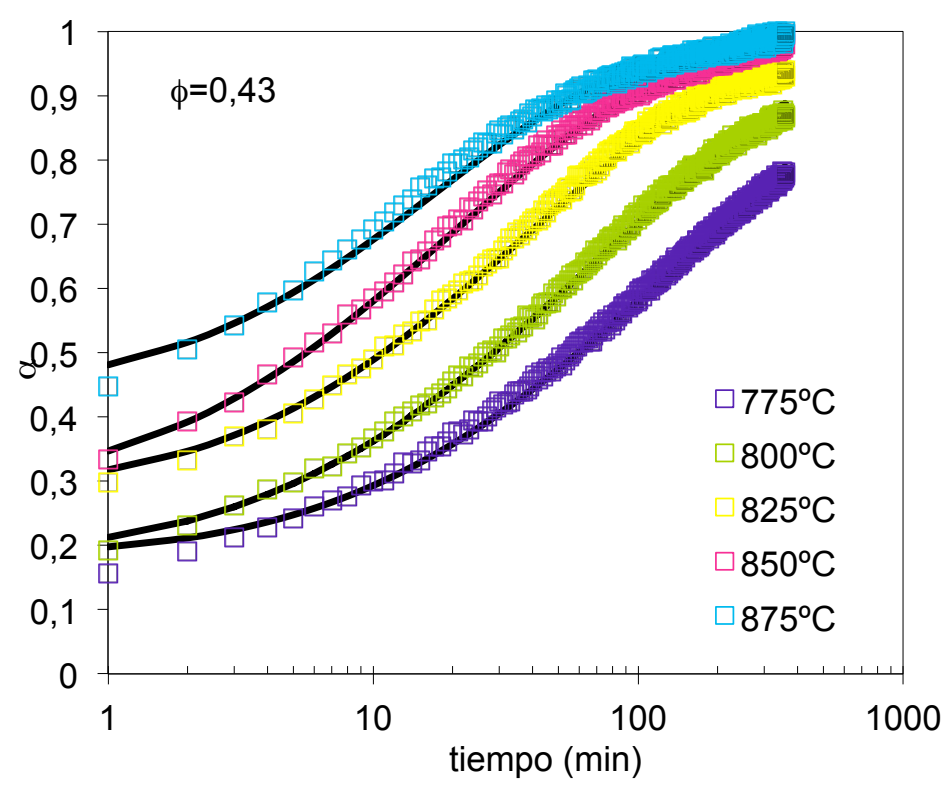

Figura 5.84. Curvas de sinterización isotermas a distintas temperaturas. Composite de la serie $F, \phi=0,43$. Comparación entre los resultados experimentales y los calculados mediante la ec. 5.14, con los valores de la Tabla 5.14. 


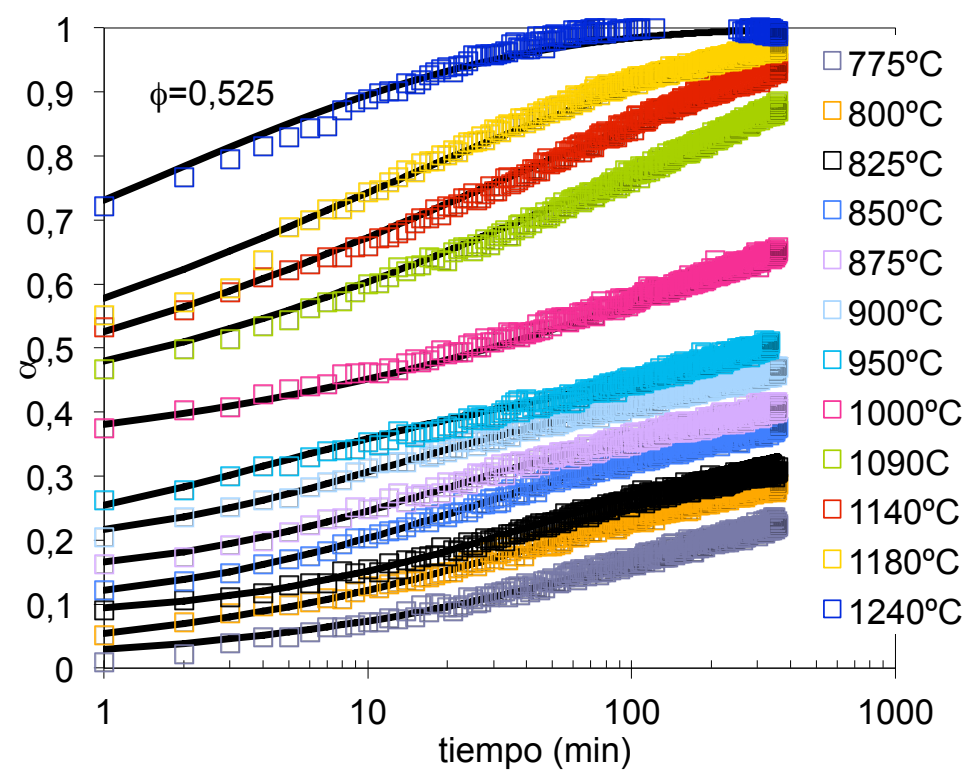

Figura 5.85. Curvas de sinterización isotermas a distintas temperaturas. Composite F2, $\phi=0,525$. Comparación entre los resultados experimentales y los calculados mediante el modelo en dos etapas (apartado 5.2.2.1.2.2).

Los valores de los parámetros cinéticos calculados, $\mathrm{n}$ y $\ln \mathrm{A}$, junto con los de la enervía de activación del flujo viscoso, Eๆ, se detallan en la Tabla 5.14.

Al comparar la Tabla 5.12 y la Tabla 5.14 se comprueba que los valores de $n$ obtenidos por un tipo u otro de experimentos, son muy similares. También se comprueba que los valores de la energía de activación del proceso son muy parecidos a los de flujo viscoso (Figura 5.86). 
Tabla 5.14. Valores de los parámetros cinéticos obtenidos al ajustar a la ec. 5.14 y la ec. 2.66 las curvas de sinterización isotermas de cada composite de la serie $F$ con $\phi \leq 0,43$. Se inlcuyen los valores de la energía de activación

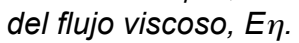

\begin{tabular}{|c|c|c|c|c|}
\hline$\phi$ & $\mathbf{n}$ & $E(\mathbf{k J} / \mathrm{mol})$ & $\operatorname{In} \mathbf{A}\left(\mathbf{s}^{-1}\right)$ & $E \eta(\mathbf{k J} / \mathrm{mol})$ \\
\hline 0,05 & $0,70 \pm 0,031$ & $365 \pm 20$ & $39,9 \pm 2,7$ & $358 \pm 2$ \\
\hline 0,106 & $0,65 \pm 0,035$ & $351 \pm 13$ & $37,2 \pm 1,7$ & $352 \pm 1$ \\
\hline 0,169 & $0,62 \pm 0,028$ & $340 \pm 22$ & $35,7 \pm 2,7$ & $333 \pm 2$ \\
\hline 0,322 & $0,51 \pm 0,04$ & $287 \pm 11$ & $26,5 \pm 1,3$ & $305 \pm 2$ \\
\hline 0,43 & $0,465 \pm 0,035$ & $270 \pm 13$ & $21,9 \pm 1,4$ & $279 \pm 2$ \\
\hline
\end{tabular}

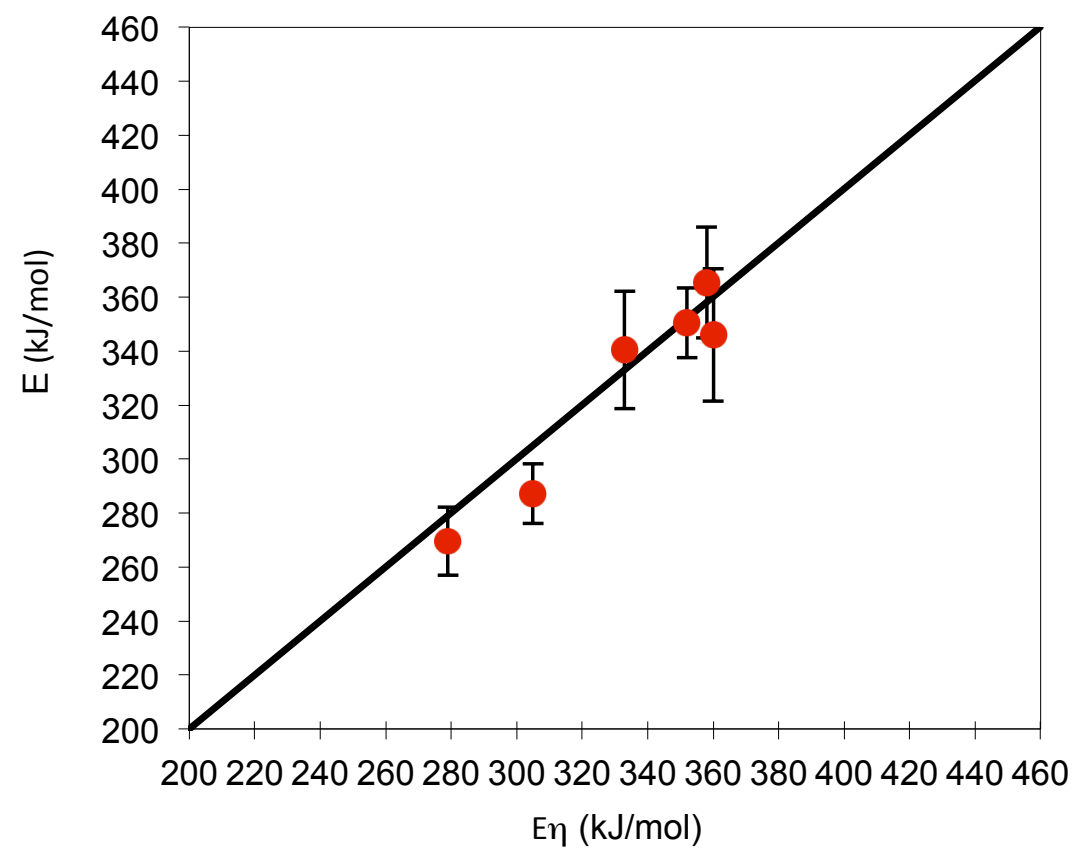

Figura 5.86. Relación entre los valore de la energía de activación de la sinterización,

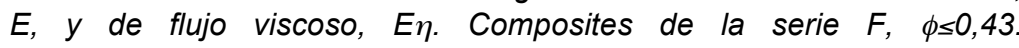
Experimentos isotermos. 
Análogamente, los valores del factor preexponencial, InA, obtenidos en experimentos isotermos, aun siendo muy diferentes (además de bastante dispersos) de los que se obtienen en experimentos a velocidad de calentamiento constante están directamente relacionados. Esto se debe a que la relación $\eta=\eta(T)$ utilizada en los experimentos no isotermos no es del tipo Arrhenius (Figura 5.87).

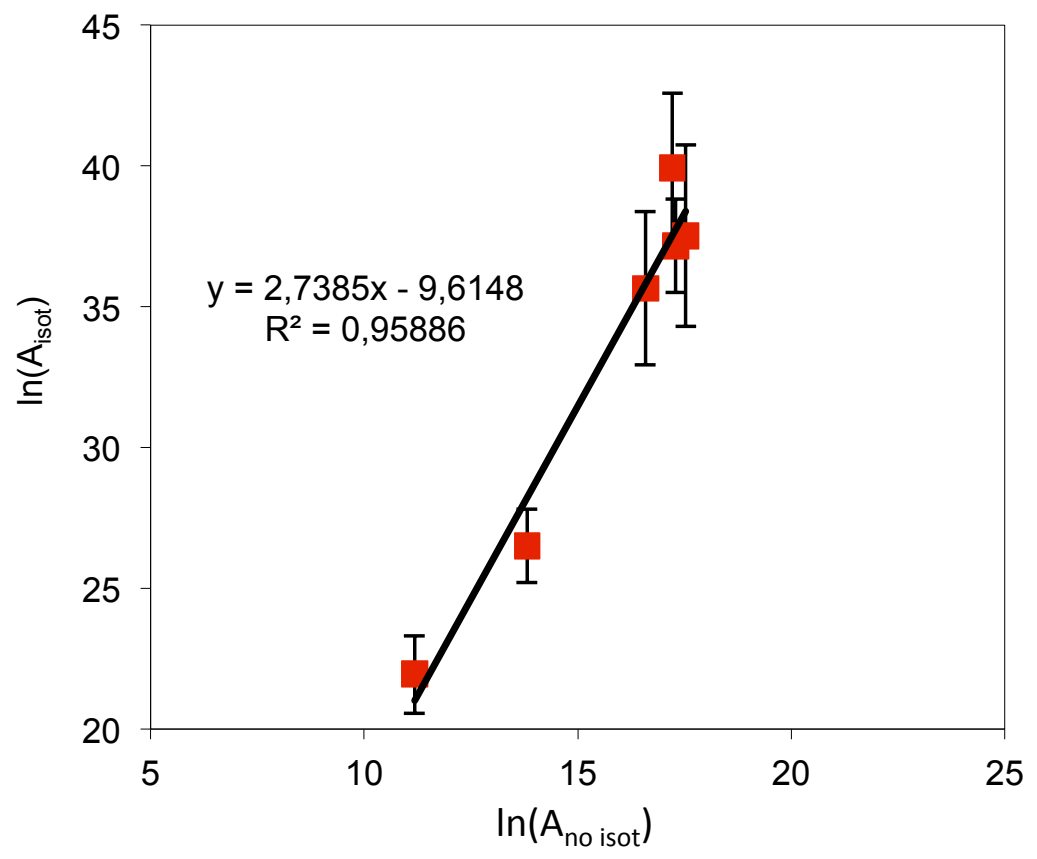

Figura 5.87. Relación entre los valores experimentales isotermos, $\ln \left(A_{i s o t}\right)$, y no isotermos, $\ln \left(A_{\text {no isot }}\right)$. Composites: Serie $F, \phi \leq 0,43$.

\subsection{Composites con alto contenido en circón, F2 $(\phi=0,525)$.}

Los resultados del apartado 5.2.2.1.2.2 han demostrado que el empleo de un modelo cinético compuesto de dos etapas individuales que se desarrollan en paralelo, descritas cada una por un modelo de Avrami-Erofeev, es apropiado para describir los resultados de este composite. En consecuencia, 
para describir los resultados obtenidos en experimentos isotermos, se ha utilizado el mismo modelo que para este tipo de experimentos:

$$
\frac{\alpha_{1}}{w_{1}}=1-\exp \left\{k_{1} t+\left[-\ln \left(1-\alpha_{0_{1}}\right)\right]^{\frac{1}{n_{1}}}\right\}^{-n_{1}}
$$

$$
\frac{\alpha_{2}}{1-w_{1}}=1-\exp \left\{k_{2} t+\left[-\ln \left(1-\alpha_{o_{2}}\right)\right]^{\frac{1}{n_{2}}}\right\}^{-n_{2}}
$$

$$
\alpha_{\text {cal }}=\alpha_{1}+\alpha_{2}
$$

Al igual que en el apartado 5.2.2.1.2.2 el ajuste del modelo se ha realizado por regresión no lineal, minimizando el cuadrado de la diferencia entre los valores experimentales, $\alpha, y$ los calculados, $\alpha_{\text {cal }}$, de acuerdo con la ec. 5.34, ec. 5.35 y ec. 5.36. Los requisitos tenidos en cuenta en el ajuste son los mismos que en el apartado 5.2.2.1.2.2.

Los resultados obtenidos a las dos temperaturas más bajas $\left(775\right.$ y $\left.800^{\circ} \mathrm{C}\right)$ y a las cuatro más altas $\left(1090-1240^{\circ} \mathrm{C}\right)$, se han ajustado a una única etapa, ya que la otra no era operativa o ya se había completado antes de alcanzar la condiciones isotermas (intervalo de temperaturas más elevado). En los demás casos se requiere la concurrencia de las dos etapas para un buen acuerdo entro los resultados experimentales y los calculados. Fijando, para todos los experimentos isotermos, los mismos valores de los parámetros $\mathrm{w}_{\mathrm{w}}$ y $n_{i}\left(w_{1}=0,3, n_{1}=0,45\right.$ y $\left.n_{2}=0,30\right)$ (apartado 5.2.2.1.2.2) se obtuvieron muy buenos resultados para todas las temperaturas ensayadas (Figura 5.85). En ningún caso $S^{2}>4 \cdot 10^{-4}$ siendo el valor más frecuente $S^{2}=2-5 \cdot 10^{-5}$. Además, las constantes de velocidad $\mathrm{k}_{1}$ y $\mathrm{k}_{2}$ que resultaban se ajustaban muy bien a dos rectas de Arrhenius (Figura 5.88). El valor de la energía de activación 
obtenido para la primera etapa $E_{1}$ (Tabla 5.15) coincide prácticamente con la de flujo viscoso y con la obtenida por métodos no isotermos (Tabla 5.13). El valor de $E_{2}$ obtenido por métodos isotermos (Tabla 5.15) también es parecido, aunque un poco menor que el obtenido por métodos no isotermos (Tabla 5.13).

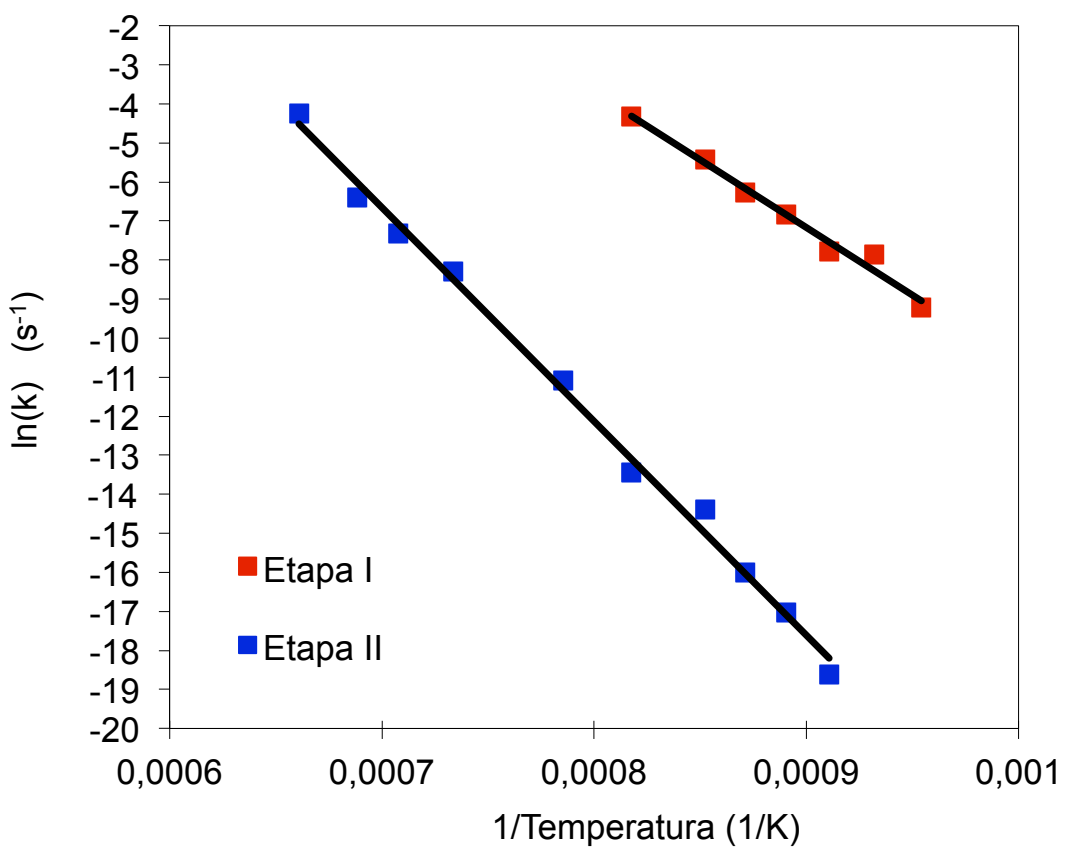

Figura 5.88. Representación de Arrhenius para el cálculo de la energía de activación de las dos etapas de sinterización.

Tabla 5.15. Valores de los parámetros cinéticos obtenidos al ajustar las curvas de sinterización isotermas al modelo en dos etapas. Composite F2 $(\phi=0,525)$

\begin{tabular}{|c|c|c|c|c|c|c|}
\hline Etapa & $\mathbf{w}_{\mathrm{i}}$ & $\mathbf{n}_{\mathrm{i}}$ & $\operatorname{In} \mathbf{A}_{\mathbf{i}}\left(\mathbf{s}^{-1}\right)$ & $\begin{array}{c}\mathbf{E}_{\mathbf{i}} \\
(\mathbf{k J / m o l})\end{array}$ & $\begin{array}{c}\mathrm{E}_{\eta} \\
(\mathbf{k J} / \mathrm{mol})\end{array}$ & $\mathbf{s}^{2}$ \\
\hline 1 & 0,3 & 0,45 & $28,1 \pm 1,8$ & $288 \pm 17$ & $294 \pm 1,8$ & 0,0015 \\
\hline 2 & 0,7 & 0,30 & $35,8 \pm 1,1$ & $456 \pm 11$ & - & 0,0011 \\
\hline
\end{tabular}


Se confirma que el modelo cinético desarrollado describe perfectamente los resultados del proceso de sinterización tanto si los experimentos son isotermos como no isotermos.

\subsubsection{Influencia del contenido en circón sobre los parámetros cinéticos.}

5.2.2.3.1 Composites con bajos o moderados contenidos en circón $(\phi \leq 0,43)$.

i) Índice de potencia de Avrami, "n".

La sinterización de todos estos composites se puede describir mediante una única etapa, utilizando el modelo de Avrami-Erofeev, con un valor del índice de potencia de Avrami, "n", que disminuye linealmente con el aumento del contenido en circón, $\phi$ (Figura 5.89). Para visualizar más claramente el efecto de " $n$ " sobre el modelo cinético, $f(\alpha)$; es decir, sobre la función que expresa la disminución de la velocidad del proceso debido exclusivamente al avance de la sinterización, en la Figura 5.90 se han representado las curvas $f(\alpha)$ vs $\alpha$ para distintos valores de "n". Se aprecia que conforme se reduce el valor de " $n$ " con el aumento de $\phi$, la caída de la velocidad de sinterización con el avance del proceso es más alta. Estos resultados son consistentes con los obtenidos experimentalmente [128][129][33] y mediante simulación [130][131] en composites de matriz vítrea. Este comportamiento refleja a su vez el efecto que ejerce el aumento del contenido en circón sobre la fuerza impulsora del proceso o presión de sinterización, y sobre la contribución del mismo al flujo viscoso o modulo viscoso. En efecto, conforme avanza el proceso de densificación el crecimiento del tamaño de poros abiertos en estados iniciales e intermedios de la sinterización y el aumento de la porosidad cerrada (con la consiguiente presión de los gases ocluidos) debe provocar una disminución de la presión de sinterización, como ya se ha indicado en apartados anteriores (apartados 5.2.1.2.3 y 5.2.1.2.4. Además, dichos fenómenos se desarrollan de forma más acusada con el aumento de 
$\phi$, por lo que la caída de la presión de sinterización y, por consiguiente, la velocidad de sinterización con el avance del proceso también lo serán.

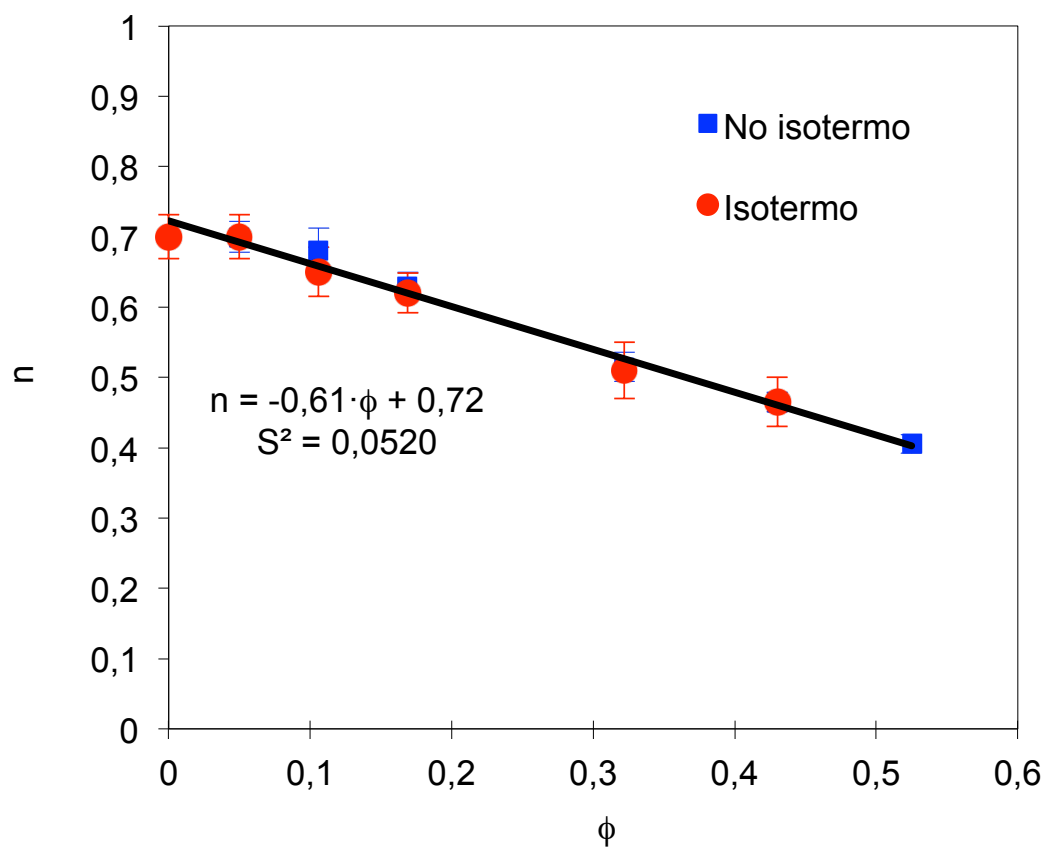

Figura 5.89. Efecto de $\phi$ sobre el índice de potencia " $n$ ". Experimentos isotermos (círculos rojos) y a velocidad de calentamiento constante (cuadrados azules). Composites de la serie $F$. 


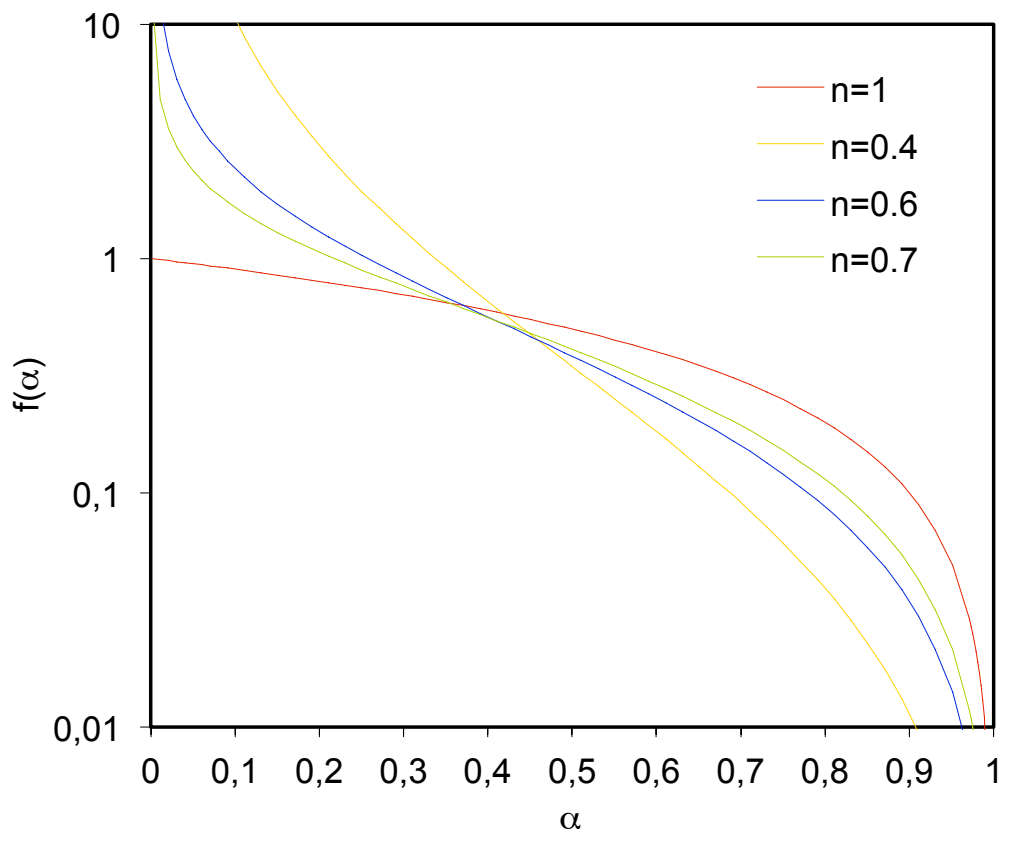

Figura 5.90. Efecto del índice de potencia " $n$ " sobre el modelo cinético, $f(\alpha)$.

En lo que respecta al comportamiento reológico del material, un aumento del contenido en sólidos suspendido o disperso en un líquido o fundido de alta viscosidad, no solo aumenta la viscosidad del sistema (o dispersión) sino que también modifica el tipo de fundido de newtoniano a seudoplástico o incluso a plástico.

En el caso de un fluido seudoplástico su viscosidad disminuye conforme se incrementa la tensión aplicada que debe estar directamente relacionada con la presión de sinterización. En consecuencia, conforme avanza el proceso, $\alpha$, se reducirá la presión de sinterización y, por consiguiente, aumentara la viscosidad del sistema. Dicho efecto, el carácter seudoplástico del material, es tanto más marcado cuanto mayor es el contenido en sólidos. En consecuencia, conforme se incrementa el contenido en sólidos, $\phi$, más acusada será el aumento de la viscosidad con el avance del proceso, $\alpha, y$, por consiguiente, la disminución de la velocidad de sinterización. 
La caída de la velocidad de sinterización con el avance del proceso y el incremento de ésta con el contenido en circón también puede interpretarse atendiendo al número de contactos: vidrio-vidrio (gg), vidrio-circón (gh) y circón-circón (hh), ya que la velocidad de sinterización de cada uno es dependiente del tipo de contacto, por lo que su relativa abundancia debe determinar la cinética del proceso y, en consecuencia, los valores de los parámetros cinéticos.

En efecto, conforme se incrementa el contenido en circón se reducen la frecuencia de contactos (gg) y aumentan los de tipo (gh) y (hh), como ha sido comprobado por simulación [132]. Además, la velocidad de sinterización del contacto (gh) suele considerarse la mitad que la del contacto (gg), mientras que la del contacto (hh) se considera despreciable [130][131][132]. En consecuencia, un aumento del contenido en circón y, por lo tanto, del número de contactos (gh) y (hh) con velocidad de sinterización más baja o despreciable, debe conducir a una reducción de la velocidad de sinterización del compacto, en cualquier grado de avance de la sinterización, y a una mayor caída de la velocidad del proceso con el avance del mismo; es decir, a una disminución de "n".

La interacción entre partículas de circón es otro factor a considerar. En efecto, la cantidad de contactos (hh) aumenta no solo con la cantidad de circón en el composite sino también con el grado de avance del proceso, llegando incluso a formar una red continua de partículas en contacto (límite de percolación) a una determinada fracción volumétrica, $\phi_{p}$ (volumen circón/volumen total), lo que puede llegar a paralizar la densificación, si dicha red es suficientemente rígida para resistir las tensiones de sinterización [128][130][131].

Además, mediante simulación [130][131] se ha comprobado que la viscosidad efectiva del composite aumenta con el número de contactos (hh), especialmente en las proximidades de la percolación; en cambio, la velocidad de sinterización disminuye en igual medida con dicha característica. 
Así pues, el número de contactos iniciales (hh) y su incremento con el grado de avance de la sinterización [129][130][131] es mayor con el contenido en circón, $\phi$, por lo que la caída de la velocidad de sinterización con el avance del proceso aumenta con $\phi$, y, en consecuencia "n" disminuye con dicha variable, de acuerdo con los resultados obtenidos.

ii) Energía de activción, "E".

De forma general, los valores de la energía de activación de la sinterización, obtenidos por el modelo de Friedman, $\bar{E}_{\alpha}$, a partir de experimentos no isotermos, (Figura 5.76) como los obtenidos en experimentos isotermos, $\mathrm{E}$ (Tabla 5.14), disminuyen con el aumento de $\phi$ (Figura 5.91). Esta variación, como ya se ha comentado anteriormente, se debe a que la energía de activación del flujo viscoso, $E_{\eta}$, también disminuye conforme se desplaza el intervalo de temperaturas a valores más altos, lo que coincide con el aumento de $\phi$. En consecuencia, los valores de $E$ y $E_{\eta}$ son prácticamente coincidentes, dentro del error experimental.

Además, al igual que se hizo en el apartado 5.1.2.2 para el vidrio, se han representado (Figura 5.92) frente a la inversa del tiempo característico de este, $\gamma / d \eta$, los valores de las constantes de velocidad, $k$, obtenidos en experimentos isotermos para todos los composites de la serie $F$ con $\phi \leq 0,43$, en escala doble logarítmica. Para el cálculo del tiempo característico de la matriz vítrea se ha considerado que solo se modifica la viscosidad con la temperatura y que esta sigue la curva de viscosidad-temperatura dada por la NBS. Al ajustar los valores correspondientes a cada composite a una ecuación del tipo: $\mathrm{k}=\lambda\left(\frac{\gamma}{\eta \cdot d}\right)$, se obtuvieron muy buenos resultados. En efecto, en la representación doble logarítmica se obtuvieron rectas paralelas, de pendiente unidad, que se desplazan tanto más a la derecha ( $\lambda$ disminuye) con el aumento del contenido en sólidos, $\phi$. El parámetro $\lambda$ debe interpretarse como una constante de velocidad adimensional. 


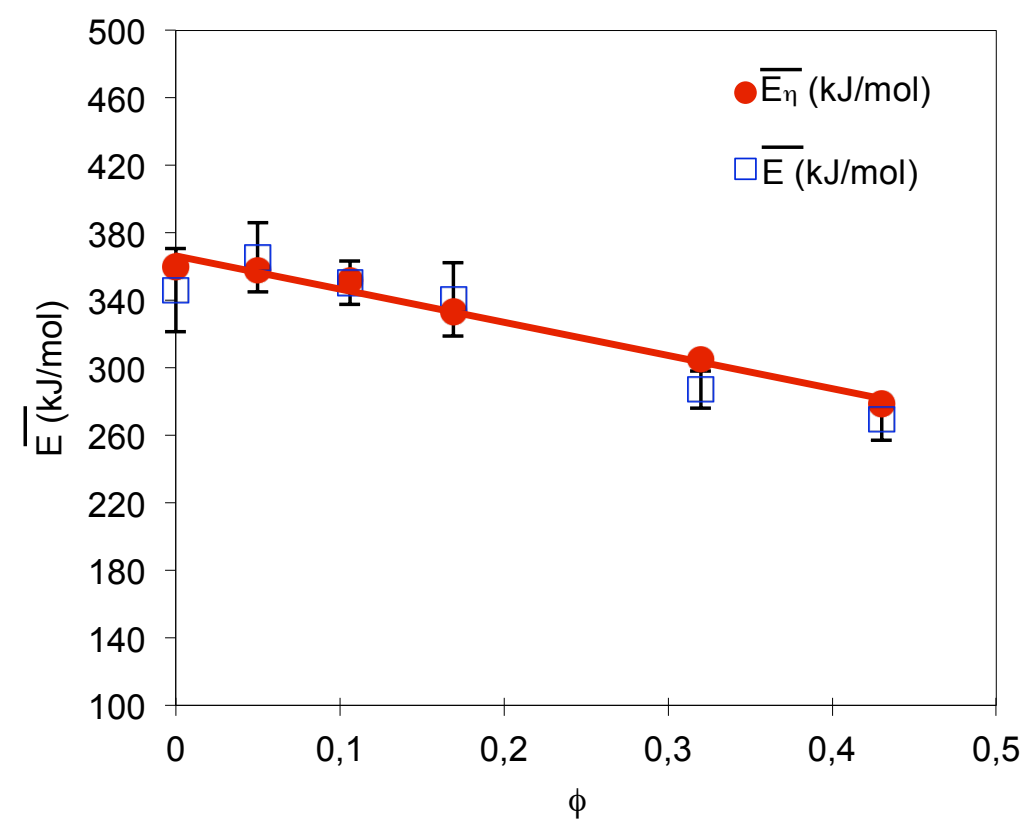

Figura 5.91. Variación de $\overline{\mathrm{E}}$ y $\overline{\mathrm{E}}_{\eta}$ con $\phi$. Experimentos isotermos. Composites: serie $F \operatorname{con} \phi \leq 0,43$.

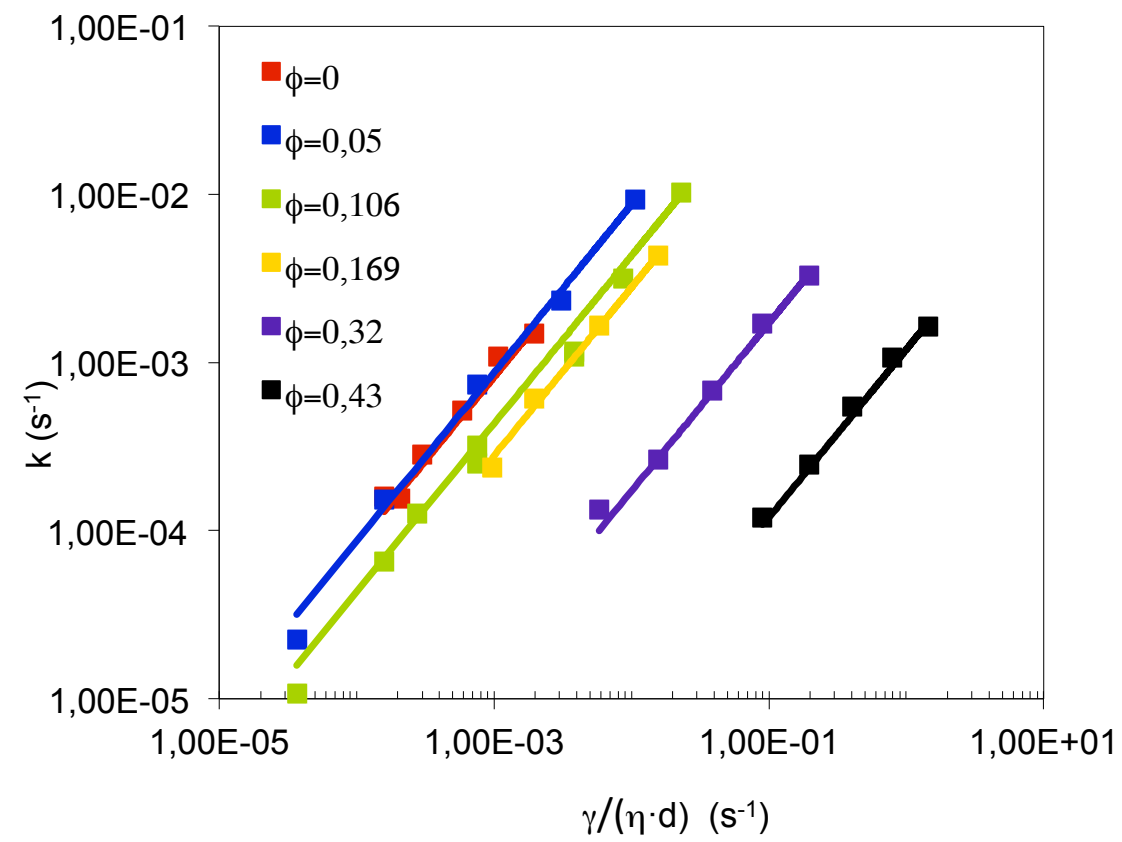

Figura 5.92. Representación de la constante de velocidad, $k$, vs la inversa del tiempo característico del vidrio, $\gamma /(\eta \cdot d)$, en escala doble logarítmica. Composites: serie $F$ con $\phi \leq 0,43$. 
La proporcionalidad entre $\mathrm{k}$ y $\frac{1}{\eta}$ confirma, una vez más, que el efecto de la temperatura sobre la velocidad del proceso se debe "exclusivamente" al efecto de dicha variable sobre la viscosidad de la matriz vítrea.

Lo propio puede concluirse de los buenos resultados obtenidos en experimentos no isotermos, en los que se ha considerado que el efecto de la temperatura sobre la velocidad del proceso se describe muy bien suponiendo que únicamente varía $\mu$ con la temperatura y que dicha relación es la V.F.T. suministrada por la NBS.

iii) Factor preexponencial, "A" y constante de velocidad adimensional, $\lambda$.

Este parámetro cinético cuantifica el efecto del contenido en circón sobre la constante de velocidad del proceso, k. En consecuencia, tanto los valores obtenidos de experimentos isotermos, $A_{\text {isot }}$, como no isotermos, $A_{\text {no isot }}$, disminuyen con el aumento de $\phi$ (Figura 5.93). Se comprueba que aunque los valores de $A_{\text {isot }}$ y $A_{\text {no isot }}$ sean muy diferentes, debido a que la relación $\eta=\eta(T)$ es bastante distinta como ya se ha indicado antes (apartado 5.2.2.2.1), la evolución que siguen ambos es similar. 


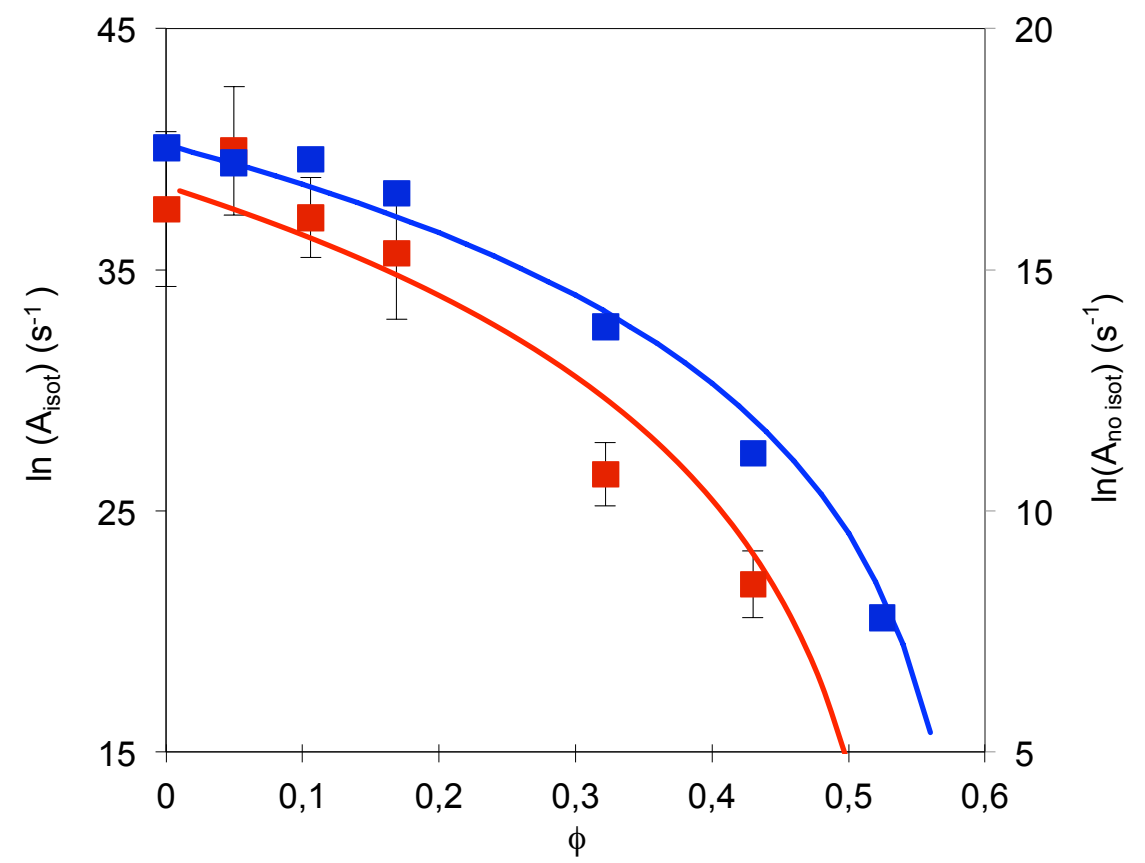

Figura 5.93. Efecto de $\phi$ sobre el factor preexponencial obtenido mediante experimentos isotermos, $A_{\text {isot, }}$ (rojo) y a velocidad de calentamiento constante, $A_{\text {no isot, }}($ azul). Composites serie $F$.

En ambos casos, el efecto del aumento del contenido en circón, $\phi$, sobre la disminución del factor preexponencial (pendiente de las curvas) es tanto mayor con el aumento de este, $\phi$. Asimismo, también se aprecia una mayor dispersión de los valores $A_{\text {isot }}$ asociada a variaciones de la energía de activación. En efecto, pequeños o moderados variación de la pendiente $(E / R)$ de la ecuación de Arrhenius conducen a grandes variaciones en la ordenada en el origen, A. Este problema no se presentaba en el caso de experimentos no isotermos en los que la relación $k=k(T) \circ \mu=\mu(T)$ se considera la misma para todos los experimentos.

Otra forma de tratar los datos de los experimentos isotermos, para determinar con mayor precisión el efecto de $\phi$ sobre $k$, que no esté afectada por la dispersión de los valores de E, consiste en utilizar la constante de 
velocidad adimensional, $\lambda$ en vez de $A_{\text {isot. }}$ En efecto, para el cálculo de $\lambda, \eta$, el único parámetro que depende de la temperatura, se calcula de la ecuación V.F.T. dada por la NBS, que es muy precisa. Al representar $\lambda$ vs $\phi$ (Figura 5.94) se confirma que los valores siguen la misma tendencia que los de $A_{\text {no isot }}$ y $A_{\text {isot }}$, pero su dispersión es bastante menor que los de $A_{\text {isot }}$.

La variación que siguen tanto el factor preexponencial $\operatorname{como} \lambda \operatorname{con} \phi$, directamente relacionada con la velocidad de sinterización (a igualdad de temperatura), es muy similar al que se obtiene por simulación para un empaquetamiento compacto de esferas del mismo tamaño, al modificar la fracción de partículas rígidas [130][131]. Por lo que dicho comportamiento puede explicarse atendiendo al efecto que $\phi$ ejerce sobre le tipo de contacto, su relativa abundancia y el efecto de éstas últimas características estructurales del empaquetamiento sobre sus propiedades reológicas, mediante la teoría de la percolación [130][131]. En efecto, por simulación se ha comprobado que la adición de esferas rígidas a un empaquetamiento de esferas de vidrio reduce la velocidad de sinterización, siendo esta disminución tanto más acusada cuanto más alto es $\phi$; es decir, cuanto menor es la diferencia entre $\phi$ y $\phi_{\mathrm{p}}$ (fracción volumétrica a la que se forma una red continua y rígida de inclusiones en contacto). Además, el valor de $\phi_{p}$ y el efecto cuantitativo de $\phi$ sobre la velocidad de sinterización, en el caso concreto de esferas, depende de que el contacto entre esferas rígidas (contacto hh) sea friccional o deslizante. En el caso de que se considere contacto friccional, que es el que debe considerarse cuando no hay sinterización sólida entre partículas de circón, el valor obtenido por simulación [131] es de $\phi_{p}=0,66$, valor muy próximo al que tienden las curvas de la Figura 5.93 y la Figura 5.94, aun cuando en nuestro caso las partículas ni son esféricas ni de tamaño uniforme. 


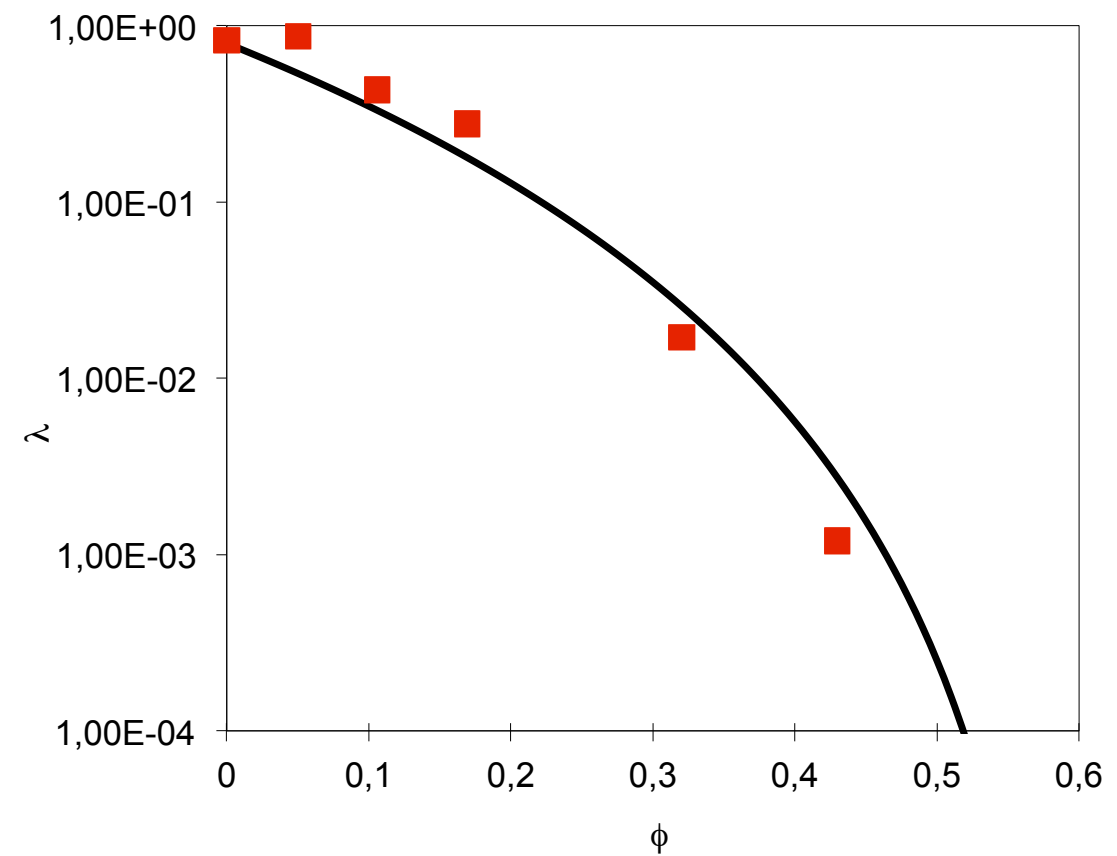

Figura 5.94. Efecto de $\phi$ sobre la constante de velocidad adimensional del composite, $\lambda=\mathrm{k}\left(\frac{\gamma}{\eta \cdot d}\right)^{-1}$. Composites: serie $F$, con $\phi \leq 0,43$.

5.2.2.3.2 Composite con alto contenido en circón, F2 $(\phi=0,525)$.

Al ajustar únicamente el primer tramo de las curvas de sinterización $(\alpha \leq 0,30)$, obtenidas en experimentos no isotermos, al modelo de una única etapa se han obtenido valores de " $n$ " y $\ln A_{\text {no isot }}$, que al representarlos en las curvas "n" vs $\phi$ (Figura 5.89) y In $A_{\text {no isot }}$ vs $\phi$ (Figura 5.93) siguen la misma tendencia que los compactos con $\phi \leq 0,43$.

A la vista de los resultados obtenidos en los apartados 5.2.2.3.1 y 5.2.2.3.2 los resultados correspondientes a las curvas " $n$ " vs $\phi$ (Figura 5.89), In $A_{\text {no isot }}$ vs $\phi$ (Figura 5.93) y $\lambda$ vs $\phi$ (Figura 5.94) se han ajustado a distintas funciones matemáticas. En los ajustes de los dos últimos se ha procurado que la función que describe las dos curvas fuera la misma, ya que tanto 
$A_{\text {no isot }}$ como $\lambda$ cuantifican el efecto del contenido en circón, $\phi$, sobre la velocidad del proceso. La expresión relacionada es del tipo de las usualmente empleadas para relacionar propiedades (reológicas y mecánicas) y velocidades de proceso (sinterización) [130][131] en composites con la fracción volumétrica de inclusiones, $\phi$, inferior a $\phi_{\mathrm{p}}$. Los valores de los ajustes se detallan en la Tabla 5.16.

Tabla 5.16. Influencia de $f$ sobre los parámetros cinéticos, $n, A_{n o}$ isot $y \lambda$. Composites: serie $F$, con $\phi \leq 0,43$.

\begin{tabular}{|c|c|}
\hline Parámetro & $\mathbf{S}^{2}$ \\
\hline $\mathrm{n}=-0,61 \cdot \phi+0,72$ & 0,052 \\
\hline $\mathrm{A}_{\text {no isot }}=4,3 \cdot 10^{7}\left(1-\frac{\phi}{0,6}\right)^{4,5}$ & 0,303 \\
\hline$\lambda=0,95\left(1-\frac{\phi}{0,6}\right)^{4,5}$ & 0,084 \\
\hline
\end{tabular}

Se comprueba que los resultados (Figura 5.89, Figura 5.93 y Figura 5.94), aunque con alguna dispersión, se ajustan aceptablemente bien a sus respectivas ecuaciones. El hecho de que los valores de los parámetros $\phi_{p} 0$ $\phi_{\max }(=0,60)$ y del exponente $(=4,5)$ obtenidos sean muy parecidos [130][131] o incluso iguales [30] a los encontrados al relacionar la viscosidad efectiva del composite con $\phi$, parece indicar que el principal factor causante de la disminución de la velocidad de sinterización con el aumento del contenido en circón, $\phi$, es probablemente el aumento de la viscosidad efectiva del sistema con $\phi$. 


\subsection{Sinterización de composites. Efecto de la distribución de tamaños}

de partícula del circón y del vidrio.

Para la realización de este apartado se han utilizado dos distribuciónes de tamaños de partícula de vidrio (GM y GF) y tres de circón micronizado (F), harina tamizada a $40 \mu \mathrm{m}(\mathrm{H})$ y mezcla de ambas (M)) (Figura 4.1).

Con estos materiales se han preparado las mezclas que se especifican en la Tabla 5.17. Se incluyen en ella el diámetro medio volumétrico del vidrio, $d_{g}^{v}$, y del circón, d d .

Así pues, con estos cinco materiales de partida se han obtenido dos series de composites: una con moderado contenido en circón $(\phi=0,322)$, serie 1 , y otra de alto contenido en circón $(\phi=0,525)$, serie 2 . Dentro de cada serie se ha modificado la distribución de tamaños de partícula del vidrio y del circón obteniendo, de este modo, seis composites con valores de la razón: diámetro medio volumétrico de circón/diámetro medio volumétrico de vidrio, $\mathrm{d}_{\mathrm{z}}^{\mathrm{v}} / \mathrm{d}_{\mathrm{g}}^{\mathrm{v}}$, comprendidos entre $\approx 0,25$ para los composites $\mathrm{F} 1$ y $\mathrm{F} 2, \mathrm{y} \approx 10$ para los $\mathrm{FH} 1$ y $\mathrm{FH} 2$. Una variación de $\mathrm{d}_{\mathrm{z}}^{\mathrm{v}} / \mathrm{d}_{\mathrm{g}}^{\mathrm{v}}$ de tal magnitud $(\approx 40)$ debe alterar considerablemente el número de contactos entre los distintos componentes (vidrio-circón, circón-circón, vidrio-vidrio). Por otra parte, con esta variación también se debe modificar de forma sustancial la compacidad en crudo y al inicio de la sinterización, $\rho_{0}$, de las probetas $y$, por consiguiente, la contracción de cocción. Análogamente, un aumento del tamaño medio y de la amplitud de la distribución de tamaños de partícula del circón, de $\mathrm{F}$ a $\mathrm{H}$ (Figura 4.1), debe incrementar el volumen y el tamaño de los poros de sus respectivos empaquetamientos y, en consecuencia, el contenido teórico de líquido requerido para rellenar los poros iniciales, y por consiguiente para conseguir la densificación completa del composite. 
Tabla 5.17. Fórmulas de carga (en peso) de los composites estudiados y diámetro medio volumétrico de cada componente.

\begin{tabular}{|c|c|c|c|c|c|c|}
\hline \multicolumn{2}{|l|}{ Vidrio } & \multicolumn{3}{|l|}{ Circón } & \multicolumn{2}{|c|}{ Composite } \\
\hline $\begin{array}{l}\text { GM } \\
d_{g}{ }^{v}=7,8 \mu \mathrm{m}\end{array}$ & $\begin{array}{l}\mathrm{GF} \\
\mathrm{d}_{\mathrm{g}}^{\mathrm{v}}=2,1 \mu \mathrm{m}\end{array}$ & $\begin{array}{l}F \\
d_{z} v_{=2,0}, 0 m\end{array}$ & $\begin{array}{l}H \\
d_{z}^{v}=21,0 \mu \mathrm{m}\end{array}$ & $\begin{array}{l}M \\
d_{z}^{v}=9,8 \mu \mathrm{m}\end{array}$ & Ref. & $\phi$ \\
\hline 100 & - & - & - & - & $\mathrm{GM}\left({ }^{*}\right)$ & 0 \\
\hline- & 100 & - & - & - & GF & 0 \\
\hline 50 & - & 50 & - & - & $\mathrm{F} 1\left(^{*}\right)$ & 0,322 \\
\hline 50 & - & - & 50 & - & $\mathrm{H} 1$ & 0,322 \\
\hline 50 & - & - & - & 50 & M1 & 0,322 \\
\hline 30 & - & 70 & - & - & $\mathrm{F} 2\left(^{*}\right)$ & 0,525 \\
\hline 30 & - & - & 70 & - & $\mathrm{H} 2$ & 0,525 \\
\hline 30 & - & - & - & 70 & M2 & 0,525 \\
\hline- & 50 & 50 & - & - & FF1 & 0,322 \\
\hline- & 50 & - & 50 & - & $\mathrm{FH} 1$ & 0,322 \\
\hline- & 50 & - & - & 50 & FM1 & 0,322 \\
\hline- & 30 & 70 & - & - & FF2 & 0,525 \\
\hline- & 30 & - & 70 & - & $\mathrm{FH} 2$ & 0,525 \\
\hline- & 30 & - & - & 70 & $\mathrm{FM} 2$ & 0,525 \\
\hline
\end{tabular}

$\left({ }^{*}\right)$ Composites estudiadas en apartados anteriores. 
Al efecto, para determinar las características de los empaquetamientos obtenidos con distintos tamaños de partículas del circón se han preparado, por colado, probetas cilíndricas a partir de suspensiones concentradas $(60 \%$ vol.) de circón, con granulometrías $\mathrm{F}, \mathrm{H}$ y $\mathrm{M}$, en medio acuoso. En las probetas secas se ha determinado su distribución del diámetro de intrusión y su porosidad por porosimetría de mercurio (Figura 5.95 y Tabla 5.18). También se ha determinado la porosidad total por empuje de mercurio (Tabla 5.18). Se comprueba, tal como se había sugerido, una notable reducción de la porosidad (más del 10\%) y un marcado incremento del tamaño medio de poro $\left(\mathrm{d}_{50}\right)$ y de la amplitud de la distribución $\left(\mathrm{d}_{84}-\mathrm{d}_{16}\right)$ al aumentar la granulometría del circón de la distribución $\mathrm{F}$ a la H.

Tabla 5.18. Porosidad y tamaños de poro correspondientes a probetas secas de circón con las granulometrías: micronizado $(F)$, harina $(H)$ y mezcla $(M)$.

\begin{tabular}{|l|l|l|l|l|l|}
\hline $\begin{array}{l}\text { Granulometría } \\
\text { Circón }\end{array}$ & $\begin{array}{l}\text { Porosidad } \\
\text { empuje } \\
\text { mercurio } \\
(\%)\end{array}$ & $\begin{array}{l}\text { Porosidad } \\
\text { intrusión } \\
(\%)\end{array}$ & $\mathbf{d}_{16}(\mu \mathrm{m})$ & $\mathbf{d}_{50}(\mu \mathrm{m})$ & $\mathbf{d}_{84}(\mu \mathrm{m})$ \\
\hline $\mathrm{F}$ & 46,5 & 46,9 & 0,27 & 0,23 & 0,14 \\
\hline $\mathrm{M}$ & 41,4 & 39,1 & 0,5 & 0,35 & 0,18 \\
\hline $\mathrm{H}$ & 35,0 & 34,3 & 2,40 & 1,47 & 0,62 \\
\hline
\end{tabular}



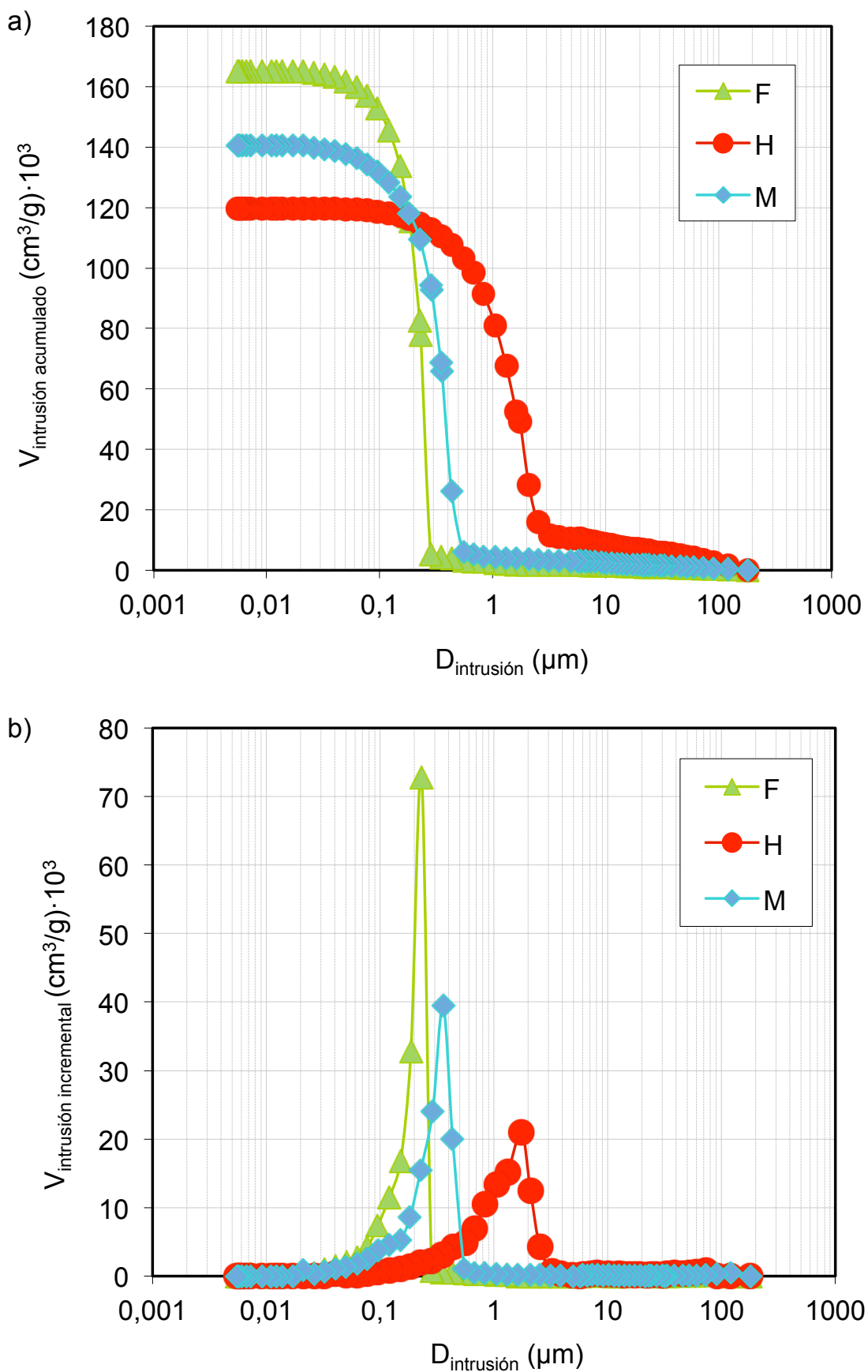

Figura 5.95. Distribuciones de tamaño de poro correspondientes a probetas secas de circón, de granulometrías: micronizado $(F)$, harina $(H)$ y mezcla $(M)$. a) acumulado y b)diferencial. 
5.3.1 Procesos físico-químicos que se desarrollan en los materiales durante su tratamiento térmico.

\subsubsection{Curvas de sinterización no isotermas.}

En la Figura 5.96 y Figura 5.97 se han representado las curvas de sinterización, en la forma $-\varepsilon_{A}=-\ln \left(A / A_{0}\right)_{S}$ vs $T$, obtenidas a $5 \mathrm{~K} / \mathrm{min}$, correspondientes a la serie $1(\phi=0,322)$ y serie $2 \quad(\phi=0,525)$, respectivamente. Se ha incluido en cada representación la curva de sinterización correspondiente a la granulometría del vidrio utilizado en el composite. De su examen se desprende lo siguiente:

i) Al comparar las curvas correspondientes a los compactos de vidrio GM y GF se aprecia que una disminución de la granulometría de las partículas de vidrio reduce significativamente (alrededor de $50^{\circ} \mathrm{C}$ ) las temperaturas de inicio y de máxima densificación. Además, con dicha modificación, el intervalo de cocción del material se reduce considerablemente. En efecto, el compacto GF, una vez alcanza su máxima densificación, $\varepsilon_{A, \max }$, comienza a expandir. Por el contrario, el compacto GM mantiene prácticamente sus dimensiones hasta unos $150^{\circ} \mathrm{C}$ después de alcanzar su máxima compacidad. Asimismo, se confirma que un aumento de la finura de la distribución de tamaño de partícula provoca un incremento de $\varepsilon_{A, \max }$, debido, principalmente, a una reducción de la compacidad del empaquetamiento en crudo de la pieza. 

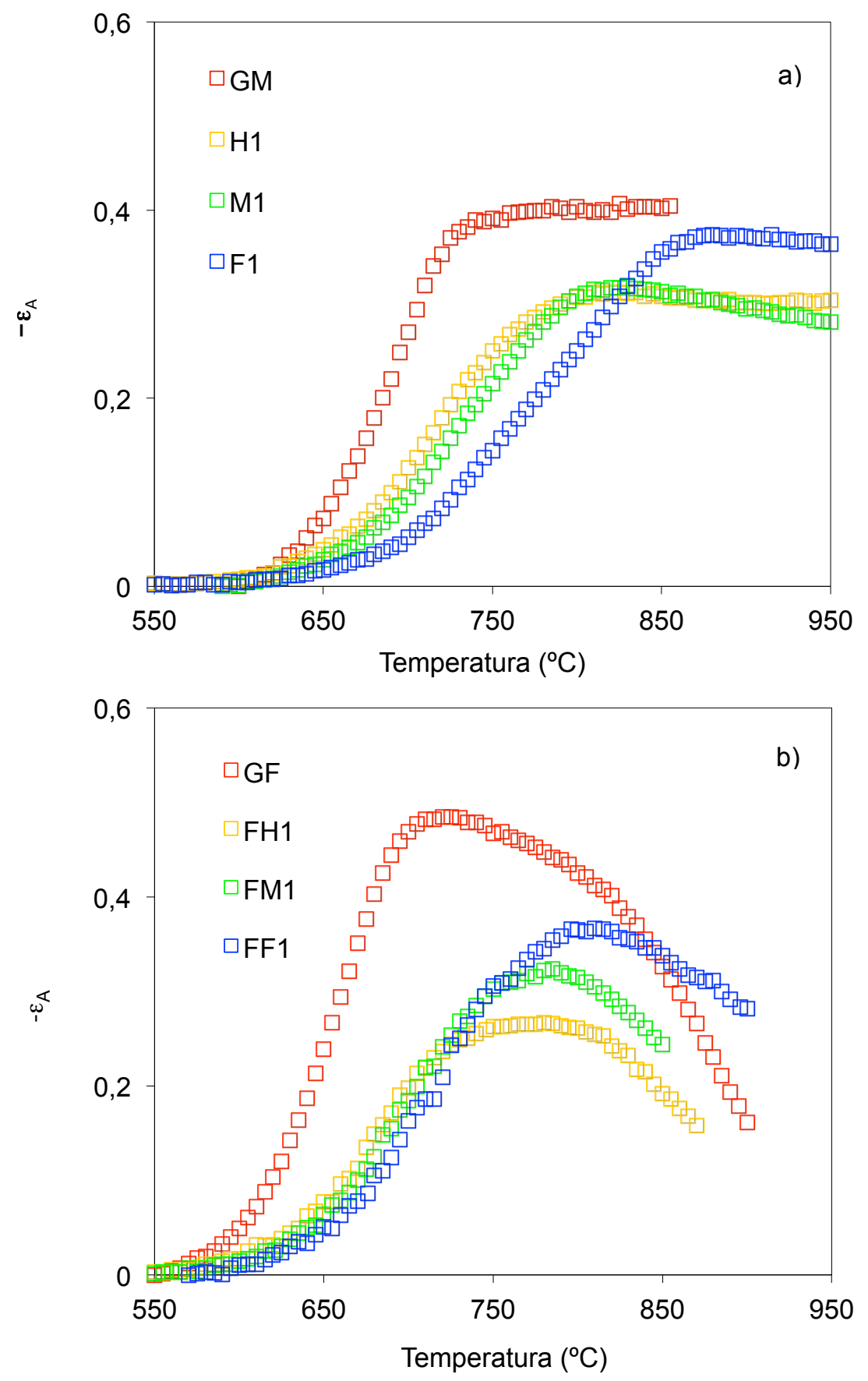

Figura 5.96. Curvas de sinterización: $-\varepsilon_{A}$ vs $T$, correspondientes a los composites de la serie $1 \quad(\phi=0,322)$. Velocidad de calentamiento $\beta=5 \mathrm{~K} / \mathrm{min}$. a) Preparados con la matriz vítrea $G M(F 1, H 1$ y $M 1), b)$ preparados con la matriz vitrea GF (FF1, FH1 y FM1). 

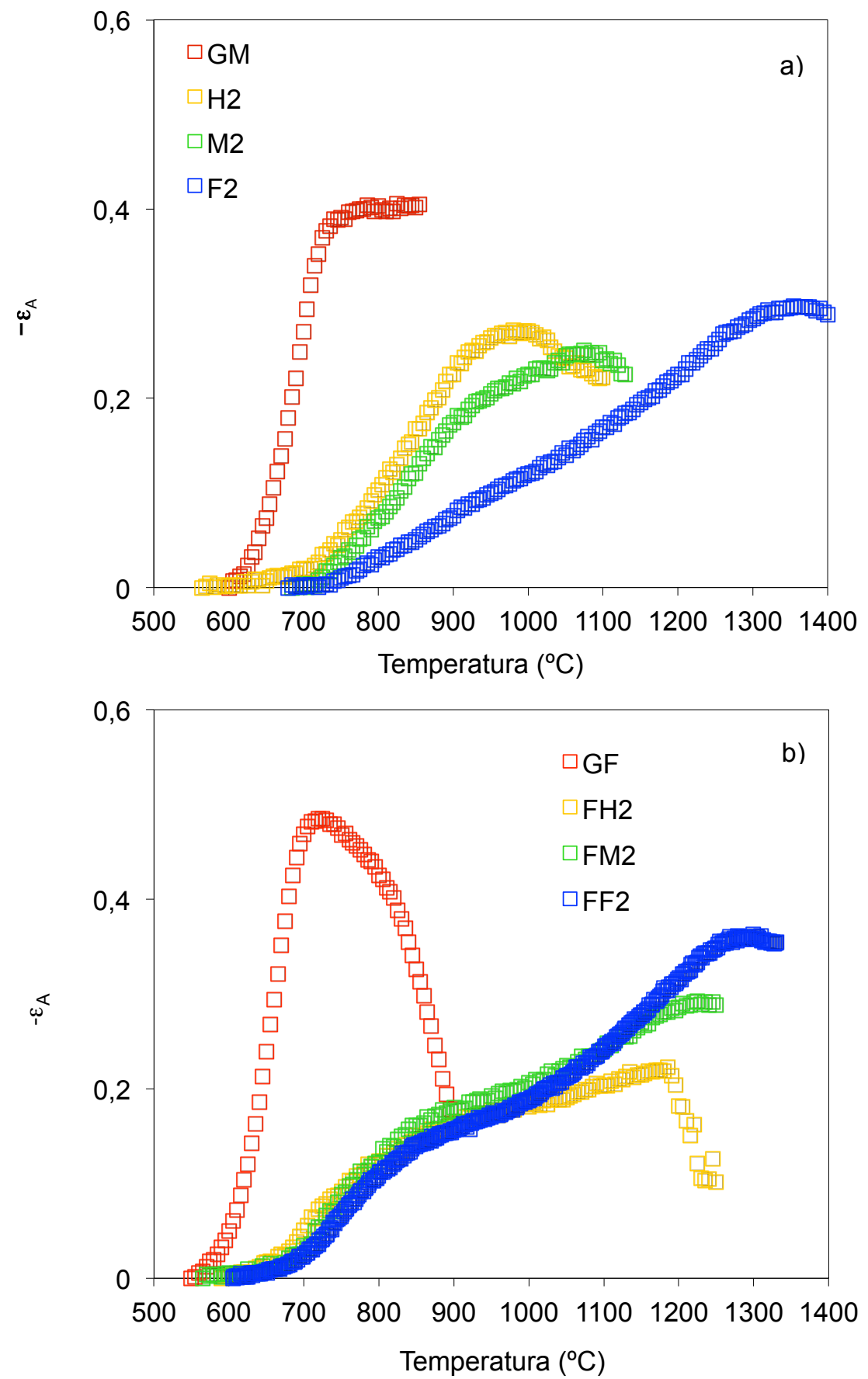

Figura 5.97. Curvas de sinterización: $-\varepsilon_{A}$ vs $T$, correspondientes a los composites de la serie $2(\phi=0,525)$. Velocidad de calentamiento $\beta=5 \mathrm{~K} / \mathrm{min}$. a) Preparados con la matriz vitrea $\mathrm{GM}$ (F2, H2 y M2), b) preparados con la matriz vitrea GF (FF2, FH2 y FM2). 
ii) Al analizar los resultados correspondientes a la serie 1 $(\phi=0,322)$ de la Figura 5.96 a y b se aprecia que, tanto para los composites obtenidos con el vidrio de granulometría GF como con el de la GM, los valores de inicio de sinterización y la máxima densificación disminuyen conforme aumenta el tamaño medio de partícula del circón utilizado. Para estos composites, con una fracción volumétrica de fase vítrea superior a la teóricamente necesaria para llenar los espacios entre partículas de circón, este comportamiento puede explicarse atendiendo, por una parte, a la variación que siguen el número de contactos vidrio-vidrio, vidrio-circón y circón-circón con el aumento del tamaño de grano del "filler". Y, por otra, al exceso de fase vítrea respecto al necesario para completar la densificación del composite. Ambos factores dependen de la distribución del tamaño de partícula. En efecto, en estos casos el mecanismo de sinterización es por flujo viscoso, cuya velocidad, en los estados inciales e intermedios del proceso, depende, entre otros factores, de la abundancia relativa de los tipos de contacto antes señalados. De forma general, el número de contactos vidriovidrio, que son los más activos, y los del tipo vidrio-circón, que también contribuyen en la sinterización, aunque en menor medida, deben de aumentar conforme lo hace el tamaño medio de circón, de acuerdo con los modelos teóricos de empaquetamiento de partículas.

Por otra parte, de forma general, la viscosidad efectiva del material (vidrio + circón), factor determinante de la velocidad de sinterización por este mecanismo, se reduce conforme aumenta el exceso de fase vítrea sobre la teóricamente necesaria para llenar los huecos entre partículas de filler, cuando estas empaquetan a su máxima densidad, de acuerdo con los modelos reológicos de suspensiones de partículas en líquidos. Los resultados de la Figura 5.95 y de la Tabla 5.18 confirman un 
aumento de la compacidad máxima de los lechos de partículas de circón obtenidos al pasar de la granulometría $\mathrm{F}$ a la $\mathrm{H}$, lo que también explicaría el comportamiento observado.

Además, tanto para los composites preparados con la matriz vítrea GM como para los obtenidos con la GF (Figura 5.96 a y b), un aumento del tamaño medio de partícula del circón (de F a $H$, pasando por $M)$ se traduce en una disminución de $\varepsilon_{A, \max }$, siendo ésta mucho más marcada en los composites obtenidos con partículas de vidrio de la granulometría GF. Este comportamiento, que puede deberse a un aumento de la compacidad en crudo de las probetas y/o de su porosidad final, con el aumento del tamaño medio de partícula del circón, será analizada posteriormente.

La influencia de la distribución de tamaños de partícula del vidrio sobre la sinterización de composites se traduce en dos aspectos fundamentales. Por una parte, al igual que ocurría en la sinterización de partículas de vidrio, los valores de la temperatura de inicio y final de la sinterización de los compactos obtenidos con matrices vítreas más finas, GF, son más bajas que las obtenidas con la más gruesa, GM. Por otra, también como ocurría en la sinterización de vidrio, la expansión de la probeta, tras alcanzar la máxima densificación, es prácticamente inmediata, para los compactos preparados con la matriz vítrea más fina, GF.

iii) A la vista de los resultados correspondientes a la serie 2 $(\phi=0,525)$, de la Figura 5.97 a y $b$, se comprueba que, al igual que ocurría en la sinterización de composites con menos circón, serie 1 , los valores de las temperaturas de inicio de la sinterización y máxima densificación disminuyen conforme aumenta el tamaño medio de partícula del circón utilizado. En lo que respecta a las etapas iniciales del proceso, el comportamiento de estos composites puede explicarse 
atendiendo al efecto que la variación de la granulometría del circón ejerce sobre el número y tipo de contactos, como ya se ha analizado en el apartado ii). Por el contrario, la sinterización en las etapas intermedia y final del proceso, y el efecto de la granulometría del vidrio y del circón sobre su desarrollo, es mucho más complejo debido a la concurrencia del proceso solución-reprecipitación del circón. Ahora bien, en el apartado 5.2.1.3.3 se estableció una clara correspondencia entre la presencia de un segundo tramo convexo en la curva $\frac{\mathrm{d} \alpha}{\mathrm{dt}}$ y la concurrencia de la sinterización por un mecanismo de soluciónreprecipitación. En consecuencia, se han calculado, siguiendo el procedimiento habitual, las curvas: $\frac{\mathrm{d} \alpha}{\mathrm{dt}}$ vs $\mathrm{T}$, correspondientes a los distintos composites de la serie 2 (Figura 5.98).

Se aprecia (Figura 5.97 y Figura 5.98) que en la sinterización de todos los composites de la serie 2 preparados con las partículas de vidrio de granulometría más fina (GF), FF2, FM2 y FH2, interviene el mecanismo de solución-reprecipitación del circón, a temperaturas superiores a los $900^{\circ} \mathrm{C}$, aunque de forma muy desigual, dependiendo de la granulometría del circón. En efecto, conforme aumenta el tamaño medio del circón la contribución de esta etapa a la densificación es menor, como puede comprobarse comparando las áreas bajo los dos tramos convexos de las curvas de sinterización (Figura 5.97b) y/o la longitud de los dos tramos de las curvas $\varepsilon_{A}$ vs $T$. Dicho comportamiento, se debe al efecto combinado de dos factores que varían simultáneamente con la modificación de la granulometría del circón. Por una parte, con el aumento de la finura del circón se reduce la compacidad en crudo de la pieza,

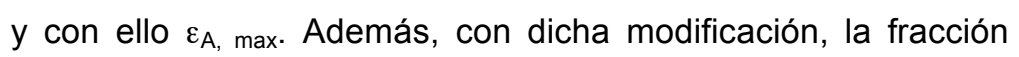
volumétrica máxima del empaquetamiento en crudo del circón 
también se reduce (aumenta la porosidad inicial) lo que implica un aumento del contenido en líquido necesario para completar la densificación por flujo viscoso. Este último factor debe compensarse con una mayor contribución al proceso de sinterización del mecanismo de solución-reprecipitación. Por otra, el proceso de solución-reprecipitación se ve favorecido con el aumento de la superficie específica del circón que se produce al reducirse su tamaño de partícula.

En lo que respecta a la sinterización de los composites preparados con partículas de vidrio más gruesas, GM, la influencia de la granulometría del circón sobre las curvas de sinterización, aun siguiendo la misma tendencia que con los preparados con las partículas de vidrio más finas, GF, es mucho más acusada tanto en lo referente a las temperaturas inicial y de máxima densificación como a la contribución del mecanismo de solución-reprecipitación a la densificación. Este último está prácticamente ausente en el composite preparado con harina de circón, H2, y es mayoritario, su contribución es del $70 \%$ (apartado 5.2.1.3) en el composite preparado con circón five (F2).

También es de destacar que, debido al marcado efecto de la granulometría de las partículas de vidrio sobre la sinterización por flujo viscoso, en las curvas de sinterización de los composites preparados con partículas de vidrio GF, los tramos correspondientes a este mecanismo y al de soluciónreprecipitación están más claramente diferenciados que en los otros composites (Figura 5.97b y Figura 5.98). 


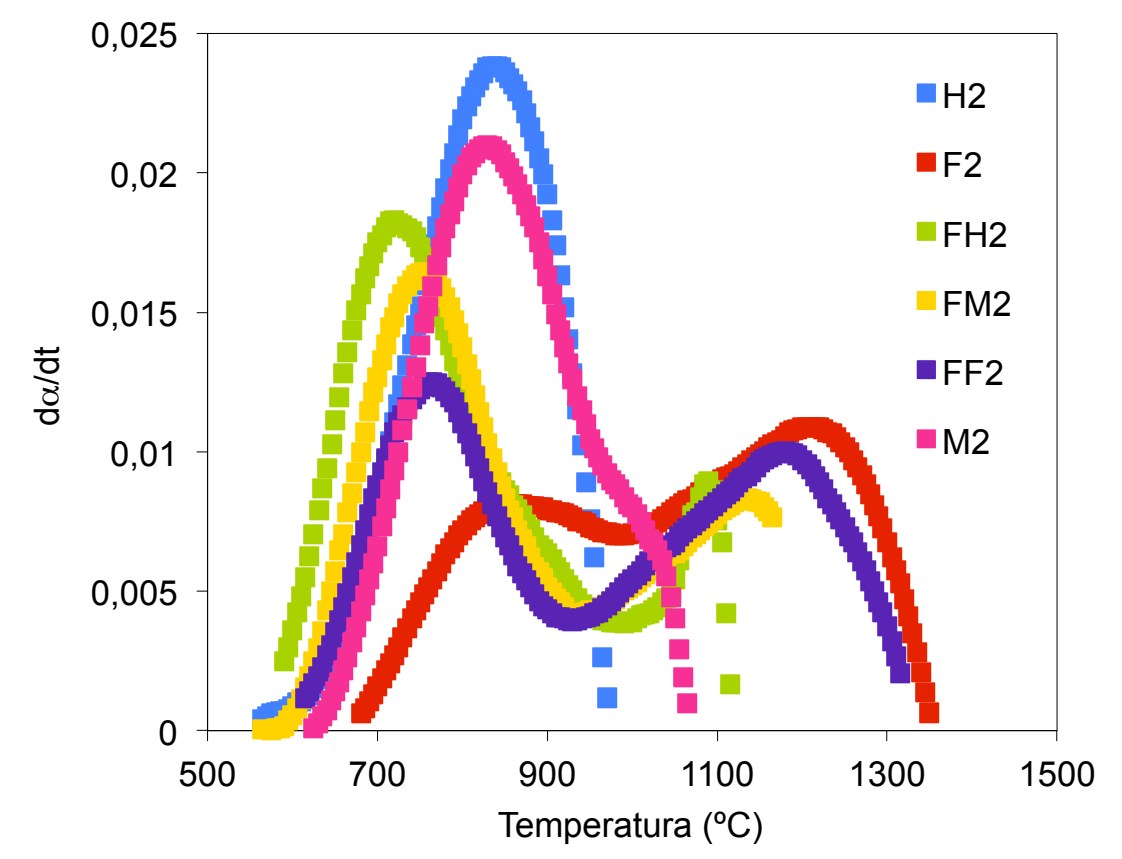

Figura 5.98. Curvas de sinterización: $\frac{\mathrm{d} \alpha}{\mathrm{dt}}$ vs $T$ correspondiente a los composites de la serie 2. Velocidad de calentamiento $\beta=5 \mathrm{~K} / \mathrm{min}$.

5.3.1.1.1 Contracción superficial máxima de la silueta, $\varepsilon_{A \text {, max }}$.

A partir de las curvas de sinterización, obtenidas para cada uno de los composites que se detallan en la Tabla 5.17, empleando cinco velocidades de calentamiento distintas, se han determinado los correspondientes valores promedio de $\varepsilon_{\mathrm{A} \text {, max }} \mathrm{y}$ su desviación estándar. En la Figura 5.99 a y b se han representado estos resultados frente a $\phi$, para los composites preparados con la granulometría del vidrio GM y GF respectivamente. De su examen se desprende lo siguiente: 

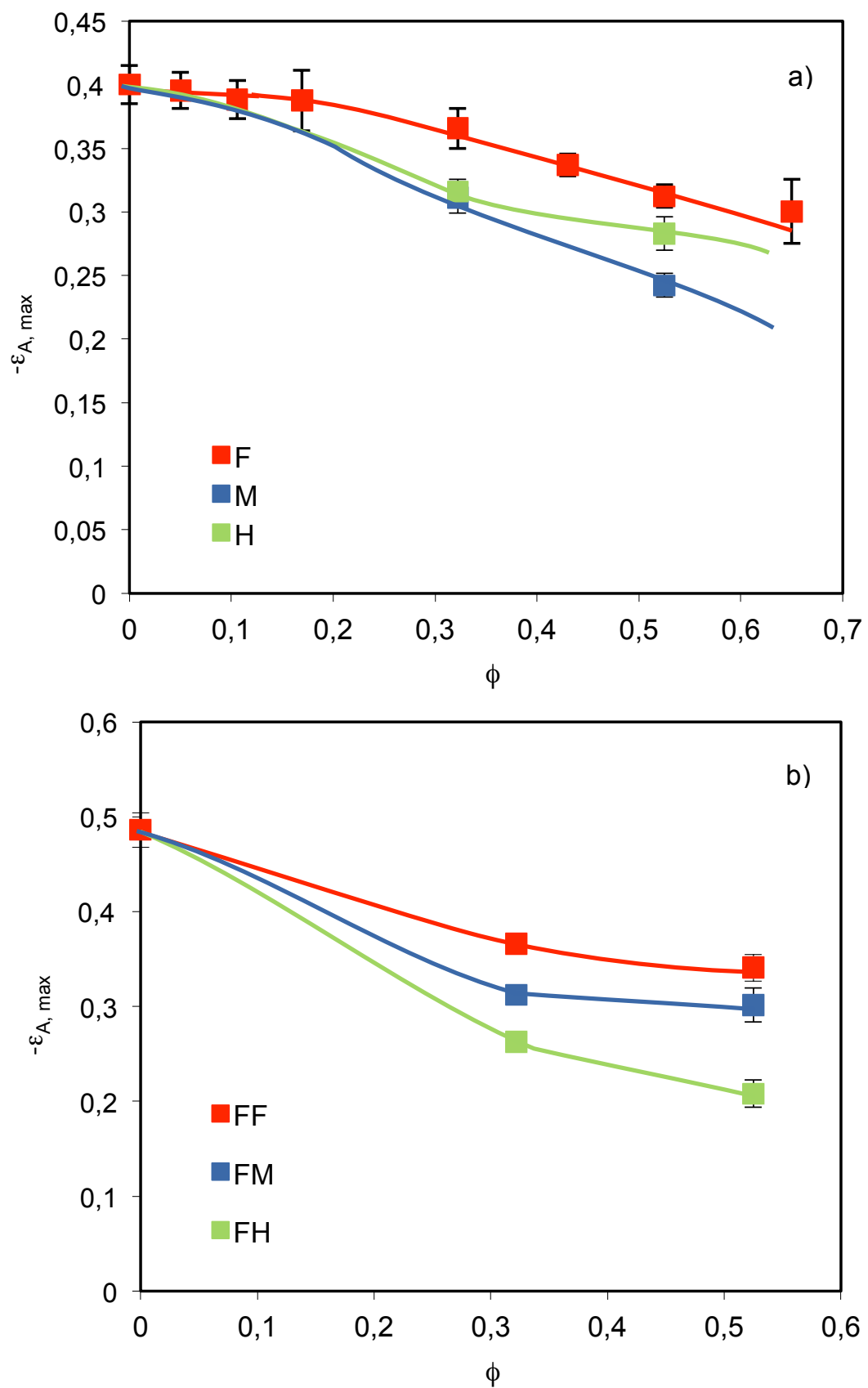

Figura 5.99. Variación de la contracción superficial máxima promedio, $\varepsilon_{A \text {, max, }}$ correspondientes a los composites de las series 1 y 2 frente a $\phi$. Velocidad de calentamiento $\beta=2,5,15,25$ y 60K/min. a) Granulometría del vidrio $G M, b)$ granulometría del vidrio GF. Se incluyen los resultados de la Figura 5.35. 
En ambos casos, un aumento del contenido en circón conduce a una reducción de la contracción máxima de cocción, $\varepsilon_{\mathrm{A} \text {, max }}$. Ahora bien, el efecto depende de las distribuciones de tamaño de partícula del vidrio y del circón. De forma general, la adición de circón a la matriz vítrea más fina, GF (Figura $5.99 b)$, ejerce un mayor efecto que la correspondiente a la matriz vítrea más gruesa, GM (Figura 5.99a). Además, este efecto es tanto mayor cuanto más gruesa es la granulometría del circón añadido. En el caso de los composites preparados con la matriz vítrea $\mathrm{GM}$, se produce una anomalía; la probeta $\mathrm{M} 2$ contrae menos que la $\mathrm{H} 2$.

Al igual que se hizo en el apartado 5.2.1.1.1, se ha calculado el grado de reducción de la contracción máxima con el aumento del contenido en circón, $\phi$, para todas las probetas, de acuerdo con la ec. 5.17 (Figura 5.100).

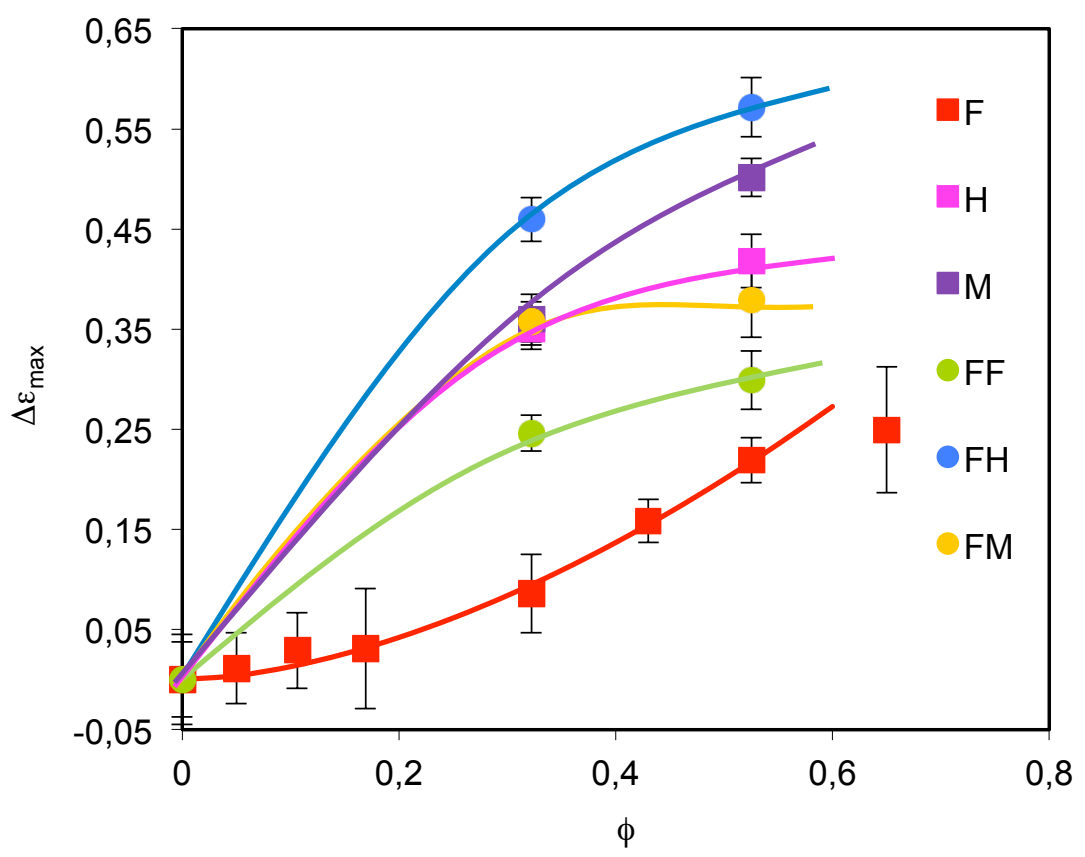

Figura 5.100. Variación del grado de reducción de la contracción máxima, $\Delta \varepsilon_{A}$, max, correspondiente a los composites de las series 1 y 2 frente a $\phi$. Velocidad de calentamiento $\beta=5 \mathrm{~K} / \mathrm{min}$. Se incluyen los resultados de la Figura 5.36. 
Al igual que ocurría con $\varepsilon_{\mathrm{A}, \max }, \Delta \varepsilon_{\mathrm{A}, \max }$ aumenta con $\phi$ dependiendo dicha variación de las granulometrías del vidrio y del circón empleados. No obstante, este efecto es tanto mayor conforme aumenta el tamaño medio de partícula del circón utilizado, si se exceptúan los resultados correspondientes a los composites M2 y H2. Este comportamiento no puede deberse exclusivamente al efecto que ejercen estas variables ( $\phi$, granulometría) sobre el incremento de la porosidad final de las probetas, sino que, en gran medida, también depende de su efecto sobre de la compacidad de las piezas en crudo (o al inicio de la contracción de cocción, $\rho_{0}$ ) ya que estas características dependen, a su vez, del tamaño y forma de las partículas de ambos componentes y de la proporción de estas últimas.

5.3.1.2 Evolución de las características texturales y microestructurales de los composites con el avance del proceso de sinterización.

\subsection{Análisis cualitativo (micrografías SEM).}

Las micrografías de las secciones pulidas de los diferentes GMC's que se han seleccionado en la Figura 5.101 a Figura 5.103 han sido obtenidas a velocidades de calentamiento de $5 \mathrm{~K} / \mathrm{min}$, hasta diferentes temperaturas máximas. Éstan ilustran, para los composites F2, FF2 y FH2, la evolución de su microestructura con el avance de la sinterización. Se han seleccionado estos composites debido, fundamentalmente, a las siguientes razones: a) el efecto de la granulometría de los componentes (matriz vítrea y circón) sobre la sinterización del composite y sobre su evolución microestructural es tanto mayor cuanto más alto es el contenido en circón, b) la variación de la razón tamaño medio volumétrico de circón/tamaño medio volumétrico del vidrio, $d_{z}^{v} / d_{g}^{v}$, es la más alta, lo que permite estudiar mejor el efecto de esta variable. En efecto, los valores de $d_{z}^{v} / d_{g}^{v}$ son: 0,25 para el composite $F 2$, 0,95 para el FF2 y 10 para el FH2. También se han seleccionado las temperaturas de cocción para hacer más hincapié en las microestructuras iniciales e intermedias, ya que las que se obtienen a alta temperatura, etapa 
final, solo se diferencian en el tamaño relativo del poro y del grano de circón. Los resultados más significativos se resumen a continuación:
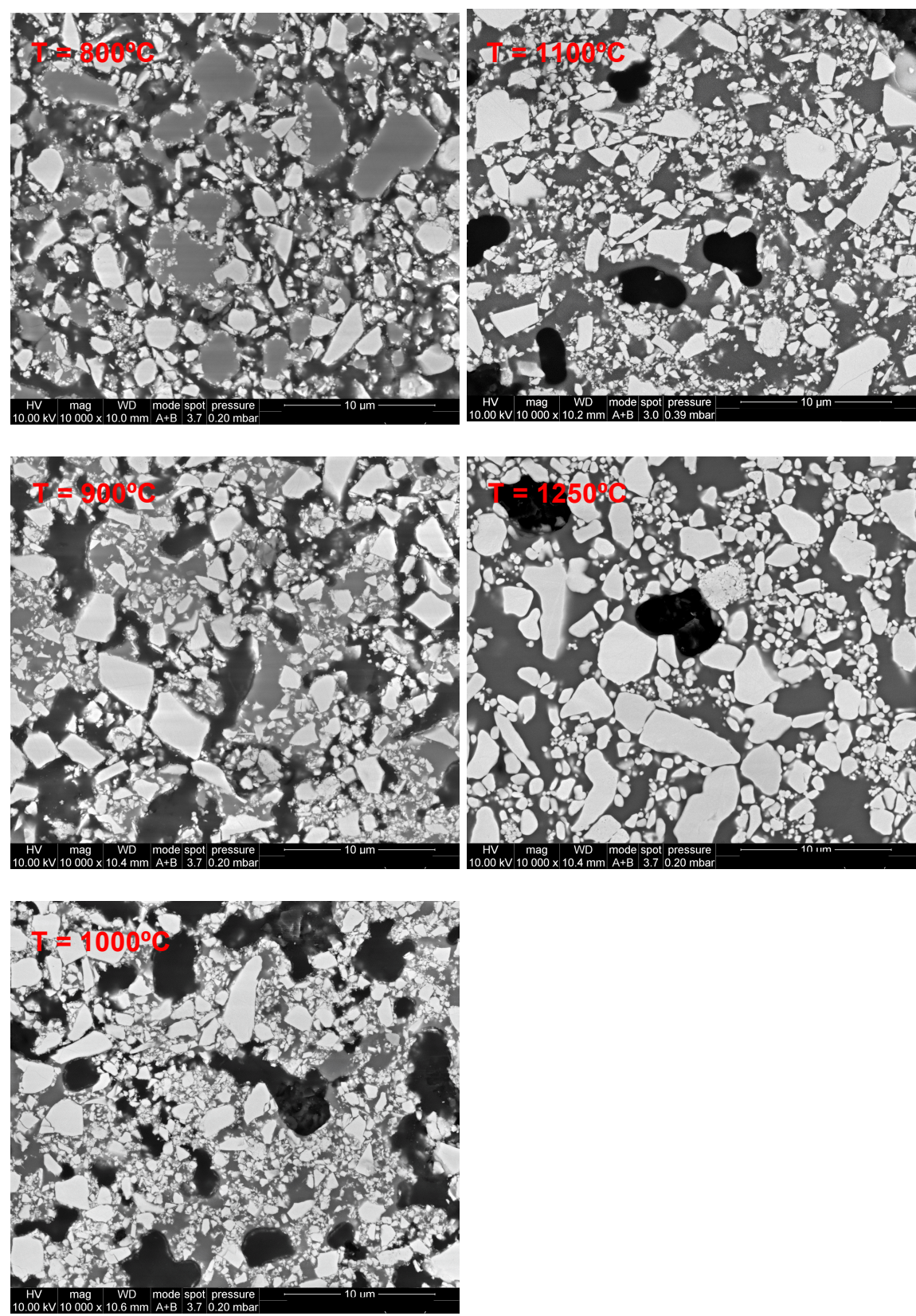

Figura 5.101. Micrografías (x10000) correspondientes a probetas del composite F2 $\left(\phi=0,525, \quad d_{z}^{v} / d_{g}^{v} \approx 0,25\right)$ cocidas a diferentes temperaturas máximas: $800^{\circ} \mathrm{C}(\alpha=0,10), 900^{\circ} \mathrm{C}(\alpha=0,24), 1000^{\circ} \mathrm{C}(\alpha=0,40), 1100^{\circ} \mathrm{C}(\alpha=0,58)$ y $1250^{\circ} \mathrm{C}(\alpha=0,86)$. Velocidad de calentamiento $\beta=5 \mathrm{~K} / \mathrm{min}$. 

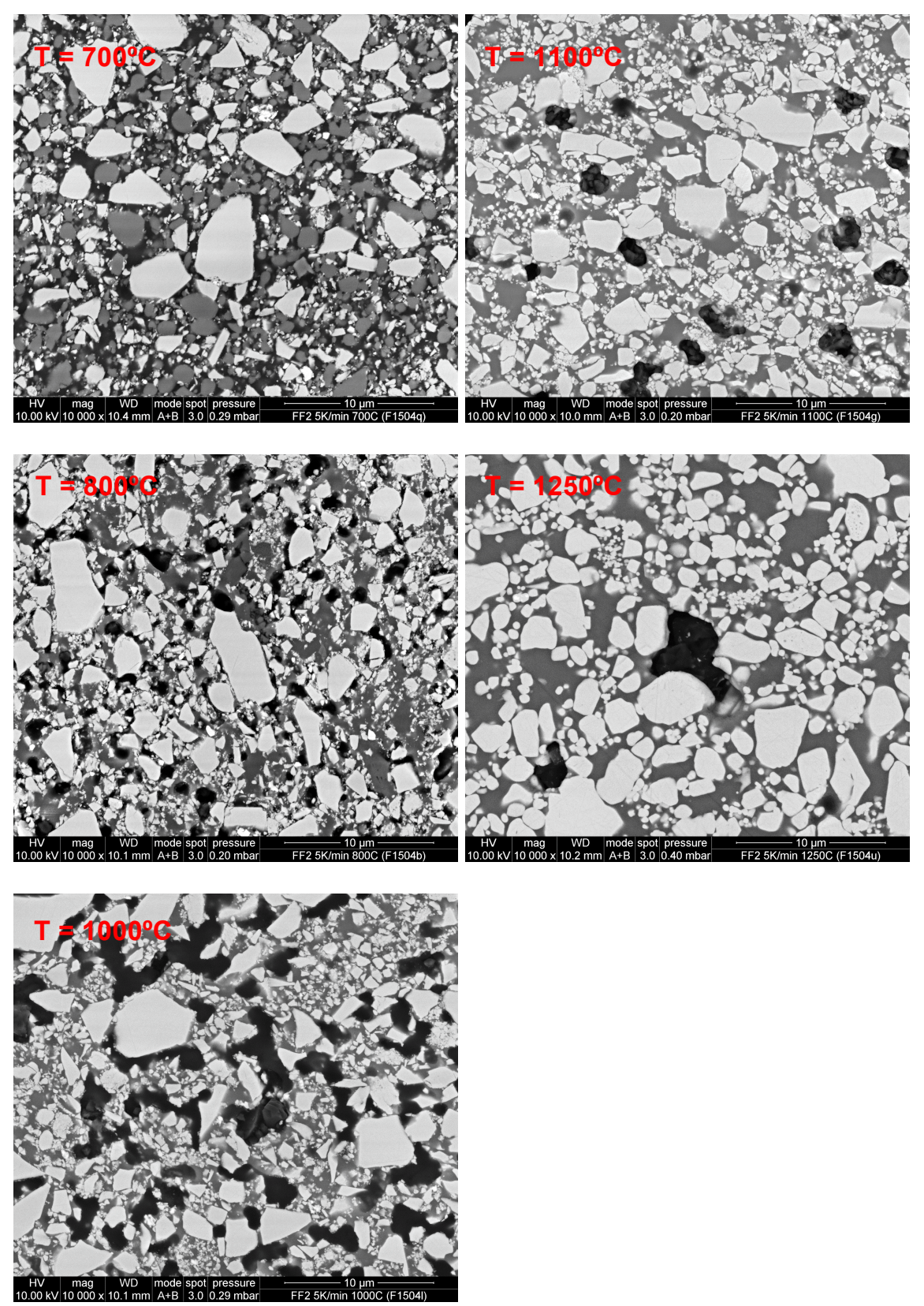

Figura 5.102. Micrografías (x10000) correspondientes a probetas del composite FF2 $\left(\phi=0,525, d_{z}^{v} / d_{g}^{v} \approx 0,95\right)$ cocidas a diferentes temperaturas máximas: $700^{\circ} \mathrm{C}(\alpha=0,10), 800^{\circ} \mathrm{C}(\alpha=0,28), 1000^{\circ} \mathrm{C}(\alpha=0,48), 1100^{\circ} \mathrm{C}(\alpha=0,60)$ y $1250^{\circ} \mathrm{C}(\alpha=0,92)$. Velocidad de calentamiento $\beta=5 \mathrm{~K} / \mathrm{min}$. 

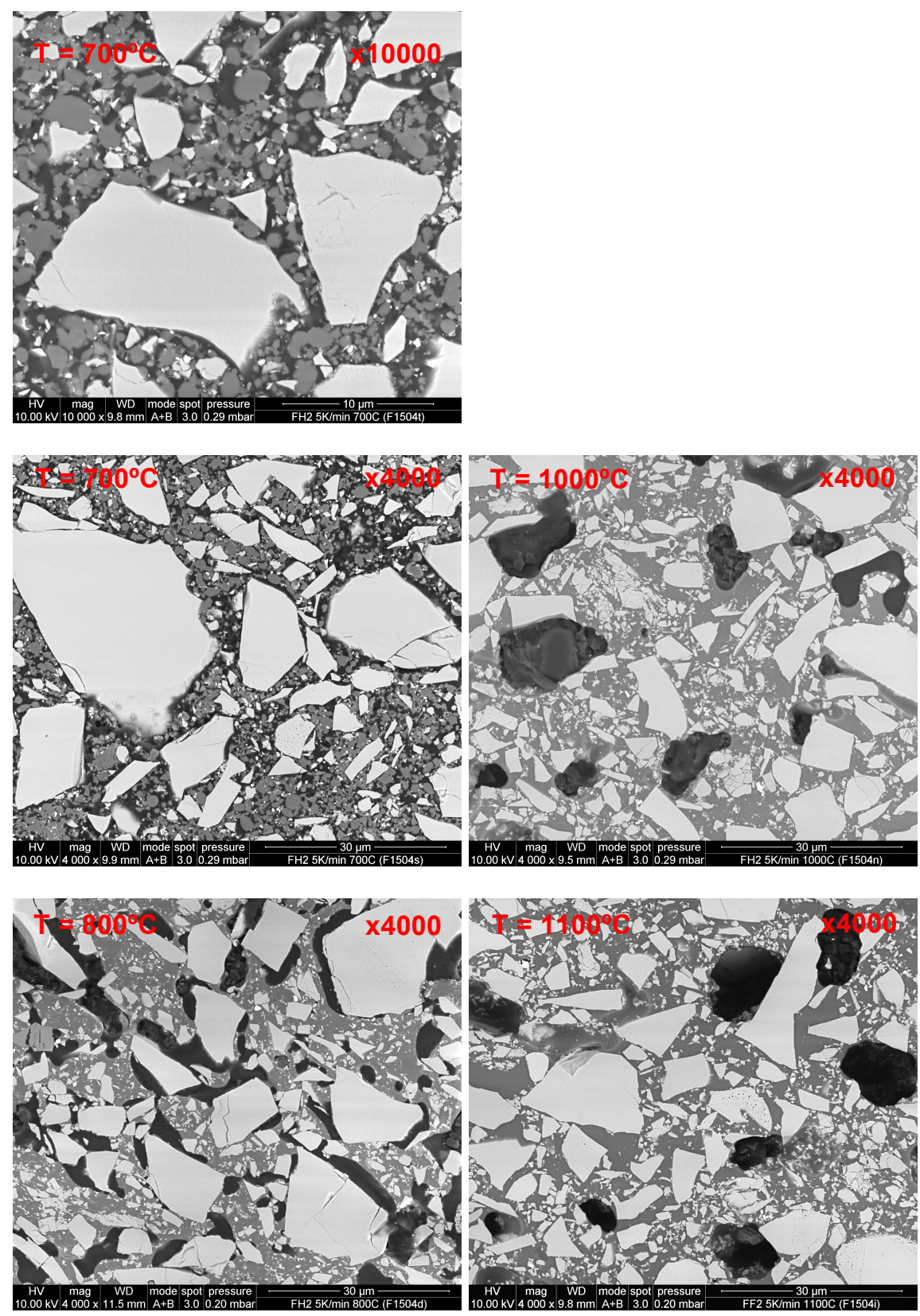

Figura 5.103. Micrografías (x4000 y x10000) correspondientes a probetas del composite $\mathrm{FH} 2\left(\phi=0,525, d_{z}^{v} / d_{g}{ }^{v} \approx 10\right)$ cocidas a diferentes temperaturas máximas: $700^{\circ} \mathrm{C}(\alpha=0,24), 800^{\circ} \mathrm{C}(\alpha=0,52), 1000^{\circ} \mathrm{C}(\alpha=0,78), 1100^{\circ} \mathrm{C}$ $(\alpha=0,92)$. Velocidad de calentamiento $\beta=5 \mathrm{~K} / \mathrm{min}$. 
i) De forma general, las distribuciones espaciales de las partículas, tanto de vidrio como de circón, en estados iniciales, intermedios y finales de la sinterización, son bastante homogéneas, lo que confirma que la preparación de los composites, especialmente el mezclado de los componentes, es apropiado.

ii) Al observar las micrografías correspondientes a estados iniciales de la sinterización $\left(800^{\circ} \mathrm{C}\right.$ para $\mathrm{F} 2$ y $700^{\circ} \mathrm{C}$ para FF2 y $\mathrm{FH} 2$ ), se aprecian microestructuras muy diferentes. En el caso de F2 (Figura $5.101,800^{\circ} \mathrm{C}$ ), las áreas vítreas consisten en partículas individualizadas de vidrio con cantos redondeados y, ocasionalmente, en zonas más irregulares, resultantes de la coalescencia de partículas originales de vidrio. Ambas están recubiertas generalmente de pequeñas partículas de circón adheridas superficialmente, formando en algunos casos una costra prácticamente continua, aunque algunas áreas irregulares contienen pequeñas partículas de circón embebidas en su interior. Las partículas de circón de mayor tamaño, que también están adheridas al vidrio, solo lo hacen por una parte de su superficie. En general, se observan más contactos circóncircón que en los otros composites. En el caso de los composites preparados con las partículas de vidrio más finas (FH2 y FF2), éstas están completamente esferoidizadas, e incluso forman áreas irregulares resultantes de su coalescencia, especialmente en la $\mathrm{FH} 2$ (Figura $5.103,700^{\circ} \mathrm{C}$ ) en el que el número de contactos vidrio-vidrio es mayor que en la FF2 (Figura $5.102,700^{\circ} \mathrm{C}$ ). Además, en el composite $\mathrm{FH} 2$, los contactos que se establecen entre el vidrio y el circón son poco numerosos y el ángulo de mojado del vidrio sobre la superficie del circón es mayor de $90^{\circ}$ indicando un pobre mojado a esta temperatura. 
iii) En un estado intermedio de sinterización, pero aún incipiente, $900^{\circ} \mathrm{C}$ para $\mathrm{F} 2$ y $800^{\circ} \mathrm{C}$ para $\mathrm{FF} 2$ y $\mathrm{FH} 2$, la matriz vítrea, con partículas de circón de tamaño pequeño y mediano, dispersas o embebidas, forma grandes áreas irregulares. El área de contacto entre las partículas de circón grandes y la matriz vítrea sigue siendo baja, especialmente para el FH2 (Figura 5.103, $800^{\circ} \mathrm{C}$ ), preparado con harina de circón. Los poros son irregulares, abundantes y grandes, a excepción del composite FF2 preparado con los componentes más finos (Figura 5.102, $\left.800^{\circ} \mathrm{C}\right)$.

iv) La evolución de la textura porosa con el avance de la sinterización en las etapas intermedia y final de la densificación, presenta rasgos comunes para todos los composites, tales como un crecimiento del tamaño de poro y una reducción de su número. Ahora bien, la magnitud del crecimiento de poro, su mecanismo y la variación de su forma parecen ser diferentes dependiendo de la granulometría de cada componente de partida (circón y vidrio). En efecto, para el composite FH2 (Figura 5.103), un incremento de la temperatura de 700 a $800^{\circ} \mathrm{C}$, lo que supone un aumento del grado de avance del proceso de $\alpha \approx 0,25$ a $\alpha \approx 0,5$ aproximadamente, provoca un considerable cambio de la forma y tamaño de los poros, pasando de irregulares, pero bastante isométricos, y pequeños (del orden de $1 \mu \mathrm{m}$ o menos) a mayoritariamente alargados y grandes (del orden $10 \mu \mathrm{m}$ o más), y estando además localizados en la superficie de las partículas de circón más grandes. A temperaturas más elevadas (Figura $5.103,1000^{\circ} \mathrm{C}$ y $1100^{\circ} \mathrm{C}$ ), la forma de estos poros se va volviendo más esférica, aunque su tamaño medio y su localización no cambian demasiado. Los posibles mecanismos de generación de estos poros grandes parecen ser la formación, en crudo, de clústers de partículas de vidrio rodeadas por grandes partículas de circón y/o la formación 
de una estructura rígida de partículas grandes de circón en cuyo interior se sitúan partículas más pequeñas de vidrio. En ambos casos, estas regiones de vidrio sinterizan de forma prácticamente independiente del resto, debido principalmente a que la presión de sinterización que se desarrolla en el empaquetamiento de estas partículas de vidrio no es suficiente para deformar la estructura y/o arrastrar las gruesas partículas de circón, lo que provoca la separación de la matriz vítrea de la partícula de circón, causando, finalmente, un poro de gran tamaño en la superficie de ésta. Pascual et al. [133] consideran también otro mecanismo de formación de grandes poros en contacto con inclusiones rígidas de gran tamaño. Según estos autores, las partículas de vidrio que están en contacto con grandes inclusiones rígidas, en su caso fibras de circona, aunque modifiquen su forma, pasando de irregulares a esféricas con el aumento de la temperatura/tiempo, no sinterizan manteniendo el volumen inicial de poros.

v) En el caso de los composites F2 (Figura 5.101) y FF2 (Figura 5.102), preparados con circón micronizado, $F$, el crecimiento del tamaño de poro y su evolución con la densificación, en la etapa intermedia de sinterización, es consecuencia de la reordenación de partículas y/o aglomerados, el habitual en este tipo de materiales. Ahora bien, debido a que en el composite F2 la distribución granulométrica de las partículas de vidrio es más ancha y su tamaño medio más grueso que en el FF2, el tamaño de poro y su crecimiento durante la etapa intermedia de sinterización es mayor. En la etapa final, la formación de poros en ambos composites está asociado, como ya se ha indicado en el apartado 5.2.1.2, a la formación de cavidades por agrupación de partículas de circón y a la generación de gas en el vidrio y/o a su expansión. 


\subsection{Compacidad máxima, $\rho_{\max }, \mathrm{e}$ incial, $\rho_{0}$.}

Siguiendo el mismo procedimiento que el utilizado en el apartado 5.2.1.2.2, a partir de las dimensiones y masa de las probetas y de la variación que sigue la superficie de la silueta y su altura en el ensayo HSM, se han calculado, para todos los composites estudiados, los valores de $\rho_{\max }$. En la Figura 5.104 se representan, frente a $\phi$, los valores de esta propiedad que resultan de promediar los cinco valores individuales correspondientes a las cinco velocidades de calentamiento utilizadas. Como ya se ha indicado antes, la precisión estimada para dicha determinación, de $\pm 0,02$, es siempre superior o muy similar a la desviación estándar que resulta de promediar los resultados individuales. Además, tampoco se apreció ninguna variación sistemática de $\rho_{\max }$ con la velocidad de calentamiento al igual que ocurría en el apartado 5.2.1.2.2.

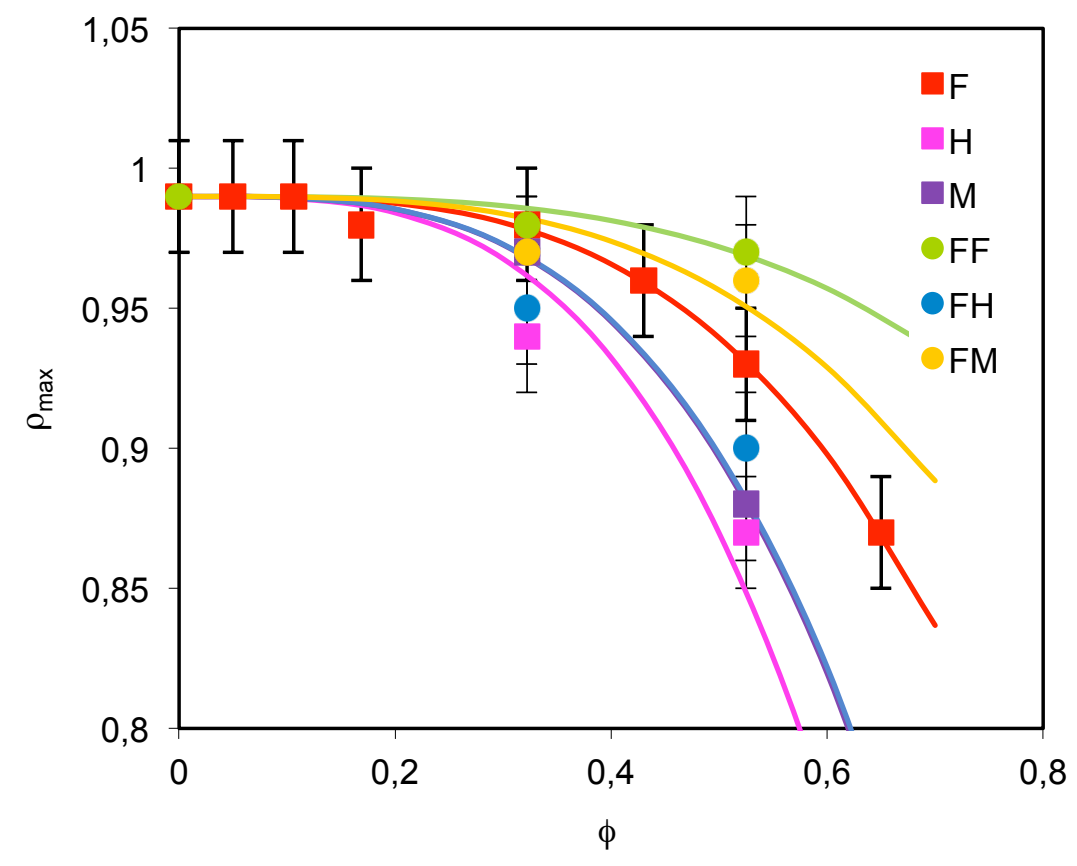

Figura 5.104. Variación de $\rho_{\max }$ promedio frente a $\phi$, correspondientes a los composites de las series 1 y 2. Velocidades de calentamiento $\beta=2,5,15$, 25 y 60K/min. Se incluyen los resultados de la Figura 5.49 . 
Se comprueba que, de forma general, la adición de circón al composite reduce su compacidad máxima, $\rho_{\max }$, siendo dicho efecto dependiente tanto de la granulometría de la matriz vítrea como del circón. En efecto, a igualdad de contenido y tamaño del circón, los composites preparados con la matriz vítrea más fina, GF, son menos porosos, $\rho_{\max }$ más alta. Análogamente, un aumento del tamaño medio del circón, manteniendo constantes las demás variables ( $\phi$ y granulometría de la matriz vítrea), provoca una disminución considerable de la compacidad máxima, $\rho_{\max }$. Estos resultados son consistentes con los obtenidos en el apartado anterior y con los mecanismos de formación de poros que allí se han descrito. En efecto, el aumento del contenido en partículas gruesas del circón, al utilizar harina de circón, $\mathrm{H}$, en vez de la mezcla harina y micronizado, $M$, o solo micronizado, F, y/o al aumentar el contenido en circón, $\phi$, en los dos primeros tipos de composites, se incrementa considerablemente la presencia de gruesos poros en contacto con grandes partículas. Análogamente, a igualdad de contenido en circón, al emplear partículas de vidrio más finas se reduce el número de contactos circón-circón, lo que a su vez también disminuye la probabilidad de que se generen cavidades formadas por pequeñas partículas de circón, responsables de la formación de porosidad fina.

Este efecto es tan marcado a altos contenidos en circón, $\phi=0,525$, que estos composites solo pueden obtenerse con baja porosidad, empleando la granulometría del circón más fina, $F$, aunque para ello se requieran temperaturas de cocción muy altas $\left(1300^{\circ} \mathrm{C}\right.$ para FF2 y $1350^{\circ} \mathrm{C}$ para F2), incluso empleando velocidades de calentamiento bajas $(5 \mathrm{~K} / \mathrm{min})$, como puede comprobarse en las Figura 5.97 a y b.

Con vistas a cuantificar el efecto combinado que ejercen el contenido en circón y las granulometrías de cada componente sobre la compacidad máxima alcanzable, $\rho_{\max }$, basándose en la ec. 5.19 , que describe el efecto de $\phi$ sobre la reducción de la compacidad máxima, para composites de la serie $\mathrm{F}$, los resultados de la Figura 5.104 se han tratado de ajustar a la ecuación: 
$\rho_{\max }=\rho_{\max }(0)-(\kappa \phi)^{3,3}$

ec. 5.37

siendo $\rho_{\max }(0)=0,99$ la compacidad máxima media de las probetas de vidrio у к un parámetro de ajuste, dependiente de la granulometría de los dos componentes, que expresa el efecto de esta variable sobre la compacidad máxima. Con los valores de $\kappa$ que se detallan en la Tabla 5.19 los resultados de la Figura 5.104 se ajustaron bien a la ec. 5.37, (Figura 5.105), resultando un valor de $S^{2}=0,00195$.

Tabla 5.19. Influencia de la granulometría del circón y del vidrio sobre el parámetro $\kappa$ de la ecuación $\rho_{\max }=0,99-(\kappa \phi)^{3,3}$.

\begin{tabular}{|l|l|l|l|}
\hline Composite & vidrio & Circón & $\boldsymbol{\kappa}$ \\
\hline F & GM & F & 0,81 \\
\hline M & GM & $0,5 \mathrm{~F}-0,5 \mathrm{H}$ & 0,97 \\
\hline H & GM & H & 1,05 \\
\hline FF & GF & F & 0,59 \\
\hline FM & GF & $0,5 \mathrm{~F}-0,5 \mathrm{H}$ & 0,71 \\
\hline FH & GF & H & 0,97 \\
\hline
\end{tabular}




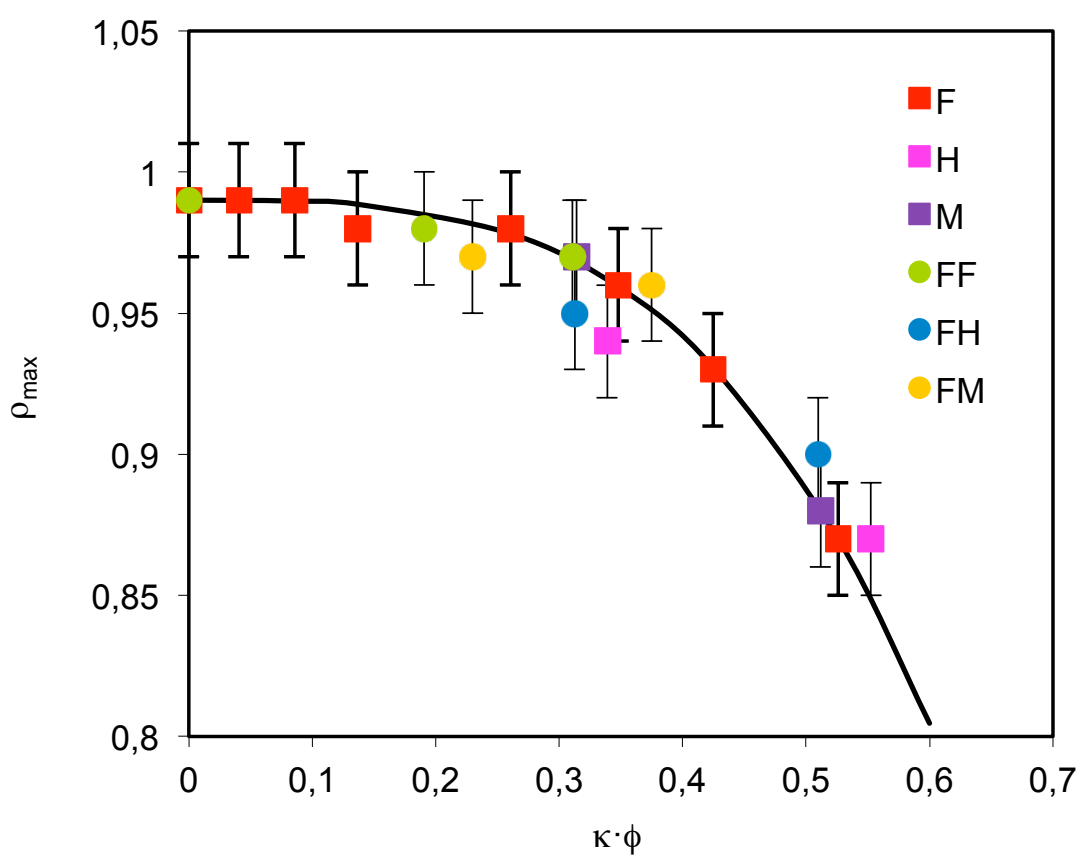

Figura 5.105. Ajuste de los resultados experimentales de la Figura 5.104 a la ec. 5.37.

Los resultados de la Tabla 5.19 confirman que la disminución de la compacidad máxima alcanzable, $\rho_{\max }$, de un composite debido al incremento del contenido en circón es tanto mayor cuanto más gruesas son las granulometrías del circón y del vidrio; es decir, conforme aumenta $\kappa$.

Así pues, el grado de reducción de la compacidad máxima de un composite por adición de circón será:

$\Delta \rho_{\max }^{*}=(\kappa \phi)^{3,3}$ ec. 5.38

Se comprueba, por tanto, que $\Delta \rho^{*}{ }_{\max }$ sigue una variación lógica con el contenido en circón, $\phi$, y con la granulometría de los componentes, a diferencia de lo que ocurría con $\Delta \varepsilon_{\mathrm{A} \text {, max. }}$ 
A partir de los valores promedio de $\rho_{\max }$ y de los valores de $\varepsilon_{\mathrm{A} \text {, max }}$ se han calculado, mediante la ec. 5.15 los valores de la compacidad al inicio de la sinterización, $\rho_{0}$, (Figura 5.106). Se comprueba que para los composites FF, FH y FM, obtenidos con las partículas de vidrio más finas, GF, un aumento del contenido en circón, $\phi$, conduce a un incremento considerable de la compacidad en crudo, $\rho_{0}$, para bajos y moderados contenidos en circón, $\phi \leq 0,322$. Este efecto es tanto mayor cuanto más gruesa es la granulometría del circón empleado. En el caso de los composites $F, H$ Y M, preparados con partículas de vidrio más gruesas, GM, el efecto del contenido en circón sobre $\rho_{0}$ es menos pronunciado, debido a que la distribución granulométrica del vidrio GM es más ancha y su tamaño medio más grande que la del GF. A diferencia del caso anterior, este efecto es mayor para la granulometría $\mathrm{M}$ que para la $\mathrm{H}$ debido a que la mezcla de la granulometría $\mathrm{M}$ y $\mathrm{GM}$ conduce a un empaquetamiento de partículas más compacto que la de la mezcla de la granulometría H y GM. Este comportamiento explica la anomalía que se observó al estudiar el efecto de la granulometría sobre $\Delta \varepsilon_{\mathrm{A}, \max }$ (Figura $5.100)$. 


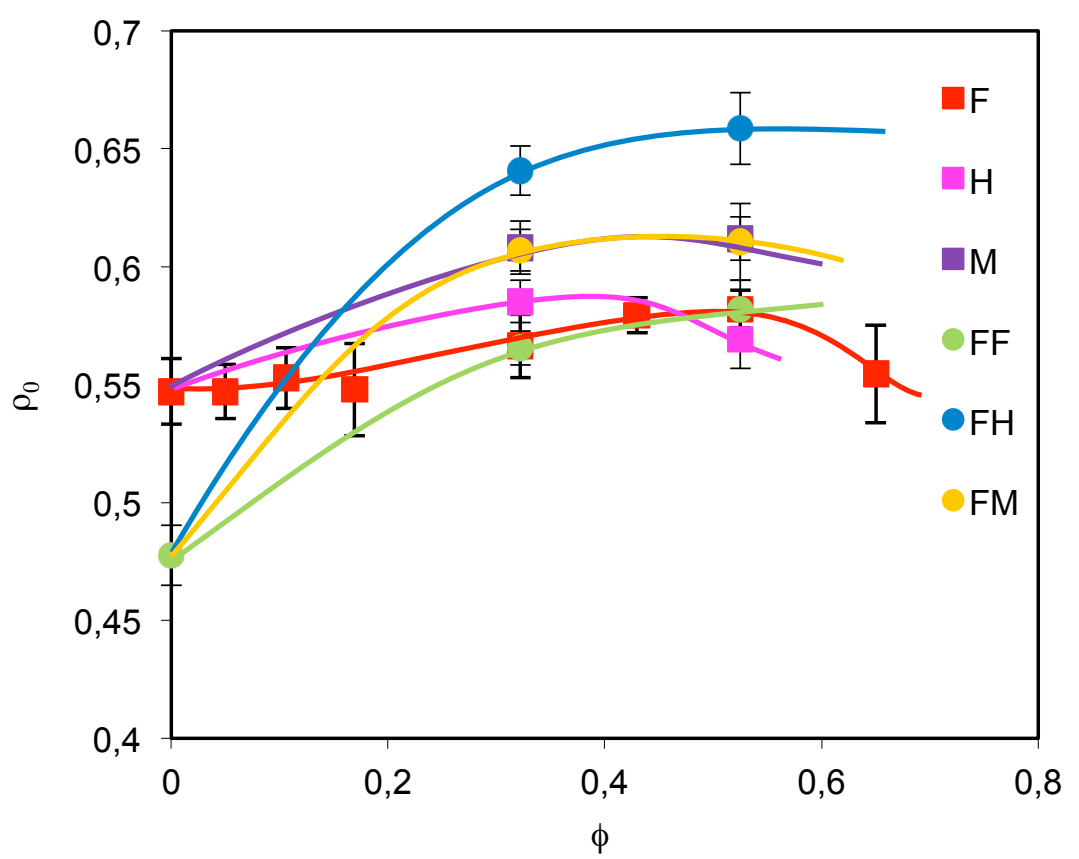

Figura 5.106. Variación con $\phi$ de los valores de la compacidad al inicio de la sinterización calculada, $\rho_{0}$, correspondientes a los composites de las series 1 y 2. Velocidades de calentamiento $\beta=2,5,15,25$ y 60K/min. Se incluyen los resultados de la Figura 5.49.

\subsubsection{Análisis cinético de la sinterización. Determinación de los parámetros cinéticos}

\subsubsection{Compactos de partículas de vidrio de la granulometría GF.}

Previamente a la determinación de los parámetros cinéticos de los distintos composites, en este apartado se han calculado los correspondientes al componente vítreo de granulometría GF.

Las curvas de sinterización correspondientes a las cinco velocidades de calentamiento habitualmente utilizadas se han representado en la Figura 5.107. La forma de éstas es sigmoidal, por lo que, de acuerdo con los resultados del apartado 5.1.2.1.3.1, y siguiendo el mismo procedimiento que el descrito allí, al ajustar los pares de valores correspondientes a cada velocidad a la ec. 5.12 , se han determinado los valores de los parámetros 
cinéticos: "n" y $\ln A_{g}$, y la varianza $S^{2}$, (Tabla 5.20). Al igual que ocurría en el apartado 5.1.2.1.3.1, el ajuste entre los resultados experimentales y los calculados es muy bueno.

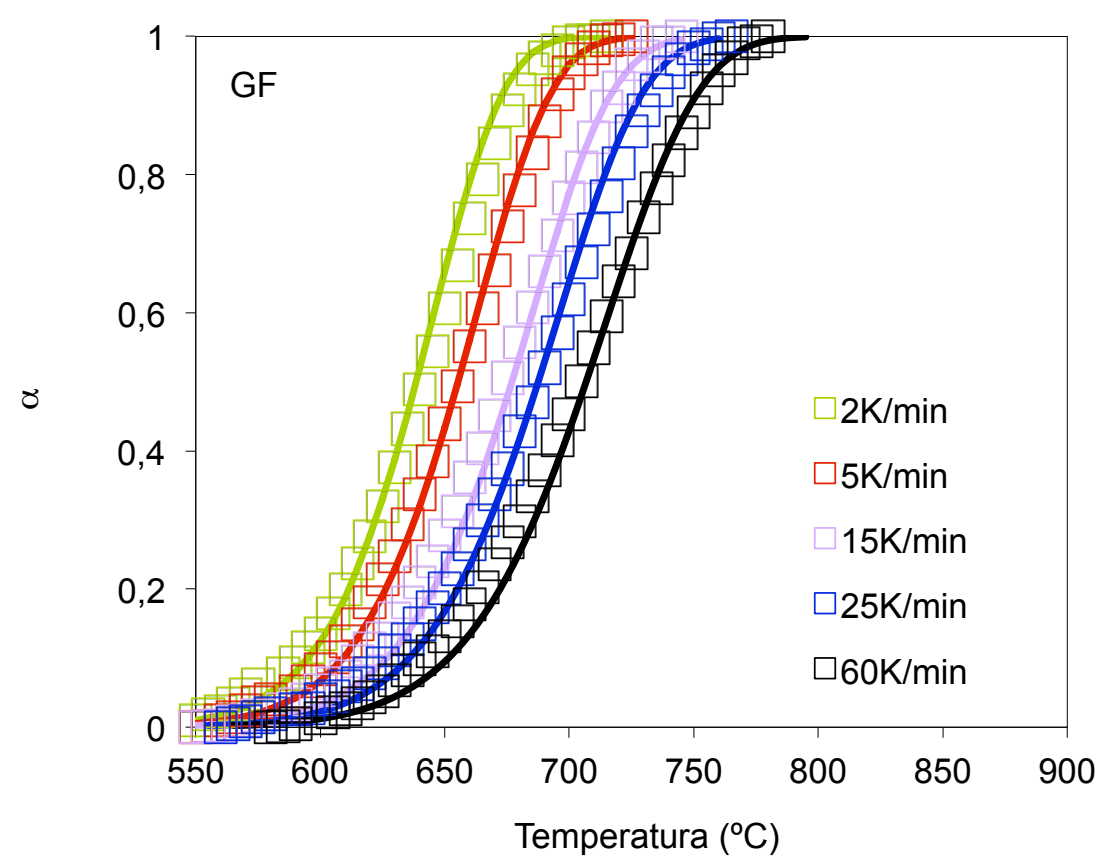

Figura 5.107. Curvas de sinterización no isotermas de compactos de vidrio de la granulometría GF, a distintas velocidades de calentamiento. Comparación entre los resultados experimentales y los calculados mediante la ec. 5.12 y los parámetros de la Tabla 5.20.

Tabla 5.20. Valores de los parámetros $\ln A_{g}, n$ y $S^{2}$ que resultan del ajuste de los valores de $\alpha$ a la ec. 5.12 para la matriz vitrea de granulometrías $G M$ y GF. Se incluyen los valores de $\ln \left(A_{g} \cdot d\right)$, siendo $d$ el diámetro medio geométrico.

\begin{tabular}{|c|c|c|c|c|}
\hline Granulometría & $\mathbf{n}$ & $\ln \mathbf{A}_{\mathrm{g}}\left(\mathbf{s}^{-1}\right)$ & $\mathbf{S}^{2}$ & $\ln \left(\mathbf{A}_{\mathrm{g}} \cdot \mathbf{d}\right)(\mathbf{m} / \mathbf{s})$ \\
\hline GF & $0,70 \pm 0,017$ & $18,9 \pm 0,18$ & 0,00032 & 5,55 \\
\hline GM & $0,71 \pm 0,019$ & $17,5 \pm 0,16$ & 0,00096 & 5,46 \\
\hline
\end{tabular}


En la Figura 5.108 se representan las curvas correspondientes a los ensayos isotermos. Los pares de valores $(\alpha, T)$, correspondientes a cada temperatura, se han ajustado a la ec. 5.14. Al fijar el valor de $\mathrm{n}$ al valor medio de todas las temperaturas se obtuvo un valor de $\mathrm{k}_{\mathrm{g}} \mathrm{y} \mathrm{x}_{0}$ para cada temperatura. El valor de la varianza, $S^{2}$, para cada ajuste individual también fue muy bajo $\left(S^{2}<0,0002\right)$ y el acuerdo entre los resultados experimentales y calculados también muy bueno (Figura 5.108, Tabla 5.21).

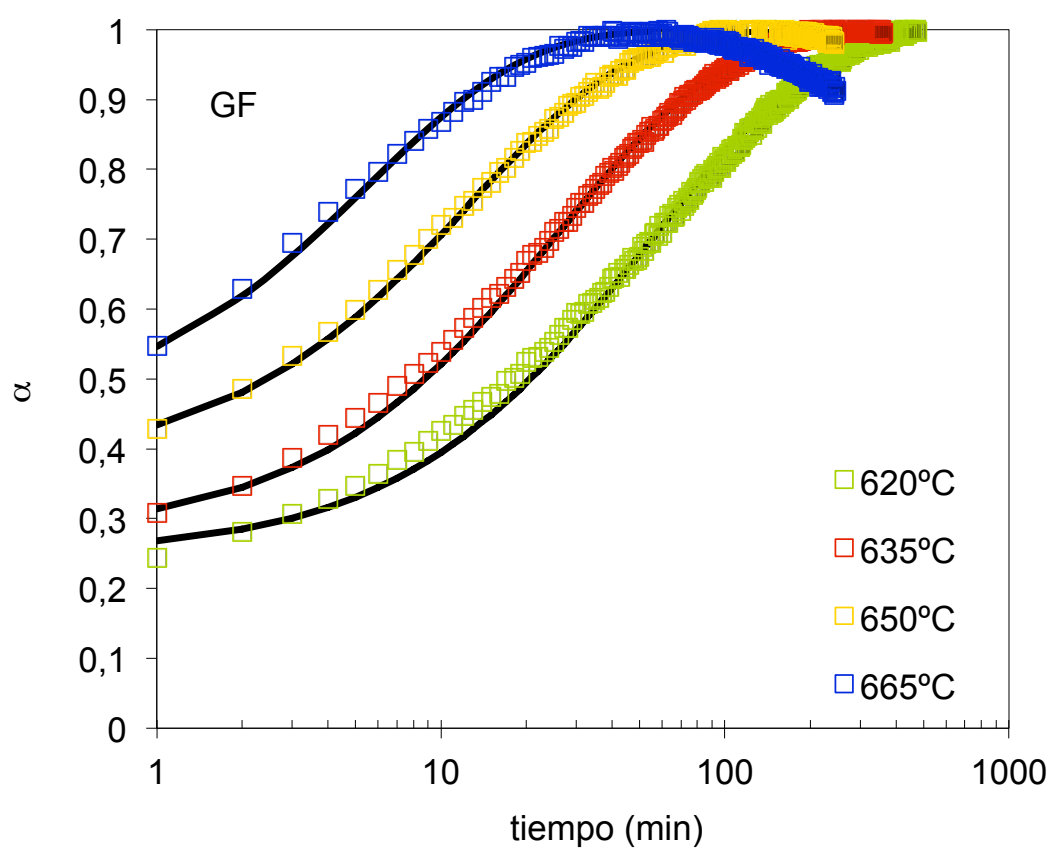

Figura 5.108. Curvas de sinterización isotermas de compactos de vidrio de la granulometría GF. Comparación entre los resultados experimentales y los calculados mediante la ec. 5.14 con los parámetros de la Tabla 5.21. 
Tabla 5.21. Valores de los parámetros cinéticos $n, \ln A_{g}, E$ y de la varianza, $S^{2}$, que resultan de ajustar simultáneamente los resulados a la ec. 5.14 y la ec.

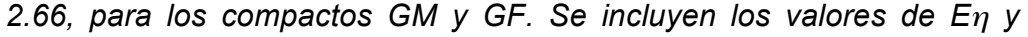
$\ln \left(A_{g} \cdot d\right)$ siendo d el diámetro medio geométrico del vídrio.

\begin{tabular}{|c|c|c|c|c|c|c|}
\hline Gran. & $\mathbf{n}$ & $\operatorname{In}_{\mathbf{g}}\left(\mathbf{s}^{-1}\right)$ & $\mathbf{s}^{2}$ & $\begin{array}{c}E \\
(\mathbf{k J} / \mathrm{mol})\end{array}$ & $\begin{array}{c}\mathrm{E} \eta \\
(\mathbf{k J} / \mathrm{mol})\end{array}$ & $\begin{array}{c}\operatorname{In}\left(\mathbf{A}_{\mathbf{g}} \cdot \mathbf{d}\right) \\
(\mathbf{m} / \mathbf{s})\end{array}$ \\
\hline GF & $0,70 \pm 0,042$ & $46,9 \pm 1,45$ & $6,5 \cdot 10^{-5}$ & $377 \pm 11$ & $363 \pm 2$ & $33,6 \pm 1,45$ \\
\hline GM & $0,71 \pm 0,035$ & $41,9 \pm 3,21$ & $6,3 \cdot 10^{-5}$ & $348 \pm 24$ & $360 \pm 2$ & $29,8 \pm 3,21$ \\
\hline
\end{tabular}

Al representar, en escala doble logarítmica, la constante de velocidad obtenida a cada temperatura, $\mathrm{k}_{\mathrm{g}}$, frente a la inversa del tiempo característico, $\gamma / \mathrm{d} \eta$ para los vidrios de granulometría GF y GM (Figura 5.109), los resultados se ajustan muy bien a una única recta de ecuación ec. 5.39, lo que confirma que el modelo cinético también describe de forma excelente el efecto de la granulometría del vidrio sobre su velocidad de sinterización.

$\mathrm{k}_{\mathrm{g}}=0,9 \cdot \frac{\gamma}{\mathrm{d} \eta} \quad \mathrm{S}^{2}=1,77 \cdot 10^{-8}$ ec. 5.39 


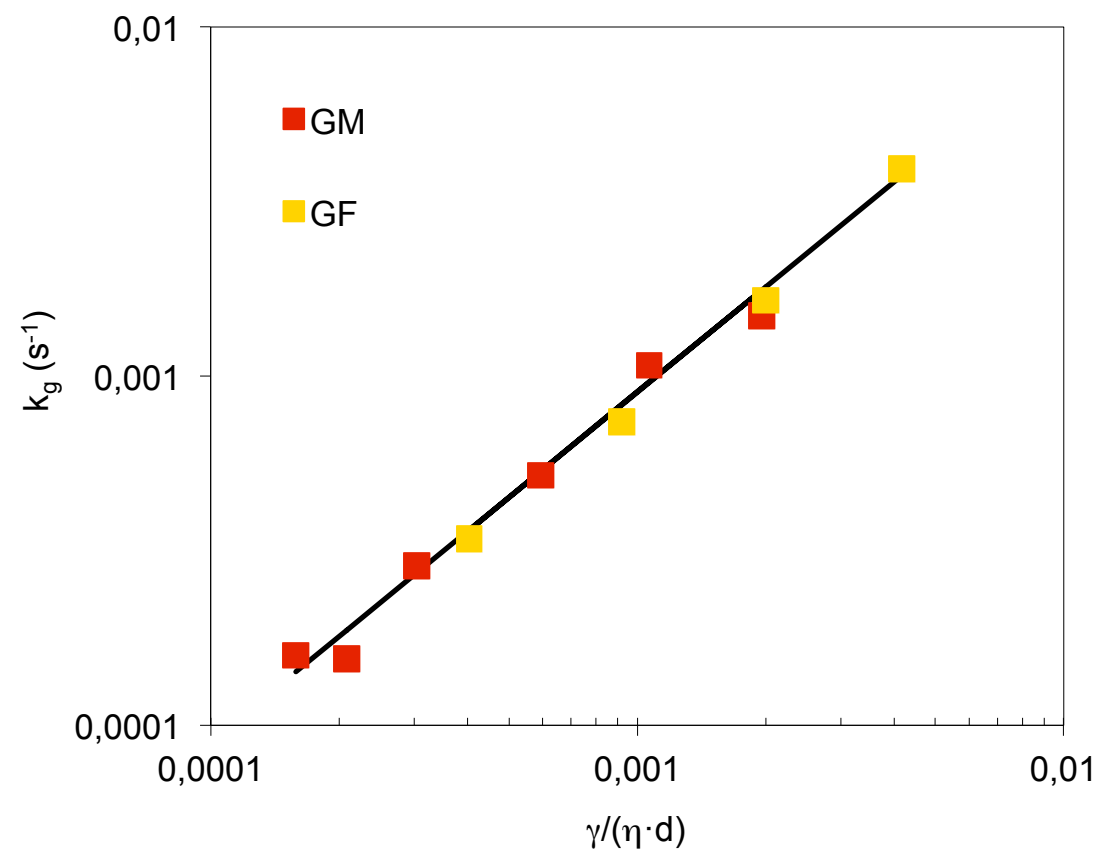

Figura 5.109. Variación de las constantes de velocidad isotermas, $\boldsymbol{k}_{g}$, frente a la inversa del tiempo característico, $\gamma / \eta d$. Compactos de vidrio de las granulometrías GM y GF.

Por otra parte, el producto $A_{g} \cdot d$ debe ser constante (solo dependerá de la naturaleza del vidrio), ya que la constante de velocidad, $k_{g}$, es proporcional a la inversa del tamaño medio geométrico, $d$, como acabamos de comprobar. Así pues, se ha multiplicado dicho parámetro por el valor de d correspondiente a cada vidrio (Tabla 5.20 y Tabla 5.21). Al realizar estos cálculos se comprueba que también en este caso los valores del producto $\ln \left(A_{g} \cdot d\right)$, tanto en los ensayos isotermos como en los no isotermos, son muy similares y/o prácticamente coincidentes, dentro del error experimental. $\mathrm{Si}$ en vez de considerar como representativo del tamaño medio del vidrio el diámetro medio geométrico, $d$, se emplea el diámetro medio volumético, $d_{g}{ }^{v}$, se obtiene resultados análogos, aunque los valores de $\ln \left(A_{g} \cdot d_{g}{ }^{v}\right)$ y de $\lambda_{g}$ serían un poco más grandes. 
5.3.2.2 Composites con moderados contenidos en circón: serie 1 ( $\phi=0,322)$.

En este apartado se estudian los composites $\mathrm{H} 1$ y $\mathrm{M} 1$ preparados con vidrio de granulometría GM, y los composites FF1, FM1 y FH1, obtenidos con el vidrio de granulometría GF (Tabla 5.17).

5.3.2.2.1 Experimentos a velocidad de calentamiento constante.

Para cada composite se han representado en la Figura 5.110 a la Figura 5.114 las curvas de sinterización correspondientes a las cinco velocidades de calentamiento habituales.

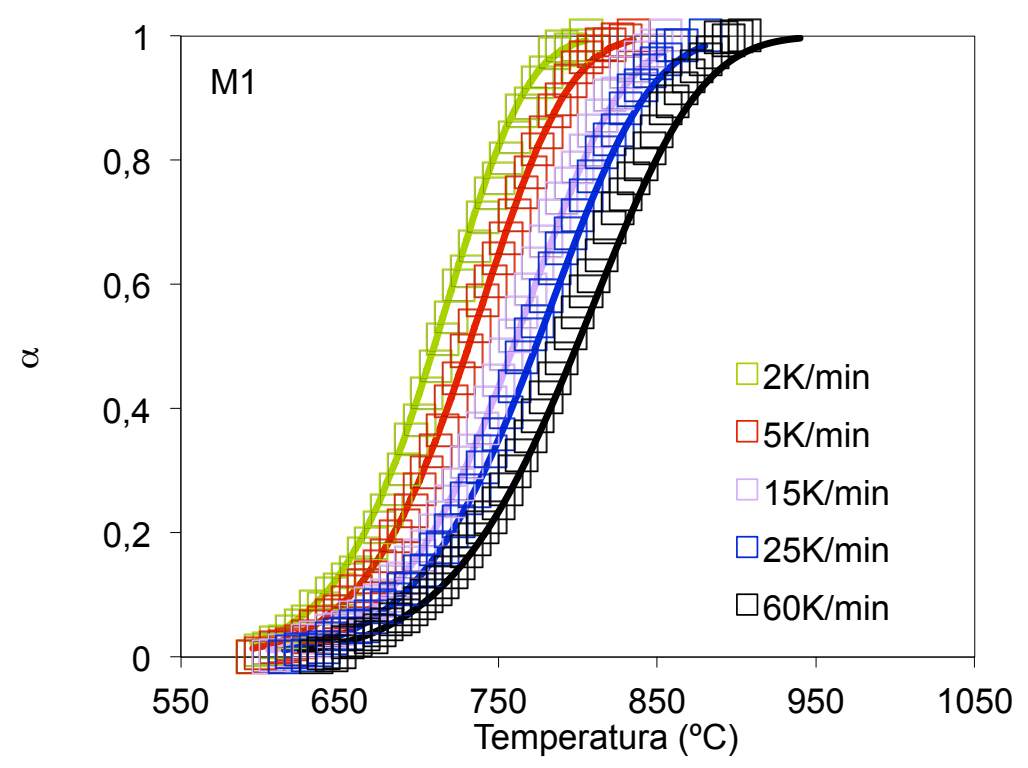

Figura 5.110. Curvas de sinterización del composite $\mathrm{M1}$, a velocidad de calentamiento $\beta=2,5,15,25$ y $60 \mathrm{~K} / \mathrm{min}$. Comparación entre los resultados experimentales y los calculados mediante la ec. 5.12 y Tabla 5.22 . 


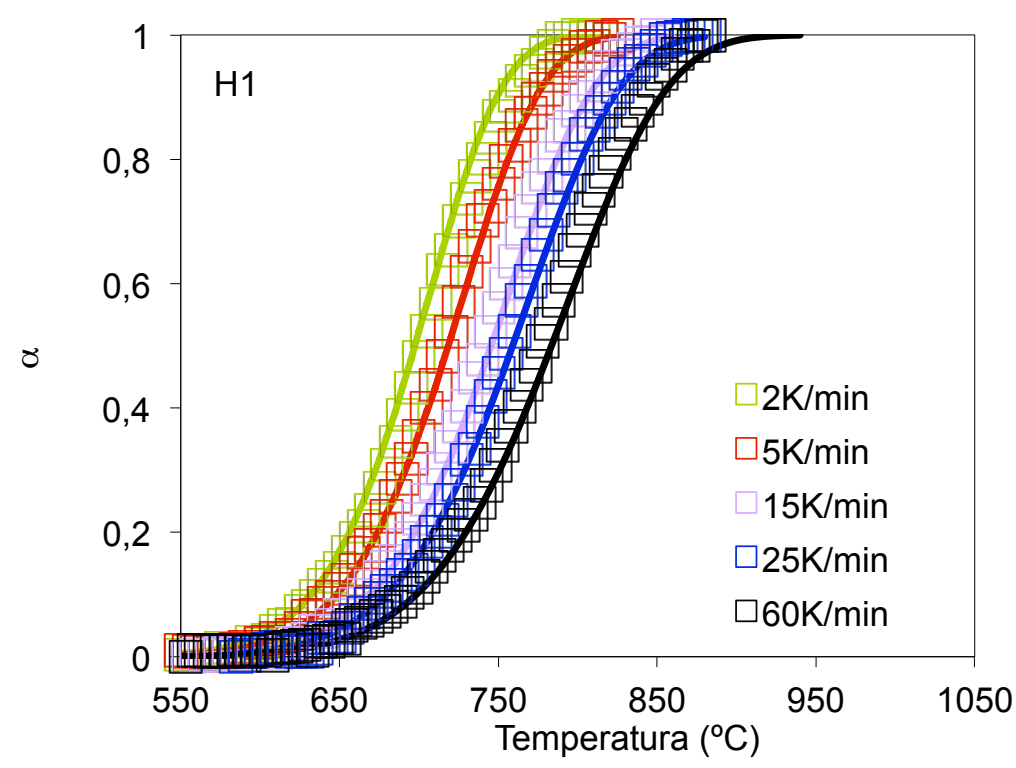

Figura 5.111. Curvas de sinterización del composite $\mathrm{H1}$, a velocidad de calentamiento $\beta=2,5,15,25$ y $60 \mathrm{~K} / \mathrm{min}$. Comparación entre los resultados experimentales y los calculados mediante la ec. 5.12 y Tabla 5.22 .

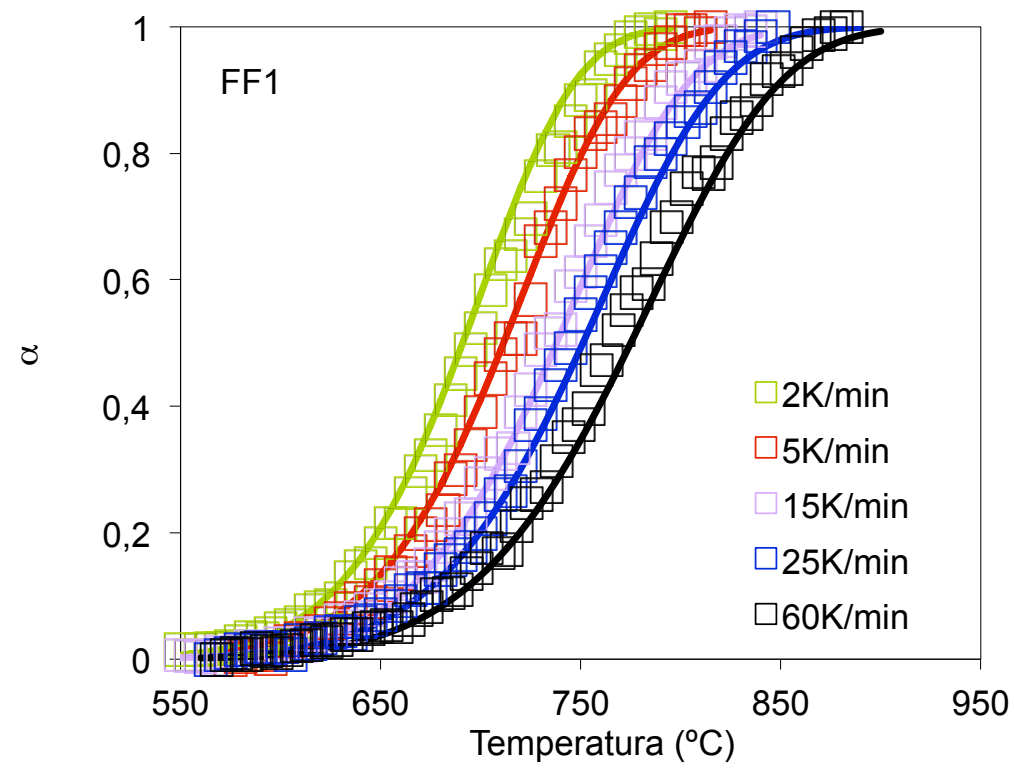

Figura 5.112. Curvas de sinterización del composite FF1, a velocidad de calentamiento $\beta=2,5,15,25$ y $60 \mathrm{~K} / \mathrm{min}$. Comparación entre los resultados experimentales y los calculados mediante la ec. 5.12 y Tabla 5.22. 


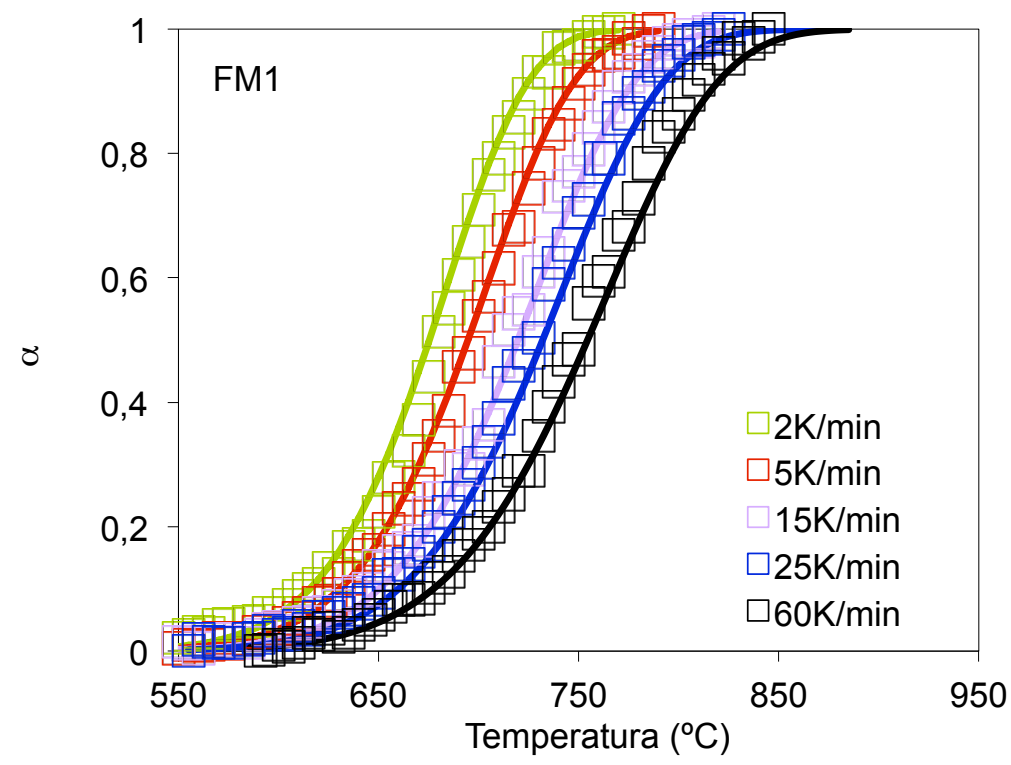

Figura 5.113. Curvas de sinterización del composite FM1, a velocidad de calentamiento $\beta=2,5,15,25$ y 60K/min. Comparación entre los resultados experimentales y los calculados mediante la ec. 5.12 y Tabla 5.22 .

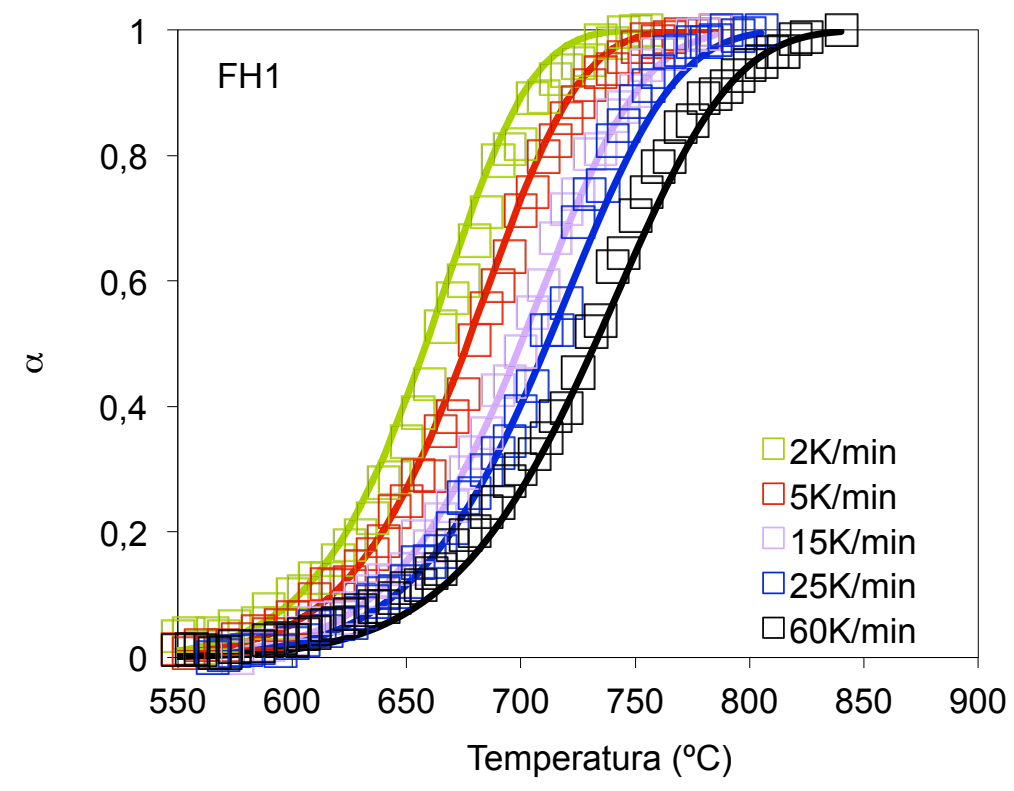

Figura 5.114. Curvas de sinterización del composite FH1, a velocidad de calentamiento $\beta=2,5,15,25$ y 60K/min. Comparación entre los resultados experimentales y los calculados mediante la ec. 5.12 y Tabla 5.22 . 
Para todos los composites, como era de esperar a la vista de los resultados analizados en el apartado 5.3.1.1, las curvas de sinterización son sigmoidales, por lo que, pueden ser descritas mediante una sola etapa asociada al flujo viscoso, al igual que ocurría para los composites de la serie $F$ para valores de $\phi \leq 0,43$ (apartado 5.2.2.1.2.1). También para estos últimos composites se comprobó (apartado 5.2.2.1.1) que los valores de $\overline{E_{\alpha}}$ y $\overline{E_{\eta}}$ para cada composite eran muy parecidos. En consecuencia, dada la similitud de comportamiento entre unos (serie F) y otros (serie 1) composites se ha considerado que no era necesario, para estos composites de la serie 1, determinar los valores de $\overline{E_{\alpha}}$ y $\overline{E_{\eta}}$, mediante el método de Friedman, y pasar directamente a la determinación del triplete cinético, como ya se ha hecho en apartados anteriores (5.1.2.1.3.1, 5.2.2.1.2.1 y 5.3.2.1), basándose en que el efecto de la temperatura sobre la velocidad del proceso es la debida al efecto de dicha variable sobre la viscosidad.

\subsection{Selección del modelo. Determinación del triplete cinético.}

Para cada composite, los pares de valores $(\alpha, T)$ correspondientes a las cinco velocidades ensayadas, se han ajustado a la ec. 5.12 y siguiendo el mismo procedimiento que el descrito en el apartado 5.1.2.1.3.1, se han determinado los valores de los parámetros cinéticos: "n" y InA, y de la varianza, $\mathrm{S}^{2}$. En la Tabla 5.22 se detallan los resultados. Se incluyen, en esta tabla, a efectos comparativos, los resultados correspondientes al vidrio de granulometría GM y GF y al composite F1.

Al igual que ocurría en el apartado 5.2.2.1.2.1 se confirma (Tabla 5.22 y Figura 5.110 a Figura 5.114) que el modelo cinético utilizado describe muy bien el proceso, aun cuando los parámetros cinéticos " $\mathrm{n}$ " y $\ln \mathrm{A}$ se han mantenido fijos en el ajuste a la ec. 5.12, independientemente de la velocidad de calentamiento, $\beta$, a pesar de que esta última se ha modificado de $\beta=2$ a $\beta=60 \mathrm{~K} / \mathrm{min}$. 
Tabla 5.22. Valores de los parámetros cinéticos, $n$ y $\ln A$, y de la varianza, $S^{2}$, obtenidos al ajustar las curvas de sinterización, $\alpha$ vs $T$, de los diferentes composites a la ec. 5.12 con $B=5495$ y $T_{0}=421 \mathrm{~K}$.

\begin{tabular}{|c|c|c|c|c|c|}
\hline Composite & Circón & Vidrio & $\mathbf{n}$ & $\operatorname{InA}\left(\mathbf{s}^{-1}\right)$ & $\mathbf{s}^{2}$ \\
\hline GM & - & GM & $0,71 \pm 0,019$ & $17,5 \pm 0,16$ & 0,000956 \\
\hline F1 & F & GM & $0,515 \pm 0,016$ & $13,8 \pm 0,16$ & 0,000754 \\
\hline M1 & $0,5 F-0,5 \mathrm{H}$ & GM & $0,55 \pm 0,023$ & $15,2 \pm 0,22$ & 0,000505 \\
\hline H1 & H & GM & $0,56 \pm 0,022$ & $15,8 \pm 0,17$ & 0,000289 \\
\hline GF & - & GF & $0,70 \pm 0,017$ & $18,9 \pm 0,18$ & 0,000315 \\
\hline FF1 & F & GF & $0,53 \pm 0,020$ & $16,0 \pm 0,32$ & 0,000601 \\
\hline FM1 & $0,5 F-0,5 H$ & GF & $0,57 \pm 0,019$ & $16,8 \pm 0,17$ & 0,000964 \\
\hline FH1 & H & GF & $0,58 \pm 0,022$ & $17,6 \pm 0,35$ & 0,000863 \\
\hline
\end{tabular}

\subsection{Experimentos isotermos.}

Para cada uno de los composites se ha realizado un conjunto de, al menos, cuatro experimentos a diferentes temperaturas. También, al igual que se hizo en el apartado 5.2.2.2, las temperaturas seleccionadas para un determinado composite son, en general, distintas a la de otro, debido a que el intervalo apropiado de temperaturas de sinterización es distinto para cada material. Los resultados se detallan en la Figura 5.115 a Figura 5.119. 


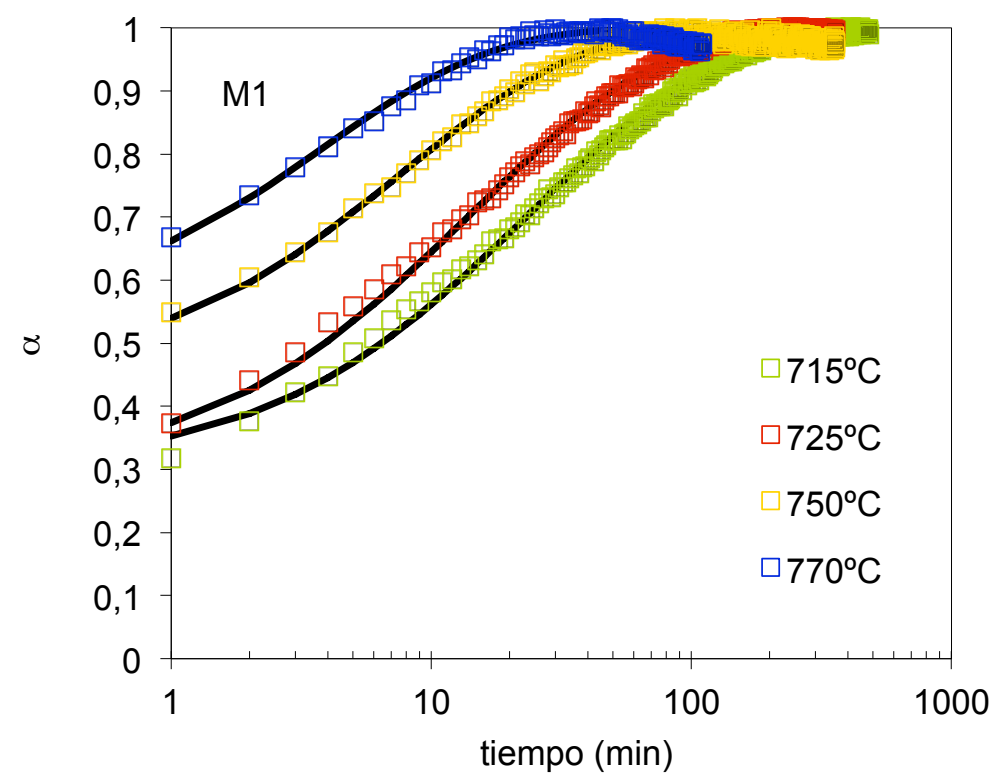

Figura 5.115. Curvas de sinterización isotermas del composite M1, a distintas temperaturas. Comparación entre los resultados experimentales y los calculados mediante la ec. 5.14 y la Tabla 5.23.

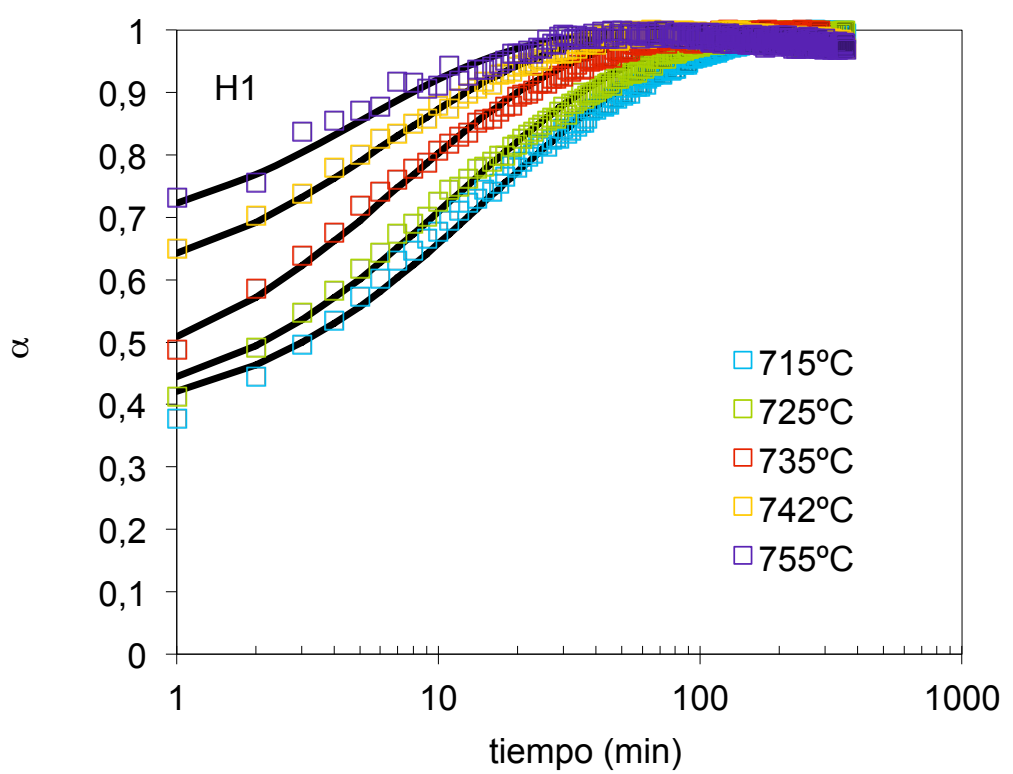

Figura 5.116. Curvas de sinterización isotermas del composite $\mathrm{H1}$, a distintas temperaturas. Comparación entre los resultados experimentales y los calculados mediante la ec. 5.14 y la Tabla 5.23 . 


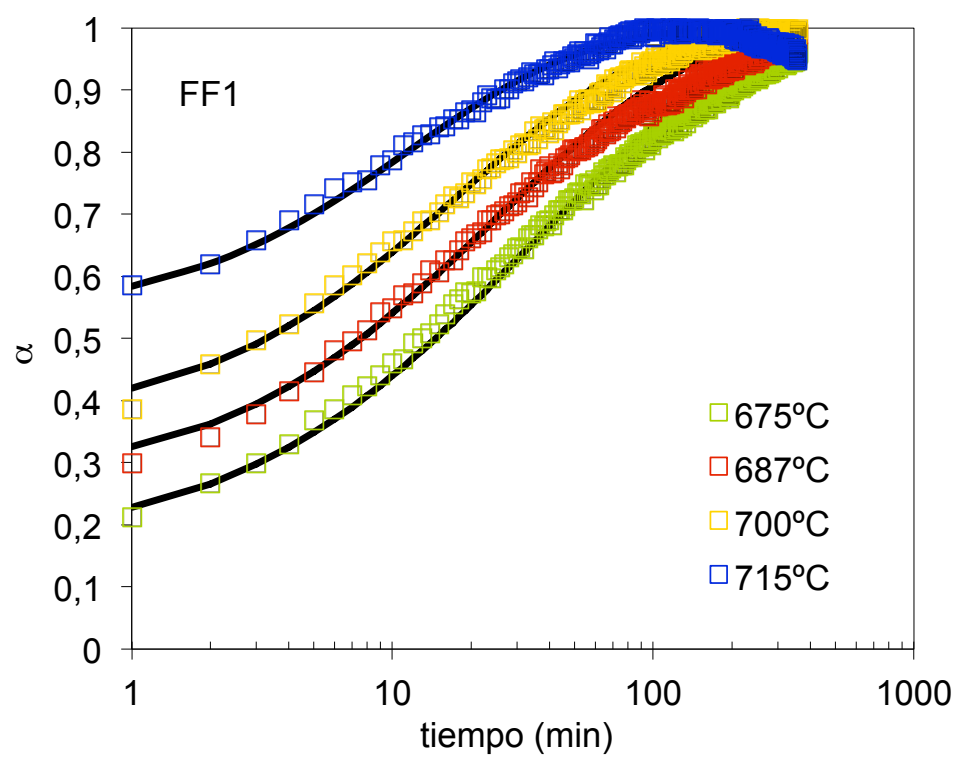

Figura 5.117. Curvas de sinterización isotermas del composite FF1, a distintas temperaturas. Comparación entre los resultados experimentales y los calculados mediante la ec. 5.14 y la Tabla 5.23.

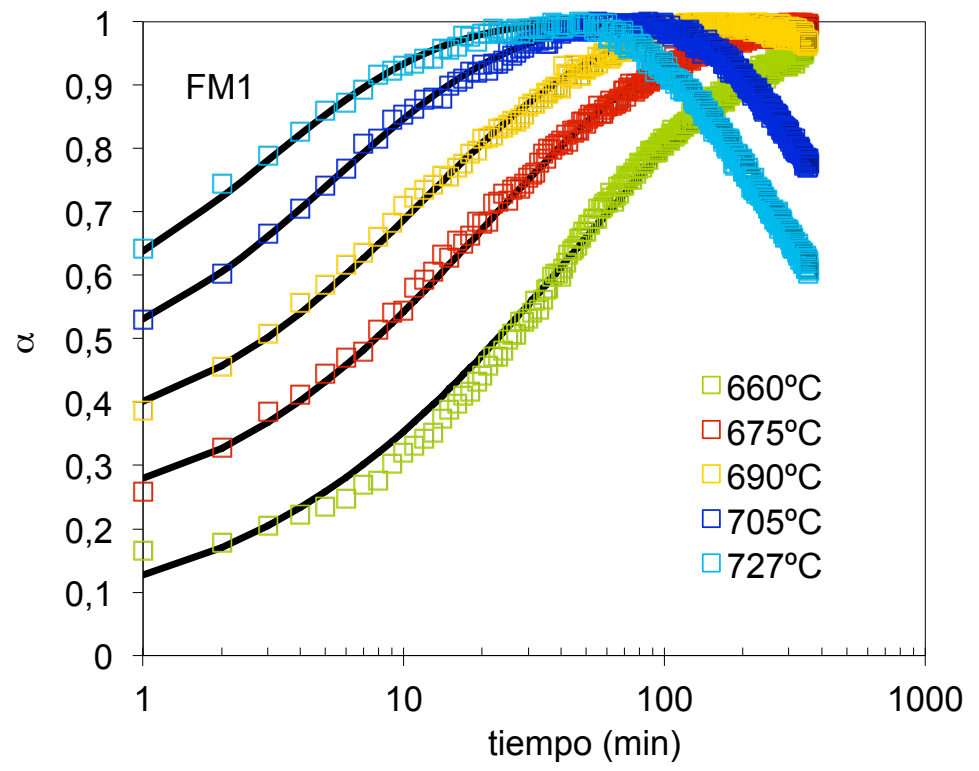

Figura 5.118. Curvas de sinterización isotermas del composite FM1, a distintas temperaturas. Comparación entre los resultados experimentales y los calculados mediante la ec. 5.14 y la Tabla 5.23. 


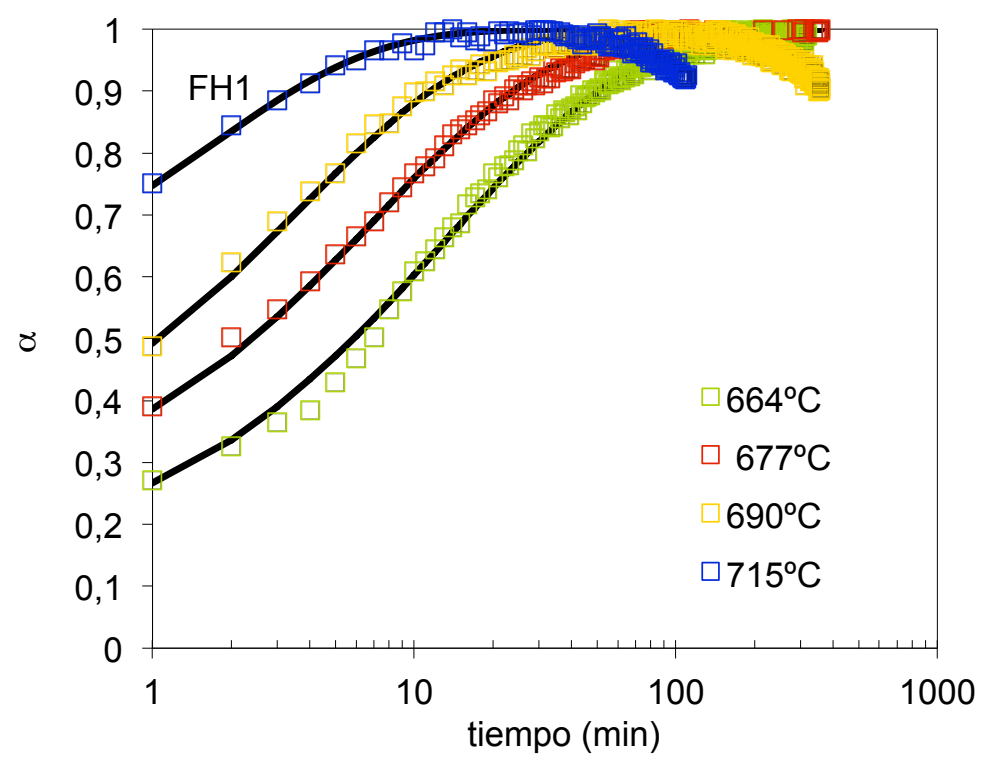

Figura 5.119. Curvas de sinterización isotermas del composite FH1, a distintas temperaturas. Comparación entre los resultados experimentales y los calculados mediante la ec. 5.14 y la Tabla 5.23.

\subsection{Selección del modelo. Determinación del triplete cinético.}

Al igual que se hizo en los apartados anteriores (5.1.2.2, 5.2.2.2.1.1 y 5.3.2.2.2.1), para cada composite, los pares de valores $(\alpha, T)$ correspondientes a cada experimento isotermo, se han ajustado a la ec. 5.14. También, en este caso, para cada composite se fijaba el mismo valor de $\mathrm{n}$ (el valor medio de todos los experimentos isotermos) resultando, para cada temperatura, un valor de $\mathrm{k}$ y $\alpha_{0}$ propios. Para todas las temperaturas $\mathrm{y}$ composites, el valor de la varianza, $S^{2}$, de cada ajuste era muy bajo $\left(S^{2}<0,0002\right)$, y el acuerdo entre los valores experimentales y los calculados excelente (Figura 5.115 a Figura 5.119). Para cada composite la energía de activación, E, se ha obtenido ajustando los pares de valores de $\mathrm{k}$ y $\mathrm{T}$ obtenidos a la ecuación de Arrhenius (ec. 2.66). En la Tabla 5.23 se detallan los valores calculados del triplete cinético: $n, E$ y $\ln A$, junto a los de la

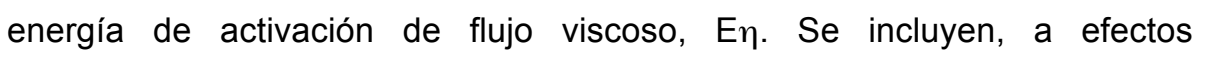


comparativos, los valores correspondientes al vidrio de granulometría GM y GF y al composite F1.

Tabla 5.23. Valores de los parámetros cinéticos obtenidos al ajustar a la ec. 5.14 y la ec. 2.66, las curvas de sinterización isotermas de cada composite de la serie 1. Se incluyen los valores de Eq.

\begin{tabular}{|c|c|c|c|c|c|c|}
\hline Comp. & Circón & Vidrio & $\mathbf{n}$ & $\operatorname{InA}\left(\mathbf{s}^{-1}\right)$ & $\mathbf{E}(\mathbf{k J} / \mathrm{mol})$ & $\mathrm{E} \eta(\mathrm{kJ} / \mathrm{mol})$ \\
\hline $\mathrm{GM}$ & - & $\mathrm{GM}$ & $0,70 \pm 0,035$ & $41,9 \pm 3,2$ & $348 \pm 24$ & $360 \pm 2$ \\
\hline $\mathrm{F} 1$ & $\mathrm{~F}$ & $\mathrm{GM}$ & $0,51 \pm 0,040$ & $30,6 \pm 1,3$ & $287 \pm 11$ & $305 \pm 2$ \\
\hline $\mathrm{M} 1$ & $0,5 \mathrm{~F}-0,5 \mathrm{H}$ & $\mathrm{GM}$ & $0,54 \pm 0,028$ & $37,7 \pm 1,9$ & $333 \pm 16$ & $310 \pm 2$ \\
\hline $\mathrm{H} 1$ & $\mathrm{H}$ & $\mathrm{GM}$ & $0,56 \pm 0,019$ & $39,4 \pm 2,9$ & $344 \pm 24$ & $313 \pm 2$ \\
\hline GF & - & GF & $0,70 \pm 0,042$ & $46,9 \pm 1,5$ & $377 \pm 11$ & $363 \pm 2$ \\
\hline FF1 & F & GF & $0,53 \pm 0,031$ & $36,7 \pm 1,8$ & $317 \pm 15$ & $327 \pm 2$ \\
\hline FM1 & $0,5 \mathrm{~F}-0,5 \mathrm{H}$ & GF & $0,57 \pm 0,028$ & $43,8 \pm 2,8$ & $368 \pm 22$ & $333 \pm 2$ \\
\hline FH1 & H & GF & $0,58 \pm 0,022$ & $46,4 \pm 1,9$ & $380 \pm 15$ & $335 \pm 2$ \\
\hline
\end{tabular}

Se confirma, de nuevo, que los valores de E, siempre con mayor dispersión

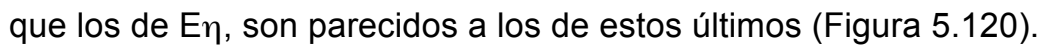




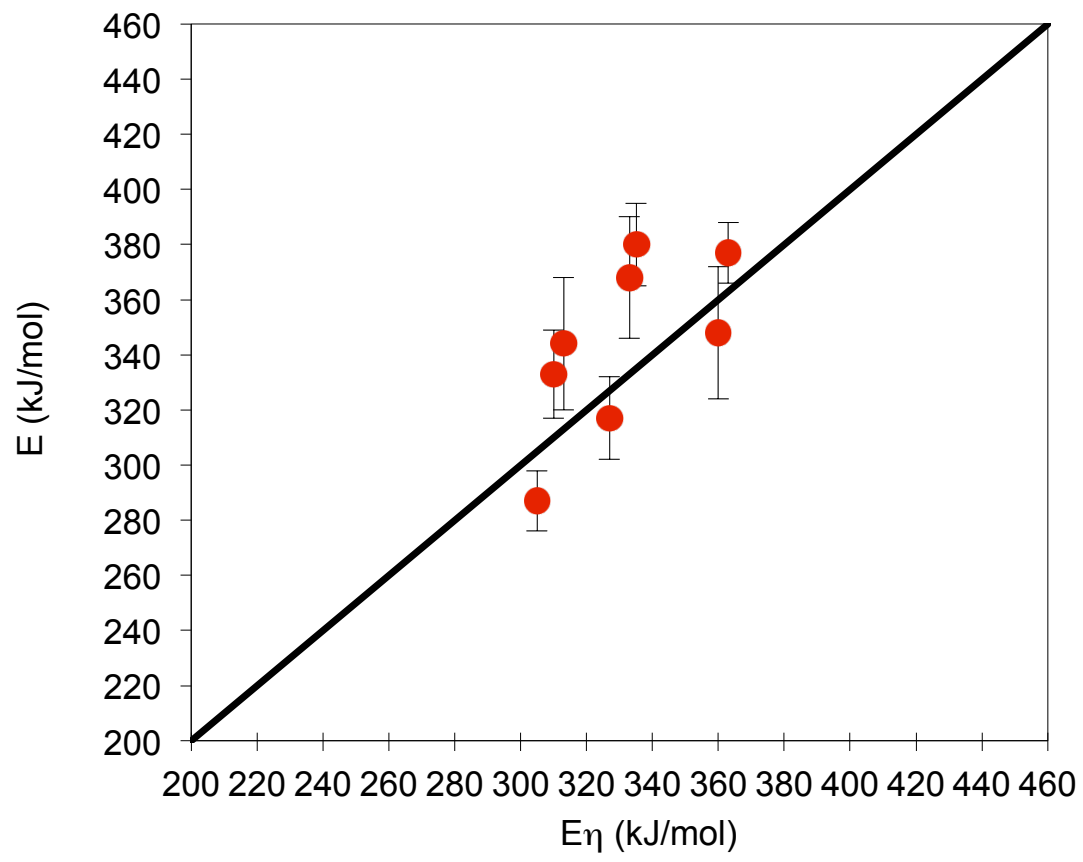

Figura 5.120. Relación entre los valores de la energía de activación de la sinterización, $E$, y los de flujo viscoso, $E_{\eta}$. Composites de la serie 1. Experimentos isotermos.

Al comparar los valores de " $\mathrm{n}$ " obtenidos por métodos isotermos (Tabla 5.23) con los que resultan de experimentos a velocidad de calentamiento constante (Tabla 5.22) se aprecia que ambos son prácticamente coincidentes. Asimismo, el factor preexponencial obtenido de experimentos isotermos, $\ln \left(\mathrm{A}_{\text {isot }}\right)$, está directamente relacionado con el que resulta de experimentos a velocidad de calentamiento constante, $\ln \left(A_{n o}\right.$ isot $)$ (Figura 5.121), al igual que ocurría en la Figura 5.87, aunque los valores obtenidos por uno y otro método sean muy distintos, como ya se ha discutido antes, en el apartado 5.2.2.2.1.1. 


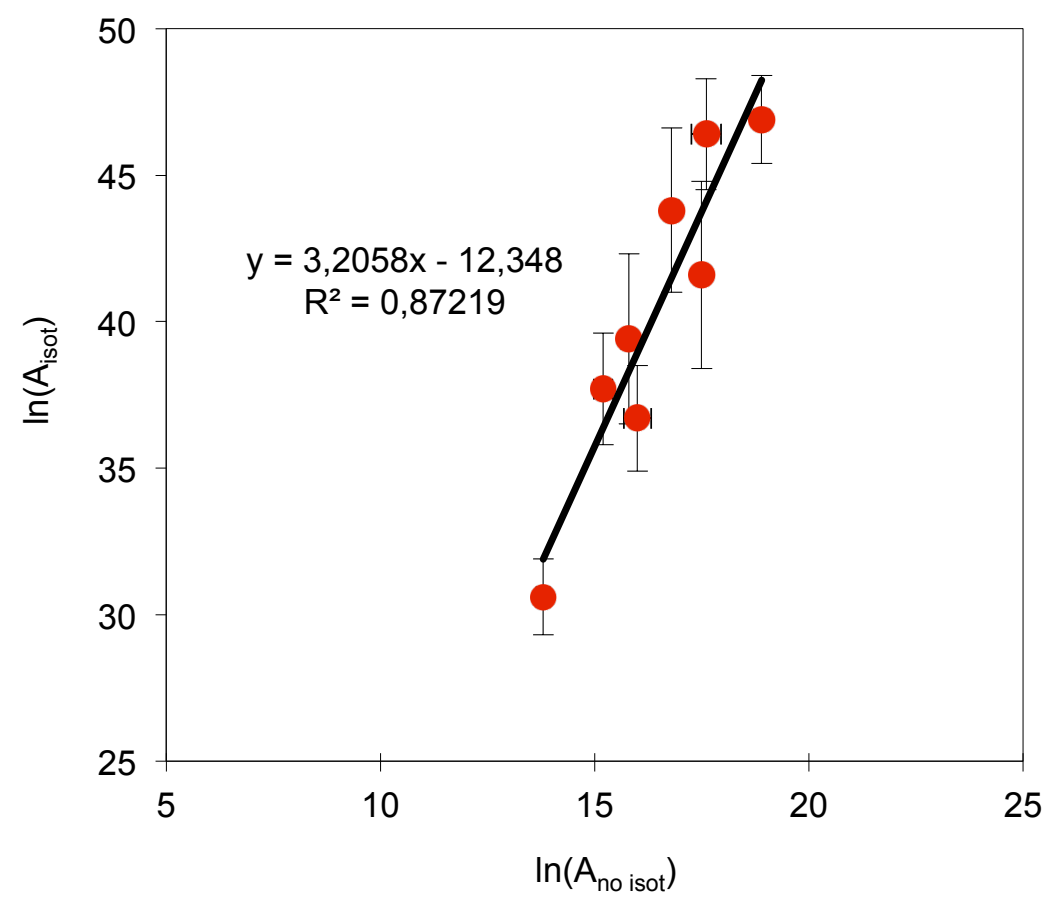

Figura 5.121. Relación entre los valores del factor preexponencial obtenido en experimentos isotermos, $A_{\text {isot, }}$ y no isotermos, $A_{\text {no isot. }}$ Composites de la serie 1.

\subsubsection{Composites con elevados contenidos en circón $(\phi=0,525)$.}

En este apartado se estudian los composites $\mathrm{H} 2$ y $\mathrm{M} 2$ preparados con el vidrio de granulometría GM, y los composites FF2, FM2 y FH2, obtenidos con el vidrio de granulometría más fina, GF (Tabla 5.17).

5.3.2.3.1 Experimentos a velocidad de calentamiento constante.

Para cada composite se han representado en la Figura 5.122 a la Figura 5.126 las curvas de sinterización correspondientes a las cinco velocidades de calentamiento habituales. A la vista de los resultados analizados en el apartado 5.3.1.1, para todos los composites, a excepción del H2, las curvas de sinterización no son sigmoidales ya que la pieza no puede alcanzar su máxima densificación, sin la intervención del mecanismo de soluciónreprecipitación del circón. En efecto, al comparar las curvas experimentales 
con las que se obtienen al ajustar los resultados experimentales, correspondientes a la etapa de flujo viscoso, se aprecia una elevada desviación entre ambas para valores de $\alpha$ superiores a un determinado valor, que depende de la granulometría del vidrio y del circón.

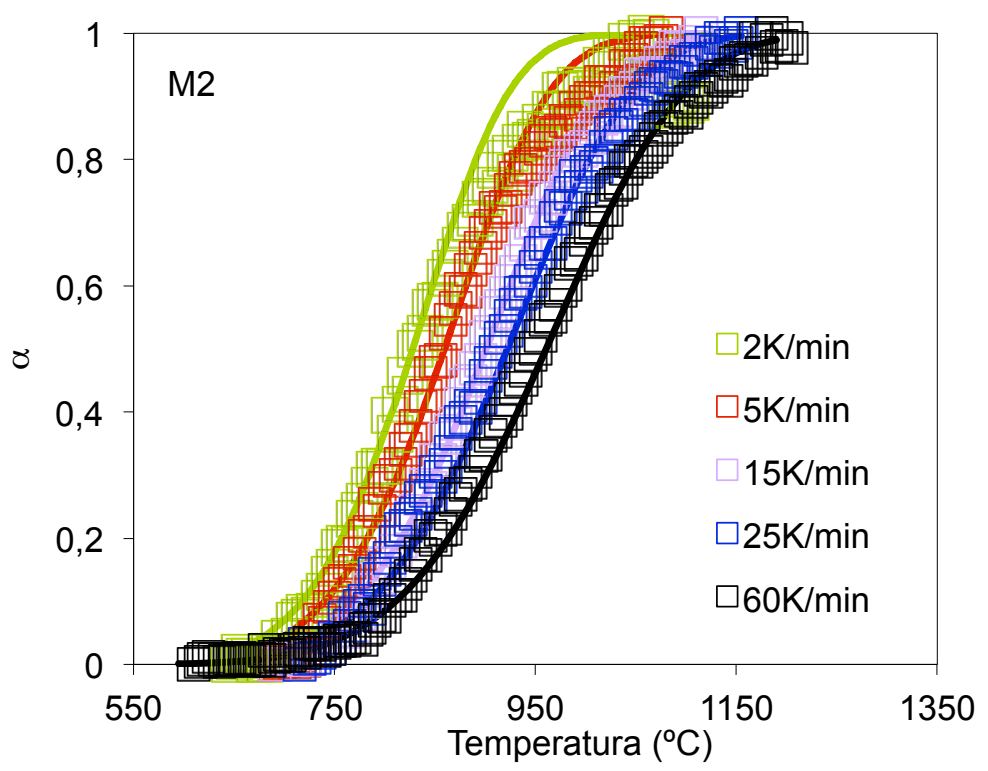

Figura 5.122. Curvas de sinterización del composite M2, a velocidad de calentamiento $\beta=2,5,15,25$ y $60 \mathrm{~K} / \mathrm{min}$. Comparación entre los resultados experimentales y los calculados mediante la ec. 5.12, con los valores de los parámetros de la Tabla 5.24. 


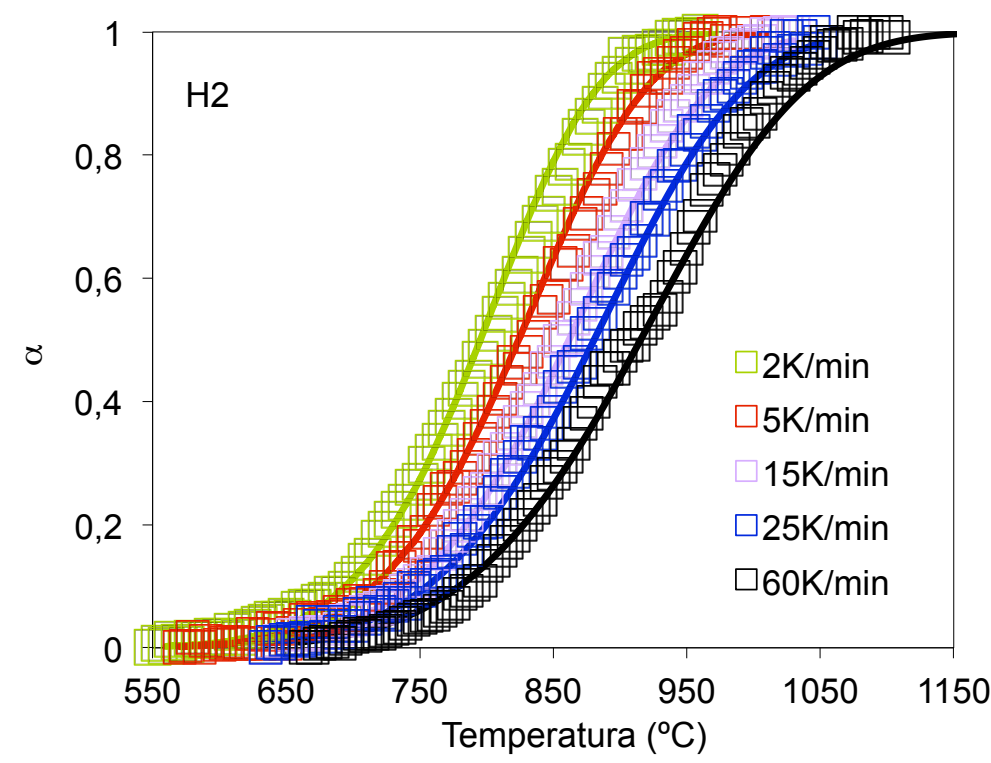

Figura 5.123. Curvas de sinterización del composite $\mathrm{H} 2$, a velocidad de calentamiento $\beta=2,5,15,25$ y $60 \mathrm{~K} / \mathrm{min}$. Comparación entre los resultados experimentales y los calculados mediante la ec. 5.12, con los valores de los parámetros de la Tabla 5.24.

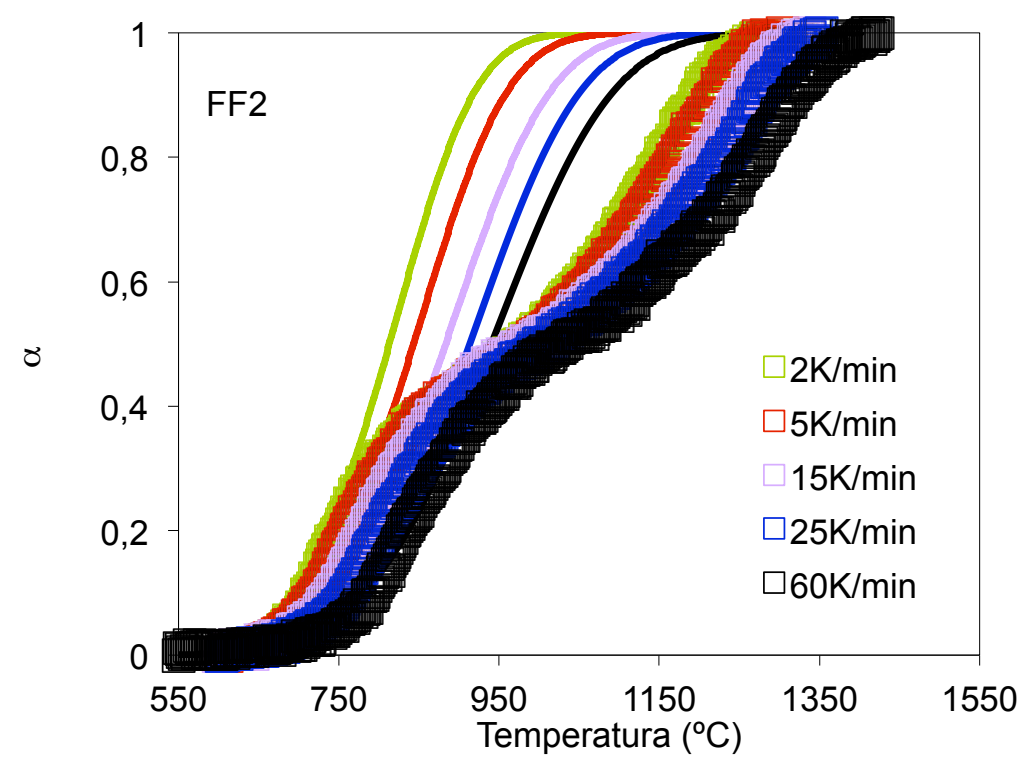

Figura 5.124. Curvas de sinterización del composite FF2, a velocidad de calentamiento $\beta=2,5,15,25$ y 60K/min. Comparación entre los resultados experimentales y los calculados mediante la ec. 5.12, con los valores de los parámetros de la Tabla 5.24. 


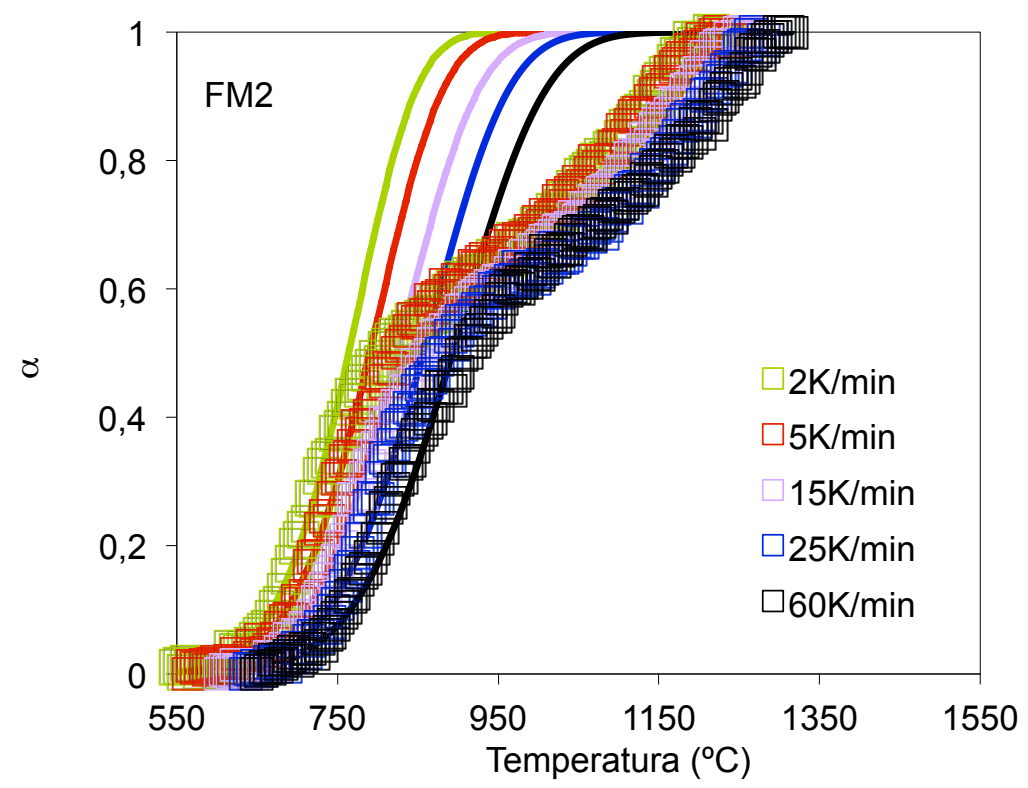

Figura 5.125. Curvas de sinterización del composite FM2, a velocidad de calentamiento $\beta=2,5,15,25$ y 60K/min. Comparación entre los resultados experimentales y los calculados mediante la ec. 5.12, con los valores de los parámetros de la Tabla 5.24.

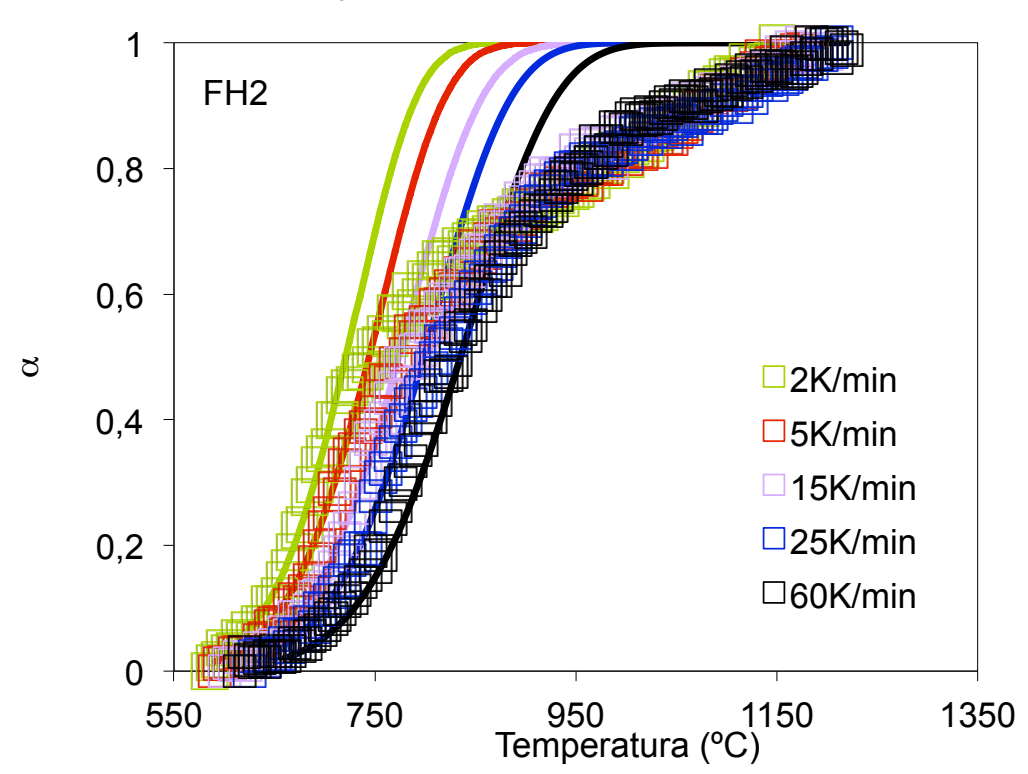

Figura 5.126. Curvas de sinterización del composite FH2, a velocidad de calentamiento $\beta=2,5,15,25$ y 60K/min. Comparación entre los resultados experimentales y los calculados mediante la ec. 5.12, con los valores de los parámetros de la Tabla 5.24. 
Por otra parte, la mayoría de estos composites con alto contenido en circón ( $\phi=0,525)$, excepto los composites $\mathrm{F} 2$ y FH2, son muy porosos, por lo que su interés tecnológico es nulo. No obstante, el efecto que la granulometría del circón y del vidrio ejercen sobre la cinética de la densificación por el mecanismo de flujo viscoso si que es interesante desde el punto de vista tanto tecnológico como teórico.

\subsection{Selección del modelo. Determinación del triplete cinético.}

De acuerdo con lo que se acaba de exponer, para todos los composites se han calculado los parámetros cinéticos correspondientes al primer tramo de la curva de sinterización.

i) $\quad 1^{\circ}$ tramo de la curva de interización.

Para cada uno de los composites, el primer tramo de la curva de sinterización obtenido a las cinco velocidades de calentamiento ensayadas se ha ajustado a la ec. 5.12. Se ha empleado el mismo procedimiento que el descrito en el apartado 5.1.2.1.3.1. Se han determinado los valores de los parámetros cinéticos: " $n$ " y $\ln A$, y de la varianza, $S^{2}$. En la Tabla 5.24 se detallan los resultados. Se incluyen en ésta, a efectos comparativos, los resultados correspondientes al vidrio de granulometría GM y GF y al composite F2.

Se comprueba (Figura 5.122 a Figura 5.126 y Tabla 5.24) que el modelo cinético utilizado describe la etapa de sinterización por flujo viscoso, que corresponde al $1^{\circ}$ tramo de la curva para todos los compactos, excepto para el $\mathrm{H} 2$ que abarca todo el proceso. En los ajustes a la ec. 5.12, al igual que en los apartados anteriores (5.2.2.1.2.1 y 5.3.2.2.1.1) los parámetros " $n$ " y InA se han mantenido fijos, independientemente de la velocidad de calentamiento, $\beta$. 
Tabla 5.24. Valores de los parámetros cinéticos, $n$ y $\ln A$, y de la varianza, $S^{2}$, obtenidos al ajustar el primer tramo de las curvas de sinterización, $\alpha$ vs $T$, de los diferentes composites a la ec. 5.12 con $B=5495$ y $T_{0}=421 \mathrm{~K}$.

\begin{tabular}{|c|c|c|c|c|c|}
\hline Composite & Circón & Vidrio & $n$ & $\operatorname{InA}\left(\mathbf{s}^{-1}\right)$ & $\mathbf{s}^{2}$ \\
\hline GM & - & GM & $0,71 \pm 0,019$ & $17,5 \pm 0,16$ & 0,000956 \\
\hline F2 & F & GM & $0,405 \pm 0,013$ & $7,8 \pm 0,11$ & 0,000697 \\
\hline M2 & $0,5 \mathrm{~F}-0,5 \mathrm{H}$ & $\mathrm{GM}$ & $0,48 \pm 0,021$ & $10,8 \pm 0,35$ & 0,000737 \\
\hline H2 & $\mathrm{H}$ & $\mathrm{GM}$ & $0,48 \pm 0,018$ & $11,9 \pm 0,26$ & 0,000517 \\
\hline GF & - & $\mathrm{GF}$ & $0,70 \pm 0,017$ & $18,9 \pm 0,18$ & 0,000315 \\
\hline FF2 & $\mathrm{F}$ & $\mathrm{GF}$ & $0,42 \pm 0,021$ & $11,2 \pm 0,25$ & 0,000645 \\
\hline FM2 & $0,5 \mathrm{~F}-0,5 \mathrm{H}$ & $\mathrm{GF}$ & $0,46 \pm 0,019$ & $12,9 \pm 0,36$ & 0,000465 \\
\hline FH2 & $\mathrm{H}$ & GF & $0,48 \pm 0,018$ & $14,6 \pm 0,30$ & 0,000563 \\
\hline
\end{tabular}

ii) Curva completa de sinterización.

Además del composite F2 (estudiado en el apartado 5.2.2.1.2.2), el único composite con $\phi=0,525$ que tiene interés tecnológico es el FF2. Los dos tramos de las curvas de sinterización se han tratado de describir mediante el modelo cinético compuesto de dos etapas o contribuciones, que se desarrollan en paralelo, descrito en el apartado (5.2.2.1.2.2). Al ajustar los resultados experimentales al modelo descrito en aquel apartado, siguiendo el procedimiento y atendiendo a los requisitos allí propuestos, se han obtenido los parámetros cinéticos que se detallan en la Tabla 5.25. 
Tabla 5.25. Parámetros cinéticos obtenidos al ajustar los resultados experimentales al modelo descrito en el apartado 5.2.2.1.2.2.

\begin{tabular}{|c|c|c|c|c|c|c|}
\hline Etapa & $\mathbf{w}_{1}$ & $\mathbf{n}_{1}$ & $\operatorname{In} \mathbf{A}_{\mathrm{i}}\left(\mathbf{s}^{-1}\right)$ & $\mathbf{E}_{\mathrm{i}}(\mathbf{k J} / \mathrm{mol})$ & ec. & $\mathbf{s}^{2}$ \\
\hline 1 & 0,4 & 0,52 & 14,2 & - & ec. 5.30 & 0,000536 \\
\hline 2 & 0,6 & 0,40 & 30,3 & 510 & ec. 5.32 & 0,000866 \\
\hline
\end{tabular}

Se comprueba (Figura 5.127 y Tabla 5.25) que, al igual que para el composite $\mathrm{F} 2$, el modelo cinético desarrollado (ec. 5.30 , ec. 5.32 y ec. 5.33 ) decribe muy bien la sinterización no isoterma de compactos con alto contenido en circón.

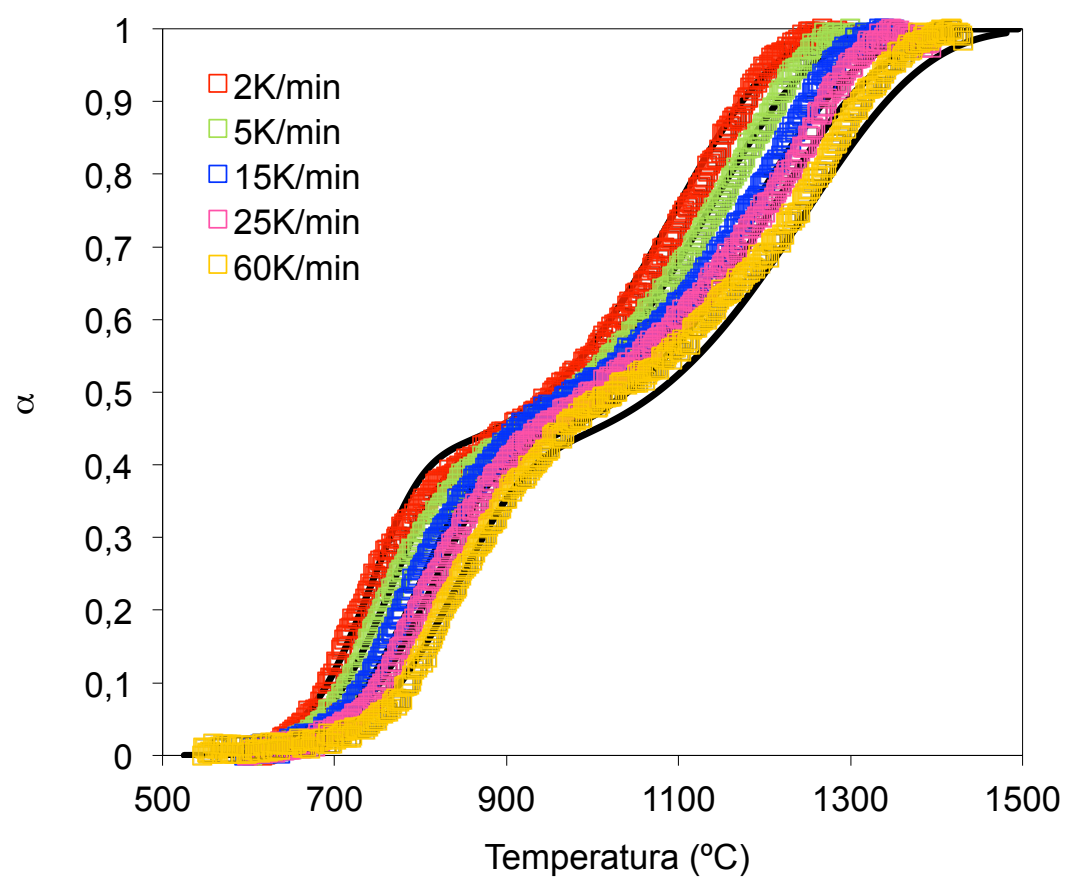

Figura 5.127. Curvas de sinterización del composite FF2, a velocidad de calentamiento $\beta=2,5,15,25$ y $60 \mathrm{~K} / \mathrm{min}$. Comparación entre los resultados experimentales y los calculados mediante el modelo desarrollado en el apartado 5.2.2.1.2.2. 


\subsection{Experimentos isotermos.}

Por los mismos motivos que se han señalado en el apartado 5.3.2.3.1 solo se han realizado experimentos isotermos con el composite FF2. En la Figura 5.128 se representan las curvas isotermas de sinterización obtenidas a 8 temperaturas diferentes.

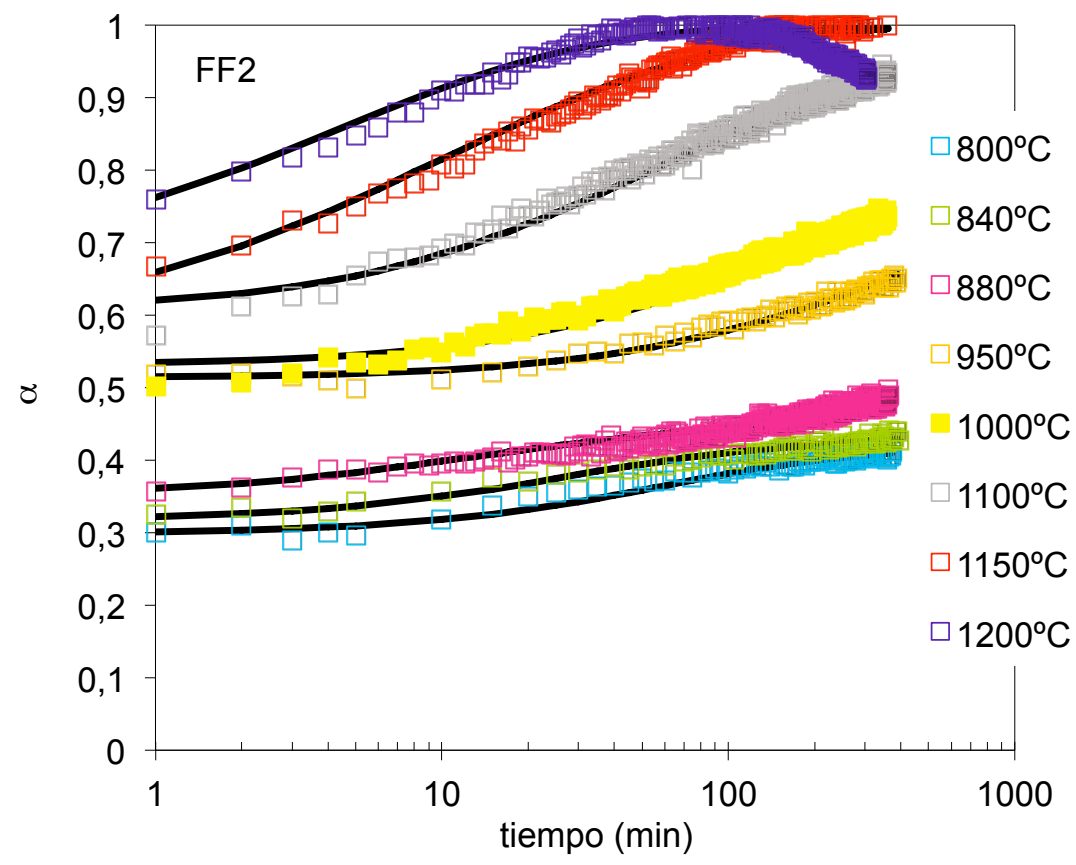

Figura 5.128. Curvas de sinterización isotermas del composite FF2, a distintas temperaturas máximas. Comparación entre los resultados experimentales y los calculados.

\subsection{Selección del modelo. Determinación del triplete cinético.}

El modelo cinético utilizado es el mismo que el empleado en el apartado 5.2.2.2.1.2 para describir, mediante dos etapas que se desarrollan en paralelo, la sinterización del composite F2. Siguiendo el mismo procedimiento que el desarrollado en aquel apartado, para todas las temperaturas ensayadas, fijando los mismos valores de los parámetros $w_{i} y$ 
$n_{i}$ que los obtenidos en los experimentos a velocidad de calentamiento constante (Tabla 5.25), $\mathrm{w}_{1}=0,4, \mathrm{n}_{1}=0,52$ y $\mathrm{n}_{2}=0,4$, se han conseguido muy buenos resultados (Figura 5.128). Para todos los ajustes individuales $S^{2} \leq 1,5 \cdot 10^{-4}$. Con las constantes de velocidad, $k_{1} y k_{2}$, que resultaron de estos ajustes se han obtenido las correspondientes rectas de Arrhenius (Figura 5.129). Se incluyen en esta las rectas correspondientes al composite F2.

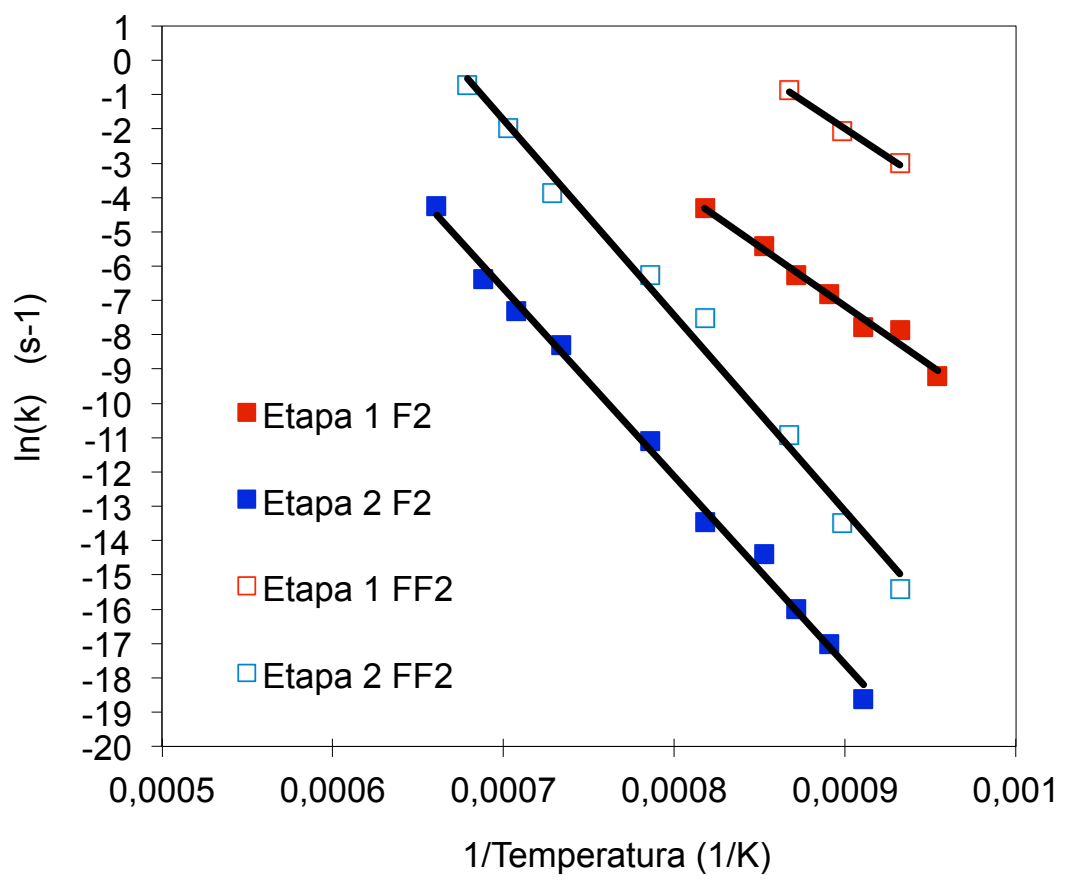

Figura 5.129. Representación de Arrhenius para el cálculo de la energía de activación de las dos etapas de sinterización, para los composites F2 y FF2.

El valor de la energía de activación obtenido para la primera etapa (Tabla 5.26), aún cuando su dispersión es alta, ya que se ha obtenido solo a partir de tres puntos, es prácticamente coincidente con el de flujo viscoso. En lo que respecta a la energía de activación del tramo 2 su valor es parecido al obtenido por el método no isotermo (Tabla 5.25) y al que resulta para el composite F2 (Tabla 5.15). 
Tabla 5.26. Valores de los parámetros cinéticos obtenidos al ajustar a la ec. 5.14 y la ec. 2.66, las curvas de sinterización isotermas del composite FF2. Se

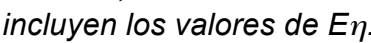

\begin{tabular}{|c|c|c|c|c|c|c|}
\hline Etapa & $w_{i}$ & $n_{i}$ & $\operatorname{InA}\left(\mathbf{s}^{-1}\right)$ & $\begin{array}{c}E_{i} \\
(\mathbf{k J} / \mathrm{mol})\end{array}$ & $\begin{array}{c}E \eta \\
(\mathrm{kJ} / \mathrm{mol})\end{array}$ & $\mathbf{s}^{2}$ \\
\hline 1 & 0,4 & 0,52 & $27,6 \pm 2,8$ & $274 \pm 26$ & $294 \pm 2$ & $6,58 \cdot 10^{-5}$ \\
\hline 2 & 0,6 & 0,40 & $38,2 \pm 1,8$ & $474 \pm 18$ & - & 0,00012 \\
\hline
\end{tabular}

Al comparar las representaciones de Arrhenius correspondientes a los composites F2 y FF2 (Figura 5.129) se confirma no solo la similitud en las energías de activación de éstos composites (rectas paralelas dos a dos), sino también el efecto que ejerce el tamaño de partícula de la matriz vítrea sobre las constantes de velocidad de cada etapa. En efecto, a igualdad de temperaturas, los valores de las constantes $k_{1}$ y $k_{2}$ correspondientes al composite FF2 preparado con vidrio de granulometría más fina (GF) son más altos que los del composite F2, obtenido con una granulometría de vidrio más gruesa $(G M)$.

5.3.2.4 Influencia de la granulometría del circón y del vidrio sobre los parámetros cinéticos. La importancia de la razón $\frac{\mathrm{d}_{\mathrm{z}}^{\mathrm{v}}}{\mathrm{d}_{\mathrm{g}}^{\mathrm{v}}}$.

A la vista de los resultados obtenidos en los apartados 5.3.2.2 y 5.3.2.3 se desprende que, de forma general, la velocidad de sinterización por flujo viscoso aumenta conforme se incrementa la granulometría del circón y/o se reduce la del vidrio. Dicha tendencia también se manifiesta en un aumento del factor preexponencial (InA) y del exponente de Avrami ( $n$ ) como puede comprobarse en la Tabla 5.22 y Tabla 5.23. Estos resultados son consistentes con los obtenidos por Dutton et al. [129] y Boccaccini [33] 
quienes observaron para contenidos moderados de inclusiones rígidas, $\phi \approx$ 20-30\%, una disminución de la velocidad de sinterización y una mayor caída de ésta con el avance del proceso, conforme se reduce el tamaño de la inclusión.

Este comportamiento debe estar también relacionado con la relativa abundancia de los distintos tipos de contactos (vidrio-vidrio, gg, vidrio-circón, gh y circón-circón, hh) existentes en los compactos y su variación con la granulometría del circón y del vidrio.

Dicha información es prácticamente imposible de obtener dada la complejidad de los sistemas de partículas utilizados (distribución de tamaños de partícula anchas e incluso bimodales, formas irregulares, etc.). No obstante, es muy probable que un tratamiento muy simplificado del problema sea suficiente para comprender el efecto de las granulometrías de los dos componentes sobre la cinética del proceso de sinterización.

En el modelo simplificado se considera que las partículas de cada componente son esféricas y del mismo tamaño, por lo que la razón entre el número de partículas de vidrio, $\mathrm{N}_{\mathrm{g}}$, y de circón, $\mathrm{N}_{\mathrm{z}}$, depende de la fracción volumétrica de circón, $\phi$, y de la razón del diámetro medio volumétrico de vidrio, $d_{g}{ }^{v}, y$ del circón, $d_{z}{ }^{v}$, mediante la ecuación:

$\frac{N_{g}}{N_{z}}=\left(\frac{1-\phi}{\phi}\right)\left(\frac{d_{z}^{v}}{d_{g}^{v}}\right)^{3}$

El número de partículas de vidrio necesarias para recubrir completamente a una partícula de circón, $\mathrm{N}_{\mathrm{g}} / \mathrm{N}_{\mathrm{z}}$, en el caso de un empaquetamiento denso e ideal, viene dado por [134]:

$$
\left(\frac{N_{g}}{N_{z}}\right)_{C r}=3,0+3,16\left(\frac{d_{z}^{v}}{d_{g}^{v}}\right)_{C r}^{2}
$$


Al igualar ec. 5.40 y ec. 5.41 se obtiene el valor de $\left(\frac{d_{z}^{v}}{d_{g}^{v}}\right)_{C r}$ al que todas las partículas de circón están recubiertas por una monocapa de partículas de vidrio sin que queden partículas de vidrio en contacto con otras. Es decir, para $\left(\frac{d_{z}^{v}}{d_{g}^{v}}\right)_{c r}$ no existirán contactos entre partículas de circón (hh), ni contactos (gg).

Así pues, operando se obtiene:

$$
\frac{1-\phi}{\phi}=\left[3+3,16\left(\frac{d_{z}^{v}}{d_{g}^{v}}\right)_{C r}^{2}\right] \cdot\left(\frac{d_{z}^{v}}{d_{g}^{v}}\right)_{C r}^{-3}
$$

Para los valores de $\phi$ utilizados para estudiar el efecto de la granulometría de cada uno de los componentes se obtienen los siguientes valores de $\left(\frac{d_{z}^{v}}{d_{g}^{v}}\right)_{C r}$

Para $\phi=0,322\left(\frac{d_{z}^{v}}{d_{g}^{v}}\right)_{C r} \approx 2$

ec. 5.43

Para $\phi=0,525\left(\frac{d_{z}^{v}}{d_{g}^{v}}\right)_{C r} \approx 5$

Así pues, para cada contenido en circón, $\phi$, existe un valor crítico de la razón de tamaños, $\left(\frac{d_{z}^{v}}{d_{g}^{v}}\right)_{C r}$, que aumenta con $\phi$, por debajo del cual todas las partículas de circón no están rodeadas completamente por partículas de vidrio, y son, por tanto, susceptibles de formar contactos (hh). Teóricamente 
la abundancia relativa de estos contactos (hh) debe aumentar conforme se incrementa la diferencia:

$\left(\frac{d_{z}^{v}}{d_{g}^{v}}\right)_{C r}-\left(\frac{d_{z}^{v}}{d_{g}^{v}}\right)$

Análogamente, para mezclas en las que $\left(\frac{d_{z}^{v}}{d_{g}^{v}}\right)$ sea mayor que la crítica $\left(\frac{d_{z}^{v}}{d_{g}^{v}}\right)_{C r}$ existirán partículas de vidrio que no rodeen a partículas de circón y que formen contactos (gg).

Así pues, al menos de forma aproximada, se desprende que con el aumento de la razón, $\left(\frac{d_{z}^{v}}{d_{g}^{v}}\right)$, para un determinado contenido de circón, $\phi$, se van reduciendo progresivamente el numero de contactos (hh) y/o (hg) y aumentan los (gg). Dicho efecto es dependiente de $\phi$; en efecto, a igualdad de $\left(\frac{d_{z}^{v}}{d_{g}^{v}}\right)$, cuanto mayor es $\phi$, más alto es el valor de $\left(\frac{d_{z}^{v}}{d_{g}^{v}}\right)_{C r}$ (ec. 5.43), lo que implica una mayor abundancia relativa de contactos (hh) y/o (hg) respecto a los $(\mathrm{gg})$.

En consecuencia, puede considerarse, al menos de forma cualitativa, que la disminución de la razón $\left(\frac{d_{z}^{v}}{d_{g}^{v}}\right)$ es equivalente, a efectos de la abudancia relativa de contactos (hh), (hg) y (gg), al aumento del contenido en sólidos $\phi$. Por otra parte, al igual que se hizo en el apartado 5.2.2.3, se ha tratado de explicar el efecto de la granulometría sobre la cinética del proceso de sinterización por flujo viscoso, basándose en la teoría de la percolación según la cual, la velocidad de sinterización y la viscosidad efectiva del 
sistema depende de la diferencia entre $\phi$ y $\phi_{p}$ (fracción volumétrica de percolación) y de la naturaleza del contacto (hh).

En este caso, el valor de $\left(\frac{d_{z}^{v}}{d_{g}^{v}}\right)$ debe modificar $\phi_{p}$, o más concretamente, la curva de probabilidad de que se forme una red continua de partículas de circón en contacto. Por simulación [135][136] y mediante el desarrollo de modelos estructurales [137] se ha comprobado que los valores de $\phi_{p}$ (y las curvas de probabilidad de percolación) se desplazan hacia valores más bajos conforme se reduce el valor de la razón $\left(\frac{d_{z}^{v}}{d_{g}^{v}}\right)$. En consecuencia, con el aumento de la razón $\left(\frac{d_{z}^{v}}{d_{g}^{v}}\right)$ debe aumentar el valor de $\phi_{p}$ y con ello $\phi_{p}-\phi$ (a igualdad de $\phi$ ) lo que debe incrementar la velocidad de sinterización.

5.3.2.4.1 Composites con moderados contenidos en circón $(\phi=0,322)$.

i) Índice de potencia de Avrami, "n".

En la Figura 5.130 se han representado los valores de "n", obtenidos en experimentos isotermos y no isotermos, frente a la razón $\left(\frac{d_{z}^{v}}{d_{g}^{v}}\right)$. Los resultados se han ajustado a una ecuación del tipo:

$$
\frac{n}{n_{g}}=\frac{1}{1+a\left(\frac{d_{z}^{v}}{d_{g}^{v}}\right)^{-b}}
$$

siendo a y b dos parámetros de ajuste. Dicha expresión es consistente con el comportamiento observado, ya que predice un aumento de "n" con la razón $\left(\frac{d_{z}^{v}}{d_{g}^{v}}\right)$, siendo el efecto de esta última tanto menor cuando mayor es la 
razón de tamaños, y tiende asistóticamente al valor de $\mathrm{n}_{\mathrm{g}}=0,7$ (el del vidrio) cuando la razón $\left(\frac{d_{z}^{v}}{d_{g}^{v}}\right)$ tiende a infinito. Se comprueba (Figura 5.130) un buen ajuste entre los resultados experimentales y los calculados según la ec. 5.40 .

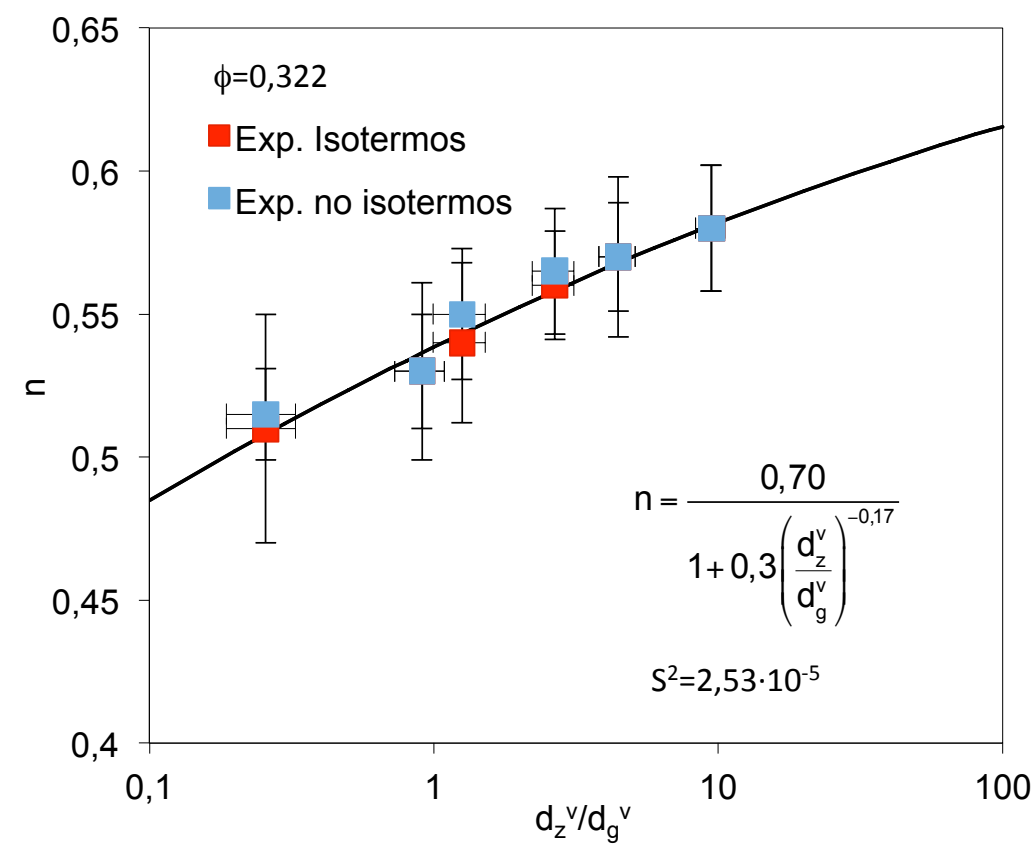

Figura 5.130. Representación de los valores de $n$ obtenidos en experimentos isotermos y no isotermos, frente a la razón $d_{z}{ }^{v} / d_{g}{ }^{\nu}$. Comparación entre los valores experimentales y los calculados mediante la ecuación que se indica.

ii) Energía de activación, "E".

Al igual que se observó en el apartado 5.2.2.3.1, la variación que experimenta $\mathrm{E}$, obtenida en experimentos isotermos, con la granulometría de los componentes (circón y vidrio), se debe, además del error experimental, a la variación que sigue la energía de activación de flujo

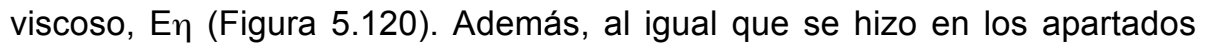
5.1.2.2 y 5.2.2.3.1 se han representado en la Figura 5.131, frente a la inversa del tiempo característico del vidrio, $\gamma / \eta \mathrm{d}$, los valores de las 
constantes de velocidad, $\mathrm{k}$, obtenidas en experimentos isotermos, en escala doble logarítmica. Al ajustar los resultados a la ecuación $k=\lambda\left(\frac{\gamma}{\eta d}\right)$, se obtuvieron muy buenos resultados, ya que los puntos experimentales se ajustaban a rectas paralelas de pendiente unidad (escala doble logarítmica) con diferentes valores de $\lambda$.

La estricta proporcionalidad entre k y $1 / \eta$ confirma, una vez más, que el efecto de la temperatura sobre la velocidad del proceso se debe "exclusivamente" al que ejerce dicha variable sobre la viscosidad de la matriz vítrea.

Lo propio se concluye de los buenos resultados obtenidos en experimentos a velocidad de calentamiento constante, considerando que el efecto de la temperatura sobre la velocidad del proceso es el debido exclusivamente al de dicha variable sobre $\eta$. 

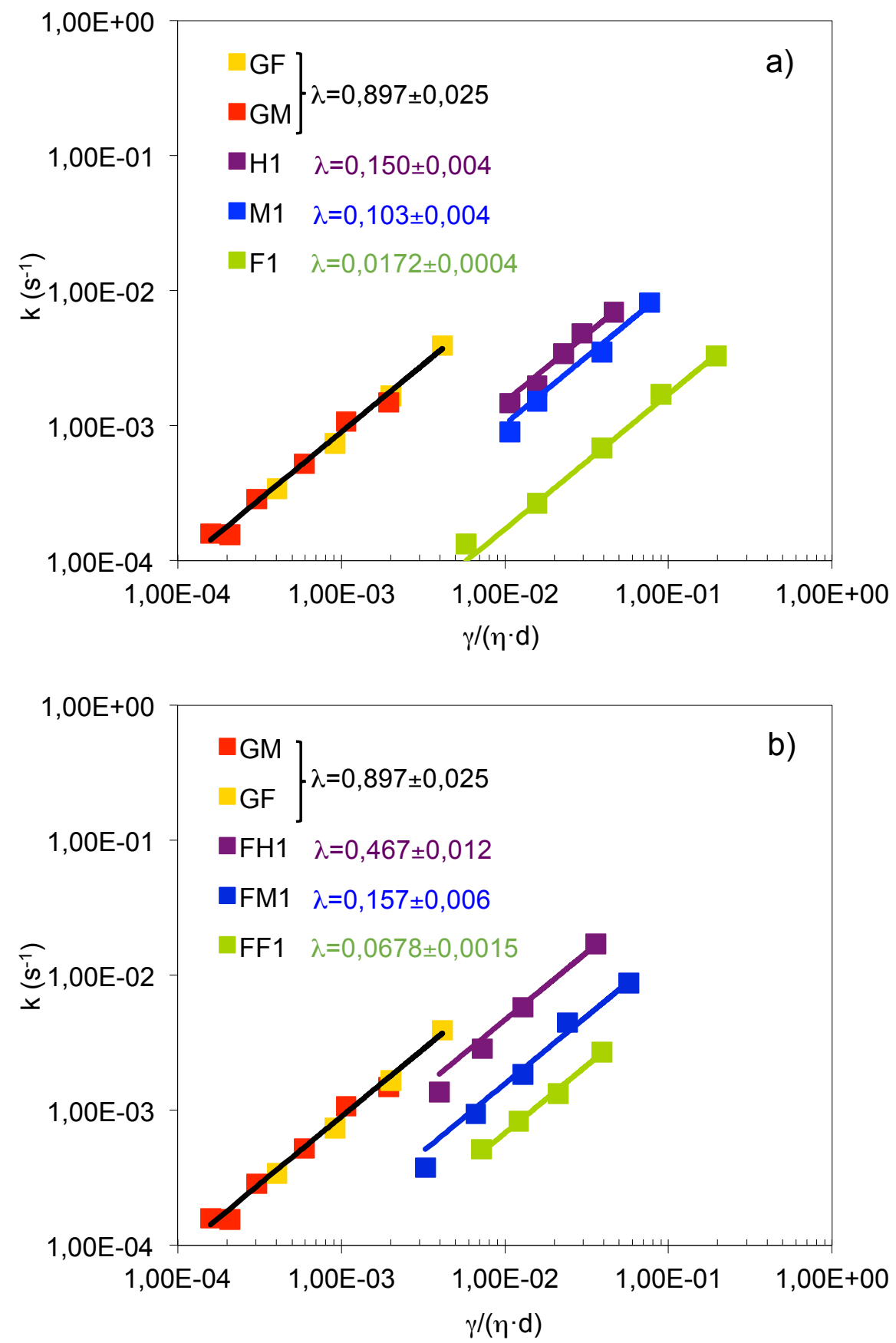

Figura 5.131. Representación de la constante de velocidad, $k$, frente a la inversa del tiempo característico del vidrio, $\gamma / \eta d$, obtenidas en experimentos isotermos, en escala doble logarítmica. a) composites de vidrio GM y b) composites de vidrio GF. 
iii) Factor preexponencial "A" y constante de velocidad adimensional, $\lambda$.

De acuerdo con lo expuesto al inicio de este apartado, la relación existente entre la razón $\left(\frac{d_{z}^{v}}{d_{g}^{v}}\right)$ y la abundancia relativa de los distintos tipos de contactos y el límite de percolación, $\phi_{p}$, determina que los valores $A / A_{g}$, obtenidos en experimentos isotermos y no isotermos aumentan conforme lo hace dicha razón (Figura 5.132 y Figura 5.133). Los resultados se han ajustado a una ecuación del mismo tipo que la ec. 5.40 y los valores de los parámetros obtenidos se han indicado en la Figura 5.132 y la Figura 5.133. Se aprecia, claramente, una gran dispersión en los valores de $\ln \left(\frac{A}{A_{g}}\right)_{\text {isot }}$, al igual que ocurría en el apartado 5.2.2.3.1, que es debido a la dispersión de los valores de la energía de activación, obtenidos en experimentos isotermos (como ya se ha comentado en el apartado 5.2.2.3.1, iii).

Para determinar más claramente, con menos dispersión, el efecto de $\left(\frac{d_{z}^{v}}{d_{g}^{v}}\right)$ sobre la velocidad de sinterización del composite, en experimentos isotermos (al igual que se hizo en el apartado 5.2.2.3.1, iii)) se han determinado los valores de la constante adimensional de velocidad: $\lambda=\mathrm{k}\left(\frac{\gamma}{\eta \mathrm{d}}\right)^{-1}$ para todos los composites y para el vidrio, $\lambda_{\mathrm{g}}$. Al representar la razón $\left(\frac{\lambda}{\lambda_{g}}\right)$ vs $\left(\frac{d_{z}^{v}}{d_{g}^{v}}\right)$ (Figura 5.134) se comprueba que los resultados se ajustan muy bien a la ecuación del tipo ec. 5.40, con valores de los parámetros idénticos a los utilizados para la razón $\left(\frac{A}{A_{g}}\right)_{\text {noisot }}$. 


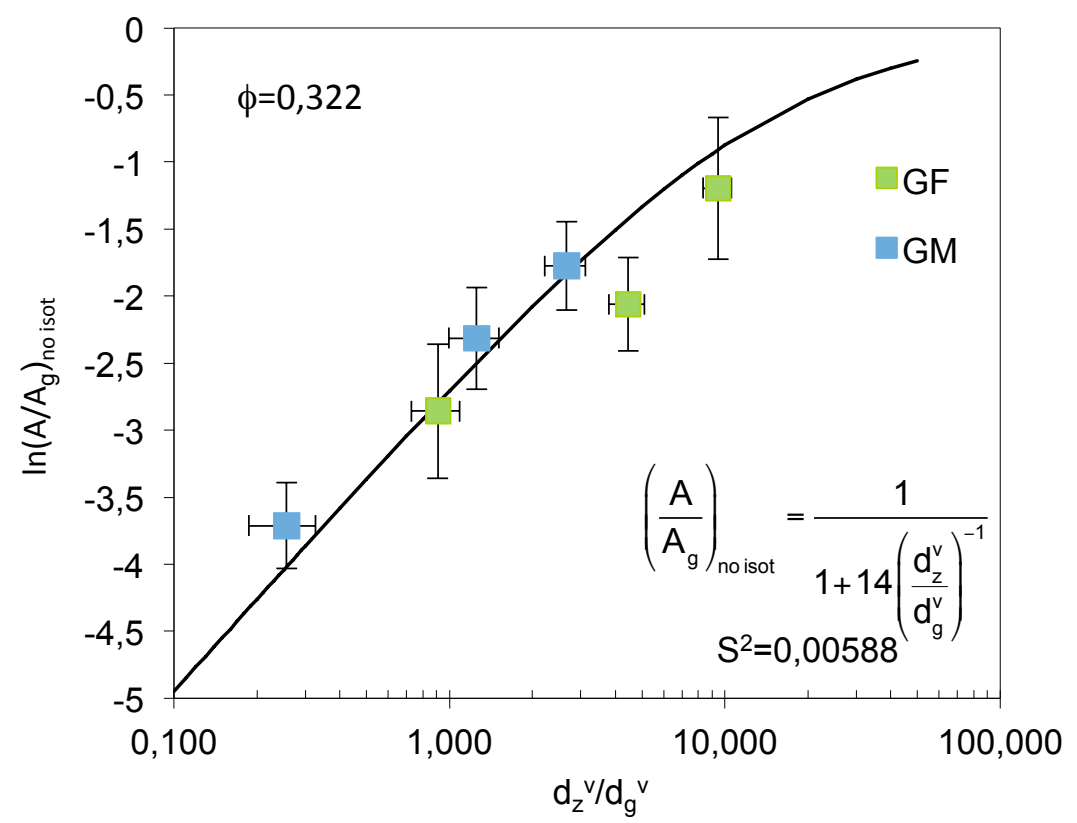

Figura 5.132. Representación de los valores de $\ln \left(A / A_{g}\right)$ obtenidos en experimentos no isotermos, frente a la razón $d_{z}^{v} / d_{g}^{v}$. Comparación entre los valores experimentales y los calculados mediante la ecuación que se indica.

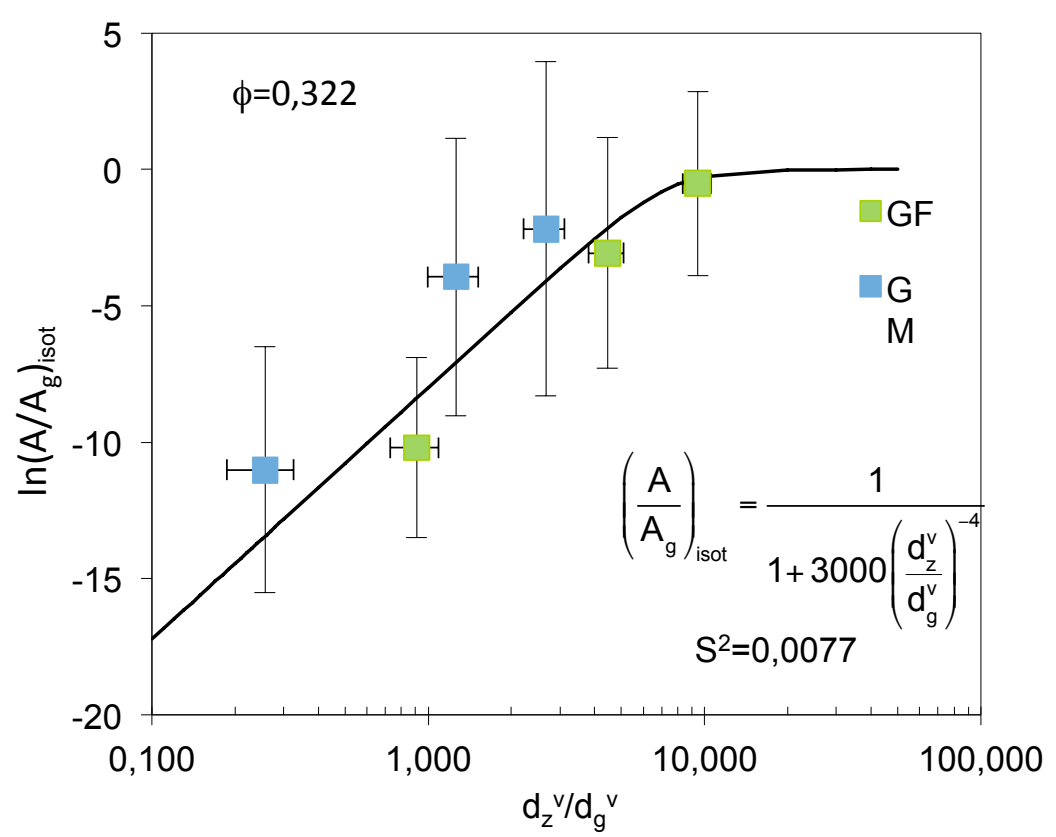

Figura 5.133. Representación de los valores de $\ln \left(A / A_{g}\right)$ obtenidos en experimentos no isotermos, frente a la razón $d_{z}^{v} / d_{g}^{v}$. Comparación entre los valores experimentales y los calculados mediante la ecuación que se indica. 


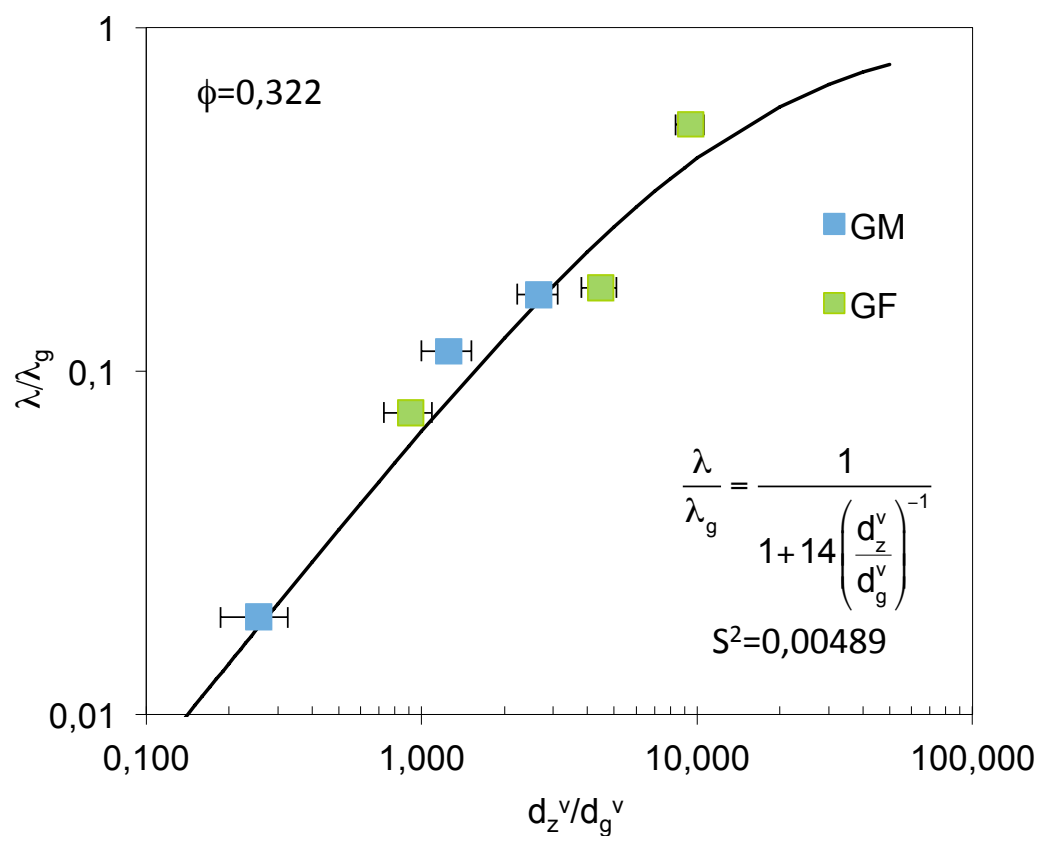

Figura 5.134. Representación de los valores de $\lambda \lambda_{g}$ obtenidos en experimentos no isotermos, frente a la razón $d_{z}^{v} / d_{g}^{v}$. Comparación entre los valores experimentales y los calculados mediante la ecuación que se indica.

Así pues, se confirma que la influencia de la granulometría de la matriz vítrea y del circón sobre la velocidad de sinterización de composites con un contenido en circón dado, tanto en experimentos isotermos como no isotermos, queda perfectamente descrito mediante una ecuación del tipo:

$\left(\frac{\chi}{\chi_{g}}\right)=\frac{1}{1+a\left(\frac{d_{z}^{v}}{d_{g}^{v}}\right)^{-b}}$

donde: $\chi$ y $\chi_{g}$ son los parámetros cinéticos, " $\mathrm{n}$ " el índice de potencia de Avrami, A el factor preexponencial y $\lambda$ la constante de velocidad adimensional del composite y del vidrio, respectivamente. "a" y "b" son los parámetros de ajuste. 
Se comprueba, asimismo, que la razón $\left(\frac{d_{z}^{v}}{d_{g}^{v}}\right)$ ha resultado ser suficiente para describir el efecto de la granulometría de los composites (vidrio y circón) sobre la velocidad de sinterización. Este hecho se explica atendiendo a la relación existente entre la razón, $\left(\frac{d_{z}^{v}}{d_{g}^{v}}\right)$, y la relativa abundancia del número de contactos $(g g),(g h)$ y $(h h)$, por una parte. $Y$, por otra, entre $\left(\frac{d_{z}^{v}}{d_{g}^{v}}\right)$ y la fracción volumétrica límite de percolación, $\phi_{p}$.

5.3.2.4.2 Composites con elevados contenidos en circón $(\phi=0,525)$.

De acuerdo con la ec. 5.42 y el sistema ec. 5.43, al pasar de contenidos moderados de circón $(\phi=0,322)$ a elevados $(\phi=0,525)$, la razón entre los tamaños $\left(\frac{d_{z}^{v}}{d_{g}^{v}}\right)_{\text {crit }}$ se incrementa de 2 a 5 , por lo que, a igualdad de granulometría o de $\left(\frac{d_{z}^{v}}{d_{g}^{v}}\right)$, la diferencia entre ambos será mayor, lo que se traduce en una mayor abundancia relativa de contactos (hh) y (gh).

Análogamente, al pasar de $\phi=0,322$ a $\phi=0,525$ la fracción volumétrica de partículas de circón, $\phi$, se aproxima más al límite de percolación, $\phi_{p}$.

Ambas consideraciones conducen a que el valor de " $n$ " para $\phi=0,525$ siga la misma tendencia con la variación de $\left(\frac{d_{z}^{v}}{d_{g}^{v}}\right)$ que la que sigue para $\phi=0,322$, pero con un valor del índice de potencia " $\mathrm{n}$ " notablemente menor (Figura 5.130 y Figura 5.135). El mismo razonamiento puede aplicarse al factor 
preexponencial no isotermo, o más precisamente a $\ln \left(\frac{A}{A_{g}}\right)_{\text {noisot }}$ (Figura 5.136). La influencia de $\left(\frac{d_{z}^{v}}{d_{g}^{v}}\right)$ sobre los dos parámetros también se ha descrito mediante la ec. 5.46, obteniendo buenos resultados.

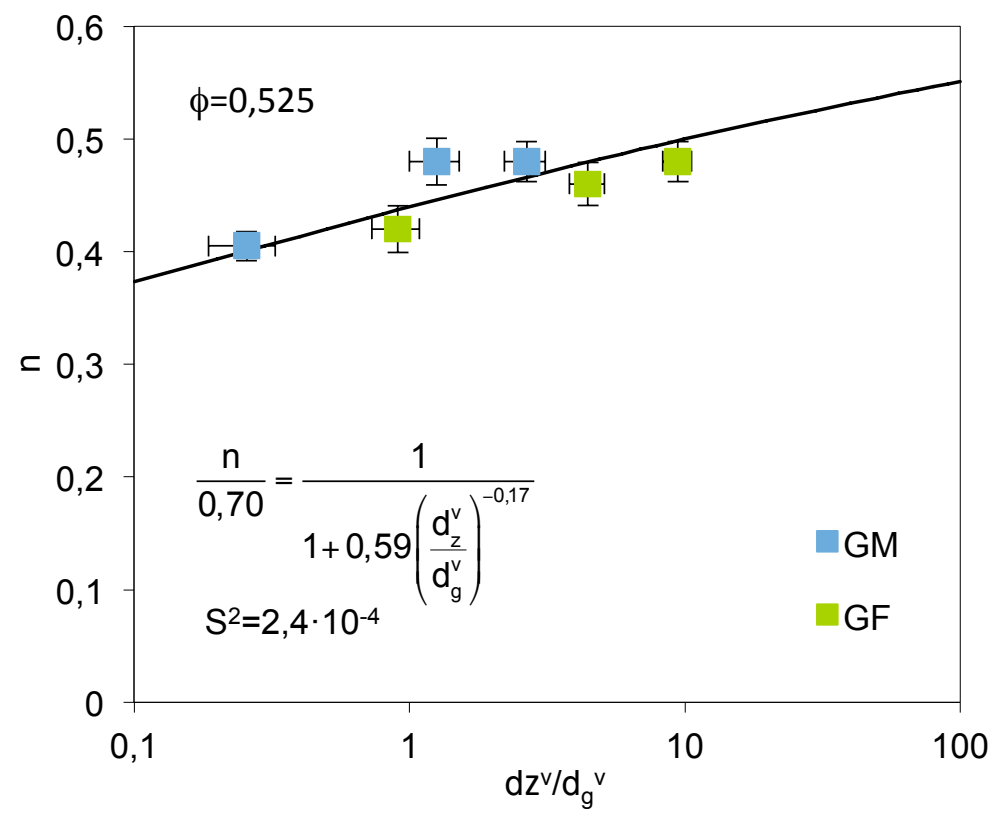

Figura 5.135. Representación de los valores de $n$ obtenidos en experimentos no isotermos, frente a la razón $d_{z}^{v} / d_{g}^{v}$. Comparación entre los valores experimentales y los calculados mediante la ecuación que se indica. 


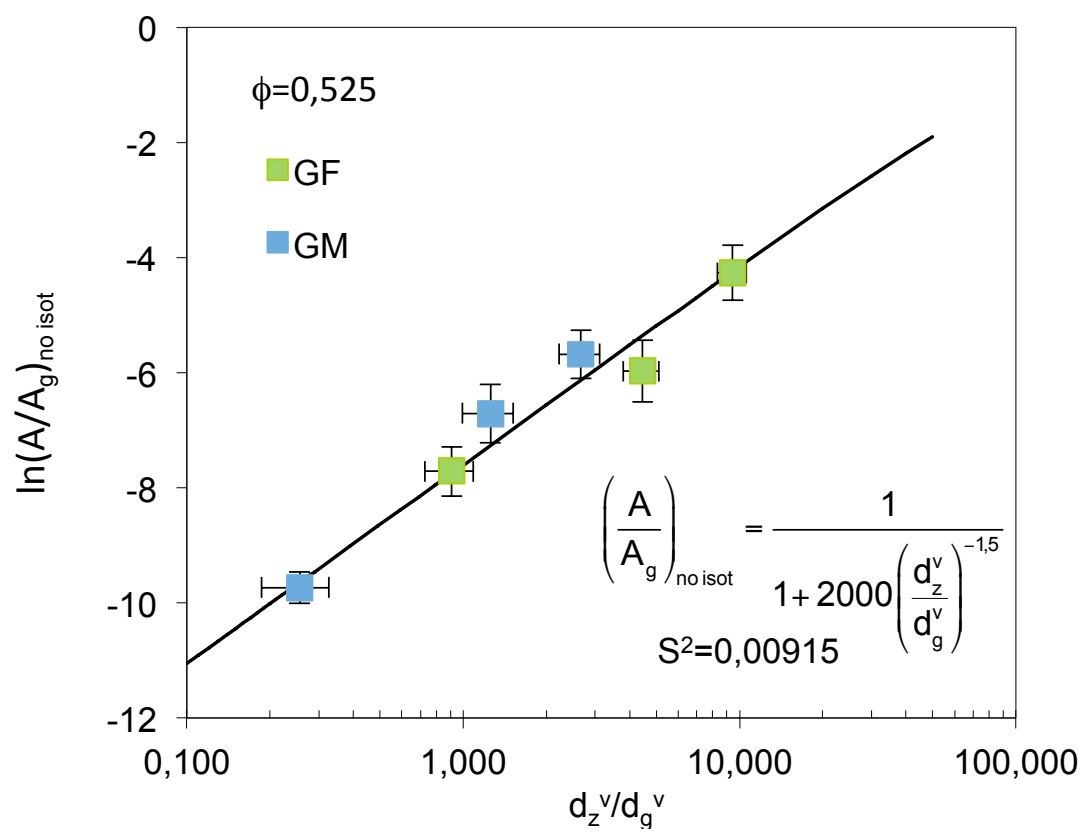

Figura 5.136. Representación de los valores de $\ln \left(A / A_{g}\right)$ obtenidos en experimentos no isotermos, frente a la razón $d_{z}^{v} / d_{g}^{v}$. Comparación entre los valores experimentales y los calculados mediante la ecuación que se indica.

5.4 Sinterización no isoterma de composites asociada a reordenación de partículas por flujo viscoso. Efecto combinado del contenido en circón y de las granulometrías del vidrio y del circón.

\subsubsection{Modelo cinético desarrollado.}

En los apartados anteriores (5.2 y 5.3) se ha comprobado lo siguiente:

- El efecto de la temperatura sobre la velocidad del proceso de sinterización por flujo viscoso es la que ejerce dicha variable sobre la viscosidad de la matriz vítrea, descrito por la expresión V.F.T. (ec. $5.1)$.

- El efecto del contenido en circón sobre la velocidad de sinterización, manteniendo constantes la granulometría del circón y de la matriz vítrea, queda perfectamente descrito mediante el efecto de $\phi$ sobre 
los parámetros cinéticos A y " $\mathrm{n}$ ", descrito en la Tabla 5.16 (apartado $5.2)$.

- La influencia de la granulometría del circón y del vidrio sobre la velocidad del proceso, manteniendo constante el contenido en circón, se ha descrito muy bien determinando la relación entre $A / A_{g}$ y la razón de tamaños $\left(\frac{d_{z}^{v}}{d_{g}^{v}}\right)$, y la de "n" con dicha razón, mediante ecuaciones del tipo de la ec. 5.46 (apartado 5.3.2.4). Además, el factor preexponencial del vidrio, $A_{g}$, está inversamente relacionado con su tamaño de partícula (Tabla 5.20) por lo que, $\left(A_{g} d_{g}^{v}\right)$ es constante.

En consecuencia, la velocidad de sinterización del composite asociada al mecanismo de flujo viscoso puede expresarse como:

$$
\frac{d \alpha}{d t}=\frac{\left(A_{g} d_{g}^{v}\right)}{d_{g}^{v}}\left(\frac{A}{A_{g}}\right) n(1-\alpha)[-\ln (1-\alpha)]^{\frac{n-1}{n}} \cdot \exp \left(\frac{-B}{T-T_{0}}\right)
$$

donde $\mathrm{B}$ y $\mathrm{T}_{0}$ son parámetros de la ecuación de V.F.T. (ec. 5.1) para el vidrio. Estos parámetros al igual que el producto $\left(A_{g} d_{g}^{v}\right)$ son solo función de la composición del vidrio.

Así pues, para disponer de una relación que exprese el efecto combinado de la granulometría de cada componente y del contenido en circón sobre la velocidad de sinterización por flujo viscoso, de acuerdo con la ec. 5.47, necesitamos determinar las relaciones: $\left(\frac{A}{A_{g}}\right)=\frac{A}{A_{g}}\left(\frac{d_{z}^{v}}{d_{g}^{v}}, \phi\right)$ y $n=n\left(\frac{d_{z}^{v}}{d_{g}^{v}}, \phi\right)$.

En la Figura 5.90 se comprueba que, para valores de $\alpha \approx 0,4$ el valor de $f(\alpha) \approx 0,55$ es independiente de $n$ para $0,4 \leq n \leq 0,7$; en consecuencia, de acuerdo con la ec. 5.47 se tiene que la razón entre la velocidad de 
sinterización del composite y la del vidrio, a cualquier temperatura, para un grado de sinterización de $\alpha \approx 0,4$ viene dada por:

$\left(\frac{\mathrm{d} \alpha}{\mathrm{dt}} / \frac{\mathrm{d} \alpha_{\mathrm{g}}}{\mathrm{dt}}\right)_{\alpha \approx 0,4}=\left(\frac{\dot{\alpha}}{\dot{\alpha_{g}}}\right)_{\alpha \approx 0,4}=\frac{\mathrm{A}}{\mathrm{A}_{\mathrm{g}}}$

Así pues, $\mathrm{A} / \mathrm{A}_{\mathrm{g}}$ también puede interpretarse como la razón de velocidades de sinterización entre el composite y el vidrio, para un grado de sinterización intermedio incipiente (Figura 5.11), en el que la textura porosa tanto del vidrio como de los composites (Figura 5.56) es todavía totalmente abierta. En consecuencia, dicha estructura puede considerarse, en lo que a la abundancia relativa de contactos (hh) se refiere, equivalente a un empaquetamiento compacto de inclusiones y vidrio al inicio del proceso [138].

Por otra parte, tanto la viscosidad efectiva de empaquetamientos compactos de partículas de composite, como la razón de velocidades de sinterización, $\left(\dot{\alpha} / \dot{\alpha}_{g}\right)$, obtenidos por simulación, y su relación con el contenido en inclusiones rígidas, $\phi$, puede describirse mediante expresiones del tipo:

$$
\left(\frac{\dot{\alpha}}{\dot{\alpha}_{g}}\right)=\left(1-\frac{\phi}{\phi_{p}}\right)^{v}
$$

Dicha expresión ya fue utilizada con éxito para describir la relación de $\mathrm{A}=\mathrm{A}(\phi)$, para una granulometría del circón y del vidrio determinada (Tabla $5.16)$, obteniéndose un valor de $\phi_{p} \approx 0,6$, próxima al teórico $\left(\phi_{p}=0,66\right)$. Este último se ha obtenido partiendo de esferas del mismo tamaño (inclusión y vidrio) suponiendo que el contacto entre inclusiones es de fricción [131]. 
También, según la teoría de la percolación [135][136], la fracción volumétrica crítica de percolación, $\phi_{\mathrm{p}}$, depende de la forma y del tamaño de las partículas, de tal modo que para mezclas binarias de esfereas, $\phi_{p}$ aumenta conforme lo hace la razón de tamaños entre la inclusión y la matriz del vidrio. En base a dicha consideración se explicaba la relación $\left(\frac{A}{A_{g}}\right)=\frac{A}{A_{g}}\left(\frac{d_{z}^{v}}{d_{s}^{v}}\right)$ y $n=n\left(\frac{d_{z}^{v}}{d_{s}^{v}}\right)$ del apartado 5.3.2.2.1.

En vista de todo lo expuesto, se pensó que la forma más sencilla y apropiada de determinar las relaciones $\left(\frac{A}{A_{g}}\right)=\frac{A}{A_{g}}\left(\frac{d_{z}^{v}}{d_{g}^{v}}, \phi\right)$ y $n=n\left(\frac{d_{z}^{v}}{d_{g}^{v}}, \phi\right)$ sería la siguiente:

En primer lugar, considerar que la razón de tamaños, $\left(\frac{d_{z}^{v}}{d_{s}^{v}}\right)$, solo afecta a $\phi_{p}$, por lo que a cada razón de diámetros, $\left(\frac{d_{z}^{v}}{d_{s}^{v}}\right)$, le corresponderá un valor de $\phi_{p}$. A partir de dicho valor se define y determina $\phi^{*}$ como la razón: $\phi^{*}=\frac{\phi}{\phi_{p}}$. $\mathrm{Al}$ introducir esta nueva variable, $\phi^{*}$, las expresiones que describen el efecto de $\phi$ sobre $A / A_{g} y$ " $n$ " (Tabla 5.16) quedarán como:

$$
\begin{aligned}
& \frac{\mathrm{A}}{\mathrm{A}_{\mathrm{g}}}=\left(1-\phi^{*}\right)^{v} \\
& \mathrm{n}=\mathrm{a}+\mathrm{b} \cdot \phi^{*}
\end{aligned}
$$

En segundo lugar, considerar que las relaciones anteriores (ec. 5.50 y ec. 5.51) no están afectadas por la razón, $\left(\frac{d_{z}^{v}}{d_{g}^{v}}\right)$, es decir, que $v$, a y b se mantienen constantes. 
Para todos los composites, los valores de $\left(\frac{A}{A_{g}}\right)_{\text {noisot }}$ y " $n$ ", obtenidos en experimentos no isotermos, se han ajustado, teniendo en cuenta estos requisitos a la ec. 5.50 y ec. 5.51 , seleccionando, para cada valor de $\left(\frac{d_{z}^{v}}{d_{g}^{v}}\right)$ el valor $\phi_{\mathrm{p}}$ más adecuado; es decir, aquel que conduzca simultáneamente al mejor ajuste de todos los valores a las relaciones de ec. 5.50 y ec. 5.51 (Figura 5.137 y Figura 5.138).

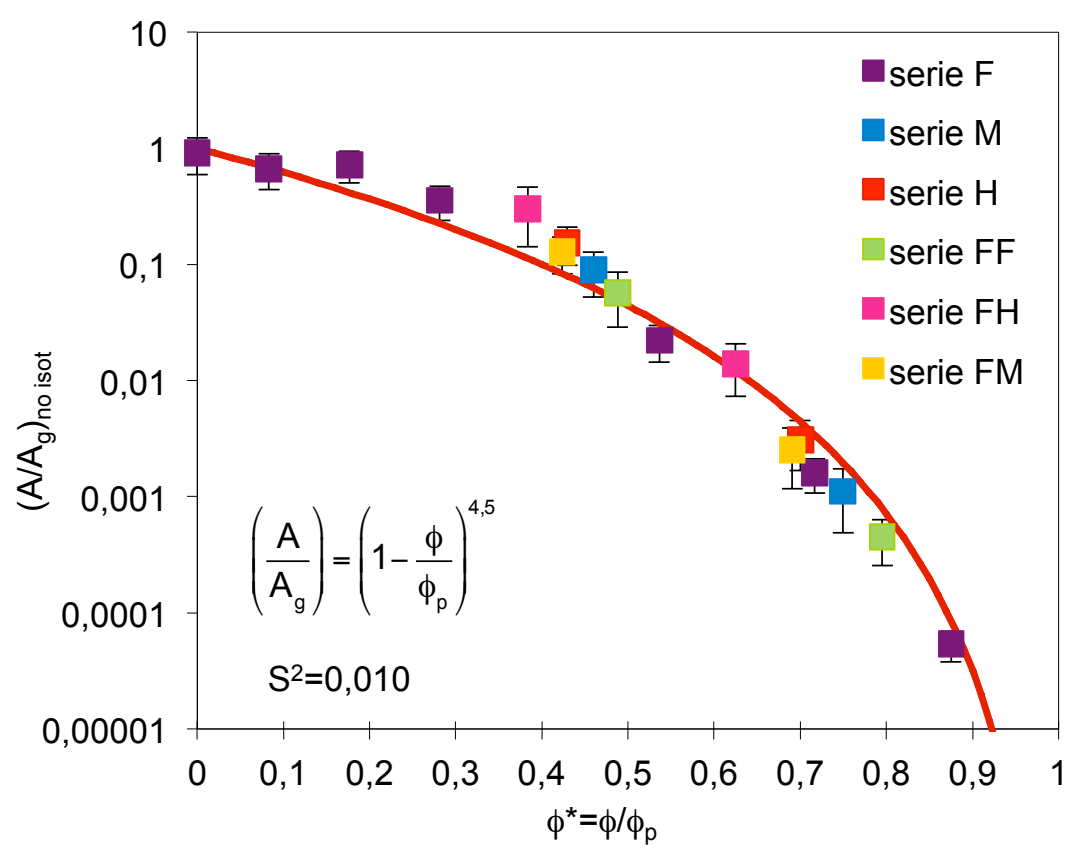

Figura 5.137. Representación de los valores de $\left(A / A_{g}\right)$ obtenidos en experimentos no isotermos, frente a $\phi^{*}=\phi / \phi_{p}$. Comparación entre los valores experimentales y los calculados mediante la ec. 5.50 y ec. 5.52 . 


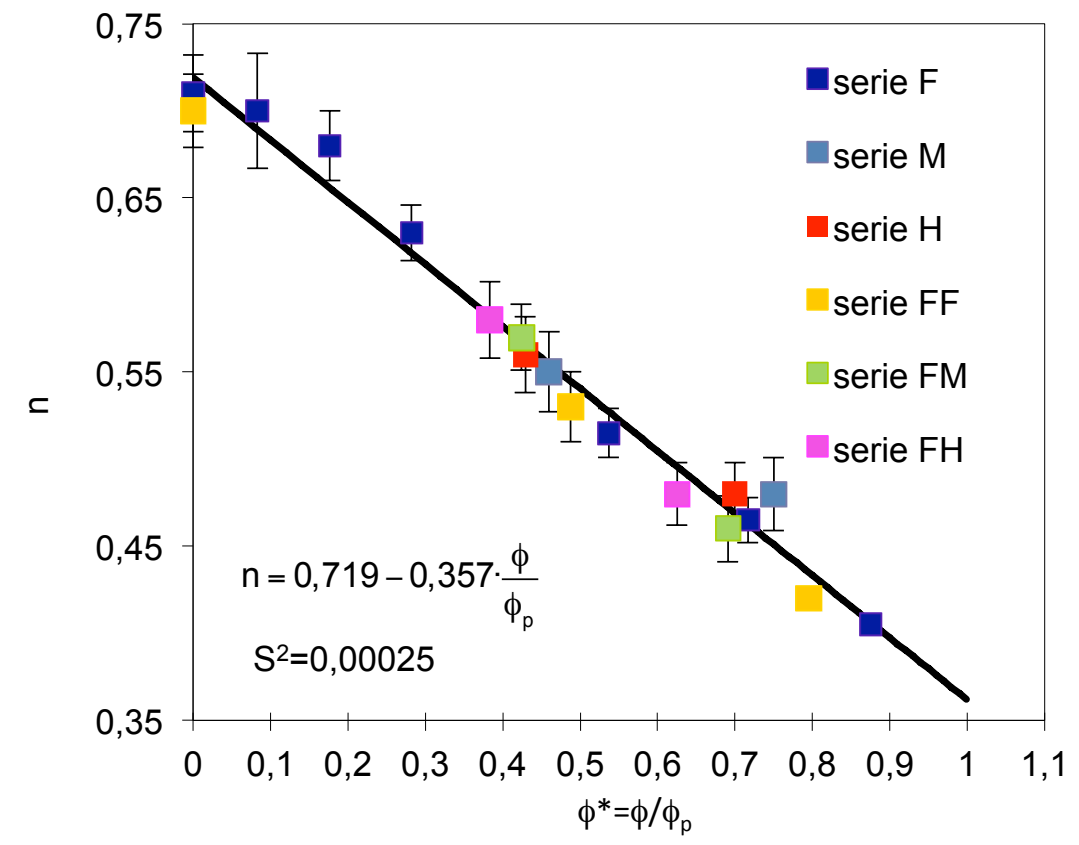

Figura 5.138. Representación de los valores de $n$ obtenidos en experimentos no isotermos, frente a $\phi^{*}=\phi / \phi_{p}$. Comparación entre los valores experimentales y los calculados mediante la ec. 2.51 y ec. 5.52 .

Se confirma que los resultados experiementales, aunque algo dispersos, se ajuntan aceptablemente bien a los calculados mediante las ec. 5.50 y ec. 5.51 , estando, además, los valores de $\phi_{p}$ relacionados con la razón $\left(\frac{d_{z}^{v}}{d_{g}^{v}}\right)$ mediante la ecuación (Figura 5.139):

$\phi_{p}=0,68+0,15 \cdot \log \left(\frac{d_{z}^{v}}{d_{g}^{v}}\right)$

Según la ec. 5.52, $\phi_{p}$, fracción volumétrica de circón, referida al sólido, volumen de circón/volumen de sólido, a la que las partículas de esta inclusión forman una red continua rígida aumenta conforme se incrementa la razón entre el tamaño de la inclusión y del vidrio. Estos resultados están de 
acuerdo con la bibliografía [135][136][137]. Además, se comprueba que para un valor de $\left(\frac{d_{z}^{v}}{d_{g}^{v}}\right)=1$ el valor que predice la ec. 5.52 es $\phi_{p}=0,68$, prácticamente coincidente al valor de $\phi_{\mathrm{p}}=0,66$, que predice el modelo de Jagota et al [131], al determinar por simulación el efecto de $\phi$ sobre las viscosidades y velocidad de sinterización de empaquetamientos de composites de partículas esféricas y del mismo tamaño.

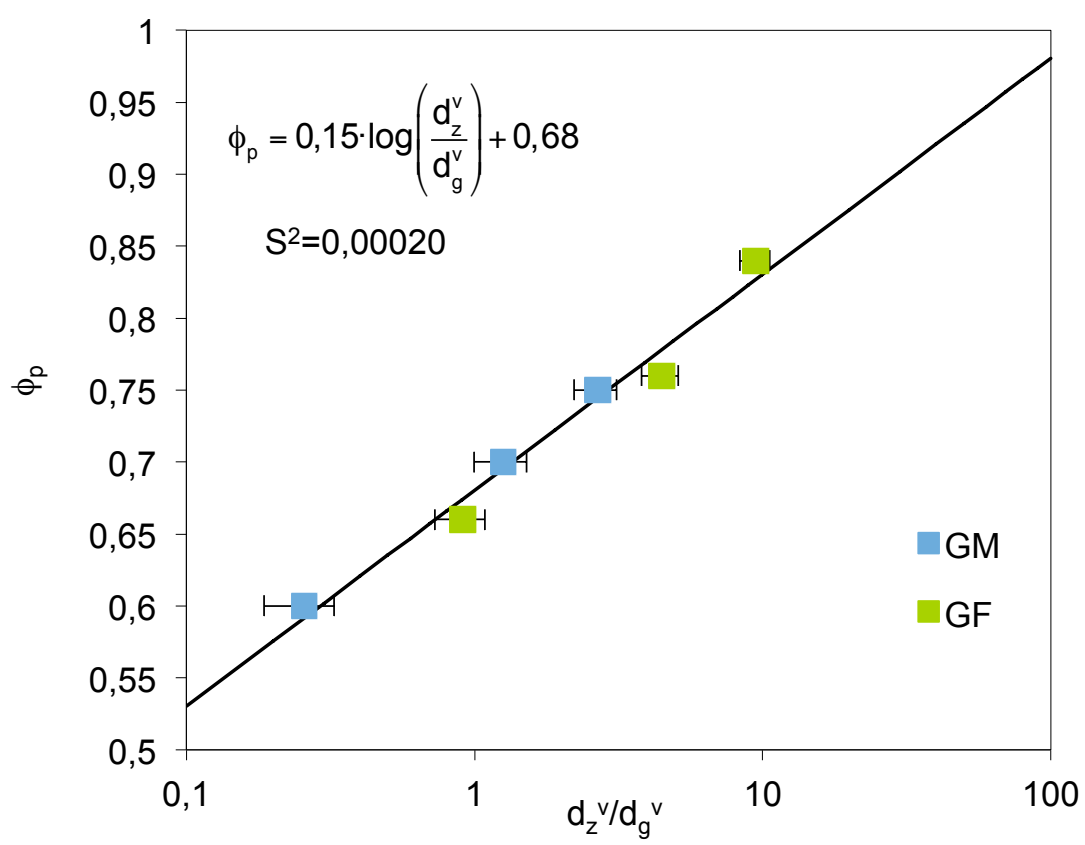

Figura 5.139. Representación de los valores de $\phi_{p}$ obtenidos en experimentos no isotermos, frente a la razón $d_{z}^{v} / d_{g}^{v}$. Comparación entre los valores experimentales y los calculados mediante la ec. 5.52.

\subsubsection{Validación del modelo.}

Para ello, se han conformado por prensado, siguiendo el procedimiento habitual (apartado 4.2), compactos con las siguientes características: $\phi=0,4$, 
$d_{z}^{v}=6,2 \mu m$ y $d_{g}^{v}=4,1 \mu \mathrm{m}$, a los que les corresponde una razón $\frac{d_{z}^{v}}{d_{g}^{v}}=1,52$.

Todas las características seleccionadas son diferentes a las utilizadas en la elaboración del modelo cinético desarrollado.

La granulometría del circón corresponde a una mezcla del $75 \%$ circón $\mathrm{F}$ (micronizado) y $25 \% \mathrm{H}$ (harina de circón). La granulometría de las partículas de vidrio se obtuvieron por molienda durante $1 \mathrm{~h}$ de la frita NBS, siguiendo el procedimiento habitual (apartado 4.2).

Los tratamientos térmicos (apartado 4.2.5.1) se realizaron a dos velocidades de calentamiento: una baja, $\beta=1 \mathrm{~K} / \mathrm{min}$, y una alta, $\beta=30 \mathrm{~K} / \mathrm{min}$, diferentes también a las empleadas habitualmente. Los resultados se detallan en la Figura 5.140.

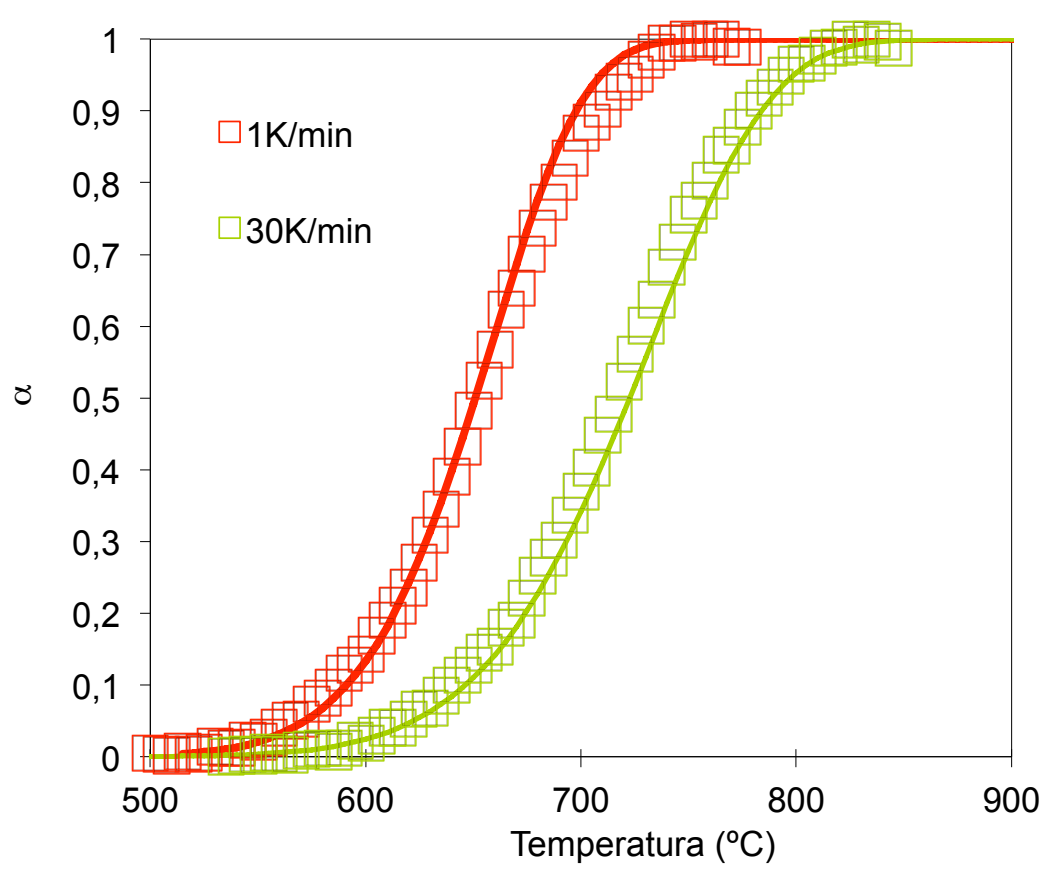

Figura 5.140. Curvas de sinterización del composite preparado para validar el modelo. Comparación entre los resultados experimentales y los calculados. 
Los valores de $\alpha$ que predice el modelo se han calculado mediante la ec. 5.12 sustituyendo los valores de A y "n" por los que resultan de aplicar las relaciones apropiadas. En efecto:

$A=\left(\frac{A}{A_{g}}\right)_{\text {no isot }} \cdot \frac{\left(A_{g} \cdot d_{g}^{v}\right)_{\text {noisot }}}{d_{g}^{v}}$

que de acuerdo con la ec. 5.50 y con un valor de $\left(A_{g} \cdot d_{g}^{v}\right)_{\text {noisot }}=325 \pm 15 \mathrm{~m} / \mathrm{s}$ (calculado de los valores de la Tabla 5.20) se transforma:

$A=\left(1-\frac{\phi}{\phi_{p}}\right)^{4,5} \cdot \frac{325}{d_{g}^{v}}$

Al sustituir los valores concretos de $\frac{d_{z}^{v}}{d_{g}^{v}}$, $\phi$ y $d_{g}^{v}(m)$ en la ec. 2.51 , ec. 2.52 y ec. 2.54 se obtienen los valores de $\mathrm{A}$ y $\mathrm{n}$ que deben introducirse en la ec. 5.12 para el cálculo de $\alpha$ a las velocidades de calentamiento de $\beta=1 \mathrm{~K} / \mathrm{min}$ y $\beta=30 \mathrm{~K} / \mathrm{min}$.

Se comprueba una buena concordancia entre los valores experimentales y los calculados según el modelo.

5.5 Interpretación de los parámetros cinéticos basándose en la teoría continua de la sinterización.

La ec. 5.47 también puede expresarse en función de la constante de velocidad como:

$\frac{d \alpha}{d t}=k_{g} \cdot\left(\frac{A}{A_{g}}\right) \cdot f(\alpha)$

o bien: 
$\frac{\mathrm{d} \alpha}{\mathrm{dt}}=\mathrm{k} \cdot \mathrm{f}(\alpha)$

ec. 5.56

Siendo $\mathrm{k}_{\mathrm{g}}$ y $\mathrm{k}$ las constantes de velocidad de sinterización del vidrio y de composite.

\subsubsection{Viscosidad efectiva del composite $\left(\eta_{c}\right)$ y viscosidad relativa $\left(\eta_{r}\right)$.}

De ec. 5.55 y ec. 5.56 resulta:

$$
\frac{\mathrm{k}}{\mathrm{k}_{\mathrm{g}}}=\frac{\mathrm{A}}{\mathrm{A}_{\mathrm{g}}}
$$

Al introducir en la ec. 5.57 para el vidrio $\left(k_{g}\right)$ y el composite $(k)$ la ec. 2.47, que relaciona la constante de velocidad del proceso de sinterización de una muestra, $K(T)$, con sus propiedades (tamaño de partícula, $r_{0}$, tensión superficial, $\gamma, y$ viscosidad, $\eta$ ) según la teoría continua de la sinterización viscosa (apartado 2.1.4.1.3.5) se tiene:

$\eta_{c}=\left(\frac{A}{A}\right) \cdot\left(\frac{\gamma}{r_{0}}\right)_{c} \cdot\left(\frac{r_{0}}{\gamma}\right)_{g} \cdot \ln \left(\frac{\rho_{\max }}{\rho_{0}}\right)_{g} \cdot \ln \left(\frac{\rho_{0}}{\rho_{\max }}\right)_{c} \cdot \eta$

El producto $\left[\ln \left(\frac{\rho_{\max }}{\rho_{0}}\right)_{g} \cdot \ln \left(\frac{\rho_{0}}{\rho_{\max }}\right)_{c}\right]$, según la ec. 5.15 es igual a la razón entre la contracción máxima de la silueta del composite, $\left(\varepsilon_{A, \max }\right)_{c}, y$ a del vidrio, $\left(\varepsilon_{A, \max }\right)_{g}, \quad\left(\varepsilon_{A, \max }\right)_{d} /\left(\varepsilon_{A}, \max \right)_{g}$. Aunque dicha razón disminuya con $\phi$, dependiendo esta reducción de la granulometría del vidrio y del circón (Figura 5.98), su magnitud es mucho menor que la que sigue $\left(A_{g} / A\right)$ con $\phi$, para todas las granulometrías, como puede comprobarse en la Figura 5.137.

Análogamente, el producto $\left(\frac{\gamma}{r_{0}}\right)_{c} \cdot\left(\frac{r_{0}}{\gamma}\right)_{g}$, que representa la razón entre la presión capilar al inicio de la sinterización, asociada al composite y la 
correspondiente al vidrio, se consideraran muy similares si el mojado del sólido por el vidrio es bueno [139][140][123].

Al considerar estas dos aproximaciones en la ec. 5.58 resulta:

$$
\eta_{c}=\left(\frac{A_{g}}{A}\right) \cdot \eta
$$

Así pues, la razón $\left(A_{g} / A\right)$ también tiene el significado físico de viscosidad relativa del composite, $\eta_{\mathrm{r}}$, definida como la razón entre la viscosidad efectiva del composite, $\eta_{c}$, y la del vidrio, $\eta$, ambos exentos de poros, y que de acuerdo con la ec. 5.50 y ec. 5.51, y la Figura 5.137 y Figura 5.139, varía con las granulometrías de los componentes (vidrio y circón) mediante la expresión de Krieger-Dougherty [141]:

$$
\eta_{\mathrm{r}}=\frac{\eta_{\mathrm{c}}}{\eta}=\left(1-\frac{\phi}{\phi_{\mathrm{p}}}\right)^{-4,5}
$$

donde $\phi_{p}$, que es equivalente a la máxima densidad de empaquetamiento, $\phi_{\max }$, está relacionado con $\left(\frac{d_{z}^{v}}{d_{g}^{v}}\right)$ mediante la ec. 5.52 .

En consecuencia, de acuerdo con la ec. 5.57, la constante de velocidad correspondiente a un composite, $\mathrm{k}$, es numéricamente igual a la de la matriz vítrea, $\mathrm{k}_{\mathrm{g}}$, dividido por la viscosidad relativa del composite, $\eta_{\mathrm{r}}$. En otras palabras, la constante de velocidad de un composite, $k$, es la resultante de sustituir en la expresión de la constante de la matriz vítrea, $\mathrm{k}_{\mathrm{g}}$, la viscosidad del vidrio, $\eta$, por la del composite, $\eta_{c}$.

Así pues, teniendo en cuenta además la ec. 5.39 resulta: 
$\mathrm{k}=\mathrm{k}_{\mathrm{g}} \cdot \eta_{\mathrm{r}}=\left(0,9 \frac{\gamma}{\eta \mathrm{d}}\right)\left(1-\frac{\phi}{\phi_{\mathrm{p}}}\right)^{-4,5}=\left(0,9 \frac{\gamma}{\eta_{\mathrm{c}} \mathrm{d}}\right)$

ec. 5.61

Los parámetros de la ec. $5.60, v=4,5$ y $0,6 \leq \phi_{p} \leq 0,83$ (ec. 5.52) obtenidos son consistentes con los resultados localizados en la bibliografía. En efecto, Müller [30], al estudiar, en composites de vidrio de borosilicato y corindón, el efecto del contenido en corindón sobre la viscosidad relativa del composite, $\eta_{\mathrm{r}}$, mediante experimentos de sinterización, utilizando un modelo cinético distinto al nuestro, obtuvo también un valor de $v=4,5$. Champellier [142] al relacionar los valores experimentales de la viscosidad efectiva de suspensiones de alúmina en líquidos magmáticos con la fracción volumétrica de sólidos, obtuvo un valor de $v=4 \pm 0,7$.

Análogamente, al estudiar la sinterización de esmaltes complejos [66] [67][68] y determinar el valor de su viscosidad efectiva a partir de los parámetros cinéticos del proceso, siguiendo un procedimiento similar al empleado en esta tesis, también se obtuvo un valor de $v=4,5$.

Los valores de $\phi_{p}$, comprendidos entre $0,6 \leq \phi \leq 0,83$, cuyo significado físico según la ec. 5.60 es el de máxima densidad de empaquetamiento de inclusiones, $\phi_{\max }$, también son consistentes con los localizados en la bibliografía. En efecto, para un vidriado complejo de aspecto mate, cuyo contenido en fases cristalinas variaba entre $0,3 \leq \phi \leq 0,6$, se ajustaba bien a la ec. 5.60 con $\phi_{p}=0,74$ [67]. Müller, en compactos vidrio-alúmina [30], con valores de $\phi \leq 0,35$ obtuvo un valor de $\phi_{p}=0,74$. En el estudio de viscosidades efectivas de suspensiones de alúmina en líquidos magmáticos, con valores de $\phi \leq 0,75$ [142] obtuvo un valor de $\phi=0,66 \pm 0,1$. En un trabajo anterior en el que se determinó la viscosidad efectiva de cinco esmaltes complejos, de composiciones distintas, sus viscosidades relativas se ajustaron bien a la ec. 5.60 con un valor crítico de 0,69 [68]. En todos estos casos no se ha tenido en cuenta las distribuciones de tamaño de partículas de las inclusiones ni la forma de las partículas. El hecho de que $\phi_{\mathrm{p}}$ aumenta con el tamaño medio 
del circón utilizado (y con la amplitud de la distribución) (Figura 5.139) se debe a que la densidad máxima de empaquetamiento de un componente aumenta con la ampliturd de su distribución granulométrica. La razón por la que a igualdad de tamaño de circón los composites preparados con una matriz vítrea más fina le corresponda un $\phi_{p}$ mayor, o lo que es lo mismo, un valor de $\eta_{\mathrm{r}}$ más bajo, se puede deber a que en éstos la interacción entre las partículas de circón en el seno de la matriz vítrea es menor (Figura 5.139).

\subsubsection{Modelo de sinterizacion, $f(\alpha)$.}

De acuerdo con la ec. 2.46, el modelo cinético $f(\alpha)$ se puede interpretar como la razón entre la presión de sinterización adimensional, $\wp_{\mathrm{L}}$, y el modulo viscoso también adimensional, $\psi$. Éstos últimos solo dependen de la compacidad del material (ec. 2.34 y Tabla 2.3; y ec. 2.43 y Tabla 2.4), por lo que para cada composite solo son función del grado de avance de la sinterización; pudiendo establecer la relación:

$f(\alpha)=\frac{\wp_{\llcorner}(\alpha)}{\Psi(\alpha)}=n \cdot(1-\alpha) \cdot[-\ln (1-\alpha)]^{\frac{n-1}{n}}$

siendo "n", función única de $\phi$ y $\left(\frac{d_{z}^{v}}{d_{g}^{v}}\right)$, el parámetro determinante del efecto de estas características sobre la caída de velocidad de sinterización con el avance del proceso.

Aunque en este trabajo no hemos determinado por separado $\wp_{L}(\alpha)$ y $\Psi(\alpha)$, a la vista de los resultados obtenidos se pueden extraer algunas consideraciones sobre dichos parámetros y sobre su variación con $\alpha$, que nos ayuden a interpretar la cinética del proceso. En efecto:

i) $\quad f(\alpha)$ también puede ser visto como la disminución de la razón entre la fuerza impulsora de la sinterización, $\wp_{L}(\alpha)$, y la resistencia viscosa a la densificación, $\Psi$, ambas adimensionales, con el avance del proceso. 
ii) La fuerza impulsora es la presión de sinterización, que de acuerdo con el apartado 5.2.1.2.3, $\sum^{S}$ (Figura 5.5), y en consecuencia $\wp_{L}(\alpha)$ disminuyen con el avance del proceso, tanto más cuanto mayor es el contenido en circón.

iii) La resistencia que se opone al flujo y a la densificación en los composites es principalmente la debida a los contactos entre partículas de inclusiones, que pueden inducir una estructura continua y rígida, que llegue a paralizar la densificación, si existe una conectividad entre partícuals rígidas suficientemente elevada. En cualquier caso, el modulo viscoso, $\Psi(\alpha)$, siempre aumenta con el avance del proceso, $\alpha$, debido, por una parte, a la disminución de la porosidad, y, por otra al crecimiento del número de contactos entre inclusiones, que es tanto mayor cuanto mayor es la fracción volumétrica de circón, $\phi$, y menor es la razón de tamaños entre el circón y el vidrio, $\left(\frac{d_{z}^{v}}{d_{g}^{v}}\right)$. Además, en estados intermedios y finales de la sinterización, en las que $\wp_{\llcorner}(\alpha)$ ya debe ser pequeña, la interacción entre partículas de inclusión rígidas es la controlante del proceso.

iv) La resultante de la disminución de la fuerza impulsora de la sinterización, $\wp_{L}(\alpha)$, por una parte y del aumento de la resistencia al flujo viscoso, $\Psi(\alpha)$, por otra, con el avance del proceso, $\alpha$, se manifiesta en una disminución de la velocidad de sinterización, $f(\alpha)$, con dicha variable, $\alpha$. Tanto para el vidrio como para todos los composites se ha obtenido un valor de $\mathrm{n}<1$ lo que implica, según la ec. 5.61, un modelo cinético de velocidad decreciente.

v) El efecto del contenido en circón, $\phi$, sobre la caída de la fuerza impulsora, $\wp_{L}(\alpha)$, y sobre el aumento de la resistencia a la 
densificación, $\Psi(\alpha)$, es tanto mayor cuanto mayor es la fracción volumétrica de circón. En consecuencia, la resultante de ambos efectos se traduce en que la caída de la velocidad de sinterización con el avance del proceso también sea más alta a mayor contenido en circón. Para composites con elevados contenidos en circón, durante su sinterización, el número de contactos entre estas partículas rígidas, que se forman a un determinado grado de avance llega a ser tan elevado que dichas partículas forman una red tridimensional rígida incompresible, que paraliza la densificación sin que se anule la fuerza impulsora, $\wp_{L}(\alpha)>0$. En efecto, para los composites de la serie $F$, para $\phi=0,525$ dicha paralización se sitúa en $\alpha \approx 0,30$ y para $\phi=0,65$, a $\alpha \approx 0,125$. En estos casos, la solubilidad parcial del circón en la matriz vítrea disminuye la rigidez de la red sólida y permite que continue la sinterización, por el mecanismo de solución-reprecipitación.

vi) El aumento que experimenta la resistencia a la densificación, $\Psi(\alpha)$, de un composite con el avance de la sinterización, $\alpha$, es tanto mayor cuanto menor es la relación entre tamaños medios del circón y del vidrio, $\left(\frac{d_{z}^{v}}{d_{g}^{v}}\right)$. Dicho comportamiento es consecuencia de que el número de contactos entre inclusiones rígidas aumenta conforme se reduce dicha razón, $\left(\frac{d_{z}^{v}}{d_{g}^{v}}\right)$. Probablemente el marcado efecto que ejerce esta variale sobre el aumento de $\Psi(\alpha)$ con $\alpha$ es el responsable de que la caída de la velocidad de sinterización con el avance del proceso sea más alta cuanto menor sea la razón $\left(\frac{d_{z}^{v}}{d_{g}^{v}}\right)$. En efecto, a igualdad de contenido en circón, el parámetro " $\mathrm{n}$ " de la ec. 5.61 disminuye 
conforme $\left(\frac{d_{z}^{v}}{d_{g}^{v}}\right)$ se reduce. Por otra parte, al comparar las curvas de sinterización de los composites F2 y FF2, que son las únicas que alcanzan una baja porosidad con altos contenidos en circón $(\phi=0,525)$, se comprueba que el $F 2$, con $\left(\frac{d_{z}^{v}}{d_{g}^{v}}\right)$ menor, presenta una menor contribución al mecanismo de sinterización por flujo viscoso $\left(w_{1}=0,30\right)$ mientras que el $F F 2$, con $\left(\frac{d_{z}^{v}}{d_{g}^{v}}\right)$ mayor, presenta un valor más alto $\left(w_{1}=0,40\right)$. El hecho de que en la sinterización de $\mathrm{F} 2$ se alcanza una estructura sólida rígida, a un grado de avance menor que en FF2, también se debe a que el número de contactos entre partículas de circón aumenta conforme se reduce la rázon $\left(\frac{d_{z}^{v}}{d_{g}^{v}}\right)$.

vii) El número de contactos entre partículas de circón, dependiente del contenido en circón, $\phi$, y de las granulometrías de los dos componentes, vidrio y circón, mediante la razón $\left(\frac{d_{z}^{v}}{d_{g}^{v}}\right)$, parece ser el parámetro microestructural clave en el proceso de sinterización por flujo viscoso de un composite, ya que es determinante tanto del modelo cinético, $f(\alpha)$, al modificar " $n$ ", como de la viscosidad relativa del material $\eta_{r}=\frac{\eta_{c}}{\eta}$. De hecho ambos factores cinéticos se han relacionado con la fracción volumétrica adimensional, $\phi^{*}$, mediante la ec. 5.50 y la ec. 5.51 , basándose en argumentos relacionados con la interacción entre partículas de circón. 


\section{Conclusiones}

\subsection{Sinterización de compactos de partículas de frita.}

Se ha obtenido, por fusión, y se ha caracterizado una frita de composición seleccionada y viscosidad conocida, que, además, no cristaliza durante los tratamientos térmicos utilizados.

El estudio del proceso principalmente ha supuesto, por una parte, la determinación de la evolución textural y microestructural de los compactos con el avance de la sinterización, $\alpha$, y por otra, su análisis cinético, lo que comprende, fundamentalmente, la selección del modelo apropiado y la determinación de los parámetros cinéticos realizando series de experimentos isotermos y a velocidad de calentamiento constante, $\beta$, con valores de $0,5 \leq \beta \leq 60 \mathrm{~K} / \mathrm{min}$, en un microscopio de calefacción (HSM). La distribución del tamaño de las partículas de vidrio utilizada es similar a la industrial (GM).

6.1.1 De los resultados obtenidos al caracterizar el comportamiento en fundido del vidrio y la evolución textural y microestructural de las probetas con el avance del proceso se han extraído las siguientes conclusiones:

i) Se ha comprobado que la curva: viscosidad en fundido de la frita temperatura, calculada a partir de los puntos fijos de viscosidad obtenidos experimentalmente, se ajustan muy bien a la ecuación de Vogel-Fulcher-Tamman (VFT) con los parámetros que recomienda la NBS (National Bureau Standard) para este vidrio (SRM 717 o SRM 731).

ii) En probetas conformadas, tanto por prensado como por colado, y cocidas, mediante tratamientos isotermos y no isotermos, se han identificado por MEB los tres estados de sinterización: inicial ( $\alpha \leq 0,3-$ $0,4)$, en el que solo las partículas más pequeñas coalescen y presentan los cantos redondeados; intermedio $(0,4 \leq \alpha \leq 0,8)$, caracterizado por una matriz vítrea continua, en la que se 
distribuyen poros de forma y tamaño irregular; final $(\alpha>0,8)$, los poros son de sección circular o cuasicircular.

iii) En probetas conformadas por colado a partir de la granulometría GM, y cocidas mediante tratamientos isotermos y a velocidad de calentamiento constante, se ha comprobado lo siguiente:

- La variación que siguen: la razón porosidad abierta/porosidad total, $\varepsilon_{a b i e r t a} / \varepsilon_{\text {total }}$, el diámetro medio de intrusión, $4 \mathrm{~V} / \mathrm{A}$, y la presión de sinterización calculada, $\Sigma_{\mathrm{S}}$, frente al grado de avance de la sinterización, $\alpha$, es independiente del tratamiento térmico seguido.

- Para valores de $\alpha<0,7$, la práctica totalidad de la porosidad de la pieza es abierta. Por contra, para valores de $\alpha>0,9$, la porosidad es mayoritariamente cerrada (estado final de la sinterización).

- El diámetro medio de intrusión, 4V/A, debido a la progresiva eliminación de los poros más pequeños, aumenta con el grado de avance del proceso, $\alpha$, en los estados iniciales de la sinterización, hasta alcanzar un máximo para $\alpha \approx 0,3-0,4$. En el estado intermedio de la sinterización, $0,3<\alpha<0,8,4 \mathrm{~V} / \mathrm{A}$ disminuye muy poco. En cambio, para valores de $\alpha>0,8$, estado final, la disminución de 4V/A es más brusca.

- La variación de la presión de sinterización, $\Sigma^{\mathrm{S}}$, calculada según Wakai, frente a $\alpha \circ \rho$, que es la imagen especular de la que sigue $4 \mathrm{~V} / \mathrm{A}$ frente a $\alpha \circ \rho$, es muy diferente en magnitud y forma de la que predicen los modelos más clásicos.

- La mínima porosidad cerrada alcanzada en las probetas fue de $\approx 2 \%$. Un posterior aumento de la temperatura provoca un ligero aumento de ésta, debido a la eliminación progresiva de los poros más pequeños $(<1 \mu \mathrm{m})$ y al crecimiento de los más grandes $(>10 \mu \mathrm{m})$. 
6.1.2 Del estudio cinético del proceso se han extraído las siguientes conclusiones:

i) Se han determinado los valores de la energía de activación del proceso, $E_{\alpha}$, en función del avance del mismo, $\alpha$, a partir de experimentos a velocidad de calentamiento constante, $\beta$, mediante el modelo de Friedman y el K.A.S., y los correspondientes al flujo viscoso, $E_{\eta}$, a partir de la curva de viscosidad. Se comprueba que los valores de $\mathrm{E}_{\alpha}$ calculados por el método de Friedman son solo un poco más grandes que los de $E_{\eta}$. Ambas, $E_{\alpha}$ y $E_{\eta}$, disminuyen ligeramente y de forma paralela con el aumento de $\alpha$, lo que confirma que la sinterización de partículas de vidrio es un proceso controlado por el mecanismo de flujo viscoso. Los valores de $\mathrm{E}_{\alpha}$ calculados por el método de K.A.S. y el obtenido por el método de Kissinger se desvían más de los valores de $\mathrm{E}_{\eta}$.

ii) Los resultados de la sinterización a seis velocidades de calentamiento distintas, $0,5 \leq \beta \leq 60 \mathrm{~K} / \mathrm{min}$, quedan perfectamente descritas por el modelo de Avrami-Erofeev, con valores del índice de potencia " $\mathrm{n}$ " y factor preexponencial, $\ln \mathrm{A}_{g}$, independientes de la velocidad de calentamiento, $\beta$, y $E$ (prácticamente coincidente con $E_{\eta}$ ), disminuyendo ligeramente con $\beta$.

iii) Al sustituir en el modelo cinético la ecuación de Arrhenius por la relación que sigue la viscosidad del vidrio con la temperatura (ecuación V.F.T.) utilizando como parámetros $\mathrm{B}$ y $\mathrm{T}_{0}$ los recomendados por la NBS, los resultados experimentales se ajustan perfectamente al modelo con solo dos parámetros de ajuste " $n$ " y $\ln \mathrm{A}_{\mathrm{g}}$, ambos independientes de la velocidad de calentamiento.

iv) Los resultados experimentales obtenidos en experimentos isotermos se han ajustado también muy bien al modelo de Avrami-Erofeev, con 
valores de " $\mathrm{n}$ " y E muy parecidos a los obtenidos por métodos no isotermos.

v) Se ha comprobado que los valores de la constante de velocidad, $\mathrm{k}_{\mathrm{g}}$, obtenidos en experimentos isotermos son proporcionales a la inversa del tiempo de sinterización característico del vidrio, $\frac{\gamma}{\mathrm{d} \cdot \eta}$, de acuerdo con los modelos teóricos, resultando una constante de proporcionalidad (constante de velocidad adimensional) de $\lambda_{\mathrm{g}} \approx 0,8$.

vi) Se ha estudiado el efecto de la compacidad en crudo de los compactos sobre el proceso de sinterización, mediante experimentos a velocidad de calentamiento $\beta=15 \mathrm{~K} / \mathrm{min}$, modificando la presión de compactación, $\mathrm{P}_{\text {comp }},(2,2$ a $50 \mathrm{MPa})$. Las conclusiones más relevantes obtenidas son:

- El valor de la compacidad máxima de los compactos, que es $\rho_{\max }=0,99 \pm 0,02$, es independiente de la presión de compactación. Por el contrario, la contracción superficial máxima de la silueta, $\varepsilon_{A}$, max, disminuye con el logaritmo de la presión de compactación, debido a que la compacidad de la probeta al inicio de la sinterización, $\rho_{0}$, aumenta con el logaritmo de dicha variable.

- El factor de anisotropía de la sinterización, $\mathrm{k}_{\mathrm{A}}$, definido como la razón: contracción axial/contracción diametral, no varía con el avance del proceso, $\alpha$, para estados iniciales e intermedios de la sinterización $(\alpha \leq 0,6-0,7)$ pero disminuye un poco con la presión de compactación $\left(0,72<\mathrm{k}_{\mathrm{A}}<0,83\right)$. En estados finales, $\mathrm{k}_{\mathrm{A}}$ aumenta con $\alpha$ hasta alcanzar prácticamente la unidad a la máxima densificación. A temperaturas superiores, $\mathrm{k}_{\mathrm{A}}$ vuelve a disminuir de nuevo, debido a que la expansión axial, provocada por la dilatación de gases ocluidos en los poros, es mayor que la diametral.

- El efecto que ejerce la compacidad en crudo $\left(\approx \rho_{0}\right)$, o la presión de compactación de la probeta, sobre la cinética de la 
sinterización es muy pequeño. Un aumento considerable de la presión de compactación (de 2,2 a 50MPa) solamente provoca un reducido incremento del factor preexponencial, $\ln \mathrm{A}_{g}$, (inferior al $3 \%$ ).

vii) Se ha estudiado la sinterización de compactos de patículas de vidrio de granulometría GF, mucho más fina que la industrial, GM, comprobándose, mediante series de experimentos isotermos y a velocidad de calentamiento constante, que los parámetros cinéticos obtenidos para cada granulometría son muy similares entre si, a excepción del factor preexponencial $\left(\ln _{g}\right)$, que varía con la inversa del tamaño de partícula del vidrio. Se confirma que la constante adimensional de velocidad obtenida a partir de experimentos isotermos, $\lambda_{\mathrm{g}}=0,9$, es independiente de las variables de operación (distribución granulométrica, temperatura, etc.).

viii) Se ha comprobado que el modelo cinético de Avrami-Erofeev con un índice de potencia " $n=0,7$ " es adecuado para describir adecuadamente la cinética de todo el proceso de sinterización, independientemente de las condiciones de operación. Este modelo predice una caída de la velocidad de sinterización con el avance del proceso, intermedio a la correspondiente a los modelos teóricos de Mackenzie [61] y Sura \& Panda [63] para vidrios.

\subsection{Sinterización de composites. Efecto del contenido en circón.}

Al efecto se han preparado mezclas de vidrio y circón con la granulometría GM para el vidrio y el micronizado para el circón (F), modificando la fracción volumétrica de circón, $\phi=$ volumen de circón/volumen de sólido, hasta valores de $\phi=0,65$. 
6.2.1 Del análisis de las curvas de sinterización no isotermas, correspondientes a los diferentes composites, obtenidas a cinco velocidades de calentamiento distintas $(2 \leq \beta \leq 60 \mathrm{~K} / \mathrm{min})$, se han obtenido las siguientes conclusiones:

i) Un aumento de $\phi$ supone un incremento de las temperaturas de inicio de sinterización y de máxima densificación; este efecto, que es más pequeño para valores de $\phi \leq 20$, se va incrementando progresivamente con $\phi$, llegando incluso a no densificar $\left(a 1600^{\circ} \mathrm{C}\right)$ para valores de $\phi=0,65$.

ii) Se ha comprobado que, a excepción del composite con $\phi=0,65$, los valores de la contracción máxima de cocción, $\varepsilon_{\mathrm{A}}$, max, son independientes de la velocidad de calentamiento y disminuyen con el aumento del contenido en circón, $\phi$. El grado de reducción de la contracción máxima, $\Delta \varepsilon_{\mathrm{A}, \max }=1-\frac{\varepsilon_{\mathrm{A}, \max }}{\varepsilon_{\mathrm{A}, \max }(0)}$, siendo $\varepsilon_{\mathrm{A}, \max }(0)$ la $\varepsilon_{\mathrm{A}, \max }$ correspondiente al vidrio, aumenta de forma potencial con $\phi$.

iii) Se ha comprobado, en composites de moderado, $\phi=0,322$, y alto contenido en circón, $\phi=0,525$, que la presión de compactación, $P_{\text {comp }}$, empleada en el conformado de las probetas, para $20 \leq P_{\text {comp }} \leq 80 \mathrm{MPa}$, no ejerce ningún efecto aparante sobre la curva de sinterización: $\alpha$ vs temperatura. En cambio, los valores de contracción, $\varepsilon_{\mathrm{A}}$ y del factor de anisotropía solo se ven ligeramene afectados.

6.2.2 Del análisis del conjunto de los resultados obtenidos al estudiar la evolución textural y microestructural de los composites con el avance del proceso, se pueden extraer las siguientes conclusiones:

i) Se ha comprobado, por MEB, que en etapas iniciales e intermedias de la sinterización, la mayoría de las partículas de circón están en contacto y/o rodeando áreas de vidrio (partículas de vidrio con los cantos redondeados o agregados vítreos resultantes de la coalescencia de partículas). Esta distribución es indicativa de un 
buen mezclado. Conforme avanza el proceso se observa que la matriz vítrea viscosa va mojando progresivamente las partículas de circón y/o penetrando por capilaridad en los poros. La cantidad de clústers de circón aumenta con $\phi$ y son muy visibles en estados intermedios de la sinterización.

De forma general con el avance del proceso se reduce el número de poros, crece su tamaño y su forma se vuelve menos irregular. La mayor parte de los poros presentes en el composite, en la etapa final de la sinterización, son esféricos o cuasiesféricos, con sus paredes constituidas mayoritariamente por partículas de circón. No obstante, también se aprecían poros esféricos en el seno de la matriz vítrea.

ii) Únicamente a temperaturas elevadas y/o tiempos de permanencia largos se aprecia claramente un cambio del tamaño y la forma de las partículas de circón, debido a su solución-reprecipitación.

iii) La compacidad máxima, $\rho_{\max }$, alcanzada para cada composite (excepto para $\phi=0,65$ ) es independiente de la velocidad de calentamiento, y disminuye con el contenido en circón, $\phi$. El grado de reducción de la compacidad máxima, $\Delta \rho_{\text {max }}^{*}$, definido como $\Delta \rho_{\max }^{*}=1-\frac{\rho_{\max }}{\rho_{\max }(0)}$, siendo $\rho_{\max }(0)$ el valor de $\rho_{\max }$ del vidrio, sigue una relación del tipo: $\Delta \rho_{\max }^{*} \alpha \phi^{3,3}$. El hecho que el exponente resultante sea alto $(>3)$ se relaciona con un buen mezclado de los componentes, buen mojado del circón por el vidrio y con la presencia de poros formados por la agrupación de al menos tres partículas de circón.

iv) Se ha comprobado, mediante porosimetría de mercurio, en composites de contenido en circón moderado $(\phi=0,322)$ y alto $(\phi=0,525)$, que conforme avanza la sinterización, $\alpha$, la estructura porosa abierta experimenta los siguientes cambios:

- En los composites con $\phi=0,525$ se observa un crecimiento continuo del diámetro medio de intrusión, 4V/A, de tipo 
exponencial en el estado inicial de la sinterización, $\alpha \leq 0,4$, y de tipo lineal en la etapa intermedia, $0,4 \leq \alpha \leq 0,8$. Esta evolución se debe a un considerable crecimiento continuo de los poros abiertos más grandes $\left(d_{16}\right.$ pasa de $0,5 \mu \mathrm{m}$ a $\left.10 \mu \mathrm{m}\right)$ y a una progresiva eliminación de los poros más pequeños.

- En el composite con $\phi=0,322$, en el estado inicial de la sinterización, solo se aprecia un ligero crecimiento de 4V/A, similar al observado para el vidrio. En la etapa intermedia se mantiene prácticamente constante. La eliminación de poros pequeños es progresiva mientras que el crecimiento de los más grandes solo se produce al inicio de la sinterización, $\alpha \leq 0,2$.

- La presión de sinterización, $\Sigma^{\mathrm{S}}$, calculada según Wakai [114] disminuye continuamente con el avance de la sinterización, $\alpha$, tendiendo asintóticamente a un valor constante, que es tanto más pequeño cuanto mayor es el contenido en circón, $\phi$.

- Para todos los composites, en estados iniciales de la sinterización, $\alpha<0,4$, toda la porosidad es abierta. Ahora bien, para el composite con alto contenido en circón, $\phi=0,525$, la porosidad cerrada comienza a aparecer para valores de $\alpha \approx 0,4$ y ya es mayoritaria en el estado intermedio de la sinterización, $\alpha \geq 0,7$. Para el composite con $\phi=0,322$, su comportamiento es parecido al del vidrio, la porosidad cerrada solo se desarrolla para valores de $\alpha \geq 0,7$, y es mayoritaria para $\alpha \geq 0,9$.

6.2.3 Se ha estudiado la solución-reprecipitación de granos de circón en la matriz vítrea en compactos con moderado $(\phi=0,322)$ y altos contenidos en circón $(\phi=0,525)$, en experimentos a velocidad de calentamiento $\beta=5 \mathrm{~K} / \mathrm{min}$. De los resultados se concluye lo siguiente:

i) Las distribuciones de tamaño de grano de circón, DTG's, (determinadas por MEB y análisis de imagen en 2D y transformadas a 3D) se ajustan muy bien a la función de distribución de Weibull. Se comprobó un aumento del tamaño medio, $G_{M}$, y del índice de 
uniformidad, $M$, de tipo exponencial con el aumento de la temperatura, siendo éste claramente evidente a temperaturas $\geq$ $1100^{\circ} \mathrm{C}$.

ii) Se ha determinado la energía aparente de activación de crecimiento cristalino, $Q_{G} \approx 170 \mathrm{~kJ} / \mathrm{mol}$, del circón. Su valor está dentro del intervalo de valores de energía de activación del proceso de difusión del circón en vidrios fundentes de composición variable (entre 140 y $250 \mathrm{~kJ} / \mathrm{mol}$ ).

iii) Por difracción de RX se ha determinado la fracción másica de circón y su tamaño de cristalito, C. Se comprobó que únicamente a temperaturas superiores a los $1100^{\circ} \mathrm{C}$ se reduce ligeramente el contenido en circón y que la evolución del tamaño de cristalito con la temperatura era análoga a la del tamaño medio de circón, $\mathrm{G}_{\mathrm{M}}$. La energía de activación calculada a partir de los valores de $C$ era prácticamente igual a la obtenida a partir de los valores de $G_{M}$.

iv) Se ha comprobado por EDS que a $1000^{\circ} \mathrm{C}$ la zona de difusión vidrio-circón es inexistente al igual que el contenido en circonio en la fase vítrea. Por contra, a $1300^{\circ} \mathrm{C}$ la capa de difusión es de alrededor de $5 \mu \mathrm{m}$ y el porcentaje de $\mathrm{ZrO}_{2}$ en el vidrio significativo.

6.2.4 De los resultados obtenidos en el estudio cinético de la sinterización de composites se concluye lo siguiente:

i) Para todos los composites con $\phi \leq 0,43$, las curvas de sinterización son sigmoidales, y pueden ser descritas por una sola etapa. Se ha determinado, por el método de Friedman, para cada composite, la variación que sigue con el grado de avance de la sinterización, $\alpha$, la energía de activación del proceso, $\mathrm{E}_{\alpha}$, comprobándose que ésta, al igual quela energía de activación del flujo viscoso, $E_{\eta}$, disminuyen conforme aumenta $\alpha$. Dicho comportamiento se debe a que $E_{\eta}$ disminuye con el aumento de la temperatura y, por tanto, con $\alpha$ en experimentos no isotermos. Al calcular los valores medios de $E_{\alpha}$, 
$\overline{E_{\alpha}}$, y de $E \eta, \overline{E_{\eta}}$, correspondientes a todo el proceso, para cada composite, se comprueba que $\bar{E}_{\alpha}$ y $\bar{E}_{\eta}$ son en todos los casos muy parecidos (aunque $\overline{E_{\alpha}}$ es siempre un poco mayor que $\overline{E_{\eta}}$ ) y disminuyen con el aumento de $\phi$, por la razón antes señalada $\left(\bar{E}_{\eta}\right.$ disminuye con el aumento de la temperatura).

ii) Se confirma, como consecuencia de lo anterior, para composites con $\phi \leq 0,43$ y con las distribuciones granulométricas empleadas (vidrio GM y circón F), que su densificación por el mecanismo de reordenación de partículas está controlado por un mecanismo de flujo viscoso, en el que el efecto de la temperatura sobre la velocidad del proceso, $\mathrm{E}_{\alpha}$, es el debido al que ejerce esta misma variable sobre la viscosidad del vidrio, $E_{\eta}$.

iii) Para el composite con alto contenido en circón $(\phi=0,525)$ la curva de sinterización no es un sigmoide, por lo que se requieren al menos dos etapas para describir la cinética del proceso. Al determinar los valores de $E_{\alpha}$ se ha comprobado que para valores de $\alpha \leq 0,3$ los valores de $E_{\alpha}$ son parecidos a los de $E_{\eta}$, lo que confirmaría que la densificación en este estado, por reordenación de partículas, está controlado por el flujo viscoso. Para $\alpha \geq 0,3$ los valores de $\mathrm{E} \alpha$ son mucho mayores que los de $E_{\eta}$, lo que demostraría que en este estado de la sinterización, el mecanismo de solución-reprecipitación del circón sería el controlante.

iv) Las curvas de sinterización obtenidas en experimentos no isotermos $(2<\beta<60 \mathrm{~K} / \mathrm{min}$ ) para composites con $\phi \leq 0,43$ (considerando que el efecto de la temperatura sobre la velocidad de sinterización es el debido al efecto de esta variable sobre la viscosidad) se ajustaron muy bien al modelo de Avrami-Erofeev. 
Los dos únicos parámetros de ajuste, " $\mathrm{n}$ " y $\ln \left(\mathrm{A}_{\mathrm{no}}\right.$ isot $)$, disminuían con el aumento de $\phi$. Los resultados obtenidos en experimentos isotermos también se ajustan muy bien al modelo, comprobándose además que los valores de "n" obtenidos por uno y otro procedimiento eran prácticamente coincidentes. Asimismo, se comprobó que los valores de la energía de activación, E, del

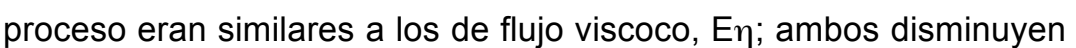
con $\phi$ como ya se ha indicado antes. También se ha comprobado que la variación que siguen la constante de velocidad adimensional, $\lambda, y$ el factor preexponencial isotermo, $\ln \left(A_{\text {isot }}\right), y$ no isotermo, $\ln \left(A_{n o}\right.$ isot), con el contenido en circón, $\phi$, es muy similar.

v) La cinética de la sinterización isoterma y no isoterma del composite con un alto contenido en circón $(\phi=0,525)$ se ha descrito muy bien mediante un modelo cinético compuesto de dos etapas que se desarrollan en paralelo: la primera se asocia a la reordenación de partículas por flujo viscoso y la segunda al mecanismo de soluciónreprecipitación del circón. Los valores de los parámetros cinéticos: contribución de cada etapa, $\mathrm{w}_{\mathrm{i}}$, índice de potencia de Avrami, $\mathrm{n}$, factor preexponencial, $\ln A_{i}$, y energía de activación, $E_{i}$, obtenidos por uno y otro método son muy parecidos.

\subsection{Sinterización de composites. Efecto de la distribución de tamaños de partícula.}

Al efecto, a partir de dos granulometrías de vidrio (GM y GF) y tres de circón $(\mathrm{F}, \mathrm{H}$ y $\mathrm{M})$ se han preparado dos series de composites: una con un contenido moderado en circón $(\phi=0,322)$, serie $1, y$ otra de alto contenido en circón $(\phi=0,525)$, serie 2 . Dentro de cada serie se ha modificado la granulometría de cada componente para conseguir seis composites con valores de la razón: diámetro medio volumétrico de circón, $\mathrm{d}_{\mathrm{z}}^{\mathrm{v}}$ /diámetro medio volumétrico de vidrio, $d_{g}^{v}$, diferentes, comprendidos entre 0,25 y 10 . 
6.3.1 De las curvas de sinterización no isotermas, obtenidas a diferentes velocidades de calentamiento $(2 \leq \beta \leq 60 \mathrm{~K} / \mathrm{min})$, se puede concluir lo siguiente:

i) Para los composites con moderado contenido en circón $(\phi=0,322)$, serie 1 , el empleo de matrices vítreas más finas se manifiesta en una disminución de las temperaturas de inicio y final de la sinterización y en una reducción del intervalo de cocción. Además, independientemente de la granulometría de la matriz, las temperaturas de inicio y de máxima densificación disminuyen con el aumento del tamaño medio de circón utilizado.

ii) Para los composites con alto contenido en circón $(\phi=0,525)$, serie 2 , se confirma que al igual que ocurría con la serie 1, las temperaturas de inicio y final de la sinterización disminuyen con el aumento del tamaño medio de partícula de circón utilizado. Asimismo, se ha observado que el empleo de la mariz vítrea más fina se traduce en una temperatura de inicio de la sinterización también más baja, al igual que en la serie 1. Ahora bien, el efecto de esta variable en estados de la sinterización más avanzados es complejo, debido a la solución-reprecipitación de circón.

iii) El aumento del contenido en circón siempre reduce la contracción superficial máxima, $\varepsilon_{\mathrm{A}, \max }$. Ahora bien, el grado de reducción de la contracción máxima debido a la adición de circón, $\Delta \varepsilon_{\mathrm{A}}$ max, aumenta con $\phi y$, por lo general, con el tamaño de partícula de circón.

6.3.2 Del estudio de la evolución textural y microestructural realizado en los composites con el avance de la sinterización, se han extraído las siguientes conclusiones:

i) Se ha comprobado, por MEB, en estados iniciales de la sinterización, que a igualdad de contenido en circón, la frecuencia de contactos circón-circón disminuye (los contactos vidrio-vidrio 
aumentan) conforme aumenta la razón: diámetro medio volumétrico de circón/diámetro medio volumétrico de vidrio, $\frac{d_{z}^{v}}{d_{g}^{v}}$.

ii) Para todos los composites, con el avance de la sinterización, $\alpha$, crece el tamaño de los poros y se reduce su número, especialmente en los composites preparados con un alto contenido $(\phi=0,525)$ del circón más grueso $(H)$. En estos últimos, la porosidad final es abundante y la consitituyen poros grandes adheridos generalmente a las partículas de circón más gruesas. En los composites preparados con el circón más fino $(F)$ la formación de poros está asociada a la de cavidades formadas por agrupación de partículas de circón y/o la generación de gas en el vidrio y/o a su expansión.

iii) Se ha comprobado que la compacidad máxima, $\rho_{\max }$, alcanzada por los distintos composites disminuye conforme se incrementa el contenido en circón, $\phi$, siendo dicho efecto tanto mayor cuanto mayor es el tamaño de partícula del vidrio y/o del circón. El grado de reducción de la compacidad de los composites por la adición de circón, $\Delta \rho^{*}{ }_{\max }$, se ha comprobado que sigue una relación: $\Delta \rho_{\max }^{*}=(\kappa \phi)^{3,3}$, siendo $\kappa$ un parámetro de ajuste que aumenta con el tamaño medio de partícula de la matriz vítrea y del circón.

6.3.3 Del estudio cinético de la sinterización de composites se concluye lo siguiente:

i) Para composites con moderado contenido en circón $(\phi=0,322)$, serie 1 , se ha comprobado que las curvas de sinterización no isotermas se ajustan muy bien al modelo de Avrami-Erofeev (considerando, como siempre, que el efecto de la temperatura sobre la velocidad de sinterización es la debida al efecto de dicha variable sobre la viscosidad del vidrio). Los valores de " $n$ " y $\ln \left(A_{n o}\right.$ isot), parámetros de ajuste, se han relacionado muy bien con los de la razón, $\frac{d_{z}^{v}}{d_{g}^{v}}$, 
mediante ecuaciones sencillas. Los resultados obtenidos en experimentos isotermos también se ajustaron muy bien al modelo y con los mismos valores de "n" que en los no isotermos. Además, se confirma que los valores de la energía de activación del proceso, E,

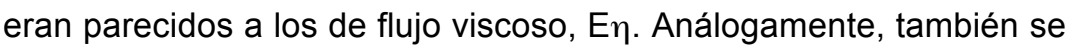
ha comprobado que la variación de la constante de velocidad adimensional, $\lambda$, y el factor preexponencial isotermo $\ln \left(A_{\text {isot }}\right)$ y no isotermo $\ln \left(A_{\text {noisot }}\right)$, con la razón $\frac{d_{z}^{v}}{d_{g}^{v}}$ siguen la misma tendencia.

ii) Para los composites con alto contenido en circón $(\phi=0,525)$, serie 2 , a excepción del composite $\mathrm{H} 2$, preparado con el vidrio (GM) y circón $(\mathrm{H})$ de granulometría más gruesa, para el que el proceso de densificación finaliza antes de que comience la soluciónreprecipitación del circón, las curvas de sinterización no isotermas presentan dos tramos; asociados, el primero a la reordenación de partículas y el segundo a la solución- reprecipitación del circón. Los resultados correspondientes al primer tramo se ajustaron muy bien al modelo de Avrami-Erofeev (considerando, como siempre, que el efecto de la temperatura sobre el proceso se debe al efecto de dicha variable sobre la viscosidad del vidrio). También los valores de " $n$ " $y$ $\ln \left(A_{\text {no isot }}\right)$, únicos parámetros de ajuste, se han relacionado con la razón $\frac{d_{z}^{v}}{d_{g}^{v}}$ mediante el mismo tipo de relaciones que las empleadas para los composites con $\phi=0,322$, serie 1 .

iii) Al comparar los resultados obtenidos al estudiar la cinética del proceso completo de sinterización, correspondientes a los dos composites con alto contenido en circón de granulometría fina (micronizado, F), los únicos que conducen a materiales de baja porosidad, se han extraido las siguientes conclusiones:

- El modelo en dos etapas describe muy bien los resultados para cada composite, con valores de los parámetros cinéticos 
obtenidos en experimentos isotermos y no isotermos muy parecidos.

- Al comparar las rectas de Arrhenius correspondientes a las dos etapas de los dos composites se confirma que las energías de activación para cada recta son muy similares (rectas paralelas dos a dos), aun cuando los valores de la contribución de cada etapa, $w_{i}$, y el índice de potencia, $n_{i}$, dependen de la granulometría de la matriz vítrea utilizada.

6.4 Sinterización no isoterma de composites asociada a la reordenación de partículas por flujo viscoso. Efecto combinado del contenido en circón, $\phi$, y de la razón, $d_{z}{ }^{v} / d_{g}{ }^{v}$.

i) Se ha desarrollado y validado una ecuación cinética que describe muy bien el primer tramo de la curva de sinterización no isoterma (que es el único para valores de $\phi$ moderados), para amplios intervalos de las variables de operación: velocidad de calentamiento $(2 \leq \beta \leq 60 \mathrm{~K} / \mathrm{min})$, contenido en circón $(\phi \leq 0,525)$ y razón de diámetro medio volumétrico de circón/diámetro medio volumétrico de vidrio $\left(0,25 \leq \frac{d_{z}^{v}}{d_{g}^{v}} \leq 10\right)$. En su desarrollo se ha considerado lo siguiente:

- El efecto de la temperatura sobre la constante de velocidad del proceso es únicamente la que ejerce dicha variable sobre la viscosidad de la matriz vítrea, descrita mediante la ecuación de Vogel-Fulcher-Tamman, con los valores de los parámetros recomendados por la NBS.

- El modelo de Avrami-Erofeev describe la cinética del proceso con un valor del índice de potencia "n" relacionado con una función de $\phi$ y $\frac{\mathrm{d}_{\mathrm{z}}^{\mathrm{v}}}{\mathrm{d}_{\mathrm{g}}^{\mathrm{v}}}$ mediante la introducción de la fracción volumétrica crítica de percolación, $\phi_{p}$, del circón.

- El factor preexponencial, $A_{n o}$ isot, de cada composite es el producto de los factores: el correspondiente a la matriz vítrea, 
$\left(\mathrm{A}_{\mathrm{g}}\right)_{\mathrm{no} \text { isot }}$, inversamente proporcional a su tamaño de partícula, $d_{g}^{v}$, y la razón $\left(\frac{A}{A_{g}}\right)_{\text {noisot }}$. Ésta última está relacionada con el contenido en circón, $\phi$, y con la razón $\frac{d_{z}^{v}}{d_{g}^{v}}$ mediante la ecuación de Krieger-Dougherty, tomando como fracción volumétrica máxima la de percolación, $\phi_{p}$, del circón.

- La fracción crítica de percolación, $\phi_{p}$, que es la fracción volumétrica del circón a la que las partículas de éste forman una red continua rígida, es una función única creciente de la razón $\frac{d_{z}^{v}}{d_{g}^{v}}$.

\subsection{Interpretación de los parámetros cinéticos basándose en la teoría continua de la sinterización.}

i) La razón entre los factores preexponencial del vidrio y del circón, $\mathrm{A}_{\mathrm{g}} / \mathrm{A}$, también tiene el significado físico de viscosidad relativa del composite, $\eta_{\mathrm{r}}$, definida como la viscosidad efectiva del composite (vidrio + circón), $\eta_{c}, y$ del vidrio, $\eta$. En consecuencia, su relación con el contenido en circón, $\phi$, y con la granulometría de sus componentes, viene dada por la ecuación de Krieger-Dougherty, relación ampliamente utilizada para determinar el efecto del contenido en sólidos y su distribución de tamaños sobre la viscosidad de suspensiones concentradas.

ii) El índice de potencia de Avrami, "n", y su variación con el contenido en circón, $\phi$, y con las granulometrías de cada componente, vidrio y circón, se ha interpretado adecuadamente en base al efecto que dichas variables de operación ejercen sobre la razón: presión de sinterización adimensional, $\wp_{\mathrm{L}}$, modelo viscoso adimensional, $\Psi$. En 
definitiva, $\frac{\wp_{L}}{\psi}$, según la teoría continua de la sinterización, es equivalente al modelo de sinterización, $f(\alpha)$. 


\section{Apéndice}

7.1 Relación entre el grado de avance de la sinterización, a, la densidad relativa, $\rho$, y las dimensiones (silueta) de la probeta.

El microscopio de calefacción (Hot Stage microscope) en adelante HSM, permite registrar en continuo la silueta de una probeta que, hasta temperaturas y/o tiempo a las que la deformación sea excesiva, puede considerarse cilíndrica.

Si h y D son la longitud y el diámetro de la probeta respectivamente, en cualquier estado del proceso, la contracción axial y radial, vendrán dadas por:

$\varepsilon_{\mathrm{z}}=\ln \frac{\mathrm{h}}{\mathrm{h}_{0}}$ y $\varepsilon_{\mathrm{R}}=\ln \frac{\mathrm{D}}{\mathrm{D}_{0}}$

ec. A1. 1

Siendo $h_{0}$ y $D_{0}$ la altura y el diámetro de la probeta al inicio de la sinterización. Análogamente, la contracción de la superficie de la silueta será:

$\varepsilon_{A}=\ln \left(\frac{A}{A_{0}}\right)_{S}=\varepsilon_{Z}+\varepsilon_{R}$

Si se considera que la contracción de la probeta es isotrópica, es decir $\varepsilon_{\mathrm{Z}}=\varepsilon_{\mathrm{R}}$, resulta que $\varepsilon_{\mathrm{A}}=2 \varepsilon_{\mathrm{Z}}=2 \varepsilon_{\mathrm{R}}$.

Análogamente la contracción volumétrica vendrá dada por:

$\varepsilon_{\mathrm{V}}=\ln \frac{\mathrm{V}}{\mathrm{V}_{0}}=3 \varepsilon_{\mathrm{z}}=3 \varepsilon_{\mathrm{R}}$ ec. A1. 3 
$\varepsilon_{\mathrm{V}}=\frac{3}{2} \varepsilon_{\mathrm{A}}$

ec. A1. 4

Basándose en estas relaciones, se puede definir un grado de avance de la sinterización, $\alpha$, en función de la contracción de la superficie de la silueta de la probeta, fácilmente medible. En efecto, el grado de avance de la sinterización $\alpha$ se puede definir como:

$\alpha=\frac{\varepsilon_{\mathrm{V}}}{\varepsilon_{\mathrm{V}, \max }}$

ec. A1. 5

Siendo $\varepsilon_{\mathrm{V} \text {,max }}$ la máxima contracción volumétrica alcanzada por la probeta, que define el comienzo de la expansión de la misma.

De la ec. A1. 3, ec. A1. 4 y ec. A1. 5 se obtiene:

$\alpha=\frac{\ln \left(A / A_{0}\right)_{S}}{\ln \left(A_{\min } / A_{0}\right)_{S}}$

De acuerdo con esta definición del grado de avance de sinterización, determinando la superficie de la silueta durante su densificación se pueden obtener la curva de sinterización ( $\alpha$ vs. t) en experimentos isotermos y no isotermos ( $\alpha$ vs. T).

Por otra parte, la densidad relativa o compacidad, $\rho$, está relacionada con la contracción volumétrica, $\varepsilon_{\mathrm{v}}$, mediante la ecuación:

$\rho=\rho_{0} \cdot \exp \left(\left|\varepsilon_{\mathrm{v}}\right|\right)$

Siendo $\rho_{0}$ la compacidad inicial.

Aplicando la ec. A1. 7 cuando la sinterización es completa, es decir, $\left|\varepsilon_{\mathrm{v}}\right|=\left|\varepsilon_{\mathrm{v} \text {,max }}\right|$ y $\rho=\rho_{\text {max }}$, se tiene: 
$\rho_{\max }=\rho_{0} \cdot \exp \left(\left|\varepsilon_{\mathrm{v}}\right|_{\max }\right)$

ec. A1. 8

De ec. A1. 5, ec. A1. 7 y ec. A1. 8 se obtiene la relación entre $\alpha$ y $\rho$.

$\rho=\rho_{0} \cdot \exp \left(\alpha \cdot \ln \frac{\rho_{\max }}{\rho_{0}}\right)$

ec. A1. 9

Tomando logaritmos naturales y derivando respecto del tiempo se obtiene:

$\frac{d \varepsilon_{v}}{d t}=\frac{d \rho}{\rho \cdot d t}=\frac{d \alpha}{d t} \cdot \ln \frac{\rho_{\max }}{\rho_{0}}$

ec. A1. 10 
7.2 Determinación del error experimental en la determinación de las curvas de sinterización a velocidad de calentamiento constante. Selección del procedimiento.

Al efecto se han obtenido cuatro probetas de $5 \mathrm{~mm}$ de diámetro y $3 \mathrm{~mm}$ de altura por prensado a 50MPa, a partir de polvo de composite $\mathrm{H} 1$ peparado de la forma habitual. A continuación, se han sometido en el microscopio de calefacción a un calentamiento a 15K/min. De los datos obtenidos del equipo se han determinado los valores de $\varepsilon_{A}=\ln \left(\frac{A}{A_{0}}\right)_{S}$, siendo $A$ la superficie de la silueta de la probeta durante la sinterización y $A_{0}$ la inicial.

En primer lugar, se ha calculado $\varepsilon_{A}(25)$, tomando como valor inicial $A_{0}$, la superficie de la silueta a $25^{\circ} \mathrm{C}$. En segundo lugar, para el cálculo de $\varepsilon_{\mathrm{A}}$ se ha tomado como $A_{0}$ el valor de la superficie de la silueta correspndiente al inicio de la sinterización.

En la Figura 7.1 y la Figura 7.2 se representan las curvas de sinterización correspondientes. Por último, se ha obtenido la curva de sinterización en la forma $\alpha$ vs temperatura (Figura 7.3). Para el cálculo de los valores del grado de avance de la sinterización: $\alpha=\frac{\varepsilon_{A}}{\varepsilon_{A, \max }}$, se ha tomado el valor de $A_{0}$ correspondiente al inico de la sinterizición. 


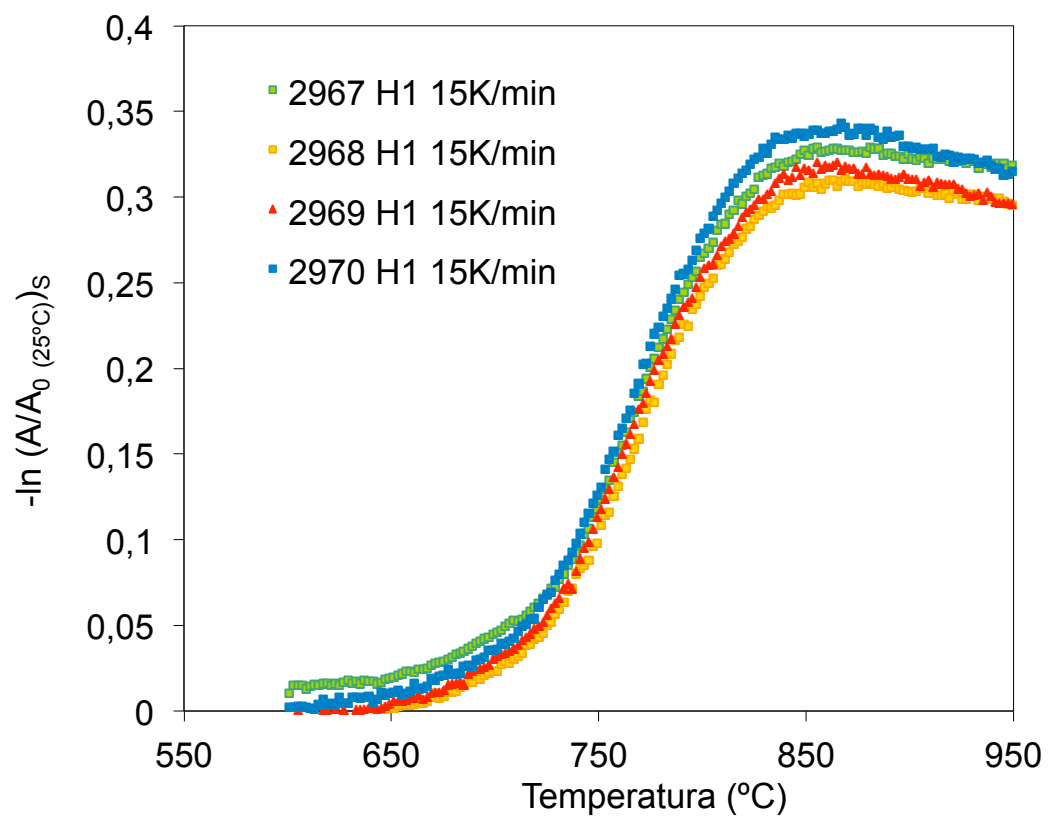

Figura 7.1. Evolución de $-\varepsilon_{A}$ vs temperatura tomando como $A_{0}$ la superficie de la silueta a $25^{\circ} \mathrm{C}$. Composite H1. Velocidad de calentamiento $\beta=15 \mathrm{~K} / \mathrm{min}$.

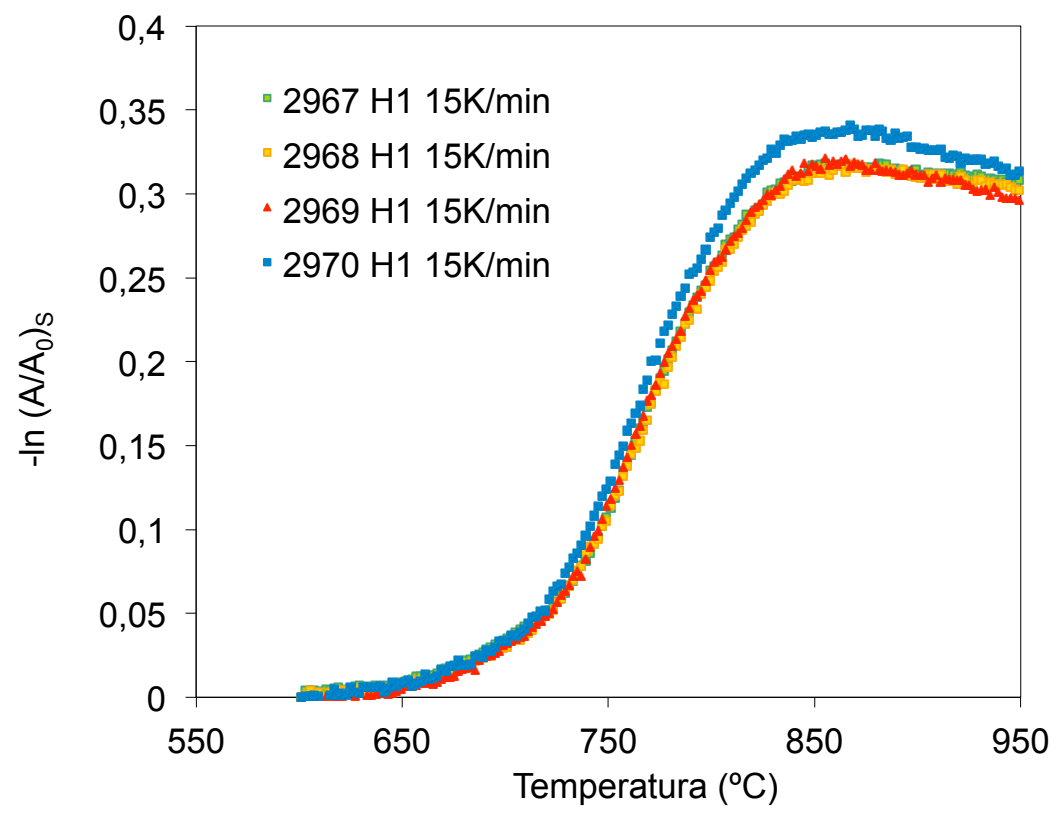

Figura 7.2. Evolución de $-\varepsilon_{A}$ vs temperatura tomando como $A_{0}$ la superficie de la silueta al inicio de la sinterización. Composite H1. Velocidad de calentamiento $\beta=15 \mathrm{~K} / \mathrm{min}$. 


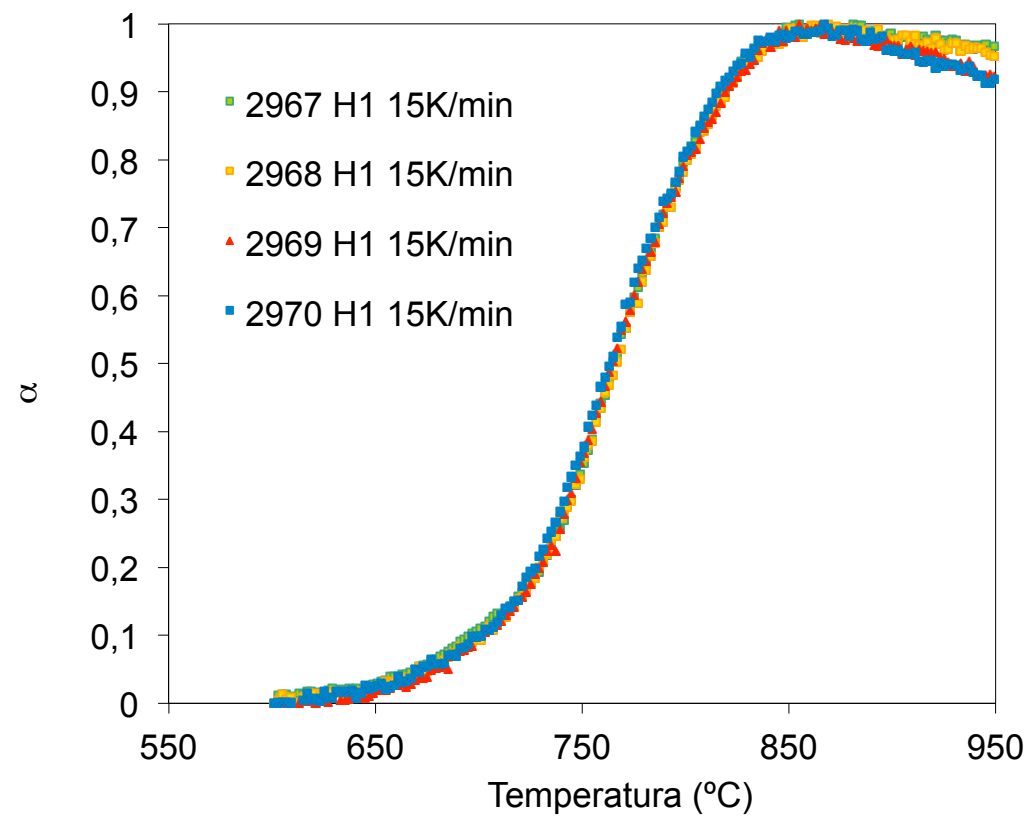

Figura 7.3. Evolución de $\alpha$ vs temperatura. Composite H1. Velocidad de calentamiento $\beta=15 \mathrm{~K} / \mathrm{min}$.

Los valores medios, desviación estándar y el coeficiente de variación de $\varepsilon_{A}(25), \varepsilon_{A}$ y $\alpha$ correspondientes a las temperaturas $660^{\circ} \mathrm{C}$ (inicio de la sinterización) y $840^{\circ} \mathrm{C}$ (final de la sinterización) para estos cuatro experimentos virtualmente idénticos se detallan en la Tabla 7.1.

Tabla 7.1. Valores medios, desviación estándar y coeficiente de variación de $\varepsilon_{A}(25)$, $\varepsilon_{A}$ y $\alpha$ a $660^{\circ} \mathrm{C}$ y $840^{\circ} \mathrm{C}$ obtenidos en cuatro experimentos idénticos.

\begin{tabular}{|c|c|c|c|c|c|c|}
\hline \multirow{2}{*}{ Parámetro } & \multicolumn{2}{|c|}{$-\varepsilon_{\mathrm{A}}(25)$} & \multicolumn{2}{c|}{$-\varepsilon_{\mathrm{A}}$} & \multicolumn{2}{c|}{$\alpha$} \\
\cline { 2 - 7 } & $\mathbf{6 6 0 ^ { \circ } \mathrm { C }}$ & $\mathbf{8 4 0 ^ { \circ } \mathrm { C }}$ & $\mathbf{6 6 0 ^ { \circ } \mathrm { C }}$ & $\mathbf{8 4 0} \mathrm{C}$ & $\mathbf{6 6 0 ^ { \circ } \mathrm { C }}$ & $\mathbf{8 4 0} \mathrm{C}$ \\
\hline Media & 0,013 & 0,317 & 0,011 & 0,315 & 0,034 & 0,972 \\
\hline $\begin{array}{c}\text { Desviación } \\
\text { estándar }\end{array}$ & 0,00837 & 0,01502 & 0,00228 & 0,01244 & 0,00620 & 0,00601 \\
\hline $\begin{array}{c}\text { Coef. de } \\
\text { variación }\end{array}$ & 0,6652 & 0,0474 & 0,2039 & 0,0394 & 0,1808 & 0,0063 \\
\hline
\end{tabular}


Del examen del conjunto de estos resultados se desprende lo siguiente:

i) La dispersión de las curvas $\varepsilon_{A}(25)$ vs temperatura es muy superior a las de $\varepsilon_{\mathrm{A}}$ vs temperatura (Figura 7.1 y Figura 7.2). En estas últimas curvas, la correspondiente al experimento 2970 (Figura 7.2) se desvía significativamente de las otras tres, prácticamente coincidentes, debido probablemente a que la compacidad en crudo de la muestra 2970 es más baja.

ii) La desviación estándar de los valores de $\varepsilon_{\mathrm{A}}$, tanto al inicio de la sinterización $\left(T=660^{\circ} \mathrm{C}\right.$ y $\left.\varepsilon_{A} \approx 0,01\right)$ como en los estados finales de la misma $\left(T=840^{\circ} \mathrm{C}\right.$ y $\left.\varepsilon_{A} \approx 0,315\right)$ es mucho menor que los correspondientes a $\varepsilon_{A}(25)$, especialmente al inicio de la sinterización. Los coeficientes de variación siguen la misma tendencia (Tabla 7.1).

iii) Las curvas de sinterización: $\alpha$ vs temperatura, calculadas a partir de $\varepsilon_{\mathrm{A}}$ son prácticamente coincidentes, excepto para temperaturas superiores a la de máxima densificación (Figura 7.3). Es decir, minimiza el efecto de la compacidad en crudo. Además, los valores del coeficiente de variación de $\alpha$ al inicio y al final de la sinterización son mucho menores que los correspondientes a $\varepsilon_{A}(25)$ y $\varepsilon_{A}$, especialmente al final de la sinterización (Tabla 7.1). 
7.3 Estimación de los valores de la diferencia entre la temperatura del horno y la temperatura media de la pieza y distribución interna de la temperatura.

\subsubsection{Desarrollo del modelo.}

Se ha considerado el modelo más sencillo, en el que no existe generación de calor, ya que un nuestro caso la única transformación es la sinterización, y esta es muy poco endotérmica. Se han asumido las siguientes hipótesis:

- La conductividad térmica, $\overline{\mathrm{k}}$, la densidad, $\bar{\rho}$, y el calor específico, $\overline{\mathrm{C}_{\mathrm{p}}}$, promedios, no se modifican al cambiar la temperatura.

- La pieza es un cilindro de diámetro $D_{0}$ y altura $h_{0}$, apoyado en una de sus caras sobre un refractario (Figura 7.4).

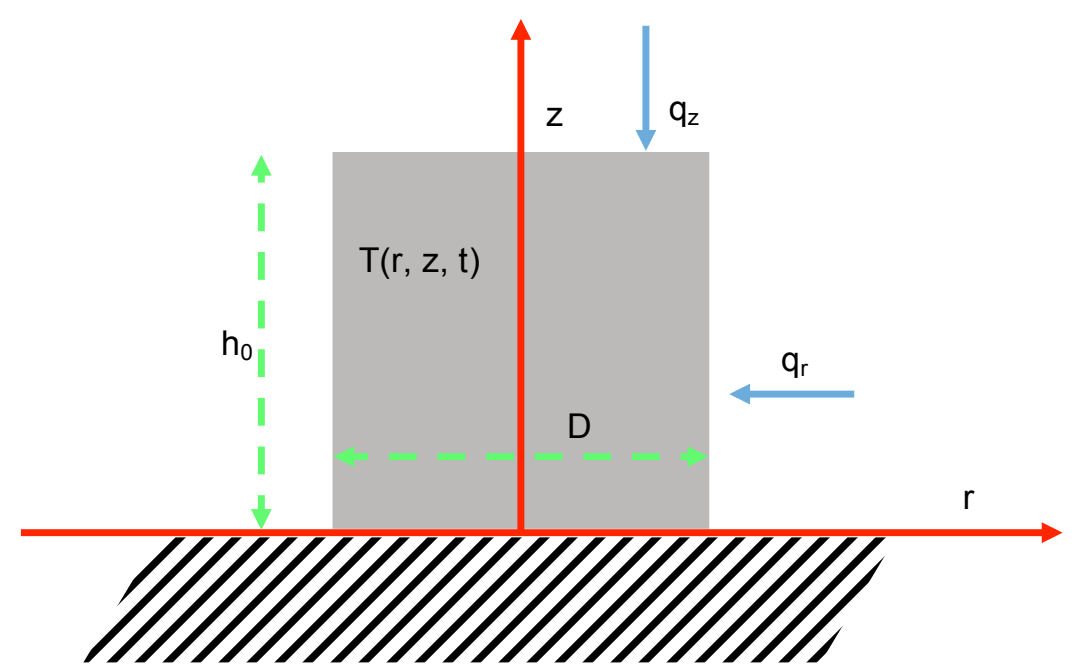

Figura 7.4. Situación de la probeta en el horno.

La ecuación diferencial que expresa la temperatura en el interior de la pieza en función del tiempo, $t$, y la posición ( $r, z$ ) adopta la forma:

$\frac{\partial T}{\partial t}=\bar{\alpha}\left[\frac{1}{r} \cdot \frac{\partial}{\partial r}\left(r \frac{\partial T}{\partial r}\right)+\frac{\partial^{2} T}{\partial z^{2}}\right]$ ec. A3. 1 
siendo $\bar{\alpha}$ la difusividad térmica definida como: $\bar{\alpha}=\frac{\overline{\mathrm{k}}}{\bar{\rho} \overline{\mathrm{C}_{\mathrm{p}}}}$, que también se considera independiente de la temperatura.

Las condiciones de contorno necesarias para la integraciñon de ec. A3. 1 son:

Para $z=0 \quad q_{z}=-\bar{k} \frac{\partial T}{\partial z}=0$

Para $z=h_{0} \quad q_{z}=-\bar{k} \frac{\partial T}{\partial z}=h^{\prime} \cdot\left(T_{h}-T\left(z, h_{0}, t\right)\right)$

Para $r=D / 2 \quad q_{r}=-\bar{k} \frac{\partial T}{\partial r}=h^{\prime} \cdot\left(T_{h}-T\left(\frac{D_{0}}{2}, z, t\right)\right)$

ec. A3. 2

y la inicial:

Para $t=0 \quad T(r, z, 0)=T_{0}$

donde: $T_{h}$ es la temperatura del horno, que consideramos que es la que mide el termopar, próximo a la pieza y protegido de la radiación; h' es el coeficiente individual de transmisión de calor, que engloba tanto la convección natural como la radiación. Dicho coeficiente se puede calcular [143] mediante la ecuación:

$h^{\prime}=h_{c}+4 \sigma \varepsilon T_{h}^{3}$ ec. A3. 3

$\mathrm{h}_{\mathrm{c}}=\mathrm{el}$ valor estimado para el coeficiente individual de transmisión de calor, para hornos radiantes $\left(\mathrm{W} / \mathrm{m}^{2} \mathrm{~K}\right) ; \mathrm{T}_{\mathrm{h}}=$ temperatura del horno $(\mathrm{K})$; $\sigma=$ constante de Stefan-Boltzman $\left(5,670 \cdot 10^{-8} \mathrm{~W} / \mathrm{m}^{2} \mathrm{~K}^{4}\right) ; \varepsilon=$ emisividad resultante.

La ec. A3. 1 con las condiciones ec. A3. 2 se ha resuelto por el método de incrementos finitos (programa Code_Aster). Para la estimación se han utilizado los valores siguientes: 
- $\bar{\rho}_{1}=1600$ y $\bar{\rho}_{2}=2500 \mathrm{Kg} / \mathrm{m}^{3}$.

- $\overline{\mathrm{k}}_{1}=0,6$ y $\overline{\mathrm{k}}_{2}=1,5 \mathrm{~W} / \mathrm{m} \cdot \mathrm{K}$

- $\overline{\mathrm{C}}_{\mathrm{p}}=1000 \mathrm{~J} / \mathrm{K} \cdot \mathrm{kg}$

- $\mathrm{h}_{\mathrm{c}}=7,69 \mathrm{~W} / \mathrm{m}^{2} \cdot \mathrm{K}$

- $\varepsilon=0,9$

Los valores de $\bar{\rho}_{1}$ y $\bar{k}_{1}$ coresponden a materiales porosos, representativos del inicio de la sinterización y los de $\bar{\rho}_{2}$ y $\bar{k}_{2}$ a piezas compactas, representativos del final de la sinterización.

\subsubsection{Resultados.}

7.3.2.1 Calentamiento a velocidad de calentamiento constante de probetas cilindricas pequeñas, $D_{0}=5 \mathrm{~mm}$ y $h_{0}=3 \mathrm{~mm}$.

Estas condiciones son similares a las que se dan en experimentos a velocidad de calentamiento constante en el microscopio de calefacción (HSM).

En la Figura 7.5 se representan, para probetas densas $\left(\bar{\rho}_{2}=2500 \mathrm{~kg} / \mathrm{m}^{3}\right.$ y $\left.\overline{\mathrm{k}}_{2}=1,5 \mathrm{~W} / \mathrm{m} \cdot \mathrm{K}\right)$ y porosas $\left(\bar{\rho}_{1}=1600 \mathrm{~kg} / \mathrm{m}^{3}\right.$ y $\left.\overline{\mathrm{k}}_{1}=0,6 \mathrm{~W} / \mathrm{m} \cdot \mathrm{K}\right)$ los valores de la diferencia entre la temperatura del horno y la temperatura media de la probeta $\left(T_{h}-T_{m}\right)$, frente a la temperatura del horno, $T_{h}$, para diferentes velocidades de calentamiento. 


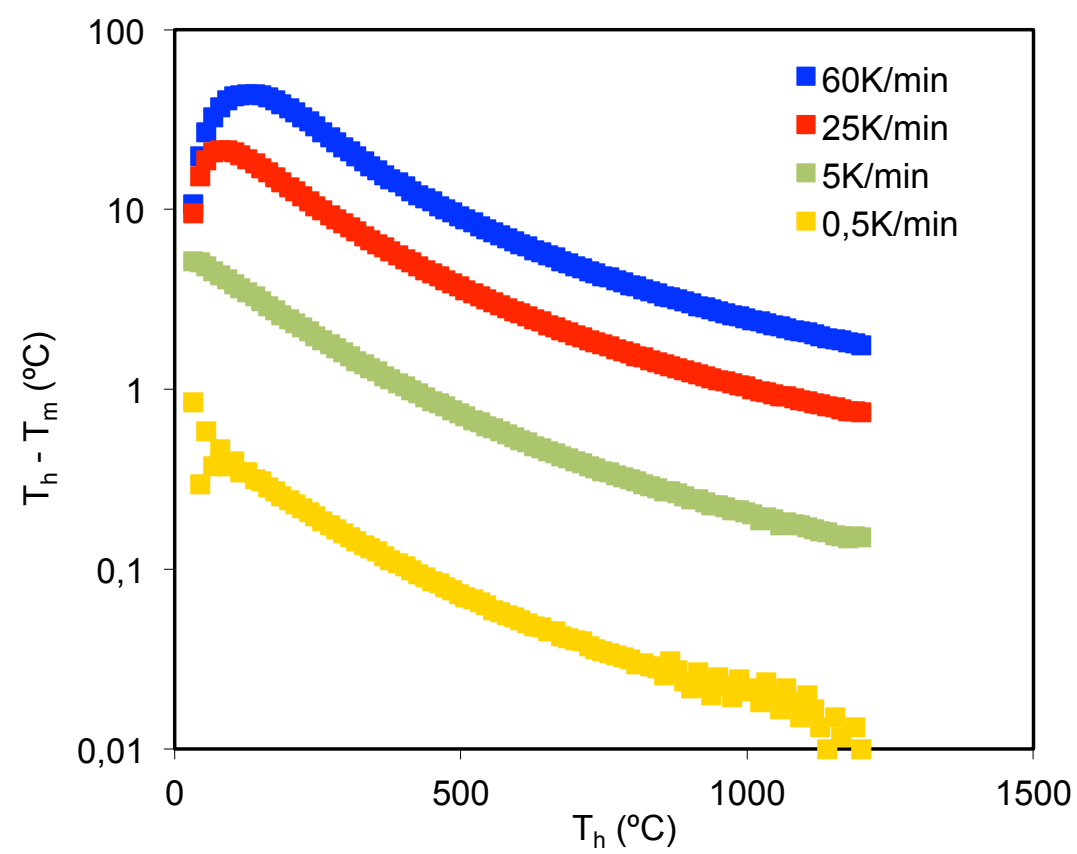

Figura 7.5. Evolución de la diferencia entre la temperatura del horno, $T_{h}$, y la media del cilindro, $T_{m}$, frente a la temperatura del horno, $T_{h}$, para distintas velocidades de calentamiento. Probetas porosas $\left(\bar{\rho}_{1}=1600 \mathrm{~kg} / \mathrm{m}^{3}\right.$ y $\left.\overline{\mathrm{k}}_{1}=0,6 \mathrm{~W} / \mathrm{m} \cdot \mathrm{K}\right)$.

Se confirma que a todas las velocidades excepto al comienzo del calentamiento, los valores de $T_{h}-T_{m}$ disminuyen conforme avanza el calentamiento. A temperaturas ya relativamente elevadas se ha podido constatar que los valores de $T_{h}-T_{m}$ son prácticamente proporcionales a la velocidad de calentamiento, siendo dicha constante de porporcionalidad (pendiente de las rectas) tanto más alta cuanto menor es la temperatura del horno, $T_{h}$ (Figura 7.6). 


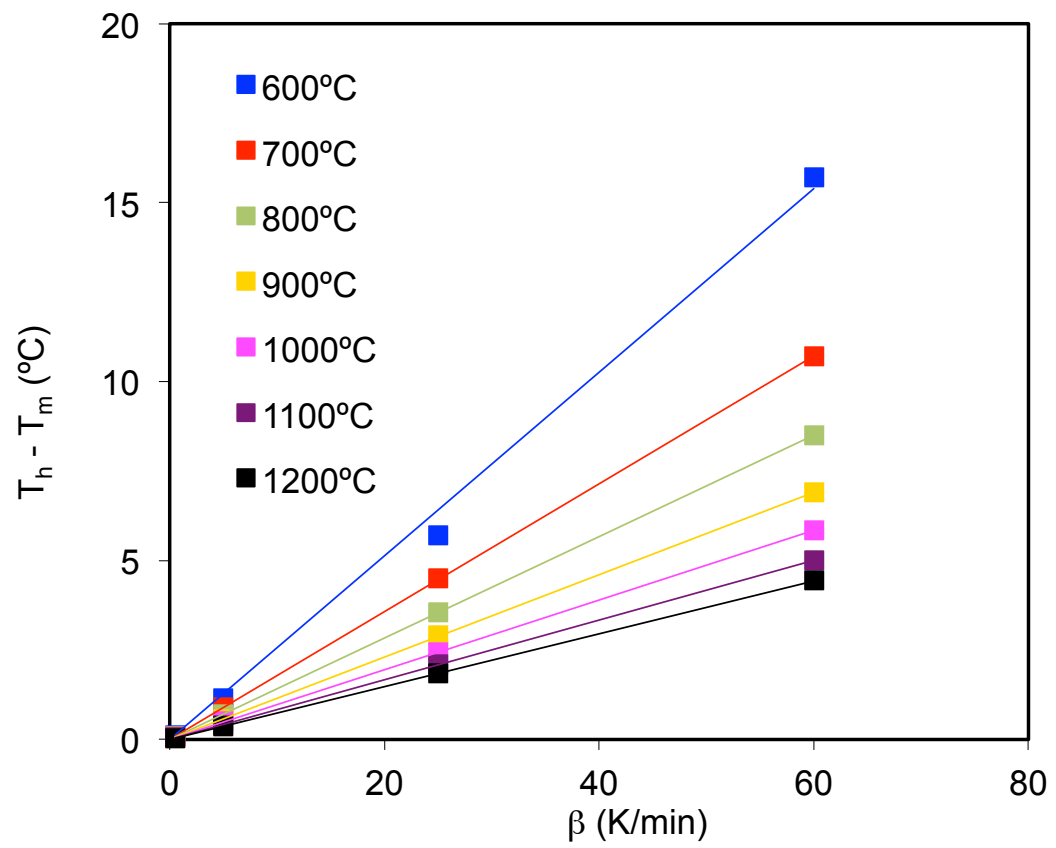

Figura 7.6. Efecto de la velocidad de calentamiento, $\beta$, sobre la diferencia entre la temperatura del horno, $T_{h}$, y la temperatura media del cilindro, $T_{m}$, a diferentes temperaturas del horno, $T_{h}$. Probetas porosas $\left(\bar{\rho}_{1}=1600 \mathrm{~kg} / \mathrm{m}^{3}\right.$ $\left.y \overline{\mathrm{k}}_{1}=0,6 \mathrm{~W} / \mathrm{m} \cdot \mathrm{K}\right)$.

En la Figura 7.7 se ha representado, para la velocidad de $60 \mathrm{~K} / \mathrm{min}$, los valores de $T_{h}-T_{m}$ vs $T_{h}$ para la probeta porosa (1) de dimensiones iniciales, $y$ para una probeta mas densa (2) y con dimensiones un $20 \%$ más pequeñas que la anterior, que representase las dimensiones y la compacidad del material final de la sinterización. Se comprueba que los valores de $T_{h}-T_{m}$ para la probeta más densa y pequeña siempre es menor que la porosa y más grande. No obstante, el efecto de las dimensiones y de la compacidad (y en consecuencia de la conductividad) es mucho menor que el de la velocidad de calentamiento. En cualquier caso, los valores de $T_{h}-T_{m}$ durante el calentamiento y sinterización de la probeta, a temperaturas bajas $\mathrm{T}<700^{\circ} \mathrm{C}$, serán muy parecidos a los de la curva del material poroso. A partir de esta temperatura se van aproximando cada vez más a los de la probeta densa y más pequeña. 


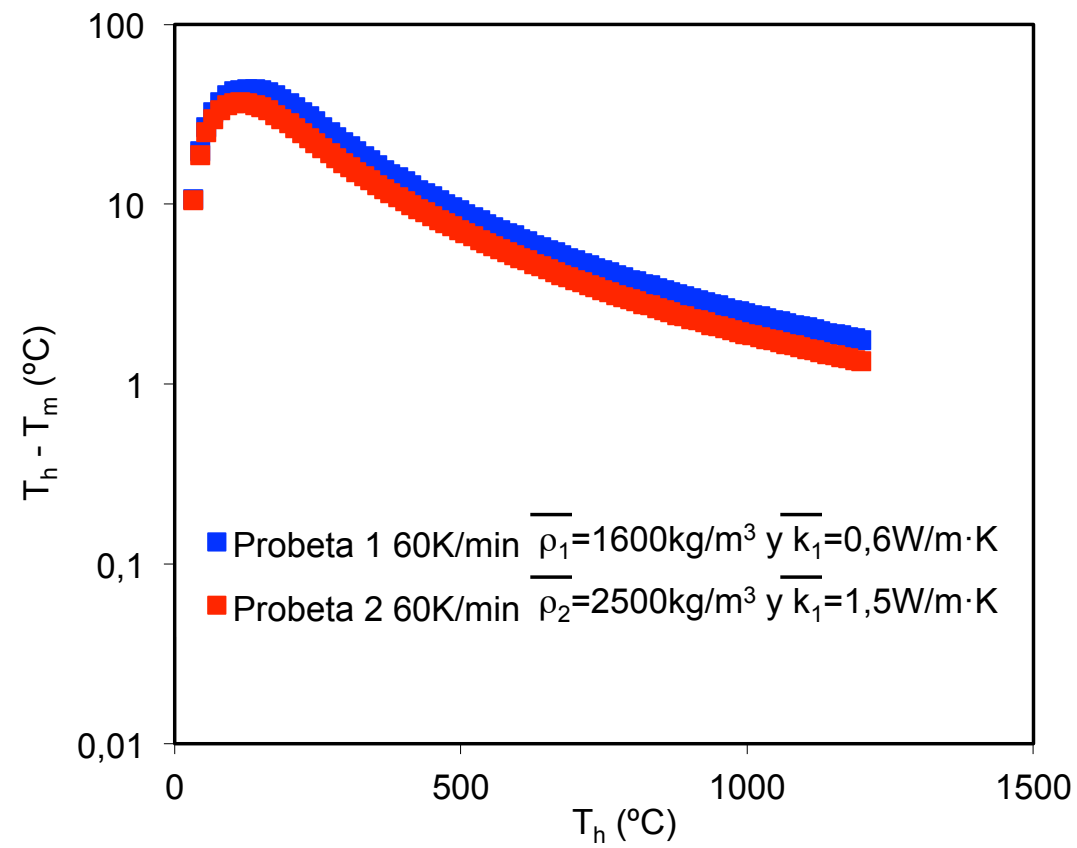

Figura 7.7. Evaluación de la diferencia entre la temperatura del horno, $T_{h}$, y la media de la pieza, $T_{m}$, frente a la temperatura del horno, $T_{h}$, a una velocidad de calentamiento de $\beta=60 \mathrm{~K} / \mathrm{min}$. Efecto de la sinterización. Probeta 1 (dimensiones iniciales, $\bar{\rho}_{1}=1600 \mathrm{~kg} / \mathrm{m}^{3}$ y $\quad \overline{\mathrm{k}}_{1}=0,6 \mathrm{~W} / \mathrm{m} \cdot \mathrm{K}$ ). Probeta 2 (dimensiones reducidas un $20 \%, \bar{\rho}_{2}=2500 \mathrm{~kg} / \mathrm{m}^{3}$ y $\overline{\mathrm{k}}_{2}=1,5 \mathrm{~W} / \mathrm{m} \cdot \mathrm{K}$ ).

7.3.2.2 Calentamiento a $5 \mathrm{~K} / \mathrm{min}$ de probetas cilíndricas grandes, $D_{0}=20 \mathrm{~mm}$ $y h_{0}=20 \mathrm{~mm}$ en horno eléctrico.

En la Figura 7.8 se representa la variación de $T_{h}-T_{m}$ para el cilindro poroso $\left(\bar{\rho}_{1}=1600 \mathrm{~kg} / \mathrm{m}^{3}\right.$ y $\left.\overline{\mathrm{k}}_{1}=0,6 \mathrm{~W} / \mathrm{m} \cdot \mathrm{K}\right)$ frente a $\mathrm{T}_{\mathrm{h}}$. Los resultados son muy parecidos a los que se obtienen con el cilindro pequeño a $\beta=25 \mathrm{~K} / \mathrm{min}$.

La variación de la distribución de la temperatura en el interior del cilindro durante el calentamiento también ha sido calculada y los resultados más significativos se representan en la Figura 7.9. En ella se han representado, frente a la temperatura del horno, $T_{h}$, los valores de $\left(T_{s}-T_{a}\right)$ y $\left(T_{m}-T_{a}\right)$, siendo $\mathrm{T}_{\mathrm{s}}$ la temperatura media de la cara del cilindro no aislada $\left(\mathrm{z}=\mathrm{h}_{0}\right.$ de la Figura 
7.1), $T_{a}$ la temperatura media de la cara aislada ( $z=0$ de la Figura 7.1) y $T_{m}$ la temperatura media del cilindro. Se aprecia que para $T_{h}>600^{\circ} \mathrm{C}$ los valores $\mathrm{T}_{\mathrm{s}}-\mathrm{T}_{\mathrm{a}} \leq 10^{\circ} \mathrm{C}$ y van disminuyendo con el aumento de la temperatura del horno hasta $\approx 7^{\circ} \mathrm{C}$ a $1200^{\circ} \mathrm{C}$. En cambio, $\mathrm{T}_{\mathrm{m}}-\mathrm{T}_{\mathrm{a}}$ se mantiene prácticamente constante para este intervalo de temperaturas, es decir, para $\mathrm{T}>600^{\circ} \mathrm{C}$ $\left(T_{m}-T_{a} \approx 5,5^{\circ} \mathrm{C}\right)$.

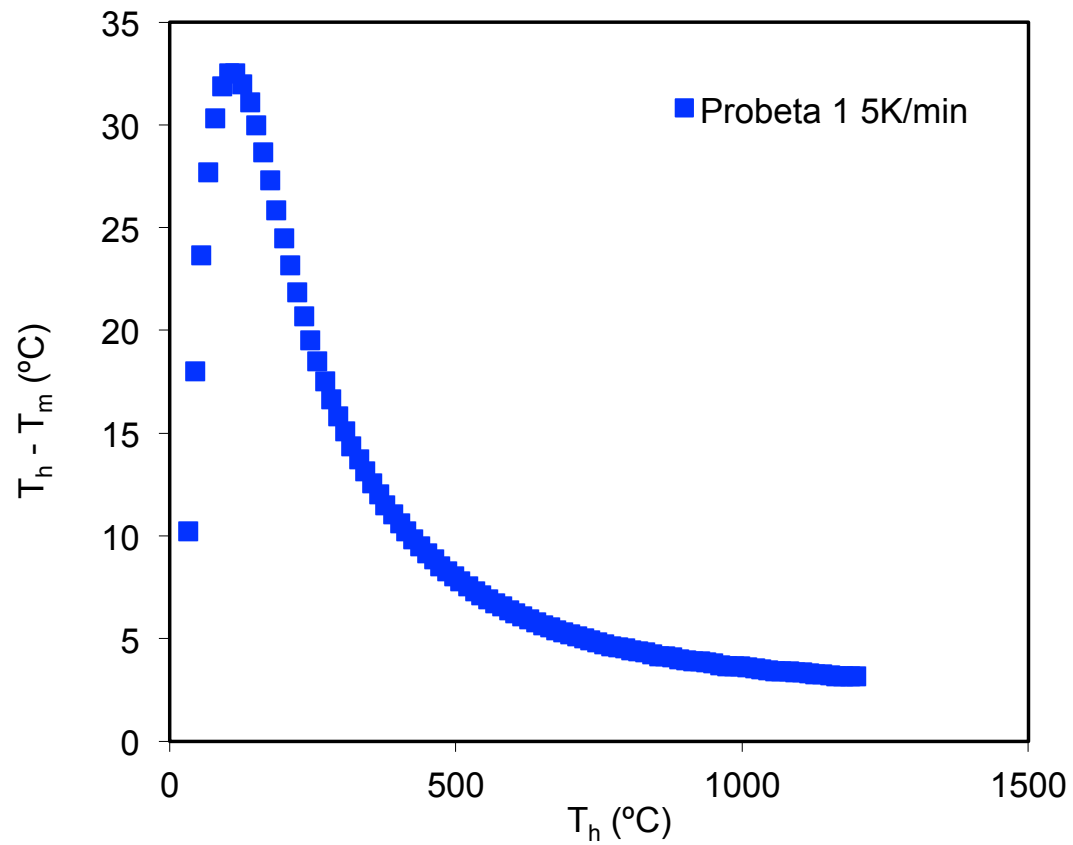

Figura 7.8. Evolución de la diferencia entre la temperatura del horno, $T_{h}$, y la media de la pieza, $T_{m}$, frente a la temperatura del horno, $T_{h}$, a una velocidad de calentamiento de $\beta=5 \mathrm{~K} / \mathrm{min}$, para un cilindro grande $\left(D_{0}=20 \mathrm{~mm}\right.$ y $\left.h_{0}=20 \mathrm{~mm}\right)$ y poroso $\left(\bar{\rho}_{1}=1600 \mathrm{~kg} / \mathrm{m}^{3}\right.$ y $\left.\overline{\mathrm{k}}_{1}=0,6 \mathrm{~W} / \mathrm{m} \cdot \mathrm{K}\right)$. 


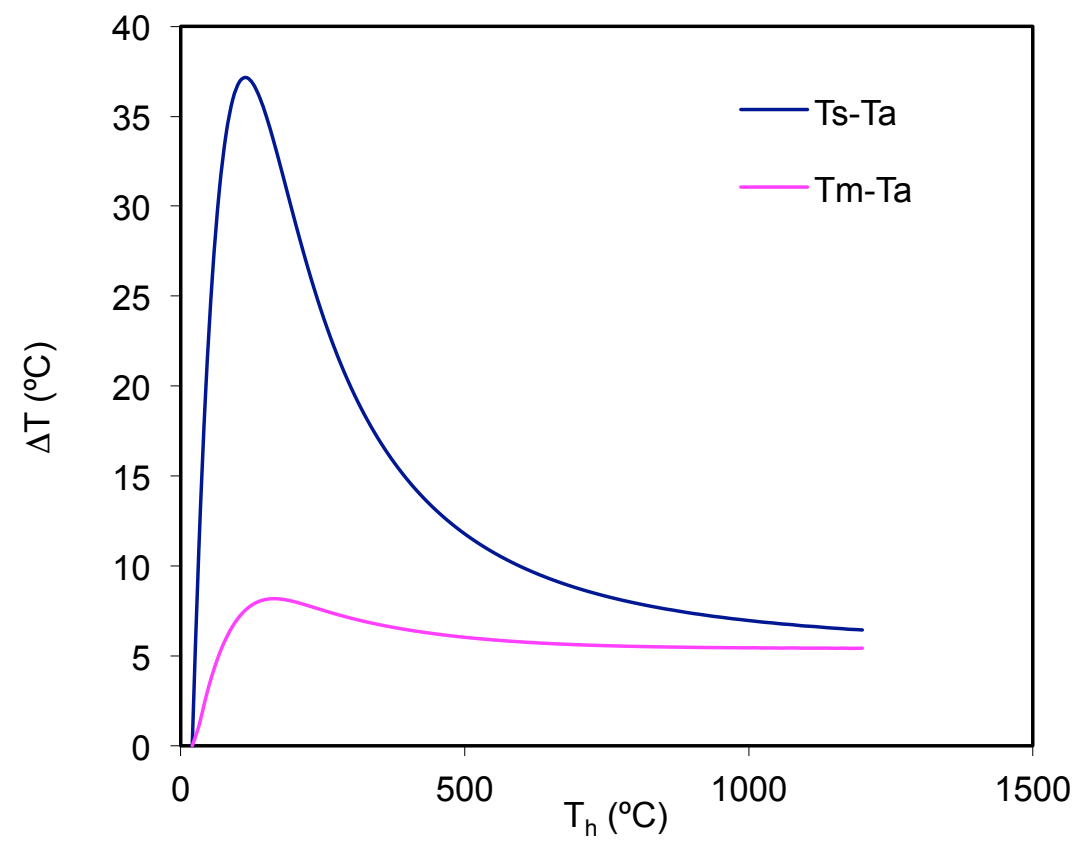

Figura 7.9. Evolución con la temperatura del horno, $T_{h}$, de la diferencia entre la temperatura media de la cara no aislada, $T_{s}$, y la aislada, $T_{a}$, y de la diferencia entre la temperatura media del cilindro, $T_{m}$, y de la cara aislada, $T_{a}$. Cilindro grande $\left(D_{0}=20 \mathrm{~mm}\right.$ y $\left.h_{0}=20 \mathrm{~mm}\right)$ y poroso $\left(\bar{\rho}_{1}=1600 \mathrm{~kg} / \mathrm{m}^{3}\right.$ y $\left.\quad \overline{\mathrm{k}}_{1}=0,6 \mathrm{~W} / \mathrm{m} \cdot \mathrm{K}\right)$. Velocidad de calentamiento de $\beta=5 \mathrm{~K} / \mathrm{min}$.

7.3.2.3 Tratamientos isotermos de probetas cilíndricas grandes $\left(D_{0}=20 \mathrm{~mm}\right.$ y $h_{0}=20 \mathrm{~mm}$ ) en horno eléctrico.

En esta simulación la temperatura del horno, $T_{h}$, se mantiene constante y a $\mathrm{t}=0$ se introduce la probeta cuya temperatura es de $20^{\circ} \mathrm{C}$.

En la Figura 7.10 se representan para las probetas porosas $\left(\rho_{1}=1600 \mathrm{~kg} / \mathrm{m}^{3}\right.$ y $\left.\overline{\mathrm{k}}_{1}=0,6 \mathrm{~W} / \mathrm{m} \cdot \mathrm{K}\right)$, la evolución de la temperatura media del cilindro, $T_{m}$, con la duración del tratamiento, $t$, para valores de $T_{h}=800$, $850,900,950$ y $1000^{\circ} \mathrm{C}$. En todos los casos, para valores de 6,5-7min, la temperatura media de la pieza, $T_{m}$, y la del horno, $T_{h}$, eran prácticamente coincidentes $T_{h}-T_{m} \leq 1^{\circ} \mathrm{C}$. Para este mismo tipo de material, en la Figura 7.11 se ha representado para $T_{h}=900^{\circ} \mathrm{C}$, la evolución que siguen con el tiempo de permanencia en el horno, $t$, los valores de la temperatura media del cilindro, 
$T_{m}$, de la cara no aislada, $T_{s},\left(z=h_{0}\right.$ de la Figura 7.1), y de la cara aislada, $T_{a}$, ( $z=0$ de la Figura 7.1). Se comprueba que para tiempos de permanencia superiores a $8 \mathrm{~min}$, la temperatura de cilindro se puede considerar uniforme, $\mathrm{T}_{\mathrm{s}}-\mathrm{T}_{\mathrm{a}} \leq 1^{\circ} \mathrm{C}$. El efecto de la sinterización se representa en la Figura 7.12. Los valores de $T_{s, p}$ y $T_{a, p}$ se refieren, para un tratamiento también a $900^{\circ} \mathrm{C}$, a los valores de la probeta anterior. Los valores $T_{s, d}$ y $T_{a, d}$ corresponden a las temperaturas medias de la cara no aislada y aislada, respectivamente, para una probeta más pequeña ( $20 \%$ de contracción) y más densa y conductora $\left(\bar{\rho}_{2}=2500 \mathrm{~kg} / \mathrm{m}^{3}\right.$ y $\left.\overline{\mathrm{k}}_{2}=1,5 \mathrm{~W} / \mathrm{m} \cdot \mathrm{K}\right)$ debido a la sinterización. En este caso, el tiempo de permanencia requerido para que la temperatura de la pieza sea homogénea se reduce a unos $5 \mathrm{~min}$.

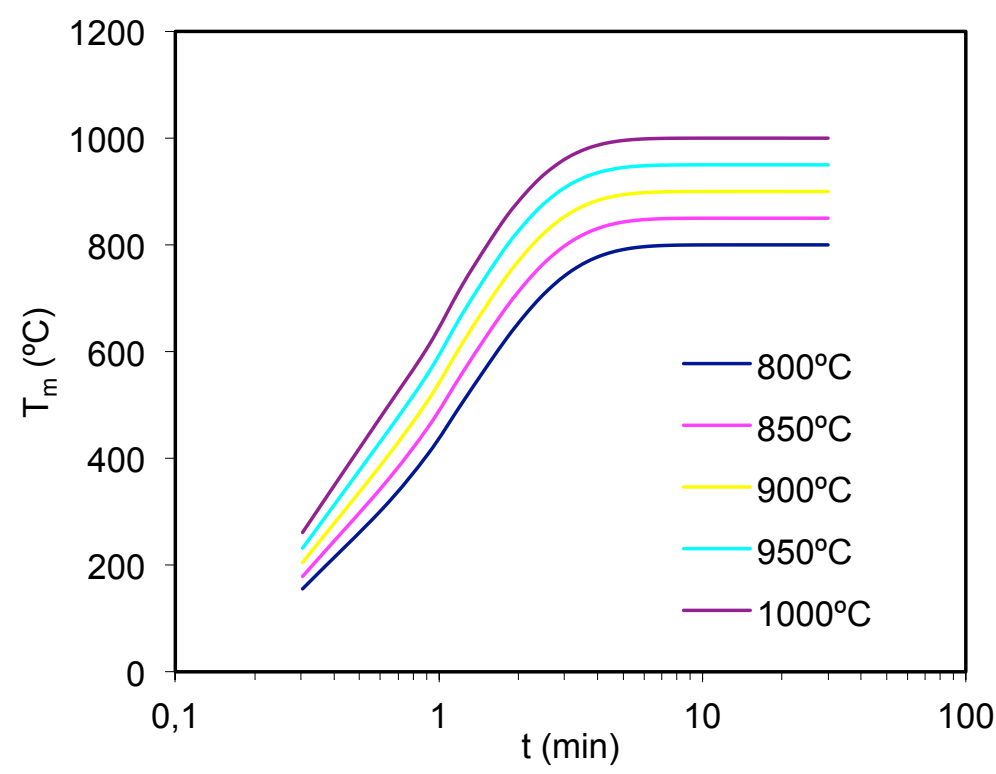

Figura 7.10. Evolución de la temperatura media, $T_{m}$, del cilindro con el tiempo de permanencia en el horno, $t$, en tratamientos isotermos para valores de $T_{h}=800,850,900,950$ y $1000^{\circ} \mathrm{C}$. Cilindros porosos: $D_{0}=20 \mathrm{~mm}$ y $h_{0}=20 \mathrm{~mm} ;\left(\bar{\rho}_{1}=1600 \mathrm{~kg} / \mathrm{m}^{3}\right.$ y $\left.\overline{\mathrm{k}}_{1}=0,6 \mathrm{~W} / \mathrm{m} \cdot \mathrm{K}\right)$. 


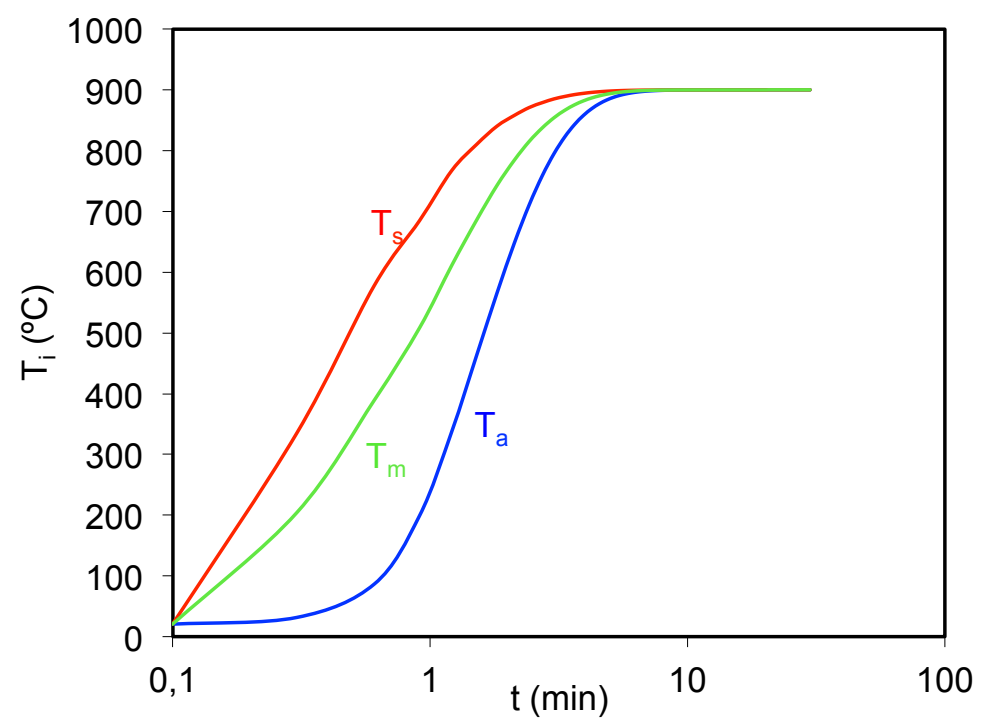

Figura 7.11. Evolución con el tiempo de permanencia en el horno, $t$, de la temperatura media de la cara no aislada para el cilindro poroso $\left(T_{s, p}\right)$ y compacto $\left(T_{s, d}\right)$ y aislada para el cilindro poroso $\left(T_{a, p}\right)$ y compacto $\left(T_{a, d}\right)$. Tratamiento isotermo a $900^{\circ} \mathrm{C}$. Cilindro poroso: $D_{0}=20 \mathrm{~mm}$ y $h_{0}=20 \mathrm{~mm} ; \bar{\rho}_{1}=1600 \mathrm{~kg} / \mathrm{m}^{3}$ y $\overline{\mathrm{k}}_{1}=0,6 \mathrm{~W} / \mathrm{m} \cdot \mathrm{K}$.

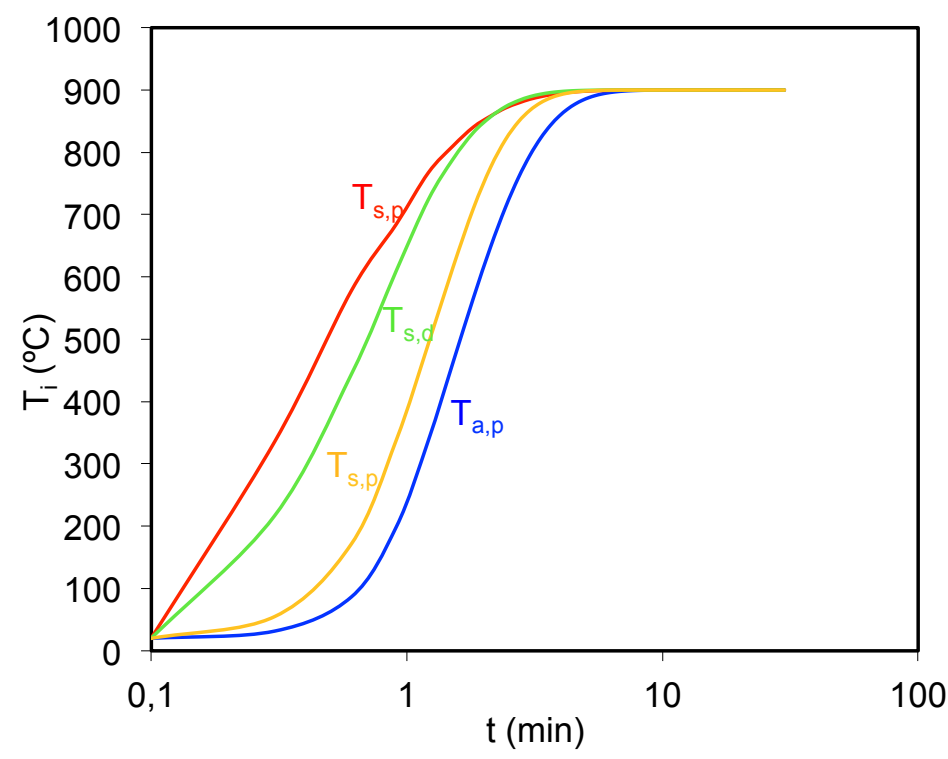

Figura 7.12. Evolución con el tiempo de permanencia en el horno, $t$, de la temperatura media del cilindro, $T_{m}$, de su cara no aislada, $T_{s}$, y de su cara aislada, $T_{a}$. Tratamiento isotermo a $900^{\circ} \mathrm{C}$. Cilindro poroso: $D_{0}=20 \mathrm{~mm}$ y $h_{0}=20 \mathrm{~mm}$; $\bar{\rho}_{1}=1600 \mathrm{~kg} / \mathrm{m}^{3}$ y $\overline{\mathrm{k}}_{1}=0,6 \mathrm{~W} / \mathrm{m} \cdot \mathrm{K}$. 


\section{Nomenclatura}

$a_{s}$

A

$\mathrm{A}_{\mathrm{g}}$

$A_{\text {min }}$

$A_{\alpha}$

$A^{*}$

$\mathrm{A}_{0}$

$A_{1}$

$A_{2}$

B

C

$\overline{C_{p}}$

d

$d_{g}^{v}$

$d_{z}^{v}$

D

$D_{S}$

$D_{Z r}$

E

$E_{1}$
Radio del cilindro del modelo de Scherer.

$\mathrm{m}$

Factor preexponencial genérico, ec. 2.66.

$\mathrm{s}^{-1}$

Factor preexponencial de la sinterización $\mathrm{s}^{-1}$ correspondiente al vidrio.

Área de la probeta cilíndrica a la máxima $\mathrm{m}^{2}$ compacidad.

Factor preexponencial para un determinado $\mathrm{s}^{-1}$ grado de avance, $\alpha$, del proceso de sinterización, ec. 2.73.

Parámetro de la ecuación de Vogel-Fulcher- Pa.s Tamman, ec. 5.1.

Área de la probeta cilíndrica al inicio de la $\mathrm{m}^{2}$ sinterización.

Factor preexponencial asociado a la $\mathrm{s}^{-1}$ reordenación de partículas por flujo viscoso, ec. 5.30 y ec. 5.31 .

Factor preexponencial asociado a la solución- $\mathrm{s}^{-1}$ reprecipitación del circón, ec. 5.32.

Parámetro de la ecuación de Vogel-Fulcher- ${ }^{\circ} \mathrm{C}$ Tamman, ec. 5.1.

Tamaño medio de cristalito.

m

Calor específico medio de la probeta.

$\mathrm{J} / \mathrm{kg} \cdot \mathrm{K}$

Diámetro medio geométrico del vidrio.

m

Diámetro medio volumétrico del vidrio.

m

Diámetro medio volumétrico del circón.

m

Diámetro de la probeta cilíndrica.

$\mathrm{m}$

Difusividad del soluto en el fundido.

$\mathrm{m}^{2} / \mathrm{s}$

Coeficiente de distribución del circón entre el sólido y el fundido.

Energía de activación genérica, ec. 2.66. $\mathrm{kJ} / \mathrm{mol}$

Energía de activación asociada a la reordenación $\mathrm{kJ} / \mathrm{mol}$ de partículas por flujo viscoso, ec. 5.31. 


\begin{tabular}{|c|c|c|}
\hline $\mathrm{E}_{2}$ & $\begin{array}{l}\text { Energía de activación asociada a la solución- } \\
\text { reprecipitación del circón, ec. } 5.32 \text {. }\end{array}$ & $\mathrm{kJ} / \mathrm{mol}$ \\
\hline $\mathrm{E}_{2}^{*}$ & Función exponencial de orden 2, ec. 2.78. & - \\
\hline $\mathrm{E}_{\alpha}$ & $\begin{array}{l}\text { Energía de activación correspondiente a un } \\
\text { determinado grado de avance, } \alpha \text {, del proceso de } \\
\text { sinterización. }\end{array}$ & $\mathrm{kJ} / \mathrm{mol}$ \\
\hline$E_{\eta}$ & $\begin{array}{l}\text { Energía de activación de flujo viscoso } \\
\text { correspondiente al vidrio, ec. 5.2. }\end{array}$ & $\mathrm{kJ} / \mathrm{mol}$ \\
\hline$f(\alpha)$ & $\begin{array}{l}\text { Modelo genérico de sinterización definido por la } \\
\text { ec. } 2.44 \text {. }\end{array}$ & - \\
\hline $\mathrm{F}_{\mathrm{S}}$ & Energía libre superficial del sistema. & $\mathrm{N} / \mathrm{m}$ \\
\hline$F(G)$ & Función de distribución de Weibull, ec. 5.24. & - \\
\hline$F\left(\rho, \rho_{0}\right)$ & Potencial de sinterización, ec. 2.60. & - \\
\hline$g(\alpha)$ & $\begin{array}{l}\text { Forma integrada del modelo genérico de } \\
\text { sinterización. }\end{array}$ & - \\
\hline G & Tamaño de grano. & $\mathrm{m}$ \\
\hline $\mathrm{G}_{\mathrm{c}}$ & $\begin{array}{l}\text { Diámetro del círculo equivalente de la sección de } \\
\text { un grano de circón. }\end{array}$ & $\mathrm{m}$ \\
\hline $\mathrm{G}_{\mathrm{M}}$ & Tamaño medio de grano. & $\mathrm{m}$ \\
\hline $\mathrm{G}^{*}$ & Fracción volumétrica de vidrio en un composite. & - \\
\hline $\mathrm{G}_{0}$ & Tamaño de grano inicial. & $\mathrm{m}$ \\
\hline h & Altura de la probeta cilíndrica. & $\mathrm{m}$ \\
\hline$h_{c}$ & $\begin{array}{l}\text { Valor estimado para el coeficiente individual de } \\
\text { transmisión de calor. }\end{array}$ & $\mathrm{W} / \mathrm{m}^{2} \cdot \mathrm{K}$ \\
\hline$h_{\min }$ & $\begin{array}{l}\text { Altura de la probeta cilíndrica a la máxima } \\
\text { compacidad. }\end{array}$ & $\mathrm{m}$ \\
\hline$h^{\prime}$ & Coeficiente de transmisión de calor. & $\mathrm{W} / \mathrm{m}^{2} \cdot \mathrm{K}$ \\
\hline$h_{0}$ & $\begin{array}{l}\text { Altura de la probeta cilíndrica al inicio de la } \\
\text { sinterización. }\end{array}$ & $\mathrm{m}$ \\
\hline$H\left(\rho ; \alpha^{\prime}, \rho_{0}\right)$ & $\begin{array}{l}\text { Función que describe el efecto del aumento de la } \\
\text { compacidad con el avance de la sinterización } \\
\text { sobre } \mathrm{K}_{\mathrm{p}} \text {, ec. } 2.61 \text {. }\end{array}$ & - \\
\hline k & $\begin{array}{l}\text { Constante de velocidad del proceso de } \\
\text { sinterización, ec. } 5.13 \text {. }\end{array}$ & $s^{-1}$ \\
\hline $\mathrm{k}_{\mathrm{A}}$ & Razón: contracción axial/contracción radial. & - \\
\hline $\mathrm{K}_{\mathrm{g}}$ & Constante de velocidad del proceso de & $s^{-1}$ \\
\hline
\end{tabular}


sinterización correspondiente al vidrio.

\begin{tabular}{|c|c|c|}
\hline$k^{*}$ & $\begin{array}{l}\text { Constante de velocidad de la sinterización de } \\
\text { orden } 1, \text { ec. } 2.8 \text {. }\end{array}$ & $s^{-1}$ \\
\hline$k^{\prime}$ & $\begin{array}{l}\text { Constante de velocidad del proceso de } \\
\text { crecimiento de grano, ec. } 2.63 \text {. }\end{array}$ & $\mathrm{m}^{3} / \mathrm{s}$ \\
\hline$k_{0}^{\prime}$ & $\begin{array}{l}\text { Factor preexponencial del proceso de crecimiento } \\
\text { de grano, ec. } 2.63 \text {. }\end{array}$ & $\mathrm{m}^{3} / \mathrm{s}$ \\
\hline$\overline{\mathrm{k}}$ & Conductividad térmica media de la probeta. & $\mathrm{W} / \mathrm{m} \cdot \mathrm{K}$ \\
\hline $\mathrm{k}_{1}$ & $\begin{array}{l}\text { Constante de velocidad de la sinterización } \\
\text { asociada a la reordenación de partículas por flujo } \\
\text { viscoso, ec. 5.34. }\end{array}$ & $s^{-1}$ \\
\hline $\mathrm{k}_{2}$ & $\begin{array}{l}\text { Constante de velocidad de la sinterización } \\
\text { asociada a la solución-reprecipitación del circón, } \\
\text { ec. } 5.35 \text {. }\end{array}$ & $s^{-1}$ \\
\hline K & $\begin{array}{l}\text { Constante de velocidad de sinterización de orden } \\
1 \text { (ec. 2.8). }\end{array}$ & $s^{-1}$ \\
\hline $\mathrm{K}_{\mathrm{SL}}$ & $\begin{array}{l}\text { Constante de velocidad de crecimiento cristalino } \\
\text { (ec. 2.6). }\end{array}$ & $\mathrm{m}^{3} / \mathrm{s}$ \\
\hline $\mathrm{K}_{\mathrm{p}}$ & Viscosidad global de un material poroso. & $\mathrm{Pa} \cdot \mathrm{s}$ \\
\hline $\mathrm{K}_{\mathrm{pm}}$ & Viscosidad global de la matriz porosa (ec. 2.50). & $\mathrm{Pa} \cdot \mathrm{s}$ \\
\hline$K(T)$ & $\begin{array}{l}\text { Constante genérica de velocidad de sinterización } \\
\text { definida por la ec. } 2.44 \text {. }\end{array}$ & $s^{-1}$ \\
\hline $\mathrm{L}_{\mathrm{s}}$ & Longitud del cilindro del modelo de Scherer. & $\mathrm{m}$ \\
\hline M & Índice de uniformidad de Weibull, ec. 5.24. & - \\
\hline$M^{*}$ & Parámetro de composición del vidrio, ec. 5.28. & - \\
\hline $\mathrm{n}$ & Índice de potencia de Avrami & - \\
\hline $\mathrm{n}^{*}$ & Concentración numérica de poros. & $n^{\circ} / m^{3}$ \\
\hline $\mathrm{n}_{1}$ & $\begin{array}{l}\text { Índice de potencia de Avrami correspondiente a } \\
\text { la reordenación de partículas por flujo viscoso, } \\
\text { ec. } 5.30 \text { y ec. } 5.31 \text {. }\end{array}$ & - \\
\hline $\mathrm{n}_{2}$ & $\begin{array}{l}\text { Índice de potencia de Avrami correspondiente a } \\
\text { la solución-reprecipitación del circón, ec. } 5.32 \text {. }\end{array}$ & - \\
\hline$\frac{N_{g}}{N_{z}}$ & $\begin{array}{l}\text { Razón numérica de partículas de vidrio/partículas } \\
\text { de circón. }\end{array}$ & - \\
\hline $\mathrm{O}$ & Número de poros por partícula. & - \\
\hline$p$ & Constante geométrica (ec. 2.5). & - \\
\hline
\end{tabular}




\begin{tabular}{|c|c|c|}
\hline $\mathrm{P}_{\text {comp }}$ & Presión de compactación de la probeta. & $\mathrm{MPa}$ \\
\hline $\mathrm{P}_{\mathrm{g}}$ & $\begin{array}{l}\text { Presión de los gases ocluídos en los poros, ec. } \\
5.8 \text {. }\end{array}$ & $\mathrm{Pa}$ \\
\hline $\mathrm{P}_{\mathrm{i}}$ & $\begin{array}{l}\text { Probabilidad de ocurrencia de cada tipo de } \\
\text { clúster, ec. } 2.54 \text {. }\end{array}$ & - \\
\hline$P_{L}$ & $\begin{array}{l}\text { Presión efectiva de Laplace o presión de } \\
\text { sinterización. }\end{array}$ & $\mathrm{Pa}$ \\
\hline$q_{r}$ & $\begin{array}{l}\text { Densidad de flujo de calor en la dirección } r \\
\text { (Figura } 7.4 \text {, ec. A3. 2). }\end{array}$ & $\mathrm{W} / \mathrm{m}^{2}$ \\
\hline$q_{z}$ & $\begin{array}{l}\text { Densidad de flujo de calor en la dirección z } \\
\text { (Figura } 7.4 \text {, ec. A3. 2). }\end{array}$ & $\mathrm{W} / \mathrm{m}^{2}$ \\
\hline$Q_{C}$ & $\begin{array}{l}\text { Energía de activación aparente del crecimiento } \\
\text { de cristalito. }\end{array}$ & $\mathrm{KJ} / \mathrm{mol}$ \\
\hline$Q_{d}$ & $\begin{array}{l}\text { Energía de activación del proceso de } \\
\text { densificación del material, ec. } 2.61 \text {. }\end{array}$ & $\mathrm{KJ} / \mathrm{mol}$ \\
\hline$Q_{G}$ & $\begin{array}{l}\text { Energía de activación del proceso de crecimiento } \\
\text { de grano, ec. } 2.63 \text {. }\end{array}$ & $\mathrm{KJ} / \mathrm{mol}$ \\
\hline$Q_{v}$ & $\begin{array}{l}\text { Energía de activación correspondiente al flujo } \\
\text { viscoso, ec. } 2.59 \text {. }\end{array}$ & $\mathrm{KJ} / \mathrm{mol}$ \\
\hline$r_{c}$ & Radio capilar de intrusión. & $\mathrm{m}$ \\
\hline$r_{\mathrm{p}}$ & Radio de poro. & $\mathrm{m}$ \\
\hline$r_{\mathrm{p} 0}$ & Radio inicial de poro. & $\mathrm{m}$ \\
\hline$r_{0}$ & Radio inicial de la partícula. & $\mathrm{m}$ \\
\hline $\mathrm{R}$ & Constante universal de los gases ideales. & $\mathrm{KJ} / \mathrm{mol} \cdot \mathrm{K}$ \\
\hline $\mathrm{s}$ & Contracción lineal. & - \\
\hline $\mathrm{s}_{\mathrm{j}}$ & $\begin{array}{l}\text { Contracción lineal debida a la fracción de } \\
\text { partículas j }\end{array}$ & - \\
\hline $\mathrm{s}_{\mathrm{ij}}$ & Sinterización de cada tipo de clúster “i”, ec. 2.54. & - \\
\hline $\mathrm{S}_{\max }$ & Contracción lineal máxima. & - \\
\hline S & Solubilidad del soluto en el fundido. & $\mathrm{Kmol} / \mathrm{m}^{3}$ \\
\hline$S_{V}$ & Superficie interfacial del compacto. & $\mathrm{m}^{2}$ \\
\hline $\mathrm{t}$ & Tiempo. & $\mathrm{s}$ \\
\hline$t_{c}$ & Tiempo característico del vidrio, ec. 5.3. & $\mathrm{S}$ \\
\hline $\mathrm{t}_{\alpha, \mathrm{i}}$ & $\begin{array}{l}\text { Tiempo al que se alcanza un determinado grado } \\
\text { de sinterización, } \alpha \text {, a la temperatura, } \mathrm{T}_{\mathrm{i}} \text {, ec. } 2.76 \text {. }\end{array}$ & $\mathrm{s}$ \\
\hline
\end{tabular}




\begin{tabular}{|c|c|c|}
\hline $\mathrm{t}^{*}$ & Tiempo de permanencia en el interior del horno. & $\mathrm{s}$ \\
\hline $\mathrm{T}$ & Temperatura. & $\mathrm{K} /{ }^{\circ} \mathrm{C}$ \\
\hline $\mathrm{T}_{\mathrm{a}}$ & $\begin{array}{l}\text { Temperatura media de la cara aislada de la } \\
\text { probeta cilíndrica. }\end{array}$ & ${ }^{\circ} \mathrm{C}$ \\
\hline$T_{a, d}$ & $\begin{array}{l}\text { Temperatura media de la cara aislada de la } \\
\text { probeta cilíndrica densa. }\end{array}$ & ${ }^{\circ} \mathrm{C}$ \\
\hline$T_{a, p}$ & $\begin{array}{l}\text { Temperatura media de la cara aislada de la } \\
\text { probeta cilíndrica porosa. }\end{array}$ & ${ }^{\circ} \mathrm{C}$ \\
\hline $\mathrm{T}_{\mathrm{c}}^{\max }$ & $\begin{array}{l}\text { Temperatura de máxima densificación del } \\
\text { composite. }\end{array}$ & ${ }^{\circ} \mathrm{C}$ \\
\hline$T_{D}$ & $\begin{array}{l}\text { Temperatura de deformación, determinada por } \\
\text { microscopía de calefacción. }\end{array}$ & ${ }^{\circ} \mathrm{C}$ \\
\hline$T_{E}$ & $\begin{array}{l}\text { Temperatura de esfera, determinada por } \\
\text { microscopía de calefacción. }\end{array}$ & ${ }^{\circ} \mathrm{C}$ \\
\hline $\mathrm{T}_{\mathrm{F}}$ & $\begin{array}{l}\text { Temperatura de flujo, determinada por } \\
\text { microscopía de calefacción. }\end{array}$ & ${ }^{\circ} \mathrm{C}$ \\
\hline $\mathrm{T}_{\mathrm{FC}}$ & $\begin{array}{l}\text { Temperatura final de contracción, determinada } \\
\text { por microscopía de calefacción. }\end{array}$ & ${ }^{\circ} \mathrm{C}$ \\
\hline $\mathrm{T}_{\mathrm{g}}^{\max }$ & Temperatura de máxima densificación del vidrio. & ${ }^{\circ} \mathrm{C}$ \\
\hline $\mathrm{T}_{\mathrm{G}}$ & $\begin{array}{l}\text { Temperatura de transformación del vidrio, } \\
\text { determinada por dilatometría. }\end{array}$ & ${ }^{\circ} \mathrm{C}$ \\
\hline$T_{h}$ & Temperatura del horno. & ${ }^{\circ} \mathrm{C}$ \\
\hline $\mathrm{T}_{\mathrm{IC}}$ & $\begin{array}{l}\text { Temperatura de incio de contracción, } \\
\text { determinada por microscopía de calefacción. }\end{array}$ & ${ }^{\circ} \mathrm{C}$ \\
\hline $\mathrm{T}_{\mathrm{m}}$ & Temperatura promedio de la probeta cilíndrica. & ${ }^{\circ} \mathrm{C}$ \\
\hline $\mathrm{T}_{\mathrm{s}}$ & $\begin{array}{l}\text { Temperatura media de la cara no aislada de la } \\
\text { probeta cilíndrica. }\end{array}$ & ${ }^{\circ} \mathrm{C}$ \\
\hline$T_{s, d}$ & $\begin{array}{l}\text { Temperatura media de la cara no aislada de la } \\
\text { probeta cilíndrica densa. }\end{array}$ & ${ }^{\circ} \mathrm{C}$ \\
\hline $\mathrm{T}_{\mathrm{s}, \mathrm{p}}$ & $\begin{array}{l}\text { Temperatura media de la cara no aislada de la } \\
\text { probeta cilíndrica porosa. }\end{array}$ & ${ }^{\circ} \mathrm{C}$ \\
\hline $\mathrm{T}_{\alpha, \mathrm{i}}$ & $\begin{array}{l}\text { Temperatura a la que el grado de sinterización } \alpha \\
\text { ha sido alcanzado, empleando el programa i, ec. } \\
2.73 \text {. }\end{array}$ & ${ }^{\circ} \mathrm{C}$ \\
\hline $\mathrm{T}_{0}$ & $\begin{array}{l}\text { Parámetro de la ecuación de Vogel-Fulcher- } \\
\text { Tamman, ec. 5.1. }\end{array}$ & ${ }^{\circ} \mathrm{C}$ \\
\hline & Temperatura de semiesfera, determinada por & ${ }^{\circ} \mathrm{C}$ \\
\hline
\end{tabular}


microscopía de calefacción.

$v_{i}$

Fracción volumétrica de inclusiones en la pieza (se incluyen poros) definida por la ec. 2.51.

Volúmen de la probeta instantáneo.

Volumen de poros.

Volumen de sólido del compacto.

$\mathrm{m}^{3}$

Volúmen de la probeta inicial.

Contribución máxima al grado de avance de la sinterización debida a la reordenación de partículas por flujo viscoso, ec. 5.30 y ec. 5.31 .

Contribución máxima al grado de avance de la sinterización debida a la solución-reprecipitación del circón, ec. 5.32.

Fracción másica de circón.

Grado de avance de la sinterización.

Parámetro efectivo del material que incluye propiedades físicas y geométricas.

Contribución al grado de avance de la sinterización debida a la reordenación de partículas por flujo viscoso, ec. 5.30 y ec. 5.31.

Contribución al grado de avance de la sinterización debida a la solución-reprecipitación del circón, ec. 5.32.

Difusividad térmica media de la probeta.

Parámetro de relajación definido por la ec. 2.53.

Velocidad de calentamiento i dentro de una serie $\mathrm{K} / \mathrm{min}$ de experimentos, ec. 2.74 .

Ensanchamiento del pico de difracción definido ${ }^{\circ}$ por la ec. 4.4.

Grado de reducción de la contracción superficial máxima de un composite debido a la introducción de circón, ec. 5.17.

Grado de reducción de la compacidad máxima de un composite debdo a la introducción de circón, ec. 5.18 .

Emisividad

Porosidad abierta. 


\begin{tabular}{|c|c|c|}
\hline$\varepsilon_{\mathrm{A}, \max }$ & $\begin{array}{l}\text { Contracción superficial máxima de la silueta de la } \\
\text { probeta. }\end{array}$ & - \\
\hline$\varepsilon_{\mathrm{A}, \max }(0)$ & $\begin{array}{l}\text { Contracción máxima } \quad \text { de } \\
\text { corresponidente al vidrio, ec. } 5.17 \text {. }\end{array}$ & - \\
\hline$\varepsilon_{A}(25)$ & $\begin{array}{l}\text { Contración superficial de la silueta tomando como } \\
\text { valor inicial del área el correspondiente a } 25^{\circ} \mathrm{C} \text {. }\end{array}$ & - \\
\hline$\varepsilon_{A}=\ln \left(A / A_{0}\right)_{S}$ & Contracción superficial de la silueta de la probeta. & - \\
\hline$\varepsilon_{C}$ & Contracción lineal de un composite (ec. 2.50). & - \\
\hline$\varepsilon_{\mathrm{fm}}$ & $\begin{array}{l}\text { Contracción lineal de la matriz de un composite } \\
\text { (ec. 2.50). }\end{array}$ & - \\
\hline$\varepsilon_{R}$ & Contracción radial, ec. A1. 1. & - \\
\hline$\varepsilon_{\text {total }}$ & Porosidad total. & - \\
\hline$\varepsilon_{\mathrm{V}}$ & Contracción volumétrica de la probeta (ec. A1. 3). & - \\
\hline$\varepsilon_{\mathrm{z}}$ & Contracción axial, ec. A1. 1. & - \\
\hline$\phi$ & $\begin{array}{l}\text { Fracción volumétrica de inclusiones o cristales, } \\
\text { referido al sólido. }\end{array}$ & - \\
\hline$\phi_{\max }$ & $\begin{array}{l}\text { Fracción volumétrica máxima de inclusiones o } \\
\text { cristales, referido al sólido, ec. } 2.55 \text {. }\end{array}$ & - \\
\hline$\phi_{\mathrm{p}}$ & Fracción volumétrica de percolación. & - \\
\hline$\phi^{*}$ & $\begin{array}{l}\text { Razón entre la fracción volumética de circón y la } \\
\text { de percolación. }\end{array}$ & - \\
\hline$\gamma$ & Tensión superficial del vidrio. & $\mathrm{N} / \mathrm{m}$ \\
\hline$\gamma_{S L}$ & Tensión superficial sólido-líquido. & $\mathrm{N} / \mathrm{m}$ \\
\hline$\eta$ & Viscosidad del vidrio. & $\mathrm{Pa} \cdot \mathrm{s}$ \\
\hline$\eta_{c}$ & Viscosidad del composite, ec. 2.56. & $\mathrm{~Pa} \cdot \mathrm{s}$ \\
\hline$\eta_{\mathrm{c}}^{\infty}$ & $\begin{array}{l}\text { Viscosidad del composite exento de porosidad, } \\
\text { ec. } 2.55 \text {. }\end{array}$ & $\mathrm{Pa} \cdot \mathrm{s}$ \\
\hline$\eta_{\mathrm{fm}}$ & Viscosidad de la matriz vítrea porosa (ec. 2.50). & $\mathrm{Pa} \cdot \mathrm{s}$ \\
\hline$\eta_{p}$ & Viscosidad de un vidrio poroso. & $\mathrm{Pa} \cdot \mathrm{s}$ \\
\hline$\eta_{\mathrm{r}}$ & $\begin{array}{l}\text { Viscosidad relativa: razón entre la viscosidad del } \\
\text { composite y la viscosidad del vidrio, ec. } 5.60 \text {. }\end{array}$ & - \\
\hline$\eta^{*}$ & $\begin{array}{l}\text { Viscosidad del vidrio a la que el composite } \\
\text { alcanza la máxima densificación. }\end{array}$ & $\mathrm{Pa} \cdot \mathrm{s}$ \\
\hline$\eta_{0}$ & $\begin{array}{l}\text { Factor preexponencial de flujo viscoso del vidrio } \\
\text { de la ec. 5.2. }\end{array}$ & $\mathrm{Pa} \cdot \mathrm{s}$ \\
\hline
\end{tabular}


$\lambda$

$\lambda_{\mathrm{g}}$

$\lambda_{\text {radiación }}$

$\lambda_{0}$

$\rho$

$\rho_{\mathrm{ap}}$

$\rho_{\text {circón }}$

$\rho_{\max }$

$\rho_{\max }(0)$

$\rho_{\text {real }}$

$\rho_{\text {vidrio }}$

$\rho_{0}$

$\bar{\rho}$

$\sigma$

$\tau$

$\tau_{0}$

$\Omega$

$\Psi$

$\Psi_{\mathrm{j}}\left(r_{0 \mathrm{j}}\right)$

$\wp_{L}$

Angúlo de contacto.

Parámetro de ajuste de la ec. 5.37.

Constante que depende de las condiciones de medida de DRX.

Constante de velocidad adimensional del composite.

Constante de velocidad adimensional del vidrio.

Longitud de onda de la radiación $k \alpha$.

$\mathrm{m}$

Radio efectivo de partícula.

$\mathrm{m}$

Compacidad o densidad relativa de la probeta.

Densidad aparente.

$\mathrm{kg} / \mathrm{m}^{3}$

Densidad real del circón.

$\mathrm{kg} / \mathrm{m}^{3}$

Compacidad o densidad relativa máxima de la probeta.

Compacidad máxima de compactos de vidrio, ec. 5.18.

Densidad real del sólido.

$\mathrm{kg} / \mathrm{m}^{3}$

Densidad real del vidrio.

$\mathrm{kg} / \mathrm{m}^{3}$

Compacidad o densidad relativa inicial de la probeta.

Densidad aparente media de la probeta. $\quad \mathrm{kg} / \mathrm{m}^{3}$

Constante de Stefan-Boltzman W/ $\quad$ $/ \mathrm{m}^{2} \mathrm{~K}^{4}$

Constante de tiempo definida en la ec. 2.52. S

Parámetro de ajuste entre la constante de $\mathrm{s}$ tiempo, $\tau$, y la temperatura, T, ec. 2.59.

Volumen molar del soluto .

$\mathrm{m}^{3} / \mathrm{kmol}$

Módulo viscoso adimensional definido por la ec. 2.42 .

Frecuencia relativa, en peso, de partículas de tamaño $r_{0 j}$.

Presión de Laplace o de sinterización adimensional definida por la ec. 2.34.

Ángulo de difracción. 
Presión de sinterización según Wakai, ec. 5.7. $\quad$ Pa

$\left(\frac{d_{g}^{v}}{d_{z}^{v}}\right)_{C r}$

Razón crítica entre el diámetro medio volumétrico del vidrio y el del circón, ec. 5.41.

$\left(\frac{N_{g}}{N_{z}}\right)_{\text {Cr }}$

Razón numérica crítica de partículas de vidrio/partículas de circón, ec. 5.41.

$\left(P_{L}\right)_{\text {cerrado }}$

Presión de sinterización correspondiente a un $\mathrm{Pa}$ poro cerrado, ec. 5.8.

$\left(\frac{\Delta \rho}{\Delta \rho_{\max }}\right)^{\lambda^{*}}$

Fracción de la superficie de las inclusiones mojada por el fundido, ec. 2.56 .

$(4 \mathrm{~V} / \mathrm{A})$

Diámetro medio de poro determinado por $\mathrm{m}$ intrusión de mercurio. 


\section{Bibliografía}

[1] R. Müller and S. Reinsch (2012). Ceramics and Composites Processing Methods. Chater 3. Viscous-phase silicate processing. Ed. Narottam P. Bansal and Aldo R. Boccaccini, pp. 75-144. Wiley.

[2] S. Kemethmüller, M. Hagymasi, A. Stiegelschmitt, A. Roosen (2007). Viscous flow as the driving forcé for the densification of low-temperature cofired ceramics. Journal of the American Ceramic Society. 90[1] 64-70.

[3] P. F. James (1995). Glass-ceramics-New compositions and uses. Journal of non-crystalline solids. 181[1-2] 1-15.

[4] K. M. Prewo (1989). Fibre reinforced glasses and glass-ceramics, in Glasses and glass-ceramics. Ed. M. H. Lewis, pp. 336-368. Chapman and Hall, London.

[5] W. D. Kingery (1959). Densification during sintering in the presence of a liquid phase. 1. Theory. Journal of Applied Physics. 30[3] 301-306.

[6] R. M. German (1985). Liquid phase sintering. Plenum Press, New York.

[7] J. Frenkel (1945). Viscous flow of crystalline bodies under the action of surface tension. Journal of Physics (Moscow). 9 796-813.

[8] J. K. Mackenzie, R. Shuttleworth (1949). A phenomenological theory of sintering. Proceedings of the Phisical Society London. 62 833-852.

[9] M. J. Pascual, A. Duran (2003). Sintering with concurrent crystallisation of a borosilicate glass. Physics and Chemistry of Glasses. 44[6] 409-415.

[10] I. B. Cutler and R. E. Henrichsen (1968). Effect of particle shape on the kinetics of sintering of glass. Journal of the American Ceramic Society. 51[10] 604-605.

[11] M. O. Prado, C. Fredericci, E. D. Zanotto (2002). Glass sintering with concurrent crystallisation. Part 2. Nonisothermal sintering of jagged polydispersed particles. Physics and Chemistry of Glasses. 43[5] 215-223. 
[12] M. O. Prado and E. D. Zanotto (2002). Glass sintering with concurrent crytallisation. Comptes Rendus Chimie. 5[11] 773-786.

[13] R. Müller (1994). On the kinetics of sintering and crystallization of glass powders. Glastechnische Berichte-Glass Science and Technology. 67C 9398.

[14] M. O. Prado and E. D. Zanotto (2001). Model for sintering polydispersed glass particles. Journal of Non-Crystalline Solids. 279[2-3] 169-178.

[15] H. E. Exner and E. A. Giess (1990). A stereology based equation for isotropic shrinkage during sintering by viscous flow. In Proceedings of the Seventh World Round Table Conference of Sintering. Ed. D. P. Uskokvic, pp. 73-82, Herceg-Novi, Yugoslavia.

[16] A. G. Evans (1982). Considerations of inhomogeneity effects in sintering. Journal of the American Ceramic Society. 65[10] 497-501.

[17] G. W. Scherer (1984). Viscous sintering of a bimodal pore-size distribution. Journal of the American Ceramic Society. 67[11] 709-715.

[18] H. E. Exner and E. A. Giess (1988). Anisotropic shrinkage of cordieritetype glass poder cylindrical compacts. Journal of Materials Research. 3[1] 122-125.

[19] E. A. Giess, J. P. Fletcher, L. W. Herron (1984). Isothermal sintering of cordierite-type glass powders. Journal of the American Ceramic Society. 67[8] 549-552.

[20] A. R. Boccaccini (1993). Anisotropic densification of glass poder compacts. Journal of the European Ceramic Society. 32[1] 27-30.

[21] A. R. Boccaccini and G. Ondracek (1992). Viscous sintering of nonspherical borosilicate glass poder. Glastechnische Berichte-Glass Science and Technology. 65[3] 73-78. 
[22] A. R. Boccaccini and E. A. Olevsky (1999). Processing of plateletreinforced glass matrix composites: Effect of inclusions on sintering anisotropy. Journal of Materials Processing Technology. 96[1-3] 92-101.

[23] A. R. Boccaccini and R. Conradt (2001). Isotropic shrinkage of platelet containing glass poder compacts during isothermal sintering. International Journal of Inorganic Materials. 3[2] 101-106.

[24] A. R. Boccaccini (1993). Anisotropic densification during sintering of glass poder compacts. Journal of Materials Science Letters. 12[12] 943-945.

[25] A. R. Boccaccini (1998). Shrinkage anisotropy of glass poder compacts sintered in dilatometers. Journal of Materials Research. 13[6] 1693-1697.

[26] A. R. Boccaccini, B. Hamann (1999). In situ high-temperature optical microscopy. Journal of Materials Science. 34[22] 5419-5436.

[27] M. Wagner, A. Roosen, A. Stiegelschmitt, D. Schwanke and F. Bechtholt (2002). In situ shrinkage measurements of LTCC multilayers by means of an dilatometer. Key Enginering Materials. 206-213 1281-1284.

[28] F. Raether, R. Springer and S. Beyer (2001). Optical dilatometry for the control of microstructure development during sintering. Materials Research Innovations. 4[4] 245-250.

[29] G. Besendörfer and A. Roosen (2008). Particle shape and size effects on anisotropic shrinkage in tape-cast ceramic layers. Journal of the American Ceramic Society. 91[8] 2514-2520.

[30] R. Müller, M. Eberstein, S. Reinsch, W. A. Schiller, J. Deubener, A. Thiel (2007). Effect of rigid inclusions on sintering of low temperatura co-fired ceramics. Physics and Chemistry of Glasses - European Journal of Glass Science and Technology Part B. 48[4] 259-266.

[31] M. Eberstein, S. Reinsch, R. Müller, J. Deubener, W. A. Schiller (2009). Sintering of glass matrix composites with small rigid inclusions. Journal of the European Ceramic Society. 29[12] 2469-2479. 
[32] M. Eberstein, R. Müller, S. Reinsch, T. Rabe, W. A. Schiller, A. Thiel, J. Deubener (2007). Kinetic modelling of LTCC shrinkage: Effect of alumina content. Journal of Microelectronics and Electronic Packaging. 4[4] 173-180.

[33] A. R. Boccaccini (1994). Sintering of glass matrix composites containing Al2O3 platelet inclusions. Journal of Materials Science. 29[16] 4273-4278.

[34] R. E. Dutton and M. N. Rahaman (1992). Sintering, creep, and electricalconuctivity of model glass-matrix composites. Journal of the American Ceramic Society. 75[8] 2146-2154.

[35] M. J. Pascual, A. Duran, L. Pascual (2002). Sintering behaviour of composite materials borosilicate glass-ZrO2 fibre composite materials. Journal of the European Ceramic Society. 22 1513-1524.

[36] A. G. Evans (1982). Considerations of inhomogeneity effects in sintering. Journal of the American Ceramic Society. 65, 497-501.

[37] R. Raj, R. K. Bordia (1984). Sintering behaviour of bimodal com- pacts. Acta Metall. 32, 1003-1019.

[38] C. H. Hsueh, A. G. Evans, R. M. Cannon, R. J. Brook (1986). Viscoelastic stresses and sintering damage in heterogeneous pow- der compacts. Acta Metall. 34, 927-936.

[39] R. Müller, R. Meszaros, B. Peplinski, S. Reinsch, M. Eberstein, W. A. Schiller, J. Deubener (2009). Dissolution of alumina, sintering, and crystallization in glass ceramic composites for LTCC. Journal of the American Ceramic Society. 92[8] 1703-1708.

[40] H. Riegger, J. A. Pask, H. E. Exner (1980). In: Kuczynski GC (ed) Sintering processes. Plenum Press, New York, 219-233.

[41] R. Warren, M. B. Waldron (1972). Microstructural development during the liquid phase sintering of cemented carbides. Powder Metall. 15 166-201. 
[42] R. K. Bordia, G. W. Scherer (1988). On constrained sintering. 3. Rigid Inclusions. Acta Metallurgica. 36[9] 2411-2416.

[43] J. N. Goodier (1936). LIII. Slow viscous flow and elastic deformation. Philosophical Magazine Series. 7 22[148] 678-681.

[44] E. A. Giess, J. P. Fletcher, L. W. Herron (1984). Isothermal sintering of cordierite-type glass powders. Journal of the American Ceramic Society. 67 549-552.

[45] E. A. Giess, C. F. Guerci, G. F. Walker, S. H. Wen (1985). Isothermal sintering os spheroidized cordierite-type glass powders. Journal of the American Ceramic Society. 68 C328-C329.

[46] R. K. Bordia, R. Raj (1986). Analysis of sintering of a composite with a glass or ceramic matrix. Journal of the American Ceramic Society. 69 C55C57.

[47] H. E. Exner, E. A. Giess (1989). A stereology-based equation for isotropic shrinkage during sintering by viscous flow. Proc. Round Table on Sintering. Herzeg-Novi. 73-79.

[48] A. R. Boccaccini. Anisotropic densification during sintering of glass poder compacts. Journal of Materials Science Letters. 1212 943-945.

[49] A. Boccaccini, J. Bossert (1994). Der einsatz des leitzErhitzungsmikroskops zur untersuchung des sinterverhaltens von pulverpresslingen. Mittelilungen für Wissenschaft und Technik Bd. X Nr. 8 S. 274-282 December.

[50] M. O. Prado, E. D. Zanotto, R. Müller (2001). Model for sintering polydispersed glass particles. Journal of Non-Crystalline Solids. 279 169178.

[51] G. W. Scherer (1977). Sintering of low-density glasses: I, theory. Journal of the American Ceramic Society. 60 236-239. 
[52] G. W. Scherer, D. L. Bachman (1977). Sintering of low-density glasses: II, Experimental study. Journal of the American Ceramic Society. 60 239243.

[53] G. W. Scherer (1977). Sintering of low-density glasses: III, Effect of a distribution of pore sizes. Journal of the American Ceramic Society. $60243-$ 246.

[54] J. Phalippou, T. Woignier, J Zarzycki (1984). Behaviour of monolitic silica aerogels at temperaturas above $1000^{\circ} \mathrm{C}$. In: Chapter 7. Ultrastructure processing of ceramics, glasses and composites. Eds. LL Hench and DR Ulrich (Wiley, New York).

[55] P. Reijnen, A. C. Firalti, H. Goerg, R. Arslan. The mechanisms of pore growth during vitrification. Ceram. Forum Int./Ver. DKG. 61 9/10 441-445.

[56] W. Kingery, H. Bowen, D. UhIman (1976). Introduction to ceramics. Wyley, New York.

[57] E. A. Olevsky (1998). Theory of sintering: from discrete to continuum. Mater. Sci. Eng. R23 41-100.

[58] V. V. Shorohod (1972). Rehological basis of the theory of sintering. Naukova Dumka. Kiev.

[59] V. C. Ducamp, R. Raj (1989). Shear and densification of glass poder compacts. Journal of the American Ceramic Society. 72[5] 789-804.

[60] G. W. Scherer (1979). Sintering inhomogeneous glasses: application to optical waveguides. J. Non.-Cryst. Solids. 34 239-256.

[61] J. K. Mackenzie (1950). Elastic constants of a solid containing spherical holes. Proc. Phys. Soc. B. 63 2-11.

[62] M. N. Rahaman, L. C. Dejonghe, G. W. Scherer, R. J. Brook (1987). Creep and densification during sintering of glass powder compacts. Journal of the American Ceramic Society. 70 766-774. 
[63] V. M. Sura, P. C. Panda (1990). Viscosity of porous glasses. Journal of American Ceramic Society. 73 2697-2701.

[64] I. Gustoz, S. Todorova, N. Jordanov (2010). Kinetics of chemical reactions and phase transitions at changing temperatura: general reconsiderations and a new approach. Bull. Chem. Commun. 42[2] 79-102.

[65] M. J. Orts, J. L. Amorós, A. Escardino, A. Gozalbo, C. Feliu (1993). Kinetic model for isothermal sintering of low porosity floor tiles. Appl. Clay Sci. 8[2/3] 231-245.

[66] J. L. Amorós, M. P. Gómez-Tena, A. Moreno, E. Blasco, E. Zumaquero, M. Galindo (2013). Dissolution, crystallization and sintering of raw matt glaze for porcelain tile. Adv. Mates. Res. 704 132-140.

[67] J. L. Amorós, M. P. Gómez-Tena, A. Moreno, E. Blasco, S. Cook, M. Galindo (2014). Formulation of porcelain tile glazes using a new boron raw material. Study of the physicochemical transformations that develop in firing. In: Procceding of the Qualicer 2014, Castellón, 17-18 February 2014.

[68] J. L. Amorós (2014). Towards rational design of porcelain tile glazes. Adv. Sci. Technol. 92 138-147.

[69] J. L. Amorós, E. Blasco, A. Moreno, M. P. Gómez-Tena (2016). Sintering of raw glazes for floor and porcelain tiles: A non-isothermal kinetic model. Ceramic International. 42 16169-16179.

[70] M. P. Gómez-Tena (2013). Esmaltes para baldosas cerámicas preparados con una nueva material prima borácica. Transformaciones físicoquímicas que se desarrollan durante la cocción. [Tesis doctoral]. Castellón: Universitat Jaume I, Departamento de Ingeniería Química.

[71] G. W. Scherer (1987). Sintering with rigid inclusions. Journal of the American Ceramic Society. 70[10] 719-725.

[72] G. W. Scherer (1988). Viscous sintering with a pore size distribution and rigid inclusions. Journal of the American Ceramic Society. 71 447-448. 
[73] A. Winkel, R. Meszaros, S. Reinsel, R. Müller, N. Travitzky, T. Fei, P. Greil, L. Wondraczev (2012). Sintering of 3D printed glass/HAp composites. J. Am. Ceram. Soc. 95[11] 3387-3393.

[74] K. R. Mikeska, G. W. Scherer, R. K. Bordia (1990). Constitutive parameters of sintering materials. Ceram. Trans. 7 200-214.

[75] S. J. Park, S. H. Chung, J. M. Martin, J. L. Johson, R. M. German (2008). Master sintering curve for densification derived from a constitutive equation with consideration of grain growth: Application to tungsten heavy alloys. Metallurgical and Materials Transactions A. 39A 2941-2948.

[76] S. Vyazowkin (2008). The handbook of termal analysis \& calorimetry. In: M. E. Brown, P. K. Gallagher (Eds.). Recent Advances, Techniques and Applications. 5503.

[77] T. Akahira, T. Sunose (1971). Method of determining activation deterioration constant of electrical insulating materials. Res. Report Chiba Inst. Technol. (Sci. Technol). 16 22-31.

[78] C. D. Doyle (1962). Estimating isothermal life from thermogravimetry data (1962). J. Appl. Polym. Sci. 6639.

[79] T. Ozawa (1965). A new method of analyzing thermogravimetry data. Bull. Chem. Soc. Japan. 381881.

[80] J. H. Flynn, L. A. Wall (1966). General treatment of the thermogravimetry of polymers. J. Res. Nat. Bur. Standards. Part A 70487.

[81] M. J. Starink (2003). The determination of activation energy from lineal heating rate experiments: a comparation of the accuracy of isoconversion methods. Thermochim. Acta 404 163-176.

[82] S. Vyazowkin, N. Sbirrazzuoli (1964). Isoconversional kinetic analysis of thermally stimulated processes in polymers. Macromol. Rapid Commun. 27 1515-1532. 
[83] S. Vyazowkin (2001). Modification of the integral isoconversional method to account for variation in the activation energy. J. Comput. Chem. 22 178183.

[84] H. L. Friedman (1964). Kinetics of thermal degradation of char-forming plastics from thermogravimetry. Application a phenolic plastic. J. Polym. Sci. Part C 6, 183-195.

[85] A. R. Boccaccini, Q. Z. Chen, L. Lefebvre, L. Gremillard, J. Chevalier (2007). Sintering, crystallization a biodegradation behavior of Bioglassderived glass-ceramics. Faraday Dis 136 27-44.

[86] M. O. Prado, C. Fredericci, E. D. Zanotto (2003). Non-isothermal sintering with concurrent crystallization of polydispersed soda-lime-silica glass beads. J. Non-Cryst. Sol. 331 (1-3) 157-167.

[87] S. Vyazowkin, I. Dranca (2006). Isoconversional analysis of combined melt and glass crystallization data. Macromol. Chem. Phys. 207 20-25.

[88] C. Alzina, N. Sbirrazzuoli, A. Mija (2010). Hybrid nanocomposites: advanced nonlinear method for calculating key kinetics parameters of complex cure kinetics. J. Phys. Chem. B 114 12480-12487.

[89] H. E. Kissinger (1957). Reaction kinetics in differential therma analysis. Anal. Chem. 29 1702-1706.

[90] T. J. W. De Bruijn, W. A. De Jong, P. J. Van Den Berg (1981). Kinetic parameters in Avrami-Erofeev type reactions fro isothermal and nonisothermal experiments. Thermochim. Acta 453 315-325.

[91] D. Chen, X. Gao, D. Dollimore (1993). A generalized form of the Kissinger equations. Thermochim. Acta 215 109-117.

[92] R. L. Braun, A. K. Kurnham (1987). Analysis of chemical reaction kinetics using a distribution of activation energies and simple models. Energy Fuels. 1 153-161. 
[93] J. M. Criado, A. Ortega (1986). A non-isothermal transformation kinetics: remarks on the Kissinger method. J. Non-Crys. Solids. 87 302-311.

[94] N. Sbirrazzuoli, Y. Girault, L. Elégant (1997). Simulations for evaluating of kinetic methods in differential scanning calorimetry. Part 3: Peak máximum evolution methods and isoconversional methods. Thermochim. Acta 29325 37.

[95] J. M. Criado, A. Ortega (1987). Non-isothermal crystallization kinetics of metal glasses: simultaneous determination of both, the activation energy and the exponent $n$ of the JMA kinetic law. Acta Metall. 35 1715-1721.

[96] P. Budrugeac, E. Segal (2007). Applicability of the Kissinger equation in thermal analysis: revisited. J. Therm. Anal. Calorim. 88 703-703.

[97] S. Vyazovkin, C. A. Wight (1999). Model-free and model fitting approaches to kinetic analysis of isothermal and non-isothermal data. Thermochim. Acta. 304-341, 53-68.

[98] M. E. Brown, M. Maciejewski, S. Vyazovkin, R. Nomen, J. Sempere, A. Burnham, J. Opfermann, R. Strey, H. L. Anderson, A. Kemmler, R. Keuleers, J. Janssens, H. O. Desseyn, C. R. Li, T. B. Tang, B. Roduit, J. Malek, T. Mitsuhashi (2000). Computational aspects of kinetics analysis. Part A: The ICTAC kinetics proyec: data, methods and results. Thermochim. Acta 355 125-143.

[99] F. M. Bertan, O. R. K. Montedo, C. R. Rambo, D. Hotza, A. P. Novaes de Oliveira (2009). Extruded $\mathrm{ZrSiO} 4$ particulated reinforced LZSA glassceramics matrix composite. J. Mater. Process. Technol. 209 1134-1142.

[100] Proyecto público (2016-2017): Desarrollo de materiales avanzados y de bajo impacto ambiental. Financiado por el Institut Valencià de Competitivitat Empresarial (IVACE), a través del Programa de Actividades para la Mejora de la Competitividad (PROMECE). 
[101] National Institute of Standards and Technology. Certificate Standard Reference Material 717a Borosilicate Glass.

[102] National Institute of Standards and Technology. Certificate Standard Reference Material 731 Borosilicate Glass-Thermal Expansion.

[103] M. J. Pascual, L. Pascual, A. Duran (2001). Determination of the Viscosity-Temperature curve for glasses on the bain of fixed viscosity points determined by hot stage microscopy. Phys. Chem. Glasses. 42[1], 61-66.

[104] M. Ahmed and D. A. Earl (2002). Characterizing glaze-melting behavior via HSM. Amer. Ceram. Soc. Bull. 81[3].

[105] M. J. Pascual, A. Duran, M. O. Prado (2005). A new method for determining fixed viscosity points of glasses. Phys. Chem. Glasses. 46[5] 512-520.

[106] F. Montanari, P. Miselli, C. Leonelli (2014). Calibration and use of the heating microscope for indirect evaluation of the viscosity and meltability of archeological glasses. International Journal of Applied Glass Science. 5[2] 161-177.

[107] R. A. Young (1996). The Rietveld method. Oxford: University Press.

[108] V. Esteve (2006). El método Rietveld. Castellón: UJI.

[109] S. F. Baktram (1967). Crystallite-size determination from line broadening and potty patterns. KAELBLE, E.F. Handbook of X-Rays. New York: McGraw-Hill. 17.1-17.8.

[110] H. Scholze (1962). Influence of viscosity and surface tension on hotstage microscopy measurements on glasses. Ver. Dtsch. Keram. Ges. 391 63-68.

[111] J. M. Fernández-Navarro (1991). El vidrio. Madrid. CSIC. 
[112] A. Van de Vorst (1998). Numerical simulation of viscous sintering by a periodic lattice of a representative unit cell. J. Am. Ceram. Soc. 81[8] 21472156.

[113] M. J. Orts, A. Escardino. J. L. Amorós, F. Negre (1993). Microstructural changes during the firing of stoneware floor tiles. Applied Clay Scince. 8 193-205.

[114] F. Wakai (2013). Mechanics of viscous sintering of the micro- and macro-scale. Acta Materialia. 61 239-247.

[115] J. L. Amorós, A. Escardino, V. Beltrán, J. E. Enrique (1984). Quality control in tile production. Interceram. $33250-54$.

[116] V. Began (1991). Efecto de las condiciones de operación en las diferentes etapas del proceso sobre las propiedades y características de un pavimento de muy baja porosidad. [Tesis doctoral]. Castellón: Universitat Jaume I, Departamento de Ingeniería Química.

[117] J. J. Jean, T. K. Gupta (1992). Densification kinetics of the initial stage of liquid phase sintering: glass-cordierite system. J. Mater. Sci. Lett. 11656.

[118] S. Kemethmüller, M. Hagymasi, A. Stiegelschmitt, A. Roosen (2007). Viscous flow as the driving force for the densification of low-temperature cofired ceramics. Journal of the American Ceramic Society. 90[1] 63-70.

[119] A. Escardino, J. L. Amorós, A. Gozalbo, M. J. Orts, J. Aparisi, F. Ferrando, L. Sánchez (2002). Porosidad superficial de vidriados pulidos: Influencia de algunas variables. In: Procceding of the Qualicer 2002, Castellón (España). 313-328.

[120] J. L. Amorós, M. J. Orts, J. Garcia-Ten, E. Sánchez (2007). Effect of the green porous texture on porcelain tile properties. Journal of the European Ceramic Society. 27[5] 2295-2301.

[121] J. Liu, R. M. German (1999). Densification and shape distortion in liquid phase sintering. Metall. Mater. Trans. A. 30A 3211-3217. 
[122] C. H. Binet, K. L. Lencoski, D. F. Heaney, R. M. German (2004). Modeling of distortion after densification during liquid-phase sintering. Metall. Mater. Trans. A. 35A, 3833-3841.

[123] R. M. German (2006). Rheological model for viscous flow densification during supersolid liquid phase sintering. Science of Sintering. 38 27-40.

[124] R. M. German (2014). Sintering from empirical observations to scientific principles. Elsevier. 115-119.

[125] P. Boehnke, E. B. Watson, D. Trail, T. M. Harrison, A. K. Schmitt (2013). Zircon saturation re-revised. Chemical Geology. 351 324-334.

[126] D. R. Baker, A. M. Conte, C. Freda, L. Ottolini (2002). The effect of halogens on $\mathrm{Zr}$ diffusion and zircón dissolution in hidrous metaluminous granitic melts. Contrib. Mineral Petrol. 142 666-678.

[127] T. M. Harrison, E. B. Watson (1983). Kinetics of zircon dissolution and zirconium diffussion in granitic melts of variable water content. Contrib. Mineral Petrol. 84 66-72.

[128] M. N. Rahaman, L. C. De Jongue (1987). Effect of rigid inclusions on sintering of glass powder compacts. J. Am. Ceram. Soc. 70[12] C348-C351.

[129] R. F. Dutton, M. N. Rahaman (1992). Sintering, creep and electrical conductivity of model glass-matrix composites. J. Am. Ceram. Soc. 75[8] 2146-2157.

[130] A. Jagota, G. W. Scherer (1993). Viscosities and sintering rates of a two-dimensional granular composite. J. Am. Ceram. Soc. 76[12] 3123-3135.

[131] A. Jagota, G. W. Scherer (1993). Viscosities and sintering rates of composite packings of spheres. J. Am. Ceram. Soc. 78[3] 521-528.

[132] Z. Yan, C. L. Martin, O. Guillon, D. Bouvard (2013). Effect of size and homogeneity of rigid inclusions on the sintering of composites. Scripta Materialia. 69 327-330. 
[133] M. J. Pascual, A. Duran, M. O. Prado, E. D. Zanotto (2005). Model for sintering devitrifying glass particles with embedded rigid fibers. J. Am. Ceram. Soc. 88[6] 1427-1434.

[134] R. Ben Aim, P. Le Goff (1968). Effet de paroi dans les empilements désordonnés de sphères et application à la porosité de mélanges binaires. Powder Technology. 15 281-290.

[135] Da He, N. N. Ekere (2004). Effect of particle size ratio on the conducting percolation threshold of granular conductive-insulating composites. J. Phys. D: Appl. Phys. 37[13] 1848-1852.

[136] D. Bouvard, F. F. Lange (1991). Relation between percolation and particle coordination in binary powder mixtures. Acta metall. Mater. 3912 3083-3090.

[137] D. Chen, Z. Lin, H. Zhu, R. J. Kee (2009). Percolation theory to predict effective properties of solid oxide fuel-cell composite electrodes. Journal of Power Sources. 191 240-252.

[138] Z. Yan, C. L. Martin, O. Guillon, D. Bouvard (2013). Effect of size and homogeneity of rigid inclusions on sintering of composites. Scripta Materialia. $69227-230$.

[139] J. Liu, R. M. German (1999). Densification and shape distortion in liquid-phase sintering. Metall. Mater. Trans. A, 30A 3211-3217.

[140] C. H. Binet, K. L. Lencoski, D. F. Heaney, R. M. German (2004). Modeling of distortion after densification during liquid-phase sintering. Metall. Mater. Trans. A, 35 3833-3841.

[141] I M. Krieger, T. J. Dougherty (1959). A mechanism for non-Newtonian flow in suspensions of rigid spheres. Trans. Soc. Rheol. 3 137-152.

[142] R. Champallier, M. Bystricky, L. Arbaret (2008). Experimental investigation of magma rheology at $300 \mathrm{MPa}$ : From pure hydrous melt to 76 vol.\% of crystals. Earth Planet Sci. Lett. 267(3-4): 13. 
[143] V. Cantavella Soler (1998). Simulación de la deformación de baldosas cerámicas durante la cocción. [Tesis doctoral]. Castellón: Universitat Jaume I, Departamento de Ingeniería Química. 